NATIONAL LABORATORY

MANAGED BY UT-BATTELLE

FOR THE DEPARTMENT OF ENERGY

\title{
Powertrain Component Inspection from Mid-Level Blends Vehicle Aging Study
}

\section{November 2010}

\author{
Prepared by \\ Brent A. Shoffner \\ Ryan D. Johnson \\ Martin J. Heimrich \\ Michael D. Lochte
}

Southwest Research Institute
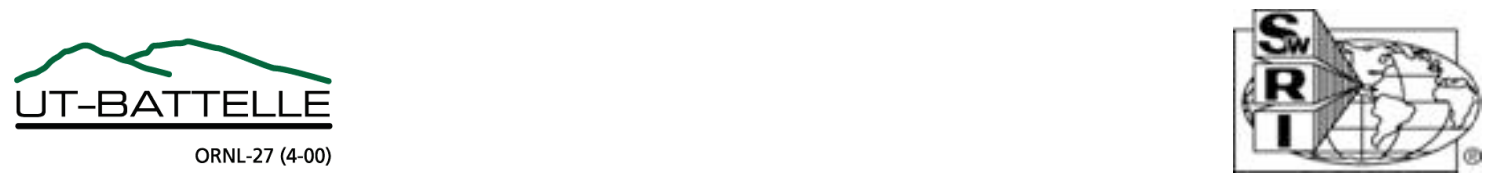


\title{
DOCUMENT AVAILABILITY
}

Reports produced after January 1, 1996, are generally available free via the U.S. Department of Energy (DOE) Information Bridge.

Website http://www.osti.gov/bridge

Reports produced before January 1, 1996, may be purchased by members of the public from the following source.

\author{
National Technical Information Service \\ 5285 Port Royal Road \\ Springfield, VA 22161 \\ Telephone 703-605-6000 (1-800-553-6847) \\ TDD 703-487-4639 \\ Fax 703-605-6900 \\ E-mail info@ntis.gov \\ Website http://www.ntis.gov/support/ordernowabout.htm
}

Reports are available to DOE employees, DOE contractors, Energy Technology Data Exchange (ETDE) representatives, and International Nuclear Information System (INIS) representatives from the following source.

Office of Scientific and Technical Information

P.O. Box 62

Oak Ridge, TN 37831

Telephone 865-576-8401

Fax 865-576-5728

E-mail reports@osti.gov

Website http://www.osti.gov/contact.html

This report was prepared as an account of work sponsored by an agency of the United States Government. Neither the United States Government nor any agency thereof, nor any of their employees, makes any warranty, express or implied, or assumes any legal liability or responsibility for the accuracy, completeness, or usefulness of any information, apparatus, product, or process disclosed, or represents that its use would not infringe privately owned rights. Reference herein to any specific commercial product, process, or service by trade name, trademark, manufacturer, or otherwise, does not necessarily constitute or imply its endorsement, recommendation, or favoring by the United States Government or any agency thereof. The views and opinions of authors expressed herein do not necessarily state or reflect those of the United States Government or any agency thereof. 


\title{
POWERTRAIN COMPONENT INSPECTION FROM MID-LEVEL BLENDS VEHICLE AGING STUDY
}

\author{
Brent A. Shoffner \\ Ryan D. Johnson \\ Martin J. Heimrich \\ Michael D. Lochte \\ Southwest Research Institute
}

Date Published: November 2010

\author{
Prepared by \\ SOUTHWEST RESEARCH INSTITUTE \\ for \\ OAK RIDGE NATIONAL LABORATORY \\ Oak Ridge, Tennessee 37831-6283 \\ managed by \\ UT-BATTELLE, LLC \\ for the \\ U.S. DEPARTMENT OF ENERGY \\ under contract DE-AC05-00OR2272
}





\section{FOREWORD}

The Energy Independence and Security Act of 2007 calls on the nation to significantly increase its use of renewable fuels to meet its transportation energy needs. The law expands the renewable fuel standard to require use of 36 billion gallons of renewable fuel by 2022. Given that ethanol is the most widely used renewable fuel in the U.S. market, ethanol will likely make up a significant portion of the 36-billiongallon requirement.

The vast majority of ethanol used in the United States is blended with gasoline to create E10 - gasoline with up to $10 \%$ ethanol. The remaining ethanol is sold in the form of E85-a gasoline blend with as much as $85 \%$ ethanol that can only be used in flexible-fuel vehicles (FFVs). Consumption of E85 is at present limited by both the size of the FFV fleet and the number of E85 fueling stations.

Gasoline consumption in the United States is currently about 140 billion gallons per year; thus the maximum use of ethanol as E10 is only about 14 billion gallons. While the U.S. Department of Energy (DOE) remains committed to expanding the E85 infrastructure, that market represented less than $1 \%$ of the ethanol consumed in 2010 and will not be able to absorb projected volumes of ethanol in the near term. Because of these factors, DOE and others have been assessing the viability of using mid-level ethanol blends (E15 or E20) as a way to accommodate growing volumes of ethanol. The DOE Mid-Level Ethanol Blends Test Program has been under way since 2007, supported jointly by the Office of the Biomass Program and the Vehicle Technologies Program. One of the larger projects, the Catalyst Durability Study, or Vehicle Aging Study, will be completed early in calendar year 2011. The following report describes a subproject of the Vehicle Aging Study in which powertrain components from 18 of the vehicles were examined at Southwest Research Institute under contract to Oak Ridge National Laboratory (ORNL). Any questions about the study can be addressed to the ORNL technical project manager, Brian West (westbh@ornl.gov). 



\author{
SOUTHWEST RESEARCH INSTITUTE ${ }^{\circledR}$ \\ 6220 Culebra Road Post Office Drawer 28510 \\ San Antonio, Texas 78228-0510
}

FUELS AND LUBRICANTS RESEARCH DIVISION

Fuels and Driveline Lubricants Research Department

Final Report

\title{
Powertrain Component Inspection from Mid-Level Blends Vehicle Aging Study
}

\author{
Conducted for \\ UT-Battelle, LLC \\ Oak Ridge, Tennessee
}

SwRI Project 08.15844

Proposal 08-58845

November 30, 2010

Submitted by:

Approved by:

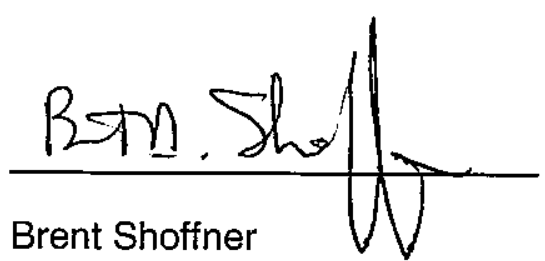

Manager

Fuel Performance Evaluations Section

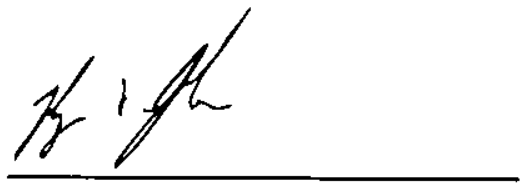

Ryan Johnson

Research Engineer

Diesel Lubricant Evaluations Section

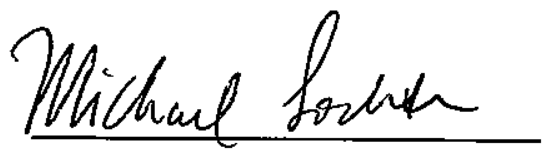

Michael Lochte

Director

Fuels and Driveline Lubricants Research Department

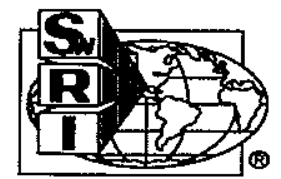

The results of this report relate only to the items tested.

This report shall not be reproduced, except in full, without the written approval of Southwest Research Institute ${ }^{\otimes}$. 



\section{TABLE OF CONTENTS}

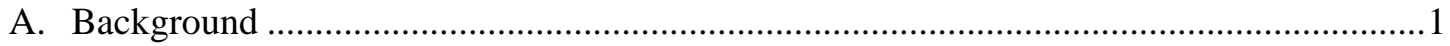

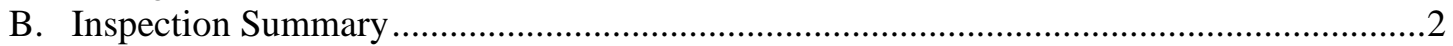

1. Evaporative Emissions System Integrity Check …....................................................

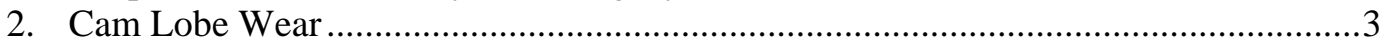

3. Valve Seat Width and Valve Surface Contour..............................................................

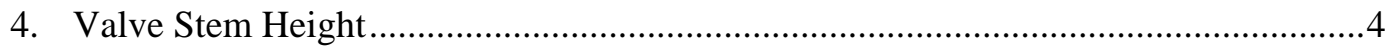

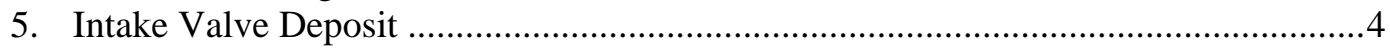

6. ASTM D5185 Analyses of Engine Oil Drain Samples.............................................5

7. Fuel Pump Performance Evaluation and Inspection ...................................................5

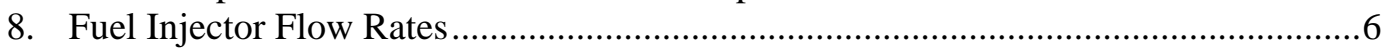

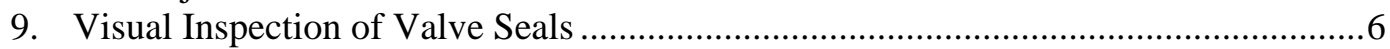

10. Evaporative Canister Working Capacity ...............................................................

11. Visual Inspections of Fuel Tanks, Fuel Lines, and Evaporative Emissions Lines ........7

\section{APPENDICES}

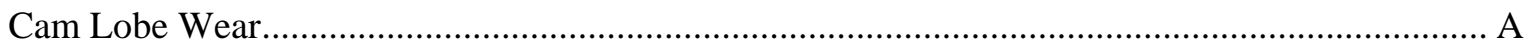

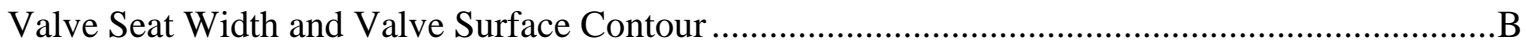

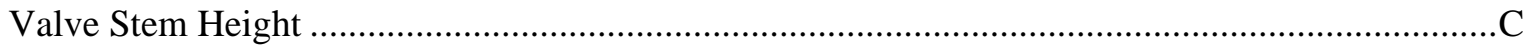

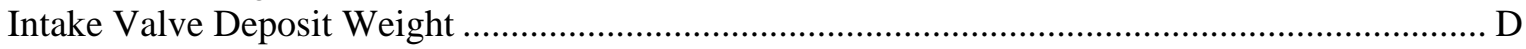

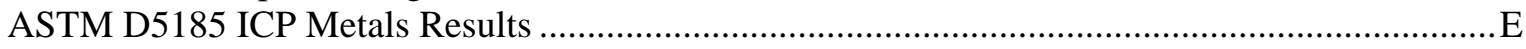

Fuel Pump Performance Evaluation and Inspection........................................................................ F

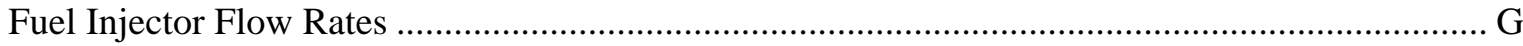

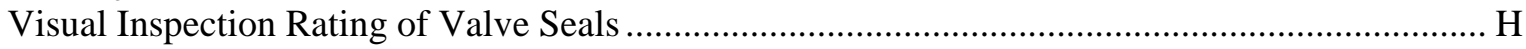

Evaporative Canister Working Capacity Test Results .................................................................... I

Visual Inspection of Fuel Tanks, Fuel Lines and Evaporative Emissions Lines............................... J 



\section{INTRODUCTION}

\section{A. Background}

The Oak Ridge National Laboratory (ORNL) project manager selected a subset of vehicles, aged in the DOE V4/CRC E-87-2 activity at Southwest Research Institute (SwRI), for powertrain component inspection. The full test-vehicle set in the program, which span a range of model years, were purchased in matched sets of three or four. Matched vehicles had the same EPA engine family, transmission, etc, and the odometer mileage range for a vehicle set was desired to be less than 10,000 miles. Actual odometers at the time of purchase for the vehicle groups involved in the inspection study are shown in Table 1. Vehicles were aged using the Standard Road Cycle (SRC) driving profile on mileage accumulation dynamometers (MADs) at SwRI. Mileage accumulation for each set was based on the highest mileage vehicle of a set, and all vehicles of a set were driven approximately the same distance, as shown in Table 1.

Each vehicle of a make/model/model year set was dedicated to a specific test fuel for aging. Aging fuels were retail top tier gasoline (RE0), and this RE0 gasoline was splash blended with ASTM D4806 ethanol to produce RE10, RE15, and RE20. Following completion of mileage accumulation and end of test (EOT) emissions tests, a subset of the vehicles entered the Powertrain Inspection Activity. The Oak Ridge contract for the E-87-2 program was modified on 6/28/2010 to include engine teardown and inspection of selected vehicles. The initial scope of work was limited to the RE0 and RE15 vehicles (listed below) of six make and model groups (total of 12 vehicles), which had recently completed mileage accumulation and emissions testing for the E-87-2 program.

1. Honda Accord ORHA0 and ORHA15

2. Chevrolet Silverado ORCS0 and ORCS15

3. Nissan Altima vehicles ORNA0 and ORNA15

4. Ford Taurus ORFT0 and ORFT15

5. Dodge Caravan ORDC0 and ORDC15

6. Chevrolet Cobalt ORCC0 and ORCC15

On 8/6/2010 the E20 vehicles from the six vehicle groups were also added to the scope of the inspection program with the direction to give the RE0 and RE15 vehicles the highest priority. This status report includes all of the results for the 18 vehicles. 
Table 1. Odometer Mileage and Test Mileage

\begin{tabular}{|c|r|r|r|r|r|c|}
\cline { 2 - 7 } & $\begin{array}{c}\text { Zero } \\
\text { Odometer } \\
\text { Miles }\end{array}$ & $\begin{array}{c}\text { First } \\
\text { Interval } \\
\text { Test } \\
\text { Miles }\end{array}$ & $\begin{array}{c}\text { First } \\
\text { Interval } \\
\text { Odometer }\end{array}$ & $\begin{array}{c}\text { Second } \\
\text { Interval } \\
\text { Test } \\
\text { Miles }\end{array}$ & $\begin{array}{c}\text { Test } \\
\text { Miles }\end{array}$ & $\begin{array}{c}\text { Final } \\
\text { Odometer } \\
\text { Miles }\end{array}$ \\
\hline ORHAO & 31,686 & 50,000 & 81,686 & 35,367 & 85,367 & 117,053 \\
\hline ORHA15 & 31,369 & 50,000 & 81,369 & 35,367 & 85,367 & 116,736 \\
\hline ORHA20 & 34,383 & 50,000 & 84,383 & 35,367 & 85,367 & 119,750 \\
\hline & \multicolumn{7}{|c|}{} & & & & \\
\hline ORCS0 & 27,606 & 50,000 & 77,606 & 42,144 & 92,144 & 119,750 \\
\hline ORCS15 & 17,152 & 50,000 & 67,152 & 42,144 & 92,144 & 109,296 \\
\hline ORCS20 & 17,103 & 50,000 & 67,103 & 42,144 & 92,144 & 109,247 \\
\hline & & & & & & \\
\hline ORNA0 & 19,284 & 50,000 & 69,284 & 50,226 & 100,226 & 119,510 \\
\hline ORNA15 & 9,950 & 50,000 & 59,950 & 50,226 & 100,226 & 110,176 \\
\hline ORNA20 & 10,307 & 50,000 & 60,307 & 50,226 & 100,226 & 110,533 \\
\hline & & & & & & \\
\hline ORFT0 & 17,231 & 50,000 & 67,231 & 52,519 & 102,519 & 119,750 \\
\hline ORFT15 & 17,099 & 50,000 & 67,099 & 52,519 & 102,519 & 119,618 \\
\hline ORFT20 & 13,082 & 50,000 & 63,082 & 52,519 & 102,519 & 115,386 \\
\hline & & & & & & \\
\hline ORDC0 & 46,473 & 44,191 & 90,664 & 24,750 & 68,941 & 115,414 \\
\hline ORDC15 & 40,031 & 44,191 & 84,222 & 24,750 & 68,941 & 108,972 \\
\hline ORDC20B & 50,809 & 44,191 & 95,000 & 24,750 & 68,941 & 119,750 \\
\hline & & & & & & \\
\hline ORCC0 & 38,894 & 47,282 & 86,176 & 24,750 & 72,032 & 110,926 \\
\hline ORCC15 & 47,718 & 47,282 & 95,000 & 24,750 & 72,032 & 119,750 \\
\hline ORCC20 & 38,499 & 47,282 & 85,781 & 24,750 & 72,032 & 110,531 \\
\hline
\end{tabular}

\section{B. Inspection Summary}

A description of the measurements and checks which were conducted are given in the following sections.

\section{Evaporative Emissions System Integrity Check}

A Smoke Pro ${ }^{\circledR}$ Total-Tech ${ }^{\mathrm{TM}}$ device was used to check the integrity of the evaporative emissions systems of each of the original 12 vehicles and the six RE20 vehicles. The evaporative emissions systems were researched in the vehicles' service manuals and the SwRI technicians introduced smoke under low pressure from the vent valve to the fuel tank and also from the vent valve to the purge valve. In every case the systems held pressure and there was no visual evidence of smoke leaking from the system. 
Because the vehicles in this program were driven on the MADs virtually around the clock, they spent very little time "soaking", which would have exercised the evaporative systems in a diurnal as they might experience in normal service. However, the evaporative systems were exercised at each refueling event due to the use of on-board refueling vapor recovery (ORVR). While mileage accumulation and fuel economy varies widely by vehicle set, a typical vehicle in this project would be refueled some 150-400 times during the mileage accumulation and emissions preps.

\section{Cam Lobe Wear}

The original plan was to measure cam lobe wear using a Precision Devices Micro/Analyzer-2000 (PDI machine). A surface trace was to be measured on the nose of each cam lobe and at a high wear area parallel to the centerline of the camshaft. The idea was to extend the trace from the front to the rear of the lobe to include the front and rear surfaces, which had not been worn. Thus the "lobe wear" would have been defined as the maximum distance from the worn surface to a straight line defined by the "unworn” edges.

However, the cam lobes of the engines either had wear at one of the edges or the lobes had a convex crown. After discussions with the ORNL Program manager, it was decided to take heel-to-toe measurements in the center of the wear area of each lobe with a calibrated micrometer. These measurements are complete for all 18 vehicles, and given in Appendix A. For vehicles whose service manual provides camshaft lobe lift specifications, base circle diameter measurements were performed to enable calculation of cam lift. Note that the Honda Accords are equipped with a primary and secondary intake camshaft lobe due to the VTEC feature of these engines.

Because the cams were not measured at the beginning of the program, and due to normal part-to-part variation, the measurements are potentially inconclusive with respect to any fuel-related differences in cam wear.

\section{Valve Seat Width and Valve Surface Contour}

The valves were measured in four radial traces at 90 degrees on the machined seating area. The width of the worn area is a measure of seating width. Refer to Appendix B for a photograph and schematic showing the measurement technique. Using a straight line datum between the un-worn areas at each end of the trace, the valve wear is computed. The measurements are complete and are given in Appendix B. No apparent trends based on differences in the aging fuel were observed. 


\section{Valve Stem Height}

The valve stem height results for all 18 vehicles are included in Appendix C. The distance from the tip of the valve was measured back to the cylinder head surface for the valve spring. In cases where a washer is inserted in production between the cylinder head surface and the spring, the washer was removed for the measurement. The exception was the Dodge Caravan which was measured with the washer in place because the valve stem height was specified in the service manual with the washer installed. The valve stem height measurement can be an indicator of valve seat wear. Because valve stem heights at the beginning of the program are not known, and because of variations in actual cylinder head geometry and valve length, it is not possible to draw any conclusions about valve seat wear from these measurements.

\section{Intake Valve Deposit}

The intake valve deposit (IVD) of the valves was photographed. These photographs have been uploaded to a password protected ftp site, which will remain in operation at least until 12/31/2012. To access the site:

\section{ftp://ftp.swri.edu}

Open site in Windows Explorer

Click File, Login As

Username: OKRD

Password: KYR745it

Two group photographs of all the intake valves from an engine were taken. For both views the valves are lined up left to right from cylinder one (front, if two intake valves per cylinder) to the last cylinder (rear, if two intake valves per cylinder). In the first view the intake valves are rotated so that the side with the most visual deposit is facing the camera. For the second view the opposite side of each valve is facing the camera. A sample of group photographs of the intake valves from Honda Accord vehicles ORHA0 and ORHA15 are included in Appendix D.

The intake valve deposit weight of each valve was measured as follows. Intake valve deposit weights for all 18 vehicles are given in Appendix D.

a. Each valve is carefully removed from the cylinder head so that no IVD is scraped from the valve.

b. The valves are then placed in a desiccator until they are photographed or prepared for weighing.

c. Each valve face is buffed.

d. The valves are rinsed with hexane.

e. The valves are dried by heating to $200+/-5^{\circ} \mathrm{F}$ for 5 minutes.

f. The valves are then placed in a desiccator cabinet for a minimum of one hour.

g. Each valve is weighed on a calibrated scale. 
h. After the photographs have been taken, the valves are cleaned of all deposits, prepared (b. through f.) and weighed again in the same manner.

i. The IVD weight is the difference between the dirty and clean valve weight.

In many cases, the IVD weight is considerably greater for the E15 engines when compared to the E0 (Top Tier fuel) counterpart. In most cases the E20 engine IVD weight is greater than the E15 engine IVD weight. While IVD levels at the beginning are not known, this increased IVD may be related to the dilution effect of ethanol on the fuel's detergent additive package. During this program, Top Tier E0 fuel was splash blended with ASTM D4806 denatured road-grade ethanol for RE10, RE15, and RE20. No attempt was made to determine the effectiveness of the detergent additive in the fuel after diluting it with ethanol.

For perspective, note that the specification limit for Top Tier gasoline with respect to the average IVD weight per valve in the ASTM D6201 2.3L IVD procedure is $50 \mathrm{mg}$ maximum. Some intake valves did exceed $50 \mathrm{mg}$, but a D6201 test result of $50 \mathrm{mg}$ does not necessarily guarantee a $50 \mathrm{mg}$ result in another vehicle, which is being run in a different driving cycle for many more hours

\section{ASTM D5185 Analyses of Engine Oil Drain Samples}

Engine oil samples of the engine oil drains were taken at each oil change interval and stored. Three samples listed below from each of the 18 SwRI RE0, RE15, and RE20 vehicles and three samples from each of 12 new 2009 vehicles tested at the Transportation Research Center were analyzed.

1. The first sample was taken during the second oil change. The first oil change defined the start of test.

2. The second oil sample was taken from the drain oil of the oil change prior to the mid-mileage emissions interval.

3. The third oil sample was taken from the drain oil of the last oil change prior to end-of test emissions.

The results are given in the tables in Appendix E. There do not appear to be any unusually high wear metal levels in any of the engine oil drains.

\section{Fuel Pump Performance Evaluation and Inspection}

The fuel pump modules removed from each vehicle have been evaluated. The results are given in Appendix F. During the program the fuel feed nipple of the fuel pump module of Chevrolet Cobalt ORCC15 cracked. The fuel pump module was replaced. This incident has been reported in a previous ORNL E-87-2 monthly status report. This relatively new pump was evaluated along with the original pump. 
The "dead-head" pressure of each module for all 18 vehicles was measured. Except for the Chevrolet Cobalt, which has an external pressure regulator, this measurement is a measure of the regulator pressure. The Chevrolet Cobalt fuel pump modules were measured with and without the external pressure regulator.

Fuel pump flow was measured at pressures above and below the mean expected pressure specified in the service manual of the vehicle. Those results for all 18 vehicles are also given in Appendix F.

The fuel pumps from the E0, E15, and E20 vehicles have been disassembled, inspected, and photographed with three exceptions. The photographs and inspection comments are also included in Appendix F. The Chevrolet Silverado truck fuel pumps from the E0 and E15 trucks as well as the Chevrolet Cobalt fuel pump with the broken connector (E15 pump) were inadvertently lost prior to disassembly/inspection.

While pump characteristics prior to the vehicle aging program are unknown, there do not appear to be any serious differences between the RE0, RE15 and RE20 fuel pumps.

\section{Fuel Injector Flow Rates}

The fuel injectors were removed from the 18 RE0, RE15, and RE20 vehicles and flow tested at three duty cycles, $25 \%, 35 \%$, and $75 \%$ using iso-octane. The fuel pressure was maintained at the mean pressure specified in the service manual of each vehicle. Flow test results of the fuel injectors for all 18 vehicles are complete and are given in Appendix G. Typical part-to-part variability for the average of the three pulse widths in gasoline engines is in the mean $+/-3 \%$ range. All injectors from a given set of vehicles fall within this range.

\section{Visual Inspection of Valve Seals}

The intake valve seals from all 18 vehicles have been visually rated by a calibrated rater (based on previous Coordinating Research Council workshops) and the results are included in Appendix H. Sample photographs of seals from Honda Accords ORHA0 and ORHA15 are included in Appendix H. The photographs were taken using the following procedure based on the ratings:

1. Only one "typical" seal was photographed for each engine.

2. The photographed "typical" seal was the one closest to the front on the cylinder head or in the case of a "V" engine, the left cylinder head.

3. Two views of the "typical" seal "front angle" and "rear angle" were taken with adjusted lighting to get a view of a portion of the inside seal lip.

4. Seals that the rater identified as being "different" than "typical" were also photographed. 
5. For each of these "different" seals the two angles, front and rear, were photographed. If those views did not pick up the "flaw" a third view was taken.

6. The size of each seal in the photographs was adjusted to be about the same for all photos (except the third view if it took a zoom in to see the "flaw")

7. Photographs for all 18 vehicles have been uploaded to the password-protected ftp site referenced above in section 5 .

\section{Evaporative Canister Working Capacity}

Evaporative canisters from the six teardown vehicle sets were removed from the vehicles and tested for working capacity at two different laboratories. Multiple laboratories were selected in order to meet the requested time schedule.

Canisters from the E0, E15, and E20 fueled Nissan Altimas, Chevrolet Cobalts, Dodge Caravans, and Chevrolet Silverados (12 canisters) were tested at Detroit Testing Laboratory (DTL) for butane or gasoline working capacity. DTL dedicated two butane working capacity test stands for our canister testing. The canisters that required shorter run times were started first so that some data would be available earlier. The butane working capacity tests require approximately five to six days to complete a full test sequence of 15 cycles.

To accelerate the time to completion, the canisters from the Dodge Caravan and the Chevrolet Silverado vehicle sets were selected for testing on two gasoline working capacity (GWC) test stands. The GWC test can be performed in significantly less time because the test cycles are shorter and the number of required cycles for a complete test sequence is fewer (only ten cycles are required for a complete GWC test compared to the 15 cycles for the BWC test).

Evaporative canisters from the E0, E15, and E20 fueled Honda Accords and Ford Tauruses (6 canisters) were tested at Environmental Testing Corporation (ETC) for butane working capacity under contract with National Renewable Energy Laboratory (NREL). Results for the Accord and Taurus vehicle canister tests are reported here for convenience.

Evaporative canister working capacity data for the six selected teardown vehicles are summarized in Appendix I, Table I-1. Test results show a slightly decreasing canister working capacity with increasing fuel ethanol content on two of the six vehicle sets tested. The other four vehicle canister data sets show neutral or slightly increasing working capacity with increasing fuel ethanol content. No statistically significant difference in evaporative canister working capacity is suggested with this small data set. Evaporative canister working capacity test reports for individual canisters are provided in Appendix I.

\section{Visual Inspections of Fuel Tanks, Fuel Lines, and Evaporative Emissions Lines}

The fuel feed lines and hoses; and evaporative emissions lines and hoses were removed from the vehicles. The inside surfaces of each end of the lines and hoses (as far inside as possible) were inspected by a CRC calibrated rater. The fuel tanks were also removed and inspected. 
The observations were recorded and are included in Appendix J. The following is a description of the observations:

a. Percent clean - No visual deposits and no distress

b. Percent white deposit - A white colored deposit is visually present on the given percent of the area. No attempt has been made to quantify the chemistry of the deposit.

c. All the o-rings appeared to be in good condition.

d. In some cases rust deposits were observed. These deposits could have formed during the life of the vehicle (either prior to acquisition for this program, or during the program), or in the time interval between part removal and rating.

e. Debris - Particles of foreign matter were observed. No attempt has been made to quantify the chemistry of the debris.

While fuel system component characteristics prior to the vehicle aging program are unknown, there do not appear to be any serious differences among the RE0, RE15 and RE20 fuel systems inspected. 


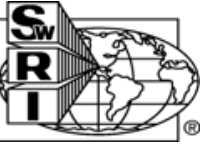

Appendix A

Cam Lobe Wear 



\section{Oak Ridge National Laboratory \\ Proposal 08-58845 Powertrain Inspection \\ Camshaft Heel to Toe Measurement}

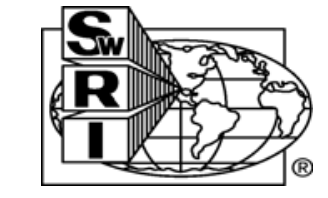

Date:

18-Aug-10

Technician:

RT

ORHA0 Micrometer: AN 003857 ORHA15/ORHA20 Micrometer: AN 005423

Standard: AN006504

Measurements in $\mathrm{mm}$

Lobes are numbered from front to rear on cam

\begin{tabular}{|c|c|r|r|r|r|r|r|}
\cline { 3 - 8 } \multicolumn{2}{c|}{} & \multicolumn{3}{c|}{ Intake Cam } & \multicolumn{3}{c|}{ Exhaust Cam } \\
\hline Cylinder & Lobe & \multicolumn{1}{c|}{ ORHA0 } & \multicolumn{1}{c|}{ ORHA15 } & \multicolumn{1}{c|}{ ORHA20 } & \multicolumn{1}{c|}{ ORHA0 } & \multicolumn{1}{c|}{ ORHA15 } & \multicolumn{1}{c|}{ ORHA20 } \\
\hline 1 & 1 & 34.235 & 34.287 & 34.244 & 34.096 & 34.114 & 34.121 \\
\hline 2 & 2 & 34.230 & 34.286 & 34.249 & 34.096 & 34.089 & 34.110 \\
\hline 3 & 3 & 34.224 & 34.279 & 34.261 & 34.079 & 34.103 & 34.110 \\
\hline 4 & 4 & 34.202 & 34.283 & 34.277 & 34.085 & 34.103 & 34.109 \\
\hline & Average & 34.223 & 34.284 & 34.258 & 34.089 & 34.102 & 34.113 \\
\cline { 2 - 8 } & StDev & 0.015 & 0.004 & 0.015 & 0.008 & 0.010 & 0.006 \\
\cline { 2 - 8 } & Max & 34.235 & 34.287 & 34.277 & 34.096 & 34.114 & 34.121 \\
\cline { 2 - 8 } & Min & 34.202 & 34.279 & 34.244 & 34.079 & 34.089 & 34.109 \\
\hline
\end{tabular}

\begin{tabular}{|c|c|r|r|r|}
\cline { 3 - 5 } \multicolumn{2}{c|}{} & \multicolumn{3}{c|}{ Intake Cam - Secondary Lobe } \\
\hline Cylinder & Lobe & \multicolumn{1}{c|}{ ORHA0 } & \multicolumn{1}{c|}{ ORHA15 } & \multicolumn{1}{c|}{ ORHA20 } \\
\hline 1 & 1 & 29.631 & 29.652 & 29.627 \\
\hline 2 & 2 & 29.623 & 29.666 & 29.635 \\
\hline 3 & 3 & 29.617 & 29.643 & 29.638 \\
\hline 4 & 4 & 29.616 & 29.658 & 29.645 \\
\hline \multirow{4}{*}{} & Average & 29.622 & 29.655 & 29.636 \\
\cline { 2 - 5 } & StDev & 0.007 & 0.010 & 0.007 \\
\cline { 2 - 5 } & Max & 29.631 & 29.666 & 29.645 \\
\cline { 2 - 5 } & Min & 29.616 & 29.643 & 29.627 \\
\cline { 2 - 5 }
\end{tabular}




\section{Oakridge National Laboratory}

2007 Honda Accord

Intake Camshaft Lobe Heel to Toe Measurements at EOT

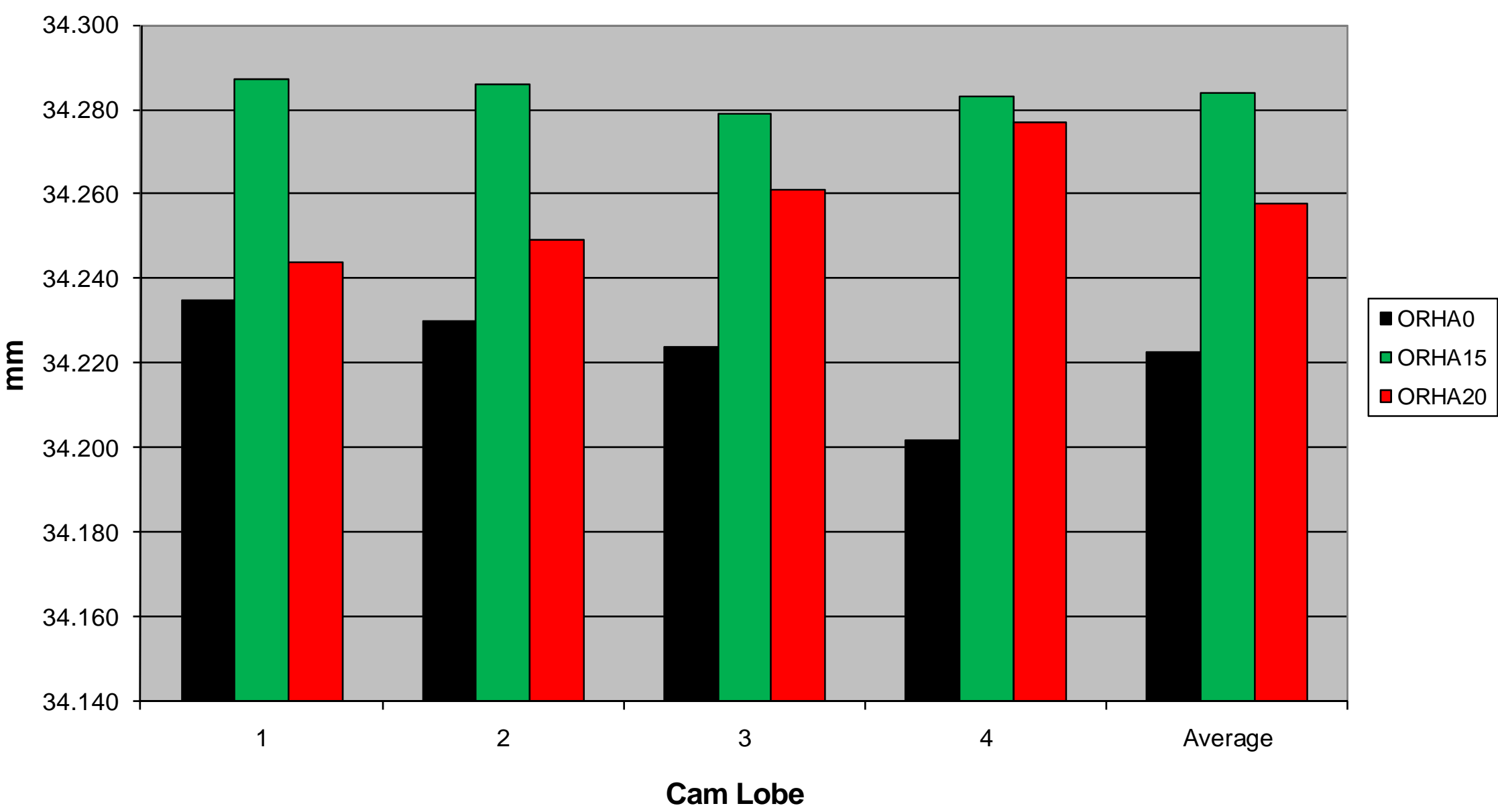


Oakridge National Laboratory

2007 Honda Accord

Intake Camshaft Secondary Lobe Heel to Toe Measurements at EOT

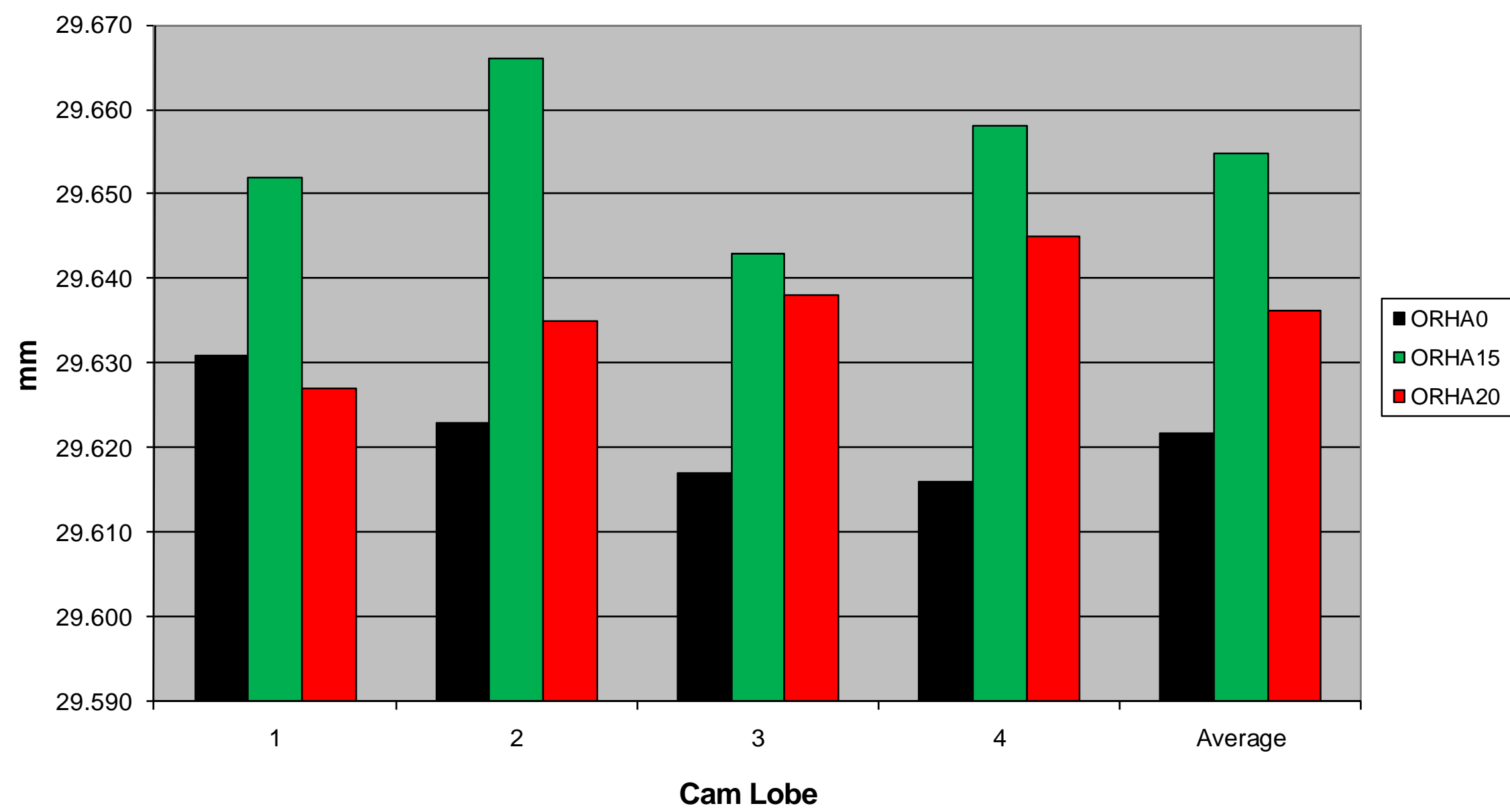


Oakridge National Laboratory

2007 Honda Accord

Exhaust Camshaft Lobe Heel to Toe Measurements at EOT

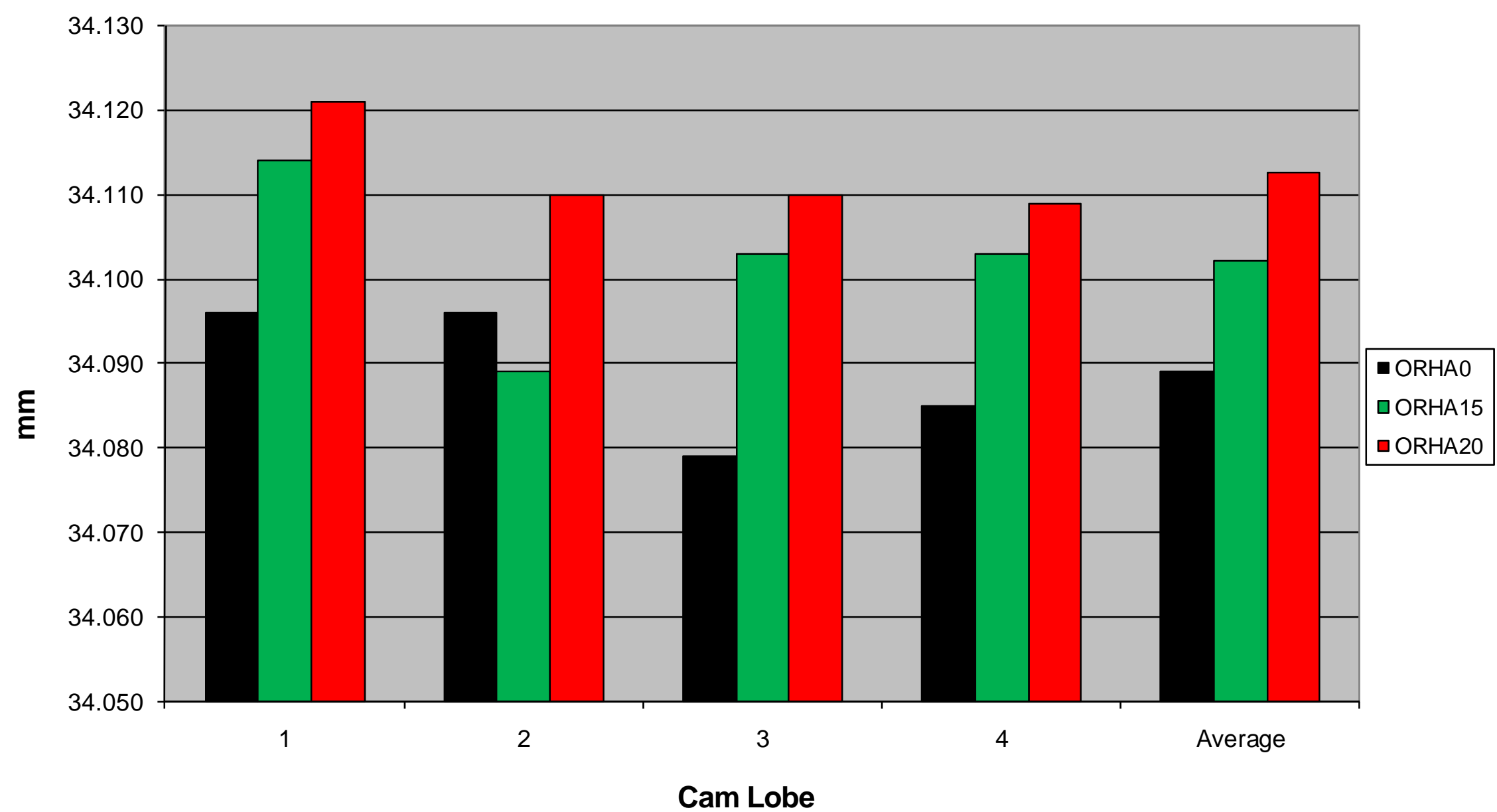




\section{Oak Ridge National Laboratory Proposal 08-58845 Powertrain Inspection Camshaft Heel to Toe Measurement}

Date:

18-Aug-10

Technician:

TP

Micrometer: AN 003857 and AN 005423

Standard: AN006504

Measurements in $\mathrm{mm}$

Lobes are numbered from front to rear on cam

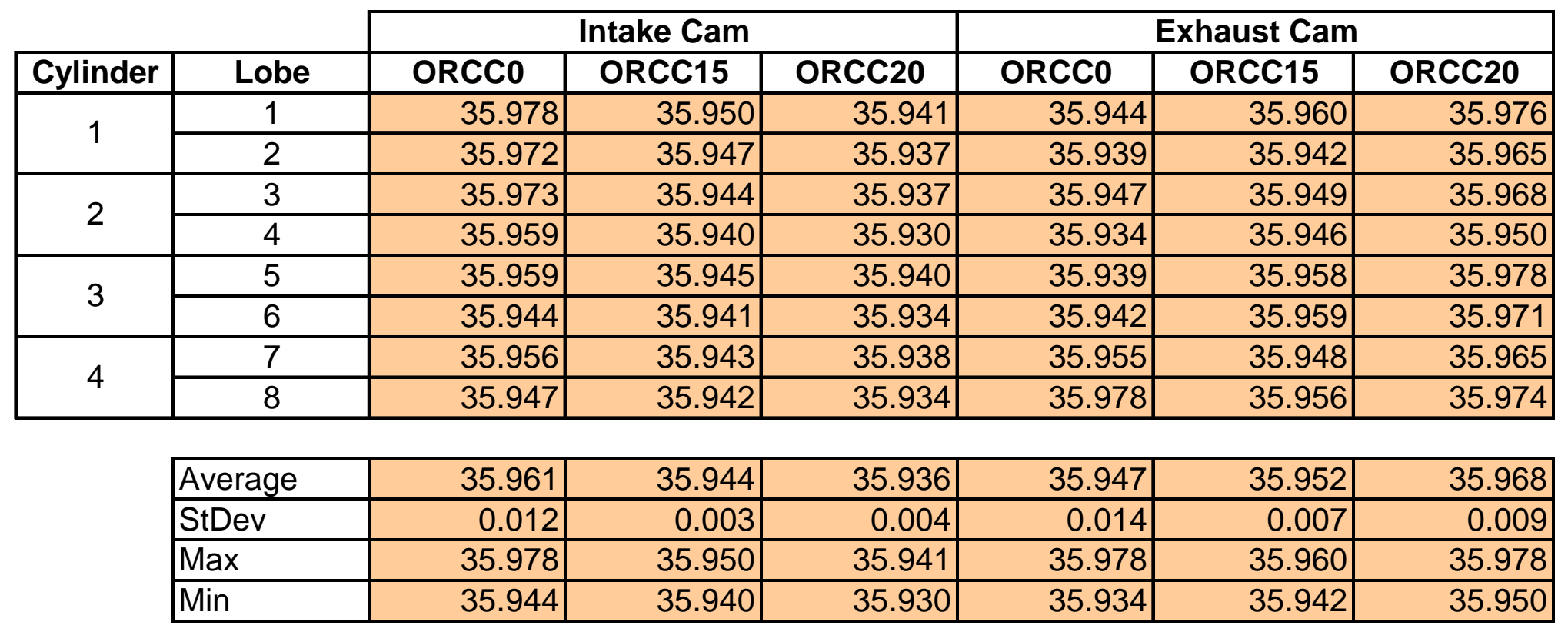


Oakridge National Laboratory

2006 Chevrolet Cobalt

Intake Camshaft Lobe Heel to Toe Measurements at EOT

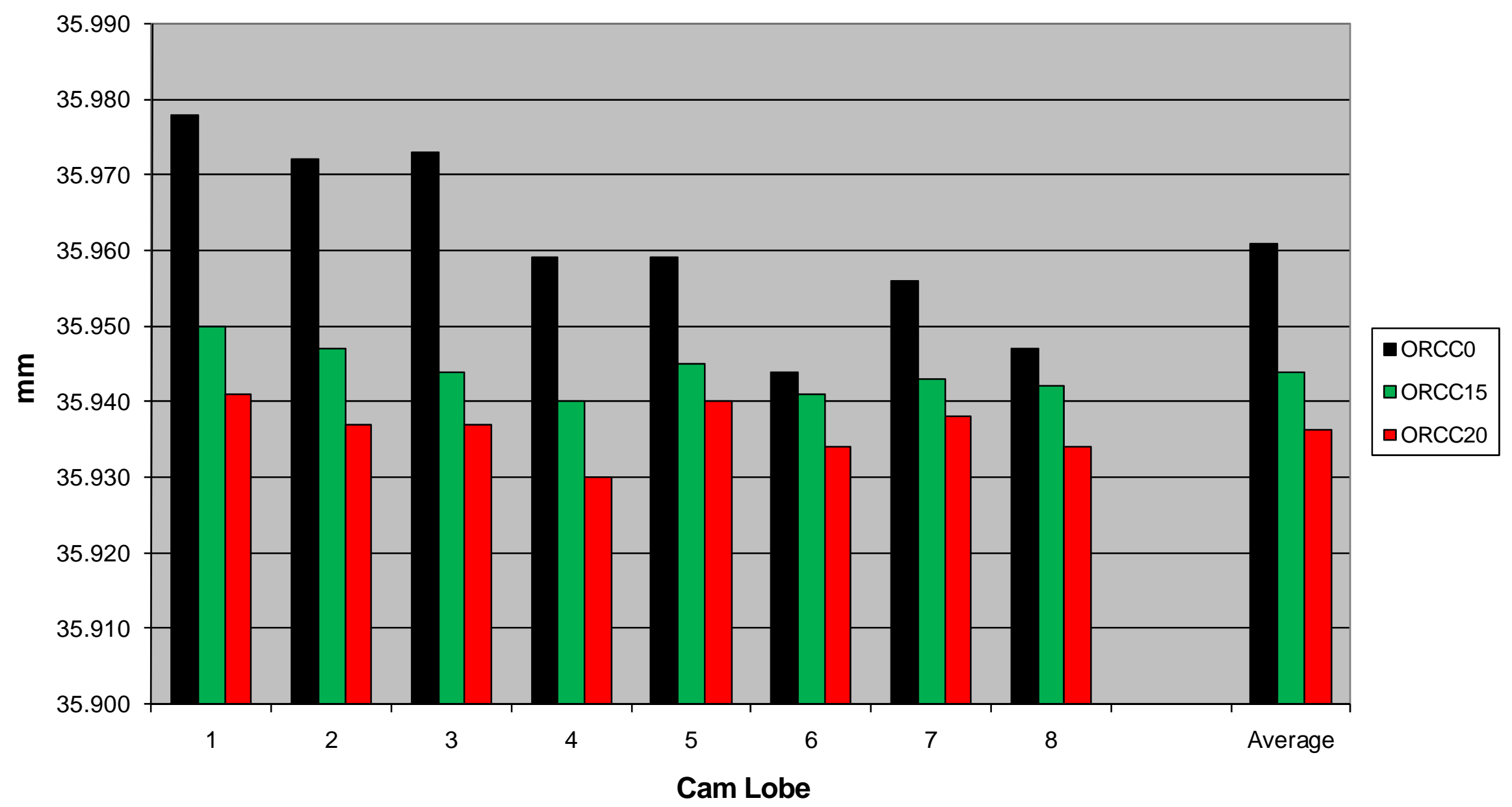


Oakridge National Laboratory

2006 Chevrolet Cobalt

Exhaust Camshaft Lobe Heel to Toe Measurements at EOT

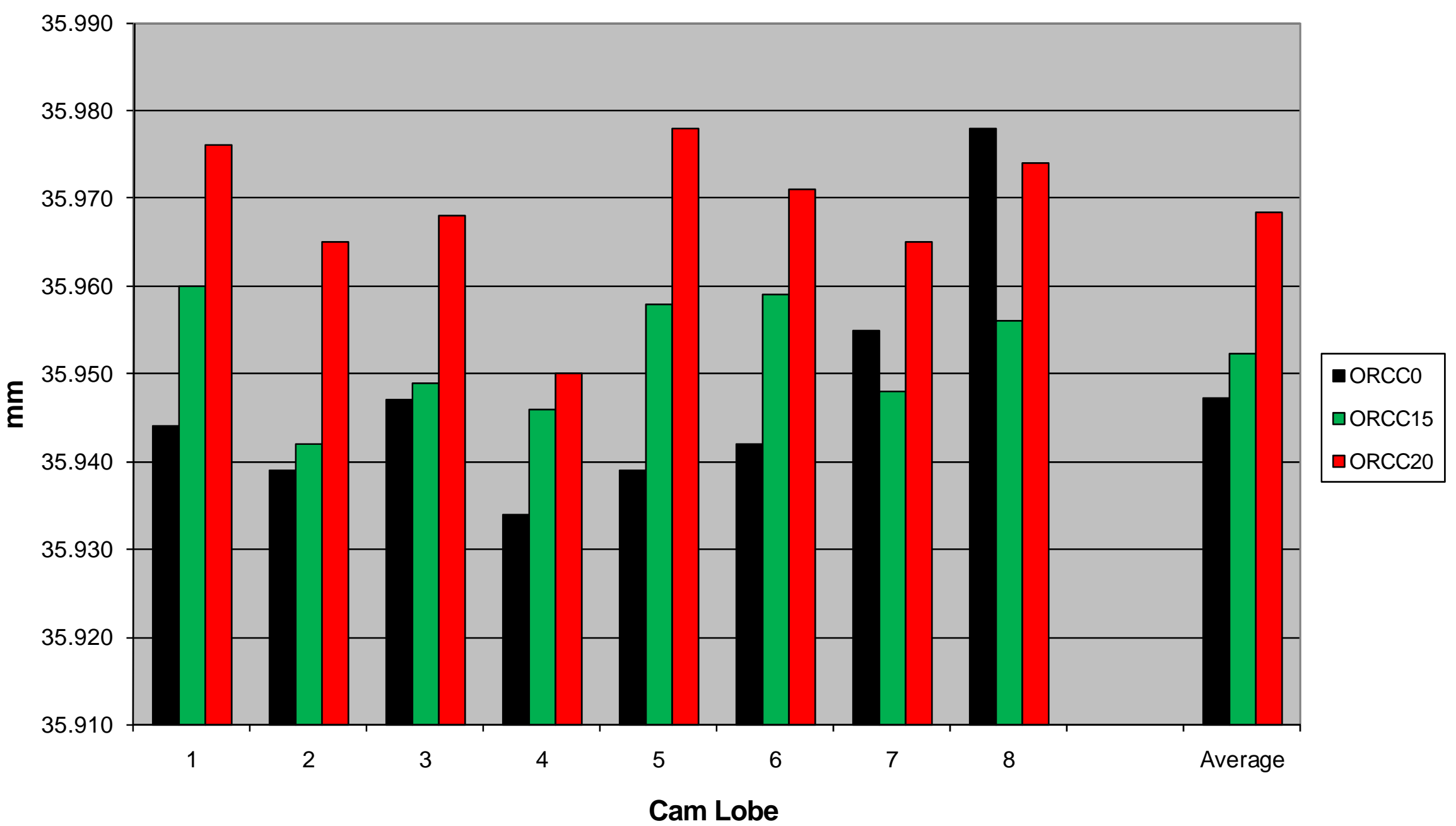




\section{Oak Ridge National Laboratory Proposal 08-58845 Powertrain Inspection Camshaft Heel to Toe Measurement}

Date:

28-Jul-10

Technician:

RT

Micrometer: AN 005423

Standard: AN006504

Measurements in $\mathrm{mm}$

Lobes are numbered from front to rear on cam

\begin{tabular}{|c|c|c|c|c|c|c|c|}
\hline \multirow{2}{*}{ Cylinder } & \multirow[b]{2}{*}{ Lobe } & \multicolumn{3}{|c|}{ Intake Cam } & \multicolumn{3}{|c|}{ Exhaust Cam } \\
\hline & & ORNAO & ORNA15 & ORNA20 & ORNAO & ORNA15 & ORNA20 \\
\hline \multirow{2}{*}{1} & 1 & 44.901 & 44.880 & 44.917 & 44.042 & 44.046 & 44.072 \\
\hline & 2 & 44.891 & 44.873 & 44.908 & 44.034 & 44.040 & 44.055 \\
\hline \multirow{2}{*}{2} & 3 & 44.896 & 44.887 & 44.908 & 44.042 & 44.041 & 44.063 \\
\hline & 4 & 44.894 & 44.882 & 44.900 & 44.041 & 44.037 & 44.062 \\
\hline \multirow{2}{*}{3} & 5 & 44.896 & 44.872 & 44.900 & 44.029 & 44.023 & 44.054 \\
\hline & 6 & 44.900 & 44.876 & 44.906 & 44.037 & 44.020 & 44.063 \\
\hline \multirow{2}{*}{4} & 7 & 44.877 & 44.867 & 44.890 & 44.025 & 44.026 & 44.041 \\
\hline & 8 & 44.885 & 44.878 & 44.904 & 44.033 & 44.033 & 44.057 \\
\hline & Average & 44.893 & 44.877 & 44.904 & 44.035 & 44.033 & 44.058 \\
\hline & StDev & 0.008 & 0.006 & 0.008 & 0.006 & 0.009 & 0.009 \\
\hline & $\operatorname{Max}$ & 44.901 & 44.887 & 44.917 & 44.042 & 44.046 & 44.072 \\
\hline & Min & 44.877 & 44.867 & 44.890 & 44.025 & 44.020 & 44.041 \\
\hline
\end{tabular}

Spec

\begin{tabular}{|l|r}
\hline Max & 45.005 \\
\hline Min & 44.815 \\
\hline
\end{tabular}

44.165




\section{Oakridge National Laboratory \\ 2008 Nissan Altima}

Intake Camshaft Lobe Heel to Toe Measurements at EOT

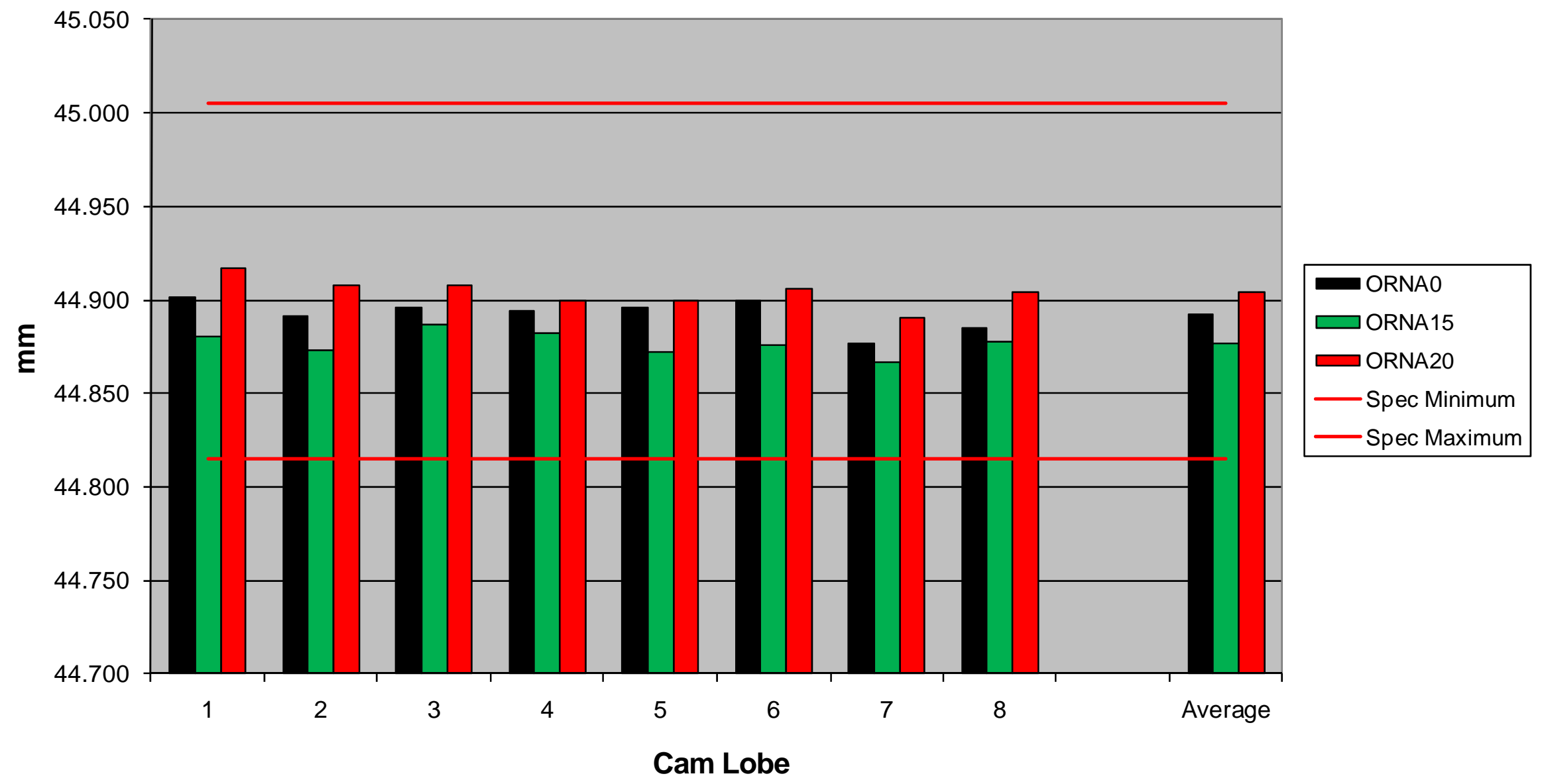


Oakridge National Laboratory

2008 Nissan Altima

Exhaust Camshaft Lobe Heel to Toe Measurements at EOT

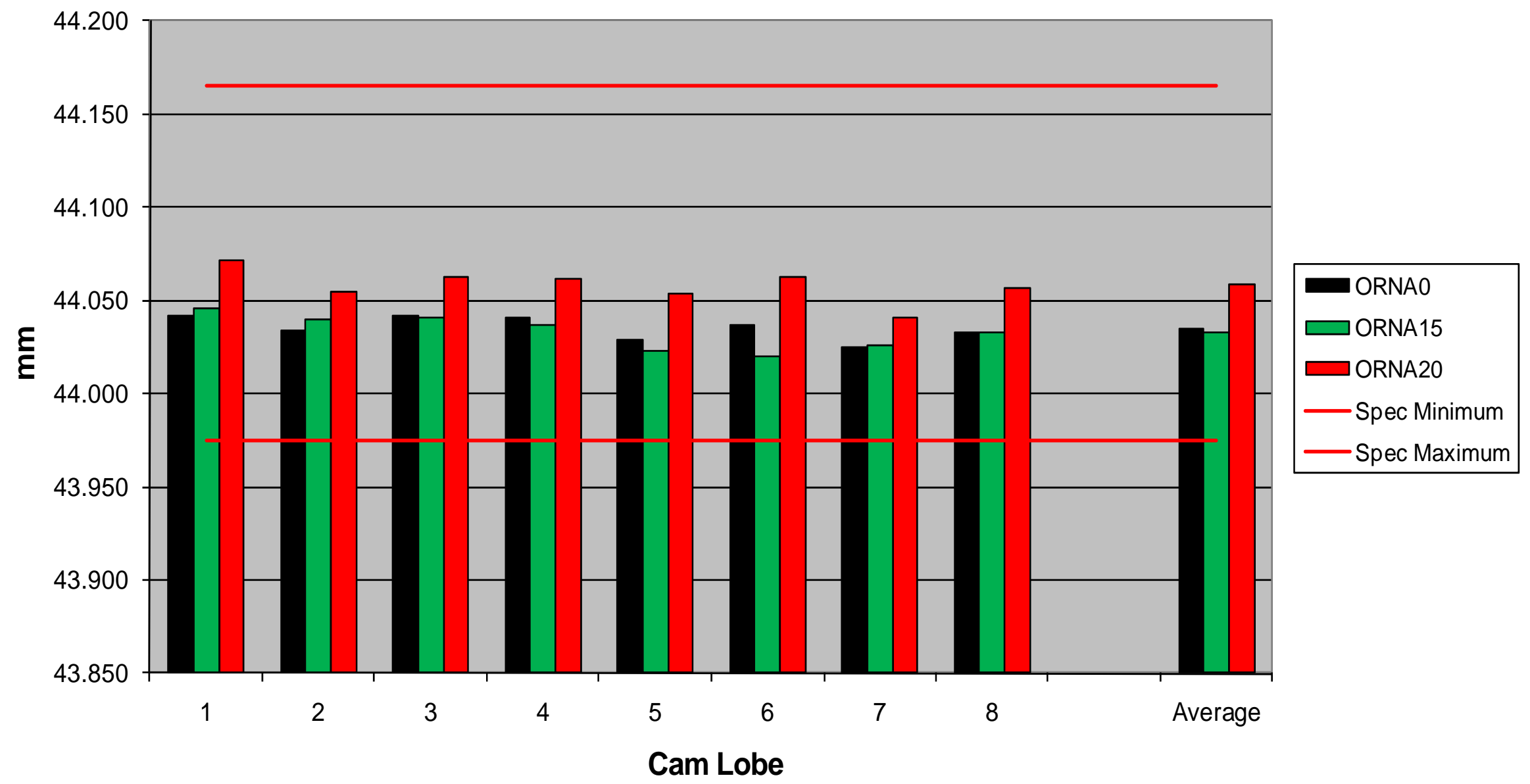




\section{Oak Ridge National Laboratory \\ Proposal 08-58845 Powertrain Inspection \\ Camshaft Heel to Toe Measurement}

Date:

$\begin{array}{ll}\text { 18-Aug-10 } & \text { Technician } \\ \text { Micrometer: AN } 005423 \\ \text { Standard: AN006504 } \\ \text { Measurements in mm }\end{array}$

Lobes are numbered from front to rear on cam

Service Manual indicates lobe lift $=9.6798 \mathrm{~mm}$, $\max$ lobe lift loss $=0.062 \mathrm{~mm}$ Lobe lift loss $=9.6798$ - (Heel to Toe - Base Circle)

\begin{tabular}{|c|c|c|c|c|c|c|c|}
\hline & \multicolumn{6}{|c|}{ ORFT0 } \\
\hline & & \multicolumn{3}{|c|}{ Intake } & \multicolumn{3}{|c|}{ Exhaust } \\
\hline Cylinder & Bank-Lobe \# & Heel-Toe & $\begin{array}{l}\text { Base } \\
\text { Circle }\end{array}$ & $\begin{array}{l}\text { Lobe Lift } \\
\text { Loss }\end{array}$ & Heel-Toe & $\begin{array}{l}\text { Base } \\
\text { Circle }\end{array}$ & $\begin{array}{l}\text { Lobe Lift } \\
\text { Loss }\end{array}$ \\
\hline \multirow{2}{*}{1} & Right-1 & 45.651 & 35.982 & 0.011 & 45.670 & 36.010 & 0.020 \\
\hline & Right-2 & 45.679 & 36.006 & 0.007 & 45.671 & 35.994 & 0.003 \\
\hline \multirow{2}{*}{2} & Right-3 & 45.656 & 35.982 & 0.006 & 45.688 & 36.020 & 0.012 \\
\hline & Right-4 & 45.676 & 36.002 & 0.006 & 45.686 & 36.024 & 0.018 \\
\hline \multirow{2}{*}{3} & Right-5 & 45.649 & 35.985 & 0.016 & 45.696 & 36.090 & 0.074 \\
\hline & Right-6 & 45.644 & 35.979 & 0.015 & 45.689 & 36.056 & 0.047 \\
\hline \multirow{2}{*}{4} & Left-1 & 45.637 & 35.972 & 0.015 & 45.712 & 36.038 & 0.006 \\
\hline & Left-2 & 45.647 & 35.978 & 0.011 & 45.710 & 36.036 & 0.006 \\
\hline \multirow{2}{*}{5} & Left-3 & 45.640 & 35.973 & 0.013 & 45.703 & 36.028 & 0.005 \\
\hline & Left-4 & 45.660 & 35.986 & 0.006 & 45.702 & 36.025 & 0.003 \\
\hline \multirow{2}{*}{6} & Left-5 & 45.651 & 35.983 & 0.012 & 45.710 & 36.033 & 0.003 \\
\hline & Left-6 & 45.647 & 35.980 & 0.013 & 45.717 & 36.039 & 0.002 \\
\hline \multicolumn{2}{|l|}{$\mid \overline{A v e r a g e}$} & 45.653 & 35.984 & 0.011 & 45.696 & 36.033 & 0.016 \\
\hline \multicolumn{2}{|l|}{ StDev } & 0.013 & 0.010 & 0.004 & 0.016 & 0.024 & 0.022 \\
\hline \multicolumn{2}{|l|}{$\operatorname{Max}$} & 45.679 & 36.006 & 0.016 & 45.717 & 36.090 & 0.074 \\
\hline \multicolumn{2}{|c|}{$\overline{M i n}$} & 45.637 & 35.972 & 0.006 & 45.670 & 35.994 & 0.002 \\
\hline
\end{tabular}




\section{Oak Ridge National Laboratory Proposal 08-58845 Powertrain Inspection Camshaft Heel to Toe Measurement}

Date:

$\begin{array}{ll}\text { 18-Aug-10 } & \text { Technician: } \\ & \text { Micrometer: AN } 005423 \\ \text { Standard: AN006504 } \\ \text { Measurements in mm }\end{array}$

Lobes are numbered from front to rear on cam

Service Manual indicates lobe lift $=9.6798 \mathrm{~mm}$, $\max$ lobe lift loss $=0.062 \mathrm{~mm}$

Lobe lift loss $=9.6798-($ Heel to Toe - Base Circle)

\begin{tabular}{|c|c|c|c|c|c|c|c|}
\hline & \multicolumn{6}{|c|}{ ORFT15 } \\
\hline & & \multicolumn{3}{|c|}{ Intake } & \multicolumn{3}{|c|}{ Exhaust } \\
\hline Cylinder & Bank-Lobe \# & Heel-Toe & $\begin{array}{l}\text { Base } \\
\text { Circle }\end{array}$ & $\begin{array}{l}\text { Lobe Lift } \\
\text { Loss }\end{array}$ & Heel-Toe & $\begin{array}{l}\text { Base } \\
\text { Circle }\end{array}$ & $\begin{array}{l}\text { Lobe Lift } \\
\text { Loss }\end{array}$ \\
\hline \multirow{2}{*}{1} & Right-1 & 45.649 & 36.013 & 0.044 & 45.651 & 36.010 & 0.039 \\
\hline & Right-2 & 45.683 & 36.006 & 0.003 & 45.647 & 35.991 & 0.024 \\
\hline \multirow{2}{*}{2} & Right-3 & 45.660 & 35.994 & 0.014 & 45.673 & 36.002 & 0.009 \\
\hline & Right-4 & 45.682 & 36.007 & 0.005 & 45.672 & 36.000 & 0.008 \\
\hline \multirow{2}{*}{3} & Right-5 & 45.658 & 35.991 & 0.013 & 45.687 & 36.018 & 0.011 \\
\hline & Right- 6 & 45.653 & 35.988 & 0.015 & 45.682 & 36.014 & 0.012 \\
\hline \multirow{2}{*}{4} & Left-1 & 45.689 & 36.026 & 0.017 & 45.694 & 36.022 & 0.008 \\
\hline & Left-2 & 45.672 & 36.006 & 0.014 & 45.703 & 36.028 & 0.005 \\
\hline \multirow{2}{*}{5} & Left-3 & 45.672 & 36.001 & 0.009 & 45.697 & 36.023 & 0.006 \\
\hline & Left-4 & 45.694 & 36.023 & 0.009 & 45.699 & 36.025 & 0.006 \\
\hline \multirow{2}{*}{6} & Left-5 & 45.670 & 36.025 & 0.035 & 45.688 & 36.018 & 0.010 \\
\hline & Left-6 & 45.693 & 36.041 & 0.028 & 45.690 & 36.018 & 0.008 \\
\hline \multicolumn{2}{|l|}{ Average } & 45.673 & 36.010 & 0.017 & 45.682 & 36.014 & 0.012 \\
\hline \multicolumn{2}{|l|}{ StDev } & 0.016 & 0.016 & 0.012 & 0.018 & 0.011 & 0.010 \\
\hline \multicolumn{2}{|l|}{$\operatorname{Max}$} & 45.694 & 36.041 & 0.044 & 45.703 & 36.028 & 0.039 \\
\hline \multicolumn{2}{|l|}{ Min } & 45.649 & 35.988 & 0.003 & 45.647| & 35.991 & 0.005 \\
\hline
\end{tabular}




\section{Oak Ridge National Laboratory \\ Proposal 08-58845 Powertrain Inspection \\ Camshaft Heel to Toe Measurement}

Date:

$\begin{array}{lll}\text { 21-Sep-10 } & \text { Technician } \\ \text { Micrometer: AN } 005423 \\ \text { Standard: AN006504 } \\ \text { Measurements in mm }\end{array}$

Lobes are numbered from front to rear on cam

Service Manual indicates lobe lift $=9.6798 \mathrm{~mm}$, $\max$ lobe lift loss $=0.062 \mathrm{~mm}$ Lobe lift loss $=9.6798$ - (Heel to Toe - Base Circle)

\begin{tabular}{|c|c|r|r|r|r|r|r|}
\cline { 3 - 8 } \multicolumn{2}{c|}{} & \multicolumn{9}{c|}{ ORFT20 } \\
\cline { 3 - 8 } \multicolumn{2}{c|}{} & \multicolumn{3}{c|}{ Intake } & \multicolumn{3}{c|}{ Exhaust } \\
\hline \multirow{3}{*}{ Cylinder } & Bank-Lobe \# & Heel-Toe & $\begin{array}{c}\text { Base } \\
\text { Circle }\end{array}$ & $\begin{array}{c}\text { Lobe Lift } \\
\text { Loss }\end{array}$ & Heel-Toe & $\begin{array}{c}\text { Base } \\
\text { Circle }\end{array}$ & $\begin{array}{c}\text { Lobe Lift } \\
\text { Loss }\end{array}$ \\
\hline \multirow{2}{*}{1} & Right-1 & 45.697 & 36.039 & 0.022 & 45.701 & 36.036 & 0.015 \\
\cline { 2 - 9 } & Right-2 & 45.681 & 36.019 & 0.018 & 45.691 & 36.028 & 0.017 \\
\hline \multirow{2}{*}{2} & Right-3 & 45.688 & 36.026 & 0.018 & 45.681 & 36.020 & 0.019 \\
\cline { 2 - 9 } & Right-4 & 45.669 & 36.011 & 0.022 & 45.679 & 36.013 & 0.014 \\
\hline \multirow{2}{*}{3} & Right-5 & 45.688 & 36.026 & 0.018 & 45.674 & 36.009 & 0.015 \\
\cline { 2 - 9 } & Right-6 & 45.682 & 36.021 & 0.019 & 45.669 & 36.006 & 0.017 \\
\hline \multirow{2}{*}{4} & Left-1 & 45.707 & 36.037 & 0.010 & 45.655 & 35.994 & 0.019 \\
\cline { 2 - 9 } & Left-2 & 45.698 & 36.033 & 0.015 & 45.640 & 35.979 & 0.019 \\
\hline \multirow{2}{*}{5} & Left-3 & 45.692 & 36.026 & 0.014 & 45.648 & 35.986 & 0.018 \\
\cline { 2 - 9 } & Left-4 & 45.686 & 36.019 & 0.013 & 45.641 & 35.980 & 0.019 \\
\hline \multirow{2}{*}{6} & Left-5 & 45.673 & 36.002 & 0.009 & 45.674 & 36.005 & 0.011 \\
\cline { 2 - 8 } & Left-6 & 45.665 & 35.997 & 0.012 & 45.680 & 36.017 & 0.017 \\
\hline Average & & 45.686 & 36.021 & 0.016 & 45.669 & 36.006 & 0.016 \\
\hline StDev & & 0.012 & 0.013 & 0.004 & 0.020 & 0.018 & 0.002 \\
\hline Max & & 45.707 & 36.039 & 0.022 & 45.701 & 36.036 & 0.019 \\
\hline Min & & 45.665 & 35.997 & 0.009 & 45.640 & 35.979 & 0.011 \\
\hline
\end{tabular}




\section{Oakridge National Laboratory \\ 2008 Ford Taurus}

Intake Camshaft Lobe Heel to Toe Measurements at EOT

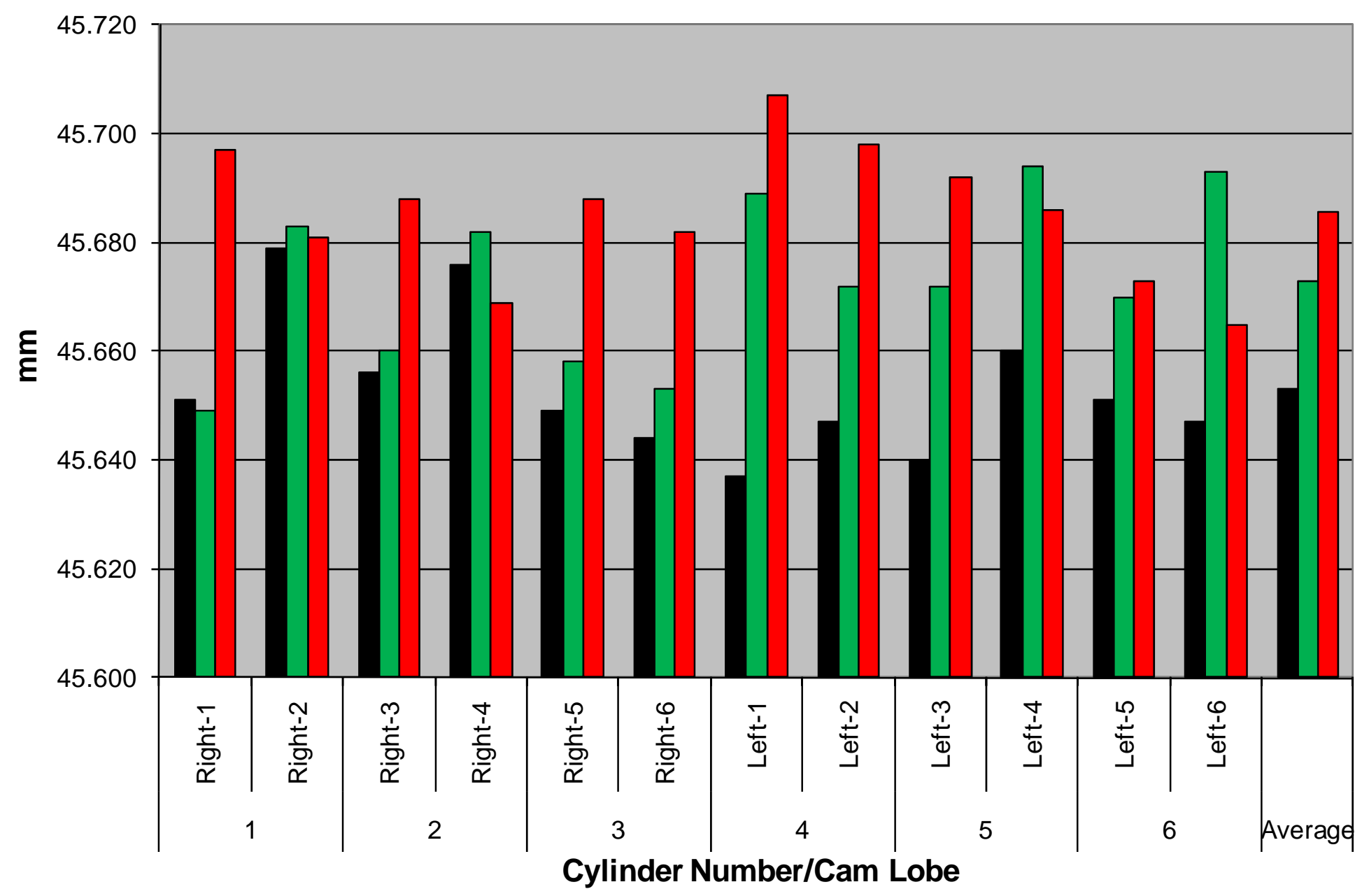


Oakridge National Laboratory

2008 Ford Taurus

Exhaust Camshaft Lobe Heel to Toe Measurements at EOT

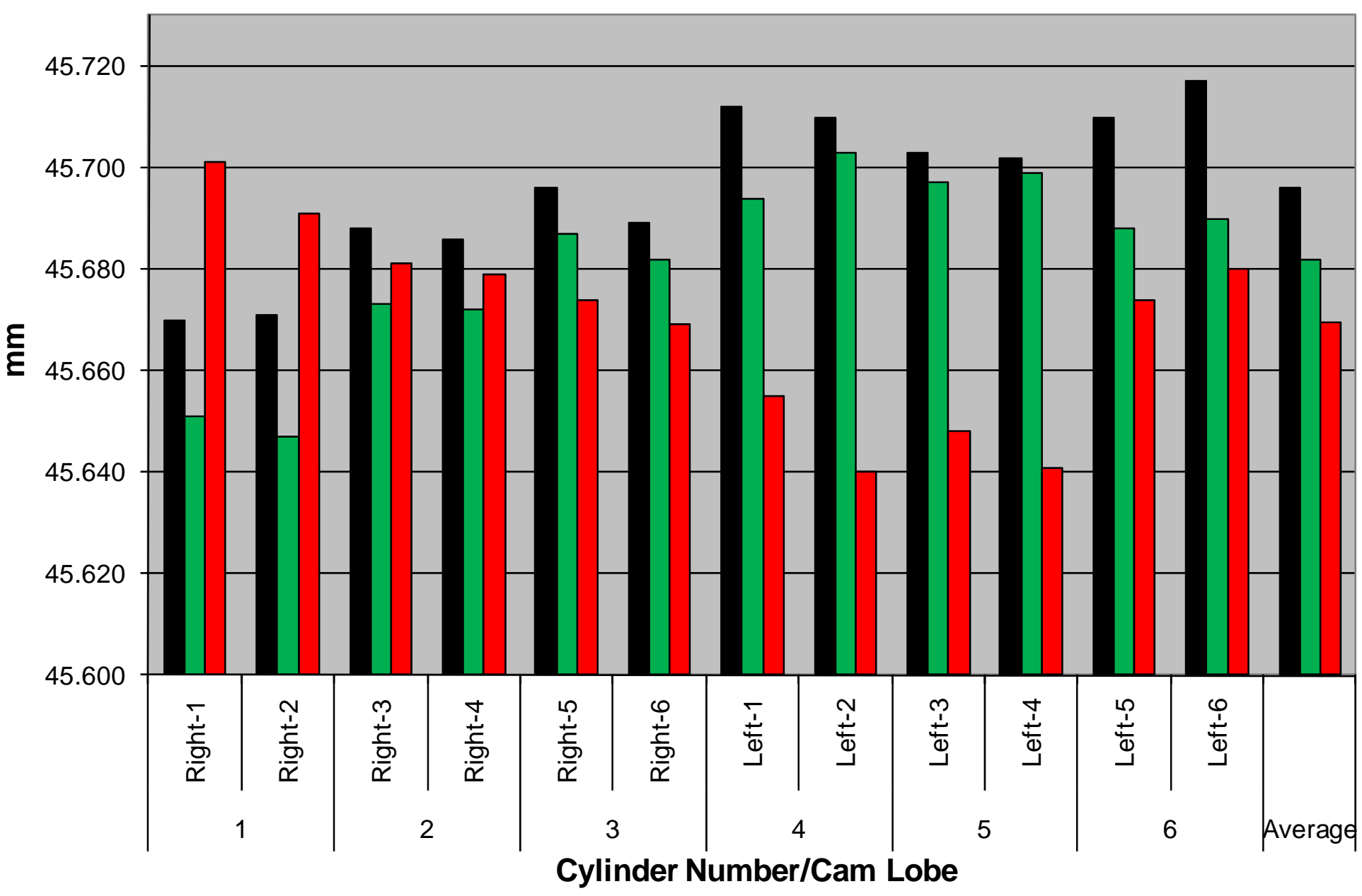


Oakridge National Laboratory

2008 Ford Taurus

Intake Camshaft Lobe Lift Loss Measurements at EOT

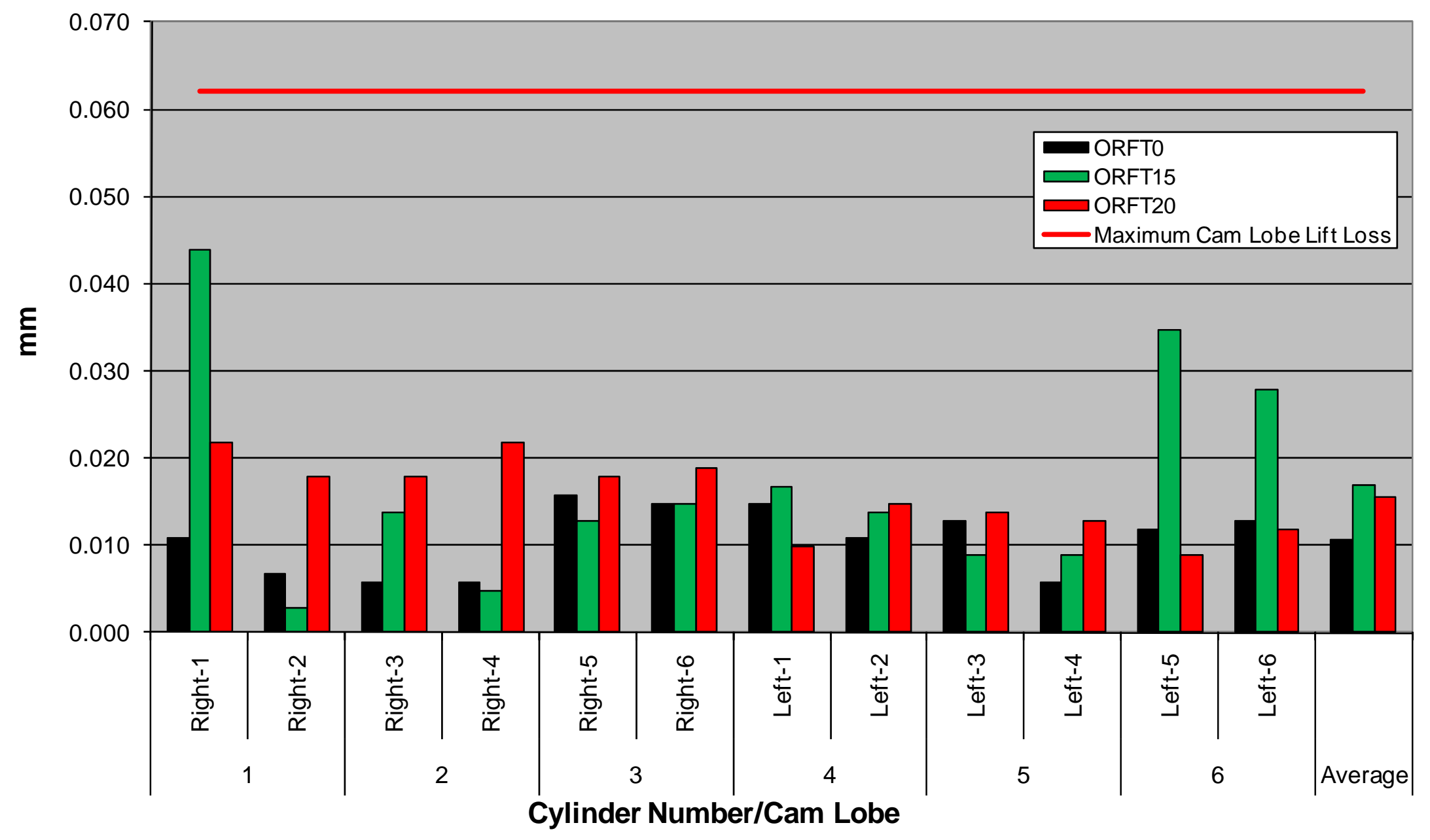




\section{Oakridge National Laboratory \\ 2008 Ford Taurus}

Exhaust Camshaft Lobe Lift Loss Measurements at EOT

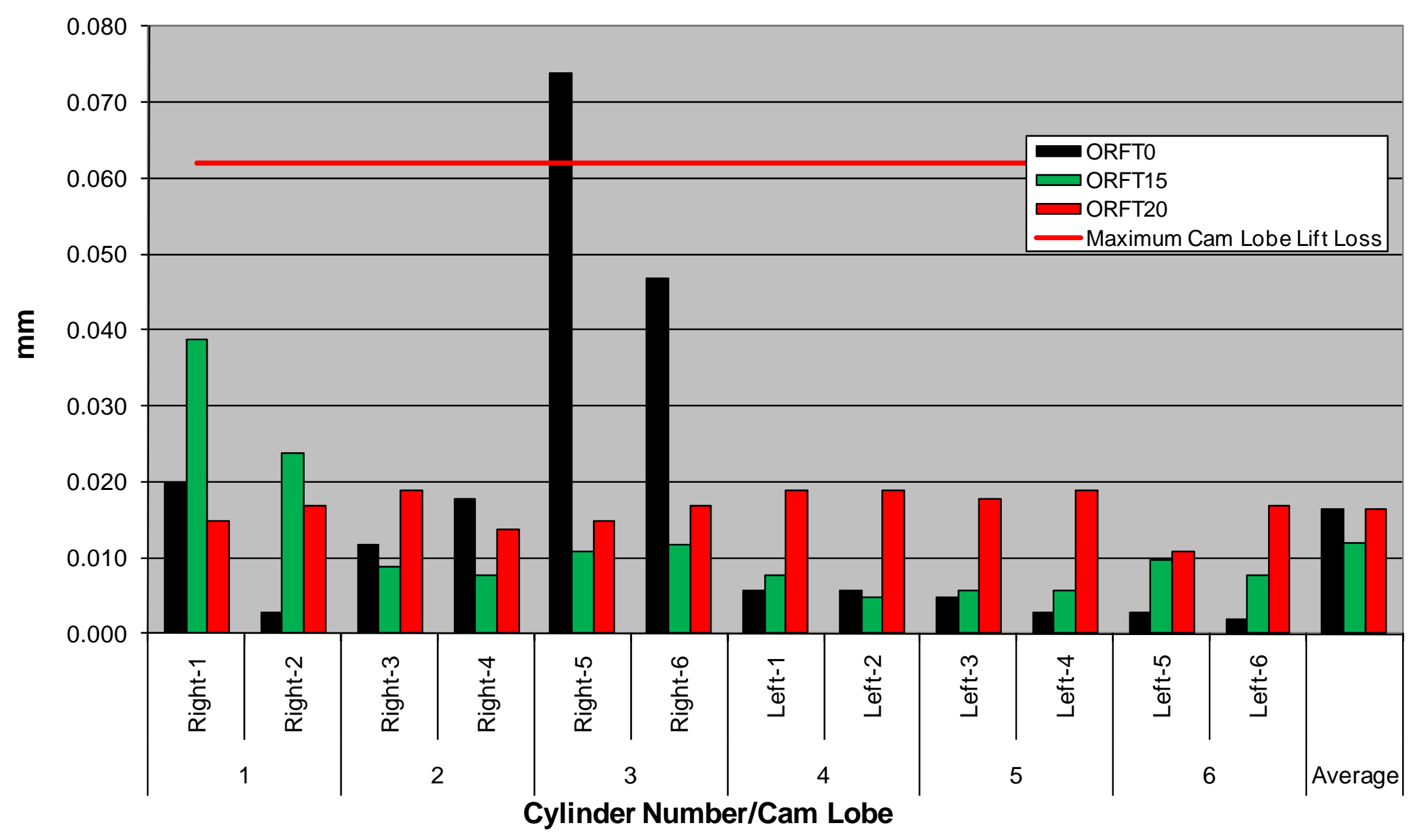




\section{Oak Ridge National Laboratory Proposal 08-58845 Powertrain Inspection Camshaft Heel to Toe Measurement}

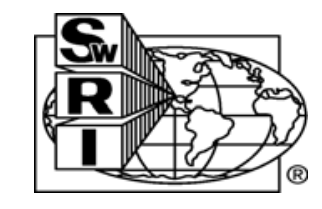

Date:

18-Aug-10

Technician:

$\mathrm{RT}$

Micrometer: AN 005423

Standard: AN006504

Measurements in $\mathrm{mm}$

Lobes are numbered from front to rear on cam

ORDC0
\begin{tabular}{|c|c|r|r|r|}
\hline \multicolumn{5}{|c|}{ Intake } \\
\hline \multicolumn{7}{|c|}{ Cylinder } & Lobe \# & \multicolumn{1}{c|}{ Heel-Toe } & Base Circle & \multicolumn{1}{c|}{ Lift } \\
\hline 1 & 3 & 40.261 & 33.926 & 6.335 \\
\hline 2 & 2 & 40.264 & 33.929 & 6.335 \\
\hline 3 & 7 & 40.255 & 33.918 & 6.337 \\
\hline 4 & 6 & 40.248 & 33.918 & 6.330 \\
\hline 5 & 11 & 40.252 & 33.914 & 6.338 \\
\hline 6 & 10 & 40.250 & 33.915 & 6.335 \\
\hline \multicolumn{7}{|r|r|}{} \\
\hline Average & 40.255 & 33.920 & 6.335 \\
\hline StDev & 0.006 & 0.006 & 0.003 \\
\hline Max & 40.264 & 33.929 & 6.338 \\
\hline Min & 40.248 & 33.914 & 6.330 \\
\hline
\end{tabular}

\begin{tabular}{|c|c|r|r|r|}
\hline \multicolumn{5}{|c|}{ Exhaust } \\
\hline Cylinder & Lobe \# & \multicolumn{1}{c|}{ Heel-Toe } & Base Circle & \multicolumn{1}{c|}{ Lift } \\
\hline 1 & 1 & 40.267 & 33.916 & 6.351 \\
\hline 2 & 4 & 40.260 & 33.911 & 6.349 \\
\hline 3 & 5 & 40.256 & 33.910 & 6.346 \\
\hline 4 & 8 & 40.248 & 33.899 & 6.349 \\
\hline 5 & 9 & 40.256 & 33.908 & 6.348 \\
\hline 6 & 12 & 40.248 & 33.906 & 6.342 \\
\hline \multicolumn{5}{|r|}{} \\
\hline Average & 40.256 & 33.908 & 6.348 \\
\hline StDev & 0.007 & 0.006 & 0.003 \\
\hline Max & 40.267 & 33.916 & 6.351 \\
\hline Min & 40.248 & 33.899 & 6.342 \\
\hline
\end{tabular}




\section{Oak Ridge National Laboratory Proposal 08-58845 Powertrain Inspection Camshaft Heel to Toe Measurement}

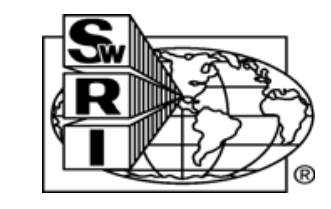

Date:

18-Aug-10

Technician:

RT

Micrometer: AN 005423

Standard: AN006504

Measurements in $\mathrm{mm}$

Lobes are numbered from front to rear on cam

\begin{tabular}{|c|c|c|c|c|}
\hline \multicolumn{5}{|c|}{ ORDC15 } \\
\hline \multicolumn{5}{|c|}{ Intake } \\
\hline Cylinder & Lobe \# & Heel-Toe & Base Circle & Lift \\
\hline 1 & 3 & 40.318 & 33.986 & 6.332 \\
\hline 2 & 2 & 40.316 & 33.981 & 6.335 \\
\hline 3 & 7 & 40.330 & 33.994 & 6.336 \\
\hline 4 & 6 & 40.324 & 33.987 & 6.337 \\
\hline 5 & 11 & 40.332 & 33.992 & 6.340 \\
\hline 6 & 10 & 40.324 & 33.991 & 6.333 \\
\hline Average & & 40.324 & 33.989 & 6.336 \\
\hline StDev & & 0.006 & 0.005 & 0.003 \\
\hline Max & & 40.332 & 33.994 & 6.340 \\
\hline Min & & 40.316 & 33.981 & 6.332 \\
\hline
\end{tabular}

\begin{tabular}{|c|c|r|r|r|}
\hline \multicolumn{5}{|c|}{ Exhaust } \\
\hline Cylinder & Lobe \# & Heel-Toe & Base Circle & \multicolumn{1}{c|}{ Lift } \\
\hline 1 & 1 & 40.314 & 33.961 & 6.353 \\
\hline 2 & 4 & 40.328 & 33.978 & 6.350 \\
\hline 3 & 5 & 40.323 & 33.983 & 6.340 \\
\hline 4 & 8 & 40.334 & 33.984 & 6.350 \\
\hline 5 & 9 & 40.329 & 33.980 & 6.349 \\
\hline 6 & 12 & 40.335 & 33.984 & 6.351 \\
\hline \multicolumn{5}{|l|}{} \\
\hline Average & 40.327 & 33.978 & 6.349 \\
\hline StDev & 0.008 & 0.009 & 0.005 \\
\hline Max & 40.335 & 33.984 & 6.353 \\
\hline Min & 40.314 & 33.961 & 6.340 \\
\hline
\end{tabular}




\section{Oak Ridge National Laboratory Proposal 08-58845 Powertrain Inspection Camshaft Heel to Toe Measurement}

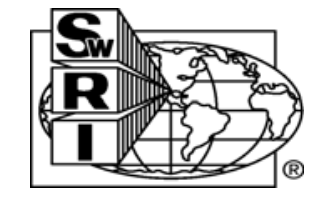

Date:

17-Sep-10

Technician:

RT

Micrometer: AN 005423
Standard: AN006504
Measurements in mm

Lobes are numbered from front to rear on cam

\begin{tabular}{l}
\hline \multicolumn{6}{|c|}{ ORDC20 } \\
\begin{tabular}{|c|c|r|r|r|}
\hline \multicolumn{7}{|c|}{ Intake } \\
\hline Cylinder & Lobe \# & \multicolumn{1}{c|}{ Heel-Toe } & Base Circle & \multicolumn{1}{c|}{ Lift } \\
\hline 1 & 3 & 40.259 & 33.924 & 6.335 \\
\hline 2 & 2 & 40.260 & 33.925 & 6.335 \\
\hline 3 & 7 & 40.274 & 33.932 & 6.342 \\
\hline 4 & 6 & 40.267 & 33.928 & 6.339 \\
\hline 5 & 11 & 40.279 & 33.939 & 6.340 \\
\hline 6 & 10 & 40.280 & 33.937 & 6.343 \\
\hline \multicolumn{7}{|c|}{} & 40.270 & 33.931 & 6.339 \\
\hline Average & 0.009 & 0.006 & 0.003 \\
\hline StDev & 40.280 & 33.939 & 6.343 \\
\hline Max & 40.259 & 33.924 & 6.335 \\
\hline Min
\end{tabular}
\end{tabular}

\begin{tabular}{|c|c|r|r|r|}
\hline \multicolumn{5}{|c|}{ Exhaust } \\
\hline Cylinder & Lobe \# & \multicolumn{1}{c|}{ Heel-Toe } & Base Circle & \multicolumn{1}{c|}{ Lift } \\
\hline 1 & 1 & 40.255 & 33.907 & 6.348 \\
\hline 2 & 4 & 40.259 & 33.912 & 6.347 \\
\hline 3 & 5 & 40.264 & 33.914 & 6.350 \\
\hline 4 & 8 & 40.267 & 33.918 & 6.349 \\
\hline 5 & 9 & 40.268 & 33.922 & 6.346 \\
\hline 6 & 12 & 40.280 & 33.933 & 6.347 \\
\hline \multicolumn{5}{|c|}{} \\
\hline Average & 40.266 & 33.918 & 6.348 \\
\hline StDev & 0.009 & 0.009 & 0.001 \\
\hline Max & 40.280 & 33.933 & 6.350 \\
\hline Min & 40.255 & 33.907 & 6.346 \\
\hline
\end{tabular}


Oakridge National Laboratory

2007 Dodge Caravan

Intake Camshaft Lobe Heel to Toe Measurements at EOT

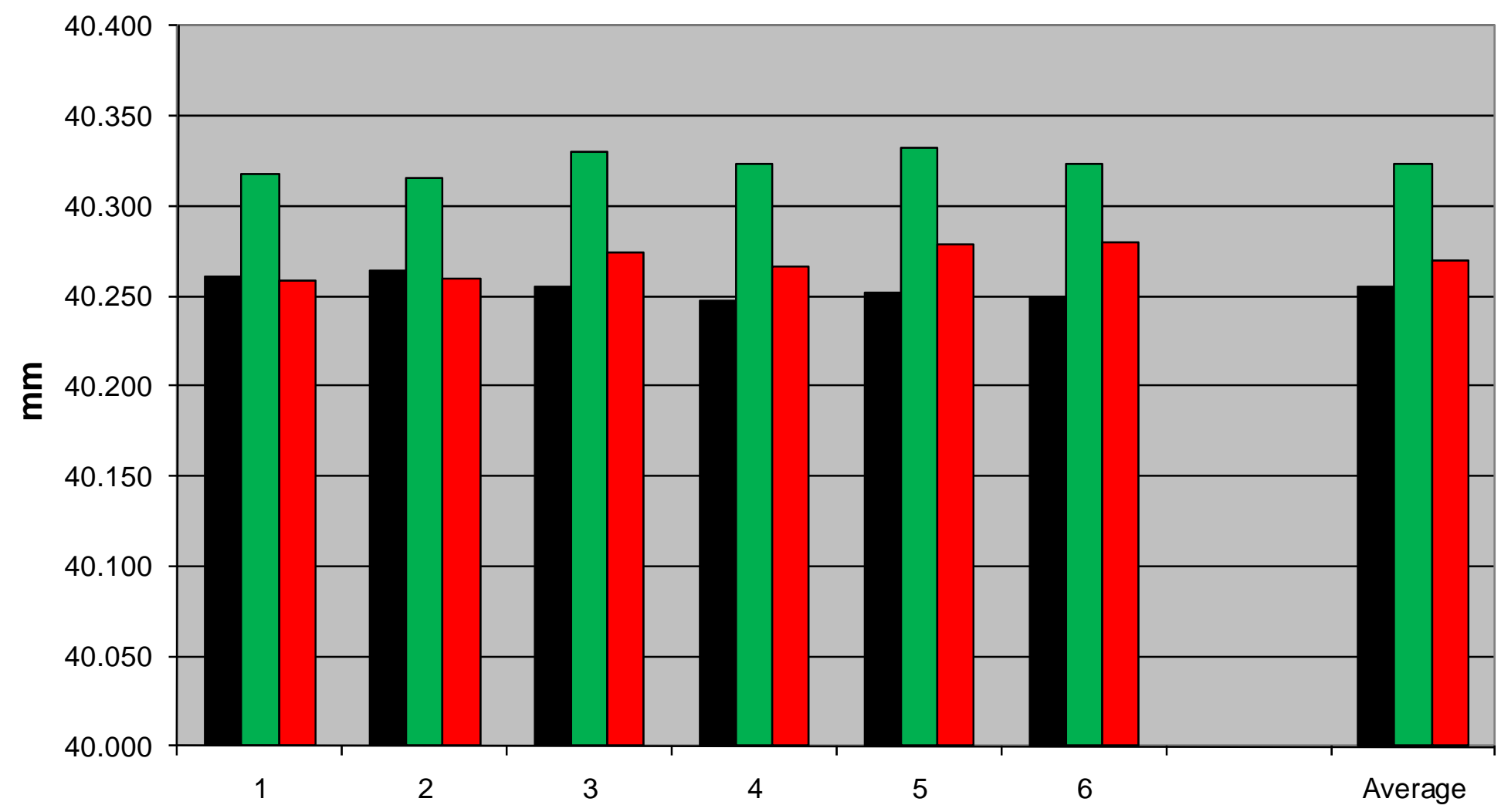

Cylinder Number 
Oakridge National Laboratory

2007 Dodge Caravan

Exhaust Camshaft Lobe Heel to Toe Measurements at EOT

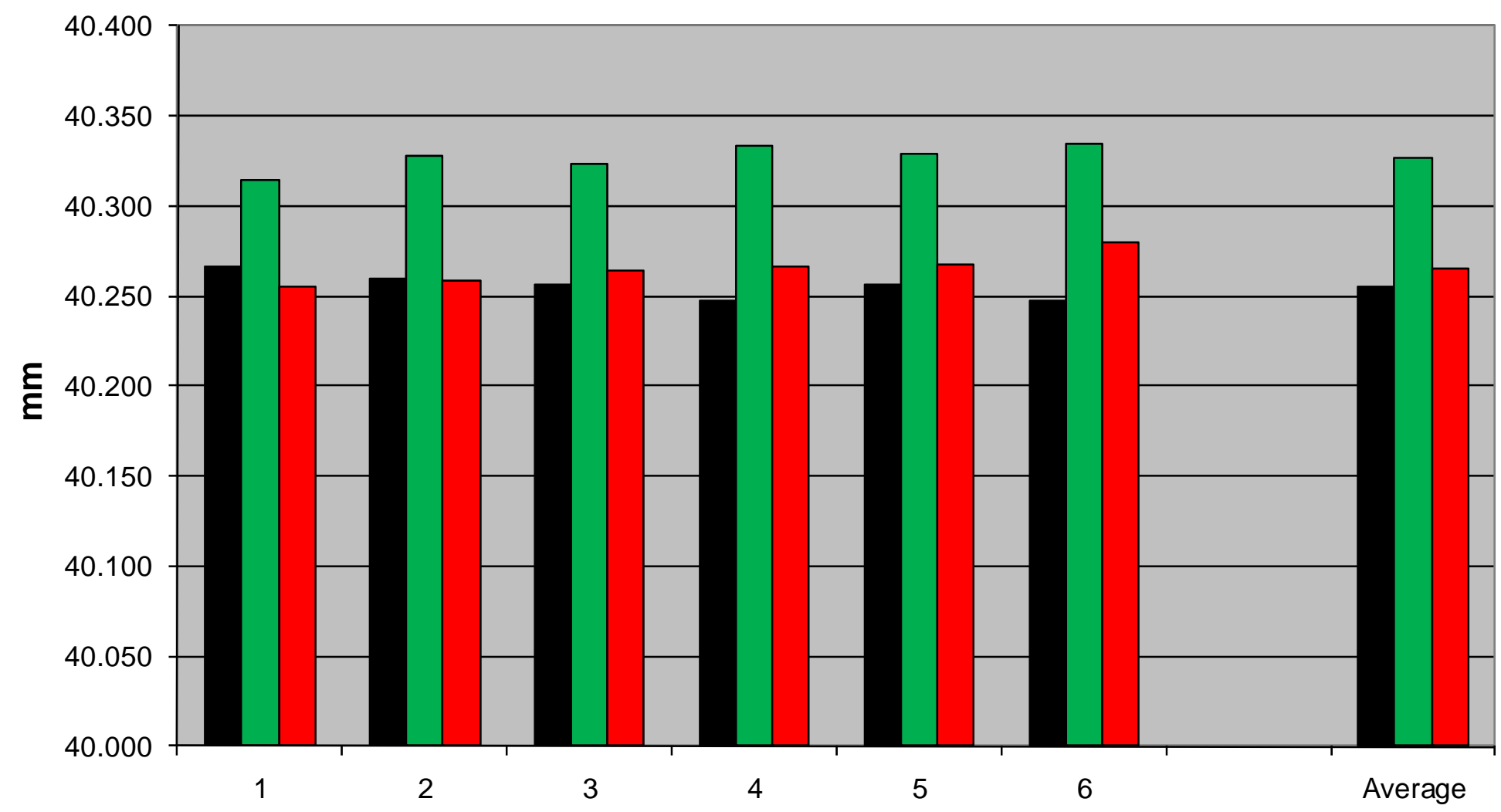

Cylinder Number 
Oakridge National Laboratory

2007 Dodge Caravan

Intake Camshaft Lobe Lift Measurements at EOT

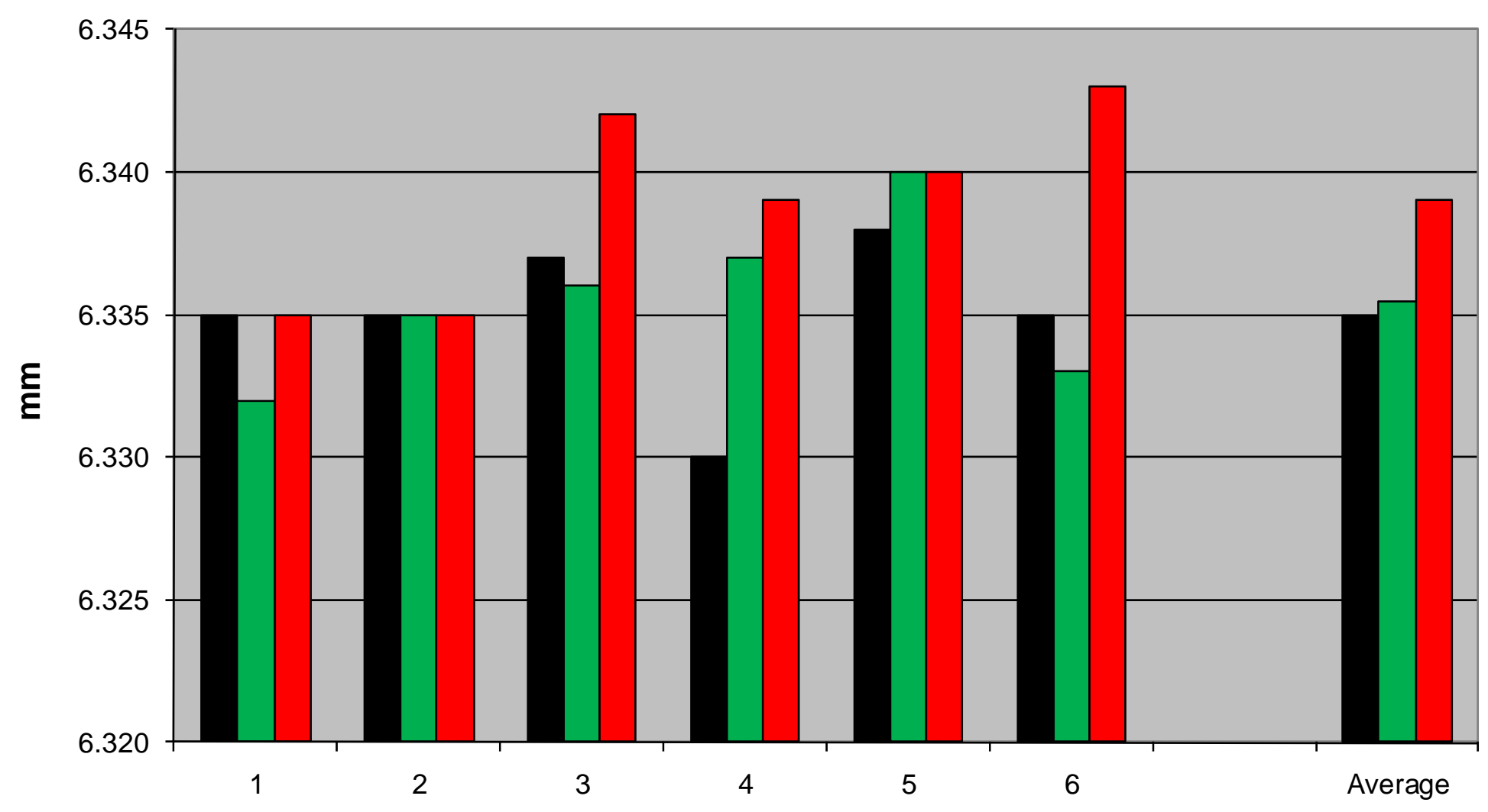

Cylinder Number 
Oakridge National Laboratory

2007 Dodge Caravan

Exhaust Camshaft Lobe Lift Measurements at EOT

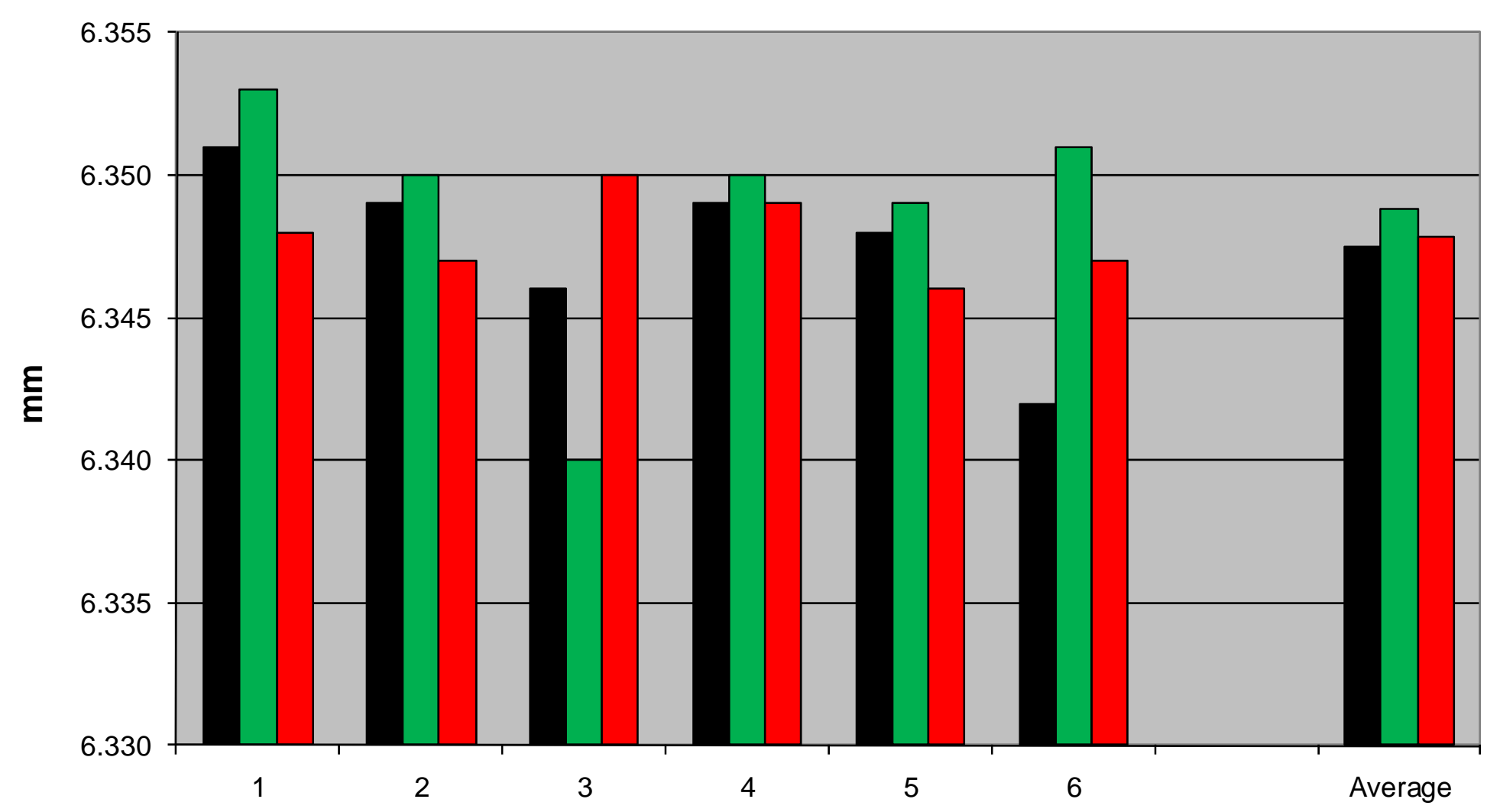

Cylinder Number 


\title{
Oak Ridge National Laboratory Proposal 08-58845 Powertrain Inspection Camshaft Heel to Toe Measurement
}

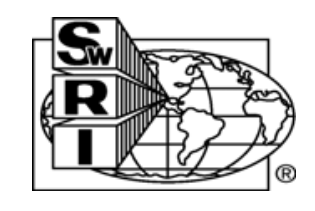

Date: 18-Aug-10

Technician: RT

\author{
Micrometer: AN 005423 \\ Standard: AN006504 \\ Measurements in $\mathrm{mm}$
}

Lobes are numbered from front to rear on cam

Service manual specification for cam lobe lift - Intake - $6.82 \mathrm{~mm}$

Service manual specification for cam lobe lift - Exhaust - $6.96 \mathrm{~mm}$

\begin{tabular}{|c|c|c|c|c|}
\hline \multicolumn{5}{|c|}{ ORCSO } \\
\hline \multicolumn{5}{|c|}{ Intake } \\
\hline Cylinder & Lobe \# & Heel-Toe & Base Circle & Lift \\
\hline 1 & 1 & 46.562 & 39.378 & 7.184 \\
\hline 2 & 4 & 46.547 & 39.394 & 7.153 \\
\hline 3 & 5 & 46.573 & 39.397 & 7.176 \\
\hline 4 & 8 & 46.549 & 39.374 & 7.175 \\
\hline 5 & 9 & 46.556 & 39.396 & 7.160 \\
\hline 6 & 12 & 46.568 & 39.398 & 7.170 \\
\hline 7 & 13 & 46.584 & 39.394 & 7.190 \\
\hline 8 & 16 & 46.566 & 39.384 & 7.182 \\
\hline Average & & 46.563 & 39.389 & 7.174 \\
\hline StDev & & 0.012 & 0.009 & 0.012 \\
\hline Max & & 46.584 & 39.398 & 7.190 \\
\hline Min & & 46.547 & 39.374 & 7.153 \\
\hline
\end{tabular}

\begin{tabular}{|c|c|r|r|r|}
\hline \multicolumn{5}{|c|}{ Exhaust } \\
\hline Cylinder & Lobe \# & Heel-Toe & Base Circle & \multicolumn{1}{c|}{ Lift } \\
\hline 1 & 3 & 46.540 & 39.391 & 7.149 \\
\hline 2 & 2 & 46.562 & 39.385 & 7.177 \\
\hline 3 & 7 & 46.540 & 39.370 & 7.170 \\
\hline 4 & 6 & 46.568 & 39.388 & 7.180 \\
\hline 5 & 11 & 46.563 & 39.392 & 7.171 \\
\hline 6 & 10 & 46.560 & 39.396 & 7.164 \\
\hline 7 & 15 & 46.562 & 39.388 & 7.174 \\
\hline 8 & 14 & 46.576 & 39.390 & 7.186 \\
\hline \multicolumn{5}{|l|}{} \\
\hline Average & 46.559 & 39.388 & 7.171 \\
\hline StDev & 0.013 & 0.008 & 0.011 \\
\hline Max & 46.576 & 39.396 & 7.186 \\
\hline Min & 46.540 & 39.370 & 7.149 \\
\hline
\end{tabular}




\section{Oak Ridge National Laboratory Proposal 08-58845 Powertrain Inspection Camshaft Heel to Toe Measurement}

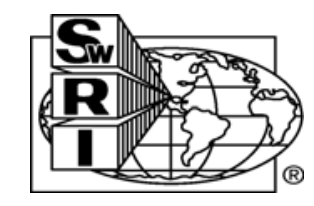

Date:

18-Aug-10

Technician:

RT

Micrometer: AN 005423

Standard: AN006504

Measurements in $\mathrm{mm}$

Lobes are numbered from front to rear on cam

Service manual specification for cam lobe lift - Intake - $6.82 \mathrm{~mm}$

Service manual specification for cam lobe lift - Exhaust - $6.96 \mathrm{~mm}$

\begin{tabular}{l}
\hline \multicolumn{7}{|c|}{ ORCS15 } \\
\begin{tabular}{|c|c|r|r|r|}
\hline \multicolumn{7}{|c|}{ Intake } \\
\hline Cylinder & Lobe \# & Heel-Toe & Base Circle & \multicolumn{1}{c|}{ Lift } \\
\hline 1 & 1 & 46.561 & 39.373 & 7.188 \\
\hline 2 & 4 & 46.580 & 39.396 & 7.184 \\
\hline 3 & 5 & 46.577 & 39.393 & 7.184 \\
\hline 4 & 8 & 46.577 & 39.384 & 7.193 \\
\hline 5 & 9 & 46.565 & 39.390 & 7.175 \\
\hline 6 & 12 & 46.582 & 39.402 & 7.180 \\
\hline 7 & 13 & 46.580 & 39.396 & 7.184 \\
\hline \multicolumn{7}{|l|}{} & 46.561 & 39.391 & 7.170 \\
\hline \multicolumn{7}{|c|}{} & 46.573 & 39.391 & 7.182 \\
\hline Average & 16 & 0.009 & 0.009 & 0.007 \\
\hline StDev & 46.582 & 39.402 & 7.193 \\
\hline Max & 46.561 & 39.373 & 7.170 \\
\hline Min
\end{tabular}
\end{tabular}

\begin{tabular}{|c|c|r|r|r|}
\hline \multicolumn{5}{|c|}{ Exhaust } \\
\hline Cylinder & Lobe \# & Heel-Toe & Base Circle & \multicolumn{1}{c|}{ Lift } \\
\hline 1 & 3 & 46.574 & 39.389 & 7.185 \\
\hline 2 & 2 & 46.564 & 39.382 & 7.182 \\
\hline 3 & 7 & 46.579 & 39.388 & 7.191 \\
\hline 4 & 6 & 46.571 & 39.390 & 7.181 \\
\hline 5 & 11 & 46.567 & 39.385 & 7.182 \\
\hline 6 & 10 & 46.570 & 39.398 & 7.172 \\
\hline 7 & 15 & 46.564 & 39.385 & 7.179 \\
\hline 8 & 14 & 46.573 & 39.388 & 7.185 \\
\hline \multicolumn{5}{|c|}{} \\
\hline Average & 46.570 & 39.388 & 7.182 \\
\hline StDev & 0.005 & 0.005 & 0.005 \\
\hline Max & 46.579 & 39.398 & 7.191 \\
\hline Min & 46.564 & 39.382 & 7.172 \\
\hline
\end{tabular}




\section{Oak Ridge National Laboratory Proposal 08-58845 Powertrain Inspection Camshaft Heel to Toe Measurement}

Date: 17-Sep-10

Technician:

RT

Micrometer: AN 005423

Standard: AN006504

Measurements in $\mathrm{mm}$

Lobes are numbered from front to rear on cam

Service manual specification for cam lobe lift - Intake - $6.82 \mathrm{~mm}$

Service manual specification for cam lobe lift - Exhaust $-6.96 \mathrm{~mm}$

\begin{tabular}{|c|c|c|c|c|}
\hline \multicolumn{5}{|c|}{ ORCS20 } \\
\hline \multicolumn{5}{|c|}{ Intake } \\
\hline Cylinder & Lobe \# & Heel-Toe & Base Circle & Lift \\
\hline 1 & 1 & 46.566 & 39.385 & 7.181 \\
\hline 2 & 4 & 46.580 & 39.397 & 7.183 \\
\hline 3 & 5 & 46.580 & 39.399 & 7.181 \\
\hline 4 & 8 & 46.582 & 39.392 & 7.190 \\
\hline 5 & 9 & 46.569 & 39.400 & 7.169 \\
\hline 6 & 12 & 46.583 & 39.403 & 7.180 \\
\hline 7 & 13 & 46.580 & 39.396 & 7.184 \\
\hline 8 & 16 & 46.587 & 39.407 & 7.180 \\
\hline Average & & 46.578 & 39.397 & 7.181 \\
\hline StDev & & 0.007 & 0.007 & 0.006 \\
\hline Max & & 46.587 & 39.407 & 7.190 \\
\hline Min & & 46.566 & 39.385 & 7.169 \\
\hline
\end{tabular}

\begin{tabular}{|c|c|r|r|r|}
\hline \multicolumn{5}{|c|}{ Exhaust } \\
\hline Cylinder & Lobe \# & Heel-Toe & Base Circle & \multicolumn{1}{c|}{ Lift } \\
\hline 1 & 3 & 46.579 & 39.396 & 7.183 \\
\hline 2 & 2 & 46.576 & 39.396 & 7.180 \\
\hline 3 & 7 & 46.578 & 39.392 & 7.186 \\
\hline 4 & 6 & 46.580 & 39.396 & 7.184 \\
\hline 5 & 11 & 46.576 & 39.398 & 7.178 \\
\hline 6 & 10 & 46.571 & 39.397 & 7.174 \\
\hline 7 & 15 & 46.597 & 39.410 & 7.187 \\
\hline 8 & 14 & 46.582 & 39.399 & 7.183 \\
\hline & \multicolumn{5}{|c}{} \\
\hline Average & \multicolumn{5}{|c|}{0} \\
\hline StDev & 46.580 & 39.398 & 7.182 \\
\hline Max & 0.008 & 0.005 & 0.004 \\
\hline Min & 46.597 & 39.410 & 7.187 \\
\hline
\end{tabular}


Oakridge National Laboratory

2006 Chevrolet Silverado

Intake Camshaft Lobe Heel to Toe Measurements at EOT

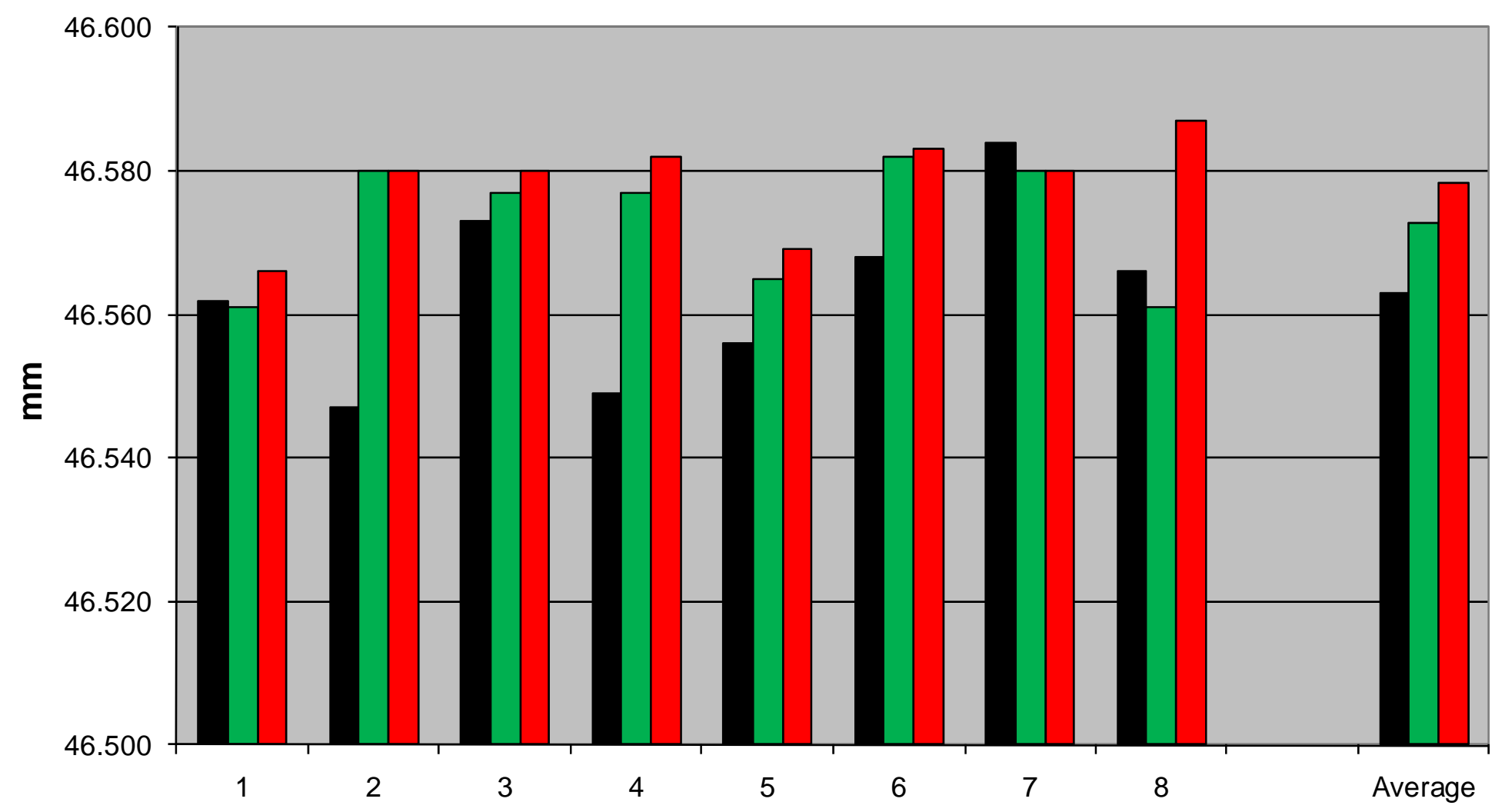

Cylinder Number 


\section{Oakridge National Laboratory \\ 2006 Chevrolet Silverado}

Exhaust Camshaft Lobe Heel to Toe Measurements at EOT

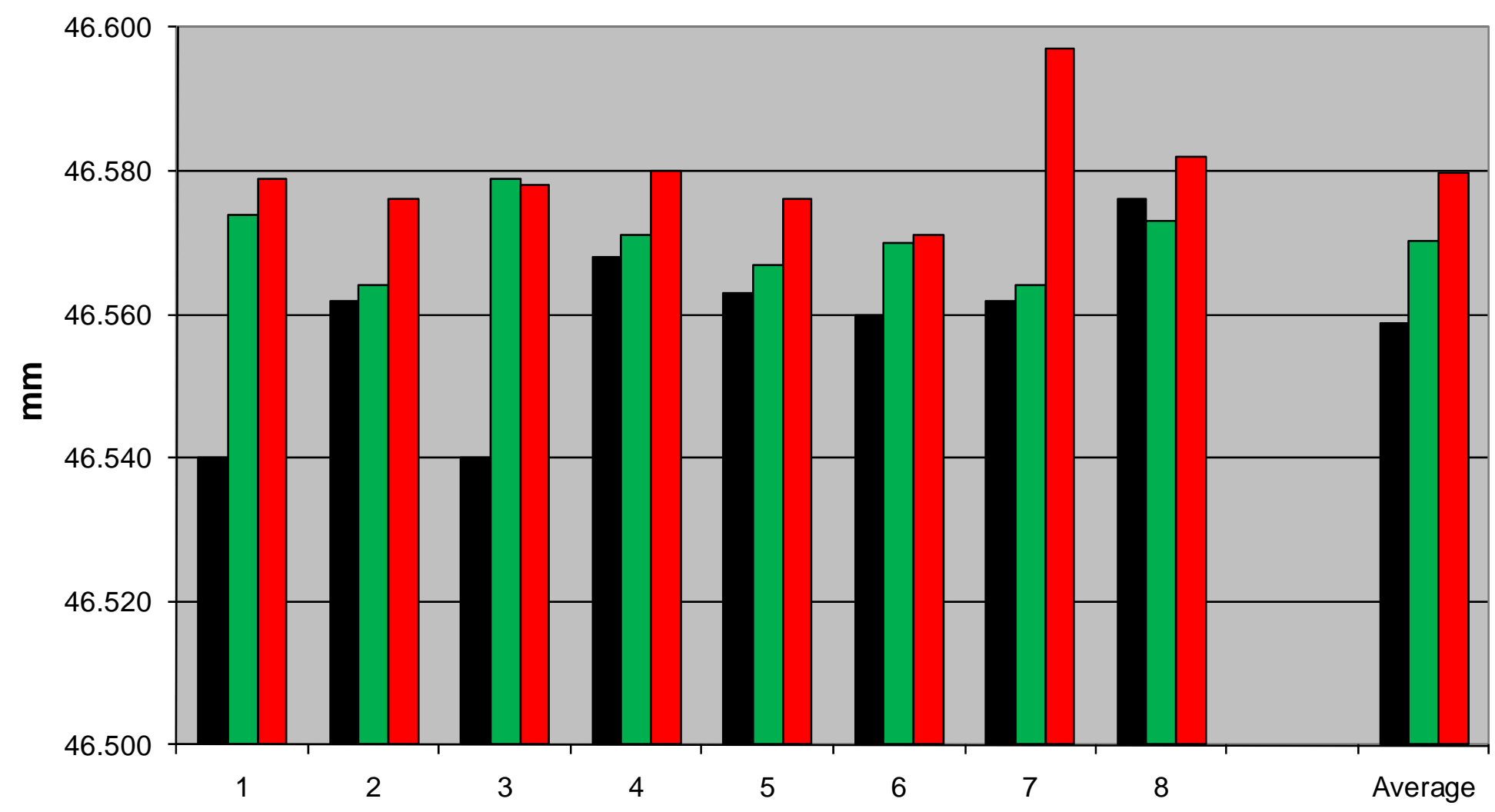

Cylinder Number 


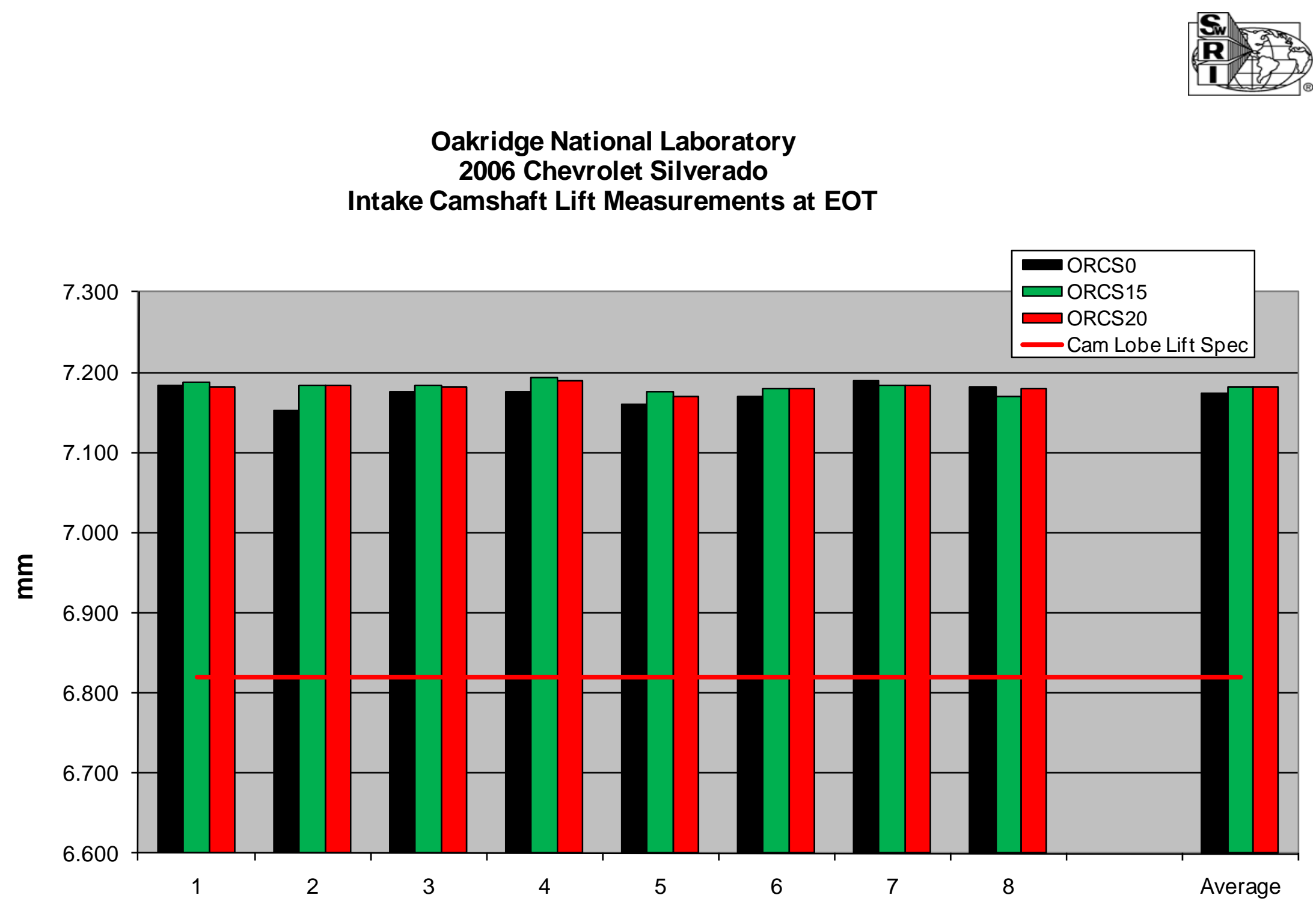

Cylinder Number 


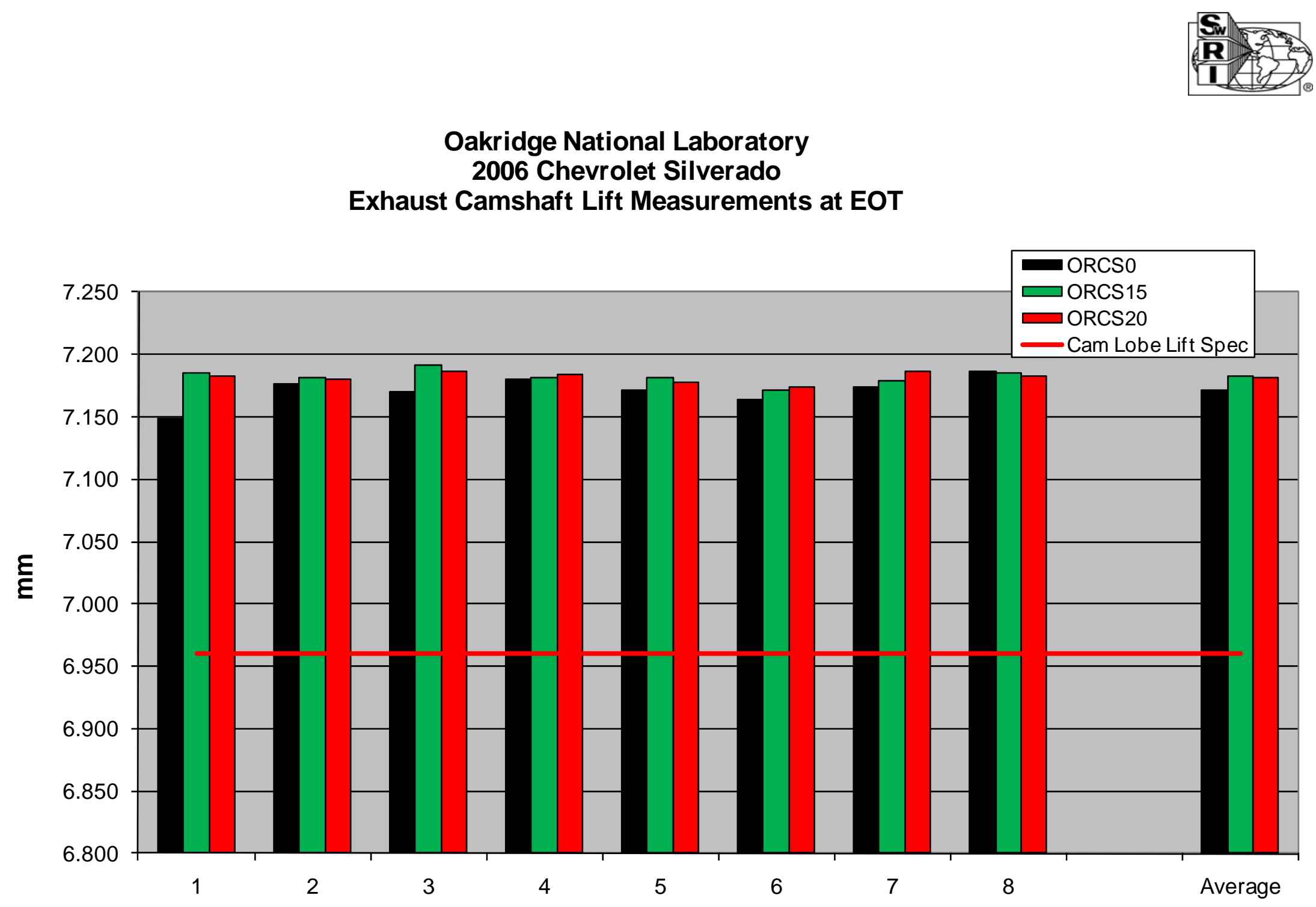

Cylinder Number 

Appendix B

Valve Seat Width and Valve Surface Contour 

Intake Valve Measured in the Precision Surface Measurement Machine

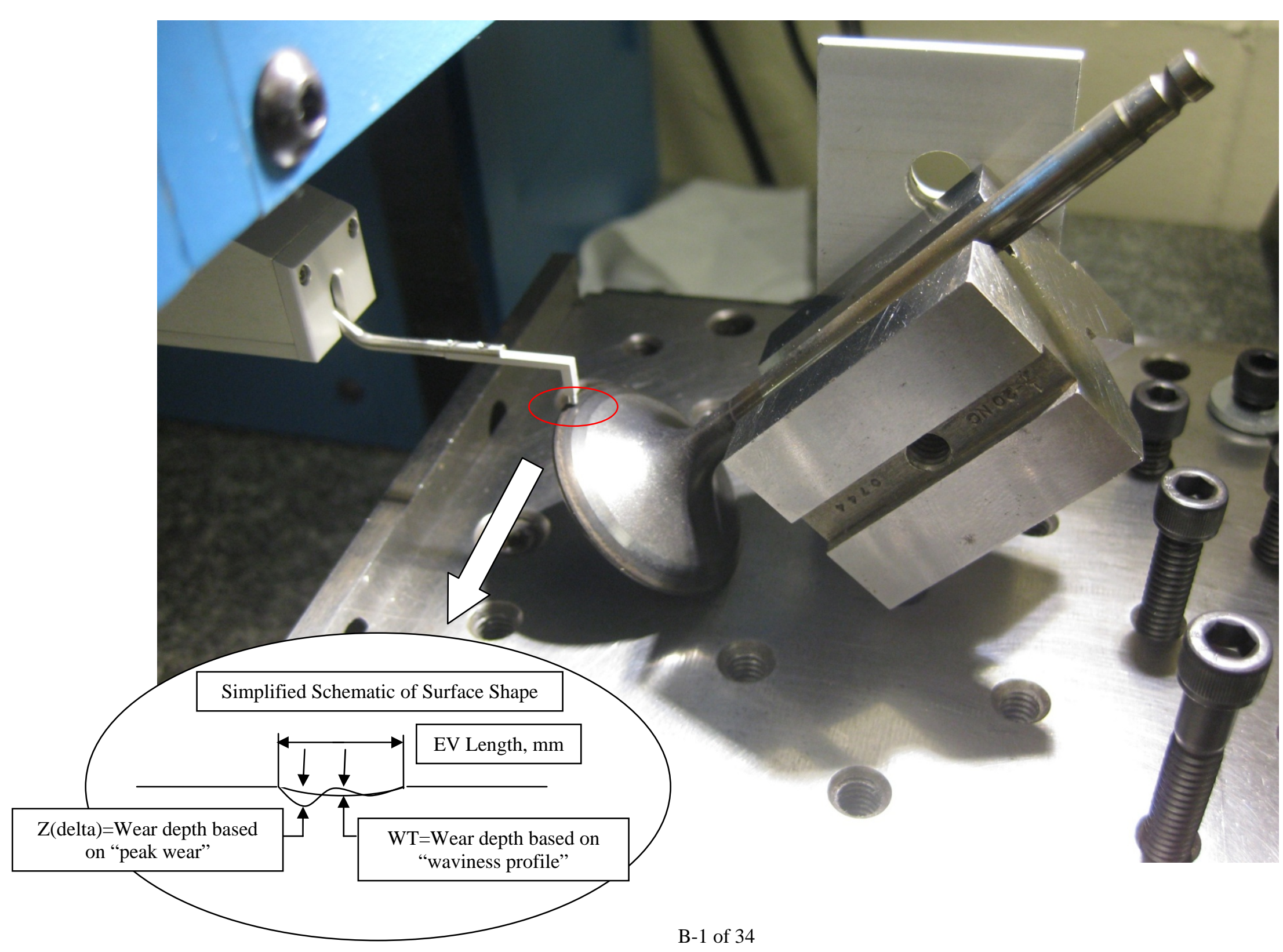




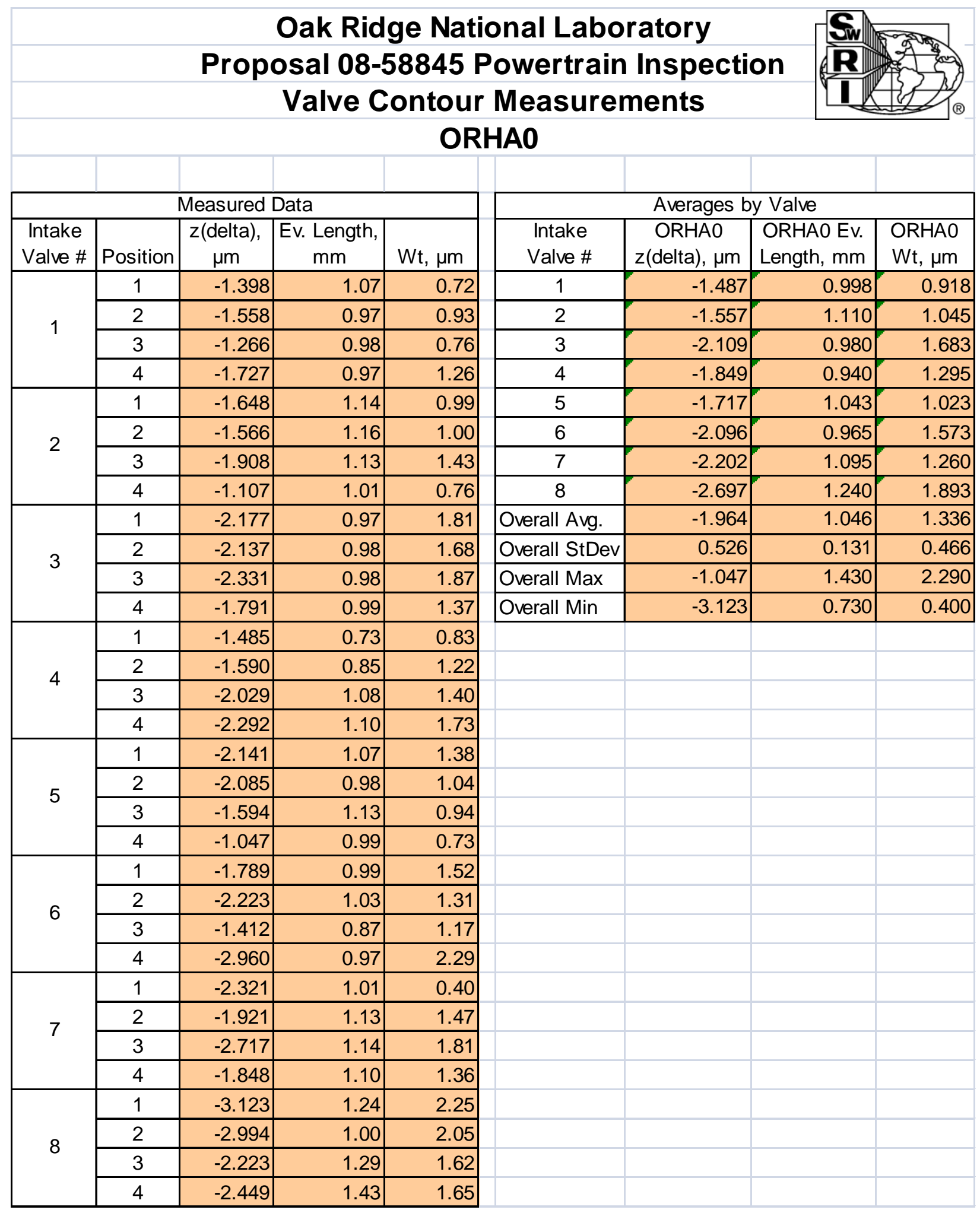




\begin{tabular}{|c|c|c|c|c|c|c|c|c|}
\hline & & & Oak Rid & ge Nat & onal Labo & ratory & & \\
\hline & & Prop & osal 08- & 58845 & owertrain & Inspecti & on & \\
\hline & & & Valve C & ontou & Measure & nents & & \\
\hline & & & & OR & HAO & & & \\
\hline & & Measured & Data & & & Averages b & y Valve & \\
\hline $\begin{array}{l}\text { Exhuast } \\
\text { Valve \# }\end{array}$ & Position & $\begin{array}{c}z \text { (delta) } \\
\mu \mathrm{m}\end{array}$ & \begin{tabular}{|c|}
$\begin{array}{c}\text { Ev. Length, } \\
\mathrm{mm}\end{array}$ \\
\end{tabular} & $\mathrm{Wt}, \mu \mathrm{m}$ & $\begin{array}{l}\text { Exhaust } \\
\text { Valve \# }\end{array}$ & $\begin{array}{c}\text { ORHAO } \\
z \text { (delta), } \mu \mathrm{m}\end{array}$ & $\begin{array}{l}\text { ORHA0 Ev. } \\
\text { Length, } \mathrm{mm}\end{array}$ & $\begin{array}{l}\text { ORHAO } \\
W t, \mu \mathrm{m}\end{array}$ \\
\hline & 1 & -20.852 & 1.52 & 20.16 & 1 & -12.218 & 1.463 & 11.770 \\
\hline 1 & 2 & -9.930 & 1.55 & 9.57 & 2 & -11.867 & 1.410 & 10.645 \\
\hline 1 & 3 & -6.215 & 1.43 & 5.97 & 3 & -12.417 & 1.488 & 11.710 \\
\hline & 4 & -11.875 & 1.35 & 11.38 & 4 & -9.097 & 1.568 & 8.168 \\
\hline & 1 & -7.420 & 1.36 & 6.47 & 5 & -8.759 & 1.645 & 8.110 \\
\hline 2 & 2 & -8.095 & 1.43 & 7.65 & 6 & -6.909 & 1.493 & 6.165 \\
\hline 2 & 3 & -9.901 & 1.28 & 8.33 & 7 & -10.038 & 1.620 & 9.448 \\
\hline & 4 & -22.050 & 1.57 & 20.13 & 8 & -8.243 & 1.573 & 7.748 \\
\hline & 1 & -17.324 & 1.23 & 16.80 & Overall Avg. & -9.943 & 1.532 & 9.220 \\
\hline 3 & 2 & -10.292 & 1.55 & 9.43 & Overall StDev & 3.825 & 0.115 & 3.719 \\
\hline 3 & 3 & -13.401 & 1.58 & 12.91 & Overall Max & -4.961 & 1.730 & 20.160 \\
\hline & 4 & -8.651 & 1.59 & 7.70 & Overall Min & -22.050 & 1.230 & 4.290 \\
\hline & 1 & -8.201 & 1.62 & 6.97 & & & & \\
\hline 4 & 2 & -10.773 & 1.64 & 9.59 & & & & \\
\hline & 3 & -7.370 & 1.48 & 6.66 & & & & \\
\hline & 4 & -10.042 & 1.53 & 9.45 & & & & \\
\hline & 1 & -10.306 & 1.68 & 9.92 & & & & \\
\hline 5 & 2 & -10.353 & 1.60 & 9.75 & & & & \\
\hline 5 & 3 & -6.717 & 1.61 & 6.05 & & & & \\
\hline & 4 & -7.660 & 1.69 & 6.72 & & & & \\
\hline & 1 & -7.126 & 1.46 & 6.61 & & & & \\
\hline 6 & 2 & -6.441 & 1.45 & 5.67 & & & & \\
\hline & 3 & -9.106 & 1.54 & 8.09 & & & & \\
\hline & 4 & -4.961 & 1.52 & 4.29 & & & & \\
\hline & 1 & -9.944 & 1.55 & 9.32 & & & & \\
\hline 7 & 2 & -7.731 & 1.52 & 7.34 & & & & \\
\hline$t$ & 3 & -11.075 & 1.73 & 10.38 & & & & \\
\hline & 4 & -11.401 & 1.68 & 10.75 & & & & \\
\hline & 1 & -8.439 & 1.61 & 7.78 & & & & \\
\hline 8 & 2 & -8.647 & 1.59 & 8.29 & & & & \\
\hline 8 & 3 & -7.663 & 1.53 & 7.19 & & & & \\
\hline & 4 & -8.222 & 1.56 & 7.73 & & & & \\
\hline
\end{tabular}




\begin{tabular}{|c|c|c|c|c|c|c|c|c|}
\hline & & & Oak Rid & ge Nat & onal Labo & ratory & & \\
\hline & & Prop & osal 08- & 58845 & owertrain & Inspecti & on & \\
\hline & & & Valve C & ontou & Measure & ments & & \\
\hline & & & & OR & A15 & & & \\
\hline & & Measured & Data & & & Averages b & y Valve & \\
\hline $\begin{array}{l}\text { Intake } \\
\text { Valve \# }\end{array}$ & Position & $\begin{array}{c}z \text { (delta) } \\
\mu \mathrm{m}\end{array}$ & \begin{tabular}{|c|}
$\begin{array}{c}\text { Ev. Length, } \\
\mathrm{mm}\end{array}$ \\
\end{tabular} & $\mathrm{Wt}, \mu \mathrm{m}$ & $\begin{array}{l}\text { Intake } \\
\text { Valve \# }\end{array}$ & \begin{tabular}{|c|} 
ORHA15 \\
$z$ (delta), $\mu \mathrm{m}$
\end{tabular} & $\begin{array}{l}\text { ORHA15 Ev. } \\
\text { Length, } \mathrm{mm}\end{array}$ & $\begin{array}{l}\text { ORHA15 } \\
W t, \mu \mathrm{m}\end{array}$ \\
\hline & 1 & -1.129 & 1.57 & 0.79 & 1 & -1.172 & 1.533 & 1.083 \\
\hline 1 & 2 & -1.041 & 1.36 & 0.94 & 2 & -1.373 & 0.975 & 1.203 \\
\hline$\perp$ & 3 & -1.272 & 1.59 & 1.28 & 3 & -1.878 & 1.175 & 1.263 \\
\hline & 4 & -1.247 & 1.61 & 1.32 & 4 & -1.681 & 0.975 & 1.390 \\
\hline & 1 & -1.270 & 0.86 & 0.87 & 5 & -2.880 & 1.628 & 2.553 \\
\hline 2 & 2 & -1.823 & 1.06 & 1.44 & 6 & -3.444 & 1.658 & 3.158 \\
\hline 2 & 3 & -1.123 & 1.12 & 1.12 & 7 & -2.262 & 1.618 & 2.163 \\
\hline & 4 & -1.274 & 0.86 & 1.38 & 8 & -2.939 & 1.693 & 2.803 \\
\hline & 1 & -2.212 & 1.19 & 1.76 & Overall Avg. & -2.203 & 1.407 & 1.952 \\
\hline 3 & 2 & -1.562 & 1.08 & 1.27 & Overall StDev & 0.870 & 0.307 & 0.820 \\
\hline 3 & 3 & -2.707 & 1.23 & 1.29 & Overall Max & -0.749 & 1.710 & 3.660 \\
\hline & 4 & -1.032 & 1.20 & 0.73 & Overall Min & -3.767 & 0.830 & 0.730 \\
\hline & 1 & -1.846 & 1.05 & 1.23 & & & & \\
\hline 4 & 2 & -2.446 & 1.03 & 1.74 & & & & \\
\hline & 3 & -0.749 & 0.83 & 1.20 & & & & \\
\hline & 4 & -1.682 & 0.99 & 1.39 & & & & \\
\hline & 1 & -2.535 & 1.70 & 2.24 & & & & \\
\hline 5 & 2 & -2.869 & 1.64 & 2.50 & & & & \\
\hline 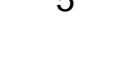 & 3 & -2.722 & 1.49 & 2.45 & & & & \\
\hline & 4 & -3.394 & 1.68 & 3.02 & & & & \\
\hline & 1 & -3.415 & 1.66 & 2.86 & & & & \\
\hline 6 & 2 & -3.382 & 1.58 & 3.66 & & & & \\
\hline & 3 & -3.212 & 1.70 & 2.82 & & & & \\
\hline & 4 & -3.767 & 1.69 & 3.29 & & & & \\
\hline & 1 & -2.821 & 1.70 & 2.35 & & & & \\
\hline 7 & 2 & -2.451 & 1.69 & 2.19 & & & & \\
\hline t & 3 & -1.669 & 1.54 & 2.14 & & & & \\
\hline & 4 & -2.105 & 1.54 & 1.97 & & & & \\
\hline & 1 & -2.752 & 1.69 & 2.55 & & & & \\
\hline 8 & 2 & -2.935 & 1.68 & 2.87 & & & & \\
\hline 8 & 3 & -2.921 & 1.71 & 2.95 & & & & \\
\hline & 4 & -3.146 & 1.69 & 2.84 & & & & \\
\hline
\end{tabular}




\begin{tabular}{|c|c|c|c|c|c|c|c|c|}
\hline & & & Oak Rid & ge Nat & onal Labo & ratory & & \\
\hline & & Prop & osal 08- & 58845 & owertrain & Inspecti & on & \\
\hline & & & Valve C & ontou & Measure & nents & & \\
\hline & & & & OR & A15 & & & \\
\hline & & Measured & Data & & & Averages b & y Valve & \\
\hline $\begin{array}{l}\text { Exhuast } \\
\text { Valve \# }\end{array}$ & Position & $\begin{array}{c}z \text { (delta) } \\
\mu \mathrm{m}\end{array}$ & \begin{tabular}{|c|}
$\begin{array}{c}\text { Ev. Length, } \\
\mathrm{mm}\end{array}$ \\
\end{tabular} & $\mathrm{Wt}, \mu \mathrm{m}$ & $\begin{array}{l}\text { Exhaust } \\
\text { Valve \# }\end{array}$ & $\begin{array}{c}\text { ORHA15 } \\
z \text { (delta), } \mu \mathrm{m}\end{array}$ & $\begin{array}{l}\text { ORHA15 Ev. } \\
\text { Length, } \mathrm{mm}\end{array}$ & $\begin{array}{l}\text { ORHA15 } \\
W t, \mu \mathrm{m}\end{array}$ \\
\hline & 1 & -9.906 & 1.55 & 9.02 & 1 & -9.406 & 1.503 & 8.423 \\
\hline 1 & 2 & -10.619 & 1.49 & 10.23 & 2 & -6.349 & 1.390 & 5.733 \\
\hline 1 & 3 & -8.937 & 1.49 & 7.04 & 3 & -7.770 & 1.433 & 7.025 \\
\hline & 4 & -8.162 & 1.48 & 7.40 & 4 & -5.697 & 1.255 & 5.158 \\
\hline & 1 & -3.326 & 1.32 & 2.98 & 5 & -6.100 & 1.315 & 5.433 \\
\hline 2 & 2 & -9.819 & 1.34 & 8.93 & 6 & -6.389 & 1.428 & 5.878 \\
\hline 2 & 3 & -8.059 & 1.42 & 7.34 & 7 & -8.416 & 1.415 & 9.605 \\
\hline & 4 & -4.193 & 1.48 & 3.68 & 8 & -9.016 & 1.428 & 8.705 \\
\hline & 1 & -11.855 & 1.34 & 11.04 & Overall Avg. & -7.393 & \begin{tabular}{l|l}
1.396 \\
\end{tabular} & 6.995 \\
\hline 3 & 2 & -4.315 & 1.45 & 3.65 & Overall StDev & 3.412 & 0.114 & 3.128 \\
\hline 3 & 3 & -9.438 & 1.44 & 8.80 & Overall Max & -2.837 & 1.570 & 14.440 \\
\hline & 4 & -5.471 & 1.50 & 4.61 & Overall Min & -15.397 & 1.190 & 2.590 \\
\hline & 1 & -5.337 & 1.31 & 4.89 & & & & \\
\hline 4 & 2 & -9.715 & 1.26 & 8.99 & & & & \\
\hline & 3 & -4.707 & 1.26 & 4.16 & & & & \\
\hline & 4 & -3.028 & 1.19 & 2.59 & & & & \\
\hline & 1 & -7.123 & 1.50 & 5.91 & & & & \\
\hline 5 & 2 & -8.530 & 1.31 & 7.58 & & & & \\
\hline 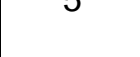 & 3 & -5.725 & 1.25 & 5.01 & & & & \\
\hline & 4 & -3.023 & 1.20 & 3.23 & & & & \\
\hline & 1 & -6.749 & 1.48 & 7.20 & & & & \\
\hline 6 & 2 & -3.823 & 1.48 & 2.76 & & & & \\
\hline & 3 & -5.724 & 1.23 & 5.06 & & & & \\
\hline & 4 & -9.258 & 1.52 & 8.49 & & & & \\
\hline & 1 & -2.837 & 1.57 & 9.97 & & & & \\
\hline 7 & 2 & -12.444 & 1.30 & 11.75 & & & & \\
\hline$t$ & 3 & -4.098 & 1.33 & 3.86 & & & & \\
\hline & 4 & -14.283 & 1.46 & 12.84 & & & & \\
\hline & 1 & -8.990 & 1.55 & 8.44 & & & & \\
\hline 8 & 2 & -15.397 & 1.52 & 14.44 & & & & \\
\hline 8 & 3 & -2.992 & 1.35 & 4.15 & & & & \\
\hline & 4 & -8.686 & 1.29 & 7.79 & & & & \\
\hline
\end{tabular}




\begin{tabular}{|c|c|c|c|c|c|c|c|c|}
\hline & & & Oak Rid & ge Nat & onal Labo & ratory & & \\
\hline & & Prop & osal 08- & 58845 & owertrain & Inspecti & on & \\
\hline & & & Valve C & ontou & Measure & ments & & \\
\hline & & & & OR & A20 & & & \\
\hline & & Measured & Data & & & Averages b & y Valve & \\
\hline $\begin{array}{l}\text { Intake } \\
\text { Valve \# }\end{array}$ & Position & $\begin{array}{c}z \text { (delta) } \\
\mu \mathrm{m}\end{array}$ & \begin{tabular}{|c|}
$\begin{array}{c}\text { Ev. Length, } \\
\mathrm{mm}\end{array}$ \\
\end{tabular} & $\mathrm{Wt}, \mu \mathrm{m}$ & $\begin{array}{l}\text { Intake } \\
\text { Valve \# }\end{array}$ & \begin{tabular}{|c|} 
ORHA20 \\
$z$ (delta), $\mu \mathrm{m}$
\end{tabular} & $\begin{array}{l}\text { ORHA20 Ev. } \\
\text { Length, } \mathrm{mm}\end{array}$ & $\begin{array}{l}\text { ORHA20 } \\
W t, \mu \mathrm{m}\end{array}$ \\
\hline & 1 & -1.443 & 0.85 & 1.17 & 1 & -1.274 & 0.765 & 1.083 \\
\hline 1 & 2 & -1.407 & 0.77 & 1.28 & 2 & -1.137 & 0.755 & 0.923 \\
\hline$\perp$ & 3 & -1.406 & 0.76 & 1.18 & 3 & -1.439 & 0.708 & 1.093 \\
\hline & 4 & -0.839 & 0.68 & 0.70 & 4 & -1.318 & 0.798 & 1.113 \\
\hline & 1 & -1.117 & 0.66 & 0.95 & 5 & -1.707 & 1.143 & 1.453 \\
\hline 2 & 2 & -1.004 & 0.71 & 0.77 & 6 & -0.986 & 0.815 & 0.698 \\
\hline 2 & 3 & -1.200 & 0.95 & 1.06 & 7 & -1.882 & 1.023 & 1.645 \\
\hline & 4 & -1.225 & 0.70 & 0.91 & 8 & -1.206 & 0.803 & 0.793 \\
\hline & 1 & -1.145 & 0.74 & 0.89 & Overall Avg. & -1.368 & 0.851 & 1.100 \\
\hline 3 & 2 & -0.897 & 0.66 & 0.71 & Overall StDev & 0.402 & 0.161 & 0.377 \\
\hline 3 & 3 & -2.557 & 0.76 & 1.93 & Overall Max & -0.720 & 1.160 & 1.930 \\
\hline & 4 & -1.157 & 0.67 & 0.84 & Overall Min & -2.557 & 0.660 & 0.610 \\
\hline & 1 & -1.389 & 0.84 & 1.15 & & & & \\
\hline 4 & 2 & -1.267 & 0.80 & 1.08 & & & & \\
\hline & 3 & -1.407 & 0.79 & 1.08 & & & & \\
\hline & 4 & -1.208 & 0.76 & 1.14 & & & & \\
\hline & 1 & -1.511 & 1.13 & 1.29 & & & & \\
\hline 5 & 2 & -2.098 & 1.15 & 1.73 & & & & \\
\hline & 3 & -1.588 & 1.16 & 1.39 & & & & \\
\hline & 4 & -1.631 & 1.13 & 1.40 & & & & \\
\hline & 1 & -1.068 & 0.80 & 0.82 & & & & \\
\hline 6 & 2 & -0.720 & 0.81 & 0.61 & & & & \\
\hline & 3 & -1.143 & 0.87 & 0.71 & & & & \\
\hline & 4 & -1.012 & 0.78 & 0.65 & & & & \\
\hline & 1 & -1.883 & 1.10 & 1.69 & & & & \\
\hline 7 & 2 & -1.646 & 0.77 & 1.46 & & & & \\
\hline$t$ & 3 & -2.133 & 1.11 & 1.88 & & & & \\
\hline & 4 & -1.867 & 1.11 & 1.55 & & & & \\
\hline & 1 & -1.167 & 0.84 & 0.62 & & & & \\
\hline 8 & 2 & -1.319 & 0.76 & 0.94 & & & & \\
\hline & 3 & -1.321 & 0.85 & 0.99 & & & & \\
\hline & 4 & -1.016 & 0.76 & 0.62 & & & & \\
\hline
\end{tabular}




\begin{tabular}{|c|c|c|c|c|c|c|c|c|}
\hline & & & Oak Rid & ge Nat & onal Labo & ratory & & \\
\hline & & Prop & osal 08- & 58845 & owertrain & Inspecti & on & \\
\hline & & & Valve C & ontou & Measure & ments & & \\
\hline & & & & OR & A20 & & & \\
\hline & & Measured & Data & & & Averages b & y Valve & \\
\hline $\begin{array}{l}\text { Exhuast } \\
\text { Valve \# }\end{array}$ & Position & $\begin{array}{c}z \text { (delta) } \\
\mu \mathrm{m}\end{array}$ & \begin{tabular}{|c|}
$\begin{array}{c}\text { Ev. Length, } \\
\mathrm{mm}\end{array}$ \\
\end{tabular} & $\mathrm{Wt}, \mu \mathrm{m}$ & $\begin{array}{l}\text { Exhaust } \\
\text { Valve \# }\end{array}$ & \begin{tabular}{|c|} 
ORHA20 \\
$z$ (delta), $\mu \mathrm{m}$
\end{tabular} & $\begin{array}{l}\text { ORHA20 Ev. } \\
\text { Length, } \mathrm{mm}\end{array}$ & $\begin{array}{l}\text { ORHA20 } \\
W t, \mu \mathrm{m}\end{array}$ \\
\hline & 1 & -7.544 & 1.61 & 6.74 & 1 & -10.457 & 1.640 & 9.433 \\
\hline 1 & 2 & -14.879 & 1.64 & 13.65 & 2 & -7.817 & 1.583 & 6.853 \\
\hline 1 & 3 & -11.177 & 1.63 & 10.48 & 3 & -10.688 & 1.680 & 10.155 \\
\hline & 4 & -8.226 & 1.68 & 6.86 & 4 & -10.663 & 1.678 & 9.853 \\
\hline & 1 & -8.910 & 1.59 & 8.09 & 5 & -14.242 & 1.888 & 13.348 \\
\hline 2 & 2 & -5.983 & 1.49 & 5.85 & 6 & -16.299 & 1.755 & 15.468 \\
\hline 2 & 3 & -5.829 & 1.62 & 4.63 & 7 & -14.522 & 1.820 & 13.688 \\
\hline & 4 & -10.544 & 1.63 & 8.84 & 8 & -12.392 & 1.795 & 11.693 \\
\hline & 1 & -14.191 & 1.68 & 13.55 & Overall Avg. & -12.135 & 1.730 & 11.311 \\
\hline 3 & 2 & -11.424 & 1.66 & 11.05 & Overall StDev & 3.526 & 0.111 & 3.489 \\
\hline 3 & 3 & -8.672 & 1.67 & 8.16 & Overall Max & -5.829 & 2.000 & 17.980 \\
\hline & 4 & -8.465 & 1.71 & 7.86 & Overall Min & -18.805 & 1.490 & 4.630 \\
\hline & 1 & -11.533 & 1.63 & 10.58 & & & & \\
\hline 4 & 2 & -8.638 & 1.69 & 7.82 & & & & \\
\hline & 3 & -11.821 & 1.72 & 10.82 & & & & \\
\hline & 4 & -10.658 & 1.67 & 10.19 & & & & \\
\hline & 1 & -16.121 & 1.92 & 15.15 & & & & \\
\hline 5 & 2 & -15.420 & 1.87 & 14.55 & & & & \\
\hline 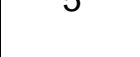 & 3 & -12.300 & 1.86 & 11.69 & & & & \\
\hline & 4 & -13.125 & 1.90 & 12.00 & & & & \\
\hline & 1 & -17.155 & 1.81 & 16.33 & & & & \\
\hline 6 & 2 & -18.205 & 1.73 & 17.35 & & & & \\
\hline 0 & 3 & -14.757 & 1.74 & 14.28 & & & & \\
\hline & 4 & -15.079 & 1.74 & 13.91 & & & & \\
\hline & 1 & -18.805 & 2.00 & 17.98 & & & & \\
\hline 7 & 2 & -16.680 & 1.82 & 15.72 & & & & \\
\hline$t$ & 3 & -9.851 & 1.66 & 9.03 & & & & \\
\hline & 4 & -12.752 & 1.80 & 12.02 & & & & \\
\hline & 1 & -8.907 & 1.79 & 8.56 & & & & \\
\hline 8 & 2 & -14.977 & 1.81 & 14.09 & & & & \\
\hline 8 & 3 & -15.031 & 1.76 & 14.23 & & & & \\
\hline & 4 & -10.652 & 1.82 & 9.89 & & & & \\
\hline
\end{tabular}




\section{Oakridge National Laboratory \\ 2007 Honda Accord \\ Intake Valve Contour Measurements at EOT}

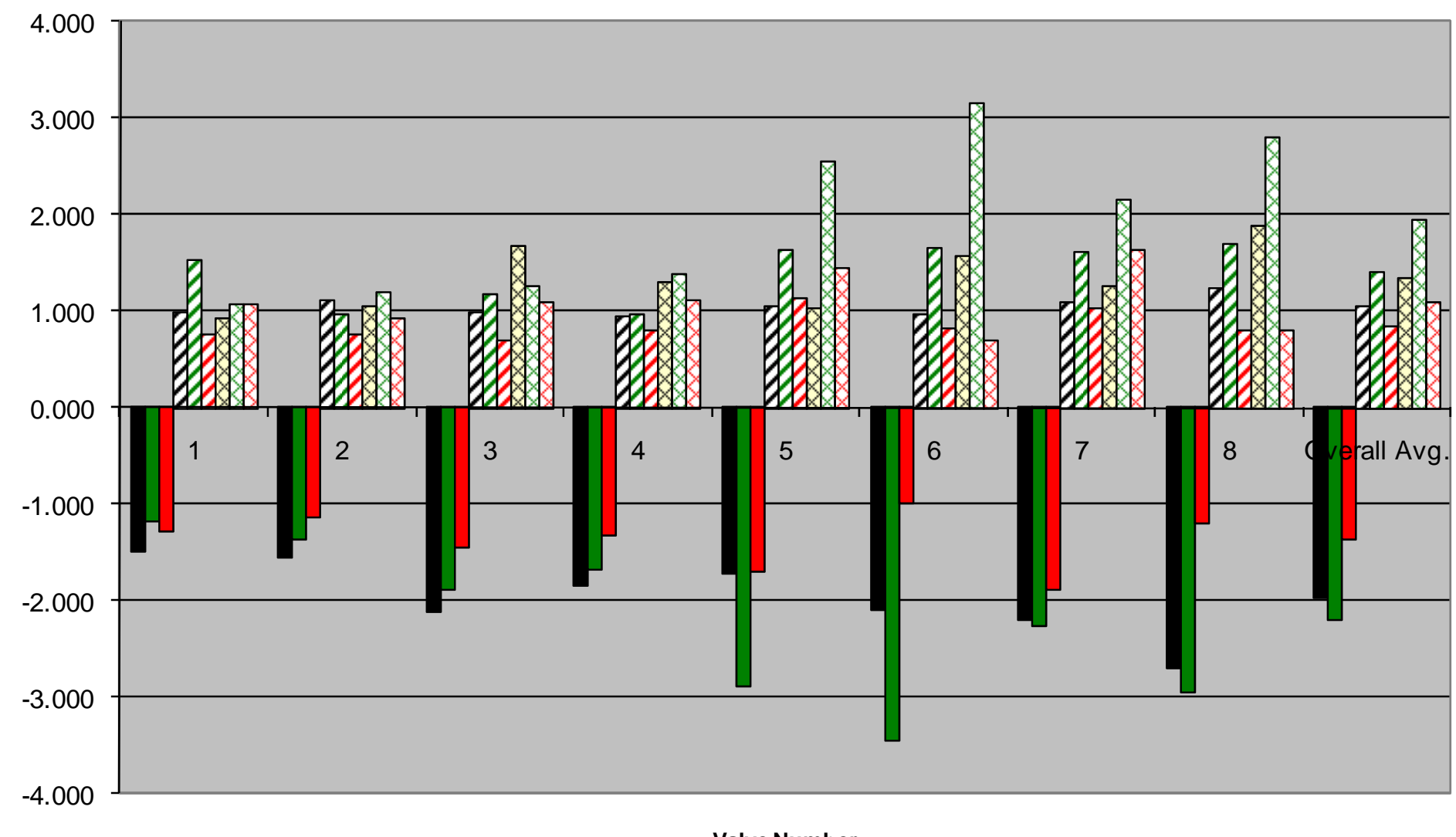

Valve Number
- ORHAO z(delta), $\mu \mathrm{m}$

口ORHA15 z(delta), $\mu \mathrm{m}$ 口ORHA20 z(delta), $\mu \mathrm{m}$ 口ORHAO Ev. Length, $\mathrm{mm}$ $\square$ ORHA15 Ev. Length, $\mathrm{mm}$ $\square O R H A 20$ Ev. Length, $\mathrm{mm}$ $\square$ ORHAO Wt, $\mu \mathrm{m}$ $\square$ ORHA15 Wt, $\mu \mathrm{m}$ QORHA20 Wt, $\mu \mathrm{m}$ 


\section{Oakridge National Laboratory \\ 2007 Honda Accord \\ Exhaust Valve Contour Measurements at EOT}

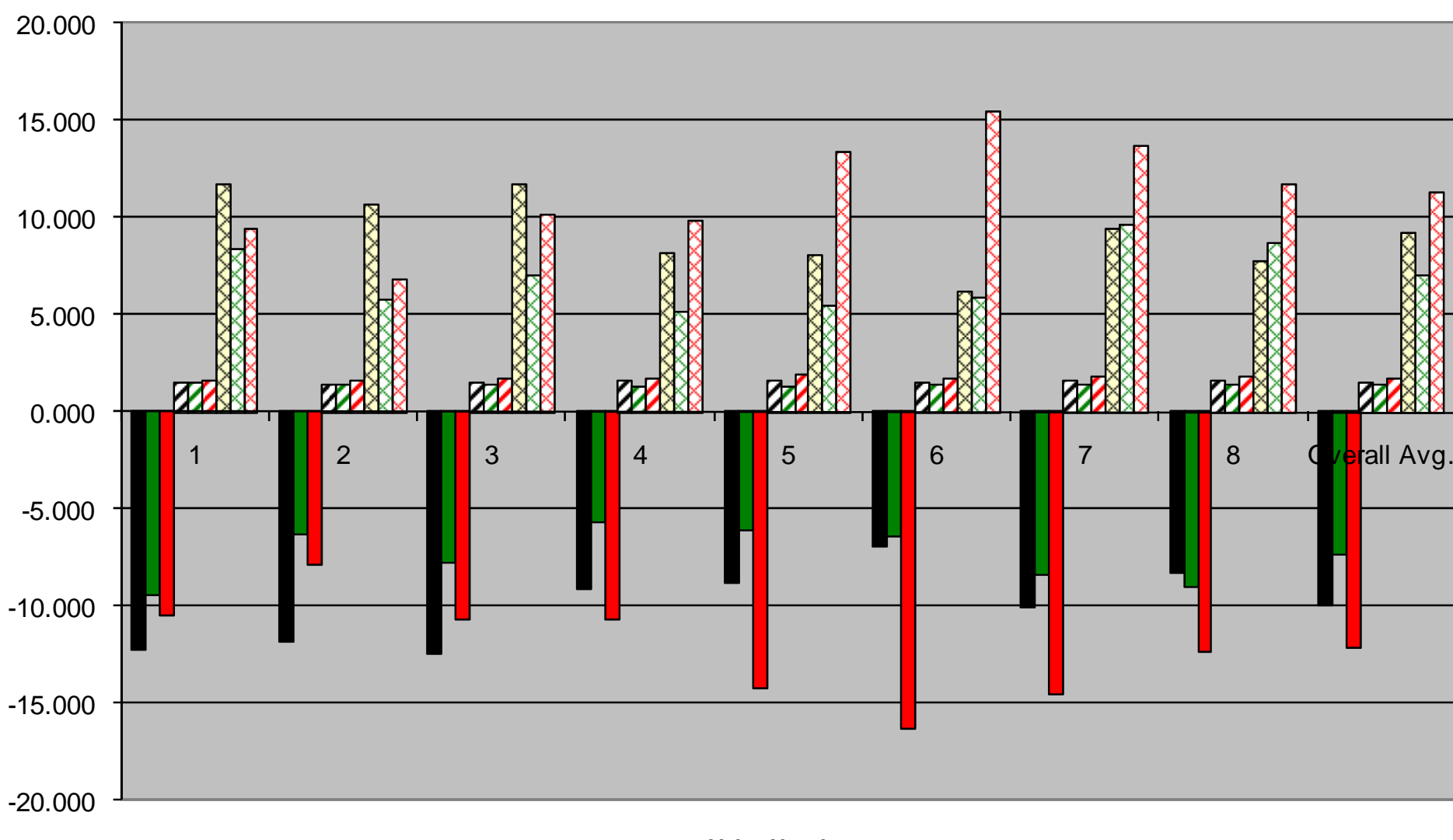

- ORHAO z(delta), $\mu \mathrm{m}$

口ORHA15 z(delta), $\mu \mathrm{m}$

-ORHA20 z(delta), $\mu \mathrm{m}$

口ORHAO Ev. Length, $\mathrm{mm}$

ఐORHA15 Ev. Length, $\mathrm{mm}$

$\square \mathrm{ORHA20}$ Ev. Length, $\mathrm{mm}$

$\square$ ORHAO Wt, $\mu \mathrm{m}$

$\triangle O R H A 15 \mathrm{Wt}, \mu \mathrm{m}$

$\triangle \mathrm{ORHA} 20 \mathrm{Wt}, \mu \mathrm{m}$

Valve Number 


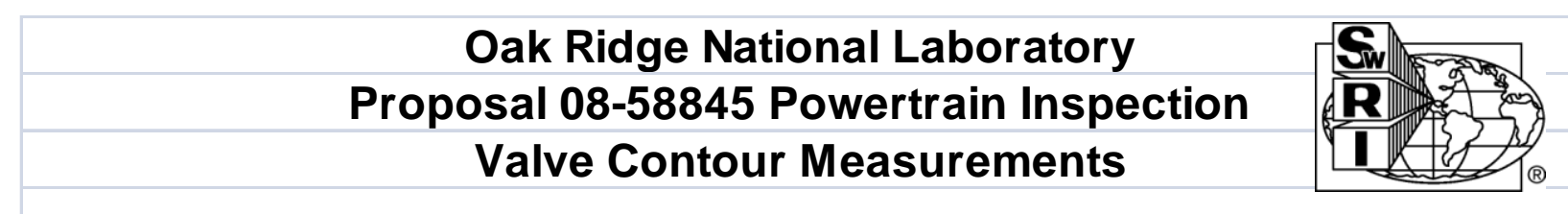

\begin{tabular}{|c|c|c|c|c|}
\hline \multicolumn{5}{|c|}{ ORDC0 } \\
\hline $\begin{array}{c}\text { Intake } \\
\text { Valve \# }\end{array}$ & Position & $\begin{array}{c}\mathrm{z}(\text { delta }) \\
\mu \mathrm{m}\end{array}$ & $\begin{array}{c}\text { Ev. } \\
\text { Length, } \\
\mathrm{mm}\end{array}$ & $\mathrm{Wt}, \mu \mathrm{m}$ \\
\hline \multirow{4}{*}{1} & 1 & -3.485 & 1.27 & 1.73 \\
\hline & 2 & -3.727 & 1.22 & 2.15 \\
\hline & 3 & -3.507 & 1.28 & 2.33 \\
\hline & 4 & -3.157 & 1.44 & 2.36 \\
\hline \multirow{4}{*}{2} & 1 & -3.497 & 1.42 & 2.54 \\
\hline & 2 & -11.840 & 1.34 & 9.84 \\
\hline & 3 & -6.574 & 1.44 & 5.24 \\
\hline & 4 & -4.129 & 1.44 & 3.48 \\
\hline \multirow{4}{*}{3} & 1 & -2.974 & 1.95 & 2.27 \\
\hline & 2 & -2.719 & 1.94 & 2.19 \\
\hline & 3 & -4.126 & 1.92 & 2.58 \\
\hline & 4 & -2.530 & 2.00 & 1.89 \\
\hline \multirow{4}{*}{4} & 1 & -2.319 & 1.10 & 1.20 \\
\hline & 2 & -1.469 & 0.97 & 1.14 \\
\hline & 3 & -2.013 & 1.13 & 1.43 \\
\hline & 4 & -3.606 & 1.17 & 1.56 \\
\hline \multirow{4}{*}{5} & 1 & -3.012 & 0.91 & 2.38 \\
\hline & 2 & -2.328 & 0.75 & 1.63 \\
\hline & 3 & -3.212 & 1.38 & 2.70 \\
\hline & 4 & -3.349 & 1.20 & 2.32 \\
\hline \multirow{4}{*}{6} & 1 & -3.604 & 1.44 & 2.12 \\
\hline & 2 & -3.491 & 1.38 & 1.84 \\
\hline & 3 & -4.366 & 1.38 & 2.75 \\
\hline & 4 & -2.635 & 1.35 & 2.04 \\
\hline
\end{tabular}

\begin{tabular}{|c|c|c|c|c|}
\hline \multicolumn{5}{|c|}{ ORDC15 } \\
\hline $\begin{array}{l}\text { Intake } \\
\text { Valve \# }\end{array}$ & Position & $\begin{array}{c}z \text { (delta), } \\
\mu \mathrm{m}\end{array}$ & $\begin{array}{c}\text { Ev. } \\
\text { Length, } \\
\mathrm{mm}\end{array}$ & $\mathrm{Wt}, \mu \mathrm{m}$ \\
\hline \multirow{4}{*}{1} & 1 & -3.401 & 1.96 & 2.43 \\
\hline & 2 & -2.485 & 2.14 & 1.64 \\
\hline & 3 & -2.429 & 2.01 & 1.49 \\
\hline & 4 & -2.924 & 2.06 & 2.25 \\
\hline \multirow{4}{*}{2} & 1 & -5.197 & 2.20 & 3.43 \\
\hline & 2 & -5.731 & 2.31 & 5.05 \\
\hline & 3 & -5.339 & 1.63 & 4.34 \\
\hline & 4 & -5.127 & 1.87 & 4.68 \\
\hline \multirow{4}{*}{3} & 1 & -5.549 & 2.06 & 4.49 \\
\hline & 2 & -3.940 & 1.98 & 3.44 \\
\hline & 3 & -4.040 & 2.51 & 3.53 \\
\hline & 4 & -5.491 & 2.24 & 4.78 \\
\hline \multirow{4}{*}{4} & 1 & -3.301 & 2.39 & 2.52 \\
\hline & 2 & -3.024 & 2.39 & 2.04 \\
\hline & 3 & -2.630 & 2.46 & 2.13 \\
\hline & 4 & -3.568 & 2.30 & 2.69 \\
\hline \multirow{4}{*}{5} & 1 & -2.990 & 2.09 & 2.28 \\
\hline & 2 & -2.444 & 2.03 & 1.76 \\
\hline & 3 & -2.730 & 2.00 & 3.46 \\
\hline & 4 & -3.550 & 1.87 & 2.50 \\
\hline \multirow{4}{*}{6} & 1 & -3.146 & 1.42 & 2.41 \\
\hline & 2 & -2.177 & 1.41 & 1.38 \\
\hline & 3 & -2.049 & 1.31 & 1.45 \\
\hline & 4 & \begin{tabular}{|c|}
-2.991 \\
\end{tabular} & 1.33 & 1.84 \\
\hline
\end{tabular}

\begin{tabular}{|l|r|r|r|}
\hline \multicolumn{5}{|c|}{ Averages by Valve } \\
\hline $\begin{array}{c}\text { Intake } \\
\text { Valve \# }\end{array}$ & $\begin{array}{c}\text { ORDC0 } \\
\text { z(delta), } \\
\mu \mathrm{m}\end{array}$ & $\begin{array}{c}\text { ORDC0 } \\
\text { Ev. } \\
\text { Length, } \\
\mathrm{mm}\end{array}$ & $\begin{array}{c}\text { ORDC0 } \\
\mathrm{Wt}, \mu \mathrm{m}\end{array}$ \\
\hline 1 & -3.469 & 1.303 & 2.143 \\
\hline 2 & -6.510 & 1.410 & 5.275 \\
\hline 3 & -3.087 & 1.953 & 2.233 \\
\hline 4 & -2.352 & 1.093 & 1.333 \\
\hline 5 & -2.975 & 1.060 & 2.258 \\
\hline 6 & -3.524 & 1.388 & 2.188 \\
\hline Average & -3.653 & 1.368 & 2.571 \\
\hline StDev & 2.002 & 0.322 & 1.754 \\
\hline Max & -1.469 & 2.000 & 9.840 \\
\hline Min & -11.840 & 0.750 & 1.140 \\
\hline
\end{tabular}

\begin{tabular}{|l|r|r|r|}
\hline \multicolumn{5}{|c|}{ Averages by Valve } \\
\hline $\begin{array}{c}\text { Intake } \\
\text { Valve \# }\end{array}$ & $\begin{array}{c}\text { ORDC15 } \\
\text { (delta), } \\
\mu \mathrm{m}\end{array}$ & $\begin{array}{c}\text { ORDC15 } \\
\text { Ev. } \\
\text { Length, } \\
\mathrm{mm}\end{array}$ & $\begin{array}{c}\text { ORDC15 } \\
\text { Wt, } \mu \mathrm{m}\end{array}$ \\
\hline 1 & -2.810 & 2.043 & 1.953 \\
\hline 2 & -5.349 & 2.003 & 4.375 \\
\hline 3 & -4.755 & 2.198 & 4.060 \\
\hline 4 & -3.131 & 2.385 & 2.345 \\
\hline 5 & -2.929 & 1.998 & 2.500 \\
\hline 6 & -2.591 & 1.368 & 1.770 \\
\hline Average & -3.594 & 1.999 & 2.834 \\
\hline StDev & 1.181 & 0.354 & 1.151 \\
\hline Max & -2.049 & 2.510 & 5.050 \\
\hline Min & -5.731 & 1.310 & 1.380 \\
\hline
\end{tabular}




\begin{tabular}{|c|c|c|c|c|c|c|c|c|c|}
\hline & & & ak Rid & ge Nati & lal Lak & orator & & $S w$ & \\
\hline & & Propo & sal 08- & 8845 P & wertra & in Insp & ection & & \\
\hline & & & Jalve C & ontour & leasur & ement & & & \\
\hline & & ORDC0 & & & & & ORDC15 & & \\
\hline $\begin{array}{l}\text { Exhaust } \\
\text { Valve \# }\end{array}$ & Position & $\begin{array}{c}z \text { (delta) } \\
\mu \mathrm{m}\end{array}$ & $\begin{array}{c}\text { Ev. } \\
\text { Length, } \\
\mathrm{mm}\end{array}$ & $\mathrm{Wt}, \mu \mathrm{m}$ & $\begin{array}{l}\text { Exhaust } \\
\text { Valve \# }\end{array}$ & Position & $\begin{array}{c}z \text { (delta) } \\
\mu m\end{array}$ & $\begin{array}{c}\text { Ev. } \\
\text { Length, } \\
\mathrm{mm}\end{array}$ & $\mathrm{Wt}, \mu \mathrm{m}$ \\
\hline & 1 & -26.525 & 1.84 & 26.90 & & 1 & -7.861 & 2.17 & 6.00 \\
\hline 1 & 2 & \begin{tabular}{l|}
-22.266 \\
\end{tabular} & 1.96 & 21.39 & 1 & 2 & -14.234 & 2.17 & 13.04 \\
\hline 1 & 3 & \begin{tabular}{|l|}
-32.723 \\
\end{tabular} & 2.02 & 31.58 & 1 & 3 & -7.819 & 2.17 & 6.82 \\
\hline & 4 & \begin{tabular}{|l|}
-31.927 \\
\end{tabular} & 2.06 & 30.99 & & 4 & -13.977 & 2.13 & 13.65 \\
\hline & 1 & -15.561 & 2.11 & 17.16 & & 1 & -12.893 & 1.97 & 15.40 \\
\hline 2 & 2 & -5.598 & 2.22 & 12.86 & 2 & 2 & -20.452 & 2.15 & 19.35 \\
\hline 2 & 3 & -19.420 & 2.15 & 18.01 & 2 & 3 & \begin{tabular}{|l|}
-9.203 \\
\end{tabular} & 1.93 & 8.33 \\
\hline & 4 & \begin{tabular}{l|}
-14.352 \\
\end{tabular} & 2.17 & 13.17 & & 4 & -13.683 & 2.11 & 11.86 \\
\hline & 1 & \begin{tabular}{|l|}
-9.563 \\
\end{tabular} & 1.95 & 12.59 & & 1 & -20.185 & 2.05 & 22.68 \\
\hline & 2 & \begin{tabular}{|c|}
-8.267 \\
\end{tabular} & 1.95 & 15.76 & 2 & 2 & \begin{tabular}{|l|}
-15.918 \\
\end{tabular} & 2.13 & 15.69 \\
\hline 3 & 3 & \begin{tabular}{|l|}
-9.070 \\
\end{tabular} & 1.67 & 8.95 & 3 & 3 & \begin{tabular}{|c|}
-15.687 \\
\end{tabular} & 2.01 & 14.97 \\
\hline & 4 & \begin{tabular}{|c|}
-18.693 \\
\end{tabular} & 1.78 & 17.68 & & 4 & $\mid-14.804$ & 2.17 & 13.79 \\
\hline & 1 & \begin{tabular}{|c|}
-14.822 \\
\end{tabular} & 2.02 & 13.45 & & 1 & -12.829 & 2.07 & 13.34 \\
\hline 1 & 2 & \begin{tabular}{|l|}
-25.436 \\
\end{tabular} & 2.06 & 22.83 & 1 & 2 & -10.545 & 2.13 & 11.19 \\
\hline 4 & 3 & -21.528 & 2.16 & 20.52 & 4 & 3 & -10.341 & 2.03 & 11.43 \\
\hline & 4 & $\mid-11.848$ & 2.16 & 12.97 & & 4 & \begin{tabular}{|l|}
-6.212 \\
\end{tabular} & 1.76 & 7.01 \\
\hline & 1 & \begin{tabular}{|l|}
-18.130 \\
\end{tabular} & 2.02 & 20.97 & & 1 & -19.312 & 2.06 & 17.95 \\
\hline & 2 & \begin{tabular}{|l|}
-34.011 \\
\end{tabular} & 1.56 & 32.67 & 5 & 2 & \begin{tabular}{|c|}
-9.403 \\
\end{tabular} & 2.05 & 7.64 \\
\hline 5 & 3 & \begin{tabular}{l|}
-13.396 \\
\end{tabular} & 2.05 & 14.52 & 5 & 3 & -9.120 & 1.90 & 8.09 \\
\hline & 4 & $\begin{array}{l}-17.132 \\
\end{array}$ & 2.10 & 15.91 & & 4 & -8.127 & 1.80 & 7.43 \\
\hline & 1 & \begin{tabular}{l|l|}
-20.460 \\
\end{tabular} & 2.10 & 19.22 & & 1 & -20.106 & 2.15 & 18.88 \\
\hline 6 & 2 & -41.688 & 2.03 & 40.61 & 6 & 2 & -24.426 & 1.59 & 23.35 \\
\hline 6 & 3 & \begin{tabular}{|l|}
-9.324 \\
\end{tabular} & 1.79 & 10.33 & 6 & 3 & -16.544 & 1.70 & 19.45 \\
\hline & 4 & -21.709 & 2.11 & 20.54 & & 4 & -14.040 & 1.63 & 13.31 \\
\hline & & & & & & & & & \\
\hline & & Averages & by Valve & & & & Averages & by Valve & \\
\hline & $\begin{array}{l}\text { Exhaust } \\
\text { Valve \# }\end{array}$ & $\begin{array}{c}\text { ORDC0 } \\
z \text { (delta) } \\
\mu \mathrm{m}\end{array}$ & $\begin{array}{l}\text { ORDC0 } \\
\text { Ev. } \\
\text { Length, } \\
\mathrm{mm}\end{array}$ & $\begin{array}{l}\text { ORDCO } \\
W \mathrm{t}, \mu \mathrm{m}\end{array}$ & & $\begin{array}{c}\text { Exhaust } \\
\text { Valve \# }\end{array}$ & $\begin{array}{c}\text { ORDC15 } \\
\text { z(delta) } \\
\mu \mathrm{m}\end{array}$ & $\begin{array}{l}\text { ORDC15 } \\
\text { Ev. } \\
\text { Length, } \\
\mathrm{mm}\end{array}$ & $\begin{array}{l}\text { ORDC15 } \\
W t, \mu \mathrm{m}\end{array}$ \\
\hline & 1 & -28.360 & 1.970 & 27.715 & & \begin{tabular}{|c|}
1 \\
\end{tabular} & -10.973 & 2.160 & 9.878 \\
\hline & 2 & \begin{tabular}{|c|}
-13.733 \\
\end{tabular} & 2.163 & 15.300 & & 2 & $\mid-14.058$ & 2.040 & 13.735 \\
\hline & 3 & $\mid-11.398$ & 1.838 & 13.745 & & 3 & \begin{tabular}{|c|}
-16.649 \\
\end{tabular} & 2.090 & 16.783 \\
\hline & 4 & \begin{tabular}{|c|}
-18.409 \\
\end{tabular} & 2.100 & 17.443 & & 4 & -9.982 & \begin{tabular}{|l|}
1.998 \\
\end{tabular} & 10.743 \\
\hline & 5 & \begin{tabular}{|l|}
-20.667 \\
\end{tabular} & 1.933 & 21.018 & & 5 & -11.491 & \begin{tabular}{|l|}
1.953 \\
\end{tabular} & 10.278 \\
\hline & 6 & -23.295 & 2.008 & 22.675 & & 6 & $\mid-18.779$ & 1.768 & 18.748 \\
\hline & Average & \begin{tabular}{|c|}
-19.310 \\
\end{tabular} & 2.002 & 19.649 & & Average & -13.655 & 2.001 & 13.360 \\
\hline & StDev & 9.165 & 0.166 & 7.911 & & StDev & 4.809 & 0.180 & 5.076 \\
\hline & Max & \begin{tabular}{l|}
-5.598 \\
\end{tabular} & 2.220 & 40.610 & & Max & \begin{tabular}{|l|}
-6.212 \\
\end{tabular} & 2.170 & 23.350 \\
\hline & Min & -41.688 & 1.560 & 8.950 & & Min & -24.426 & \begin{tabular}{l|}
1.590 \\
\end{tabular} & 6.000 \\
\hline
\end{tabular}




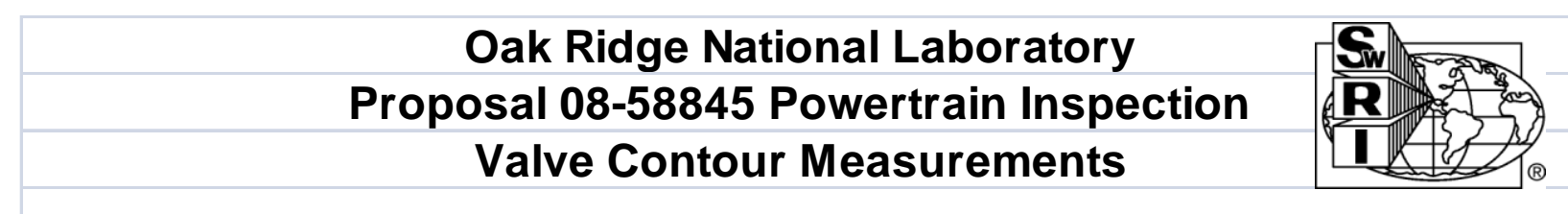

\begin{tabular}{|c|c|c|c|c|}
\hline \multicolumn{5}{|c|}{ ORDC20B } \\
\hline $\begin{array}{c}\text { Intake } \\
\text { Valve \# }\end{array}$ & Position & $\begin{array}{c}\mathrm{z}(\text { delta }) \\
\mu \mathrm{m}\end{array}$ & $\begin{array}{c}\text { Ev. } \\
\text { Length, } \\
\mathrm{mm}\end{array}$ & $\mathrm{Wt}, \mu \mathrm{m}$ \\
\hline \multirow{4}{*}{1} & 1 & -5.163 & 2.25 & 3.84 \\
\hline & 2 & -3.807 & 2.22 & 2.49 \\
\hline & 3 & -4.253 & 2.16 & 2.88 \\
\hline & 4 & -3.637 & 2.10 & 2.69 \\
\hline \multirow{4}{*}{2} & 1 & -7.067 & 1.44 & 6.20 \\
\hline & 2 & -6.161 & 1.38 & 5.15 \\
\hline & 3 & -8.106 & 1.46 & 7.19 \\
\hline & 4 & -7.953 & 1.53 & 5.97 \\
\hline \multirow{4}{*}{3} & 1 & -6.708 & 2.35 & 4.83 \\
\hline & 2 & -5.074 & 2.29 & 3.44 \\
\hline & 3 & -5.578 & 2.20 & 3.67 \\
\hline & 4 & -4.781 & 1.97 & 3.19 \\
\hline \multirow{4}{*}{4} & 1 & -2.515 & 1.44 & 2.81 \\
\hline & 2 & -1.826 & 1.19 & 1.71 \\
\hline & 3 & -2.410 & 1.30 & 1.67 \\
\hline & 4 & -3.402 & 1.29 & 2.15 \\
\hline \multirow{4}{*}{5} & 1 & -4.514 & 1.79 & 3.98 \\
\hline & 2 & -4.575 & 1.79 & 3.32 \\
\hline & 3 & -4.131 & 1.51 & 3.19 \\
\hline & 4 & -7.221 & 1.37 & 5.47 \\
\hline \multirow{4}{*}{6} & 1 & -2.968 & 1.21 & 2.00 \\
\hline & 2 & -3.762 & 1.11 & 2.25 \\
\hline & 3 & -4.192 & 1.13 & 2.44 \\
\hline & 4 & -3.183 & 1.24 & 2.45 \\
\hline
\end{tabular}

\begin{tabular}{|c|c|c|c|c|}
\hline \multicolumn{5}{|c|}{ ORDC20B } \\
\hline $\begin{array}{l}\text { Exhaust } \\
\text { Valve \# }\end{array}$ & Position & $\begin{array}{c}z \text { (delta), } \\
\mu \mathrm{m}\end{array}$ & $\begin{array}{c}\text { Ev. } \\
\text { Length, } \\
\mathrm{mm}\end{array}$ & $\mathrm{Wt}, \mu \mathrm{m}$ \\
\hline \multirow{4}{*}{1} & 1 & -17.187 & 2.09 & 15.83 \\
\hline & 2 & -17.645 & 1.84 & 17.01 \\
\hline & 3 & -11.615 & 1.94 & 10.34 \\
\hline & 4 & -14.395 & 1.59 & 12.27 \\
\hline \multirow{4}{*}{2} & 1 & -23.509 & 2.13 & 23.07 \\
\hline & 2 & -27.055 & 2.26 & 25.52 \\
\hline & 3 & -17.626 & 2.19 & 16.85 \\
\hline & 4 & -29.492 & 1.96 & 27.85 \\
\hline \multirow{4}{*}{3} & 1 & -17.716 & 2.01 & 16.31 \\
\hline & 2 & \begin{tabular}{|c|}
-17.576 \\
\end{tabular} & 1.92 & 16.53 \\
\hline & 3 & -31.377 & 2.06 & 30.52 \\
\hline & 4 & -15.049 & 2.00 & 16.78 \\
\hline \multirow{4}{*}{4} & 1 & -14.180 & 1.83 & 13.49 \\
\hline & 2 & -12.732 & 1.94 & 15.10 \\
\hline & 3 & -19.513 & 2.07 & 23.59 \\
\hline & 4 & -16.881 & 1.93 & 15.93 \\
\hline \multirow{4}{*}{5} & 1 & -13.022 & 2.03 & 16.39 \\
\hline & 2 & \begin{tabular}{|c|}
-15.027 \\
\end{tabular} & 2.03 & 18.39 \\
\hline & 3 & -20.056 & 2.07 & 21.22 \\
\hline & 4 & -20.366 & 1.99 & 19.53 \\
\hline \multirow{4}{*}{6} & 1 & -13.572 & 1.81 & 12.62 \\
\hline & 2 & -31.321 & 2.19 & 28.90 \\
\hline & 3 & -20.677 & 2.11 & 25.81 \\
\hline & 4 & -27.028 & 2.06 & 26.58 \\
\hline
\end{tabular}

\begin{tabular}{|c|r|r|r|}
\hline \multicolumn{5}{|c|}{ Averages by Valve } \\
\hline $\begin{array}{c}\text { Intake } \\
\text { Valve \# }\end{array}$ & $\begin{array}{c}\text { ORDC20B } \\
\text { (delta), } \\
\mu \mathrm{m}\end{array}$ & $\begin{array}{c}\text { ORDC20B } \\
\text { Ev. } \\
\text { Length, } \\
\mathrm{mm}\end{array}$ & $\begin{array}{c}\text { ORDC20B } \\
\mathrm{Wt}, \mu \mathrm{m}\end{array}$ \\
\hline 1 & -4.215 & 2.183 & 2.975 \\
\hline 2 & -7.322 & 1.453 & 6.128 \\
\hline 3 & -5.535 & 2.203 & 3.783 \\
\hline 4 & -2.538 & 1.305 & 2.085 \\
\hline 5 & -5.110 & 1.615 & 3.990 \\
\hline 6 & -3.526 & 1.173 & 2.285 \\
\hline Average & -4.708 & 1.655 & 3.541 \\
\hline StDev & 1.749 & 0.427 & 1.516 \\
\hline Max & -1.826 & 2.350 & 7.190 \\
\hline Min & -8.106 & 1.110 & 1.670 \\
\hline
\end{tabular}

\begin{tabular}{|l|r|r|r|}
\hline \multicolumn{5}{|c|}{ Averages by Valve } \\
\hline $\begin{array}{c}\text { Exhaust } \\
\text { Valve \# }\end{array}$ & $\begin{array}{c}\text { ORDC20B } \\
\text { (delta), } \\
\mu \mathrm{m}\end{array}$ & $\begin{array}{c}\text { ORDC20B } \\
\text { Ev. } \\
\text { Length, } \\
\mathrm{mm}\end{array}$ & $\begin{array}{c}\text { ORDC20B } \\
\text { Wt, } \mu \mathrm{m}\end{array}$ \\
\hline 1 & -15.211 & 1.865 & 13.863 \\
\hline 2 & -24.421 & 2.135 & 23.323 \\
\hline 3 & -20.430 & 1.998 & 20.035 \\
\hline 4 & -15.827 & 1.943 & 17.028 \\
\hline 5 & -17.118 & 2.030 & 18.883 \\
\hline 6 & -23.150 & 2.043 & 23.478 \\
\hline Average & -19.359 & 2.002 & 19.435 \\
\hline StDev & 5.963 & 0.144 & 5.725 \\
\hline Max & -11.615 & 2.260 & 30.520 \\
\hline Min & -31.377 & 1.590 & 10.340 \\
\hline
\end{tabular}




\section{Oakridge National Laboratory \\ 2007 Dodge Caravan \\ Intake Valve Contour Measurements at EOT}

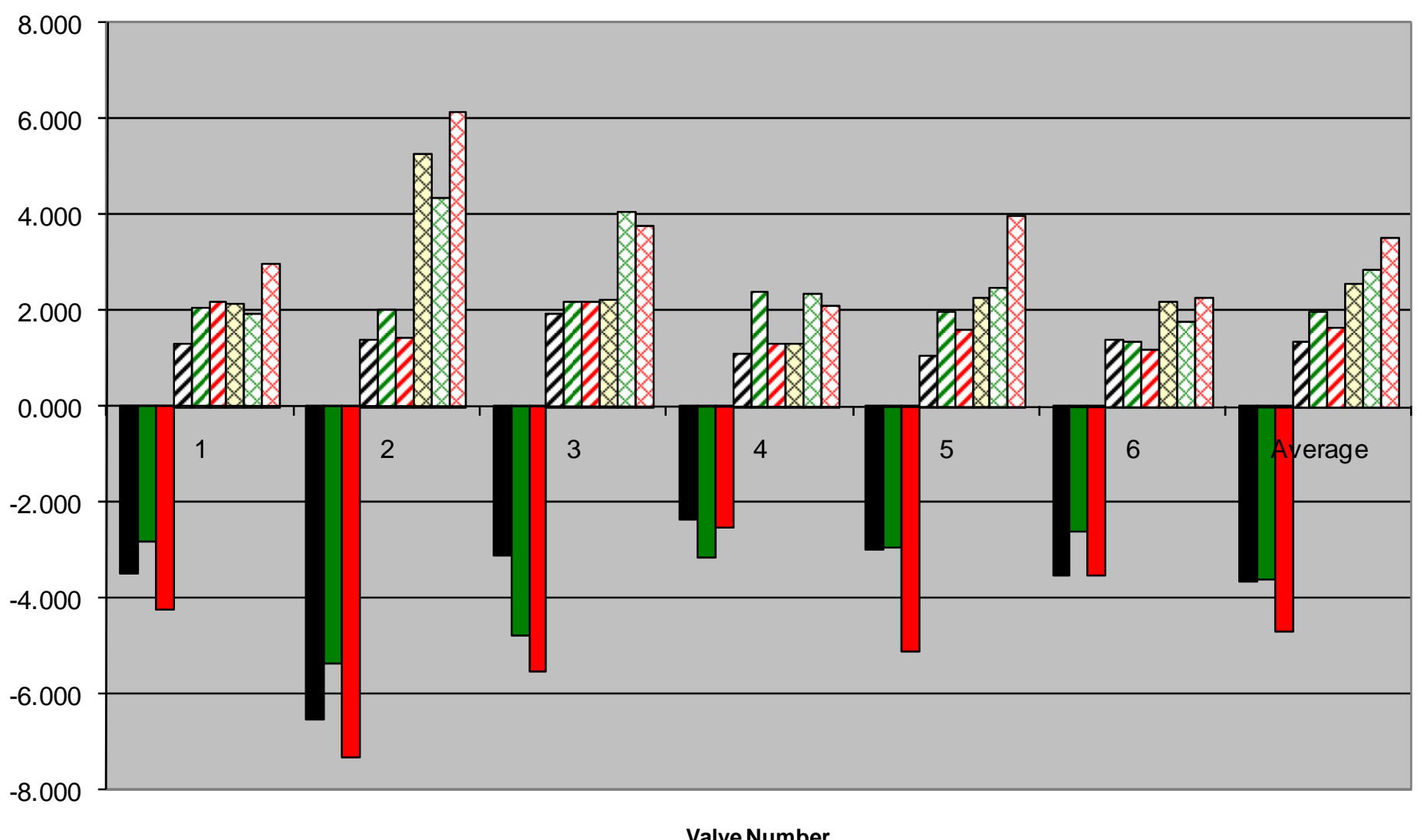

- ORDCO z(delta), $\mu \mathrm{m}$

口ORDC15 z(delta), $\mu \mathrm{m}$

口ORDC20B z(delta), $\mu \mathrm{m}$ चORDCO Ev. Length, $\mathrm{mm}$ DORDC15 Ev. Length, $\mathrm{mm}$ 口ORDC20B Ev. Length, mm 口ORDCO $\mathrm{Wt}, \mu \mathrm{m}$ ๑ORDC15 Wt, $\mu \mathrm{m}$

ఐORDC20B Wt, $\mu \mathrm{m}$ 


\section{Oakridge National Laboratory \\ 2007 Dodge Caravan \\ Exhaust Valve Contour Measurements at EOT}

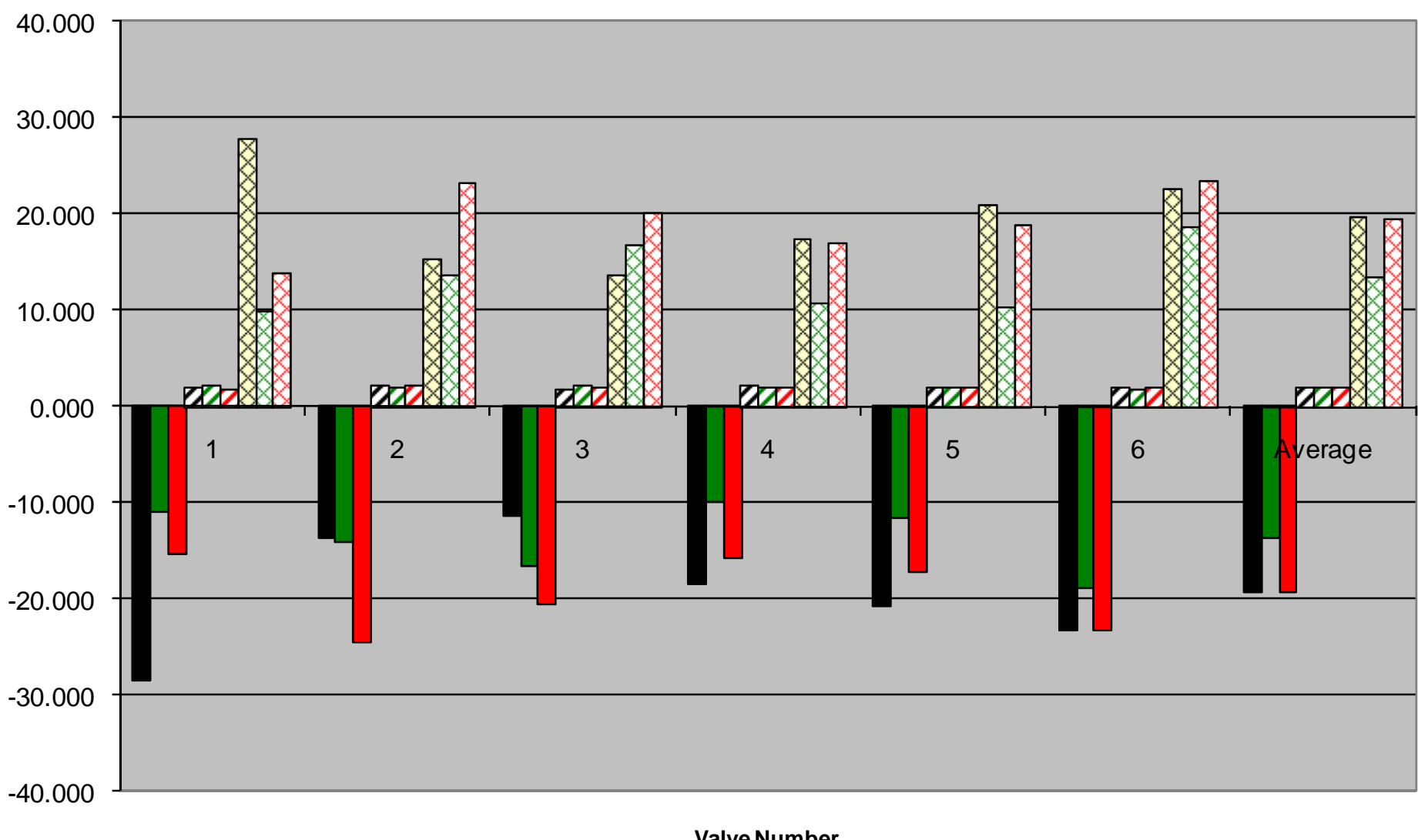

- ORDCO z(delta), $\mu \mathrm{m}$ 口ORDC15 z(delta), $\mu \mathrm{m}$ 口ORDC20B z(delta), $\mu \mathrm{m}$ चORDCO Ev. Length, $\mathrm{mm}$ DORDC15 Ev. Length, mm 口ORDC20B Ev. Length, mm 口ORDC0 $\mathrm{Wt}, \mu \mathrm{m}$ ๑ORDC15 Wt, $\mu \mathrm{m}$ OORDC20B Wt, $\mu \mathrm{m}$

Valve Number 


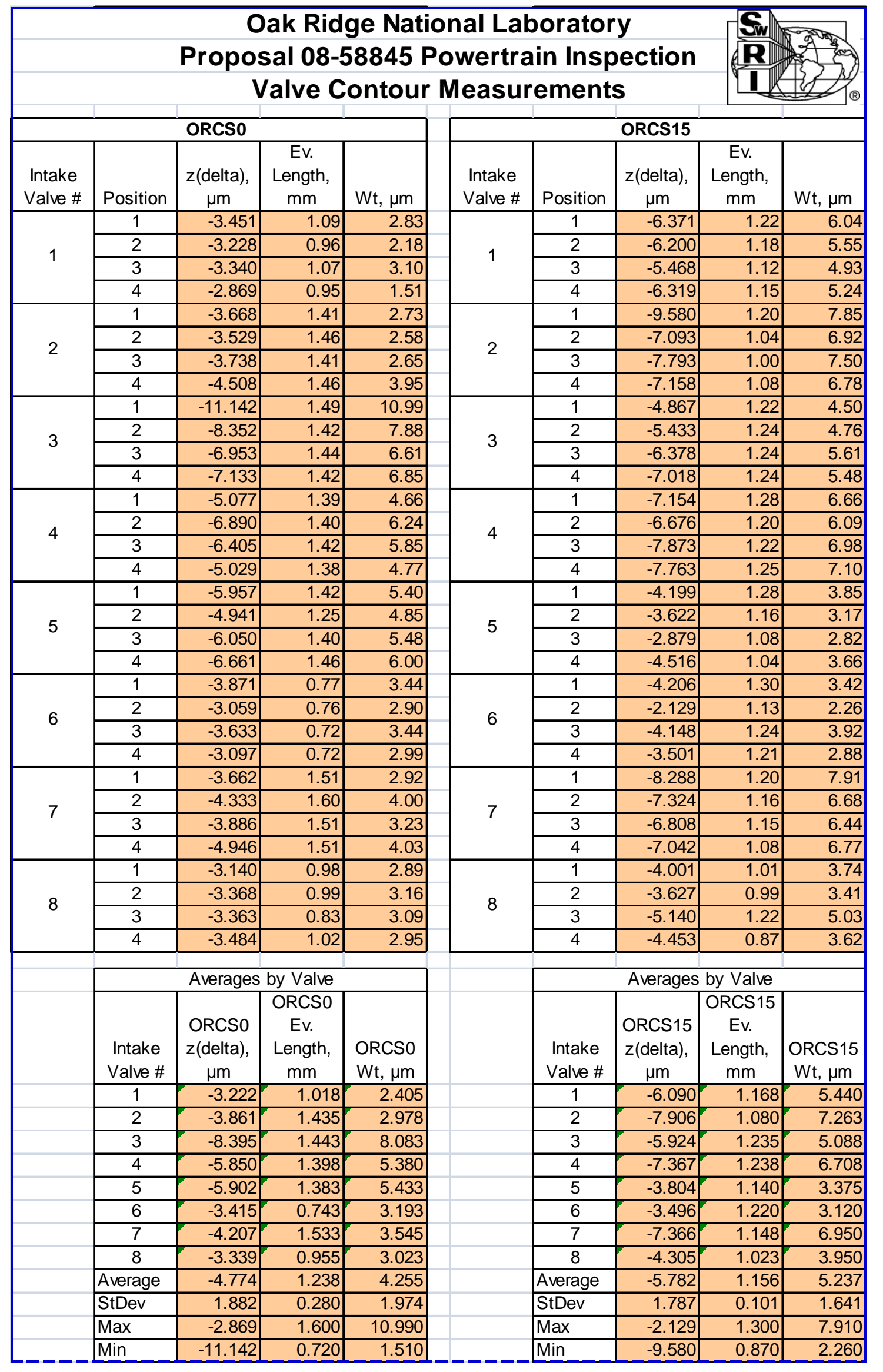

B-15 of 34 


\begin{tabular}{|c|c|c|c|c|c|c|c|c|c|}
\hline & & Propo & $\begin{array}{l}\text { ak Rid } \\
\text { sal 08- }\end{array}$ & $\begin{array}{l}\text { ge Nati } \\
\text {;8845 F }\end{array}$ & $\begin{array}{l}\text { nal La } \\
\text { wertra }\end{array}$ & $\begin{array}{l}\text { oorator } \\
\text { in Insp }\end{array}$ & $\begin{array}{l}y \\
\text { ection }\end{array}$ & Rw & \\
\hline & & & alve C & ontour & Measu & ement & & & \\
\hline & & ORCS0 & & & & & ORCS15 & & \\
\hline $\begin{array}{l}\text { Exhaust } \\
\text { Valve \# }\end{array}$ & Position & $\begin{array}{c}z \text { (delta), } \\
\mu \mathrm{m}\end{array}$ & $\begin{array}{c}\text { Ev. } \\
\text { Length, } \\
\mathrm{mm}\end{array}$ & $\mathrm{Wt}, \mu \mathrm{m}$ & $\begin{array}{l}\text { Exhaust } \\
\text { Valve \# }\end{array}$ & Position & $\begin{array}{c}z \text { (delta) } \\
\mu \mathrm{m}\end{array}$ & $\begin{array}{c}\text { Ev. } \\
\text { Length, } \\
\mathrm{mm}\end{array}$ & $\mathrm{Wt}, \mu \mathrm{m}$ \\
\hline & 1 & -16.330 & 1.80 & 15.26 & & 1 & -22.636 & 1.80 & 21.29 \\
\hline 1 & 2 & -16.626 & 1.68 & 14.83 & 1 & 2 & -28.347 & 1.79 & 27.29 \\
\hline 1 & 3 & -21.172 & 1.82 & 20.61 & 1 & 3 & -43.478 & 1.73 & 41.76 \\
\hline & 4 & -14.499 & 1.82 & 12.89 & & 4 & -27.519 & 1.41 & 26.48 \\
\hline & 1 & -21.144 & 1.90 & 19.69 & & 1 & -58.818 & 1.80 & 57.63 \\
\hline 2 & 2 & -28.255 & 1.83 & 26.48 & 2 & 2 & -22.309 & 1.96 & 20.86 \\
\hline 2 & 3 & -23.895 & 1.74 & 23.15 & 2 & 3 & -26.097 & 1.98 & 25.44 \\
\hline & 4 & -21.677 & 1.55 & 20.06 & & 4 & -21.148 & 1.76 & 20.47 \\
\hline & 1 & -21.100 & 1.66 & 20.57 & & 1 & -24.515 & 1.49 & 23.15 \\
\hline 3 & 2 & -32.367 & 2.19 & 31.89 & 2 & 2 & -24.166 & 1.41 & 23.04 \\
\hline 3 & 3 & -10.650 & 1.39 & 9.18 & 3 & 3 & -16.096 & 1.07 & 14.29 \\
\hline & 4 & -14.832 & 1.38 & 13.89 & & 4 & -16.493 & 1.00 & 15.22 \\
\hline & 1 & -25.062 & 1.84 & 24.21 & & 1 & -25.910 & 1.63 & 24.86 \\
\hline 1 & 2 & -11.630 & 1.59 & 9.75 & 4 & 2 & -29.314 & 1.52 & 28.39 \\
\hline 4 & 3 & -20.025 & 1.75 & 18.64 & 4 & 3 & -21.953 & 1.48 & 20.23 \\
\hline & 4 & -18.593 & 1.62 & 17.92 & & 4 & -16.838 & 1.56 & 15.90 \\
\hline & 1 & -7.855 & 1.85 & 7.17 & & 1 & -20.700 & 1.53 & 18.61 \\
\hline 5 & 2 & -15.268 & 1.82 & 14.74 & 5 & 2 & -25.348 & 1.64 & 24.10 \\
\hline 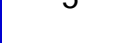 & 3 & -12.618 & 1.76 & 11.43 & $\checkmark$ & 3 & -29.293 & 1.98 & 27.62 \\
\hline & 4 & -27.399 & 1.98 & 26.76 & & 4 & -24.043 & 1.95 & 23.22 \\
\hline & 1 & -22.268 & 1.77 & 20.83 & & 1 & -20.946 & 1.76 & 19.82 \\
\hline & 2 & -25.892 & 1.81 & 25.06 & & 2 & -26.362 & 1.87 & 25.52 \\
\hline 0 & 3 & -24.874 & 1.95 & 24.78 & 6 & 3 & -12.792 & 1.78 & 11.81 \\
\hline & 4 & -21.773 & 1.86 & 20.71 & & 4 & -27.597 & 1.95 & 25.89 \\
\hline & 1 & -11.782 & 2.01 & 10.41 & & 5 & -23.327 & 1.91 & 23.16 \\
\hline 7 & 2 & -27.189 & 2.06 & 26.34 & 7 & 6 & -23.091 & 1.84 & 20.81 \\
\hline t & 3 & -17.471 & 1.67 & 16.34 & $t$ & 7 & -24.037 & 1.77 & 22.66 \\
\hline & 4 & -21.954 & 1.76 & 20.39 & & 8 & -34.526 & 1.66 & 33.74 \\
\hline & 1 & -16.574 & 1.94 & 15.33 & & 9 & -21.970 & 1.77 & 19.58 \\
\hline 8 & 2 & -11.279 & 1.36 & 10.61 & 8 & 10 & -8.770 & 1.37 & 7.98 \\
\hline 8 & 3 & -18.911 & 1.43 & 17.71 & 8 & 11 & -5.981 & 1.60 & 5.62 \\
\hline & 4 & -23.563 & 2.03 & 23.20 & & 12 & -11.251 & 1.81 & 11.71 \\
\hline & & Averages & by Valve & & & & Averages & by Valve & \\
\hline & \begin{tabular}{|l} 
Exhaust \\
Valve \#
\end{tabular} & $\begin{array}{c}\text { ORCSO } \\
\mathrm{z} \text { (delta) } \\
\mu \mathrm{m}\end{array}$ & $\begin{array}{l}\text { ORCSO } \\
\text { Ev. } \\
\text { Length, } \\
\mathrm{mm} \\
\end{array}$ & $\begin{array}{l}\text { ORCSO } \\
\mathrm{Wt}, \mu \mathrm{m}\end{array}$ & & $\begin{array}{l}\text { Exhaust } \\
\text { Valve \# }\end{array}$ & $\begin{array}{c}\text { ORCS15 } \\
\text { z(delta), } \\
\mu \mathrm{m}\end{array}$ & $\begin{array}{c}\text { ORCS15 } \\
\text { Ev. } \\
\text { Length, } \\
\mathrm{mm}\end{array}$ & $\begin{array}{l}\text { ORCS15 } \\
W \mathrm{t}, \mu \mathrm{m}\end{array}$ \\
\hline & 1 & -17.157 & 1.780 & 15.898 & & 1 & -30.495 & 1.683 & 29.205 \\
\hline & 2 & -23.743 & 1.755 & 22.345 & & 2 & -32.093 & 1.875 & 31.100 \\
\hline & 3 & -19.737 & 1.655 & 18.883 & & 3 & -20.318 & 1.243 & 18.925 \\
\hline & 4 & -18.828 & 1.700 & 17.630 & & 4 & -23.504 & 1.548 & 22.345 \\
\hline & 5 & -15.785 & 1.853 & 15.025 & & 5 & -24.846 & 1.775 & 23.388 \\
\hline & 6 & -23.702 & 1.848 & 22.845 & & 6 & -21.924 & 1.840 & 20.760 \\
\hline & 7 & -19.599 & 1.875 & 18.370 & & 7 & -26.245 & 1.795 & 25.093 \\
\hline & 8 & -17.582 & 1.690 & 16.713 & & 8 & -11.993 & 1.638 & 11.223 \\
\hline & Average & -19.516 & 1.769 & 18.463 & & Average & -23.927 & 1.674 & 22.755 \\
\hline & StDev & 5.901 & 0.201 & 6.048 & & StDev & 9.632 & 0.244 & 9.513 \\
\hline & Max & -7.855 & 2.190 & 31.890 & & Max & -5.981 & 1.980 & 57.630 \\
\hline & Min & -32.367 & 1.360 & 7.170 & & Min & -58.818 & 1.000 & 5.620 \\
\hline
\end{tabular}

B-16 of 34 


\begin{tabular}{|c|c|c|c|c|c|c|c|c|c|}
\hline \multicolumn{10}{|c|}{ Oak Ridge National Laboratory } \\
\hline \multicolumn{10}{|c|}{ Proposal 08-58845 Powertrain Inspection } \\
\hline \multicolumn{10}{|c|}{ Valve Contour Measurements } \\
\hline \multicolumn{5}{|c|}{ ORCS20 } & \multicolumn{5}{|c|}{ ORCS20 } \\
\hline $\begin{array}{c}\text { Intake } \\
\text { Valve \# }\end{array}$ & Position & $\begin{array}{c}z \text { (delta) } \\
\mu \mathrm{m}\end{array}$ & $\begin{array}{c}\text { Ev. } \\
\text { Length, } \\
\mathrm{mm} \\
\end{array}$ & $\mathrm{Wt}, \mu \mathrm{m}$ & $\begin{array}{l}\text { Exhaust } \\
\text { Valve \# }\end{array}$ & Position & $\begin{array}{c}z \text { (delta) } \\
\mu \mathrm{m}\end{array}$ & \begin{tabular}{|c|} 
Ev. \\
Length, \\
$\mathrm{mm}$ \\
\end{tabular} & $\mathrm{Wt}, \mu \mathrm{m}$ \\
\hline \multirow{4}{*}{1} & 1 & -3.860 & 0.70 & 3.27 & \multirow[t]{4}{*}{ - } & 1 & -14.988 & 1.51 & 13.10 \\
\hline & 2 & -3.617 & 0.58 & 3.40 & & 2 & -6.880 & 1.61 & 6.68 \\
\hline & 3 & -4.742 & 0.78 & 3.94 & & 3 & -9.563 & 1.59 & 8.59 \\
\hline & 4 & -2.899 & 0.51 & 2.42 & & 4 & -20.964 & 1.78 & 21.57 \\
\hline \multirow{4}{*}{2} & 1 & -13.962 & 1.15 & 11.96 & \multirow{4}{*}{2} & 1 & -20.750 & 1.89 & 20.13 \\
\hline & 2 & -11.332 & 1.26 & 10.53 & & 2 & -11.808 & 1.94 & 11.68 \\
\hline & 3 & -10.119 & 1.08 & 9.87 & & 3 & -18.332 & 1.98 & 17.26 \\
\hline & 4 & -15.798 & 1.07 & 13.43 & & 4 & -22.527 & 1.97 & 22.24 \\
\hline \multirow{4}{*}{3} & 1 & -11.124 & 1.12 & 9.86 & \multirow{4}{*}{3} & 1 & -24.090 & 2.21 & 22.29 \\
\hline & 2 & -9.956 & 1.02 & 9.31 & & 2 & -13.739 & 2.09 & 12.62 \\
\hline & 3 & -9.342 & 1.08 & 8.74 & & 3 & -15.820 & 1.77 & 15.39 \\
\hline & 4 & -8.068 & 1.06 & 7.75 & & 4 & -21.821 & 1.94 & 20.70 \\
\hline \multirow{4}{*}{4} & 1 & -3.631 & 1.23 & 3.16 & \multirow{4}{*}{4} & 1 & -19.223 & 1.60 & 19.24 \\
\hline & 2 & -2.959 & 1.09 & 2.73 & & 2 & -26.374 & 1.67 & 25.86 \\
\hline & 3 & -3.999 & 1.00 & 2.71 & & 3 & -15.056 & 1.94 & 14.61 \\
\hline & 4 & -3.182 & 1.13 & 2.81 & & 4 & -17.430 & 1.85 & 15.82 \\
\hline \multirow{4}{*}{5} & 1 & -2.314 & 1.03 & 2.10 & \multirow{4}{*}{5} & 1 & -18.974 & 1.83 & 18.90 \\
\hline & 2 & -3.397 & 0.91 & 2.10 & & 2 & -11.186 & 1.79 & 9.67 \\
\hline & 3 & -3.930 & 0.97 & 3.09 & & 3 & -7.913 & 1.61 & 8.19 \\
\hline & 4 & -3.594 & 1.08 & 2.69 & & 4 & -29.496 & 2.09 & 28.46 \\
\hline \multirow{4}{*}{6} & 1 & -6.303 & 1.14 & 5.81 & \multirow{4}{*}{6} & 1 & -15.110 & 2.03 & 15.73 \\
\hline & 2 & -14.539 & 1.39 & 12.73 & & 2 & -15.224 & 2.60 & 14.39 \\
\hline & 3 & -12.735 & 1.12 & 11.28 & & 3 & -17.807 & 1.48 & 17.37 \\
\hline & 4 & -5.440 & 1.11 & 4.85 & & 4 & -17.030 & 1.89 & 18.66 \\
\hline & 1 & -7.598 & 1.03 & 6.81 & & 1 & -29.749 & 1.57 & 29.27 \\
\hline 7 & 2 & -6.793 & 1.22 & 6.19 & 7 & 2 & -40.270 & 2.10 & 39.79 \\
\hline$r$ & 3 & -7.490 & 1.05 & 7.08 & 7 & 3 & -16.526 & 1.45 & 15.77 \\
\hline & 4 & -8.767 & 1.05 & 8.36 & & 4 & -22.256 & 1.34 & 21.17 \\
\hline & 1 & -8.091 & 1.23 & 7.63 & & 1 & -27.011 & 1.47 & 25.44 \\
\hline 8 & 2 & -7.376 & 1.04 & 6.62 & 8 & 2 & -15.249 & 1.80 & 14.35 \\
\hline 8 & 3 & -6.369 & 1.04 & 5.94 & 8 & 3 & -8.970 & 1.71 & 8.61 \\
\hline & 4 & -7.308 & 1.00 & 6.77 & & 4 & -15.321 & 1.71 & 16.07 \\
\hline & & & & & & & & & \\
\hline & & Averages & by Valve & & & & Averages & by Valve & \\
\hline & $\begin{array}{c}\text { Intake } \\
\text { Valve \# }\end{array}$ & $\begin{array}{c}\text { ORCS20 } \\
\mathrm{z} \text { (delta) } \\
\mu \mathrm{m}\end{array}$ & $\begin{array}{c}\text { ORCS20 } \\
\text { Ev. } \\
\text { Length, } \\
\mathrm{mm}\end{array}$ & $\begin{array}{l}\text { ORCS20 } \\
\mathrm{Wt}, \mu \mathrm{m}\end{array}$ & & $\begin{array}{l}\text { Exhaust } \\
\text { Valve \# }\end{array}$ & $\begin{array}{c}\text { ORCS20 } \\
z \text { (delta) } \\
\mu \mathrm{m}\end{array}$ & \begin{tabular}{|c|} 
ORCS20 \\
Ev. \\
Length, \\
$\mathrm{mm}$
\end{tabular} & $\begin{array}{l}\text { ORCS20 } \\
W t, \mu \mathrm{m}\end{array}$ \\
\hline & 1 & -3.780 & 0.643 & 3.258 & & 1 & \begin{tabular}{|r|}
-13.099 \\
\end{tabular} & 1.623 & 12.485 \\
\hline & 2 & -12.803 & 1.140 & 11.448 & & 2 & -18.354 & 1.945 & 17.828 \\
\hline & 3 & -9.623 & 1.070 & 8.915 & & 3 & -18.868 & 2.003 & 17.750 \\
\hline & 4 & -3.443 & 1.113 & 2.853 & & 4 & -19.521 & 1.765 & 18.883 \\
\hline & 5 & -3.309 & 0.998 & 2.495 & & 5 & -16.892 & 1.830 & 16.305 \\
\hline & 6 & -9.754 & 1.190 & 8.668 & & 6 & -16.293 & 2.000 & 16.538 \\
\hline & 7 & -7.662 & 1.088 & 7.110 & & 7 & -27.200 & 1.615 & 26.500 \\
\hline & 8 & -7.286 & 1.078 & 6.740 & & 8 & -16.638 & 1.673 & 16.118 \\
\hline & Average & -7.207 & 1.040 & 6.436 & & Average & -18.358 & 1.807 & 17.801 \\
\hline & StDev & 3.742 & 0.183 & 3.422 & & StDev & 7.095 & 0.262 & 7.022 \\
\hline & Max & -2.314 & 1.390 & 13.430 & & Max & -6.880 & 2.600 & 39.790 \\
\hline & Min & -15.798 & 0.510 & 2.100 & & Min & -40.270 & 1.340 & 6.680 \\
\hline
\end{tabular}




\section{Oakridge National Laboratory \\ 2006 Chevrolet Silverado \\ Intake Valve Contour Measurements at EOT}

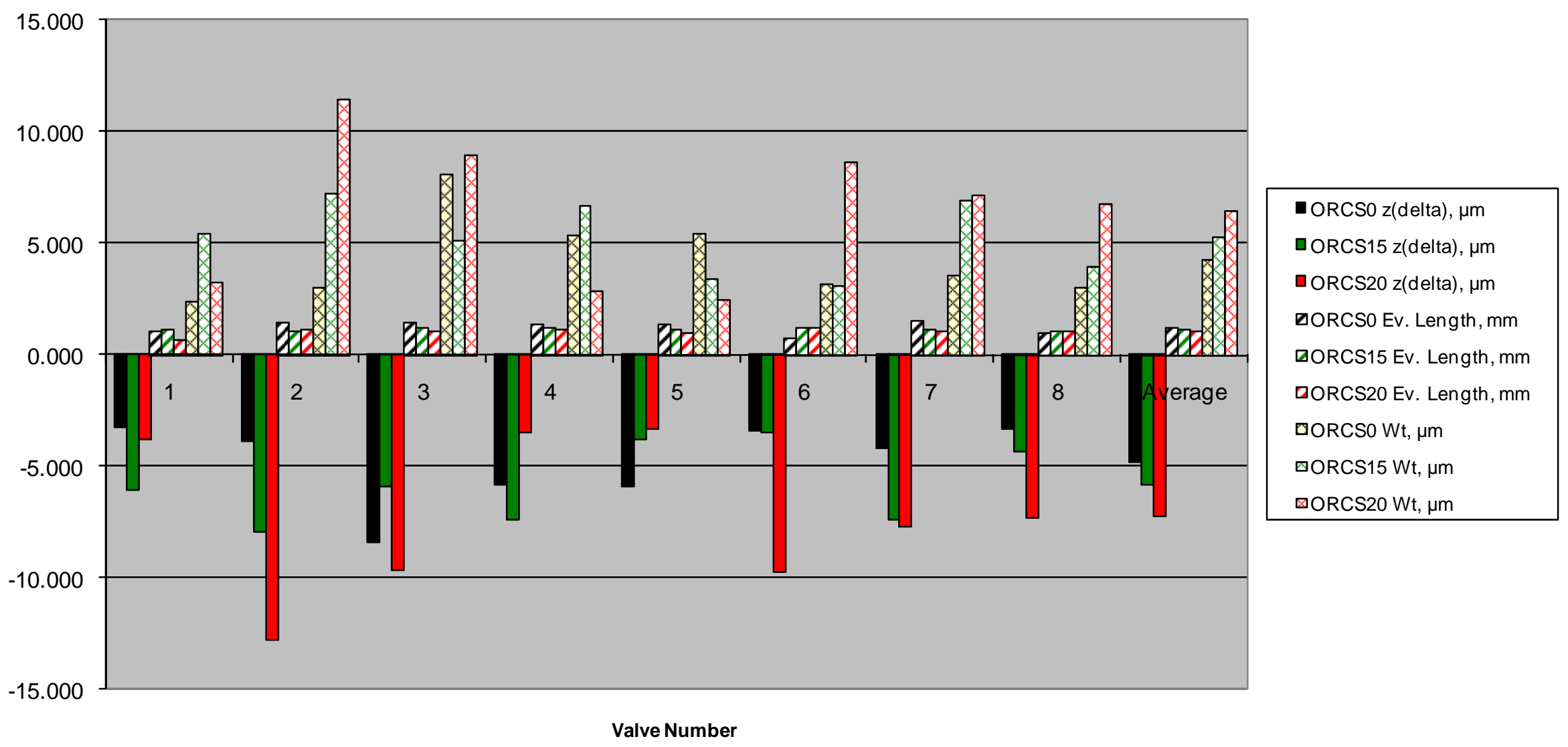




\section{Oakridge National Laboratory \\ 2006 Chevrolet Silverado \\ Exhaust Valve Contour Measurements at EOT}

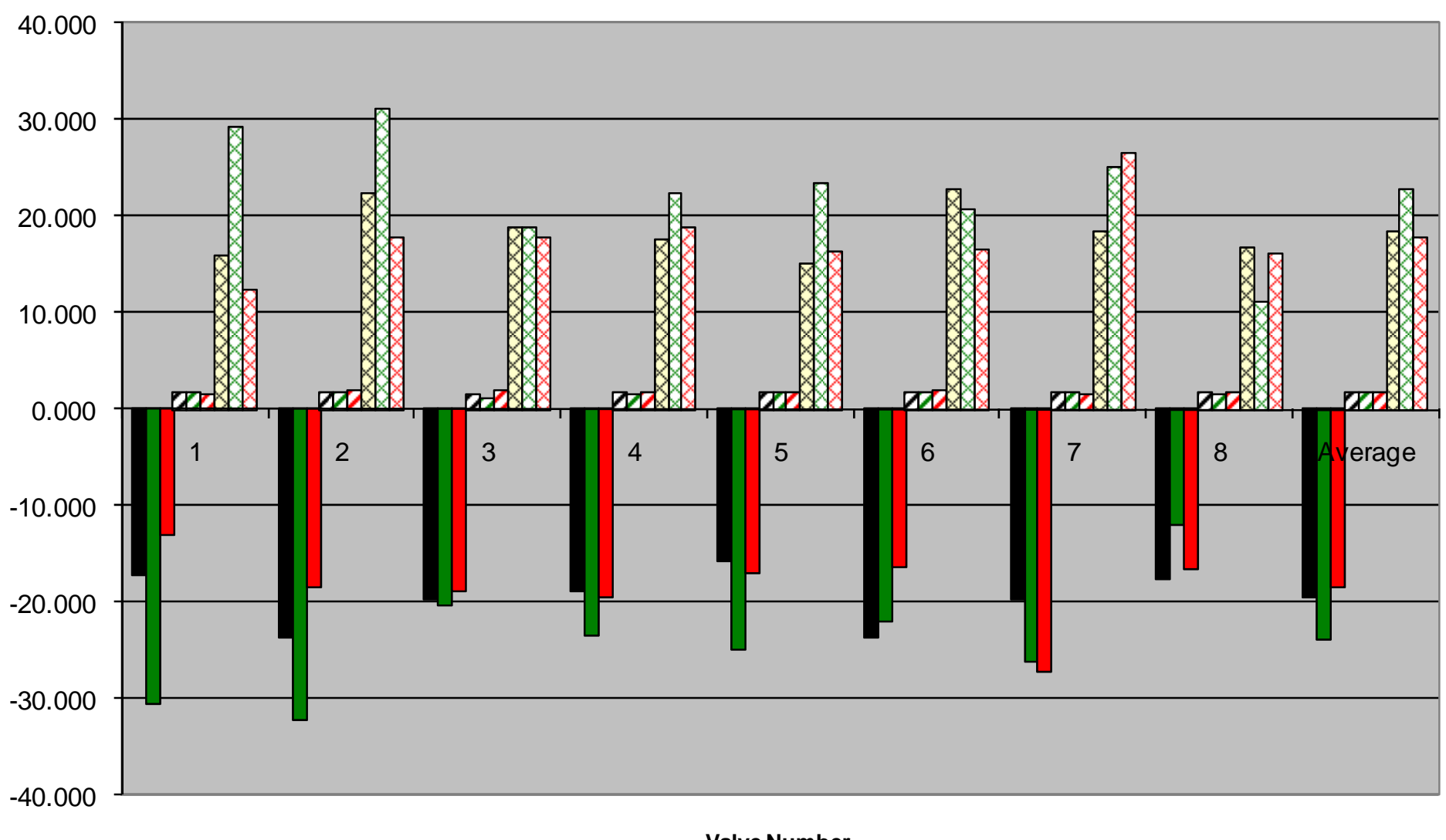

- ORCSO z(delta), $\mu \mathrm{m}$

口ORCS15 z(delta), $\mu \mathrm{m}$

口ORCS20 z(delta), $\mu \mathrm{m}$ चORCSO Ev. Length, $\mathrm{mm}$ 口ORCS15 Ev. Length, mm 口ORCS20 Ev. Length, mm DORCSO Wt, $\mu \mathrm{m}$ बORCS15 Wt, $\mu \mathrm{m}$ ๑ORCS20 Wt, $\mu \mathrm{m}$

Valve Number 


\section{Oak Ridge National Laboratory Proposal 08-58845 Powertrain Inspection Valve Contour Measurements}

\begin{tabular}{|c|c|c|c|c|c|c|c|c|c|}
\hline \multicolumn{5}{|c|}{ ORCC0 } & \multicolumn{5}{|c|}{ ORCC15 } \\
\hline $\begin{array}{l}\text { Intake } \\
\text { Valve \# }\end{array}$ & Position & $\begin{array}{c}z \text { (delta) } \\
\mu \mathrm{m}\end{array}$ & $\begin{array}{c}\text { Ev. } \\
\text { Length, } \\
\mathrm{mm}\end{array}$ & $\mathrm{Wt}, \mu \mathrm{m}$ & $\begin{array}{l}\text { Intake } \\
\text { Valve \# }\end{array}$ & Position & $\begin{array}{c}z \text { (delta) } \\
\mu \mathrm{m}\end{array}$ & $\begin{array}{c}\text { Ev. } \\
\text { Length, } \\
\mathrm{mm}\end{array}$ & $\mathrm{Wt}, \mu \mathrm{m}$ \\
\hline \multirow{4}{*}{1} & 1 & -3.445 & 1.29 & 3.25 & \multirow{4}{*}{1} & 1 & -1.576 & \begin{tabular}{|l|}
1.12 \\
\end{tabular} & 1.17 \\
\hline & 2 & -4.164 & 1.23 & 3.94 & & 2 & -2.626 & 0.94 & 2.39 \\
\hline & 3 & $\mid-3.554$ & 1.30 & 3.02 & & 3 & -3.156 & 1.48 & 2.93 \\
\hline & 4 & -3.338 & 1.29 & 2.73 & & 4 & -3.569 & 1.79 & 3.02 \\
\hline \multirow{4}{*}{2} & 1 & -2.047 & 1.04 & 3.02 & \multirow{4}{*}{2} & 1 & 3.046 & 1.18 & 3.02 \\
\hline & 2 & -4.833 & 1.08 & 5.89 & & 2 & 2.273 & 1.12 & 2.24 \\
\hline & 3 & -3.135 & 1.02 & 2.59 & & 3 & 3.375 & 1.28 & 3.99 \\
\hline & 4 & -3.344 & 1.06 & 2.93 & & 4 & 2.713 & \begin{tabular}{|l|}
1.27 \\
\end{tabular} & 4.19 \\
\hline \multirow{4}{*}{3} & 1 & -2.662 & 0.93 & 2.43 & \multirow{4}{*}{3} & 1 & 2.662 & 0.93 & 3.95 \\
\hline & 2 & -4.338 & 1.01 & 5.39 & & 2 & 2.524 & \begin{tabular}{|c|}
0.97 \\
\end{tabular} & 3.96 \\
\hline & 3 & -5.611 & 1.05 & 5.12 & & 3 & 2.718 & \begin{tabular}{|c|}
0.97 \\
\end{tabular} & 4.20 \\
\hline & 4 & -6.099 & 0.97 & 4.80 & & 4 & 1.271 & 0.97 & 3.72 \\
\hline \multirow{4}{*}{4} & 1 & -5.946 & 1.32 & 7.05 & \multirow{4}{*}{4} & 1 & -1.810 & 0.92 & 1.17 \\
\hline & 2 & -4.656 & 1.30 & 3.78 & & 2 & -2.345 & \begin{tabular}{|l|}
1.33 \\
\end{tabular} & 2.04 \\
\hline & 3 & -5.425 & 1.30 & 6.17 & & 3 & -1.134 & 1.16 & 0.90 \\
\hline & 4 & -4.806 & 1.35 & 4.06 & & 4 & -1.270 & 1.20 & 0.87 \\
\hline \multirow{4}{*}{5} & 1 & -3.195 & 1.16 & 2.69 & \multirow{4}{*}{5} & 1 & 4.650 & 1.20 & 4.44 \\
\hline & 2 & -4.538 & 1.30 & 4.13 & & 2 & 5.440 & 1.22 & 5.10 \\
\hline & 3 & -5.635 & 1.41 & 5.10 & & 3 & 6.155 & 1.29 & 5.10 \\
\hline & 4 & -4.258 & 1.17 & 3.33 & & 4 & 4.785 & 1.21 & 4.45 \\
\hline \multirow{4}{*}{6} & 1 & $\mid-1.147$ & 0.91 & 0.96 & \multirow{4}{*}{6} & 1 & -1.661 & 1.17 & 1.59 \\
\hline & 2 & \begin{tabular}{|c|}
-1.066 \\
\end{tabular} & 0.91 & 0.99 & & 2 & 2.481 & 1.10 & 2.59 \\
\hline & 3 & -1.914 & 1.20 & 1.81 & & 3 & 2.745 & 1.18 & 2.89 \\
\hline & 4 & -2.114 & 1.20 & 1.95 & & 4 & 1.608 & \begin{tabular}{|c|}
0.78 \\
\end{tabular} & 1.44 \\
\hline \multirow{4}{*}{7} & 1 & -5.005 & 1.39 & 4.54 & \multirow{4}{*}{7} & 1 & 2.258 & 1.29 & 1.86 \\
\hline & 2 & -3.399 & 1.28 & 3.23 & & 2 & 3.182 & 1.30 & 2.81 \\
\hline & 3 & -3.901 & \begin{tabular}{|l|}
1.37 \\
\end{tabular} & 4.60 & & 3 & -2.739 & 1.41 & 2.37 \\
\hline & 4 & -4.922 & 1.35 & 4.18 & & 4 & -3.097 & 1.36 & 2.76 \\
\hline \multirow{4}{*}{8} & 1 & -4.396 & 1.36 & 3.41 & \multirow{4}{*}{8} & 1 & -2.167 & \begin{tabular}{|c|}
1.17 \\
\end{tabular} & 1.43 \\
\hline & 2 & -1.421 & 1.38 & 4.53 & & 2 & -3.259 & 1.24 & 2.79 \\
\hline & 3 & -4.062 & 1.31 & 4.40 & & 3 & -1.715 & 0.97 & 1.33 \\
\hline & 4 & -2.452 & 1.25 & 4.17 & & 4 & -1.197 & 1.06 & 0.94 \\
\hline & \multicolumn{4}{|c|}{ Averages by Valve } & & \multicolumn{4}{|c|}{ Averages by Valve } \\
\hline & \begin{tabular}{|c} 
Intake \\
Valve \# \\
\end{tabular} & $\begin{array}{c}\text { ORCCO } \\
\mathrm{z} \text { (delta) } \\
\mu \mathrm{m}\end{array}$ & $\begin{array}{c}\text { ORCC0 } \\
\text { Ev. } \\
\text { Length, } \\
\mathrm{mm}\end{array}$ & $\begin{array}{l}\text { ORCCO } \\
\mathrm{Wt}, \mu \mathrm{m}\end{array}$ & & \begin{tabular}{|c} 
Intake \\
Valve \# \\
\end{tabular} & $\begin{array}{c}\text { ORCC15 } \\
\text { z(delta), } \\
\mu \mathrm{m}\end{array}$ & \begin{tabular}{|c|} 
ORCC15 \\
Ev. \\
Length, \\
$\mathrm{mm}$ \\
\end{tabular} & $\begin{array}{l}\text { ORCC15 } \\
\mathrm{Wt}, \mu \mathrm{m}\end{array}$ \\
\hline & 1 & -3.625 & 1.278 & 3.235 & & 1 & -2.732 & 1.333 & 2.378 \\
\hline & 2 & -3.340 & 1.050 & 3.608 & & 2 & 2.852 & 1.213 & 3.360 \\
\hline & 3 & -4.678 & 0.990 & 4.435 & & 3 & 2.294 & 0.960 & 3.958 \\
\hline & 4 & -5.208 & 1.318 & 5.265 & & 4 & -1.640 & 1.153 & 1.245 \\
\hline & 5 & -4.407 & 1.260 & 3.813 & & 5 & 5.258 & 1.230 & 4.773 \\
\hline & 6 & -1.560 & 1.055 & 1.428 & & 6 & 1.293 & 1.058 & 2.128 \\
\hline & 7 & -4.307 & 1.348 & 4.138 & & 7 & -0.099 & 1.340 & 2.450 \\
\hline & 8 & -3.083 & 1.325 & 4.128 & & 8 & -2.085 & 1.110 & 1.623 \\
\hline & Average & -3.776 & 1.203 & 3.756 & & Average & 0.643 & 1.174 & 2.739 \\
\hline & StDev & 1.398 & 0.156 & 1.415 & & StDev & 2.947 & 0.196 & 1.276 \\
\hline & Max & -1.066 & 1.410 & 7.050 & & Max & 6.155 & 1.790 & 5.100 \\
\hline & Min & -6.099 & 0.910 & 0.960 & & Min & -3.569 & 0.780 & 0.870 \\
\hline
\end{tabular}




\section{Oak Ridge National Laboratory Proposal 08-58845 Powertrain Inspection Valve Contour Measurements}

\begin{tabular}{|c|c|c|c|c|}
\hline \multicolumn{5}{|c|}{ ORCCO } \\
\hline $\begin{array}{l}\text { Exhaust } \\
\text { Valve \# }\end{array}$ & Position & $\begin{array}{c}z \text { (delta) } \\
\mu \mathrm{m}\end{array}$ & $\begin{array}{c}\text { Ev. } \\
\text { Length, } \\
\mathrm{mm}\end{array}$ & $\mathrm{Wt}, \mu \mathrm{m}$ \\
\hline \multirow{4}{*}{1} & 1 & -7.924 & 1.58 & 8.26 \\
\hline & 2 & -4.627 & 1.55 & 4.91 \\
\hline & 3 & -2.289 & 1.57 & 2.62 \\
\hline & 4 & -2.491 & 1.61 & 3.04 \\
\hline \multirow{4}{*}{2} & 1 & -7.135 & 1.60 & 7.32 \\
\hline & 2 & -11.942 & 1.54 & 12.09 \\
\hline & 3 & -6.414 & 1.34 & 6.77 \\
\hline & 4 & -6.848 & 1.30 & 7.17 \\
\hline \multirow{4}{*}{3} & 1 & -8.209 & 1.74 & 7.54 \\
\hline & 2 & -6.707 & 1.79 & 7.90 \\
\hline & 3 & -8.308 & 1.64 & 9.09 \\
\hline & 4 & -9.698 & 1.82 & 9.35 \\
\hline \multirow{4}{*}{4} & 1 & -5.227 & 1.42 & 5.55 \\
\hline & 2 & -5.798 & 1.51 & 7.31 \\
\hline & 3 & -7.288 & 1.46 & 6.72 \\
\hline & 4 & -3.408 & 1.55 & 4.18 \\
\hline \multirow{4}{*}{5} & 1 & -3.887 & 1.52 & 4.41 \\
\hline & 2 & -2.423 & 1.49 & 5.04 \\
\hline & 3 & -4.013 & 1.53 & 7.60 \\
\hline & 4 & -3.154 & 1.40 & 5.20 \\
\hline \multirow{4}{*}{6} & 1 & -2.715 & 1.65 & 4.03 \\
\hline & 2 & -5.698 & 1.83 & 5.33 \\
\hline & 3 & -3.095 & 1.60 & 2.88 \\
\hline & 4 & -3.450 & 1.39 & 4.66 \\
\hline \multirow{4}{*}{7} & 1 & -13.041 & 1.82 & 14.05 \\
\hline & 2 & -2.776 & 1.48 & 2.78 \\
\hline & 3 & \begin{tabular}{|l|}
-6.773 \\
\end{tabular} & 1.52 & 6.53 \\
\hline & 4 & -7.144 & 1.55 & 6.91 \\
\hline \multirow{4}{*}{8} & 1 & -1.471 & 1.48 & 3.19 \\
\hline & 2 & -10.943 & 1.85 & 11.69 \\
\hline & 3 & -5.005 & 1.53 & 5.29 \\
\hline & 4 & -4.877 & 1.36 & 5.27 \\
\hline
\end{tabular}

\begin{tabular}{|c|c|c|c|c|}
\hline \multicolumn{5}{|c|}{ ORCC15 } \\
\hline $\begin{array}{l}\text { Exhaust } \\
\text { Valve \# }\end{array}$ & Position & $\begin{array}{c}z \text { (delta), } \\
\mu \mathrm{m}\end{array}$ & $\begin{array}{c}\text { Ev. } \\
\text { Length, } \\
\text { mm }\end{array}$ & $\mathrm{Wt}, \mu \mathrm{m}$ \\
\hline \multirow{4}{*}{1} & 1 & -7.656 & 1.57 & 6.47 \\
\hline & 2 & -0.719 & 1.72 & 5.91 \\
\hline & 3 & -15.562 & 1.86 & 15.20 \\
\hline & 4 & -6.167 & 1.76 & 6.30 \\
\hline \multirow{4}{*}{2} & 1 & -4.431 & 1.57 & 4.39 \\
\hline & 2 & -2.278 & 1.85 & 4.01 \\
\hline & 3 & -3.606 & 1.39 & 3.10 \\
\hline & 4 & -8.248 & 1.49 & 8.14 \\
\hline \multirow{4}{*}{3} & 1 & -4.779 & 1.66 & 4.46 \\
\hline & 2 & -5.011 & 1.72 & 4.76 \\
\hline & 3 & -12.213 & 1.08 & 11.78 \\
\hline & 4 & -5.397 & 1.74 & 5.04 \\
\hline \multirow{4}{*}{4} & 1 & -10.636 & 1.99 & 9.64 \\
\hline & 2 & -4.487 & 1.83 & 3.90 \\
\hline & 3 & -7.505 & 1.23 & 7.19 \\
\hline & 4 & -7.081 & 2.00 & 6.79 \\
\hline \multirow{4}{*}{5} & 1 & -2.587 & 1.65 & 1.92 \\
\hline & 2 & -6.035 & 1.65 & 5.57 \\
\hline & 3 & -6.567 & 1.54 & 5.93 \\
\hline & 4 & -4.932 & 1.77 & 4.61 \\
\hline \multirow{4}{*}{6} & 1 & -3.557 & 1.73 & 6.23 \\
\hline & 2 & -3.951 & 1.58 & 4.67 \\
\hline & 3 & -3.180 & 1.79 & 5.14 \\
\hline & 4 & -2.069 & 1.83 & 2.63 \\
\hline \multirow{4}{*}{7} & 1 & -3.031 & 1.59 & 3.70 \\
\hline & 2 & -5.222 & 1.56 & 5.79 \\
\hline & 3 & -5.867 & 1.69 & 6.86 \\
\hline & 4 & -8.538 & 1.79 & 9.44 \\
\hline \multirow{4}{*}{8} & 1 & -2.857 & 1.07 & 2.37 \\
\hline & 2 & -4.627 & 1.61 & 4.23 \\
\hline & 3 & -4.654 & 1.60 & 4.30 \\
\hline & 4 & -4.765 & 1.50 & 4.14 \\
\hline
\end{tabular}

\begin{tabular}{|c|r|r|r|}
\hline \multicolumn{4}{|c|}{ Averages by Valve } \\
\hline $\begin{array}{c}\text { Exhaust } \\
\text { Valve \# }\end{array}$ & $\begin{array}{c}\text { ORCC0 } \\
\text { (delta), } \\
\mu \mathrm{m}\end{array}$ & $\begin{array}{c}\text { ORCC0 } \\
\text { Ev. } \\
\text { Length, } \\
\mathrm{mm}\end{array}$ & $\begin{array}{r}\text { ORCC0 } \\
\text { Wt, } \mu \mathrm{m}\end{array}$ \\
\hline 1 & -4.333 & 1.578 & 4.708 \\
\hline 2 & -8.085 & 1.445 & 8.338 \\
\hline 3 & -8.231 & 1.748 & 8.470 \\
\hline 4 & -5.430 & 1.485 & 5.940 \\
\hline 5 & -3.369 & 1.485 & 5.563 \\
\hline 6 & -3.740 & 1.618 & 4.225 \\
\hline 7 & -7.434 & 1.593 & 7.568 \\
\hline 8 & -5.574 & 1.555 & 6.360 \\
\hline Average & -5.774 & 1.563 & 6.396 \\
\hline StDev & 2.924 & 0.146 & 2.752 \\
\hline Max & -1.471 & 1.850 & 14.050 \\
\hline Min & -13.041 & 1.300 & 2.620 \\
\hline
\end{tabular}

\begin{tabular}{|c|r|r|r|}
\hline \multicolumn{4}{|c|}{ Averages by Valve } \\
\hline $\begin{array}{c}\text { Exhaust } \\
\text { Valve \# }\end{array}$ & $\begin{array}{c}\text { ORCC15 } \\
\text { (delta), } \\
\mu \mathrm{m}\end{array}$ & $\begin{array}{c}\text { ORCC15 } \\
\text { Ev. } \\
\text { Length, } \\
\mathrm{mm}\end{array}$ & $\begin{array}{c}\text { ORCC15 } \\
\text { Wt, } \mu \mathrm{m}\end{array}$ \\
\hline 1 & -7.526 & 1.728 & 8.470 \\
\hline 2 & -4.641 & 1.575 & 4.910 \\
\hline 3 & -6.850 & 1.550 & 6.510 \\
\hline 4 & -7.427 & 1.763 & 6.880 \\
\hline 5 & -5.030 & 1.653 & 4.508 \\
\hline 6 & -3.189 & 1.733 & 4.668 \\
\hline 7 & -5.665 & 1.658 & 6.448 \\
\hline 8 & -4.226 & 1.445 & 3.760 \\
\hline Average & -5.569 & 1.638 & 5.769 \\
\hline StDev & 3.056 & 0.218 & 2.747 \\
\hline Max & -0.719 & 2.000 & 15.200 \\
\hline Min & -15.562 & 1.070 & 1.920 \\
\hline \multicolumn{4}{|c}{}
\end{tabular}




\section{Oak Ridge National Laboratory Proposal 08-58845 Powertrain Inspection Valve Contour Measurements}

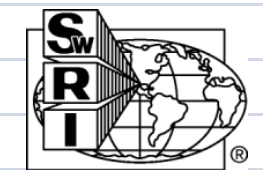

\begin{tabular}{|c|c|c|c|c|}
\hline \multicolumn{5}{|c|}{ ORCC20 } \\
\hline $\begin{array}{c}\text { Intake } \\
\text { Valve \# }\end{array}$ & Position & $\begin{array}{c}\mathrm{z} \text { (delta) } \\
\mu \mathrm{m}\end{array}$ & $\begin{array}{c}\text { Ev. } \\
\text { Length, } \\
\mathrm{mm}\end{array}$ & $\mathrm{Wt}, \mu \mathrm{m}$ \\
\hline \multirow{4}{*}{1} & 1 & -3.273 & 1.39 & 2.41 \\
\hline & 2 & -4.040 & 1.45 & 3.20 \\
\hline & 3 & -3.835 & 1.53 & 3.26 \\
\hline & 4 & -5.948 & 1.45 & 5.13 \\
\hline \multirow{4}{*}{2} & 1 & -4.783 & 1.19 & 4.26 \\
\hline & 2 & -3.596 & 1.21 & 2.90 \\
\hline & 3 & -3.880 & 1.13 & 3.41 \\
\hline & 4 & -3.657 & 1.06 & 3.36 \\
\hline \multirow{4}{*}{3} & 1 & -2.495 & 0.51 & 1.62 \\
\hline & 2 & 4.177 & 0.98 & 2.99 \\
\hline & 3 & 4.287 & 0.94 & 3.23 \\
\hline & 4 & 1.908 & 0.64 & 1.39 \\
\hline \multirow{4}{*}{4} & 1 & -2.767 & 1.11 & 2.17 \\
\hline & 2 & -1.883 & 1.06 & 1.33 \\
\hline & 3 & -3.379 & 1.09 & 2.52 \\
\hline & 4 & -2.457 & 1.19 & 2.07 \\
\hline \multirow{4}{*}{5} & 1 & -3.125 & 1.04 & 2.82 \\
\hline & 2 & -3.935 & 1.41 & $\overline{3.56}$ \\
\hline & 3 & -4.840 & 1.38 & 4.17 \\
\hline & 4 & -3.824 & 1.33 & 3.17 \\
\hline \multirow{4}{*}{6} & 1 & -8.064 & 1.51 & 7.10 \\
\hline & 2 & -7.310 & 1.37 & 6.12 \\
\hline & 3 & -6.192 & 1.31 & 5.34 \\
\hline & 4 & -5.858 & 1.12 & 5.28 \\
\hline \multirow{4}{*}{7} & 1 & 2.482 & 0.92 & 1.84 \\
\hline & 2 & 2.994 & 0.98 & 2.20 \\
\hline & 3 & 2.802 & 0.84 & 1.66 \\
\hline & 4 & 1.769 & 0.82 & 1.87 \\
\hline \multirow{4}{*}{8} & 1 & 3.156 & 1.21 & 2.46 \\
\hline & 2 & 2.643 & 1.06 & 2.01 \\
\hline & 3 & 3.505 & 1.32 & 2.73 \\
\hline & 4 & 2.857 & 1.31 & 2.06 \\
\hline
\end{tabular}

\begin{tabular}{|c|c|c|c|c|}
\hline \multicolumn{5}{|c|}{ ORCC20 } \\
\hline $\begin{array}{l}\text { Exhaust } \\
\text { Valve \# }\end{array}$ & Position & $\begin{array}{c}z \text { (delta) } \\
\mu \mathrm{m}\end{array}$ & $\begin{array}{c}\text { Ev. } \\
\text { Length, } \\
\mathrm{mm}\end{array}$ & $\mathrm{Wt}, \mu \mathrm{m}$ \\
\hline \multirow{4}{*}{1} & 1 & -4.477 & 1.72 & 4.06 \\
\hline & 2 & -4.214 & 1.81 & 3.58 \\
\hline & 3 & -4.609 & 1.91 & 4.01 \\
\hline & 4 & -5.814 & 1.83 & 5.15 \\
\hline \multirow{4}{*}{2} & 1 & 8.012 & 1.52 & 7.21 \\
\hline & 2 & 7.592 & 1.63 & 6.96 \\
\hline & 3 & -8.704 & 0.77 & 8.63 \\
\hline & 4 & 5.704 & 1.19 & 5.87 \\
\hline \multirow{4}{*}{3} & 1 & 8.582 & 1.95 & 7.71 \\
\hline & 2 & 4.351 & 1.97 & 4.22 \\
\hline & 3 & 7.914 & 2.03 & 6.62 \\
\hline & 4 & 12.804 & 1.99 & 12.03 \\
\hline \multirow{4}{*}{4} & 1 & 4.652 & 1.91 & 4.46 \\
\hline & 2 & 3.772 & 1.72 & 3.79 \\
\hline & 3 & 12.755 & 1.89 & 12.32 \\
\hline & 4 & 9.335 & 1.92 & 8.16 \\
\hline \multirow{4}{*}{5} & 1 & -3.732 & 1.24 & 3.12 \\
\hline & 2 & -6.533 & 1.20 & 6.23 \\
\hline & 3 & -3.222 & 1.17 & 2.90 \\
\hline & 4 & -4.070 & 1.42 & 3.00 \\
\hline \multirow{4}{*}{6} & 1 & 5.086 & 1.44 & 4.00 \\
\hline & 2 & 4.152 & 1.41 & 3.79 \\
\hline & 3 & 7.886 & 1.92 & 7.32 \\
\hline & 4 & 6.892 & 1.85 & 6.34 \\
\hline \multirow{4}{*}{7} & 1 & -5.893 & 1.30 & 5.86 \\
\hline & 2 & -6.028 & 1.73 & 5.18 \\
\hline & 3 & -3.022 & 1.80 & 2.25 \\
\hline & 4 & -5.128 & 1.45 & 4.22 \\
\hline \multirow{4}{*}{8} & 1 & 4.821 & 1.68 & 4.56 \\
\hline & 2 & 7.341 & 1.67 & 6.67 \\
\hline & 3 & 5.331 & 0.99 & 5.10 \\
\hline & 4 & 2.365 & 0.85 & 2.13 \\
\hline
\end{tabular}

\begin{tabular}{|c|r|r|r|}
\hline \multicolumn{4}{|c|}{ Averages by Valve } \\
\hline $\begin{array}{c}\text { Intake } \\
\text { Valve \# }\end{array}$ & $\begin{array}{c}\text { ORCC20 } \\
\text { (delta), } \\
\mu m\end{array}$ & $\begin{array}{c}\text { ORCC20 } \\
\text { Ev. } \\
\text { Length, } \\
\mathrm{mm}\end{array}$ & $\begin{array}{c}\text { ORCC20 } \\
\text { Wt, } \mu \mathrm{m}\end{array}$ \\
\hline 1 & -4.274 & 1.455 & 3.500 \\
\hline 2 & -3.979 & 1.148 & 3.483 \\
\hline 3 & 1.969 & 0.768 & 2.308 \\
\hline 4 & -2.622 & 1.113 & 2.023 \\
\hline 5 & -3.931 & 1.290 & 3.430 \\
\hline 6 & -6.856 & 1.328 & 5.960 \\
\hline 7 & 2.512 & 0.890 & 1.893 \\
\hline 8 & 3.040 & 1.225 & 2.315 \\
\hline Average & -1.768 & 1.152 & 3.114 \\
\hline StDev & 3.740 & 0.246 & 1.412 \\
\hline Max & 4.287 & 1.530 & 7.100 \\
\hline Min & -8.064 & 0.510 & 1.330 \\
\hline
\end{tabular}

\begin{tabular}{|c|r|r|r|}
\hline \multicolumn{4}{|c|}{ Averages by Valve } \\
\hline $\begin{array}{c}\text { Exhaust } \\
\text { Valve \# }\end{array}$ & $\begin{array}{c}\text { ORCC20 } \\
\text { Odelta), } \\
\mu \mathrm{m}\end{array}$ & $\begin{array}{c}\text { ORCC20 } \\
\text { Ev. } \\
\text { Length, } \\
\mathrm{mm}\end{array}$ & $\begin{array}{c}\text { ORCC20 } \\
\text { Wt, } \mu \mathrm{m}\end{array}$ \\
\hline 1 & -4.779 & 1.818 & 4.200 \\
\hline 2 & 3.151 & 1.278 & 7.168 \\
\hline 3 & 8.413 & 1.985 & 7.645 \\
\hline 4 & 7.629 & 1.860 & 7.183 \\
\hline 5 & -4.389 & 1.258 & 3.813 \\
\hline 6 & 6.004 & 1.655 & 5.363 \\
\hline 7 & -5.018 & 1.570 & 4.378 \\
\hline 8 & 4.965 & 1.298 & 4.615 \\
\hline Average & 1.997 & 1.590 & 5.545 \\
\hline StDev & 6.361 & 0.351 & 2.447 \\
\hline Max & 12.804 & 2.030 & 12.320 \\
\hline Min & -8.704 & 0.770 & 2.130 \\
\hline & \multicolumn{3}{|c}{}
\end{tabular}




\section{Oakridge National Laboratory \\ 2006 Chevrolet Cobalt \\ Intake Valve Contour Measurements at EOT}

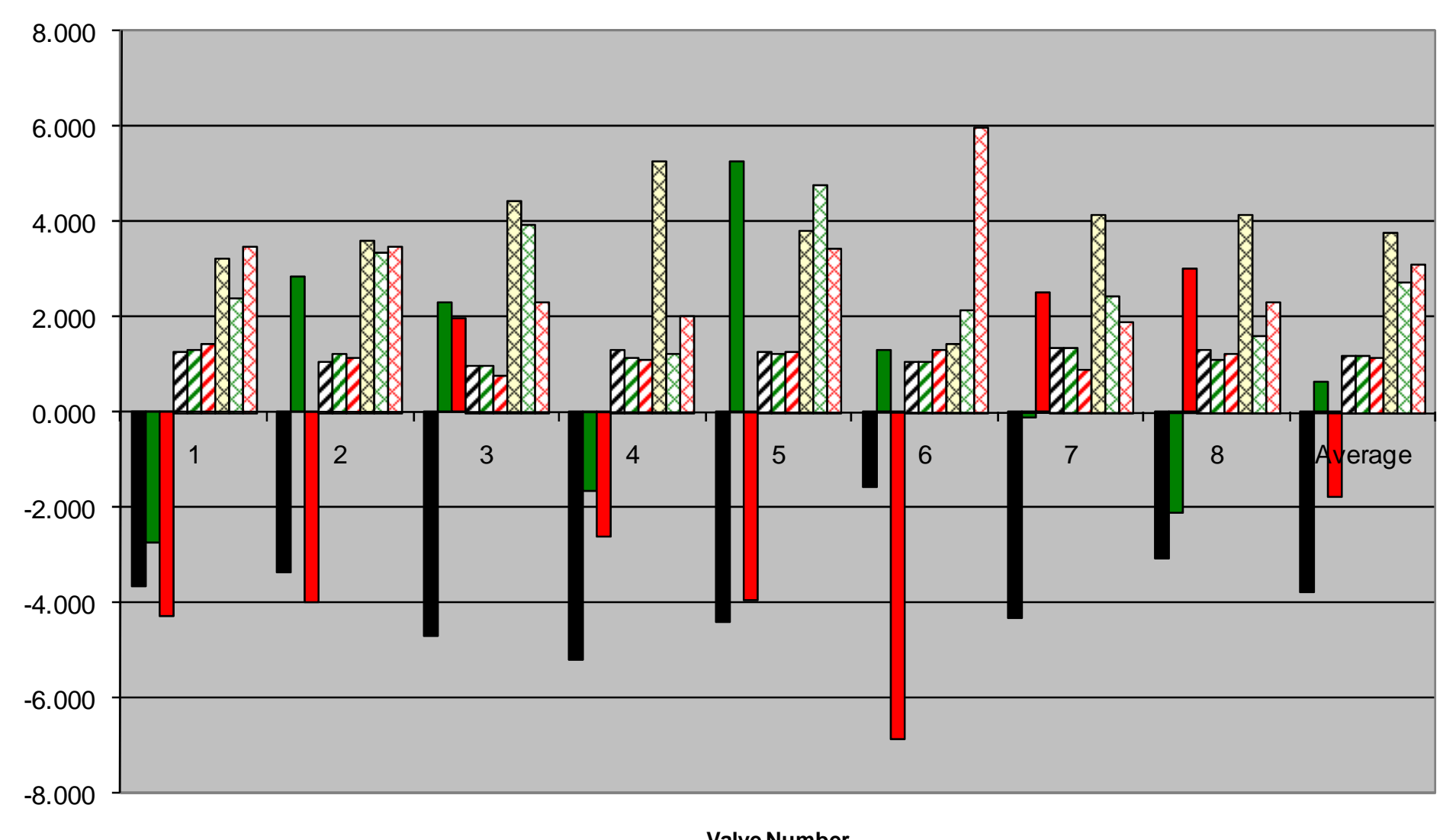

Valve Number

- ORCC0 z(delta), $\mu \mathrm{m}$ 口ORCC15 z(delta), $\mu \mathrm{m}$ 口ORCC20 z(delta), $\mu \mathrm{m}$ $\square$ ORCCO Ev. Length, $\mathrm{mm}$ घORCC15 Ev. Length, mm a ORCC20 Ev. Length, mm DORCCO Wt, $\mu \mathrm{m}$ घORCC15 Wt, $\mu \mathrm{m}$ ๑ORCC20 Wt, $\mu \mathrm{m}$ 


\section{Oakridge National Laboratory \\ 2006 Chevrolet Cobalt \\ Exhaust Valve Contour Measurements at EOT}

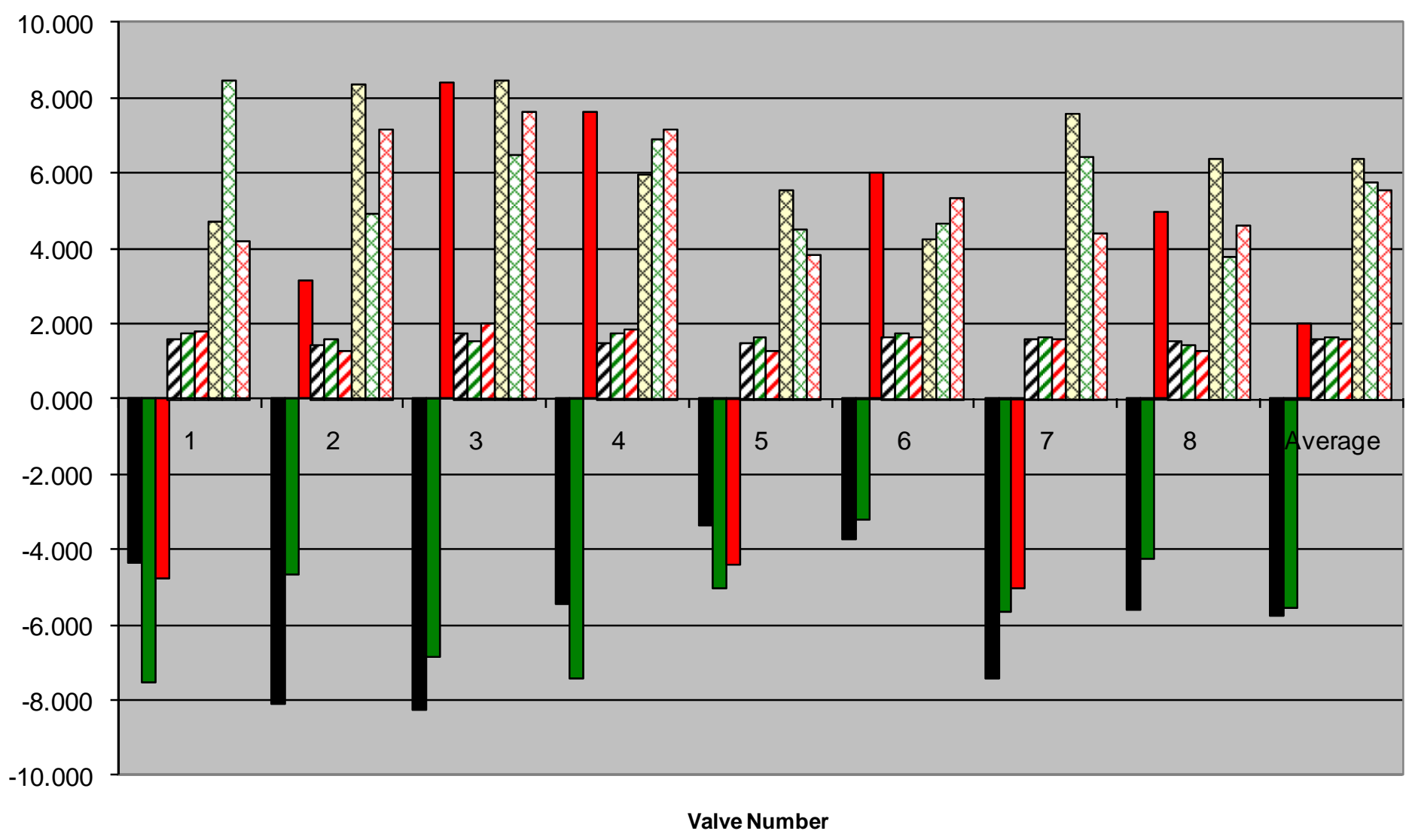

- ORCC0 z(delta), $\mu \mathrm{m}$

DORCC15 z(delta), $\mu \mathrm{m}$

口ORCC20 z(delta), $\mu \mathrm{m}$

$\triangle O R C C 0$ Ev. Length, $\mathrm{mm}$

口ORCC15 Ev. Length, $\mathrm{mm}$

口ORCC20 Ev. Length, $\mathrm{mm}$

DORCCO Wt, $\mu \mathrm{m}$

口ORCC15 Wt, $\mu \mathrm{m}$

$\triangle \mathrm{ORCC} 20 \mathrm{Wt}, \mu \mathrm{m}$

Valve Number 


\section{Oak Ridge National Laboratory Proposal 08-58845 Powertrain Inspection Valve Contour Measurements}

\begin{tabular}{|c|c|c|c|c|c|c|c|c|c|}
\hline \multicolumn{5}{|c|}{ ORNA0 } & \multicolumn{5}{|c|}{ ORNA15 } \\
\hline $\begin{array}{l}\text { Intake } \\
\text { Valve \# }\end{array}$ & Position & $\begin{array}{c}z \text { (delta) } \\
\mu \mathrm{m}\end{array}$ & $\begin{array}{c}\text { Ev. } \\
\text { Length, } \\
\mathrm{mm}\end{array}$ & $\mathrm{Wt}, \mu \mathrm{m}$ & $\begin{array}{c}\text { Intake } \\
\text { Valve \# }\end{array}$ & Position & $\begin{array}{c}z \text { (delta) } \\
\mu \mathrm{m}\end{array}$ & $\begin{array}{c}\text { Ev. } \\
\text { Length, } \\
\mathrm{mm}\end{array}$ & $\mathrm{Wt}, \mu \mathrm{m}$ \\
\hline \multirow{4}{*}{1} & 1 & -1.051 & 0.42 & 0.79 & \multirow{4}{*}{1} & 1 & -2.663 & 1.04 & 1.46 \\
\hline & 2 & $\mid-2.744$ & 0.66 & 2.26 & & 2 & -3.835 & 1.06 & 2.76 \\
\hline & 3 & -3.262 & 0.55 & 2.43 & & 3 & -2.213 & 0.95 & 1.60 \\
\hline & 4 & -2.059 & 0.67 & 1.47 & & 4 & -2.190 & 0.94 & 1.78 \\
\hline \multirow{4}{*}{2} & 1 & -1.593 & 0.68 & 1.37 & \multirow{4}{*}{2} & 1 & -1.363 & 0.72 & 1.24 \\
\hline & 2 & \begin{tabular}{|c|}
-1.628 \\
\end{tabular} & 0.49 & 1.38 & & 2 & -1.689 & 0.40 & 0.88 \\
\hline & 3 & -1.456 & 0.46 & 0.79 & & 3 & -2.029 & 0.47 & 1.16 \\
\hline & 4 & \begin{tabular}{|c|}
-1.943 \\
\end{tabular} & 0.49 & 1.40 & & 4 & -2.448 & 0.53 & 1.71 \\
\hline \multirow{4}{*}{3} & 1 & -3.180 & 0.73 & 2.92 & \multirow{4}{*}{3} & 1 & -1.554 & 0.40 & 1.08 \\
\hline & 2 & -1.834 & 0.69 & 1.30 & & $\overline{2}$ & -1.531 & 0.47 & 0.86 \\
\hline & 3 & -3.180 & 0.63 & 2.31 & & 3 & -0.841 & 0.53 & 0.44 \\
\hline & 4 & \begin{tabular}{|c|}
-1.888 \\
\end{tabular} & 0.55 & 1.49 & & 4 & -1.782 & 0.51 & 1.12 \\
\hline \multirow{4}{*}{4} & 1 & -1.635 & 0.49 & 1.10 & \multirow{4}{*}{4} & 1 & -2.301 & 0.65 & 1.32 \\
\hline & 2 & -3.491 & 0.66 & 2.88 & & 2 & -1.509 & 0.57 & 0.99 \\
\hline & 3 & -2.228 & 0.69 & 2.48 & & 3 & -3.513 & 0.79 & 3.11 \\
\hline & 4 & -3.100 & 0.51 & 2.37 & & 4 & \begin{tabular}{|c|}
-1.376 \\
\end{tabular} & 0.51 & 0.99 \\
\hline \multirow{4}{*}{5} & 1 & \begin{tabular}{|c|}
-2.663 \\
\end{tabular} & 0.67 & 2.13 & \multirow{4}{*}{5} & 1 & -1.800 & 0.42 & 1.60 \\
\hline & 2 & -2.303 & 0.59 & 1.79 & & 2 & -4.045 & 0.69 & 3.58 \\
\hline & 3 & $\mid-2.013$ & 0.61 & 1.78 & & 3 & -1.431 & 0.35 & 0.71 \\
\hline & 4 & -3.993 & 0.72 & 3.10 & & 4 & -1.120 & 0.35 & 0.40 \\
\hline \multirow{4}{*}{6} & 1 & -4.504 & 0.84 & 3.44 & \multirow{4}{*}{6} & 1 & -1.441 & 0.48 & 0.81 \\
\hline & 2 & $\mid-3.371$ & 0.76 & 2.62 & & 2 & -1.479 & 0.41 & 1.38 \\
\hline & 3 & -2.442 & \begin{tabular}{|c|}
0.74 \\
\end{tabular} & 2.17 & & 3 & -2.359 & \begin{tabular}{|l|}
0.48 \\
\end{tabular} & 1.88 \\
\hline & 4 & -3.498 & 0.71 & 2.56 & & 4 & -1.313 & 0.44 & 0.94 \\
\hline \multirow{4}{*}{7} & 1 & -2.432 & 0.75 & 1.61 & \multirow{4}{*}{7} & 1 & $\mid-1.713$ & 0.53 & 1.24 \\
\hline & 2 & -2.310 & 0.82 & 2.11 & & 2 & -2.157 & 0.46 & 1.62 \\
\hline & 3 & -4.199 & \begin{tabular}{|c|}
0.73 \\
\end{tabular} & 3.18 & & 3 & \begin{tabular}{|l|}
-1.163 \\
\end{tabular} & 0.55 & 1.31 \\
\hline & 4 & -2.385 & 0.68 & 1.72 & & 4 & \begin{tabular}{|c|}
-1.307 \\
\end{tabular} & 0.41 & 0.93 \\
\hline \multirow{18}{*}{8} & 1 & \begin{tabular}{|c|}
-2.288 \\
\end{tabular} & 0.76 & 1.94 & \multirow{4}{*}{8} & 1 & \begin{tabular}{|c|}
-3.548 \\
\end{tabular} & 0.72 & 2.49 \\
\hline & 2 & -3.124 & 0.96 & 2.97 & & 2 & -1.254 & 0.65 & 0.77 \\
\hline & 3 & -1.596 & 0.58 & 1.36 & & 3 & -2.441 & 0.66 & 1.60 \\
\hline & 4 & \begin{tabular}{|c|}
-1.543 \\
\end{tabular} & 0.75 & 1.84 & & 4 & -1.250 & 0.58 & 1.04 \\
\hline & \multicolumn{4}{|c|}{ Averages by Valve } & & \multicolumn{4}{|c|}{ Averages by Valve } \\
\hline & \begin{tabular}{|c} 
Intake \\
Valve \#
\end{tabular} & $\begin{array}{c}\text { ORNAO } \\
z \text { (delta) } \\
\mu \mathrm{m}\end{array}$ & $\begin{array}{c}\text { ORNA0 } \\
\text { Ev. } \\
\text { Length, } \\
\mathrm{mm}\end{array}$ & $\begin{array}{l}\text { ORNAO } \\
\mathrm{Wt}, \mu \mathrm{m}\end{array}$ & & $\begin{array}{l}\text { Intake } \\
\text { Valve \# }\end{array}$ & $\begin{array}{l}\text { ORNA15 } \\
\text { z(delta) } \\
\mu \mathrm{m}\end{array}$ & $\begin{array}{l}\text { ORNA15 } \\
\text { Ev. } \\
\text { Length, } \\
\text { mm }\end{array}$ & $\begin{array}{l}\text { ORNA15 } \\
\mathrm{Wt}, \mu \mathrm{m}\end{array}$ \\
\hline & 1 & -2.279 & 0.575 & 1.738 & & 1 & -2.725 & 0.998 & 1.900 \\
\hline & 2 & -1.655 & 0.530 & 1.235 & & 2 & -1.882 & 0.530 & 1.248 \\
\hline & 3 & -2.521 & 0.650 & 2.005 & & 3 & \begin{tabular}{|c|}
-1.427 \\
\end{tabular} & 0.478 & 0.875 \\
\hline & 4 & -2.614 & 0.588 & 2.208 & & 4 & -2.175 & 0.630 & 1.603 \\
\hline & 5 & -2.743 & 0.648 & 2.200 & & 5 & -2.099 & 0.453 & 1.573 \\
\hline & 6 & -3.454 & 0.763 & 2.698 & & 6 & -1.648 & 0.453 & 1.253 \\
\hline & 7 & -2.832 & 0.745 & 2.155 & & 7 & -1.585 & 0.488 & 1.275 \\
\hline & 8 & -2.138 & 0.763 & 2.028 & & 8 & -2.123 & 0.653 & 1.475 \\
\hline & Average & -2.529 & 0.658 & 2.033 & & Average & -1.958 & 0.585 & 1.400 \\
\hline & StDev & 0.865 & 0.121 & 0.704 & & StDev & 0.820 & 0.194 & 0.724 \\
\hline & Max & -1.051 & 0.960 & 3.440 & & Max & -0.841 & 1.060 & 3.580 \\
\hline & Min & -4.504 & 0.420 & 0.790 & & Min & -4.045 & 0.350 & 0.400 \\
\hline
\end{tabular}




\section{Oak Ridge National Laboratory Proposal 08-58845 Powertrain Inspection Valve Contour Measurements}

\begin{tabular}{|c|c|c|c|c|}
\hline \multicolumn{5}{|c|}{ ORNA0 } \\
\hline $\begin{array}{l}\text { Exhaust } \\
\text { Valve \# }\end{array}$ & Position & $\begin{array}{c}z \text { (delta) } \\
\mu \mathrm{m}\end{array}$ & $\begin{array}{c}\text { Ev. } \\
\text { Length, } \\
\mathrm{mm}\end{array}$ & $\mathrm{Wt}, \mu \mathrm{m}$ \\
\hline \multirow{4}{*}{1} & 1 & -4.582 & 1.38 & 4.06 \\
\hline & 2 & -9.665 & 1.45 & 9.46 \\
\hline & 3 & -12.477 & 1.49 & 18.62 \\
\hline & 4 & -15.625 & 1.40 & 15.00 \\
\hline \multirow{4}{*}{2} & 1 & -15.327 & 1.79 & 17.11 \\
\hline & 2 & -9.870 & 1.66 & 9.40 \\
\hline & 3 & -17.179 & 1.44 & 21.90 \\
\hline & 4 & -30.462 & 1.76 & 29.72 \\
\hline \multirow{4}{*}{3} & 1 & -17.691 & 1.21 & 15.68 \\
\hline & 2 & -16.194 & 1.57 & 17.46 \\
\hline & 3 & -15.443 & 1.64 & 16.46 \\
\hline & 4 & \begin{tabular}{|c|}
-9.187 \\
\end{tabular} & 1.59 & 10.01 \\
\hline \multirow{4}{*}{4} & 1 & -15.601 & 1.46 & 15.18 \\
\hline & 2 & -16.764 & 1.75 & 16.60 \\
\hline & 3 & -9.465 & 1.63 & 11.84 \\
\hline & 4 & -10.515 & 1.69 & 11.58 \\
\hline \multirow{4}{*}{5} & 1 & -6.958 & 1.26 & 6.42 \\
\hline & 2 & -16.216 & 1.61 & 16.36 \\
\hline & 3 & -13.695 & 1.65 & 16.33 \\
\hline & 4 & -11.639 & 1.05 & 11.49 \\
\hline \multirow{4}{*}{6} & 1 & -6.352 & 1.15 & 6.27 \\
\hline & 2 & -5.842 & 1.41 & 7.46 \\
\hline & 3 & -5.523 & 1.08 & 5.26 \\
\hline & 4 & -4.659 & 1.62 & 4.75 \\
\hline \multirow{4}{*}{7} & 1 & -7.892 & 1.46 & 7.68 \\
\hline & 2 & -7.252 & 1.39 & 6.40 \\
\hline & 3 & -6.129 & 1.48 & 5.85 \\
\hline & 4 & -16.531 & 1.10 & 16.14 \\
\hline \multirow{4}{*}{8} & 1 & -22.623 & 1.64 & 22.38 \\
\hline & 2 & -19.952 & 1.70 & 19.57 \\
\hline & 3 & -24.253 & 1.13 & 23.74 \\
\hline & 4 & -19.589 & 1.85 & 18.67 \\
\hline
\end{tabular}

\begin{tabular}{|c|c|c|c|c|}
\hline \multicolumn{5}{|c|}{ ORNA15 } \\
\hline $\begin{array}{l}\text { Exhaust } \\
\text { Valve \# }\end{array}$ & Position & $\begin{array}{c}z \text { (delta) } \\
\mu \mathrm{m}\end{array}$ & $\begin{array}{c}\text { Ev. } \\
\text { Length, } \\
\mathrm{mm}\end{array}$ & $\mathrm{Wt}, \mu \mathrm{m}$ \\
\hline \multirow{4}{*}{1} & 1 & -12.968 & 1.57 & 12.03 \\
\hline & 2 & -18.614 & 1.58 & 17.91 \\
\hline & 3 & -25.610 & 1.73 & 27.53 \\
\hline & 4 & -12.959 & 1.53 & 12.18 \\
\hline \multirow{4}{*}{2} & 1 & -16.505 & 1.57 & 15.83 \\
\hline & 2 & -18.029 & 1.23 & 19.03 \\
\hline & 3 & -15.298 & 0.86 & 15.22 \\
\hline & 4 & -24.416 & 1.75 & 23.00 \\
\hline \multirow{4}{*}{3} & 1 & -4.075 & 1.29 & 4.00 \\
\hline & 2 & -3.251 & 1.13 & 3.44 \\
\hline & 3 & -5.793 & 1.29 & 5.79 \\
\hline & 4 & -5.605 & 1.37 & 5.27 \\
\hline \multirow{4}{*}{4} & 1 & -7.847 & 1.29 & 7.27 \\
\hline & 2 & -3.582 & 1.22 & 3.10 \\
\hline & 3 & -2.340 & 1.15 & 2.04 \\
\hline & 4 & -3.413 & 1.31 & 2.83 \\
\hline \multirow{4}{*}{5} & 1 & -12.169 & 1.06 & 11.79 \\
\hline & 2 & -2.332 & 1.10 & 2.47 \\
\hline & 3 & -4.994 & 1.62 & 4.86 \\
\hline & 4 & -5.162 & 1.46 & 6.10 \\
\hline \multirow{4}{*}{6} & 1 & -4.936 & 1.01 & 4.75 \\
\hline & 2 & -8.765 & 1.41 & 8.07 \\
\hline & 3 & -5.363 & 1.29 & 4.47 \\
\hline & 4 & -7.137 & 1.20 & 6.99 \\
\hline \multirow{4}{*}{7} & 1 & -9.135 & 1.23 & 8.48 \\
\hline & 2 & -5.116 & 1.01 & 5.46 \\
\hline & 3 & -9.279 & 1.32 & 9.69 \\
\hline & 4 & -6.682 & 1.60 & 8.02 \\
\hline \multirow{4}{*}{8} & 1 & -12.443 & 1.52 & 13.53 \\
\hline & 2 & -4.124 & 1.09 & 3.48 \\
\hline & 3 & -8.404 & 1.45 & 8.89 \\
\hline & 4 & -10.178 & 1.63 & 9.84 \\
\hline
\end{tabular}

\begin{tabular}{|c|r|r|r|}
\hline \multicolumn{4}{|c|}{ Averages by Valve } \\
\hline $\begin{array}{c}\text { Exhaust } \\
\text { Valve \# }\end{array}$ & $\begin{array}{c}\text { ORNA0 } \\
\text { (delta), } \\
\mu \mathrm{m}\end{array}$ & $\begin{array}{c}\text { ORNA0 } \\
\text { Ev. } \\
\text { Length, } \\
\mathrm{mm}\end{array}$ & $\begin{array}{c}\text { ORNA0 } \\
\mathrm{Wt}, \mu \mathrm{m}\end{array}$ \\
\hline 1 & -10.587 & 1.430 & 11.785 \\
\hline 2 & -18.210 & 1.663 & 19.533 \\
\hline 3 & -14.629 & 1.503 & 14.903 \\
\hline 4 & -13.086 & 1.633 & 13.800 \\
\hline 5 & -12.127 & 1.393 & 12.650 \\
\hline 6 & -5.594 & 1.315 & 5.935 \\
\hline 7 & -9.451 & 1.358 & 9.018 \\
\hline 8 & -21.604 & 1.580 & 21.090 \\
\hline Average & -13.161 & 1.484 & 13.589 \\
\hline StDev & 6.269 & 0.223 & 6.370 \\
\hline Max & -4.582 & 1.850 & 29.720 \\
\hline Min & -30.462 & 1.050 & 4.060 \\
\hline
\end{tabular}

\begin{tabular}{|c|r|r|r|}
\hline \multicolumn{4}{|c|}{ Averages by Valve } \\
\hline $\begin{array}{c}\text { Exhaust } \\
\text { Valve \# }\end{array}$ & $\begin{array}{c}\text { ORNA15 } \\
\text { (delta), } \\
\mu \mathrm{m}\end{array}$ & $\begin{array}{c}\text { ORNA15 } \\
\text { Ev. } \\
\text { Length, } \\
\mathrm{mm}\end{array}$ & $\begin{array}{c}\text { ORNA15 } \\
\text { Wt, } \mu \mathrm{m}\end{array}$ \\
\hline 1 & -17.538 & 1.603 & 17.413 \\
\hline 2 & -18.562 & 1.353 & 18.270 \\
\hline 3 & -4.681 & 1.270 & 4.625 \\
\hline 4 & -4.296 & 1.243 & 3.810 \\
\hline 5 & -6.164 & 1.310 & 6.305 \\
\hline 6 & -6.550 & 1.228 & 6.070 \\
\hline 7 & -7.553 & 1.290 & 7.913 \\
\hline 8 & -8.787 & 1.423 & 8.935 \\
\hline Average & -9.266 & 1.340 & 9.168 \\
\hline StDev & 6.155 & 0.228 & 6.255 \\
\hline Max & -2.332 & 1.750 & 27.530 \\
\hline Min & -25.610 & 0.860 & 2.040 \\
\hline
\end{tabular}




\section{Oak Ridge National Laboratory Proposal 08-58845 Powertrain Inspection Valve Contour Measurements}

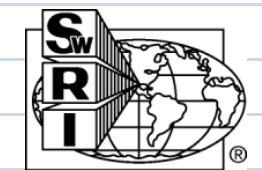

\begin{tabular}{|c|c|c|c|c|}
\hline \multicolumn{5}{|c|}{ ORNA20 } \\
\hline $\begin{array}{c}\text { Intake } \\
\text { Valve \# }\end{array}$ & Position & $\begin{array}{c}z \text { (delta) } \\
\mu \mathrm{m}\end{array}$ & $\begin{array}{c}\text { Ev. } \\
\text { Length, } \\
\mathrm{mm}\end{array}$ & $\mathrm{Wt}, \mu \mathrm{m}$ \\
\hline \multirow{4}{*}{1} & 1 & -2.369 & 0.87 & 2.14 \\
\hline & 2 & -2.758 & 0.91 & 2.17 \\
\hline & 3 & -2.446 & 0.91 & 1.89 \\
\hline & 4 & -2.260 & 0.90 & 1.43 \\
\hline \multirow{4}{*}{2} & 1 & -2.614 & 1.00 & 2.17 \\
\hline & 2 & -2.802 & 0.88 & 2.26 \\
\hline & 3 & -0.491 & 0.79 & 0.91 \\
\hline & 4 & -1.357 & 0.59 & 1.20 \\
\hline \multirow{4}{*}{3} & 1 & -1.809 & 0.92 & 1.55 \\
\hline & 2 & -1.508 & 0.64 & 1.16 \\
\hline & 3 & -1.388 & 0.68 & 0.96 \\
\hline & 4 & -1.695 & 0.84 & 1.32 \\
\hline \multirow{4}{*}{4} & 1 & -3.131 & 0.69 & 2.58 \\
\hline & 2 & -0.728 & 0.67 & 0.39 \\
\hline & 3 & -1.831 & 0.95 & 1.26 \\
\hline & 4 & -1.535 & 0.63 & 0.96 \\
\hline \multirow{4}{*}{5} & 1 & -2.204 & 0.80 & 1.51 \\
\hline & 2 & -1.401 & 0.62 & 1.00 \\
\hline & 3 & -2.331 & 0.66 & 1.68 \\
\hline & 4 & -3.565 & 0.85 & 2.74 \\
\hline \multirow{4}{*}{6} & 1 & -3.196 & 0.90 & 2.55 \\
\hline & 2 & -1.797 & 0.94 & 1.44 \\
\hline & 3 & -2.249 & 0.82 & 1.60 \\
\hline & 4 & -2.366 & 0.84 & 1.55 \\
\hline \multirow{4}{*}{7} & 1 & -3.041 & 0.72 & 2.25 \\
\hline & 2 & -2.404 & 0.74 & 1.58 \\
\hline & 3 & -2.184 & 0.74 & 1.80 \\
\hline & 4 & -1.870 & 0.66 & 1.33 \\
\hline \multirow{4}{*}{8} & 1 & -3.229 & 0.70 & 2.71 \\
\hline & 2 & -0.780 & 0.51 & 0.55 \\
\hline & 3 & -1.667 & 0.51 & 1.16 \\
\hline & 4 & -2.221 & 0.88 & 1.96 \\
\hline
\end{tabular}

\begin{tabular}{|c|c|c|c|c|}
\hline \multicolumn{5}{|c|}{ ORNA20 } \\
\hline $\begin{array}{l}\text { Exhaust } \\
\text { Valve \# }\end{array}$ & Position & $\begin{array}{c}z \text { (delta) } \\
\mu \mathrm{m}\end{array}$ & $\begin{array}{c}\text { Ev. } \\
\text { Length, } \\
\mathrm{mm}\end{array}$ & $\mathrm{Wt}, \mu \mathrm{m}$ \\
\hline \multirow{4}{*}{1} & 1 & -4.554 & 1.70 & 4.22 \\
\hline & 2 & -25.494 & 1.79 & 28.33 \\
\hline & 3 & -8.465 & 1.46 & 9.27 \\
\hline & 4 & -18.606 & 1.51 & 21.04 \\
\hline \multirow{4}{*}{2} & 1 & -14.848 & 1.51 & 16.35 \\
\hline & 2 & -15.794 & 1.71 & 16.44 \\
\hline & 3 & -23.593 & 1.67 & 22.76 \\
\hline & 4 & -12.086 & 1.73 & 13.90 \\
\hline \multirow{4}{*}{3} & 1 & -9.013 & 1.63 & 12.25 \\
\hline & 2 & -7.173 & 1.71 & 11.23 \\
\hline & 3 & -6.468 & 1.31 & 7.98 \\
\hline & 4 & -12.880 & 1.78 & 16.74 \\
\hline \multirow{4}{*}{4} & 1 & -28.396 & 1.62 & 32.90 \\
\hline & 2 & -11.171 & 1.37 & 13.52 \\
\hline & 3 & -21.730 & 1.88 & 24.43 \\
\hline & 4 & -15.081 & 1.76 & 15.98 \\
\hline \multirow{4}{*}{5} & 1 & -19.357 & 1.23 & 18.85 \\
\hline & 2 & -5.631 & 1.57 & 5.89 \\
\hline & 3 & -7.295 & 1.43 & 7.27 \\
\hline & 4 & -16.249 & 1.71 & 17.21 \\
\hline \multirow{4}{*}{6} & 1 & -24.117 & 1.48 & 22.71 \\
\hline & 2 & -28.639 & 1.57 & 28.96 \\
\hline & 3 & -16.797 & 1.24 & 15.71 \\
\hline & 4 & -16.141 & 1.50 & 16.40 \\
\hline \multirow{4}{*}{7} & 1 & -3.078 & 1.87 & 2.72 \\
\hline & 2 & -3.266 & 1.82 & 3.98 \\
\hline & 3 & -6.081 & 1.84 & 8.80 \\
\hline & 4 & -3.440 & 2.04 & 2.88 \\
\hline \multirow{4}{*}{8} & 1 & -8.870 & 1.72 & 8.40 \\
\hline & 2 & -4.576 & 1.28 & 6.23 \\
\hline & 3 & -12.222 & 1.86 & 13.33 \\
\hline & 4 & -10.333 & 1.77 & 13.86 \\
\hline
\end{tabular}

\begin{tabular}{|c|r|r|r|}
\hline \multicolumn{4}{|c|}{ Averages by Valve } \\
\hline $\begin{array}{c}\text { Intake } \\
\text { Valve \# }\end{array}$ & $\begin{array}{c}\text { ORNA20 } \\
\text { (delta), } \\
\mu \mathrm{m}\end{array}$ & $\begin{array}{c}\text { ORNA20 } \\
\text { Ev. } \\
\text { Length, } \\
\mathrm{mm}\end{array}$ & $\begin{array}{c}\text { ORNA20 } \\
\text { Wt, } \mu \mathrm{m}\end{array}$ \\
\hline 1 & -2.458 & 0.898 & 1.908 \\
\hline 2 & -1.816 & 0.815 & 1.635 \\
\hline 3 & -1.600 & 0.770 & 1.248 \\
\hline 4 & -1.806 & 0.735 & 1.298 \\
\hline 5 & -2.375 & 0.733 & 1.733 \\
\hline 6 & -2.402 & 0.875 & 1.785 \\
\hline 7 & -2.375 & 0.715 & 1.740 \\
\hline 8 & -1.974 & 0.650 & 1.595 \\
\hline Average & -2.101 & 0.774 & 1.618 \\
\hline StDev & 0.748 & 0.133 & 0.610 \\
\hline Max & -0.491 & 1.000 & 2.740 \\
\hline Min & -3.565 & 0.510 & 0.390 \\
\hline
\end{tabular}

\begin{tabular}{|c|r|r|r|}
\hline \multicolumn{4}{|c|}{ Averages by Valve } \\
\hline $\begin{array}{c}\text { Exhaust } \\
\text { Valve \# }\end{array}$ & $\begin{array}{c}\text { ORNA20 } \\
\text { O(delta), } \\
\mu \mathrm{m}\end{array}$ & $\begin{array}{c}\text { ORNA20 } \\
\text { Ev. } \\
\text { Length, } \\
\mathrm{mm}\end{array}$ & $\begin{array}{c}\text { ORNA20 } \\
\text { Wt, } \mu \mathrm{m}\end{array}$ \\
\hline 1 & -14.280 & 1.615 & 15.715 \\
\hline 2 & -16.580 & 1.655 & 17.363 \\
\hline 3 & -8.884 & 1.608 & 12.050 \\
\hline 4 & -19.095 & 1.658 & 21.708 \\
\hline 5 & -12.133 & 1.485 & 12.305 \\
\hline 6 & -21.424 & 1.448 & 20.945 \\
\hline 7 & -3.966 & 1.893 & 4.595 \\
\hline 8 & -9.000 & 1.658 & 10.455 \\
\hline Average & -13.170 & 1.627 & 14.392 \\
\hline StDev & 7.566 & 0.205 & 7.838 \\
\hline Max & -3.078 & 2.040 & 32.900 \\
\hline Min & -28.639 & 1.230 & 2.720 \\
\hline
\end{tabular}




\section{Oakridge National Laboratory \\ 2008 Nissan Altima \\ Intake Valve Contour Measurements at EOT}

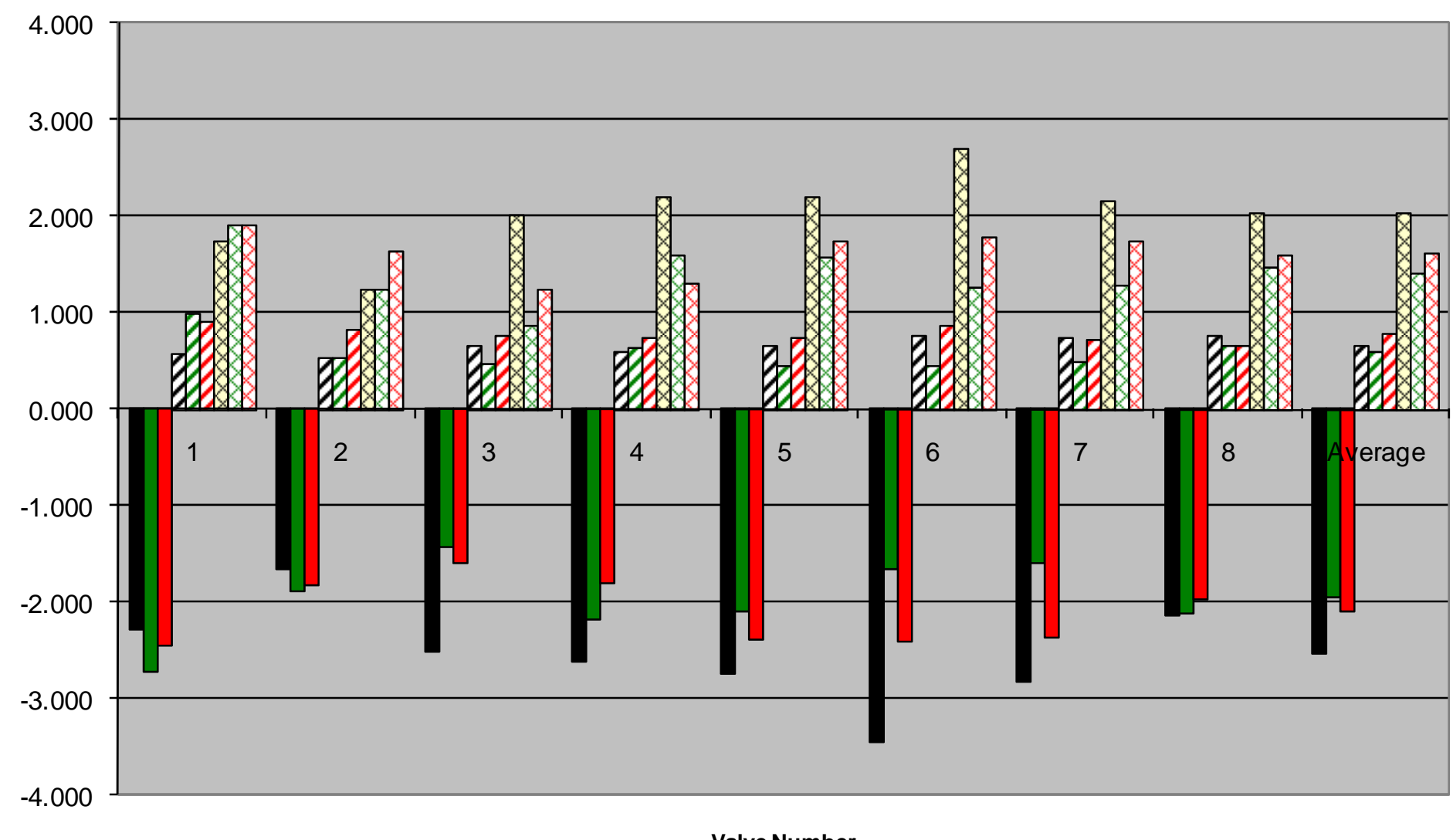

- ORNAO z(delta), $\mu \mathrm{m}$ 口ORNA15 z(delta), $\mu \mathrm{m}$ 口ORNA20 z(delta), $\mu \mathrm{m}$ DORNAO Ev. Length, $\mathrm{mm}$ 口ORNA15 Ev. Length, mm DORNA20 Ev. Length, mm DORNAO Wt, $\mu \mathrm{m}$ 口ORNA15 Wt, $\mu \mathrm{m}$ ๑ORNA20 Wt, $\mu \mathrm{m}$ 


\section{Oakridge National Laboratory \\ 2008 Nissan Altima \\ Exhaust Valve Contour Measurements at EOT}

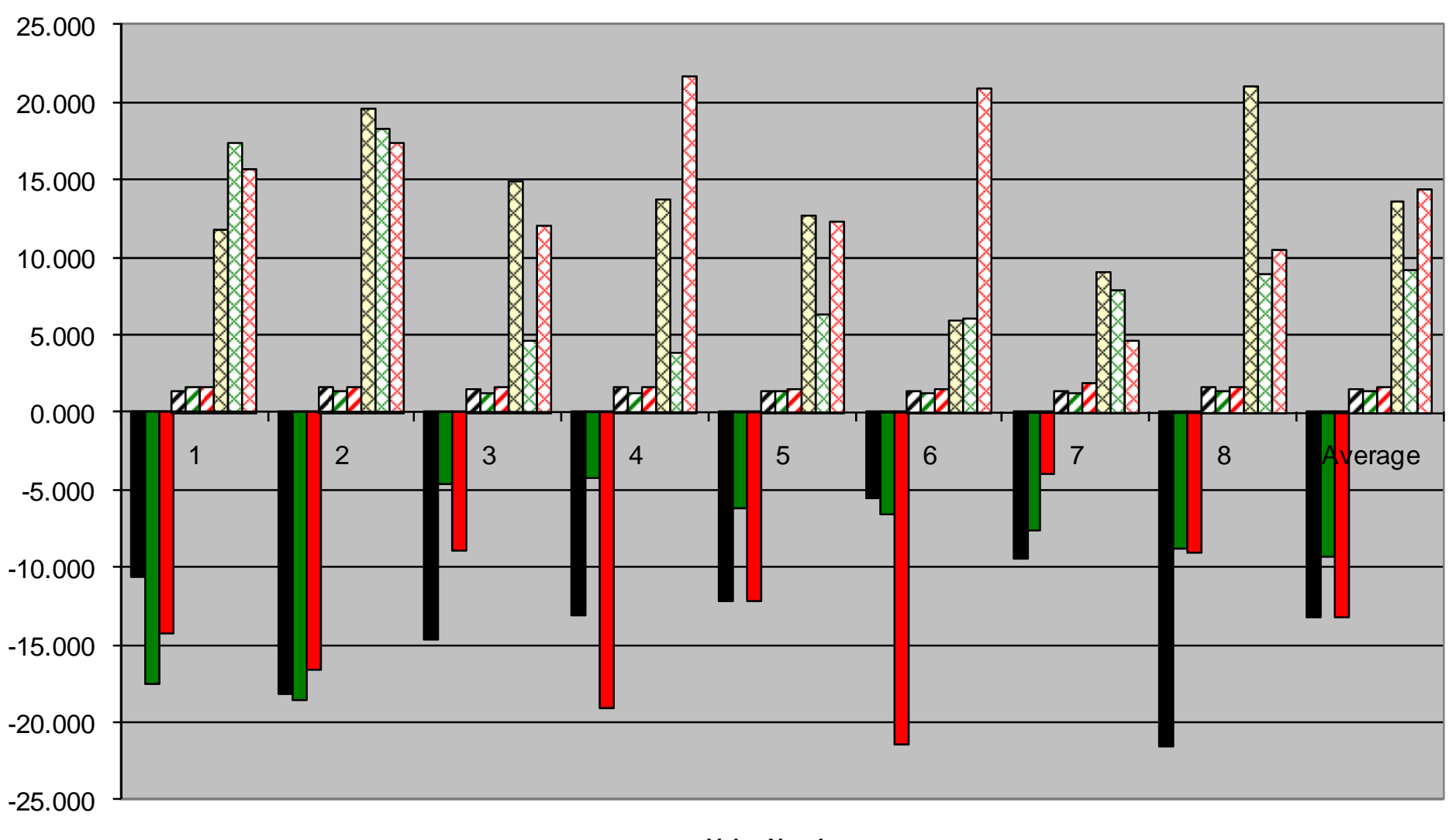

- ORNAO z(delta), um

口 ORNA15 $z$ (delta), $\mu \mathrm{m}$

口 ORNA20 z(delta), $\mu \mathrm{m}$ DORNA0 Ev. Length, $\mathrm{mm}$ 口ORNA15 Ev. Length, mm DORNA20 Ev. Length, $\mathrm{mm}$ DORNAO Wt, $\mu \mathrm{m}$ ๑ORNA15 Wt, $\mu \mathrm{m}$ ๑ORNA20 Wt, $\mu \mathrm{m}$

Valve Number 


\begin{tabular}{|c|c|c|c|c|c|c|c|c|c|}
\hline & & & Dak Rid & $\overline{\text { lge Nat }}$ & nal La & borato & & Sw & \\
\hline & & Propo & sal 08- & 58845 & wertr & ain Ins & oection & 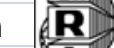 & \\
\hline & & & Valve C & Jontou & Aeasu & remen & & & \\
\hline & & ORFT0 & & & & & ORFT15 & & \\
\hline $\begin{array}{c}\text { Intake } \\
\text { Valve \# }\end{array}$ & Position & $\begin{array}{c}z \text { (delta) } \\
\mu \mathrm{m}\end{array}$ & $\begin{array}{c}\text { Ev. } \\
\text { Length, } \\
\mathrm{mm}\end{array}$ & $\mathrm{Wt}, \mu \mathrm{m}$ & $\begin{array}{c}\text { Intake } \\
\text { Valve \# }\end{array}$ & Position & $\begin{array}{c}z \text { (delta) } \\
\mu \mathrm{m}\end{array}$ & $\begin{array}{c}\text { Ev. } \\
\text { Length, } \\
\mathrm{mm}\end{array}$ & $\mathrm{Wt}, \mu \mathrm{m}$ \\
\hline & \begin{tabular}{|l|}
1 \\
\end{tabular} & \begin{tabular}{|l|}
-3.211 \\
\end{tabular} & \begin{tabular}{r|}
1.43 \\
\end{tabular} & 2.32 & & 1 & $\begin{array}{r}-3.141 \\
\end{array}$ & 0.75 & 2.44 \\
\hline & 2 & -2.387 & 1.20 & 1.72 & & 2 & -2.278 & 1.62 & 1.60 \\
\hline 1 & 3 & \begin{tabular}{l|}
-2.702 \\
\end{tabular} & 1.23 & 2.20 & 1 & 3 & -2.848 & 1.41 & 2.31 \\
\hline & 4 & -2.863 & 1.29 & 2.46 & & 4 & \begin{tabular}{l|}
-3.466 \\
\end{tabular} & 1.36 & 2.84 \\
\hline & 1 & -3.075 & 0.95 & 2.79 & & 1 & \begin{tabular}{l|}
-2.559 \\
\end{tabular} & 1.53 & 2.05 \\
\hline 2 & 2 & \begin{tabular}{|l|}
-2.969 \\
\end{tabular} & 0.66 & 1.77 & 2 & 2 & \begin{tabular}{|l|}
-4.439 \\
\end{tabular} & 1.17 & 3.51 \\
\hline 2 & 3 & -3.158 & 0.66 & 2.16 & 2 & 3 & \begin{tabular}{|c|}
-3.398 \\
\end{tabular} & 0.68 & 2.49 \\
\hline & 4 & \begin{tabular}{l|}
-3.722 \\
\end{tabular} & 0.65 & 2.19 & & 4 & \begin{tabular}{|l|}
-3.149 \\
\end{tabular} & 0.96 & 3.11 \\
\hline & 1 & -4.066 & 0.50 & 3.55 & & 1 & -2.894 & 0.75 & 2.73 \\
\hline & 2 & -4.898 & 0.73 & 4.07 & 3 & 2 & \begin{tabular}{|c|}
-2.383 \\
\end{tabular} & 0.79 & 2.05 \\
\hline 3 & 3 & -2.724 & 0.70 & 1.84 & 3 & 3 & -3.275 & 1.29 & 2.53 \\
\hline & 4 & -2.491 & 0.77 & 1.77 & & 4 & -3.938 & 1.06 & 3.34 \\
\hline & 1 & -3.147 & 0.71 & 2.48 & & 1 & \begin{tabular}{|l|}
-3.321 \\
\end{tabular} & 1.48 & 3.14 \\
\hline 4 & 2 & -3.290 & 0.65 & 2.46 & 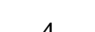 & 2 & \begin{tabular}{l|}
-4.082 \\
\end{tabular} & 0.97 & 3.59 \\
\hline 4 & 3 & -2.506 & 0.64 & 1.83 & 4 & 3 & \begin{tabular}{|c|}
-4.298 \\
\end{tabular} & 1.03 & 3.35 \\
\hline & 4 & -2.209 & 0.73 & 1.87 & & 4 & -3.598 & 1.01 & 2.59 \\
\hline & 1 & -3.284 & 0.95 & 2.16 & & 1 & \begin{tabular}{|l|}
-2.030 \\
\end{tabular} & 1.18 & 1.48 \\
\hline 5 & 2 & -3.183 & 0.66 & 2.39 & 5 & 2 & -1.380 & 0.61 & 1.61 \\
\hline 5 & 3 & -3.220 & 0.87 & 2.55 & 5 & 3 & -2.469 & 0.89 & 1.62 \\
\hline & 4 & -2.403 & 0.89 & 2.13 & & 4 & -2.257 & 1.01 & 1.86 \\
\hline & 1 & \begin{tabular}{|c|}
-3.349 \\
\end{tabular} & 0.75 & 2.31 & & 1 & \begin{tabular}{|c|}
-3.276 \\
\end{tabular} & 1.27 & 2.27 \\
\hline 6 & 2 & -4.264 & 0.72 & 3.20 & 6 & 2 & \begin{tabular}{|c|}
-3.859 \\
\end{tabular} & 1.20 & 2.87 \\
\hline 6 & 3 & -3.597 & 1.05 & 2.86 & 6 & 3 & \begin{tabular}{|c|}
-2.398 \\
\end{tabular} & 0.84 & 1.61 \\
\hline & 4 & -3.619 & 1.12 & 2.54 & & 4 & -2.171 & 1.03 & 1.56 \\
\hline & 1 & -2.890 & 0.50 & 2.19 & & 1 & -2.467 & 0.97 & 2.03 \\
\hline & 2 & \begin{tabular}{|c|}
-2.357 \\
\end{tabular} & 0.93 & 1.43 & & 2 & -2.506 & 1.58 & 2.14 \\
\hline 7 & 3 & -2.341 & 0.98 & 1.69 & 7 & 3 & \begin{tabular}{|c|}
-3.319 \\
\end{tabular} & 1.40 & 1.84 \\
\hline & 4 & -3.869 & 1.60 & 2.69 & & 4 & -3.128 & 1.58 & 2.20 \\
\hline & 1 & \begin{tabular}{|c|}
-2.789 \\
\end{tabular} & 1.57 & 2.28 & & 1 & \begin{tabular}{|c|}
-2.776 \\
\end{tabular} & 1.12 & 2.52 \\
\hline & 2 & \begin{tabular}{|c|}
-2.343 \\
\end{tabular} & 1.56 & 1.87 & & 2 & \begin{tabular}{|c|}
-3.091 \\
\end{tabular} & 1.02 & 2.38 \\
\hline 8 & 3 & -2.246 & 0.82 & 1.46 & 8 & 3 & \begin{tabular}{|c|}
-3.043 \\
\end{tabular} & 0.74 & 2.32 \\
\hline & 4 & -2.102 & \begin{tabular}{l|}
1.37 \\
\end{tabular} & 1.59 & & 4 & -2.167 & 0.92 & 1.65 \\
\hline & 1 & -3.043 & 1.50 & 2.30 & & 1 & \begin{tabular}{|c|}
-3.518 \\
\end{tabular} & 0.82 & 2.71 \\
\hline 9 & 2 & -2.513 & 1.23 & 2.16 & 9 & 2 & -6.120 & 1.46 & 5.53 \\
\hline 9 & 3 & -4.432 & 1.58 & 3.84 & 9 & 3 & \begin{tabular}{|c|}
-3.409 \\
\end{tabular} & 1.41 & 2.63 \\
\hline & 4 & -2.436 & 0.78 & 2.40 & & 4 & \begin{tabular}{|c|}
-3.741 \\
\end{tabular} & 1.04 & 2.98 \\
\hline & 1 & \begin{tabular}{|c|}
-2.839 \\
\end{tabular} & 1.05 & 2.30 & & 1 & -4.196 & 2.08 & 3.69 \\
\hline 10 & 2 & -3.447 & 1.48 & 2.40 & 10 & 2 & -4.332 & 1.94 & 3.64 \\
\hline 10 & 3 & -2.972 & 1.05 & 2.07 & 10 & 3 & -3.158 & 1.35 & 2.37 \\
\hline & 4 & -4.357 & 1.96 & 3.12 & & 4 & \begin{tabular}{|c|}
-3.907 \\
\end{tabular} & 1.08 & 2.96 \\
\hline & 1 & -4.623 & 1.32 & 3.89 & & 1 & -2.451 & 0.71 & 1.95 \\
\hline 11 & 2 & \begin{tabular}{|c|}
-2.189 \\
\end{tabular} & 0.72 & 1.70 & 11 & 2 & -2.600 & 1.13 & 1.83 \\
\hline 11 & 3 & -4.685 & 1.34 & 3.95 & 11 & 3 & \begin{tabular}{|c|}
-2.747 \\
\end{tabular} & 1.37 & 3.39 \\
\hline & 4 & -2.954 & \begin{tabular}{l|}
0.74 \\
\end{tabular} & 2.59 & & 4 & \begin{tabular}{|c|}
-2.448 \\
\end{tabular} & 1.11 & 1.82 \\
\hline & 1 & -3.374 & 1.08 & 2.50 & & 1 & -3.786 & 1.31 & 2.58 \\
\hline & 2 & -2.676 & 0.98 & 1.76 & 12 & 2 & -2.627 & 0.94 & 1.97 \\
\hline 12 & 3 & \begin{tabular}{|c|}
-2.443 \\
\end{tabular} & 0.76 & 1.65 & 12 & 3 & -4.595 & 1.09 & 3.06 \\
\hline & 4 & -3.022 & 1.39 & 2.34 & & 4 & -3.029 & 1.21 & 2.47 \\
\hline & & & & & & & & & \\
\hline & & Averages & by Valve & & & & Averages & by Valve & \\
\hline & $\begin{array}{c}\text { Intake } \\
\text { Valve \# }\end{array}$ & $\begin{array}{c}\text { ORFTO } \\
\text { z(delta), } \\
\mu \mathrm{m}\end{array}$ & $\begin{array}{l}\text { ORFT0 } \\
\text { Ev. } \\
\text { Length, } \\
\mathrm{mm}\end{array}$ & $\begin{array}{l}\text { ORFTO } \\
\mathrm{Wt}, \mu \mathrm{m}\end{array}$ & & $\begin{array}{c}\text { Intake } \\
\text { Valve \# }\end{array}$ & $\begin{array}{c}\text { ORFT15 } \\
\text { z(delta), } \\
\mu \mathrm{m}\end{array}$ & $\begin{array}{l}\text { ORFT15 } \\
\text { Ev. } \\
\text { Length, } \\
\text { mm }\end{array}$ & $\begin{array}{l}\text { ORFT15 } \\
\mathrm{Wt}, \mu \mathrm{m}\end{array}$ \\
\hline & 1 & -2.791 & 1.288 & 2.175 & & 1 & -2.933 & 1.285 & 2.298 \\
\hline & 2 & -3.231 & 0.730 & 2.228 & & 2 & -3.386 & 1.085 & 2.790 \\
\hline & 3 & -3.545 & 0.675 & 2.808 & & 3 & \begin{tabular}{|c|}
-3.123 \\
\end{tabular} & 0.973 & 2.663 \\
\hline & 4 & -2.788 & 0.683 & 2.160 & & 4 & -3.825 & 1.123 & 3.168 \\
\hline & 5 & -3.023 & \begin{tabular}{l|}
0.843 \\
\end{tabular} & 2.308 & & 5 & -2.034 & 0.923 & 1.643 \\
\hline & 6 & \begin{tabular}{|c|}
-3.707 \\
\end{tabular} & \begin{tabular}{l|}
0.910 \\
\end{tabular} & 2.728 & & 6 & -2.926 & 1.085 & 2.078 \\
\hline & 7 & -2.864 & 1.003 & 2.000 & & 7 & -2.855 & 1.383 & 2.053 \\
\hline & 8 & -2.370 & 1.330 & 1.800 & & 8 & -2.769 & 0.950 & 2.218 \\
\hline & 9 & -3.106 & 1.273 & 2.675 & & 9 & \begin{tabular}{|l|}
-4.197 \\
\end{tabular} & 1.183 & 3.463 \\
\hline & 10 & -3.404 & 1.385 & 2.473 & & 10 & \begin{tabular}{|c|}
-3.898 \\
\end{tabular} & 1.613 & 3.165 \\
\hline & 11 & -3.613 & 1.030 & 3.033 & & 11 & \begin{tabular}{l|}
-2.562 \\
\end{tabular} & 1.080 & 2.248 \\
\hline & 12 & -2.879 & 1.053 & 2.063 & & 12 & -3.509 & 1.138 & 2.520 \\
\hline & \begin{tabular}{|l|} 
Average \\
\end{tabular} & -3.110 & 1.017 & 2.371 & & Average & -3.168 & 1.151 & 2.525 \\
\hline & StDev & 0.720 & 0.349 & 0.651 & & StDev & 0.839 & 0.317 & 0.763 \\
\hline & Max & \begin{tabular}{l|}
-2.102 \\
\end{tabular} & 1.960 & 4.070 & & Max & $\begin{array}{l}-1.380 \\
\end{array}$ & 2.080 & 5.530 \\
\hline & Min & \begin{tabular}{|l|}
-4.898 \\
\end{tabular} & 0.500 & 1.430 & & Min & \begin{tabular}{|l|}
-6.120 \\
\end{tabular} & 0.610 & 1.480 \\
\hline
\end{tabular}

B-30 of 34 


\begin{tabular}{|c|c|c|c|c|c|c|c|c|c|}
\hline \multicolumn{10}{|c|}{ Oak Ridge National Laboratory } \\
\hline \multicolumn{10}{|c|}{ Proposal 08-58845 Powertrain Inspection } \\
\hline \multicolumn{10}{|c|}{ Valve Contour Measurements } \\
\hline \multicolumn{5}{|c|}{ ORFT0 } & \multicolumn{5}{|c|}{ ORFT15 } \\
\hline & & & Ev. & & & & שגורו & Ev. & \\
\hline $\begin{array}{l}\text { Exhaust } \\
\text { Valve \# }\end{array}$ & Position & $\begin{array}{c}z \text { (delta) } \\
\mu \mathrm{m}\end{array}$ & $\begin{array}{c}\text { Length, } \\
\mathrm{mm}\end{array}$ & $\mathrm{Wt}, \mu \mathrm{m}$ & $\begin{array}{l}\text { Exhaust } \\
\text { Valve \# }\end{array}$ & Position & $\begin{array}{c}\mathrm{z} \text { (delta) } \\
\mu \mathrm{m}\end{array}$ & $\begin{array}{c}\text { Length, } \\
\text { mm }\end{array}$ & $\mathrm{Wt}, \mu \mathrm{m}$ \\
\hline \multirow{4}{*}{1} & \begin{tabular}{|l|}
1 \\
\end{tabular} & \begin{tabular}{|r|}
-4.883 \\
\end{tabular} & 2.02 & 4.40 & \multirow{4}{*}{1} & 1 & -11.421 & 1.50 & 11.62 \\
\hline & 2 & \begin{tabular}{|c|}
-2.966 \\
\end{tabular} & 2.17 & 3.19 & & 2 & -23.019 & 1.65 & 22.10 \\
\hline & 3 & -4.119 & 2.22 & 3.59 & & 3 & -11.562 & 1.61 & 13.21 \\
\hline & 4 & -4.428 & 1.96 & 3.88 & & 4 & $\begin{array}{l}-3.502 \\
\end{array}$ & 1.79 & 3.92 \\
\hline \multirow{4}{*}{2} & 1 & -12.791 & 1.89 & 12.19 & & 1 & -11.294 & 1.89 & 13.35 \\
\hline & 2 & -5.794 & 1.94 & 4.82 & 2 & 2 & -10.566 & 1.37 & 11.62 \\
\hline & 3 & -16.221 & 2.05 & 15.86 & 2 & 3 & -5.784 & 1.74 & 7.52 \\
\hline & 4 & -13.069 & 2.02 & 12.38 & & 4 & \begin{tabular}{|l|}
-9.489 \\
\end{tabular} & 1.65 & 9.29 \\
\hline & 1 & -11.412 & 1.95 & 11.28 & & 1 & -6.289 & 1.74 & 7.39 \\
\hline 3 & 2 & -6.616 & 1.74 & 4.22 & 3 & 2 & \begin{tabular}{l|l|}
-4.772 \\
\end{tabular} & 1.83 & 7.51 \\
\hline 3 & 3 & -12.227 & 1.81 & 11.82 & 3 & 3 & -7.026 & 1.20 & 6.45 \\
\hline & 4 & -6.448 & 2.00 & 6.28 & & 4 & \begin{tabular}{|l|}
-7.777 \\
\end{tabular} & 1.84 & 8.24 \\
\hline & 1 & $\begin{array}{l}-16.381 \\
\end{array}$ & 2.16 & 16.09 & & 1 & -5.484 & 1.60 & 5.25 \\
\hline & 2 & -11.577 & 1.92 & 11.02 & & 2 & -3.248 & 1.67 & 6.22 \\
\hline 4 & 3 & -6.929 & 1.93 & 6.43 & 4 & 3 & -5.773 & 1.93 & 6.25 \\
\hline & 4 & -10.968 & 1.84 & 10.81 & & 4 & 16.678 & 2.05 & 16.34 \\
\hline & 1 & -19.103 & 1.72 & 18.48 & & 1 & -10.278 & 1.58 & 9.22 \\
\hline & 2 & -11.220 & 1.91 & 10.47 & & 2 & 4.724 & 1.62 & 5.89 \\
\hline 5 & 3 & $\begin{array}{r}-7.683 \\
\end{array}$ & 1.74 & 6.87 & 5 & 3 & -5.981 & 1.69 & 5.23 \\
\hline & 4 & -12.722 & 1.82 & 11.78 & & 4 & -9.051 & 1.72 & 8.90 \\
\hline & 1 & -14.001 & 1.87 & 14.26 & & 1 & -3.876 & 1.59 & 2.98 \\
\hline & 2 & -12.593 & 1.80 & 12.57 & & 2 & -4.022 & 1.71 & 3.56 \\
\hline 6 & 3 & -5.338 & 1.99 & 4.69 & 6 & 3 & -5.627 & 1.73 & 5.87 \\
\hline & 4 & -9.235 & 1.78 & 8.36 & & 4 & -3.542 & 1.81 & 2.95 \\
\hline & 1 & -8.661 & 1.70 & 8.93 & & 1 & -4.129 & 1.39 & 3.03 \\
\hline & 2 & -20.228 & 1.74 & 19.65 & & 2 & -6.856 & 1.96 & 6.15 \\
\hline 7 & 3 & -20.746 & 1.93 & 20.94 & 7 & 3 & \begin{tabular}{|l|}
-6.367 \\
\end{tabular} & 1.96 & 5.39 \\
\hline & 4 & $\begin{array}{l}-24.783 \\
\end{array}$ & 1.70 & 23.81 & & 4 & -6.048 & 1.76 & 5.61 \\
\hline & 1 & -21.697 & 1.61 & 21.32 & & 1 & -14.962 & 1.88 & 14.22 \\
\hline 8 & 2 & -11.065 & 1.98 & 10.55 & 8 & 2 & -7.965 & 2.07 & 7.52 \\
\hline 8 & 3 & -15.471 & 1.92 & 16.94 & o & 3 & -10.580 & 1.95 & 10.02 \\
\hline & 4 & -14.366 & 1.90 & 16.69 & & 4 & -4.603 & 1.93 & 4.53 \\
\hline & 1 & -16.957 & 1.27 & 16.17 & & 1 & \begin{tabular}{|c|}
-9.188 \\
\end{tabular} & 1.66 & 9.84 \\
\hline 0 & 2 & -18.092 & 1.18 & 17.95 & 0 & 2 & -9.954 & 1.80 & 9.27 \\
\hline$y$ & 3 & -6.773 & 1.91 & 8.21 & $y$ & 3 & -10.781 & 1.90 & 9.76 \\
\hline & 4 & -18.569 & 1.50 & 17.96 & & 4 & -11.020 & 1.95 & 10.65 \\
\hline & 1 & -6.557 & 1.63 & 7.24 & & 1 & -3.074 & 1.95 & 2.64 \\
\hline & 2 & \begin{tabular}{|l|}
-6.241 \\
\end{tabular} & 1.83 & 5.52 & & 2 & -12.099 & 1.82 & 12.81 \\
\hline 10 & 3 & -12.112 & 1.59 & 10.82 & 10 & 3 & -5.152 & 1.76 & 4.66 \\
\hline & 4 & -6.381 & 1.41 & 6.10 & & 4 & -10.359 & 1.87 & 12.23 \\
\hline & 1 & -4.508 & 1.82 & 4.02 & & 1 & -17.835 & 1.75 & 17.05 \\
\hline 11 & 2 & -23.711 & 1.85 & 23.15 & & 2 & -10.722 & 1.57 & 11.90 \\
\hline 11 & 3 & \begin{tabular}{l|}
-7.512 \\
\end{tabular} & 1.87 & 7.55 & 11 & 3 & -12.497 & 1.74 & 10.95 \\
\hline & 4 & -17.606 & 1.74 & 17.27 & & 4 & -6.687 & 1.31 & 5.94 \\
\hline & 1 & -9.213 & 1.87 & 8.48 & & 1 & -15.467 & 1.50 & 14.92 \\
\hline & 2 & -8.106 & 1.93 & 7.09 & & 2 & -12.157 & 1.62 & 11.56 \\
\hline 12 & 3 & -11.069 & 1.75 & 10.82 & 12 & 3 & -3.064 & 1.57 & 2.48 \\
\hline & 4 & $\begin{array}{l}-7.772 \\
\end{array}$ & 2.18 & 8.68 & & 4 & -4.114 & 1.44 & 4.18 \\
\hline & & & & & & & & & \\
\hline & & Averages & 5 by Valve & & & & Averages & by Valve & \\
\hline & $\begin{array}{c}\text { Exhaust } \\
\text { Valve \# }\end{array}$ & $\begin{array}{c}\text { ORFT0 } \\
\text { z(delta), } \\
\mu \mathrm{m}\end{array}$ & \begin{tabular}{|c|} 
ORFT0 \\
Ev. \\
Length, \\
$\mathrm{mm}$
\end{tabular} & $\begin{array}{l}\text { ORFTO } \\
\mathrm{Wt}, \mu \mathrm{m}\end{array}$ & & \begin{tabular}{|c|} 
Exhaust \\
Valve \#
\end{tabular} & $\begin{array}{c}\text { ORFT15 } \\
\text { z(delta), } \\
\mu \mathrm{m}\end{array}$ & $\begin{array}{l}\text { ORFT15 } \\
\text { Ev. } \\
\text { Length, } \\
\mathrm{mm} \\
\end{array}$ & $\begin{array}{l}\text { ORFT15 } \\
\mathrm{Wt}, \mu \mathrm{m}\end{array}$ \\
\hline & 1 & -4.099 & 2.093 & 3.765 & & 1 & -12.376 & 1.638 & 12.713 \\
\hline & 2 & -11.969 & 1.975 & 11.313 & & 2 & -9.283 & 1.663 & 10.445 \\
\hline & 3 & -9.176 & 1.875 & 8.400 & & 3 & -6.466 & 1.653 & 7.398 \\
\hline & 4 & -11.464 & 1.963 & 11.088 & & 4 & 0.543 & 1.813 & 8.515 \\
\hline & 5 & $\begin{array}{l}-12.682 \\
\end{array}$ & 1.798 & 11.900 & & 5 & -5.147 & 1.653 & 7.310 \\
\hline & 6 & -10.292 & 1.860 & 9.970 & & 6 & -4.267 & 1.710 & 3.840 \\
\hline & 7 & -18.605 & 1.768 & 18.333 & & 7 & -5.850 & 1.768 & 5.045 \\
\hline & 8 & -15.650 & 1.853 & 16.375 & & 8 & -9.528 & 1.958 & 9.073 \\
\hline & 9 & -15.098 & 1.465 & 15.073 & & 9 & -10.236 & 1.828 & 9.880 \\
\hline & 10 & -7.823 & 1.615 & 7.420 & & 10 & \begin{tabular}{l|l|}
-7.671 \\
\end{tabular} & 1.850 & 8.085 \\
\hline & 11 & -13.334 & 1.820 & 12.998 & & 11 & -11.935 & 1.593 & 11.460 \\
\hline & 12 & -9.040 & 1.933 & 8.768 & & 12 & -8.701 & 1.533 & 8.285 \\
\hline & Average & -11.603 & 1.835 & 11.283 & & Average & -7.576 & 1.721 & 8.504 \\
\hline & StDev & 5.611 & 0.210 & 5.671 & & StDev & 5.796 & 0.193 & 4.303 \\
\hline & Max & -2.966 & 2.220 & 23.810 & & Max & 16.678 & 2.070 & 22.100 \\
\hline & Min & -24.783 & 1.180 & 3.190 & & Min & -23.019 & 1.200 & 2.480 \\
\hline
\end{tabular}

B-31 of 34 


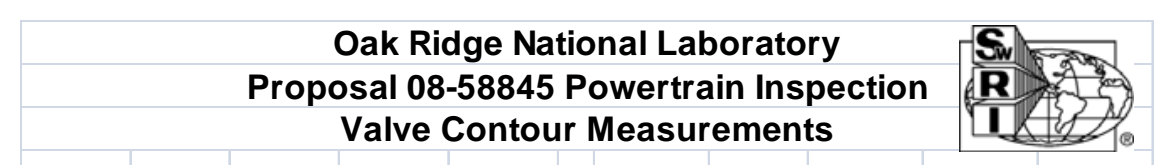

\begin{tabular}{|c|c|c|c|c|}
\hline \multicolumn{5}{|c|}{ ORFT20 } \\
\hline $\begin{array}{c}\text { Intake } \\
\text { Valve \# }\end{array}$ & Position & $\begin{array}{c}\text { z(delta), } \\
\mu \mathrm{m}\end{array}$ & $\begin{array}{c}\text { Ev. } \\
\text { Length, } \\
\text { mm }\end{array}$ & $\mathrm{Wt}, \mu \mathrm{m}$ \\
\hline \multirow{4}{*}{1} & 1 & 2.508 & 0.38 & 1.79 \\
\hline & 2 & 4.512 & 0.52 & 4.61 \\
\hline & 3 & 3.027 & 1.54 & 2.37 \\
\hline & 4 & 3.911 & 0.75 & 3.07 \\
\hline \multirow{4}{*}{2} & 1 & 2.347 & 0.66 & 1.79 \\
\hline & 2 & 2.619 & 0.25 & 2.03 \\
\hline & 3 & 2.963 & 0.79 & 2.33 \\
\hline & 4 & 2.808 & 0.45 & 1.93 \\
\hline \multirow{4}{*}{3} & 1 & 2.997 & 0.32 & 2.06 \\
\hline & 2 & 1.989 & 0.37 & 1.57 \\
\hline & 3 & 1.602 & 0.52 & 1.20 \\
\hline & 4 & 0.865 & 0.37 & 1.24 \\
\hline \multirow{4}{*}{4} & 1 & 0.934 & 0.34 & 1.12 \\
\hline & 2 & 2.691 & 0.65 & 2.56 \\
\hline & 3 & 1.728 & 0.40 & 1.50 \\
\hline & 4 & 1.722 & 0.43 & 1.30 \\
\hline \multirow{4}{*}{5} & 1 & 2.653 & 0.65 & 2.04 \\
\hline & 2 & 2.159 & 0.60 & 1.78 \\
\hline & 3 & 2.166 & 0.59 & 1.82 \\
\hline & 4 & 1.943 & 0.39 & 1.58 \\
\hline \multirow{4}{*}{6} & 1 & 1.588 & 0.55 & 1.46 \\
\hline & 2 & 2.753 & 0.61 & 2.76 \\
\hline & 3 & -1.566 & 0.52 & 1.90 \\
\hline & 4 & 4.537 & 0.68 & 3.73 \\
\hline \multirow{4}{*}{7} & 1 & 1.460 & 0.38 & 1.23 \\
\hline & 2 & -2.370 & 0.43 & 2.27 \\
\hline & 3 & 1.537 & 0.28 & 1.44 \\
\hline & 4 & -1.896 & 0.80 & 1.88 \\
\hline \multirow{4}{*}{8} & 1 & 2.629 & 0.64 & 2.41 \\
\hline & 2 & 2.163 & 0.45 & 1.92 \\
\hline & 3 & 1.526 & 0.38 & 1.38 \\
\hline & 4 & -2.775 & 0.71 & 2.68 \\
\hline \multirow{4}{*}{9} & 1 & -2.504 & 0.48 & 2.19 \\
\hline & 2 & 2.212 & 0.45 & 2.00 \\
\hline & 3 & -1.787 & 0.25 & 1.48 \\
\hline & 4 & -2.935 & 0.25 & 2.14 \\
\hline \multirow{4}{*}{10} & 1 & \begin{tabular}{|c|}
-1.111 \\
\end{tabular} & 0.28 & 0.82 \\
\hline & 2 & -2.225 & 0.53 & 1.99 \\
\hline & 3 & 3.596 & 0.75 & 3.22 \\
\hline & 4 & 2.076 & 0.37 & 1.89 \\
\hline \multirow{4}{*}{11} & 1 & 1.759 & 0.45 & 1.47 \\
\hline & 2 & 2.276 & 0.51 & 1.88 \\
\hline & 3 & 3.343 & 0.58 & 3.14 \\
\hline & 4 & 1.135 & 0.35 & 0.90 \\
\hline \multirow{4}{*}{12} & 1 & 1.936 & 0.51 & 1.71 \\
\hline & 2 & 2.476 & 0.76 & 2.37 \\
\hline & 3 & 2.823 & 0.68 & 2.43 \\
\hline & 4 & 2.943 & 0.63 & 2.32 \\
\hline
\end{tabular}

\begin{tabular}{|c|c|c|c|c|}
\hline \multicolumn{5}{|c|}{ ORFT20 } \\
\hline $\begin{array}{l}\text { Exhaust } \\
\text { Valve \# }\end{array}$ & Position & $\begin{array}{c}z \text { (delta), } \\
\mu \mathrm{m}\end{array}$ & $\begin{array}{c}\text { Ev. } \\
\text { Length, } \\
\text { mm }\end{array}$ & $\mathrm{Wt}, \mu \mathrm{m}$ \\
\hline \multirow{4}{*}{1} & 1 & -9.363 & 1.61 & 9.35 \\
\hline & 2 & -16.665 & 1.90 & 17.16 \\
\hline & 3 & -9.278 & 1.46 & 9.51 \\
\hline & 4 & -9.255 & 1.92 & 9.83 \\
\hline \multirow{4}{*}{2} & 1 & -12.672 & 1.73 & 12.63 \\
\hline & 2 & -11.424 & 2.05 & 12.78 \\
\hline & 3 & -8.554 & 1.87 & 6.09 \\
\hline & 4 & -16.057 & 1.83 & 14.95 \\
\hline \multirow{4}{*}{3} & 1 & -2.840 & 1.99 & 3.09 \\
\hline & 2 & -3.006 & 1.80 & 2.55 \\
\hline & 3 & -3.024 & 1.60 & 2.23 \\
\hline & 4 & -8.453 & 1.50 & 7.79 \\
\hline \multirow{4}{*}{4} & 1 & -8.120 & 1.11 & 7.11 \\
\hline & 2 & -7.278 & 1.78 & 6.94 \\
\hline & 3 & -6.011 & 1.74 & 5.42 \\
\hline & 4 & -6.341 & 1.82 & 5.59 \\
\hline \multirow{4}{*}{5} & 1 & -11.504 & 1.76 & 11.07 \\
\hline & 2 & -8.032 & 1.79 & 8.31 \\
\hline & 3 & -10.789 & 1.77 & 9.98 \\
\hline & 4 & -3.892 & 1.85 & 3.04 \\
\hline \multirow{4}{*}{6} & 1 & -12.411 & 1.65 & 13.48 \\
\hline & 2 & -6.453 & 1.87 & 5.68 \\
\hline & 3 & -7.913 & 1.40 & 7.12 \\
\hline & 4 & -3.658 & 1.74 & 3.77 \\
\hline \multirow{4}{*}{7} & 1 & -6.665 & 1.37 & 5.60 \\
\hline & 2 & -6.769 & 1.62 & 8.63 \\
\hline & 3 & -8.118 & 1.67 & 10.13 \\
\hline & 4 & -3.660 & 1.92 & 3.59 \\
\hline \multirow{4}{*}{8} & 1 & -12.501 & 1.77 & 11.49 \\
\hline & 2 & -17.090 & 1.62 & 16.65 \\
\hline & 3 & -7.098 & 1.53 & 6.47 \\
\hline & 4 & -17.457 & 1.87 & 17.12 \\
\hline \multirow{4}{*}{9} & 1 & -3.606 & 1.97 & 2.25 \\
\hline & 2 & -3.773 & 1.45 & 2.97 \\
\hline & 3 & -5.331 & 1.60 & 4.78 \\
\hline & 4 & -4.372 & 1.96 & 4.15 \\
\hline \multirow{4}{*}{10} & 1 & -3.091 & 1.44 & 2.64 \\
\hline & 2 & -3.231 & 1.43 & 2.80 \\
\hline & 3 & -5.874 & 1.84 & 4.35 \\
\hline & 4 & -4.229 & 1.82 & 5.56 \\
\hline \multirow{4}{*}{11} & 1 & -6.942 & 1.43 & 5.75 \\
\hline & 2 & -12.309 & 1.68 & 12.02 \\
\hline & 3 & -5.037 & 1.63 & 4.23 \\
\hline & 4 & -7.426 & 1.52 & 8.06 \\
\hline \multirow{4}{*}{12} & 1 & -7.294 & 1.81 & 6.42 \\
\hline & 2 & -12.790 & 1.58 & 11.72 \\
\hline & 3 & -10.043 & 1.82 & 9.97 \\
\hline & 4 & -10.499 & 1.94 & 10.46 \\
\hline
\end{tabular}

\begin{tabular}{|c|r|r|r|}
\hline \multicolumn{4}{|c|}{ Averages by Valve } \\
\hline & $\begin{array}{c}\text { ORFT20 } \\
\text { ORFT20 } \\
\text { Intelta), } \\
\text { Valve \# } \\
\mu \mathrm{m}\end{array}$ & $\begin{array}{c}\text { Ev. } \\
\text { Length, } \\
\mathrm{mm}\end{array}$ & $\begin{array}{c}\text { ORFT20 } \\
\text { Wt, } \mu \mathrm{m}\end{array}$ \\
\hline 1 & 3.490 & 0.798 & 2.960 \\
\hline 2 & 2.684 & 0.538 & 2.020 \\
\hline 3 & 1.863 & 0.395 & 1.518 \\
\hline 4 & 1.769 & 0.455 & 1.620 \\
\hline 5 & 2.230 & 0.558 & 1.805 \\
\hline 6 & 1.828 & 0.590 & 2.463 \\
\hline 7 & -0.317 & 0.473 & 1.705 \\
\hline 8 & 0.886 & 0.545 & 2.098 \\
\hline 9 & -1.254 & 0.358 & 1.953 \\
\hline 10 & 0.584 & 0.483 & 1.980 \\
\hline 11 & 2.128 & 0.473 & 1.848 \\
\hline 12 & 2.545 & 0.645 & 2.208 \\
\hline Average & 1.536 & 0.526 & 2.015 \\
\hline StDev & 1.954 & 0.215 & 0.718 \\
\hline Max & 4.537 & 1.540 & 4.610 \\
\hline Min & -2.935 & 0.250 & 0.820 \\
\hline
\end{tabular}

\begin{tabular}{|c|r|r|r|}
\hline \multicolumn{4}{|c|}{ Averages by Valve } \\
\hline $\begin{array}{c}\text { Exhaust } \\
\text { Valve \# }\end{array}$ & $\begin{array}{c}\text { ORFT20 } \\
\text { (delta), } \\
\mu \mathrm{m}\end{array}$ & $\begin{array}{c}\text { ORFT20 } \\
\text { Ev. } \\
\text { Length, } \\
\mathrm{mm}\end{array}$ & $\begin{array}{c}\text { ORFT20 } \\
\text { Wt, } \mu \mathrm{m}\end{array}$ \\
\hline 1 & -11.140 & 1.723 & 11.463 \\
\hline 2 & -12.177 & 1.870 & 11.613 \\
\hline 3 & -4.331 & 1.723 & 3.915 \\
\hline 4 & -6.938 & 1.613 & 6.265 \\
\hline 5 & -8.554 & 1.793 & 8.100 \\
\hline 6 & -7.609 & 1.665 & 7.513 \\
\hline 7 & -6.303 & 1.645 & 6.988 \\
\hline 8 & -13.537 & 1.698 & 12.933 \\
\hline 9 & -4.271 & 1.745 & 3.538 \\
\hline 10 & -4.106 & 1.633 & 3.838 \\
\hline 11 & -7.929 & 1.565 & 7.515 \\
\hline 12 & -10.157 & 1.788 & 9.643 \\
\hline Average & -8.087 & 1.705 & 7.777 \\
\hline StDev & 3.971 & 0.197 & 4.130 \\
\hline Max & -2.840 & 2.050 & 17.160 \\
\hline Min & -17.457 & 1.110 & 2.230 \\
\hline
\end{tabular}

B-32 of 34 


\section{Oakridge National Laboratory \\ 2008 Ford Taurus \\ Intake Valve Contour Measurements at EOT}

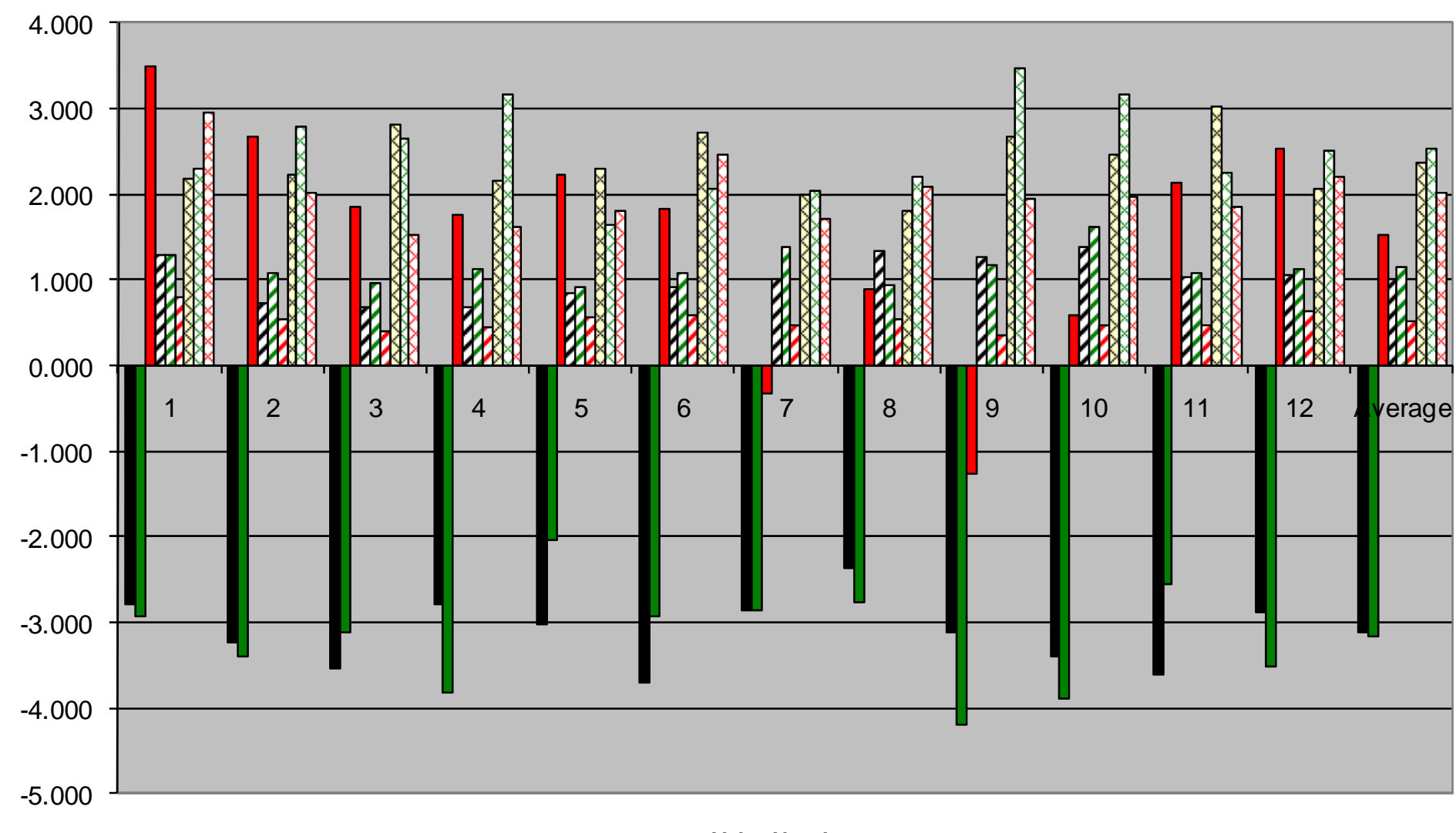

-ORFTOz(delta), $\mu \mathrm{m}$ 口ORFT15z(delta), $\mu \mathrm{m}$ 口ORFT20 z(delta), $\mu \mathrm{m}$ DORFTO Ev. Length, $\mathrm{mm}$ चORFT15Ev. Length, $\mathrm{mm}$ DORFT20 Ev. Length, mm BORFTOWt, $\mu \mathrm{m}$ $\triangle$ ORFT15 Wt, $\mu \mathrm{m}$

口ORFT20Wt, $\mu \mathrm{m}$

Valve Number 


\section{Oakridge National Laboratory \\ 2008 Ford Taurus \\ Exhaust Valve Contour Measurements at EOT}

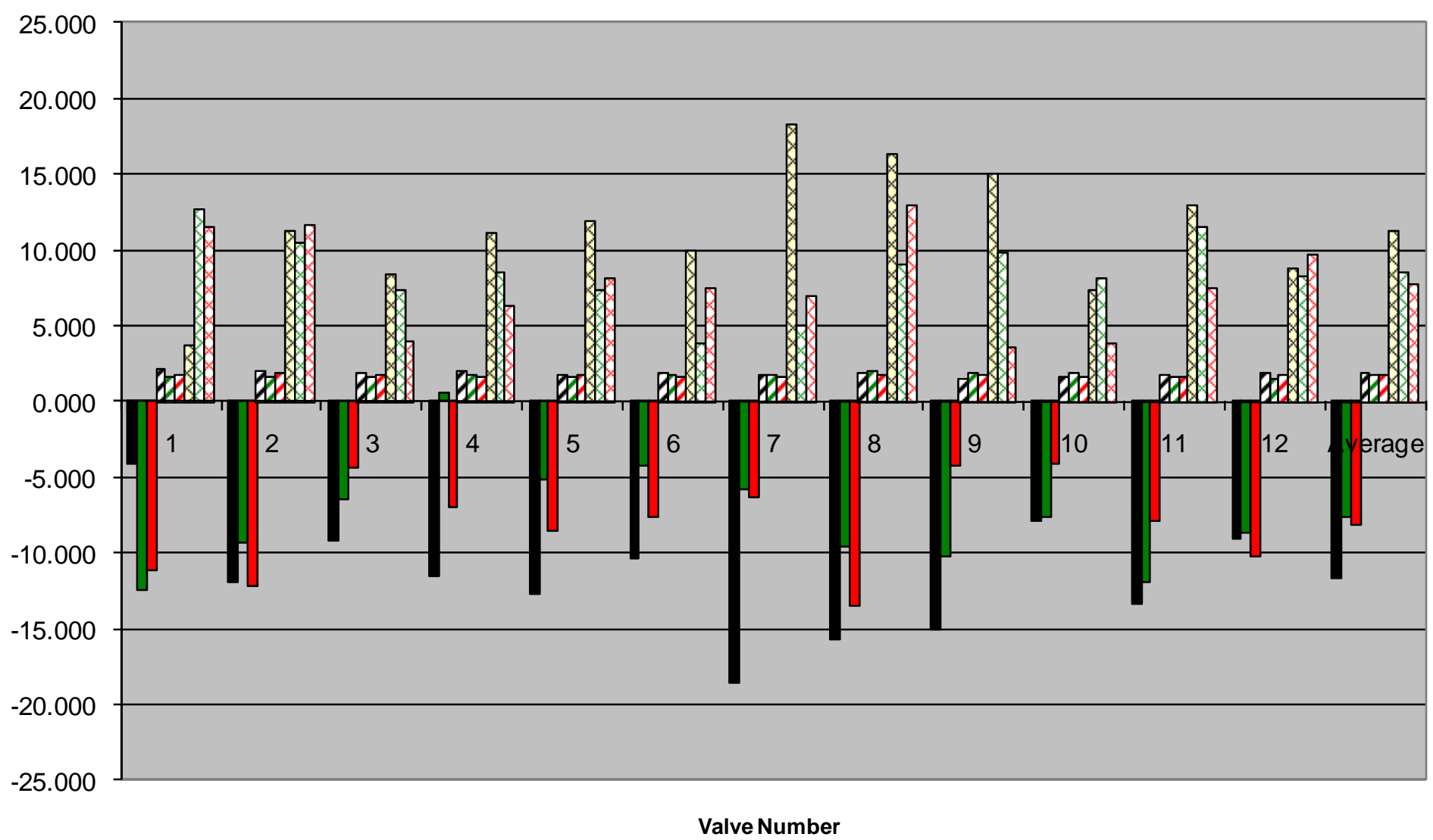

-ORFTOz(delta), $\mu \mathrm{m}$ 口ORFT15 z(delta), $\mu \mathrm{m}$ 口ORFT20 z(delta), $\mu \mathrm{m}$ DORFTO Ev. Length, $\mathrm{mm}$ DORFT15 Ev. Length, mm صORFT20 Ev. Length, mm هORFTOWt, $\mu \mathrm{m}$ $\square$ ORFT15 Wt, $\mu \mathrm{m}$

口ORFT20Wt, $\mu \mathrm{m}$ 
Appendix C

Valve Stem Height 



\section{Oak Ridge National Laboratory Proposal 08-58845 Powertrain Inspection Valve Stem Height}

Date:

31-Aug-10

Technician:

LJ

Measured from tip of valve stem to cylinder head

Measurements in inches

No specified service limit

\begin{tabular}{|c|c|c|c|c|c|c|c|}
\hline \multirow[b]{2}{*}{ Cylinder \# } & \multirow[b]{2}{*}{ Valve \# } & \multicolumn{3}{|c|}{ Intake } & \multicolumn{3}{|c|}{ Exhaust } \\
\hline & & ORHAO & ORHA15 & ORHA20 & ORHAO & ORHA15 & ORHA20 \\
\hline \multirow{2}{*}{1} & 1 & 1.7424 & 1.7413 & 1.7347 & 1.7493 & 1.7384 & 1.7338 \\
\hline & 2 & 1.7505 & 1.7394 & 1.7366 & 1.7499 & 1.7345 & 1.7413 \\
\hline \multirow{2}{*}{2} & 3 & 1.7478 & 1.7394 & 1.7378 & 1.7458 & 1.7329 & 1.7316 \\
\hline & 4 & 1.7417 & 1.7690 & 1.7340 & 1.7471 & 1.7358 & 1.7360 \\
\hline \multirow{2}{*}{3} & 5 & 1.7370 & 1.7378 & 1.7389 & 1.7511 & 1.7365 & 1.7367 \\
\hline & 6 & 1.7405 & 1.7338 & 1.7354 & 1.7499 & 1.7370 & 1.7374 \\
\hline \multirow{2}{*}{4} & 7 & 1.7445 & 1.7383 & 1.7402 & 1.7520 & 1.7361 & 1.7374 \\
\hline & 8 & 1.7427 & 1.7348 & 1.7379 & 1.7495 & 1.7320 & 1.7363 \\
\hline \multicolumn{2}{|l|}{ Average } & 1.7434 & 1.7417 & 1.7369 & 1.7493 & 1.7354 & 1.7363 \\
\hline \multicolumn{2}{|l|}{ Stdev } & 0.0042 & 0.0113 & 0.0021 & 0.0020 & 0.0021 & 0.0028 \\
\hline \multicolumn{2}{|l|}{$\operatorname{Max}$} & 1.7505 & 1.7690 & 1.7402 & 1.7520 & 1.7384 & 1.7413 \\
\hline \multicolumn{2}{|c|}{ Min } & 1.7370 & 1.7338 & 1.7340 & 1.7458 & 1.7320 & 1.7316 \\
\hline
\end{tabular}


Oakridge National Laboratory

2007 Hond Accord

Intake Valve Stem Height Measurements at EOT

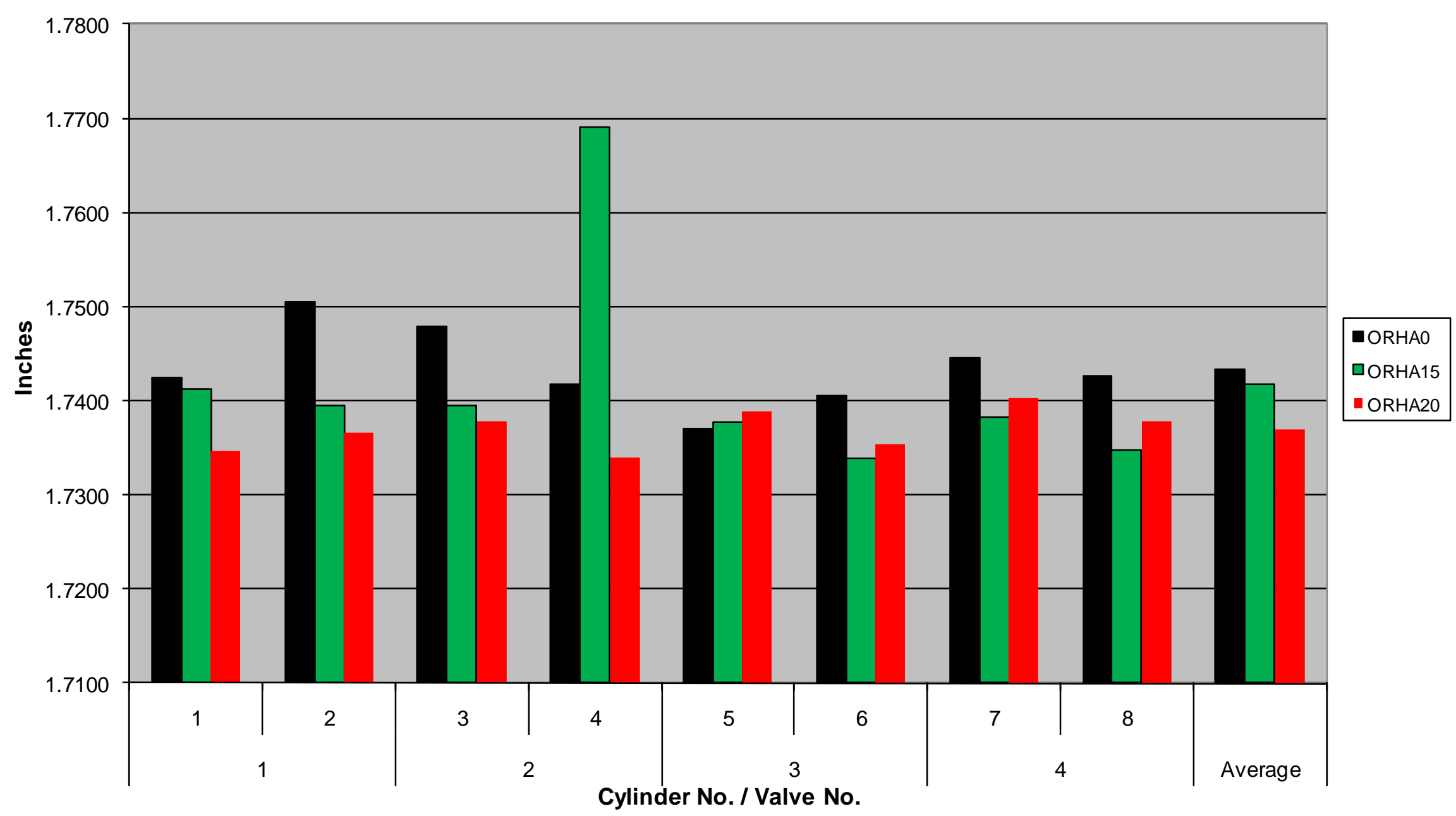


Oakridge National Laboratory

2007 Hond Accord

Exhaust Valve Stem Height Measurements at EOT

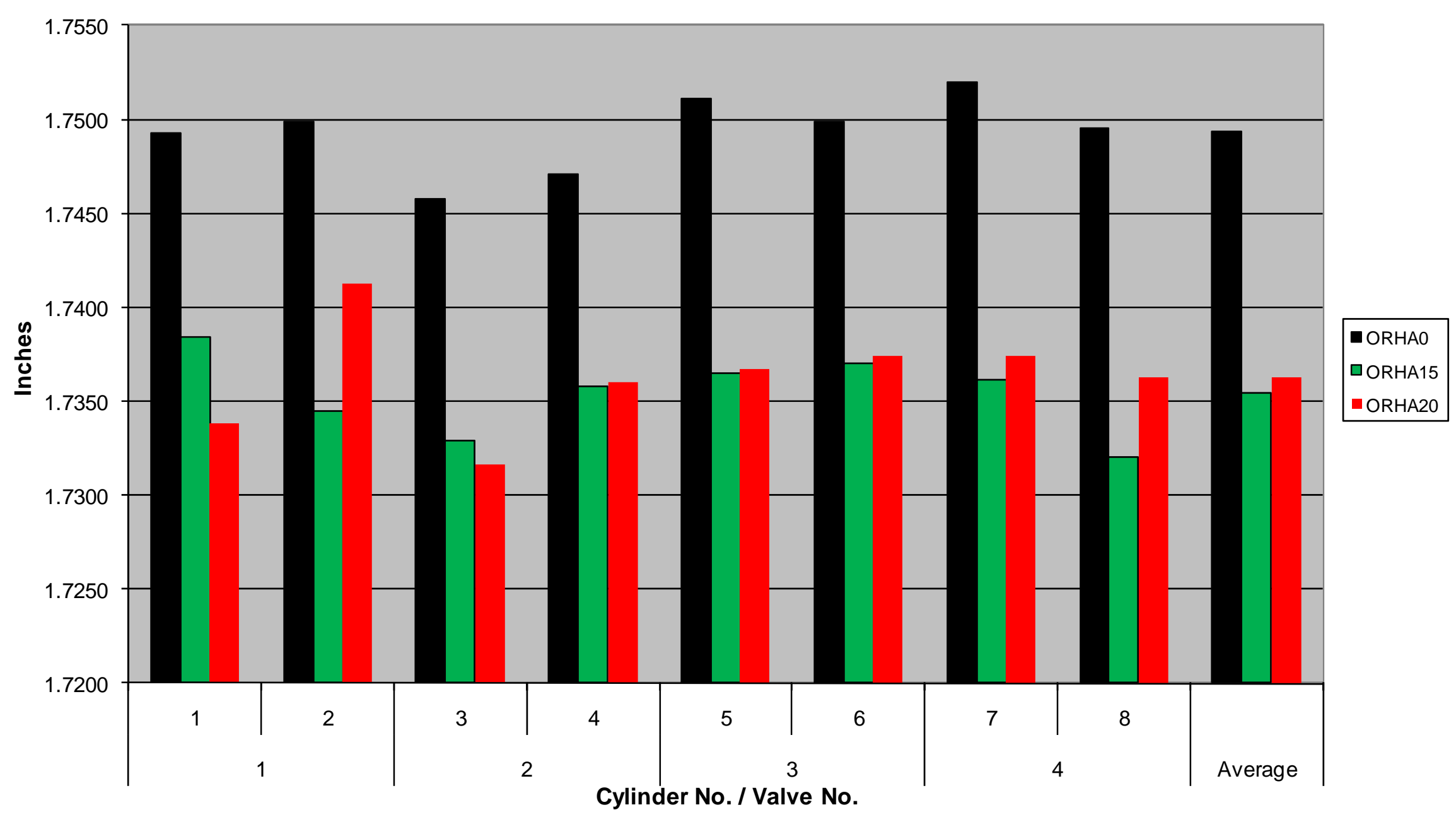




\section{Oak Ridge National Laboratory Proposal 08-58845 Powertrain Inspection Valve Stem Height}

Date:

28-Aug-10

Technician:

Measured from tip of valve stem to cylinder head Measurements in inches No specified service limit

\begin{tabular}{|c|c|r|r|r|r|r|r|}
\cline { 2 - 8 } \multicolumn{2}{c|}{} & \multicolumn{3}{c|}{ Intake } & \multicolumn{3}{c|}{ Exhaust } \\
\hline \multirow{2}{*}{1} & & & & & & & \\
& 1 & 1.59070 & 1.59435 & 1.59080 & 1.59700 & 1.60050 & 1.59860 \\
\cline { 2 - 8 } & 2 & 1.58195 & 1.59800 & 1.58640 & 1.59610 & 1.60090 & 1.59910 \\
\hline \multirow{2}{*}{2} & 3 & 1.59140 & 1.59175 & 1.59280 & 1.59680 & 1.59900 & 1.59510 \\
\cline { 2 - 9 } & 4 & 1.58305 & 1.59350 & 1.59120 & 1.59865 & 1.59970 & 1.59970 \\
\hline \multirow{2}{*}{3} & 5 & 1.58775 & 1.59270 & 1.59640 & 1.59905 & 1.60030 & 1.59980 \\
\cline { 2 - 9 } & 6 & 1.58400 & 1.59300 & 1.58990 & 1.60290 & 1.60080 & 1.59880 \\
\hline \multirow{2}{*}{4} & 7 & 1.59270 & 1.59000 & 1.59170 & 1.60360 & 1.59915 & 1.59740 \\
\cline { 2 - 8 } & 8 & 1.58985 & 1.59305 & 1.59630 & 1.60520 & 1.60130 & 1.60250 \\
\hline Average & & 1.58768 & 1.59329 & 1.59194 & 1.59991 & 1.60021 & 1.59888 \\
\hline Stdev & & 0.00415 & 0.00230 & 0.00330 & 0.00350 & 0.00084 & 0.00211 \\
\hline Max & & 1.59270 & 1.59800 & 1.59640 & 1.60520 & 1.60130 & 1.60250 \\
\hline Min & & 1.58195 & 1.59000 & 1.58640 & 1.59610 & 1.59900 & 1.59510 \\
\hline
\end{tabular}


Oakridge National Laboratory

2006 Chevrolet Cobalt

Intake Valve Stem Height Measurements at EOT

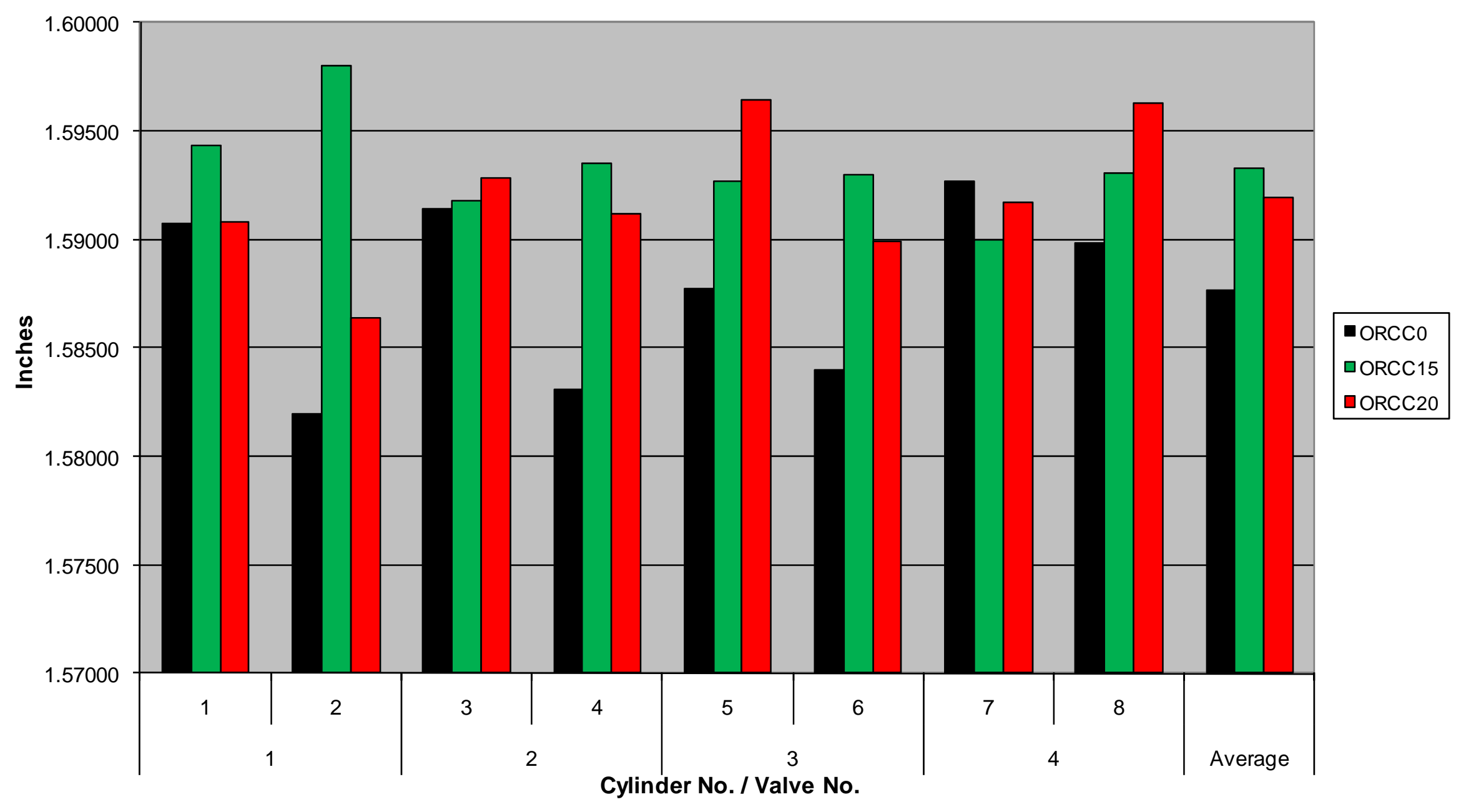


Oakridge National Laboratory

2006 Chevrolet Cobalt

Exhaust Valve Stem Height Measurements at EOT

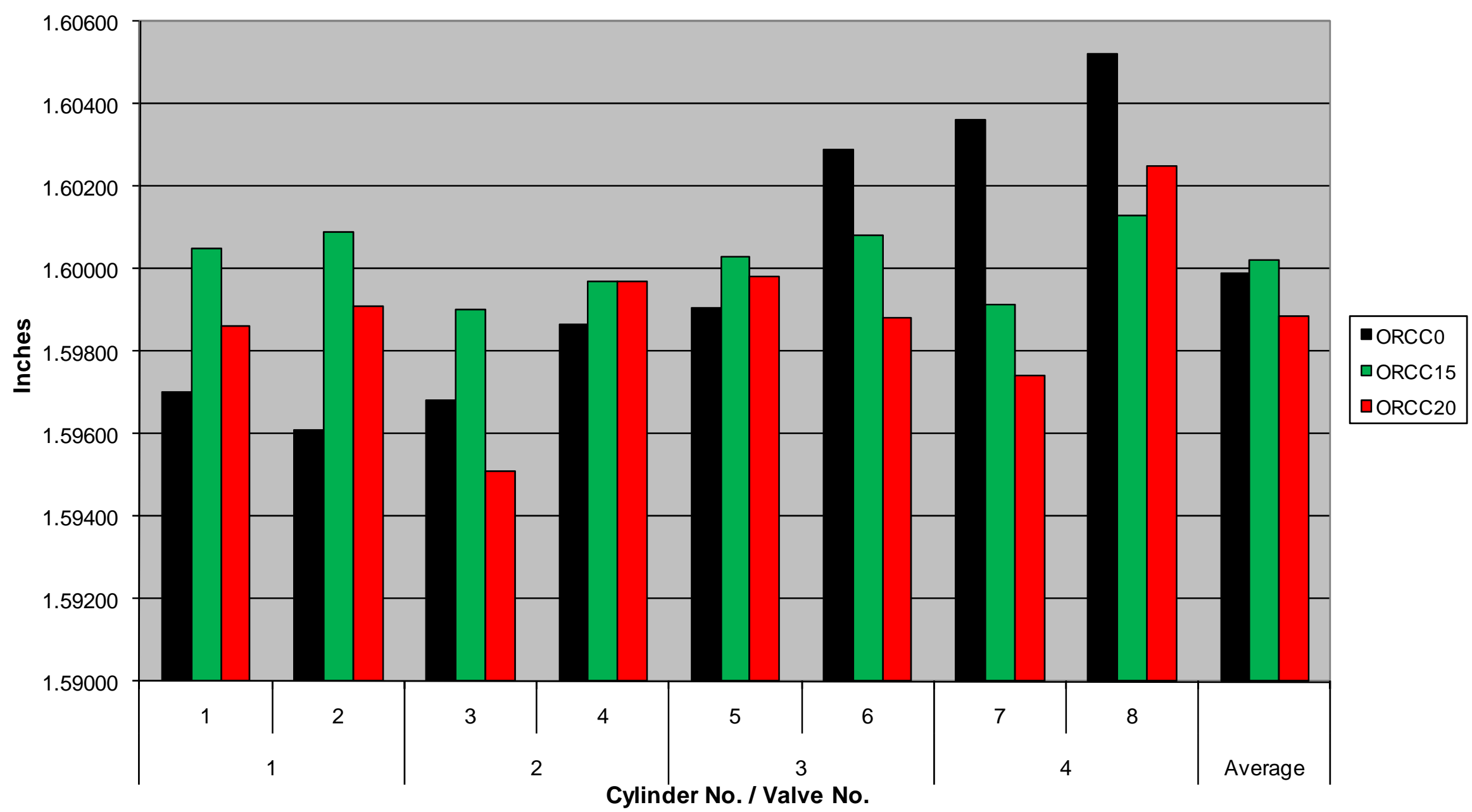




\section{Oak Ridge National Laboratory \\ Proposal 08-58845 Powertrain Inspection \\ Valve Stem Height}

Date:

28-Aug-10

Technician:

JM

Measured from tip of valve stem to cylinder head

Measurements in inches

No specified service limit

\begin{tabular}{|c|c|c|c|c|c|c|c|}
\hline \multirow[b]{2}{*}{ Cylinder \# } & \multirow[b]{2}{*}{ Valve \# } & \multicolumn{3}{|c|}{ Intake } & \multicolumn{3}{|c|}{ Exhaust } \\
\hline & & ORNAO & ORNA15 & ORNA20 & ORNAO & ORNA15 & ORNA20 \\
\hline \multirow{2}{*}{1} & 1 & 1.48240 & 1.48345 & 1.48230 & 1.47660 & 1.47765 & 1.47720 \\
\hline & 2 & 1.48340 & 1.48070 & 1.48120 & 1.47590 & 1.48000 & 1.47780 \\
\hline \multirow{2}{*}{2} & 3 & 1.48400 & 1.48440 & 1.48310 & 1.47735 & 1.47730 & 1.47820 \\
\hline & 4 & 1.48285 & 1.48220 & 1.48240 & 1.47890 & 1.47660 & 1.47830 \\
\hline \multirow[t]{2}{*}{3} & 5 & 1.48345 & 1.48420 & 1.48320 & 1.47840 & 1.47830 & 1.47870 \\
\hline & 6 & 1.48400 & 1.48270 & 1.48210 & 1.47865 & 1.47865 & 1.47890 \\
\hline \multirow{2}{*}{4} & 7 & 1.48270 & 1.48400 & 1.48160 & 1.47735 & 1.47700 & 1.47850 \\
\hline & 8 & 1.48395 & 1.48250 & 1.48100 & 1.47915 & 1.47630 & 1.47690 \\
\hline \multicolumn{2}{|l|}{ Average } & 1.48334 & 1.48302 & 1.48211 & 1.47779 & 1.47773 & 1.47806 \\
\hline \multicolumn{2}{|l|}{ Stdev } & 0.00063 & 0.00125 & 0.00081 & 0.00117 & 0.00122 & 0.00071 \\
\hline \multicolumn{2}{|l|}{ Max } & 1.48400 & 1.48440 & 1.48320 & 1.47915 & 1.48000 & 1.47890 \\
\hline \multicolumn{2}{|l|}{ Min } & 1.48240 & 1.48070 & 1.48100 & 1.47590 & 1.47630 & 1.47690 \\
\hline
\end{tabular}


Oakridge National Laboratory

2008 Nissan Altima

Intake Valve Stem Height Measurements at EOT

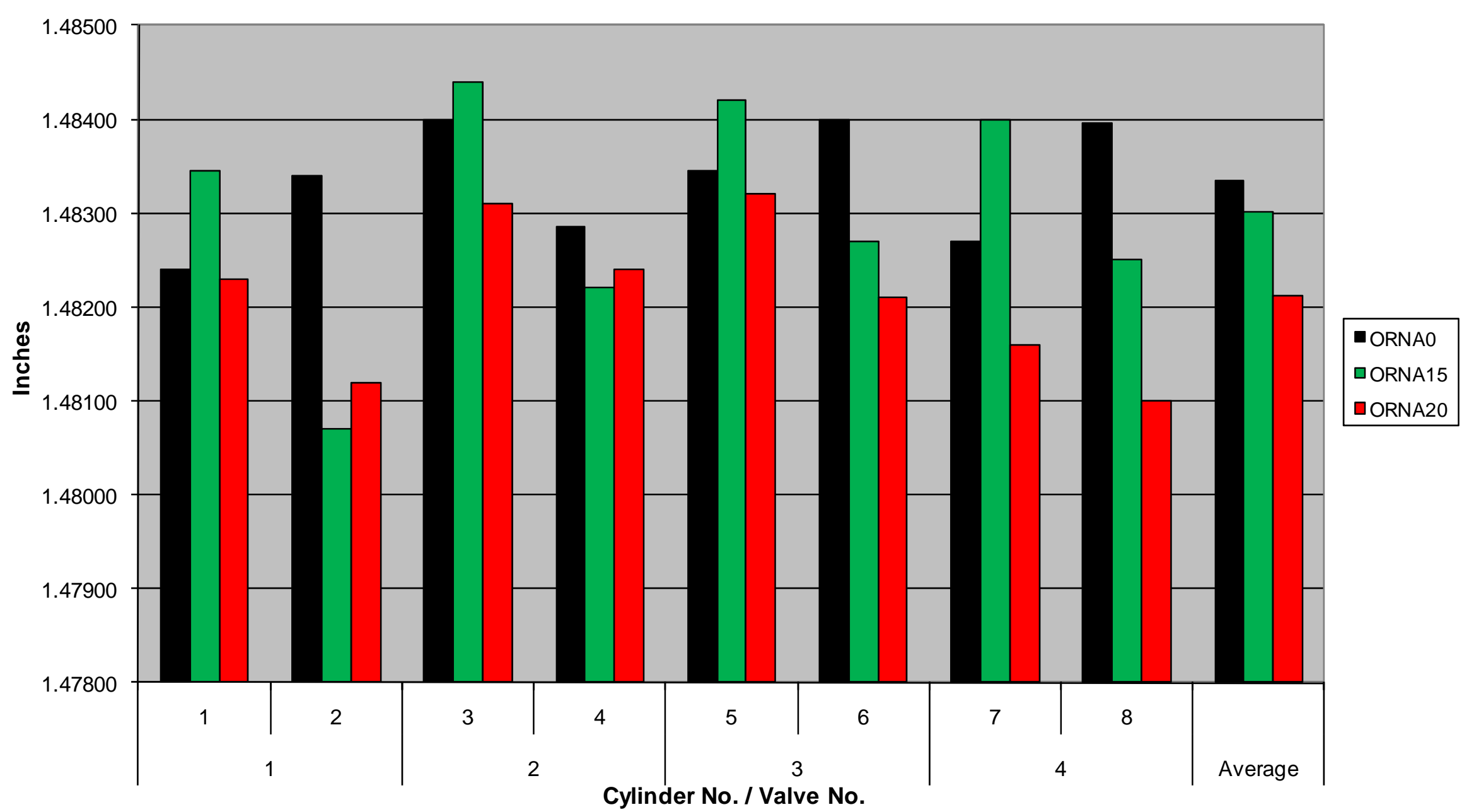


Oakridge National Laboratory

2008 Nissan Altima

Exhaust Valve Stem Height Measurements at EOT

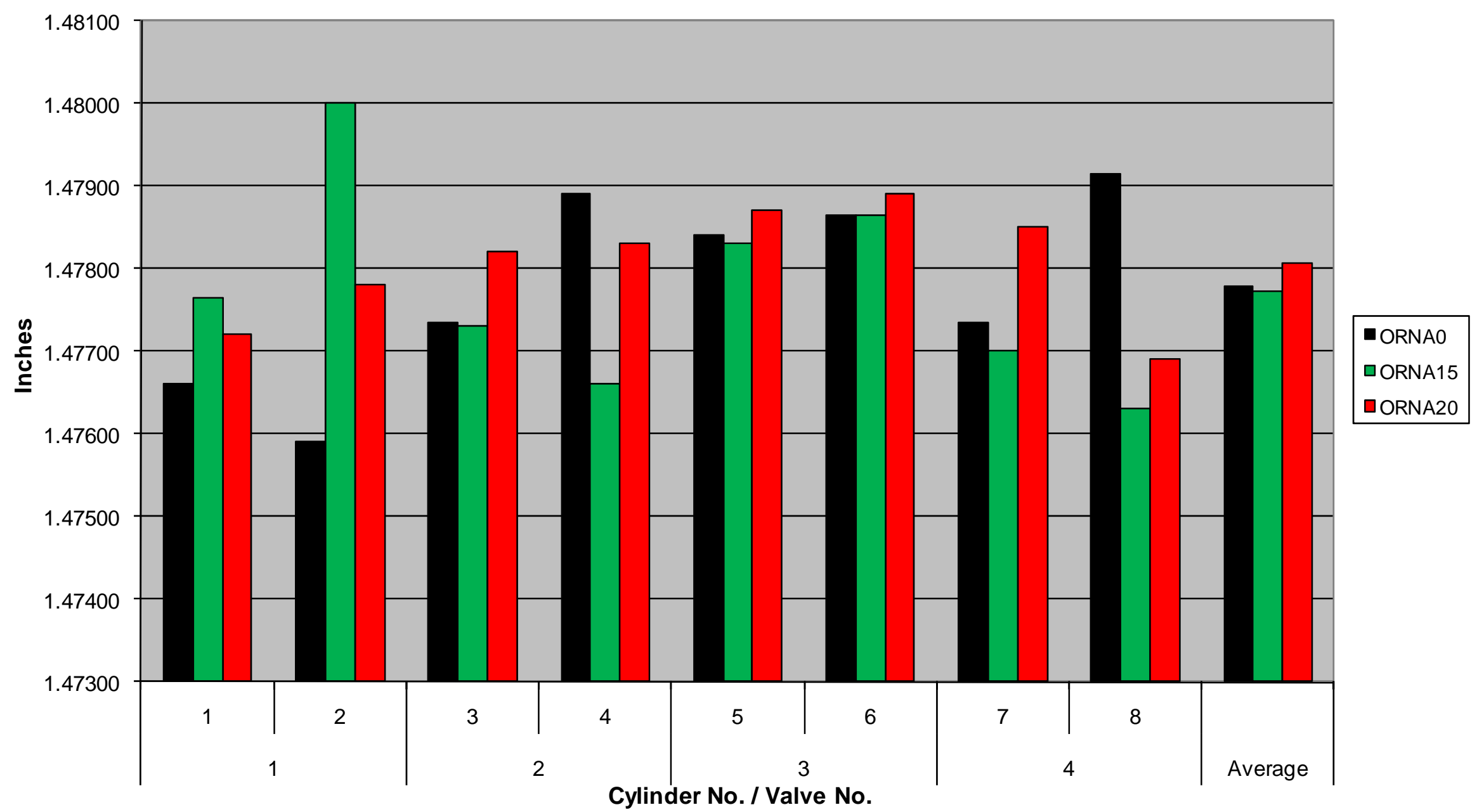




\section{Oak Ridge National Laboratory Proposal 08-58845 Powertrain Inspection Valve Stem Height}

Date:

31-Aug-10

Technician:

$\mathrm{JM}$

Measured from tip of valve stem to cylinder head

Measurements in inches

No specified service limit

\begin{tabular}{|c|c|c|c|c|c|c|c|}
\hline \multirow[b]{2}{*}{ Cylinder \# } & \multirow[b]{2}{*}{ Valve \# } & \multicolumn{3}{|c|}{ Intake } & \multicolumn{3}{|c|}{ Exhaust } \\
\hline & & ORFT0 & ORFT15 & ORFT20 & ORFTO & ORFT15 & ORFT20 \\
\hline \multirow{2}{*}{1} & 1 & 1.62170 & 1.61825 & 1.62440 & 1.62075 & 1.62300 & 1.62400 \\
\hline & 2 & 1.62175 & 1.62140 & 1.62460 & 1.62095 & 1.62030 & 1.62250 \\
\hline \multirow{2}{*}{2} & 3 & 1.62265 & 1.61975 & 1.62480 & 1.62230 & 1.62430 & 1.62360 \\
\hline & 4 & 1.62175 & 1.61950 & 1.62400 & 1.62190 & 1.62330 & 1.62450 \\
\hline \multirow{2}{*}{3} & 5 & 1.62260 & 1.62140 & 1.62700 & 1.62145 & 1.62060 & 1.62290 \\
\hline & 6 & 1.62320 & 1.62120 & 1.62580 & 1.62080 & 1.62270 & 1.62470 \\
\hline \multirow{2}{*}{4} & 7 & 1.62215 & 1.62090 & 1.62370 & 1.62175 & 1.62280 & 1.62360 \\
\hline & 8 & 1.62300 & 1.62175 & 1.62290 & 1.62175 & 1.62020 & 1.62370 \\
\hline \multirow{2}{*}{5} & 9 & 1.62305 & 1.62140 & 1.62560 & 1.62170 & 1.62055 & 1.62220 \\
\hline & 10 & 1.62365 & 1.62165 & 1.62510 & 1.62025 & 1.62165 & 1.62530 \\
\hline \multirow{2}{*}{6} & 11 & 1.62060 & 1.61585 & 1.62330 & 1.62025 & 1.62175 & 1.62270 \\
\hline & 12 & 1.62160 & 1.62110 & 1.62290 & 1.62170 & 1.61875 & 1.62080 \\
\hline \multicolumn{2}{|l|}{ Average } & 1.62231 & 1.62035 & 1.62451 & 1.62130 & 1.62166 & 1.62338 \\
\hline \multicolumn{2}{|l|}{ Stdev } & 0.00087 & 0.00177 & 0.00125 & 0.00067 & 0.00161 & 0.00124 \\
\hline \multicolumn{2}{|l|}{ Max } & 1.62365 & 1.62175 & 1.62700 & 1.62230 & 1.62430 & 1.62530 \\
\hline \multicolumn{2}{|l|}{ Min } & 1.62060 & 1.61585 & 1.62290 & 1.62025 & 1.61875 & 1.62080 \\
\hline
\end{tabular}


Oakridge National Laboratory

2008 Ford Taurus

Intake Valve Stem Height Measurements at EOT

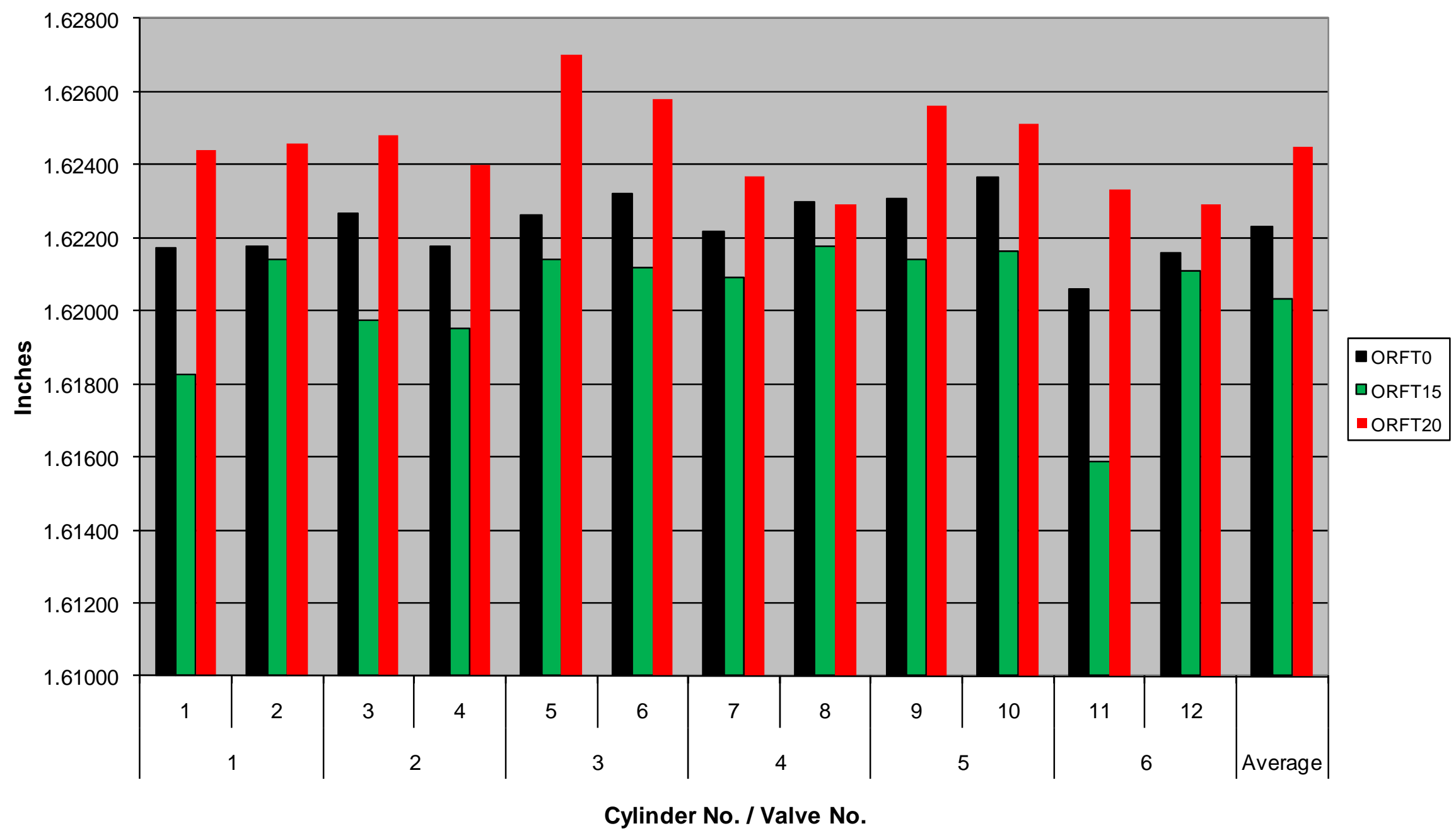




\section{Oakridge National Laboratory}

2008 Ford Taurus

Exhaust Valve Stem Height Measurements at EOT

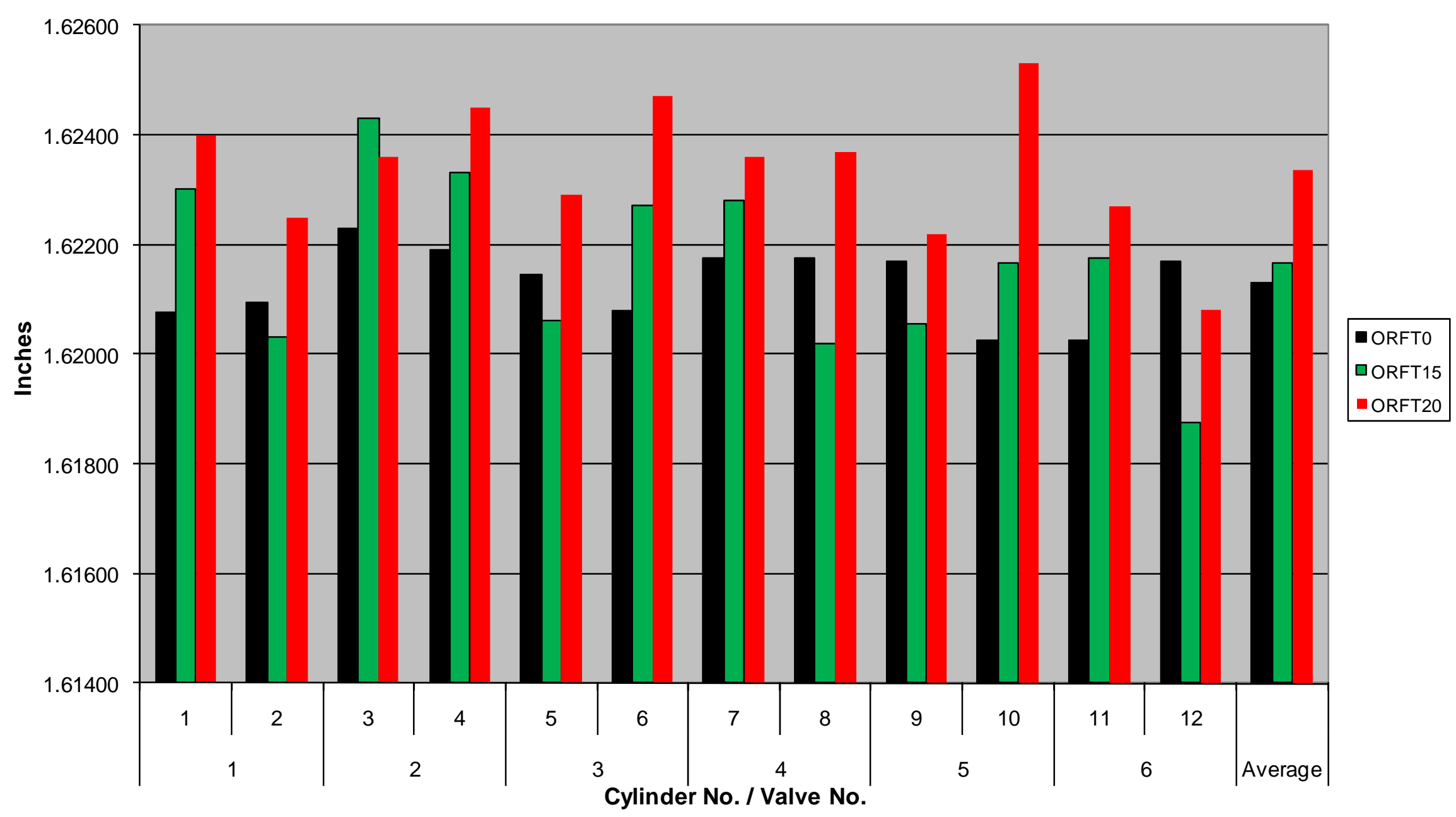




\section{Oak Ridge National Laboratory Proposal 08-58845 Powertrain Inspection Valve Stem Height}
Date: 26-Aug-10
Technician:
Measured from tip of valve stem to cylinder head Measurements in inches No specified service limit

LJ

\begin{tabular}{|c|r|r|r|r|r|r|}
\cline { 2 - 7 } \multicolumn{1}{c|}{} & \multicolumn{3}{c|}{ Intake } & \multicolumn{2}{c|}{ Exhaust } \\
\hline Cylinder \# & \multicolumn{1}{c|}{ ORCS0 } & \multicolumn{1}{c|}{ ORCS15 } & \multicolumn{1}{c|}{ ORCS20 } & \multicolumn{1}{c|}{ ORCS0 } & \multicolumn{1}{c|}{ ORCS15 } & ORCS20 \\
\hline 1 & 1.9987 & 2.0032 & 2.0065 & 1.9987 & 1.9997 & 2.0013 \\
\hline 2 & 2.0021 & 1.9973 & 2.0022 & 2.0017 & 2.0059 & 2.0025 \\
\hline 3 & 1.9992 & 2.0040 & 2.0048 & 2.0049 & 2.0014 & 2.0073 \\
\hline 4 & 2.0012 & 1.9984 & 2.0019 & 2.0012 & 2.0010 & 2.0009 \\
\hline 5 & 2.0006 & 1.9980 & 2.0012 & 2.0031 & 2.0010 & 2.0006 \\
\hline 6 & 2.0010 & 2.0001 & 2.0054 & 2.0016 & 2.0034 & 2.0078 \\
\hline 7 & 2.0008 & 2.0040 & 2.0030 & 2.0016 & 1.9984 & 2.0011 \\
\hline 8 & 2.0000 & 2.0012 & 2.0066 & 2.0013 & 1.9991 & 2.0035 \\
\hline Average & 2.0005 & 2.0008 & 2.0040 & 2.0018 & 2.0012 & 2.0031 \\
\hline Stdev & 0.0011 & 0.0027 & 0.0021 & 0.0018 & 0.0024 & 0.0029 \\
\hline Max & 2.0021 & 2.0040 & 2.0066 & 2.0049 & 2.0059 & 2.0078 \\
\hline Min & 1.9987 & 1.9973 & 2.0012 & 1.9987 & 1.9984 & 2.0006 \\
\hline
\end{tabular}




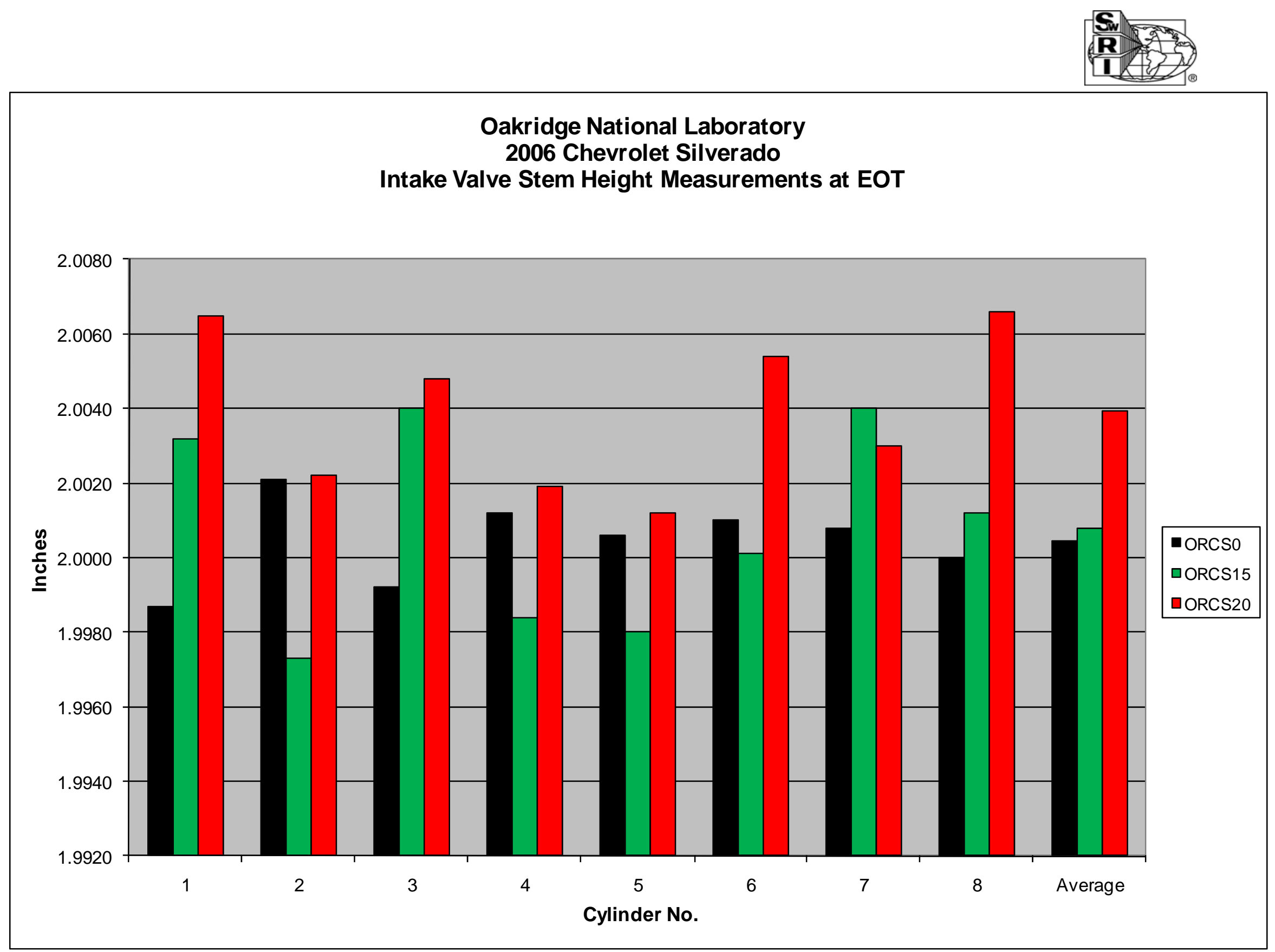




\section{Oakridge National Laboratory}

2006 Chevrolet Silverado

Exhaust Valve Stem Height Measurements at EOT

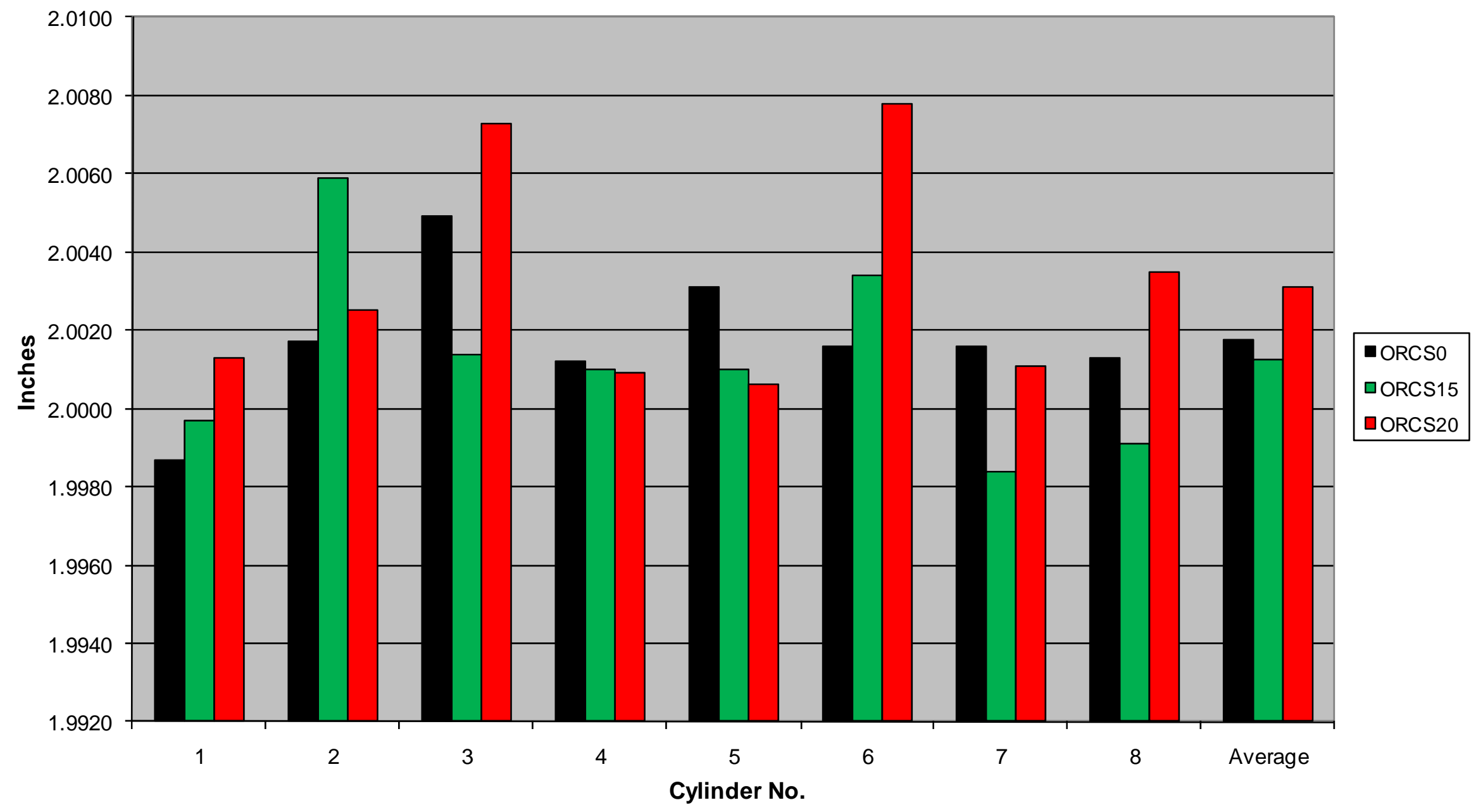




\section{Oak Ridge National Laboratory Proposal 08-58845 Powertrain Inspection Valve Stem Height}

Date:

12-Aug-10

Technician:

LJ

Measured from tip of valve stem to the spring seat washer Measurements in inches

Service Limits: Intake $=1.89 "-1.95 " \quad$ Exhaust $=1.91 "-1.97 "$

\begin{tabular}{|c|r|r|r|r|r|r|}
\cline { 2 - 7 } \multicolumn{1}{c|}{} & \multicolumn{3}{c|}{ Intake } & \multicolumn{2}{c|}{ Exhaust } \\
\hline Cylinder \# & \multicolumn{1}{c|}{ ORDC0 } & \multicolumn{1}{c|}{ ORDC15 } & \multicolumn{1}{c|}{ ORDC20 } & \multicolumn{1}{c|}{ ORDC0 } & \multicolumn{1}{c|}{ ORDC15 } & ORDC20 \\
\hline 1 & 1.93940 & 1.94975 & 1.9557 & 1.96270 & 1.95975 & 1.9640 \\
\hline 2 & 1.94270 & 1.94620 & 1.9552 & 1.96880 & 1.96825 & 1.9730 \\
\hline 3 & 1.94560 & 1.94850 & 1.9583 & 1.97075 & 1.95910 & 1.9626 \\
\hline 4 & 1.94735 & 1.94895 & 1.9532 & 1.96975 & 1.96230 & 1.9762 \\
\hline 5 & 1.94595 & 1.94445 & 1.9509 & 1.96545 & 1.95585 & 1.9687 \\
\hline 6 & 1.94840 & 1.94610 & 1.9518 & 1.96825 & 1.96805 & 1.9720 \\
\hline Average & 1.94490 & 1.94733 & 1.9542 & 1.96762 & 1.96222 & 1.9694 \\
\hline Stdev & 0.0033 & 0.0020 & 0.0027 & 0.0030 & 0.0050 & 0.0053 \\
\hline Max & 1.94840 & 1.94975 & 1.9583 & 1.97075 & 1.96825 & 1.9762 \\
\hline Min & 1.93940 & 1.94445 & 1.9509 & 1.96270 & 1.95585 & 1.9626 \\
\hline
\end{tabular}


Oakridge National Laboratory

2007 Dodge Caravan

Intake Valve Stem Height Measurements at EOT

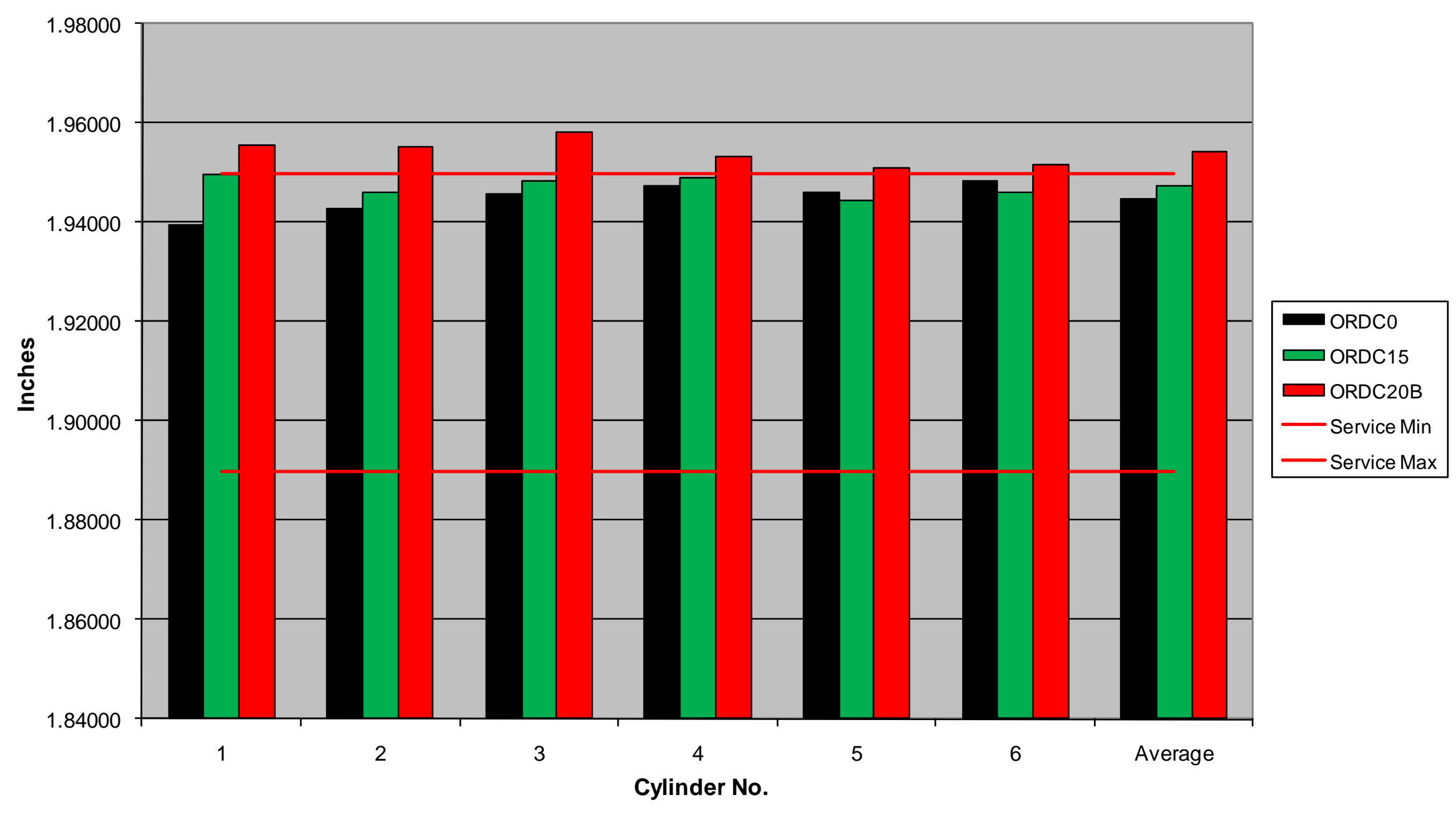

C-17 of 18 
Oakridge National Laboratory

2007 Dodge Caravan

Exhaust Valve Stem Height Measurements at EOT

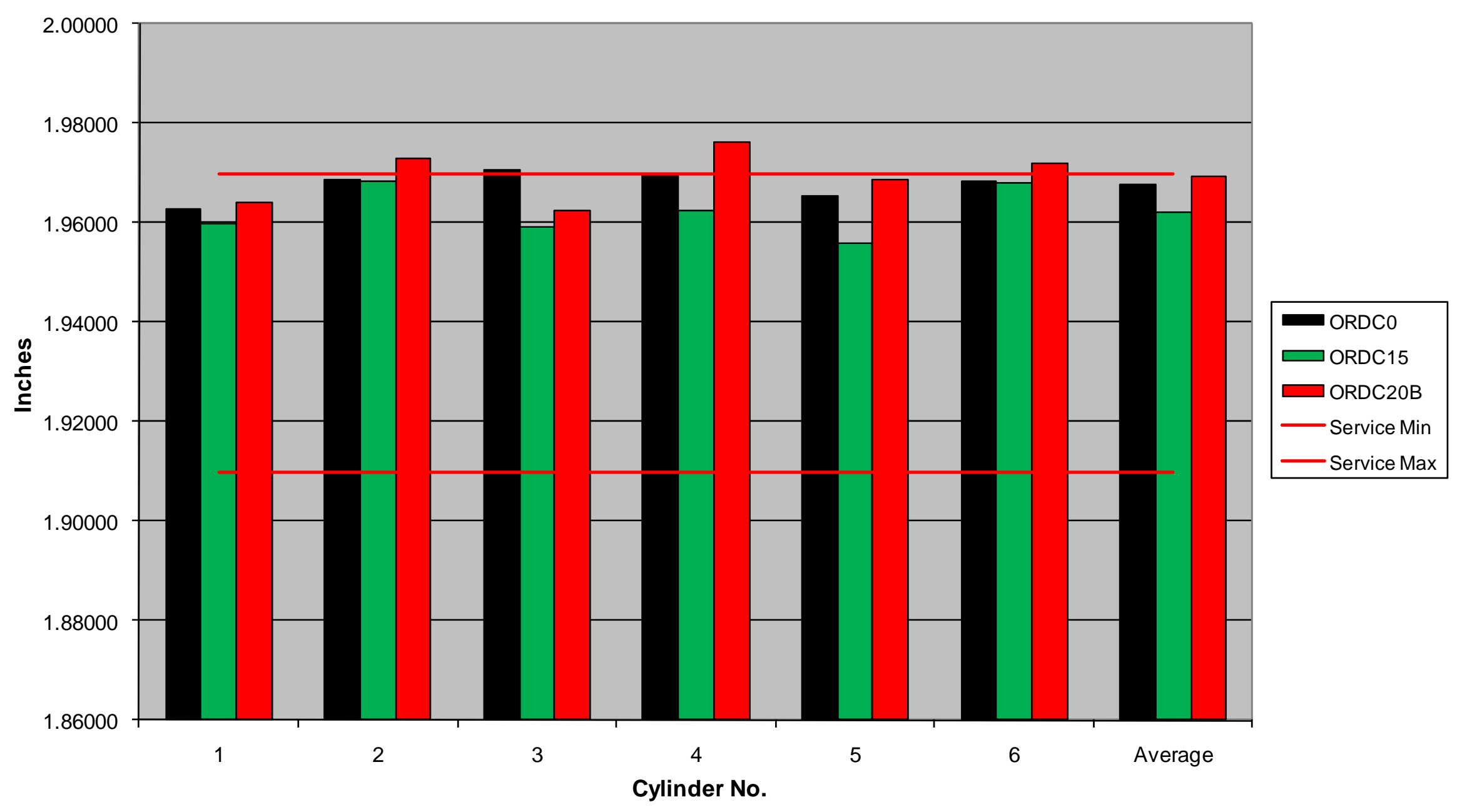

C-18 of 18 
Appendix D

Intake Valve Deposit Weight 

Oak Ridge Program E-87-2

Intake Valve Deposit Weigh in Grams

\begin{tabular}{|c|c|c|c|c|c|c|c|c|c|c|c|c|c|c|c|c|}
\hline \multirow{2}{*}{\begin{tabular}{|l} 
Vehicle \\
Number \\
\end{tabular}} & \multicolumn{2}{|c|}{ Cylinder 1} & \multicolumn{2}{|c|}{ Cylinder 2} & \multicolumn{2}{|c|}{ Cylinder 3} & \multicolumn{2}{|c|}{ Cylinder 4} & \multicolumn{2}{|c|}{ Cylinder 5} & \multicolumn{2}{|c|}{ Cylinder 6} & \multicolumn{2}{|c|}{ Cylinder 7} & \multicolumn{2}{|c|}{ Cylinder 8} \\
\hline & Front & Rear & Front & Rear & Front & Rear & Front & Rear & Front & Rear & Front & Rear & Front & Rear & Front & Rear \\
\hline ORHAO & 0.0059 & 0.0068 & 0.0071 & 0.0057 & 0.0075 & 0.0057 & 0.0046 & 0.0063 & & & & & & & & \\
\hline ORHA15 & 0.0261 & 0.0399 & 0.0365 & 0.0505 & 0.0354 & 0.0448 & 0.0348 & 0.0525 & & & & & & & & \\
\hline ORHA20 & 0.0457 & 0.0411 & 0.0500 & 0.0527 & 0.0337 & 0.0636 & 0.0366 & 0.0672 & & & & & & & & \\
\hline ORCSO & 0.0030 & & 0.0018 & & 0.0018 & & 0.0025 & & 0.0027 & & 0.0025 & & 0.0019 & & 0.0018 & \\
\hline ORCS15 & 0.0489 & & 0.0720 & & 0.0565 & & \begin{tabular}{|l|}
0.0510 \\
\end{tabular} & & 0.0325 & & 0.0270 & & 0.0482 & & 0.0454 & \\
\hline ORCS20 & 0.0420 & & 0.0866 & & 0.0758 & & 0.1527 & & 0.0596 & & 0.0622 & & 0.0404 & & 0.0262 & \\
\hline ORNAO & 0.0055 & 0.0039 & 0.0042 & 0.0046 & 0.0036 & 0.0044 & 0.0034 & 0.0117 & & & & & & & & \\
\hline ORNA15 & 0.0080 & 0.0064 & 0.0067 & 0.0055 & 0.0084 & 0.0117 & 0.0303 & 0.0531 & & & & & & & & \\
\hline ORNA20 & 0.0098 & 0.0095 & 0.0136 & 0.0113 & 0.0144 & 0.0197 & 0.0612 & 0.0493 & & & & & & & & \\
\hline ORFTO & 0.0082 & 0.0084 & 0.0067 & 0.0066 & 0.0048 & 0.0066 & 0.0076 & 0.0093 & 0.0090 & 0.0082 & 0.0147 & 0.0118 & & & & \\
\hline ORFT15 & 0.0094 & 0.0223 & 0.0199 & 0.0263 & 0.0188 & 0.0156 & 0.0200 & 0.0264 & 0.0260 & 0.0185 & 0.0208 & 0.0241 & & & & \\
\hline ORFT20 & 0.0130 & 0.1253 & 0.0335 & 0.1168 & 0.0290 & 0.0144 & 0.0484 & 0.0347 & 0.0181 & 0.0116 & 0.0417 & 0.0475 & & & & \\
\hline ORDCO & 0.0228 & & 0.0060 & & 0.0622 & & 0.1156 & & 0.0092 & & 0.0502 & & & & & \\
\hline ORDC15 & 0.0136 & & 0.0467 & & 0.2921 & & 0.1148 & & 0.0100 & & 0.0381 & & & & & \\
\hline ORDC2OB & 0.0243 & & 0.0195 & & 0.1096 & & 0.3216 & & 0.0115 & & 0.0402 & & & & & \\
\hline ORCCO & 0.0101 & 0.0077 & 0.0110 & \begin{tabular}{|l|}
0.0071 \\
\end{tabular} & 0.0091 & 0.0073 & \begin{tabular}{|l|}
0.0108 \\
\end{tabular} & 0.0120 & & & & & & & & \\
\hline ORCC15 & \begin{tabular}{|l|}
0.0274 \\
\end{tabular} & 0.0217 & 0.0119 & 0.0276 & 0.0295 & 0.0178 & 0.0296 & 0.0216 & & & & & & & & \\
\hline ORCC20 & 0.0133 & 0.0178 & 0.0090 & 0.0220 & 0.0174 & 0.0097 & 0.0263 & 0.0175 & & & & & & & & \\
\hline
\end{tabular}




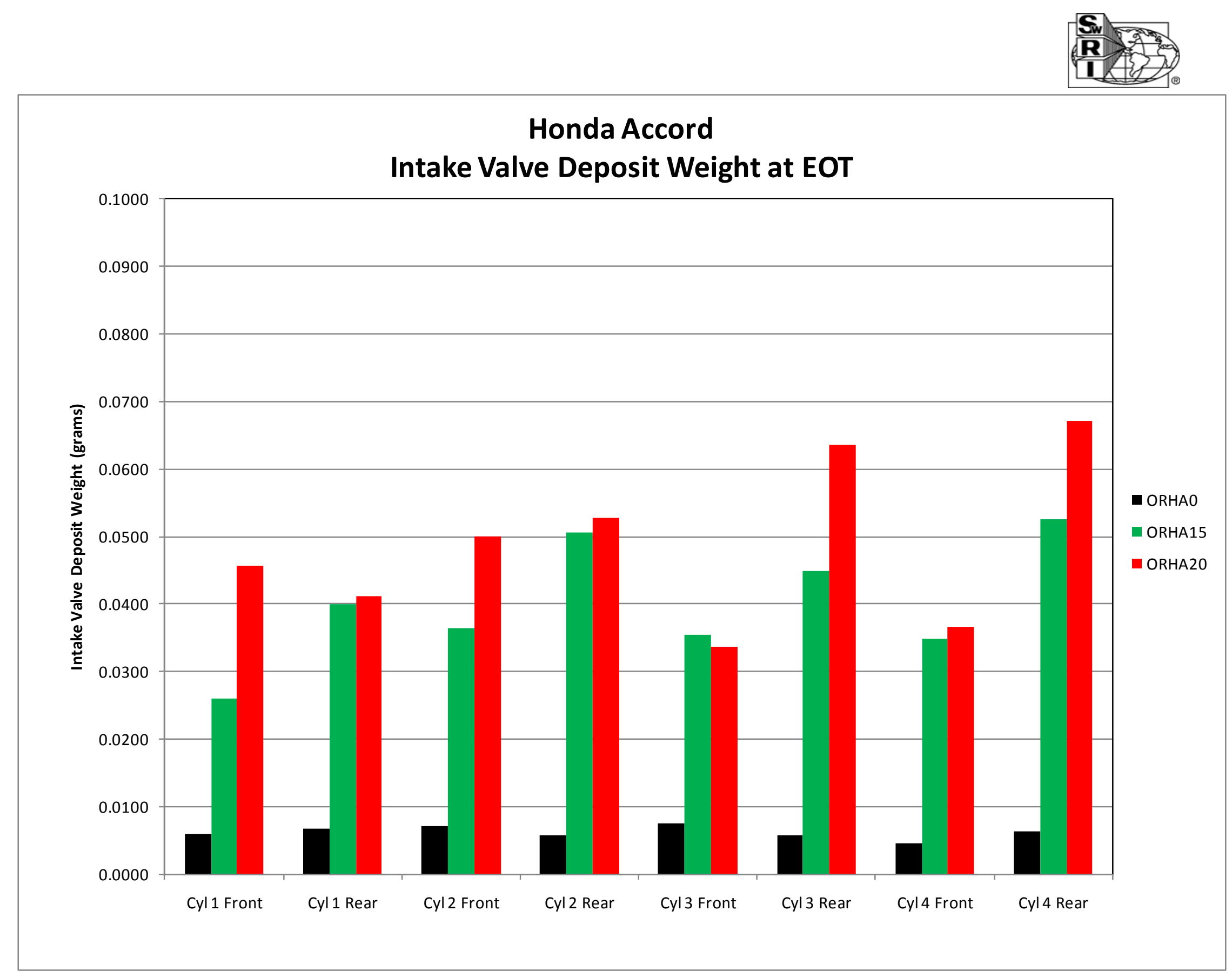


Oak Ridge Program E-87-2

\begin{tabular}{|l|l|}
\hline Vehicle: Honda Accord ORHA0 & \\
\hline End of Test & Fuel: E0 \\
\hline
\end{tabular}

Intake Valves 1 Front Through 4 Rear Maximum IVD is Facing Camera

Front View

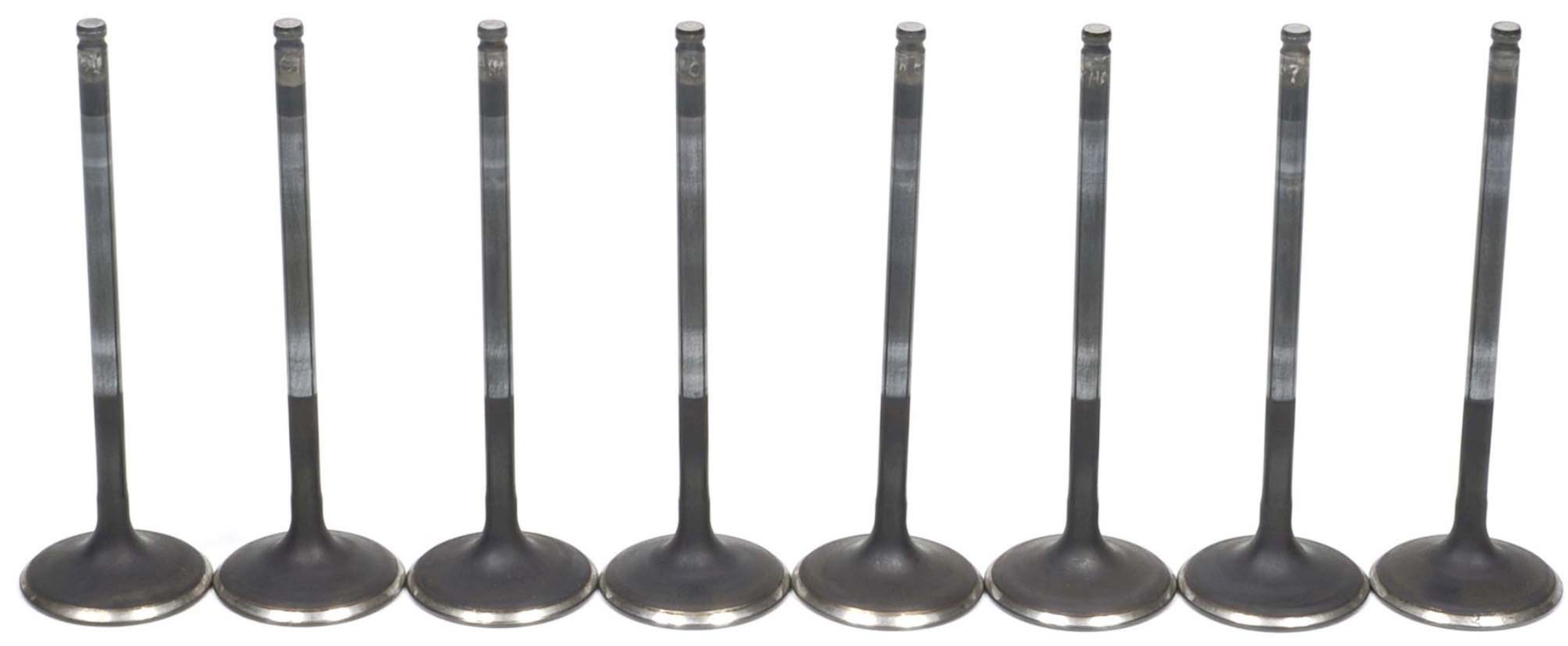

D-3 of 11 
Oak Ridge Program E-87-2

\begin{tabular}{|l|l|}
\hline Vehicle: $\quad$ Honda Accord ORHA0 & \\
\hline End of Test & Fuel: $\quad$ E0 \\
\hline
\end{tabular}

Intake Valves 1 Front Through 4 Rear Minimum IVD is Facing Camera

\author{
Back View
}

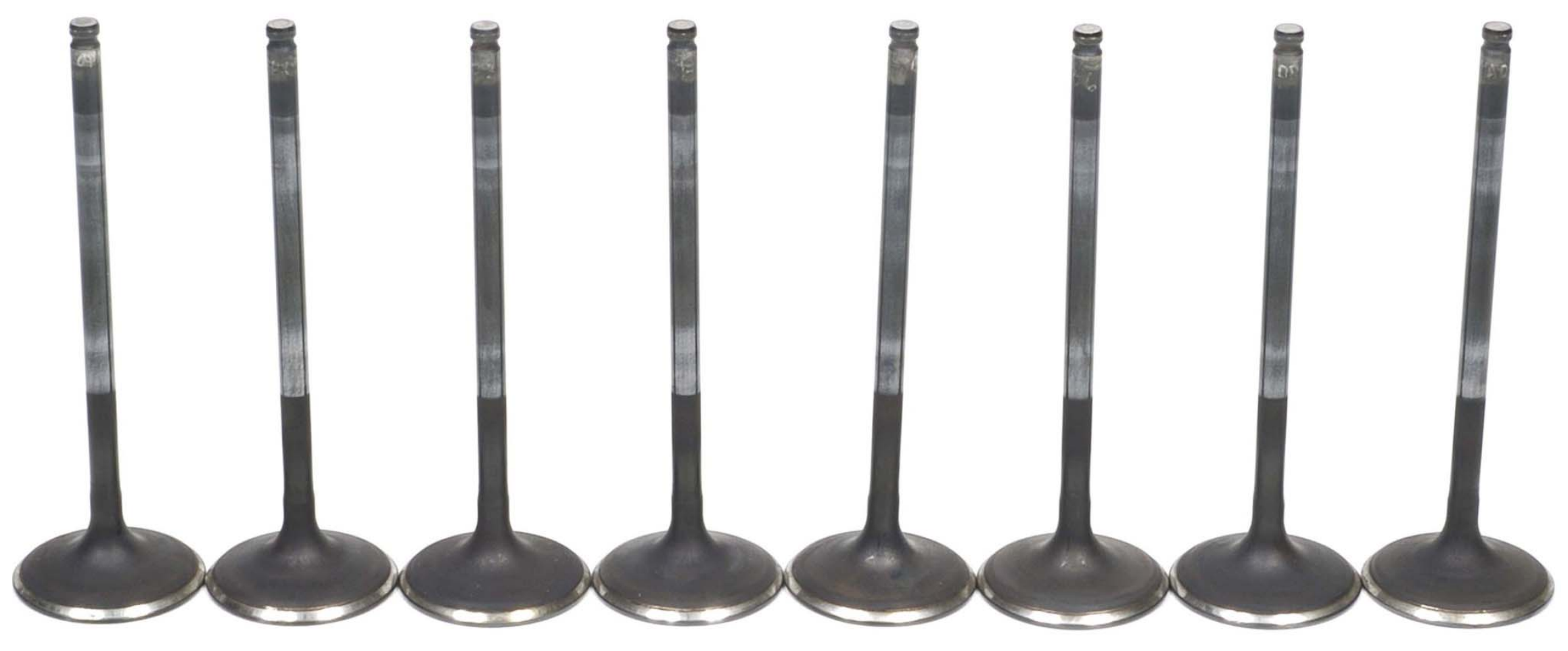

D-4 of 11 
Oak Ridge Program E-87-2

Vehicle:

Honda Accord ORHA15

End of Test Fuel: E15

Intake Valves 1 Front Through 4 Rear Maximum IVD is Facing Camera

Front View

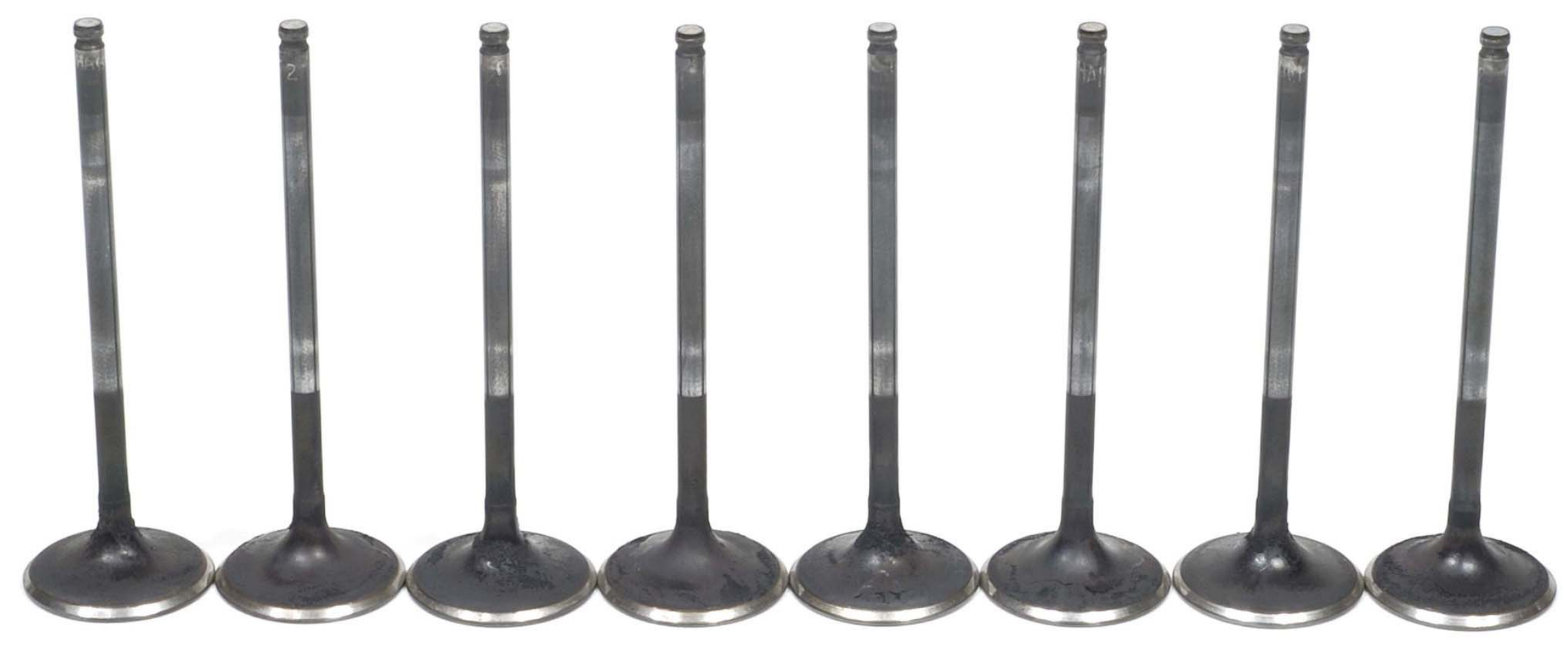

D-5 of 11 
Oak Ridge Program E-87-2

Vehicle: Honda Accord ORHA15

End of Test

Fuel:

E15

Intake Valves 1 Front Through 4 Rear

Minimum IVD is Facing Camera

Back View

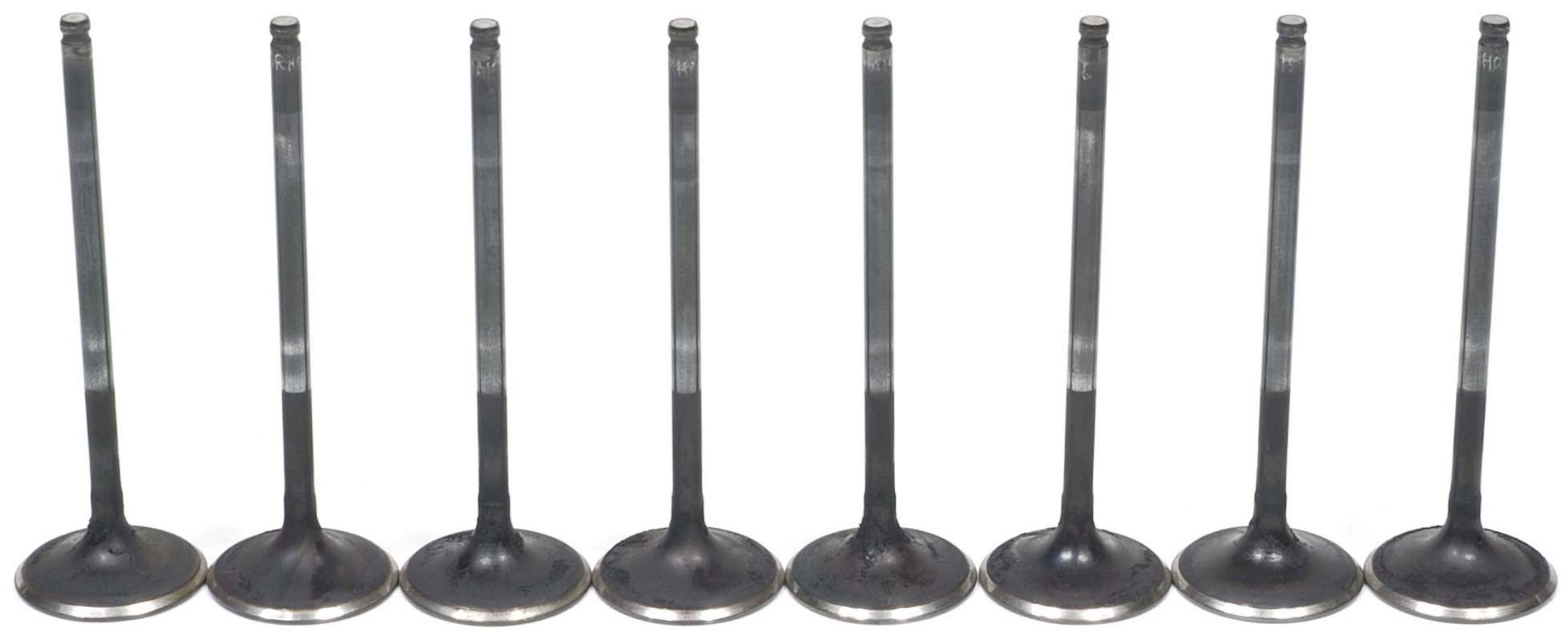

D-6 of 11 


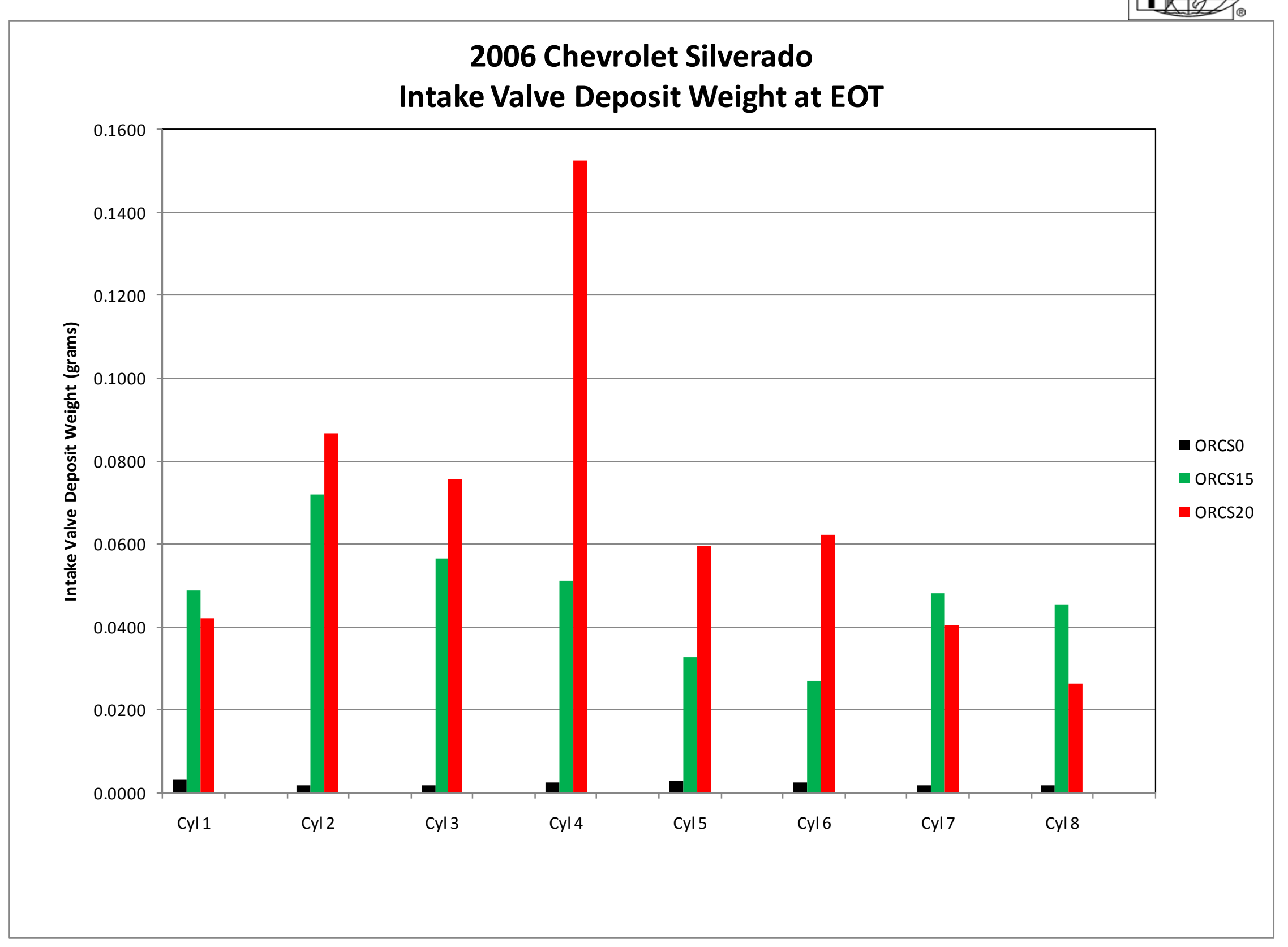

D-7 of 11 


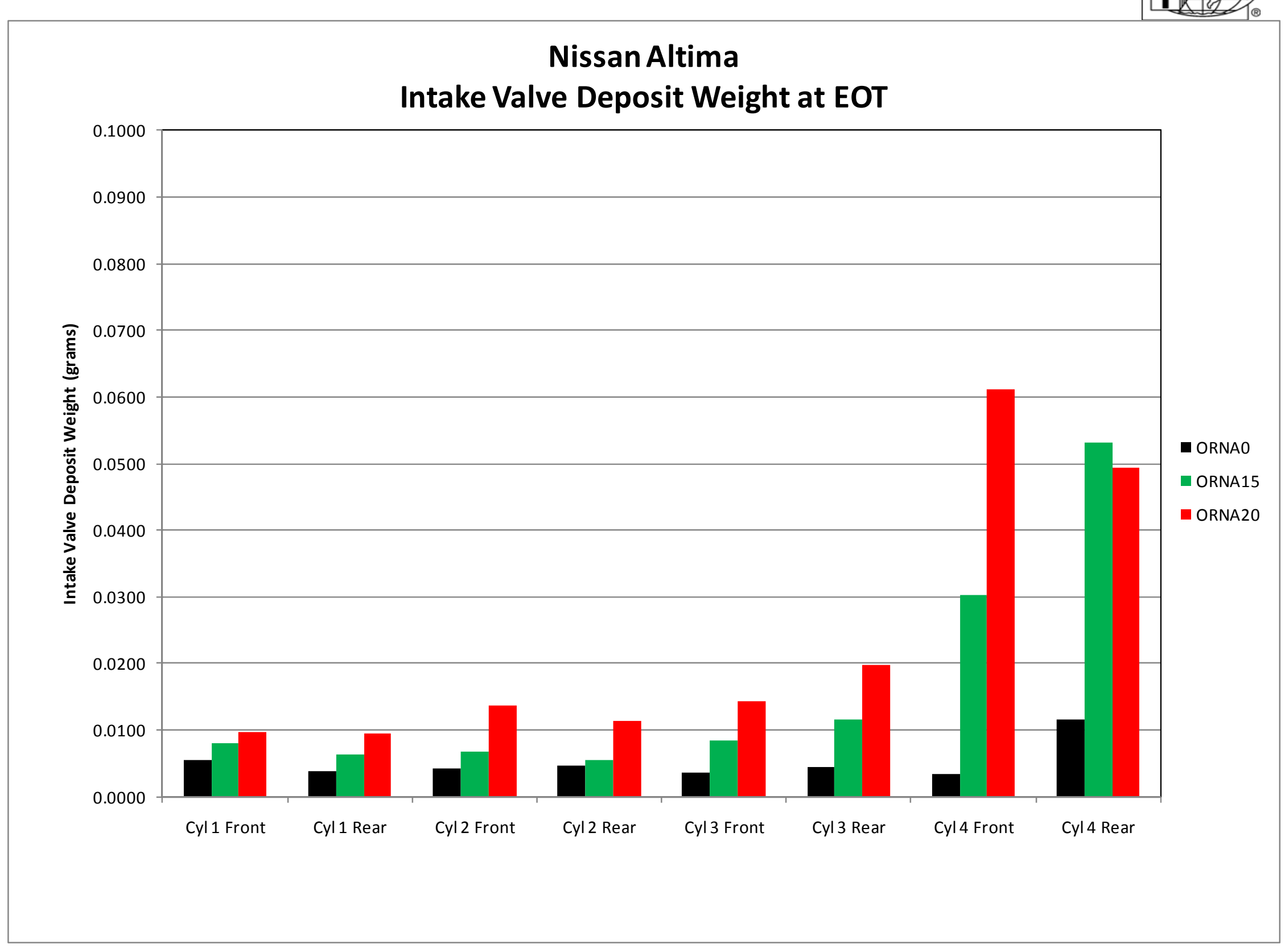




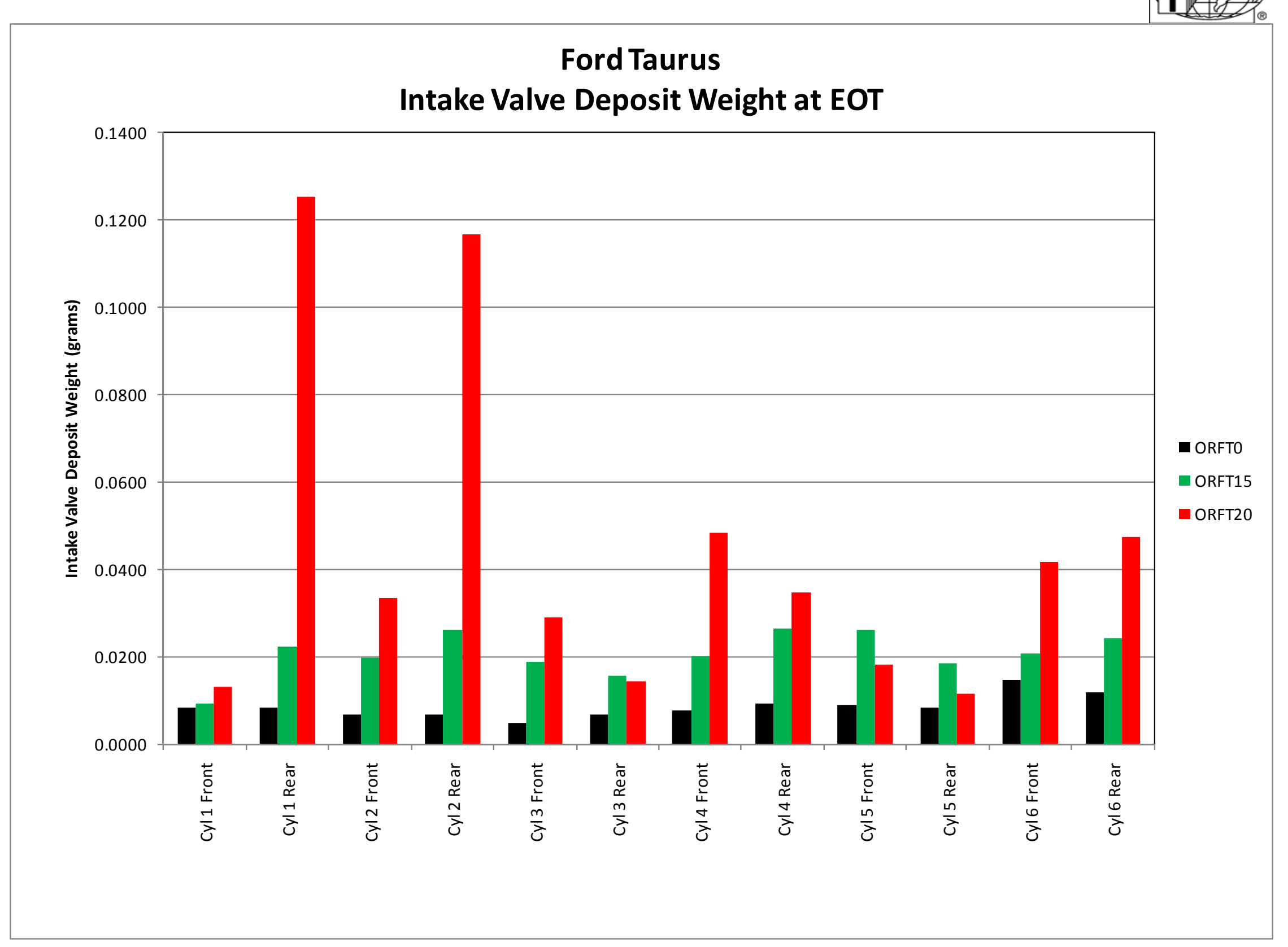




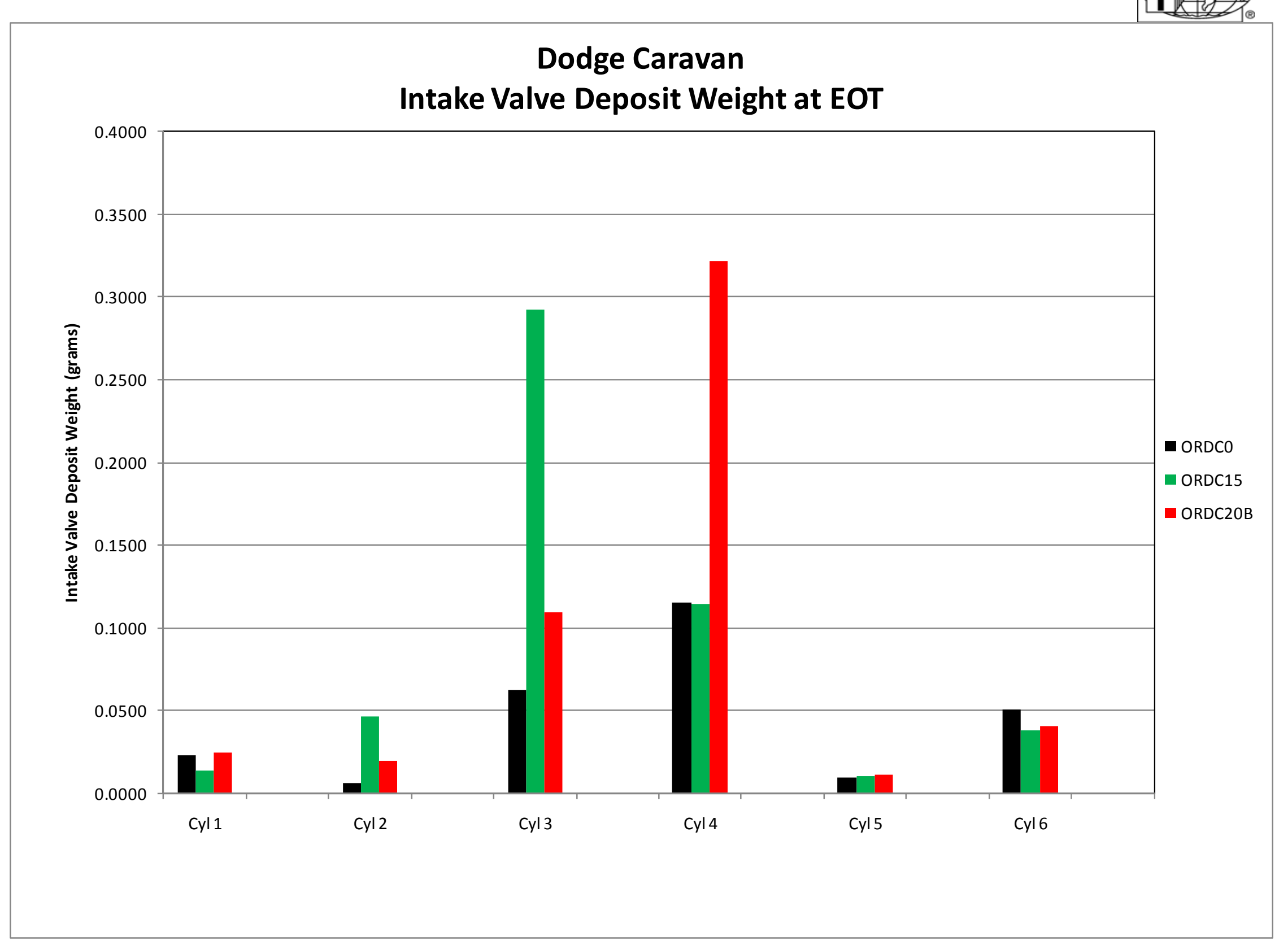

D-10 of 11 


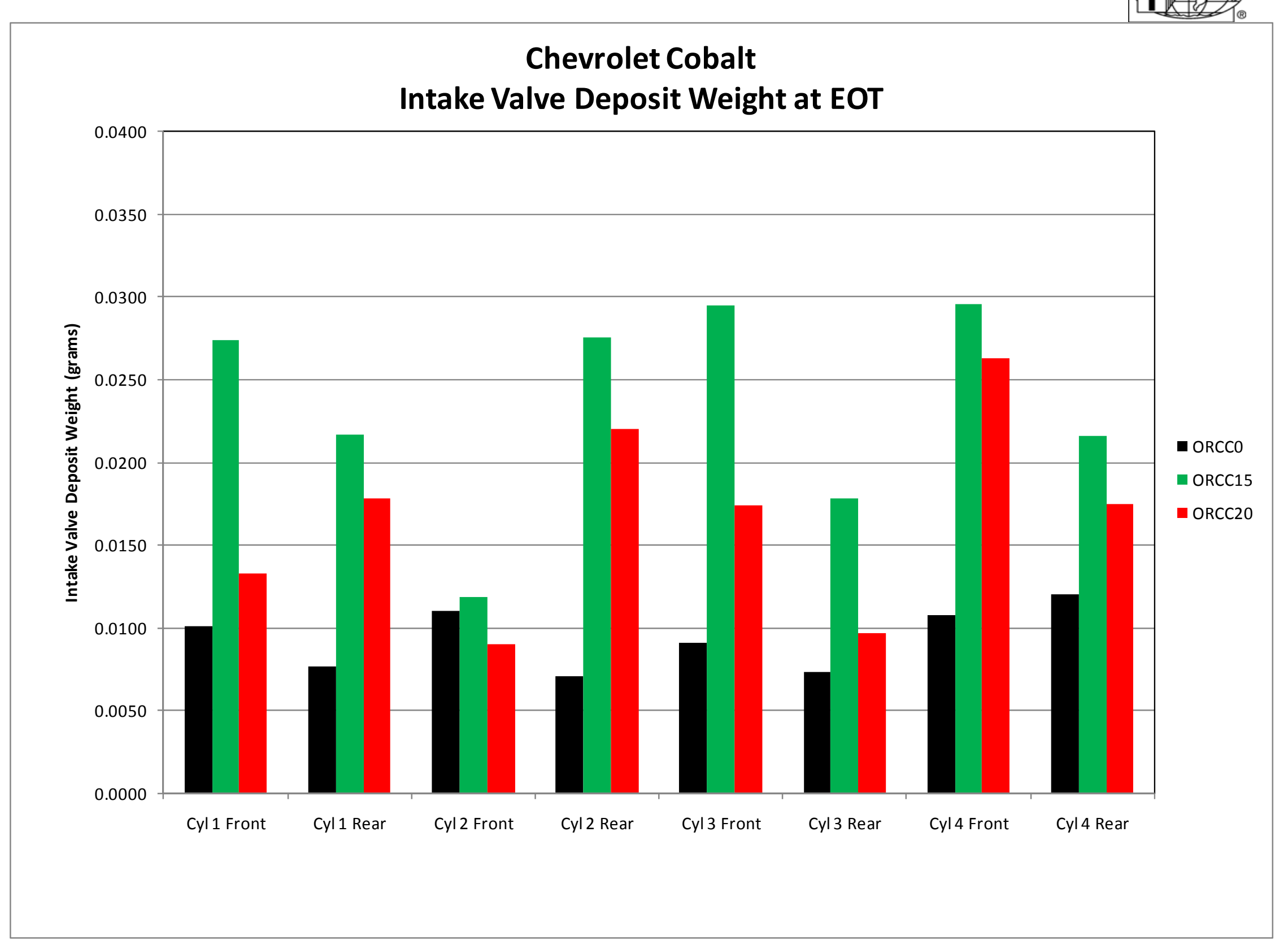

D-11 of 11 

Appendix E

ASTM D5185 ICP Metals Results 

Oak Ridge Used Oil Samples

August 4, 2010

\begin{tabular}{|c|c|c|c|c|c|c|c|c|c|c|c|c|c|c|c|c|c|c|c|c|c|c|c|c|c|c|c|c|}
\hline & & & & & \multicolumn{9}{|c|}{ ASTM D-5185 Elemental Analysis by ICP } & \multicolumn{15}{|c|}{ ASTM D-5185 Elemental Analysis by ICP } \\
\hline $\begin{array}{l}\text { Vehicle } \\
\text { Number }\end{array}$ & $\begin{array}{l}\text { Vehicle } \\
\text { Number }\end{array}$ & $\begin{array}{l}\text { SOT } \\
\text { Odo. }\end{array}$ & $\begin{array}{c}\text { Oil } \\
\text { Sample } \\
\text { Odometer } \\
\text { (miles) }\end{array}$ & \begin{tabular}{|c|} 
Oil \\
Sample \\
Test \\
Miles
\end{tabular} & 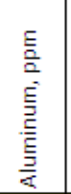 & 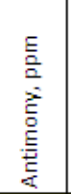 & 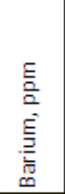 & 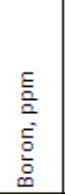 & 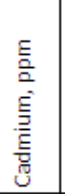 & $\begin{array}{l}\text { ⿳ㅡㅁ } \\
\text { E } \\
\frac{\bar{g}}{\sqrt{J}} \\
\end{array}$ & 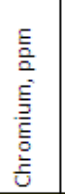 & 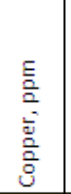 & 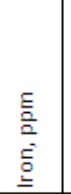 & 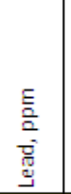 & 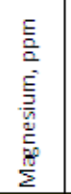 & 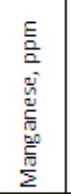 & 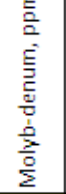 & $\begin{array}{l}\frac{E}{2} \\
\frac{\bar{a}}{\mathrm{i}} \\
\frac{\mathrm{i}}{2}\end{array}$ & 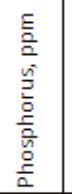 & 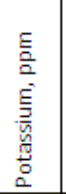 & 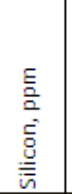 & 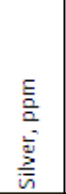 & 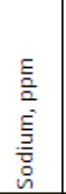 & 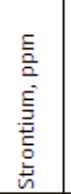 & $\begin{array}{l}E \\
\bar{E} \\
\hat{E}\end{array}$ & 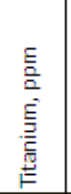 & 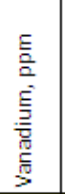 & 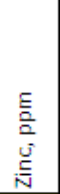 \\
\hline ORHAO & ORHAO & 31,686 & 38,603 & 6,917 & 2 & $<1$ & $<1$ & 76 & $<1$ & 2068 & $<1$ & 2 & 7 & 1 & 24 & $<1$ & 5 & $<1$ & 668 & 6 & 12 & $<1$ & $<5$ & $<1$ & $<1$ & $<1$ & $<1$ & 850 \\
\hline ORHAO & ORHAO & 31,686 & 75,460 & 43,774 & 1 & $<1$ & $<1$ & 58 & $<1$ & 2111 & $<1$ & $<1$ & 5 & $<1$ & 10 & $<1$ & $<1$ & $<1$ & 694 & 6 & 14 & $<1$ & $<5$ & $<1$ & $<1$ & $<1$ & $<1$ & 858 \\
\hline ORHAO & ORHAO & 31,686 & 115,233 & 83,547 & 1 & $<1$ & $<1$ & 54 & $<1$ & 2091 & $<1$ & 1 & 6 & $<1$ & 10 & $<1$ & $<1$ & $<1$ & 705 & 8 & 18 & $<1$ & $<5$ & $<1$ & $<1$ & $<1$ & $<1$ & 863 \\
\hline ORHA15 & ORHA15 & 31,369 & 9,220 & 7,851 & 2 & $<1$ & $<1$ & 65 & $<1$ & 2033 & $<1$ & 5 & 8 & $<1$ & 10 & $<1$ & 3 & $<1$ & 661 & 5 & 16 & $<1$ & 6 & $<1$ & $<1$ & $<1$ & $<1$ & 846 \\
\hline ORHA 15 & ORHA15 & 31,369 & 7,277 & 45,908 & 1 & $<1$ & $<1$ & 44 & $<1$ & 2081 & $<1$ & 2 & f & $<1$ & 10 & $<1$ & 1 & $<1$ & 710 & 6 & 15 & $<1$ & $<5$ & $<1$ & $<1$ & $<1$ & $<1$ & 847 \\
\hline ORHA15 & ORHA15 & 31,369 & 110,419 & 9,050 & 1 & $<1$ & $<1$ & 36 & $<1$ & 2065 & $<1$ & 2 & 0 & $<1$ & 11 & $<1$ & $<1$ & $<1$ & 685 & 8 & 18 & $<1$ & $<5$ & $<1$ & $<1$ & $<1$ & $<1$ & 856 \\
\hline ORCSO & ORCSO & 27,606 & 39,679 & 12,073 & 4 & 1 & $<1$ & 44 & $<1$ & 2054 & $<1$ & 28 & 23 & 9 & 4 & 3 & 7 & 2 & 666 & 6 & 18 & $<1$ & $<5$ & $<1$ & $<1$ & $<1$ & $<1$ & 858 \\
\hline ORCSO & ORCSC & 27,606 & 65,566 & 37,960 & 2 & $<1$ & $<1$ & 24 & $<1$ & 2068 & $<1$ & 12 & 13 & $<1$ & 8 & $<1$ & 4 & $<1$ & 681 & 7 & 27 & $<1$ & $<5$ & $<1$ & $<1$ & $<1$ & $<1$ & 859 \\
\hline ORCSO & ORCSO & 27,606 & 116,347 & 88,741 & 2 & 1 & $<1$ & 27 & $<1$ & 2052 & $<1$ & 10 & 12 & $<1$ & & $<1$ & 4 & $<1$ & 664 & 6 & 23 & $<1$ & $<5$ & $<1$ & $<1$ & $<1$ & $<1$ & 839 \\
\hline ORCS 15 & ORCS 15 & 17,152 &, 410 & 12,258 & 3 & $<1$ & $<1$ & 37 & $<1$ & 2048 & $<1$ & 76 & 26 & 6 & 9 & 2 & 14 & 3 & 653 & II & 12 & $<1$ & 56 & $<1$ & $<1$ & $<1$ & $<1$ & 849 \\
\hline ORCS 15 & ORCS15 & 17,152 &, 297 & 38,145 & 2 & $<1$ & $<1$ & 22 & $<1$ & 2073 & $<1$ & 27 & 20 & 2 & 8 & $<1$ & 6 & 1 & 656 & 12 & 12 & $<1$ & 32 & $<1$ & $<1$ & $<1$ & $<1$ & 872 \\
\hline ORCS 15 & ORCS15 & 17,152 & 106,079 & 88,927 & 2 & $<1$ & $<1$ & 19 & $<1$ & 2044 & $<1$ & 20 & 25 & 1 & of & $<1$ & 6 & 1 & 655 & 11 & 15 & $<1$ & 19 & $<1$ & $<1$ & $<1$ & $<1$ & 847 \\
\hline ORNAO & ORNA & 19,284 & 784 & -8 & 5 & $<1$ & $<1$ & 59 & $<1$ & 2080 & $<1$ & 3 & & 1 & - & $<1$ & 4 & $<1$ & 685 & 6 & 21 & $<1$ & $<5$ & $<1$ & $<1$ & $<1$ & $<1$ & 841 \\
\hline ORNAO & ORNAO & 19,284 & 64,284 & 45,000 & $<1$ & $<1$ & $<1$ & 28 & $<1$ & 2066 & $<1$ & 1 & 4 & $<1$ & & $<1$ & $<1$ & $<1$ & 641 & 6 & 20 & $<1$ & $<5$ & $<1$ & $<1$ & $<1$ & $<1$ & 839 \\
\hline ORNAO & ORNAO & 19,284 & 116,784 & 97,500 & $<1$ & 1 & $<1$ & 44 & $<1$ & 2064 & $<1$ & 1 & 5 & $<1$ & 9 & $<1$ & 1 & $<1$ & 671 & 11 & 26 & $<1$ & $<5$ & $<1$ & $<1$ & $<1$ & $<1$ & 866 \\
\hline ORNA15 & ORNA15 & 9,950 & 450 & 7,500 & 3 & $<1$ & $<1$ & 58 & $<1$ & 2023 & $<1$ & 6 & 7 & 2 & 8 & 2 & 20 & $<1$ & 640 & 8 & 29 & $<1$ & 56 & $<1$ & $<1$ & $<1$ & $<1$ & 850 \\
\hline ORNA15 & ORNA15 & 9,950 & 4,950 & 45,000 & 2 & $<1$ & $<1$ & 34 & $<1$ & 2080 & $<1$ & 2 & 6 & $<1$ & 8 & $<1$ & $<1$ & $<1$ & 703 & 6 & 21 & $<1$ & $<5$ & $<1$ & $<1$ & $<1$ & $<1$ & 846 \\
\hline ORNA15 & ORNA15 & 9,950 & 107,450 & 97,500 & $<1$ & $<1$ & $<1$ & 47 & $<1$ & 2066 & $<1$ & 2 & 4 & $<1$ & af & $<1$ & $<1$ & $<1$ & 721 & 8 & 29 & $<1$ & $<5$ & $<1$ & $<1$ & $<1$ & $<1$ & 859 \\
\hline ORFTO & ORFTO & 17,231 & 731 & 500 & 4 & $<1$ & $<1$ & 90 & $<1$ & 2008 & $<1$ & 44 & 15 & 2 & 10 & 10 & 6 & $<1$ & 662 & 5 & 30 & $<1$ & & $<1$ & $<1$ & $<1$ & $<1$ & 812 \\
\hline ORFTO & ORFTO & 17,231 & 231 & 45,000 & 2 & $<1$ & $<1$ & 62 & $<1$ & 2010 & $<1$ & 7 & 8 & $<1$ & 10 & 2 & 1 & $<1$ & 685 & 7 & 27 & $<1$ & $<5$ & $<1$ & $<1$ & $<1$ & $<1$ & 825 \\
\hline ORFTO & ORFTO & 17,231 & 113,231 & 96,000 & 1 & $<1$ & $<1$ & 50 & $<1$ & 1992 & $<1$ & 4 & 5 & $<1$ & 8 & $<1$ & 1 & $<1$ & 655 & 7 & 21 & $<1$ & $<5$ & $<1$ & $<1$ & $<1$ & $<1$ & 831 \\
\hline ORFT15 & ORFT15 & 17,099 & 24,599 & 7,500 & 4 & $<1$ & $<1$ & 80 & $<1$ & 2001 & $<1$ & 43 & 15 & 1 & 10 & 9 & 6 & $<1$ & 647 & 1 & 24 & $<1$ & 6 & $<1$ & $<1$ & $<1$ & $<1$ & 812 \\
\hline ORFT 15 & ORFT15 & 17,099 & 62,099 & 5,000 & 2 & $<1$ & $<1$ & 62 & $<1$ & 2022 & $<1$ & 9 & 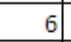 & 1 & 10 & 2 & 1 & $<1$ & 639 & 1 & 22 & $<1$ & $<5$ & $<1$ & $<1$ & $<1$ & $<1$ & 831 \\
\hline ORFT15 & ORFT15 & 17,099 & 113,099 & 96,000 & 1 & $<1$ & $<1$ & 57 & $<1$ & 2033 & $<1$ & 6 & 6 & $<1$ & 0 & $<1$ & $<1$ & $<1$ & 689 & $<5$ & 18 & $<1$ & 5 & $<1$ & $<1$ & $<1$ & $<1$ & 832 \\
\hline ORDCO & ORDCO & 46,473 & 52,473 & 6,000 & 2 & 2 & $<1$ & 111 & $<1$ & 2090 & $<1$ & 32 & 16 & $<1$ & 9 & $<1$ & 7 & $<1$ & 629 & 5 & 12 & $<1$ & 6 & $<1$ & $<1$ & $<1$ & $<1$ & 849 \\
\hline ORDCO & ORDCO & 46,473 & 88,474 & 42,001 & 1 & $<1$ & $<1$ & 78 & $<1$ & 2117 & $<1$ & 7 & 11 & $<1$ & 8 & $<1$ & 3 & $<1$ & 697 & 7 & 13 & $<1$ & $<5$ & $<1$ & $<1$ & $<1$ & $<1$ & 867 \\
\hline ORDCO & ORDCO & 46,473 & 106,473 & 60,000 & $<1$ & $<1$ & $<1$ & 76 & $<1$ & 2132 & $<1$ & 6 & 10 & $<1$ & 0 & $<1$ & 2 & $<1$ & 693 & 8 & 11 & $<1$ & $<5$ & $<1$ & $<1$ & $<1$ & $<1$ & 875 \\
\hline ORDC15 & ORDC15 & 40,031 & 46,031 & 6,000 & 4 & $<1$ & $<1$ & 105 & $<1$ & 2147 & $<1$ & 19 & 18 & $<1$ & 9 & $<1$ & 17 & $<1$ & 694 & 8 & 13 & $<1$ & 6 & $<1$ & $<1$ & $<1$ & $<1$ & 870 \\
\hline ORDC15 & ORDC15 & 40,031 & 82,031 & 42,000 & 1 & $<1$ & $<1$ & 75 & $<1$ & 2177 & $<1$ & 6 & 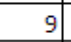 & $<1$ & 8 & $<1$ & 2 & $<1$ & 699 & 7 & 13 & $<1$ & $<5$ & $<1$ & $<1$ & $<1$ & $<1$ & 878 \\
\hline ORDC15 & ORDC15 & 40,031 & 100,031 & 60,000 & 1 & $<1$ & $<1$ & 55 & $<1$ & 2194 & $<1$ & 6 & 8 & $<1$ & 8 & $<1$ & 3 & $<1$ & 674 & 7 & 13 & $<1$ & 6 & $<1$ & $<1$ & $<1$ & $<1$ & 894 \\
\hline ORCCO & ORCCO & 38,894 & 46,784 & 7,890 & 4 & $<1$ & $<1$ & 1 & $<1$ & 2337 & $<1$ & 7 & 5 & 6 & 6 & $<1$ & $<1$ & $<1$ & 616 & $<5$ & 19 & $<1$ & 18 & $<1$ & $<1$ & $<1$ & $<1$ & 720 \\
\hline ORCCO & ORCCO & 38,894 & 84,176 & 45,282 & 1 & $<1$ & $<1$ & 65 & $<1$ & 1999 & $<1$ & $<1$ & 5 & $<1$ & 8 & $<1$ & 1 & $<1$ & 644 & 7 & 11 & $<1$ & $<5$ & $<1$ & $<1$ & $<1$ & $<1$ & 817 \\
\hline ORCCO & ORCCO & 38,894 & 106,843 & 67,949 & 1 & $<1$ & $<1$ & 28 & $<1$ & 2113 & $<1$ & $<1$ & 8 & $<1$ & 8 & $<1$ & 1 & $<1$ & 665 & 5 & 23 & $<1$ & $<5$ & $<1$ & $<1$ & $<1$ & $<1$ & 851 \\
\hline & & & & & & & & & & & & & & & & & & & & & & & & & & & & \\
\hline
\end{tabular}


Oak Ridge Used Oil Samples

September 9, 2010

\begin{tabular}{|c|c|c|c|c|c|c|c|c|c|c|c|c|c|c|c|c|c|c|c|c|c|c|c|c|c|c|c|c|}
\hline \multirow[b]{2}{*}{$\begin{array}{l}\text { Vehicle } \\
\text { Number }\end{array}$} & \multirow[b]{2}{*}{$\begin{array}{l}\text { SOT } \\
\text { Odo. }\end{array}$} & \multirow[b]{2}{*}{$\begin{array}{c}\text { Oil } \\
\text { Sample } \\
\text { Odometer } \\
\text { (miles) }\end{array}$} & \multirow[b]{2}{*}{$\begin{array}{c}\text { Oil } \\
\text { Sample } \\
\text { Test } \\
\text { Miles }\end{array}$} & \multicolumn{9}{|c|}{ ASTM D-5185 Elemental Analysis by ICP } & \multicolumn{16}{|c|}{ ASTM D-5185 Elemental Analysis by ICP } \\
\hline & & & & 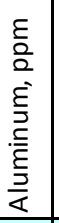 & 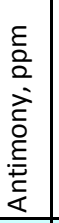 & 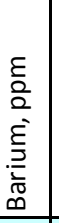 & 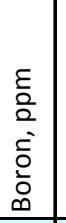 & 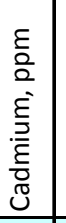 & $\begin{array}{l}\frac{\varepsilon}{0} \\
\frac{0}{2} \\
\frac{\varepsilon}{J} \\
\frac{\bar{U}}{\sqrt[U]{U}} \\
\end{array}$ & 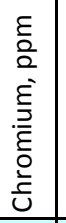 & $\begin{array}{l}\varepsilon \\
\frac{a}{2} \\
\check{\overline{0}} \\
\frac{0}{0} \\
0\end{array}$ & 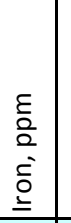 & $\begin{array}{l}\varepsilon \\
\frac{0}{2} \\
\frac{0}{0} \\
\stackrel{\Xi}{\Xi}\end{array}$ & 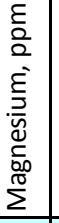 & 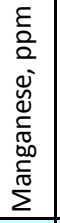 & 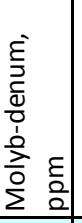 & 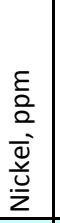 & 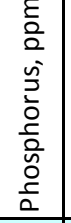 & 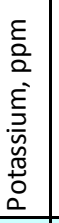 & 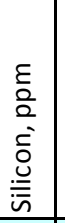 & $\begin{array}{l}\frac{\varepsilon}{2} \\
\frac{0}{2} \\
\frac{\bar{\Phi}}{\grave{i}}\end{array}$ & 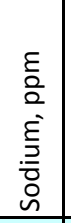 & 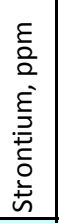 & 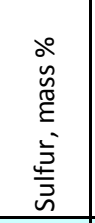 & $\begin{array}{l}\varepsilon \\
\frac{\partial}{2} \\
\dot{\xi} \\
\xi\end{array}$ & 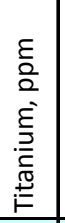 & 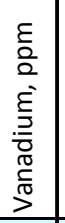 & 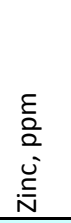 \\
\hline ORHA20 & 34383 & 41556 & 7173 & 3 & $<1$ & $<1$ & 78 & $<1$ & 2086 & $<1$ & 3 & 8 & $<1$ & 12 & $<1$ & 13 & $<1$ & 706 & 6 & 14 & $<1$ & 14 & $<1$ & 0.314 & $<1$ & $<1$ & $<1$ & 890 \\
\hline ORHA20 & 34383 & 78425 & 44042 & 1 & 1 & $<1$ & 45 & $<1$ & 2106 & $<1$ & 1 & 6 & $<1$ & 10 & $<1$ & 1 & $<1$ & 703 & 6 & 14 & $<1$ & 9 & $<1$ & 0.314 & $<1$ & $<1$ & $<1$ & 888 \\
\hline ORHA20 & 34383 & 118198 & 83815 & 1 & $<1$ & $<1$ & 34 & $<1$ & 2098 & $<1$ & 3 & 6 & $<1$ & 10 & $<1$ & 1 & $<1$ & 712 & 9 & 15 & $<1$ & 13 & $<1$ & 0.315 & $<1$ & $<1$ & $<1$ & 886 \\
\hline ORCS20 & 17287 & 29413 & 12126 & 3 & $<1$ & $<1$ & 38 & $<1$ & 2067 & $<1$ & 49 & 20 & 10 & 9 & 2 & 9 & $<1$ & 658 & $<5$ & 13 & $<1$ & 11 & $<1$ & 0.287 & $<1$ & $<1$ & $<1$ & 892 \\
\hline ORCS20 & 17287 & 55300 & 38013 & 2 & $<1$ & $<1$ & 17 & $<1$ & 2065 & $<1$ & 22 & 15 & 3 & 8 & $<1$ & 5 & $<1$ & 660 & 5 & 12 & $<1$ & 10 & $<1$ & 0.301 & $<1$ & $<1$ & $<1$ & 867 \\
\hline ORCS20 & 17287 & 93338 & 76051 & 2 & $<1$ & $<1$ & 20 & $<1$ & 2033 & $<1$ & 18 & 11 & 2 & 8 & $<1$ & 3 & $<1$ & 663 & 10 & 15 & $<1$ & 12 & $<1$ & 0.308 & $<1$ & $<1$ & $<1$ & 885 \\
\hline ORNA20 & 10307 & 17807 & 7500 & 2 & $<1$ & $<1$ & 48 & $<1$ & 2028 & $<1$ & 5 & 8 & $<1$ & 12 & $<1$ & 21 & $<1$ & 703 & 12 & 23 & $<1$ & 66 & $<1$ & 0.31 & $<1$ & $<1$ & $<1$ & 888 \\
\hline ORNA20 & 10307 & 5307 & 45000 & 2 & $<1$ & $<1$ & 21 & $<1$ & 2084 & $<1$ & 2 & 6 & $<1$ & 9 & $<1$ & $<1$ & $<1$ & 701 & 12 & 25 & $<1$ & 12 & $<1$ & 0.327 & $<1$ & $<1$ & $<1$ & 903 \\
\hline ORNA20 & 10307 & 107808 & 97501 & $<1$ & $<1$ & $<1$ & 35 & $<1$ & 2073 & $<1$ & 2 & 5 & $<1$ & 9 & $<1$ & $<1$ & $<1$ & 728 & 8 & 29 & $<1$ & 10 & $<1$ & 0.328 & $<1$ & $<1$ & $<1$ & 886 \\
\hline ORFT20 & 13082 & 20582 & 7500 & 4 & $<1$ & $<1$ & 63 & $<1$ & 1967 & $<1$ & 64 & 17 & 1 & 12 & 17 & 17 & $<1$ & 673 & 8 & 28 & $<1$ & 53 & $<1$ & 0.270 & $<1$ & $<1$ & $<1$ & 822 \\
\hline ORFT20 & 13082 & 58082 & 45000 & 1 & $<1$ & $<1$ & 46 & $<1$ & 2015 & $<1$ & 10 & 6 & $<1$ & 10 & 2 & 1 & $<1$ & 672 & 6 & 18 & $<1$ & 12 & $<1$ & 0.312 & $<1$ & $<1$ & $<1$ & 839 \\
\hline ORFT20 & 13082 & 108868 & 95786 & $<1$ & $<1$ & $<1$ & 44 & $<1$ & 2036 & $<1$ & 7 & 6 & 1 & 8 & 1 & $<1$ & $<1$ & 685 & $<5$ & 20 & $<1$ & 9 & $<1$ & 0.352 & $<1$ & $<1$ & $<1$ & 846 \\
\hline ORDC2OB & 50,809 & 56902 & 6093 & 2 & $<1$ & $<1$ & 86 & $<1$ & 2114 & $<1$ & 12 & 12 & $<1$ & 10 & $<1$ & 6 & $<1$ & 700 & 7 & 13 & $<1$ & 69 & $<1$ & 0.297 & $<1$ & $<1$ & $<1$ & 894 \\
\hline ORDC2OB & 50,809 & 92809 & 42000 & 1 & $<1$ & $<1$ & 67 & $<1$ & 2147 & $<1$ & 7 & 7 & $<1$ & 8 & $<1$ & 2 & $<1$ & 692 & 8 & 11 & $<1$ & 13 & $<1$ & 0.318 & $<1$ & $<1$ & $<1$ & 894 \\
\hline ORDC2OB & 50,809 & 110809 & 60000 & 1 & $<1$ & $<1$ & 51 & $<1$ & 2107 & $<1$ & 6 & 9 & $<1$ & 8 & $<1$ & 3 & $<1$ & 683 & 9 & 14 & $<1$ & 12 & $<1$ & 0.324 & $<1$ & $<1$ & $<1$ & 881 \\
\hline ORCC20 & 38499 & 46389 & 7890 & 2 & $<1$ & $<1$ & 55 & $<1$ & 2040 & $<1$ & 2 & 11 & $<1$ & 9 & $<1$ & 2 & $<1$ & 710 & 8 & 11 & $<1$ & 132 & $<1$ & 0.307 & $<1$ & $<1$ & $<1$ & 838 \\
\hline ORCC20 & 38499 & 83781 & 45282 & $<1$ & $<1$ & $<1$ & 53 & $<1$ & 2027 & $<1$ & 2 & 5 & $<1$ & 8 & $<1$ & $<1$ & $<1$ & 713 & 8 & 12 & $<1$ & 11 & $<1$ & 0.328 & $<1$ & $<1$ & $<1$ & 838 \\
\hline ORCC20 & 38499 & 106448 & 67949 & 1 & $<1$ & $<1$ & 26 & $<1$ & 2046 & $<1$ & 1 & 6 & $<1$ & 8 & $<1$ & 1 & $<1$ & 699 & 8 & 17 & $<1$ & 9 & $<1$ & 0.330 & $<1$ & $<1$ & $<1$ & 843 \\
\hline
\end{tabular}


Oak Ridge Used Oil Samples

August 4, 2010

\begin{tabular}{|c|c|c|c|c|c|c|c|c|c|c|c|c|c|c|c|c|c|c|c|c|c|c|c|c|c|c|c|c|}
\hline & & & & \multicolumn{9}{|c|}{ ASTM D-5185 Elemental Analysis by ICP } & \multicolumn{16}{|c|}{ ASTM D-5185 Elemental Analysis by ICP } \\
\hline $\begin{array}{c}\text { Vehicle } \\
\text { Number }\end{array}$ & $\begin{array}{l}\text { SOT } \\
\text { Odo. }\end{array}$ & $\begin{array}{c}\text { Oil } \\
\text { Sample } \\
\text { Odometer } \\
\text { (miles) }\end{array}$ & \begin{tabular}{|c|} 
Oil \\
Sample \\
Test \\
Miles \\
\end{tabular} & 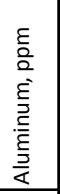 & 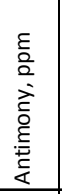 & 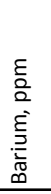 & 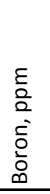 & 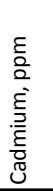 & 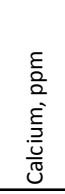 & 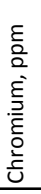 & 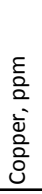 & 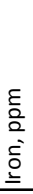 & تे & 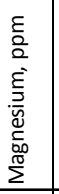 & 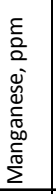 & 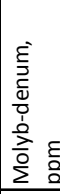 & $\begin{array}{l}\frac{E}{0} \\
\frac{0}{0} \\
\overline{\tilde{d}} \\
\frac{\underline{u}}{z}\end{array}$ & 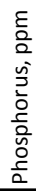 & & 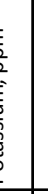 & 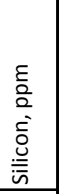 & $\begin{array}{l}\varepsilon \\
\frac{0}{2} \\
\dot{0} \\
\stackrel{\bar{\nu}}{\bar{n}} \\
\end{array}$ & 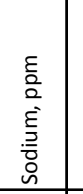 & 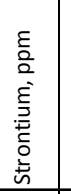 & $\begin{array}{l}\varepsilon \\
\frac{\varepsilon}{2} \\
\hat{E} \\
\hat{E}\end{array}$ & 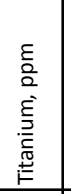 & 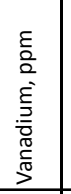 & 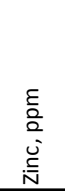 \\
\hline OR1N & 299 & 4,861 & 4,562 & 16 & $<1$ & 4 & 82 & $<1$ & 1969 & $<$ & 10 & 2 & & 11 & 3 & 64 & $<$ & 64 & & $<5$ & 241 & $<1$ & 11 & $<1$ & 1 & $<1$ & $<1$ & 793 \\
\hline OR1N & 299 & 59,889 & 59,590 & 5 & $<1$ & $<1$ & 47 & $<1$ & 2024 & $<$ & $<$ & & & 40 & $<1$ & 21 & $\leq$ & 74 & & 5 & 8 & $<1$ & 5 & $<1$ & $<1$ & $<1$ & $<1$ & 888 \\
\hline OR1N & 299 & 116,610 & 116,311 & 4 & $<1$ & $<1$ & 37 & $<1$ & 2032 & $<1$ & $<$ & & & 41 & $<1$ & 20 & $<$ & 71 & & $<5$ & 6 & $<1$ & 5 & $<1$ & $<1$ & $<1$ & $<1$ & 876 \\
\hline OR2N & 151 & 7,138 & 6,987 & 30 & $<1$ & 5 & 247 & $<1$ & 1848 & 1 & 16 & 2 & & 11 & 4 & 37 & $<$ & 68 & & $<5$ & 126 & $<1$ & 13 & $<1$ & 1 & $<1$ & $<1$ & 798 \\
\hline OR2N & 151 & 62,865 & 62,714 & 16 & $<1$ & $<1$ & 43 & $<1$ & 2027 & $<$ & 2 & 1 & & 41 & $<1$ & 22 & $<$ & 78 & & $<5$ & 12 & $<1$ & 5 & $<1$ & $<1$ & $<1$ & $<1$ & 897 \\
\hline OR2N & 151 & 114,402 & 114,251 & 13 & $<1$ & $<1$ & 28 & $<1$ & 2034 & $<$ & $<$ & & & 39 & $<1$ & 21 & $<$ & 73 & & 5 & 15 & $<1$ & 6 & $<1$ & $<1$ & $<1$ & $<1$ & 884 \\
\hline OR3N & 127 & 6,220 & 6,093 & 9 & $<1$ & $<1$ & 49 & $<1$ & 1960 & -2 & -3 & 3 & & 37 & 2 & 21 & & 7 & & 8 & 4 & $<1$ & 46 & $<1$ & 1 & $<1$ & $<1$ & 888 \\
\hline OR3N & 127 & 61,890 & 61,763 & 8 & $<1$ & $<1$ & 43 & $<1$ & 2251 & 1 & 2 & 3 & & 46 & $<1$ & 25 & & 82 & & 6 & 5 & $<1$ & 11 & $<1$ & $<1$ & $<1$ & $<1$ & 1010 \\
\hline OR3N & 127 & 112,124 & \begin{tabular}{|l|}
111,997 \\
\end{tabular} & 7 & $<1$ & $<1$ & 26 & $<1$ & 2169 & $<$ & 2 & 2 & & 44 & $<1$ & 23 & & 75 & & 5 & 5 & $<1$ & 8 & $<1$ & $<1$ & $<1$ & $<1$ & 946 \\
\hline OR4N & 268 & 7,635 & 7,367 & 34 & $<1$ & 3 & 5 & $<1$ & 1490 & 2 & 3 & 9 & & 14 & 10 & 7 & & 67 & & 9 & 23 & $<1$ & 11 & $<1$ & 1 & $<1$ & $<1$ & 784 \\
\hline OR4N & 268 & 60,025 & & 9 & $<1$ & $<1$ & 37 & $<1$ & 1903 & $<$ & 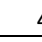 & 1 & & 40 & $<1$ & 19 & $<$ & 66 & & 5 & 11 & $<1$ & 9 & $<1$ & $<1$ & $<1$ & $<1$ & 815 \\
\hline OR4N & 268 & 113,006 & & 11 & $<1$ & $<1$ & 30 & $<1$ & 2036 & $<$ & & 1 & & 44 & $<1$ & 22 & $\leq$ & 73 & & 5 & 12 & $<1$ & 10 & $<1$ & $<1$ & $<1$ & $<1$ & 896 \\
\hline OR1E & 122 & 958 & 4,836 & 16 & $<1$ & 5 & 84 & $<1$ & 2292 & $<$ & 12 & 2 & & 13 & 3 & 63 & $<$ & 64 & & $<5$ & 237 & $<1$ & 13 & $<1$ & 1 & $<1$ & $<1$ & 776 \\
\hline OR1E & 122 & 60,084 & 59,962 & 5 & $<1$ & $<1$ & 47 & $<1$ & 2049 & $<$ & 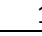 & & & 40 & $<1$ & 22 & $\leq$ & 75 & & $<5$ & 8 & $<1$ & 8 & $<1$ & $<1$ & $<1$ & $<1$ & 883 \\
\hline OR1E & 122 & 116,710 & 116,588 & 5 & $<1$ & $<1$ & 35 & $<1$ & 2067 & $<$ & $<$ & & & 42 & $<1$ & 21 & $<$ & 73 & & $<5$ & 7 & $<1$ & 7 & $<1$ & $<1$ & $<1$ & $<1$ & 883 \\
\hline OR2E & 70 & 7,247 & 7,177 & 35 & $<1$ & 5 & 239 & $<1$ & 1844 & 1 & 12 & 2 & & 11 & 4 & 37 & $<$ & 6 & & 5 & 119 & $<1$ & 14 & $<1$ & 2 & $<1$ & $<1$ & 794 \\
\hline OR2E & 70 & , 081 & 63,011 & 20 & $<1$ & $<1$ & 38 & $<1$ & 2100 & $<$ & 2 & 1 & & 42 & $<1$ & 22. & $\leq$ & 72 & & 5 & 14 & $<1$ & 8 & $<1$ & $<1$ & $<1$ & $<1$ & 915 \\
\hline OR2E & 70 & 5,190 & \begin{tabular}{|l|}
115,120 \\
\end{tabular} & 17 & $<1$ & $<1$ & 24 & $<1$ & 2026 & $<$ & - & & & 38 & $<1$ & 21 & $\leq$ & 70 & & $<5$ & 16 & $<1$ & 8 & $<1$ & $<1$ & $<1$ & $<1$ & 863 \\
\hline OR3E & 227 & 6,402 & 6,175 & 15 & $<1$ & $<1$ & 51 & $<1$ & 2007 & 2 & 10 & 4 & & 38 & 2 & 22 & & 76 & & 7 & 5 & $<1$ & 53 & $<1$ & $<1$ & $<1$ & $<1$ & 906 \\
\hline OR3E & 227 & 62,002 & 61,775 & 12 & $<1$ & $<1$ & 34 & $<1$ & 2277 & 2 & & 4 & & 45 & $<1$ & 25 & & 79 & & 5 & 6 & $<1$ & 12 & $<1$ & 1 & $<1$ & $<1$ & 991 \\
\hline OR3E & 227 & 112,216 & \begin{tabular}{|l|}
111,989 \\
\end{tabular} & 8 & $<1$ & $<1$ & 24 & $<1$ & 2216 & $<$ & 5 & 2 & & 47 & $<1$ & 24 & & $7 \varepsilon$ & & 5 & 5 & $<1$ & 11 & $<1$ & $<1$ & $<1$ & $<1$ & 956 \\
\hline OR4E & 313 & 7,735 & 7,422 & 20 & $<1$ & 4 & 4 & $<1$ & 1925 & - & 3 & 8 & & 17 & 14 & 8 & $\leq$ & 67 & & 10 & 26 & $<1$ & 15 & $<1$ & 4 & $<1$ & $<1$ & 835 \\
\hline OR4E & 313 & 60,220 & 59,907 & 12 & $<1$ & $<1$ & 33 & $<1$ & 1997 & $<$ & 2 & 1 & & 41 & $<1$ & 20 & $<$ & 70 & & 6 & 12 & $<1$ & 11 & $<1$ & $<1$ & $<1$ & $<1$ & 850 \\
\hline OR4E & 313 & 113,151 & 112,838 & 12 & $<1$ & $<1$ & 28 & $<1$ & 2062 & $<$ & & & & 45 & $<1$ & 21 & $<$ & 72 & & 6 & 12 & $<1$ & 12 & $<1$ & $<1$ & $<1$ & $<1$ & 901 \\
\hline OR1H & 641 & 4,837 & 4,196 & 15 & $<1$ & 5 & 78 & $<1$ & 2218 & $<$ & 15 & 2 & & 12 & 3 & 59 & $<$ & 62 & & 5 & 228 & $<1$ & 14 & $<1$ & 1 & $<1$ & $<1$ & 794 \\
\hline OR1H & 641 & 911 & 9,270 & 5 & $<1$ & $<1$ & 43 & $<1$ & 2072 & $<$ & $<$ & & & 40 & $<1$ & 21 & $\leq$ & & & 5 & 7 & $<1$ & 11 & $<1$ & $<1$ & $<1$ & $<1$ & 895 \\
\hline $\mathrm{OR} 1 \mathrm{H}$ & 641 & 116,486 & 115,845 & 5 & $<1$ & $<1$ & 34 & $<1$ & 2077 & $<$ & $<$ & & & 43 & $<1$ & 22 & $<$ & & & 5 & 7 & $<1$ & 9 & $<1$ & $<1$ & $<1$ & $<1$ & 895 \\
\hline $\mathrm{OR} 2 \mathrm{H}$ & 128 & 7,222 & 7,094 & 32 & $<1$ & 5 & 242 & $<1$ & 1894 & $<$ & 10 & 2 & & 11 & 3 & 37. & $\leq$ & & & $<5$ & 119 & $<1$ & 17 & $<1$ & 1 & $<1$ & $<1$ & 813 \\
\hline $\mathrm{OR} 2 \mathrm{H}$ & 128 & 63,058 & 62,930 & 9 & $<1$ & $<1$ & 41 & $<1$ & 2136 & $<$ & 1 & 1 & & 43 & $<1$ & 23 & $<$ & & & 5 & 13 & $<1$ & 9 & $<1$ & $<1$ & $<1$ & $<1$ & 936 \\
\hline $\mathrm{OR} 2 \mathrm{H}$ & 128 & 114,831 & \begin{tabular}{|l|}
114,703 \\
\end{tabular} & 6 & $<1$ & $<1$ & 27 & $<1$ & 2060 & $<$ & $<$ & & & 39 & $<1$ & 22 & $<$ & & & 5 & 13 & $<1$ & 10 & $<1$ & $<1$ & $<1$ & $<1$ & 882 \\
\hline $\mathrm{OR} 3 \mathrm{H}$ & 375 & 6,290 & 5,915 & 12 & $<1$ & $<1$ & 50 & $<1$ & 2005 & & $s$ & 3 & & 38 & 2 & 21 & & 7. & & 8 & 5 & $<1$ & 55 & $<1$ & $<1$ & $<1$ & $<1$ & 908 \\
\hline $\mathrm{OR} 3 \mathrm{H}$ & 375 & 61,944 & 61,569 & 14 & $<1$ & $<1$ & 36 & $<1$ & 2290 & 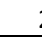 & 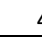 & 5 & & 46 & $<1$ & 25 & & 8 & & 5 & 6 & $<1$ & 16 & $<1$ & 1 & $<1$ & $<1$ & 1011 \\
\hline $\mathrm{OR} 3 \mathrm{H}$ & 375 & 112,371 & 111,996 & 8 & $<1$ & $<1$ & 22 & $<1$ & 2230 & $<$ & 3 & 2 & & 47 & $<1$ & 24 & & & & 5 & 5 & $<1$ & 12 & $<1$ & $<1$ & $<1$ & $<1$ & 958 \\
\hline OR4H & 231 & 7,803 & 7,572 & 26 & $<1$ & 4 & 5 & $<1$ & 1898 & & 36 & 9 & & 16 & 13 & 8 & $<$ & & & 10 & 27 & $<1$ & 15 & $<1$ & 1 & $<1$ & $<1$ & 853 \\
\hline OR4H & 231 & 60,292 & 60,061 & 9 & $<1$ & $<1$ & 31 & $<1$ & 2041 & $<$ & 3 & 1 & & 42 & $<1$ & 21 & $<$ & 7 & & 6 & 13 & $<1$ & 12 & $<1$ & $<1$ & $<1$ & $<1$ & 875 \\
\hline $\mathrm{OR} 4 \mathrm{H}$ & 231 & 113,187 & \begin{tabular}{|l|}
112,956 \\
\end{tabular} & 7 & 1 & $<1$ & 26 & $<1$ & 2115 & $<$ & 2 & 1 & & 45 & $<1$ & 22 & $<$ & & & 6 & 12 & $<1$ & 12 & $<1$ & $<1$ & $<1$ & $<1$ & 919 \\
\hline
\end{tabular}


ORNL D5185 Used Oil Analysis - Selected Metals 2006 Chevrolet Cobalt

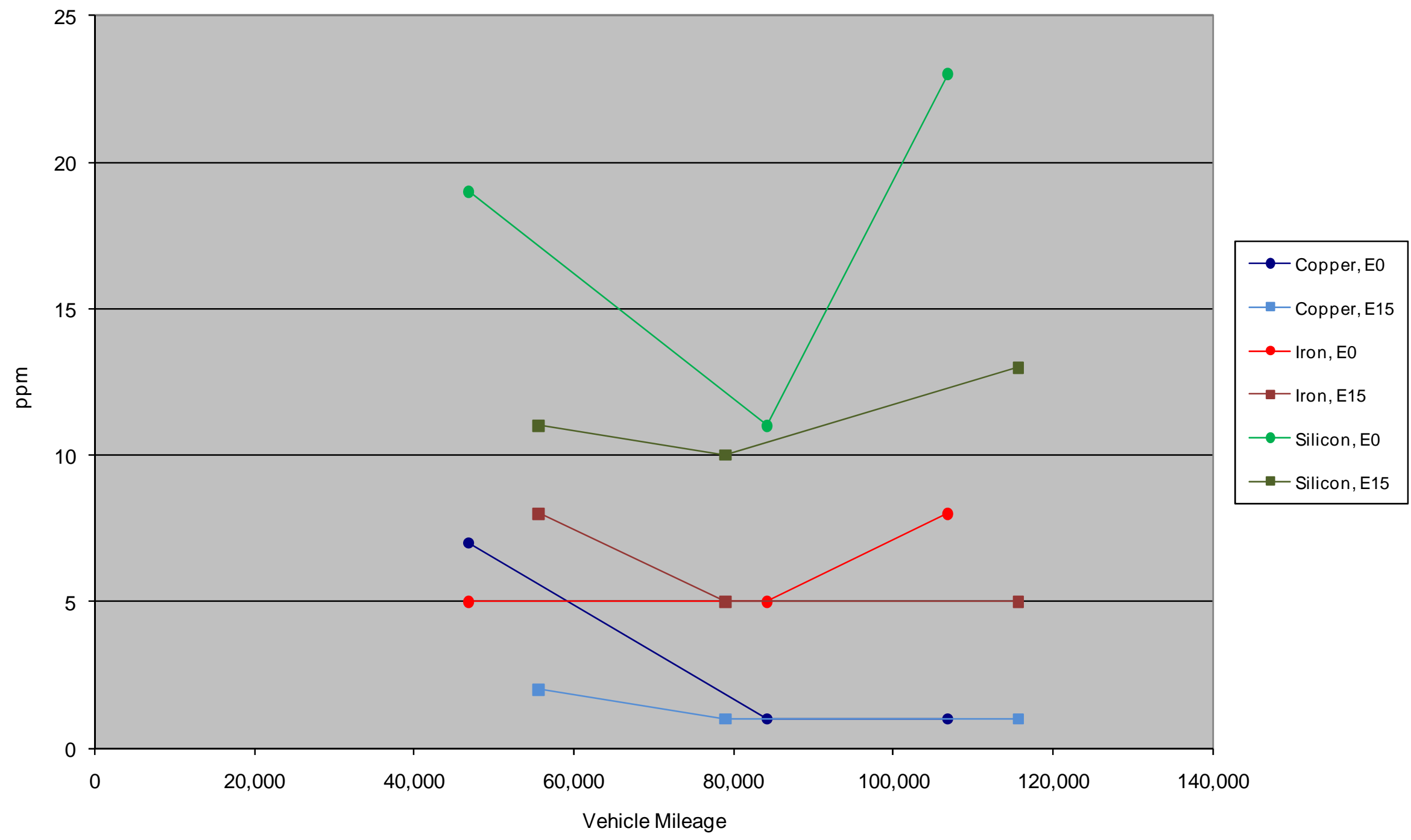




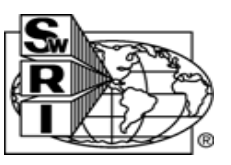

ORNL D5185 Used Oil Analysis - Selected Metals

2007 Dodge Caravan

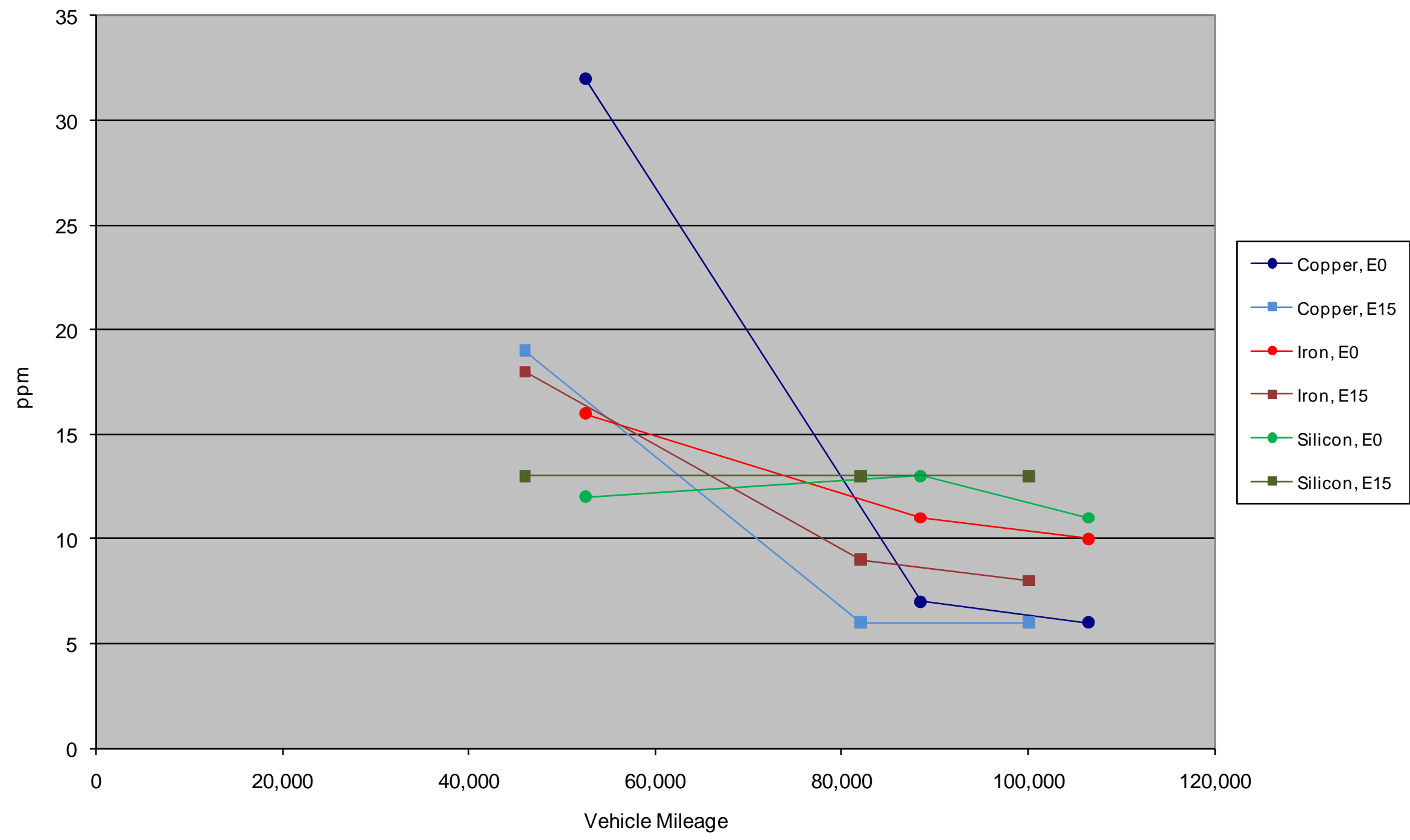


ORNL D5185 Used Oil Analysis - Selected Metals

2008 Ford Taurus

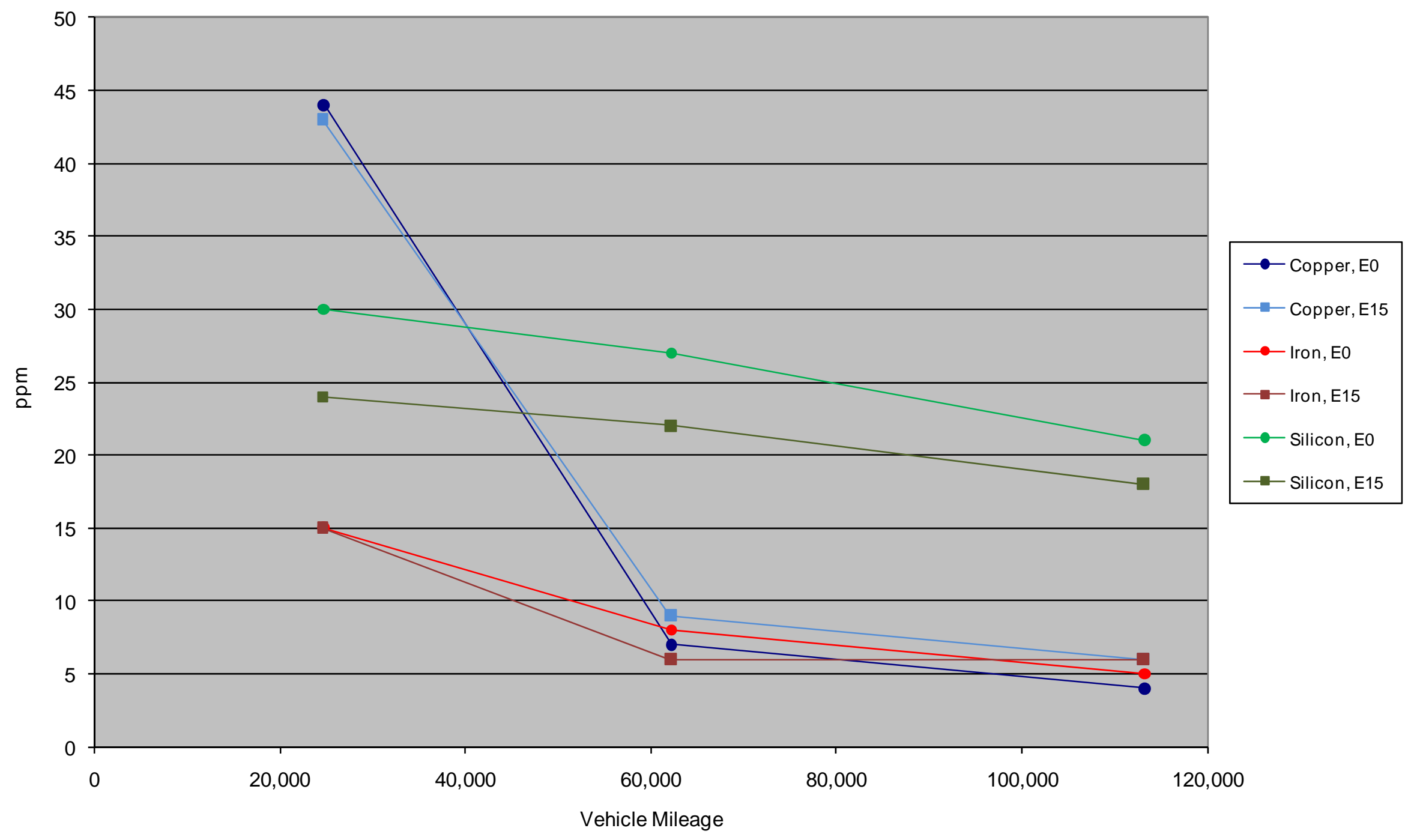




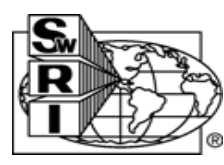

ORNL D5185 Used Oil Analysis - Selected Metals 2008 Nissan Altima

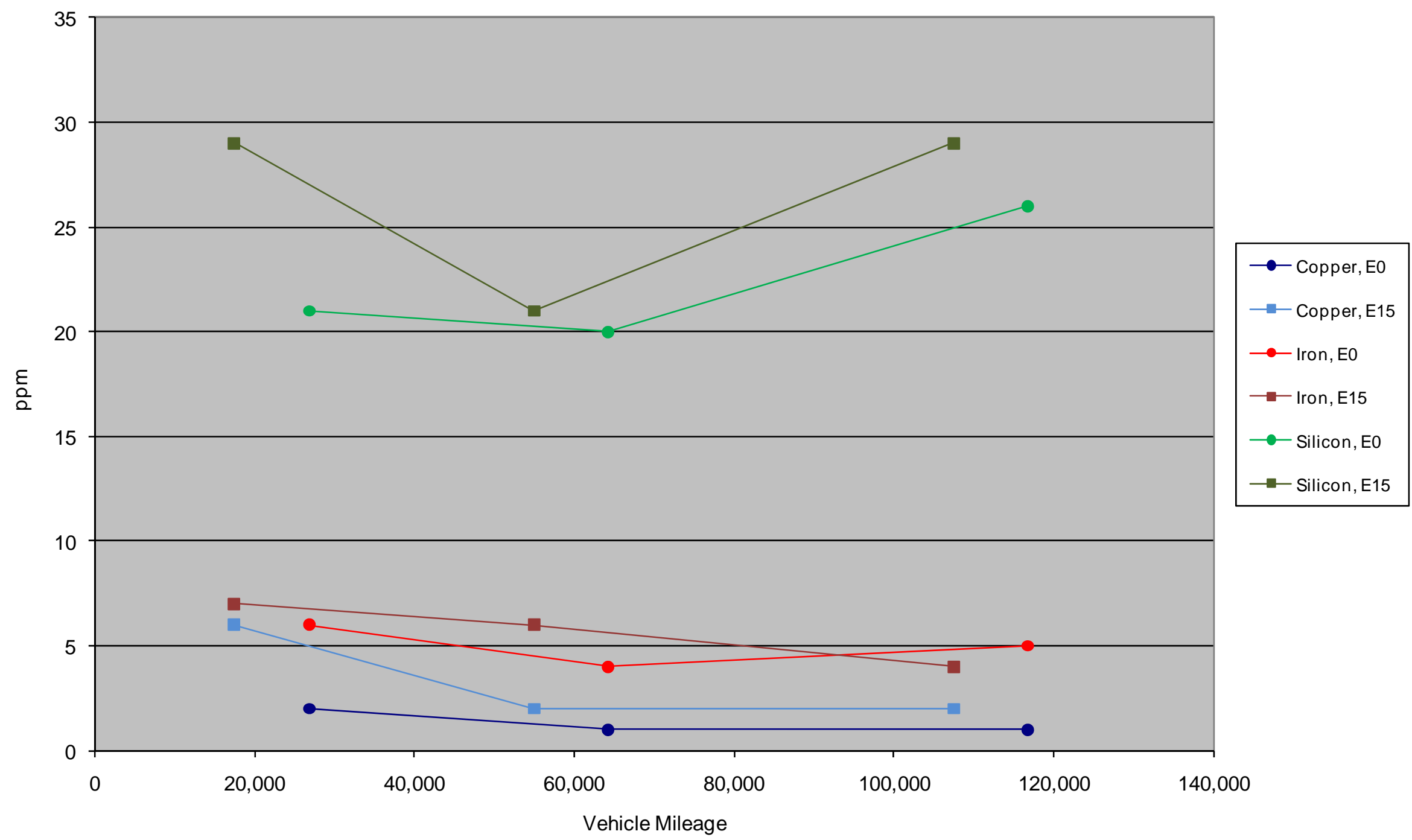


ORNL D5185 Used Oil Analysis - Selected Metals 2006 Chevrolet Silverado

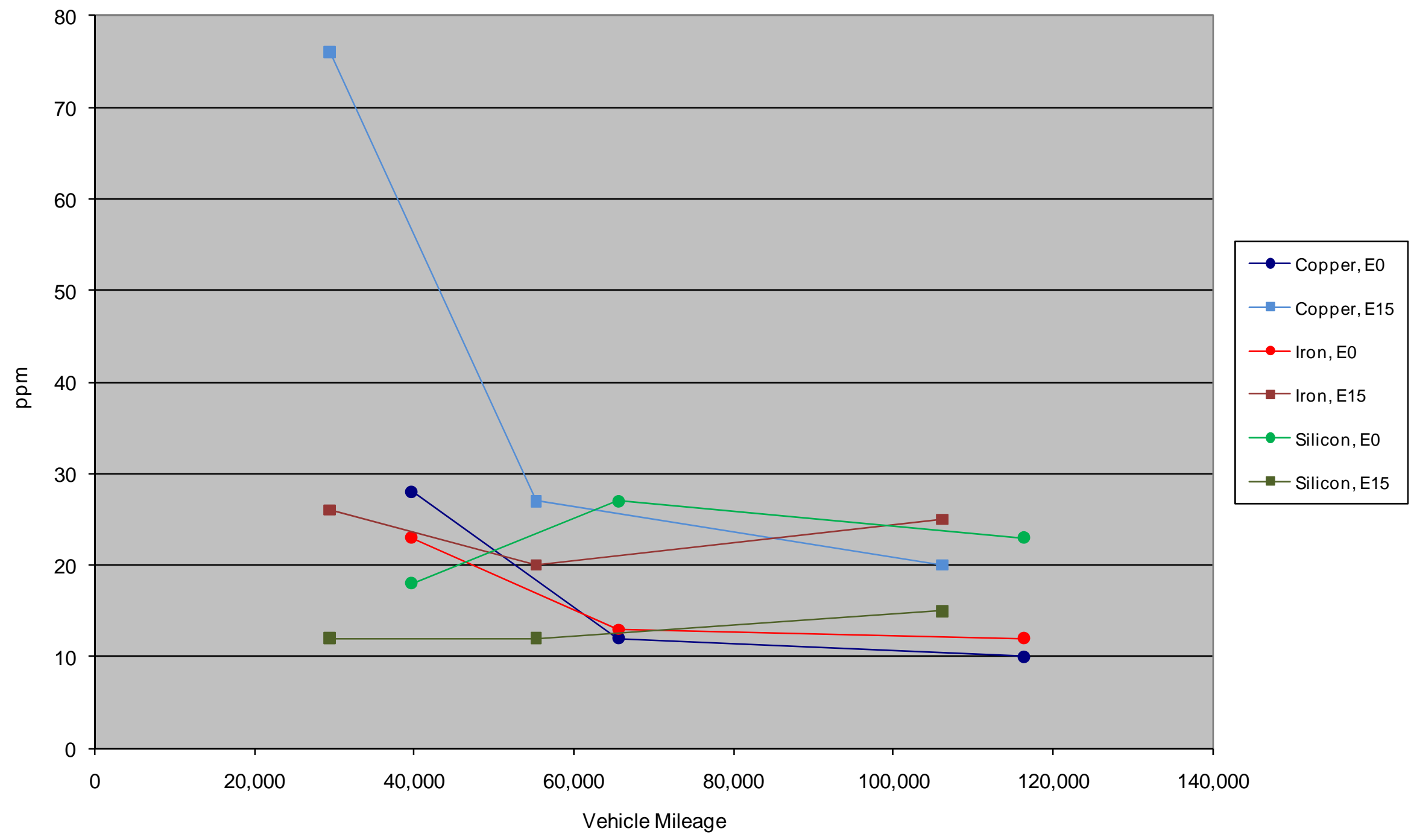


ORNL D5185 Used Oil Analysis - Selected Metals 2007 Honda Accord

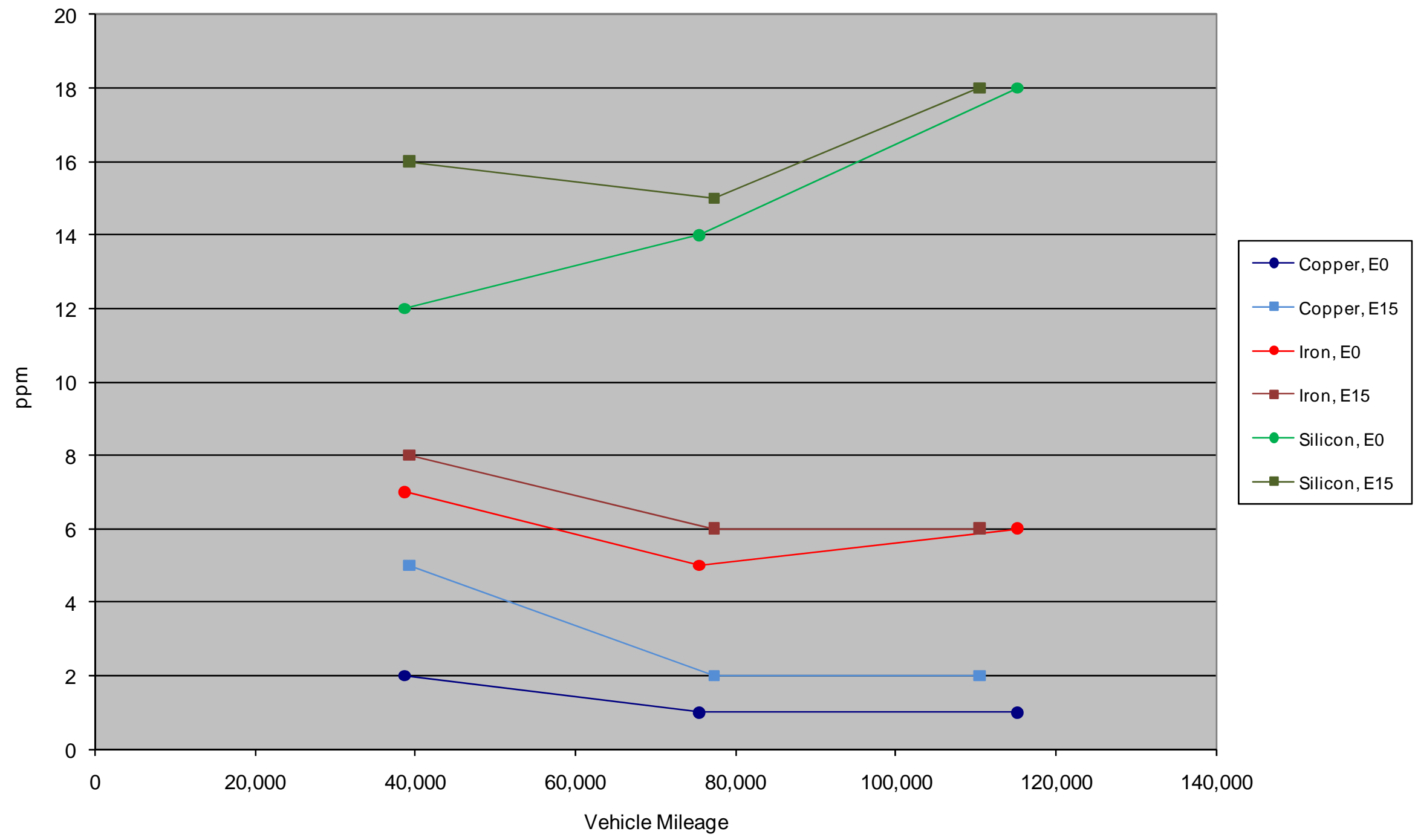





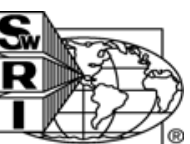

Appendix F

Fuel Pump Performance Evaluation and Inspection 



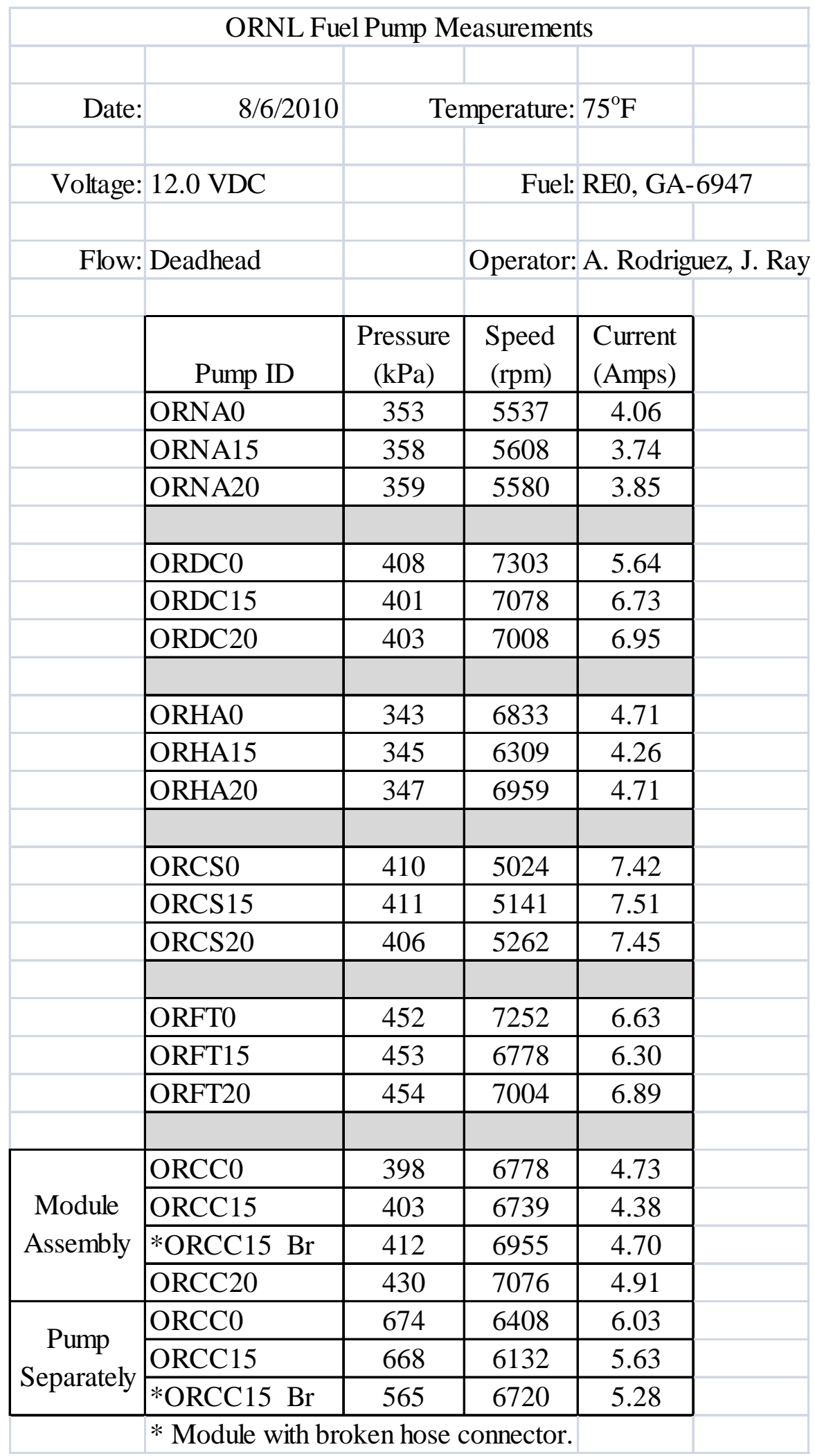




\section{ORNL Fuel Pump Measurements}

Date: $8 / 20 / 10$ to $\quad$ Temperature: $75^{\circ} \mathrm{F}$

$8 / 24 / 2010$

Voltage: 12.0 VDC

Fuel: RE0, GA-6947

Operator: A. Rodriguez, J. Ray, S. Valentine

\begin{tabular}{|c|c|c|c|c|c|}
\hline Pump ID & $\begin{array}{c}\text { Pressure } \\
(\mathrm{kPa})\end{array}$ & $\begin{array}{c}\text { Flow } \\
\text { (liters/hr) }\end{array}$ & $\begin{array}{l}\text { Speed } \\
\text { (rpm) }\end{array}$ & $\begin{array}{l}\text { Current } \\
\text { (Amps) }\end{array}$ & \% Efficiency \\
\hline \multirow{5}{*}{$\begin{array}{l}\text { ORNA0 } \\
\text { (Nissan } \\
\text { Altima) }\end{array}$} & 353 & 0.0 & 5537 & 4.06 & - \\
\hline & 350 & 18.9 & 5416 & 4.11 & 3.73 \\
\hline & 345 & 42.2 & 5617 & 4.05 & 8.32 \\
\hline & 330 & 79.2 & 5639 & 3.99 & 15.16 \\
\hline & 310 & 87.6 & 5710 & 3.84 & 16.37 \\
\hline \multirow{5}{*}{$\begin{array}{c}\text { ORNA15 } \\
\text { (Nissan } \\
\text { Altima) }\end{array}$} & 358 & 0.0 & 5608 & 3.74 & - \\
\hline & 350 & 53.9 & 5680 & 3.71 & 11.77 \\
\hline & 345 & 71.9 & 5784 & 3.73 & 15.39 \\
\hline & 330 & 107.3 & 5798 & 3.67 & 22.33 \\
\hline & 310 & 114.3 & 5843 & 3.56 & 23.04 \\
\hline & & & & & \\
\hline \multirow{5}{*}{$\begin{array}{c}\text { ORNA20 } \\
\text { (Nissan } \\
\text { Altima) }\end{array}$} & 359 & 0.0 & 5580 & 3.85 & - \\
\hline & 350 & 52.5 & 5600 & 3.83 & 11.11 \\
\hline & 345 & 75.5 & 5618 & 3.78 & 15.95 \\
\hline & 330 & 106.4 & 5653 & 3.71 & 21.91 \\
\hline & 310 & 111.3 & 5682 & 3.60 & 22.19 \\
\hline
\end{tabular}

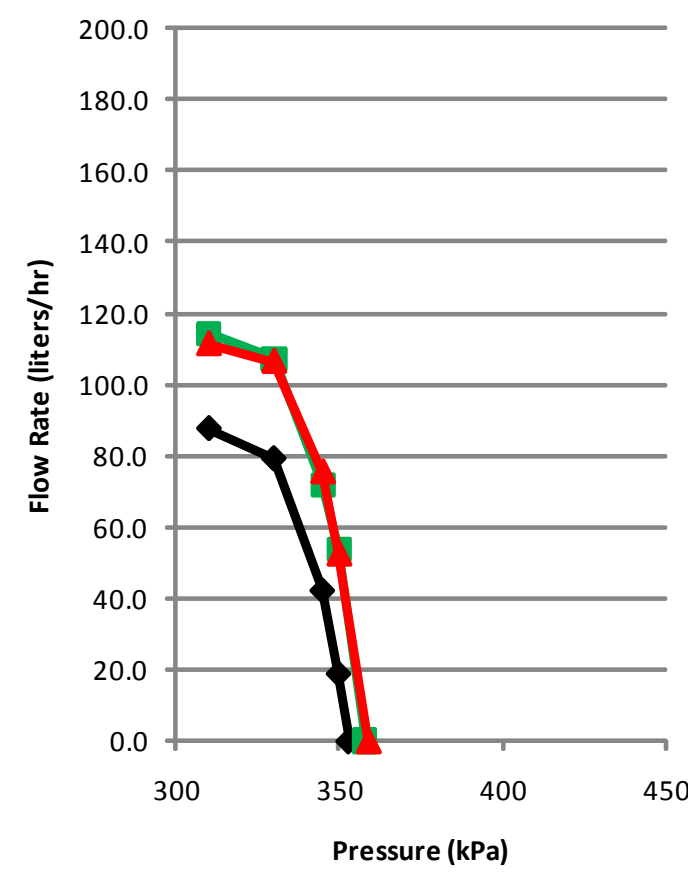

-ORNAO (Nissan Altima)

- ORNA15 (Nissan Altima)

- ORNA20 (Nissan Altima)

Pressure $(\mathrm{kPa})$ 


\begin{tabular}{|c|c|c|c|c|c|}
\hline \multirow{6}{*}{$\begin{array}{l}\text { ORDC0 } \\
\text { (Dodge } \\
\text { Caravan) }\end{array}$} & 408 & 0.0 & 7303 & 5.64 & - \\
\hline & 400 & 71.6 & 7380 & 6.51 & 10.18 \\
\hline & 380 & 101.1 & 7393 & 6.23 & 14.27 \\
\hline & 345 & 109.6 & 7410 & 5.95 & 14.71 \\
\hline & 330 & 115.6 & 7468 & 5.84 & 15.12 \\
\hline & 310 & 120.9 & 7536 & 5.72 & 15.17 \\
\hline \multirow{6}{*}{$\begin{array}{l}\text { ORDC15 } \\
\text { (Dodge } \\
\text { Caravan) }\end{array}$} & 401 & 0.0 & 7078 & 6.73 & - \\
\hline & 400 & 19.7 & 7087 & 6.67 & 2.73 \\
\hline & 380 & 85.3 & 7102 & 6.52 & 11.51 \\
\hline & 345 & 98.2 & 7258 & 6.25 & 12.55 \\
\hline & 330 & 102.5 & 7350 & 6.18 & 12.67 \\
\hline & 310 & 108.3 & 7414 & 6.06 & 12.82 \\
\hline \multirow{6}{*}{$\begin{array}{l}\text { ORDC20 } \\
\text { (Dodge } \\
\text { Caravan) }\end{array}$} & 403 & 0.0 & 7008 & 6.95 & - \\
\hline & 400 & 25.6 & 7013 & 6.86 & 3.46 \\
\hline & 380 & 92.6 & 7038 & 6.58 & 12.38 \\
\hline & 345 & 115.0 & 7445 & 6.34 & 14.49 \\
\hline & 330 & 119.0 & 7503 & 6.23 & 14.59 \\
\hline & 310 & 125.0 & 7608 & 6.13 & 14.63 \\
\hline & & & & & \\
\hline \multirow{4}{*}{$\begin{array}{l}\text { ORHA0 } \\
\text { (Honda } \\
\text { Accord) }\end{array}$} & 343 & 0.0 & 6833 & 4.71 & - \\
\hline & 345 & 0.0 & 6833 & 4.71 & 0.00 \\
\hline & 330 & 62.8 & 6890 & 4.72 & 10.16 \\
\hline & 310 & 87.1 & 6944 & 4.59 & 13.62 \\
\hline \multirow{4}{*}{$\begin{array}{c}\text { ORHA15 } \\
\text { (Honda } \\
\text { Accord) }\end{array}$} & 345 & 0.0 & 6309 & 4.17 & - \\
\hline & 345 & 0.0 & 6309 & 4.17 & 0.00 \\
\hline & 330 & 49.8 & 6171 & 4.02 & 9.46 \\
\hline & 310 & 65.7 & 6190 & 3.89 & 12.12 \\
\hline \multirow{4}{*}{$\begin{array}{c}\text { ORHA20 } \\
\text { (Honda } \\
\text { Accord) }\end{array}$} & 347 & 0.0 & 6959 & 4.71 & - \\
\hline & 345 & 14.9 & 6980 & 4.68 & 2.54 \\
\hline & 330 & 74.0 & 7038 & 4.56 & 12.40 \\
\hline & 310 & 95.0 & 7112 & 4.40 & 15.49 \\
\hline
\end{tabular}

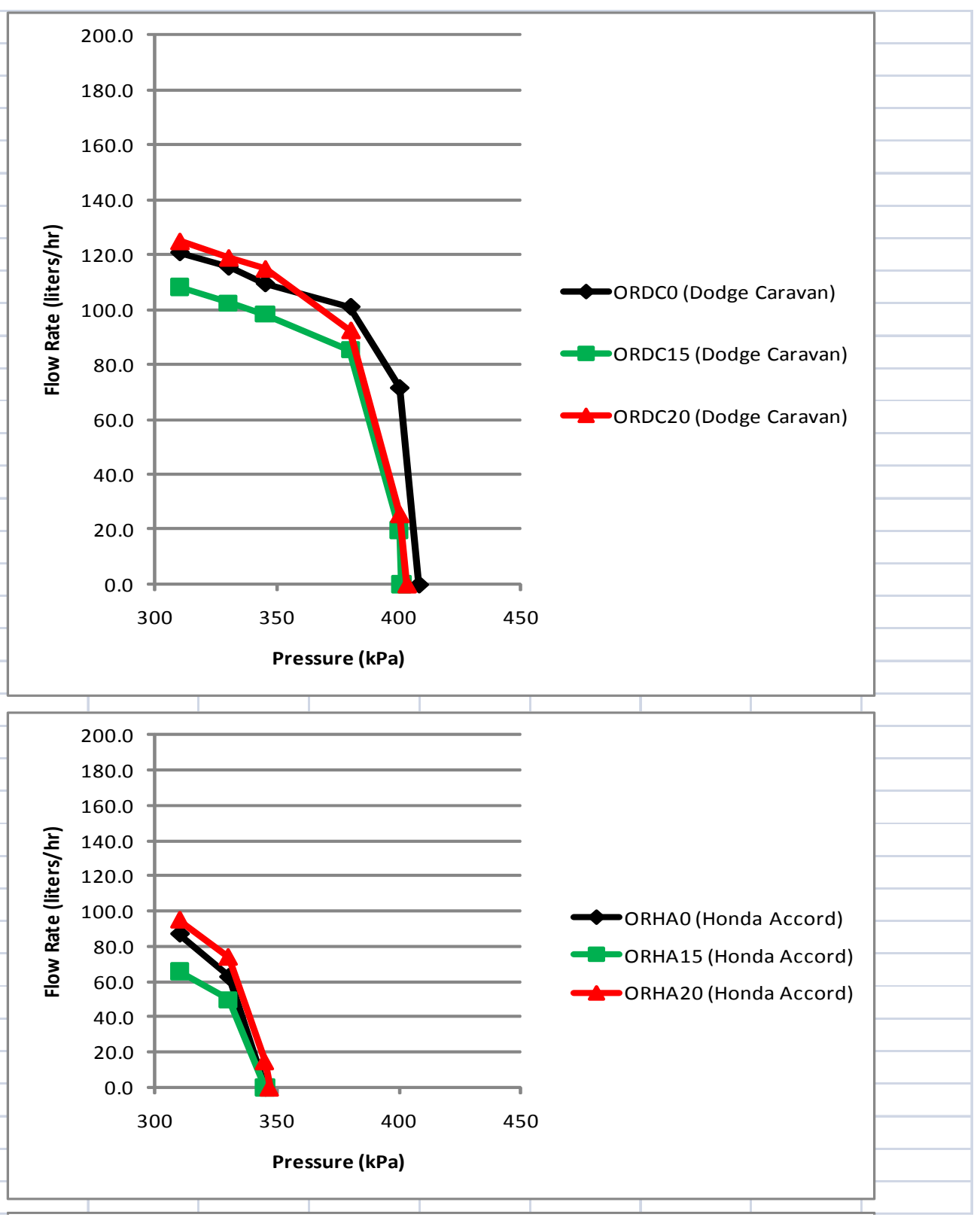




\begin{tabular}{|c|c|c|c|c|c|}
\hline \multirow{4}{*}{$\begin{array}{c}\text { ORCS0 } \\
\text { (Chevy }\end{array}$} & 410 & 0.0 & 5024 & 7.42 & - \\
\cline { 2 - 6 } & 405 & 73.4 & 5039 & 7.41 & 9.29 \\
\cline { 2 - 6 } & 380 & 153.9 & 4988 & 7.27 & 18.62 \\
\cline { 2 - 6 } & 345 & 162.5 & 5068 & 7.12 & 18.23 \\
\cline { 2 - 6 } & 330 & 166.0 & 5070 & 6.88 & 18.43 \\
\cline { 2 - 6 } & 310 & 166.4 & 5122 & 6.83 & 17.48 \\
\hline & & & & & \\
\hline \multirow{4}{*}{$\begin{array}{c}\text { ORCS15 } \\
\text { (Chevy }\end{array}$} & 411 & 0.0 & 5141 & 7.51 & - \\
\cline { 2 - 6 } Silverado) & 305 & 71.9 & 5165 & 7.52 & 8.96 \\
\cline { 2 - 6 } & 380 & 154.1 & 5402 & 7.71 & 17.58 \\
\cline { 2 - 6 } & 330 & 174.9 & 5470 & 7.49 & 18.65 \\
\cline { 2 - 6 } & 310 & 174.4 & 5513 & 7.40 & 18.00 \\
\hline \multirow{4}{*}{$\begin{array}{c}\text { ORCS20 } \\
\text { (Chevy }\end{array}$} & 406 & 0.0 & 5563 & 7.22 & 17.59 \\
\cline { 2 - 6 } & 405 & 4.0 & 5250 & 7.45 & - \\
\cline { 2 - 6 } & 380 & 160.0 & 5270 & 7.35 & 19.15 \\
\cline { 2 - 6 } & 345 & 177.0 & 5340 & 7.07 & 19.99 \\
\cline { 2 - 6 } & 330 & 179.7 & 5368 & 7.00 & 19.61 \\
\cline { 2 - 6 } & 310 & 184.3 & 5424 & 6.88 & 19.22 \\
\hline
\end{tabular}

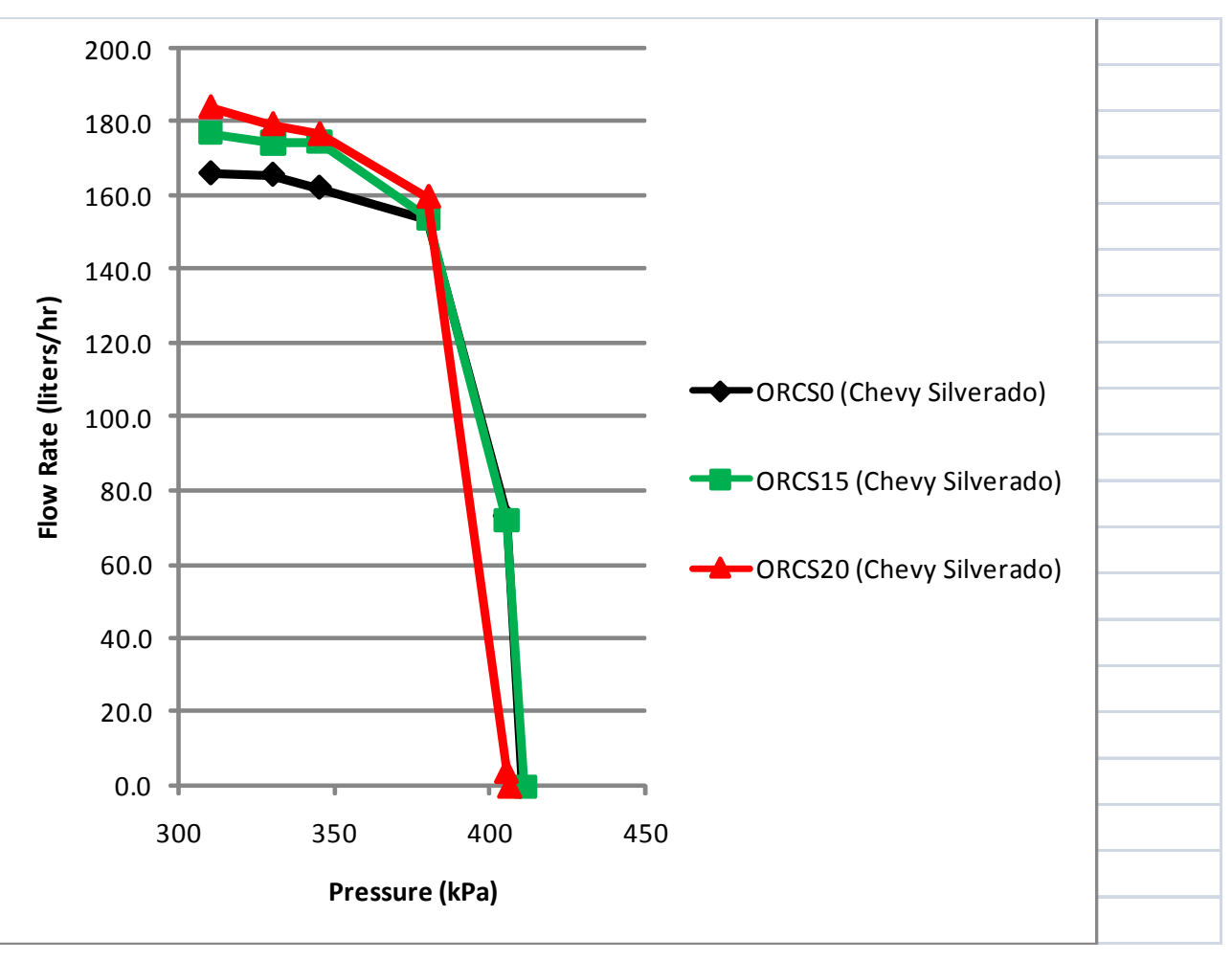




\begin{tabular}{|c|c|c|c|c|c|}
\hline \multirow{8}{*}{$\begin{array}{l}\text { ORFT0 (Ford } \\
\text { Taurus) }\end{array}$} & 452 & 0.0 & 7252 & 6.63 & - \\
\hline & 448 & 27.0 & 7220 & 6.62 & 4.23 \\
\hline & 440 & 60.9 & 7268 & 6.61 & \begin{tabular}{|c|}
9.38 \\
\end{tabular} \\
\hline & 405 & 126.7 & 7366 & 6.34 & 18.74 \\
\hline & 380 & 133.1 & 7464 & 6.16 & 19.01 \\
\hline & 345 & 144.3 & 7610 & 5.88 & 19.60 \\
\hline & 330 & 145.8 & 7674 & 5.76 & 19.34 \\
\hline & 310 & 150.5 & 7723 & 5.58 & 19.35 \\
\hline \multirow{8}{*}{$\begin{array}{c}\text { ORFT15 } \\
\text { (Ford Taurus) }\end{array}$} & 453 & 0.0 & 6778 & 6.30 & - \\
\hline & 448 & 32.2 & 6769 & 6.40 & 5.22 \\
\hline & 440 & 68.9 & 6876 & 6.27 & 11.19 \\
\hline & 405 & 129.2 & 7003 & 6.07 & 19.95 \\
\hline & 380 & 133.3 & 7087 & 5.89 & 19.91 \\
\hline & 345 & 136.2 & 7185 & 5.64 & 19.29 \\
\hline & 330 & 145.4 & 7248 & 5.54 & 20.05 \\
\hline & 310 & 147.9 & 7331 & 5.40 & 19.65 \\
\hline \multirow{8}{*}{$\begin{array}{c}\text { ORFT20 } \\
\text { (Ford Taurus) }\end{array}$} & 454 & 0.0 & 7004 & 6.89 & - \\
\hline & 448 & 44.1 & 7068 & 6.85 & 6.68 \\
\hline & 440 & 75.2 & 7025 & 6.80 & 11.26 \\
\hline & 405 & 120.4 & 7136 & 6.52 & 17.31 \\
\hline & 380 & 127.2 & 7233 & 6.30 & 17.76 \\
\hline & 345 & 135.8 & 7366 & 6.00 & 18.08 \\
\hline & 330 & 140.0 & 7440 & 5.85 & 18.28 \\
\hline & 310 & 144.3 & 7505 & 5.69 & 18.20 \\
\hline
\end{tabular}

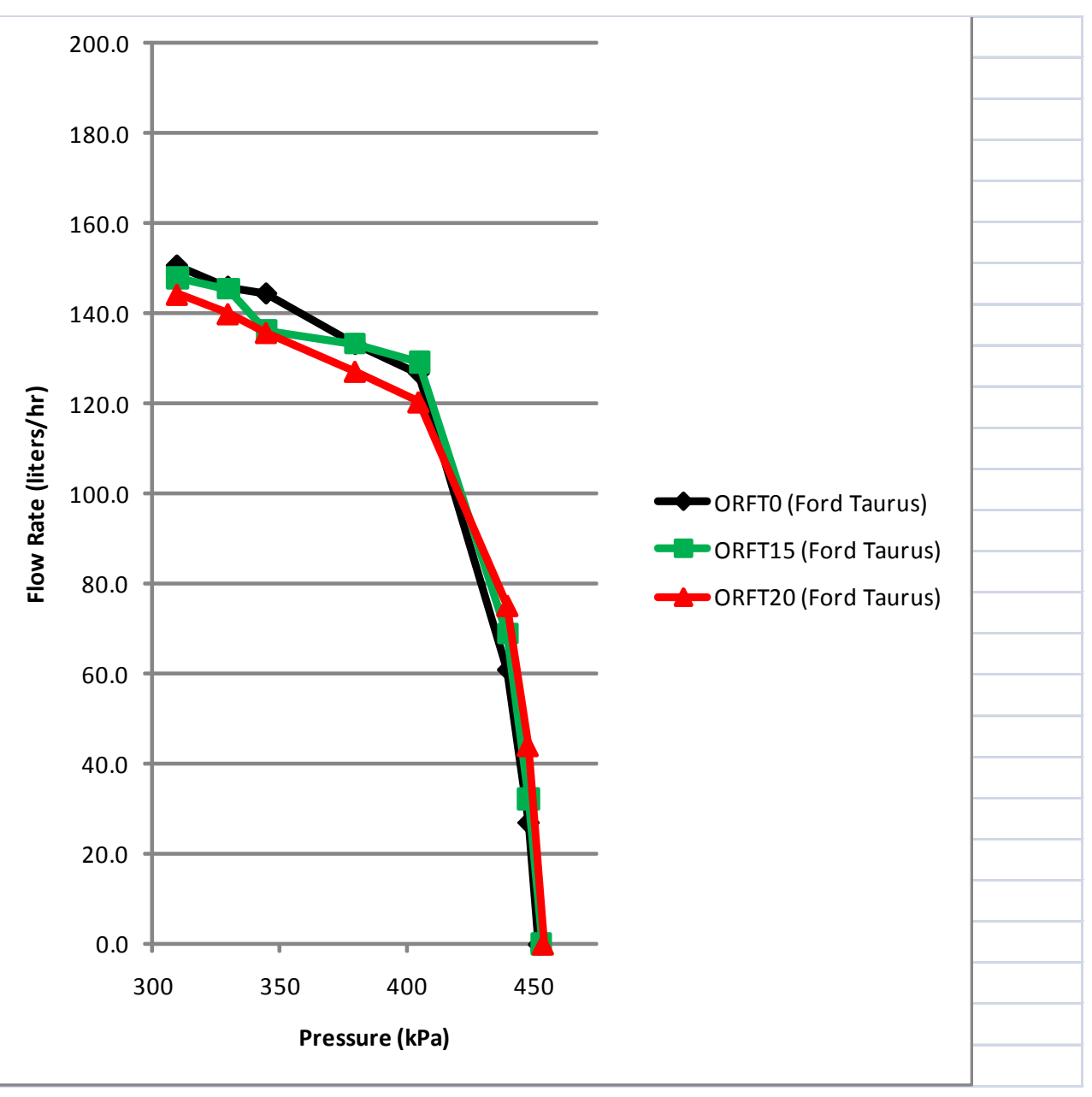




\begin{tabular}{|c|c|c|c|c|c|}
\hline \multirow{5}{*}{$\begin{array}{l}\text { ORCC0 } \\
\text { (Chevy } \\
\text { Cobalt) }\end{array}$} & 398 & 0.0 & 6778 & 4.73 & - \\
\hline & 380 & 67.8 & 6857 & 4.70 & 12.69 \\
\hline & 345 & 118.3 & 6886 & 4.45 & 21.23 \\
\hline & 330 & 122.9 & 6930 & 4.37 & 21.48 \\
\hline & 310 & 129.4 & 6974 & 4.28 & 21.70 \\
\hline \multirow{5}{*}{$\begin{array}{c}\text { ORCC15 } \\
\text { (Chevy } \\
\text { Cobalt) }\end{array}$} & 403 & 0.0 & 6739 & 4.38 & - \\
\hline & 380 & 79.0 & 6803 & 4.27 & 16.27 \\
\hline & 345 & 124.9 & 6876 & 4.11 & 24.27 \\
\hline & 330 & 127.7 & 6905 & 4.03 & 24.21 \\
\hline & 310 & 131.4 & 6954 & 3.93 & 23.99 \\
\hline \multirow{6}{*}{$\begin{array}{c}\text { *ORCC15 Br } \\
\text { (Chevy } \\
\text { Cobalt) }\end{array}$} & & & & & \\
\hline & 412 & 0.0 & 6955 & 4.70 & - \\
\hline & 380 & 93.3 & 7082 & 4.53 & 18.12 \\
\hline & 345 & 112.2 & 7155 & 4.36 & 20.55 \\
\hline & 330 & 121.7 & 7150 & 4.27 & 21.77 \\
\hline & 310 & 127.3 & 7194 & 4.19 & 21.80 \\
\hline \multirow{5}{*}{$\begin{array}{c}\text { ORCC20 } \\
\text { (Chevy } \\
\text { Cobalt) }\end{array}$} & 430 & 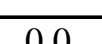 & 7076 & 491 & - \\
\hline & 380 & 123.0 & 7200 & 4.65 & 23.27 \\
\hline & 345 & 130.0 & 7259 & 4.47 & 23.23 \\
\hline & 330 & 134.0 & 7285 & 4.38 & 23.37 \\
\hline & 310 & 138.0 & 7322 & 4.27 & 23.19 \\
\hline
\end{tabular}

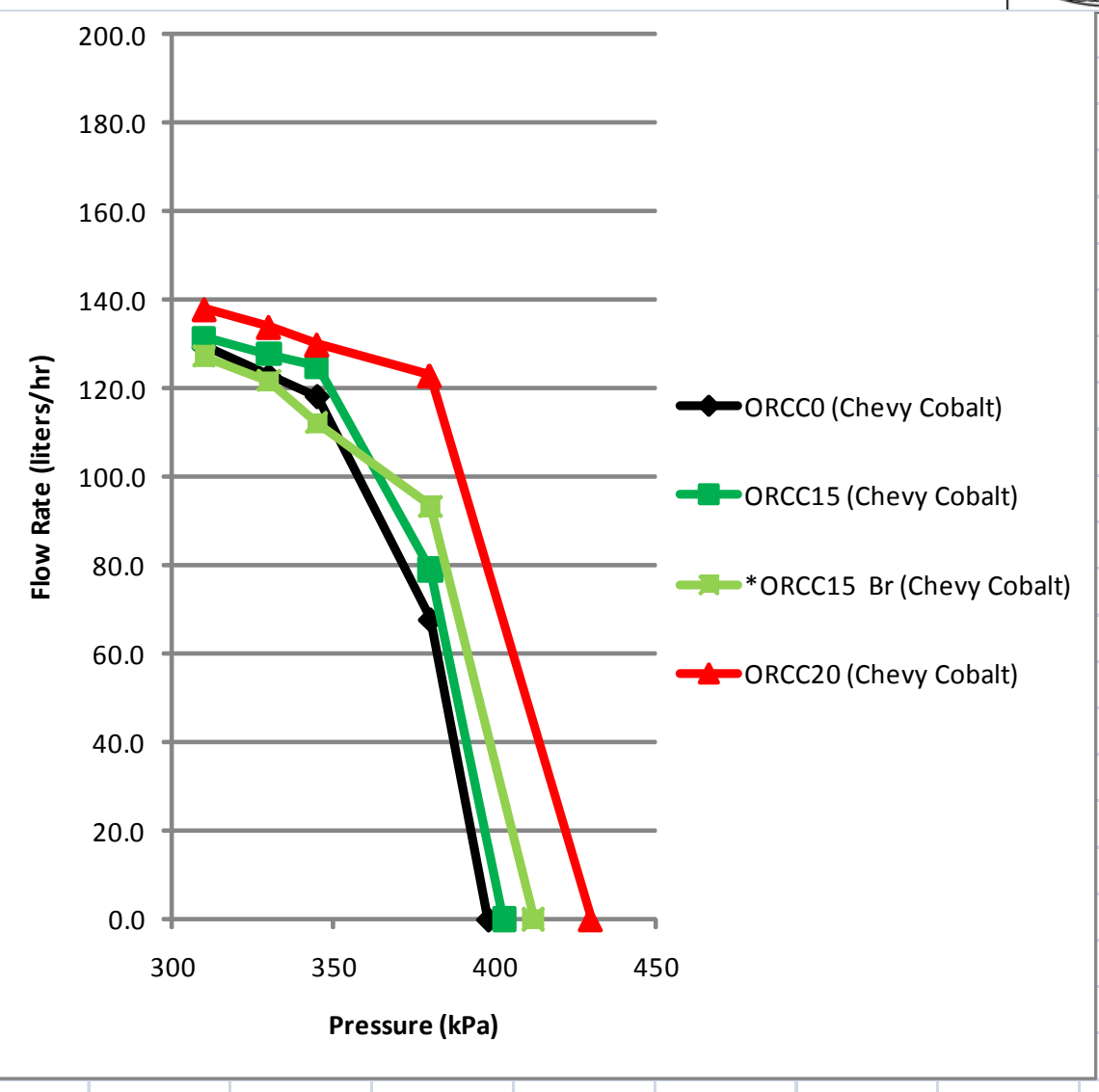

* Module with broken hose connector. 


\section{Fuel Pump Teardown Comments}

From the flow performance measurements, no major flow problems were uncovered.

The results of the teardown evaluation revealed no significant defects or wear.

Some parts had wear indications that would likely continue and eventually

lead to poor pump performance given continued service of the pump. The

parts noted having relatively greater or less wear (shown in their respective photo

in the following pages) are listed below.

Pumps ORCS0, ORCS15, and ORCC15 Br were not included in the teardown evaluation.

These three pumps were misplaced at SwRI and not available for teardown.

\begin{tabular}{|c|l|l|}
\hline Pump & \multicolumn{1}{|c|}{ Component } & \multicolumn{1}{c|}{ Comment } \\
\hline ORNA0 & Commutator & Heavy wear on trailing edge of commutator segments. \\
\hline & Brushes & Wear on commutator NOT seen on mating brushes. \\
\hline & & \\
\hline ORNA15 & Impeller Cover, Outlet Side & Surface polishing at outer radius between high and low pressure area. \\
\hline & Commutator & Heavy wear NOT seen on trailing edge of commutator segments. \\
\hline & & \\
\hline ORNA20 & Commutator & Heavy wear on outer radius and trailing edge of commutator segments. \\
\hline & Brushes & Normal wear on brushes. \\
\hline & & \\
\hline ORDC0 & Impeller Cover, Inlet and Outlet & Surface polishing at outer radius between high and low pressure area. \\
\hline & Sock Filter & Significant discoloration of sock filter. \\
\hline & & \\
\hline ORDC15 & Impeller Cover, Inlet and Outlet & Less surface polishing than seen on ORDC0. \\
\hline & Commutator & Deeper radial wear grooves than ORDC0 or ORDC20. \\
\hline & & \\
\hline ORDC20 & Commutator & Normal to light wear on commutator. \\
\hline & Brushes & Normal to light wear on brushes. \\
\hline
\end{tabular}




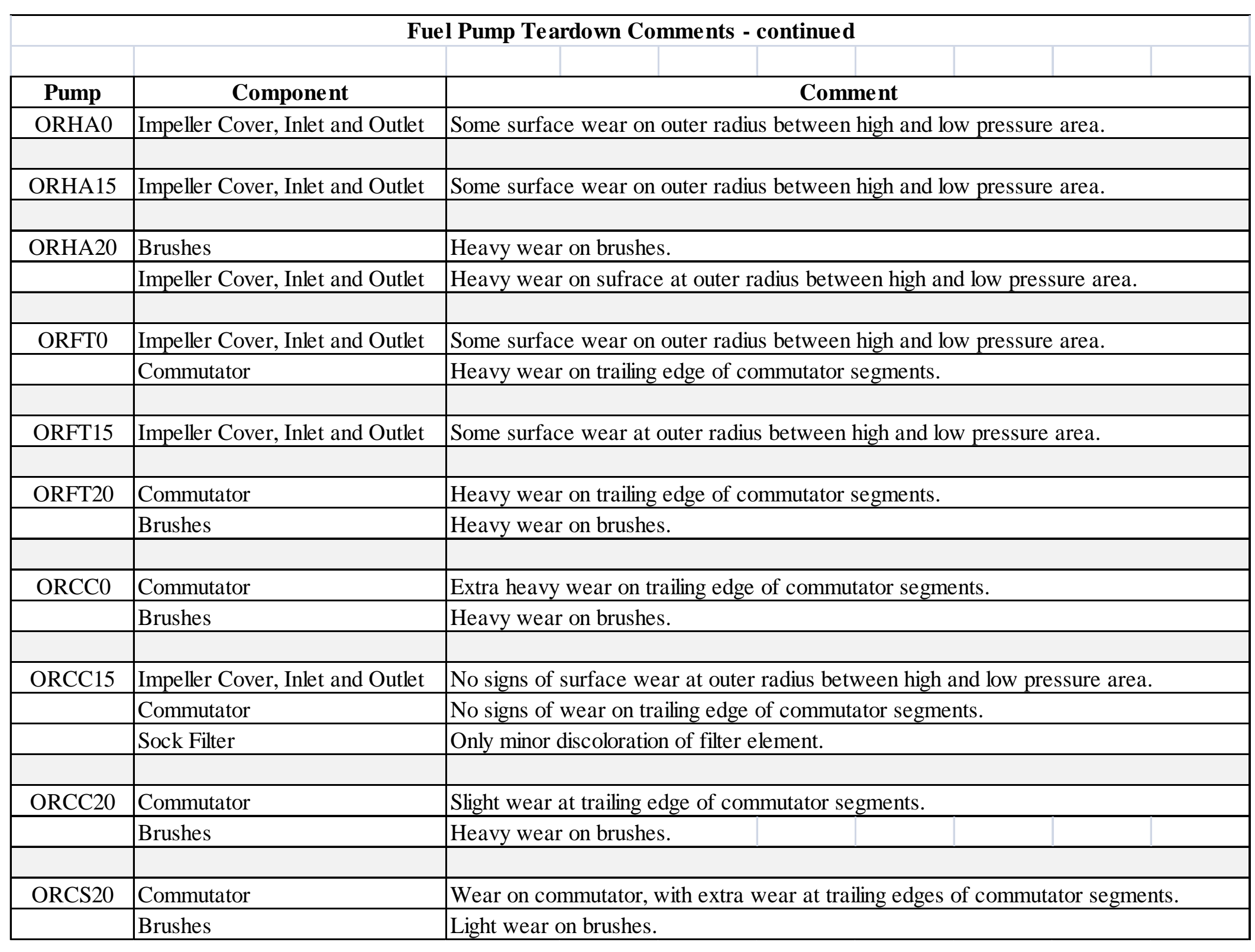




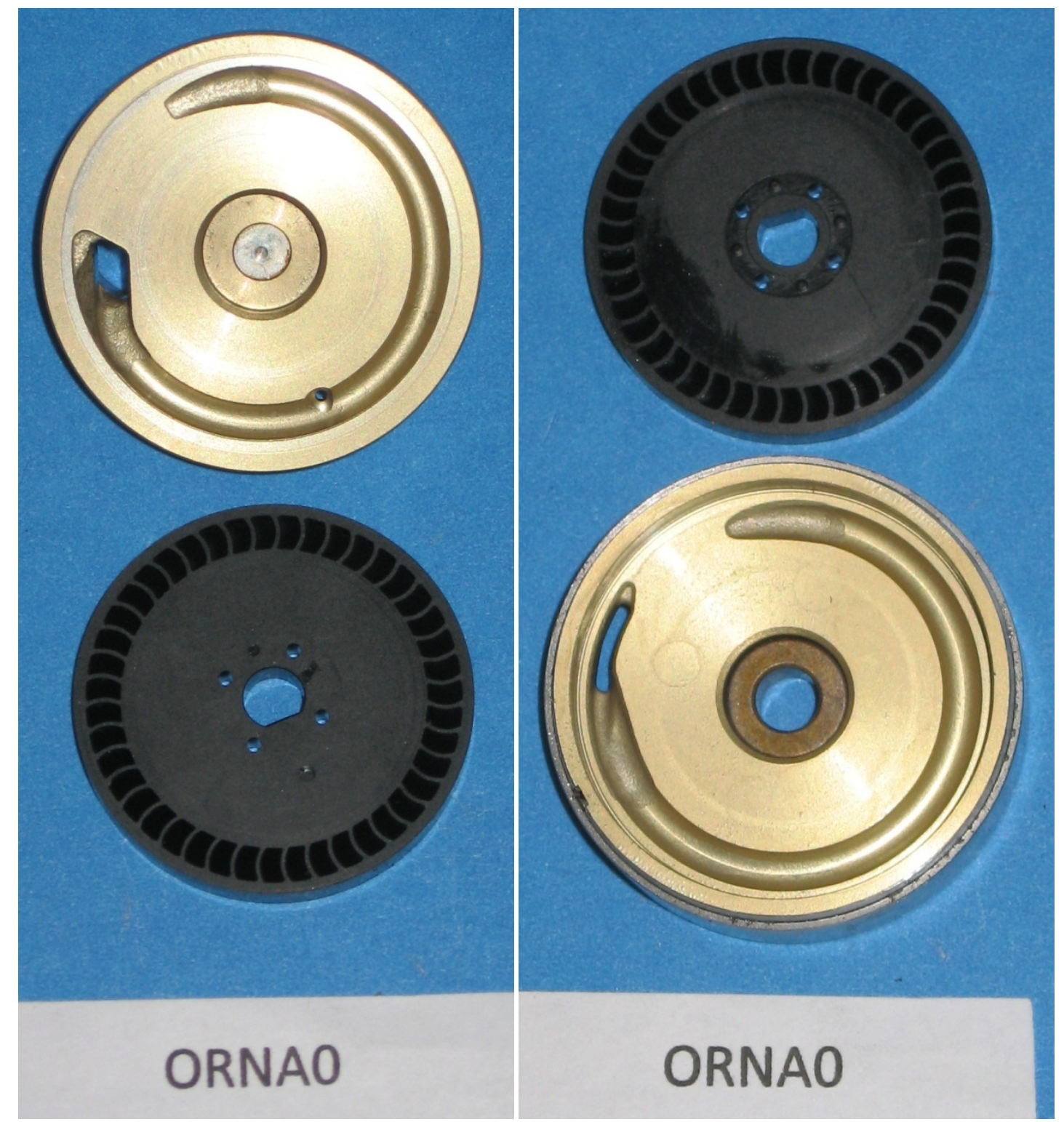

Figure F1. ORNA0 Fuel Pump Impeller with Inlet (Left) and Outlet (Right) Mating Cover. 


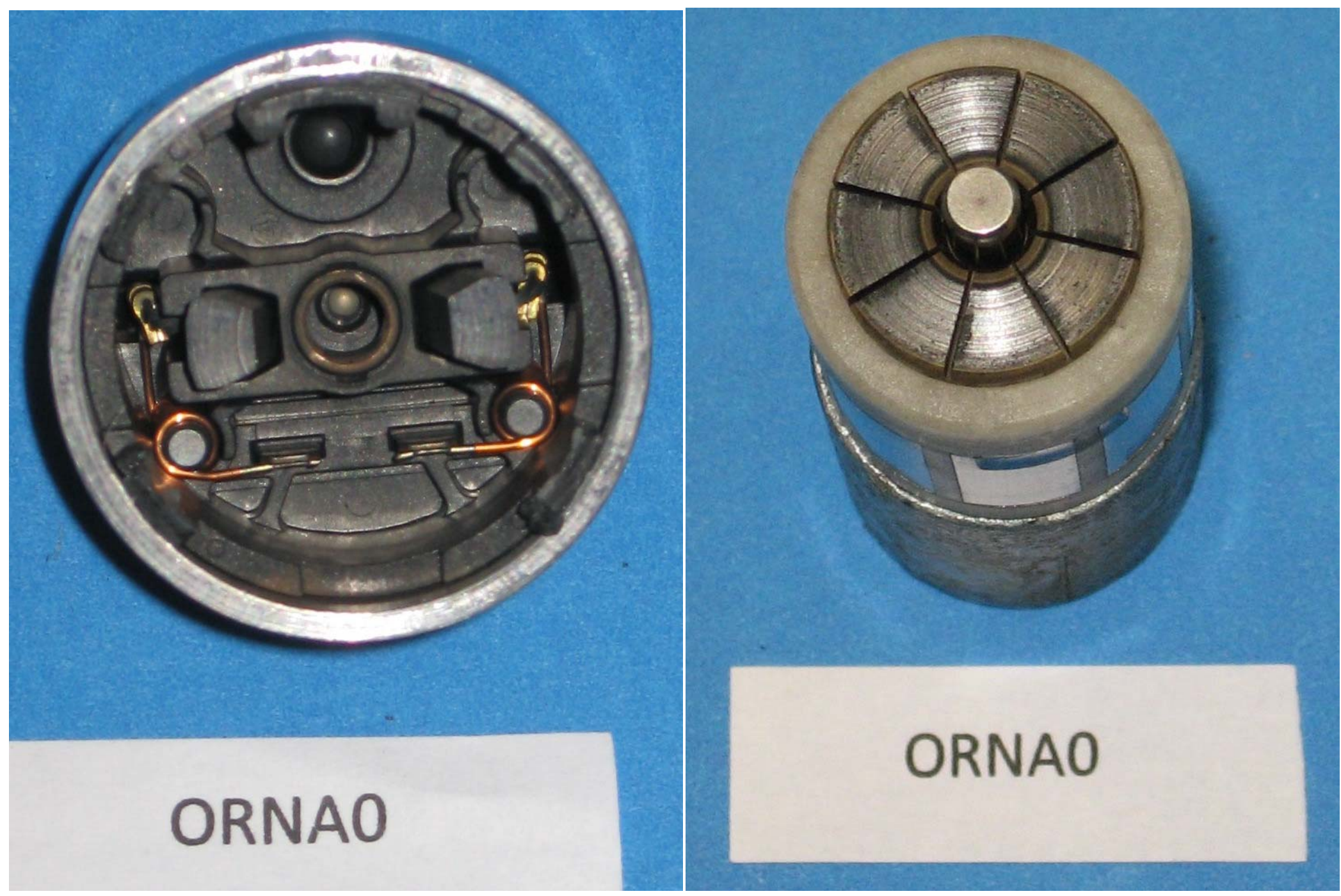

Figure F2. ORNA0 Fuel Pump Brushes (Left) and Commutator (Right). 


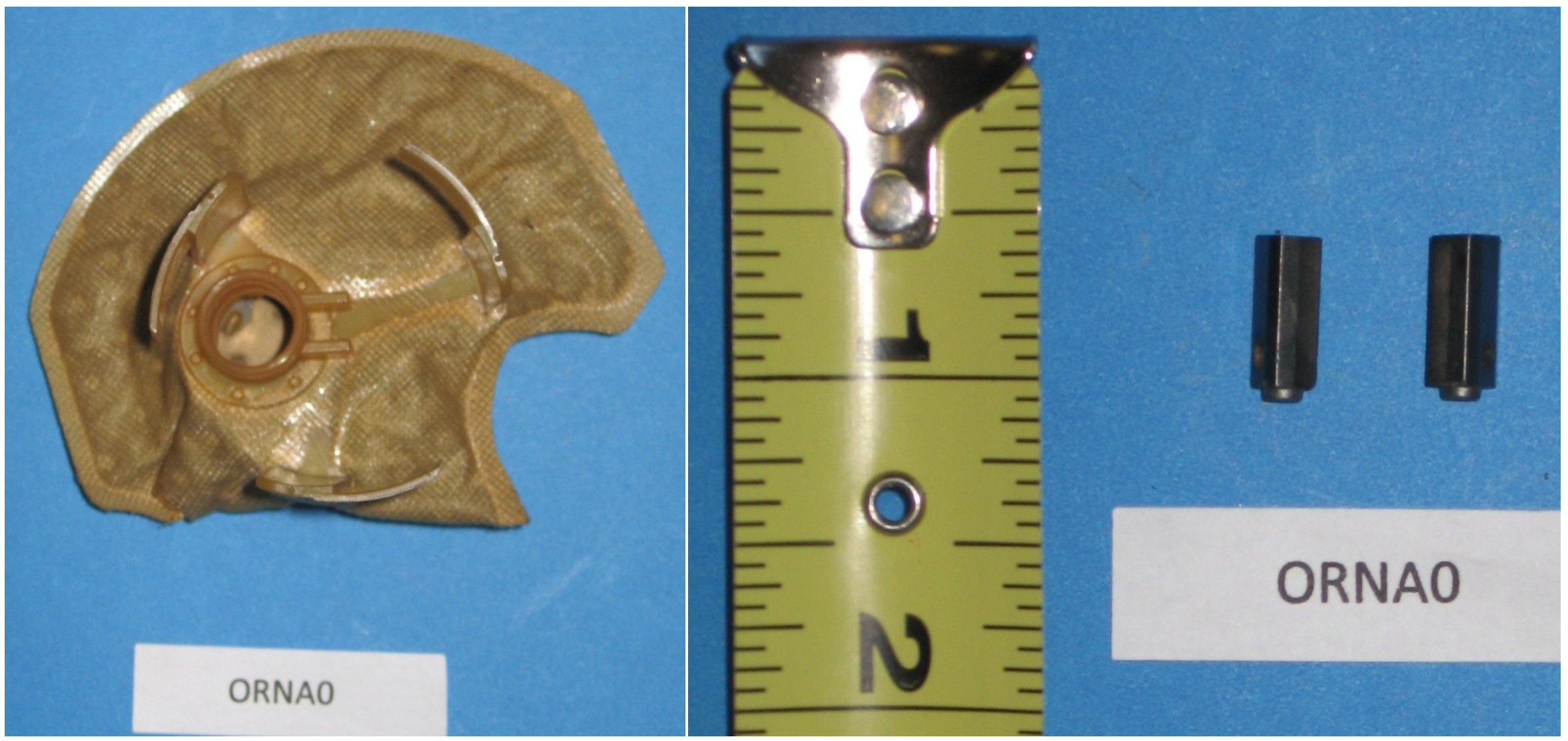

Figure F3. ORNA0 Fuel Pump Sock Filter (Left) and Brushes (Right). 


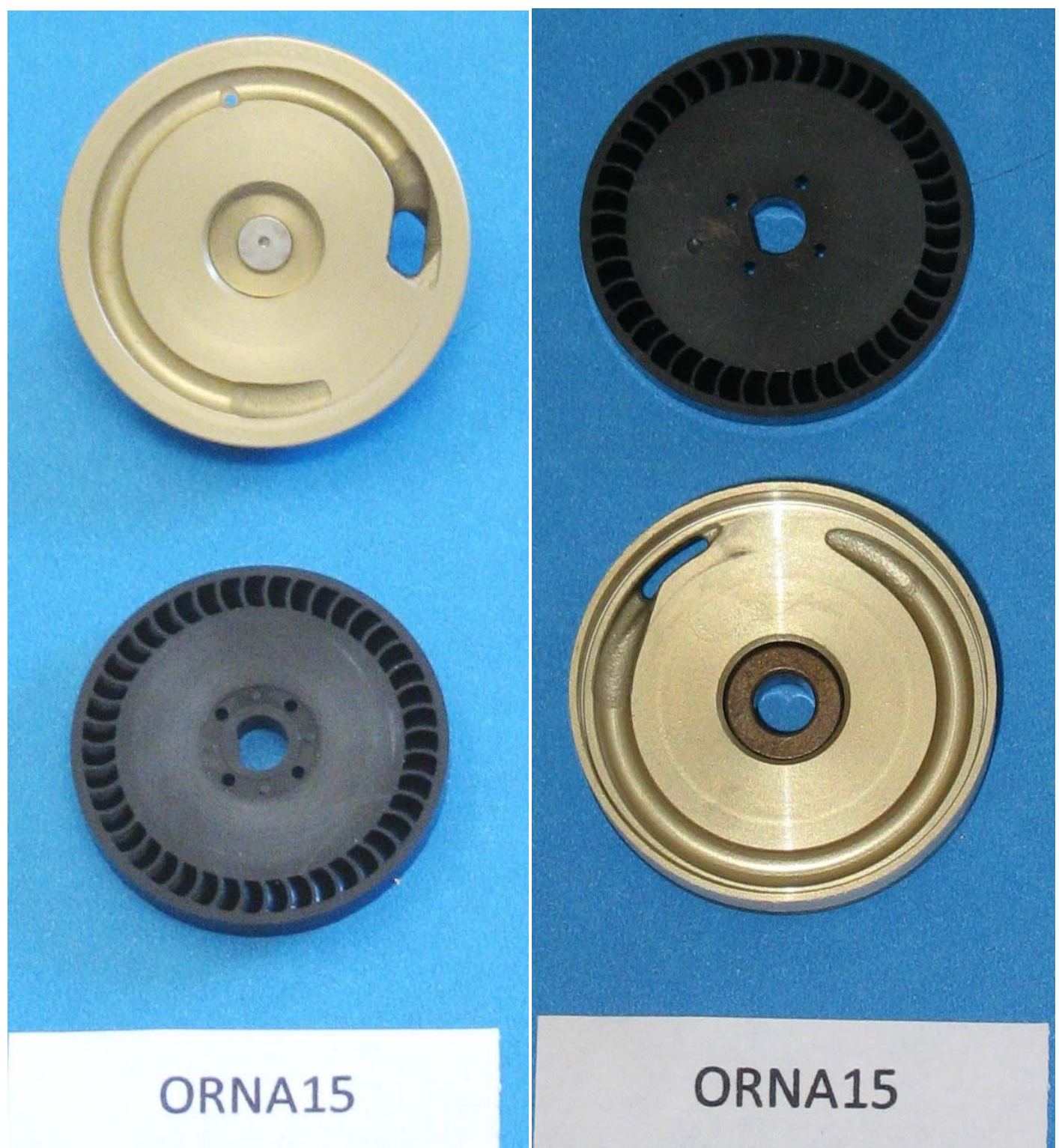

Figure F4. ORNA15 Fuel Pump Impeller with Inlet (Left) and Outlet (Right) Mating Cover. 


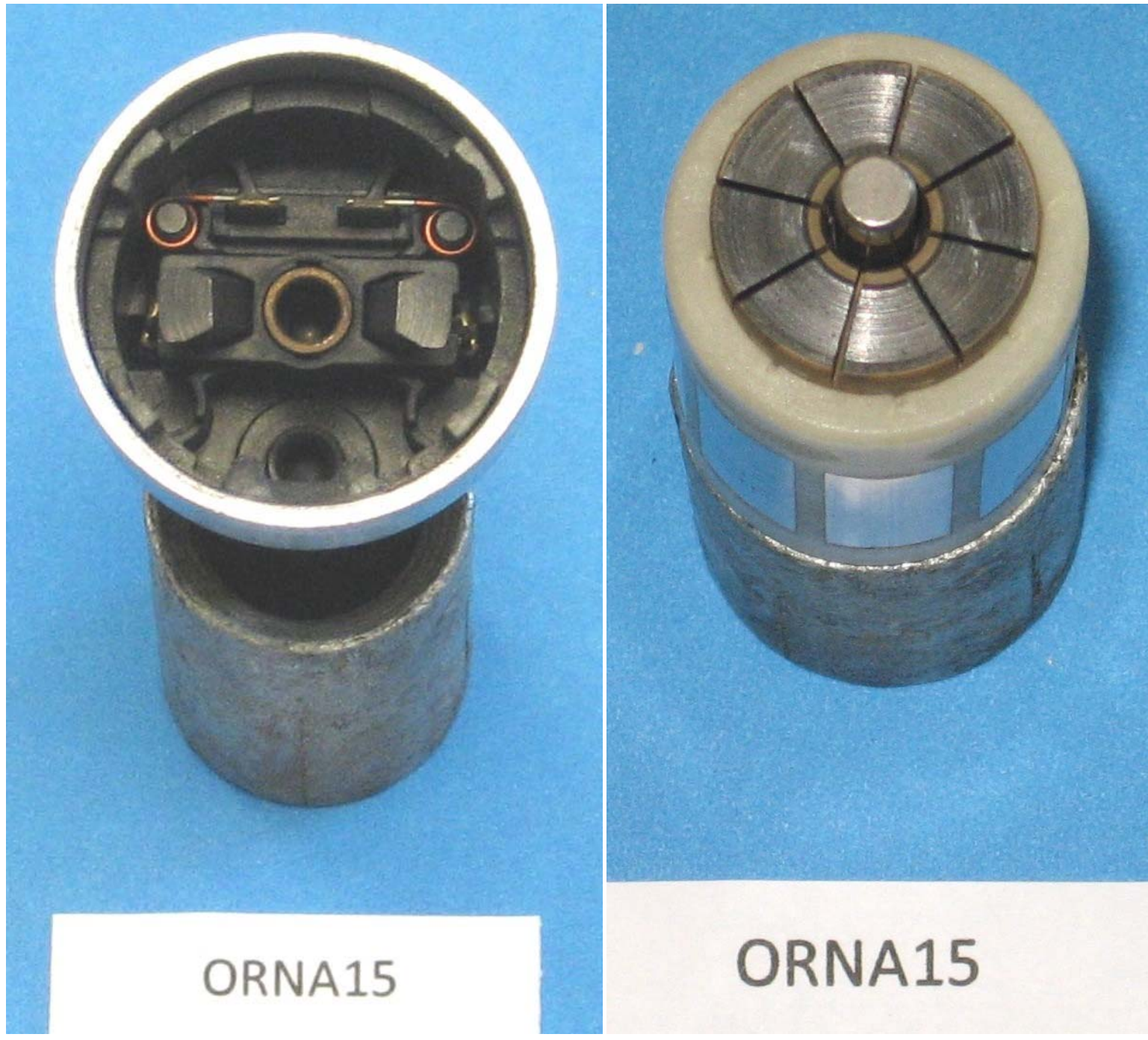

Figure F5. ORNA15 Fuel Pump Brushes (Left) and Commutator (Right). 


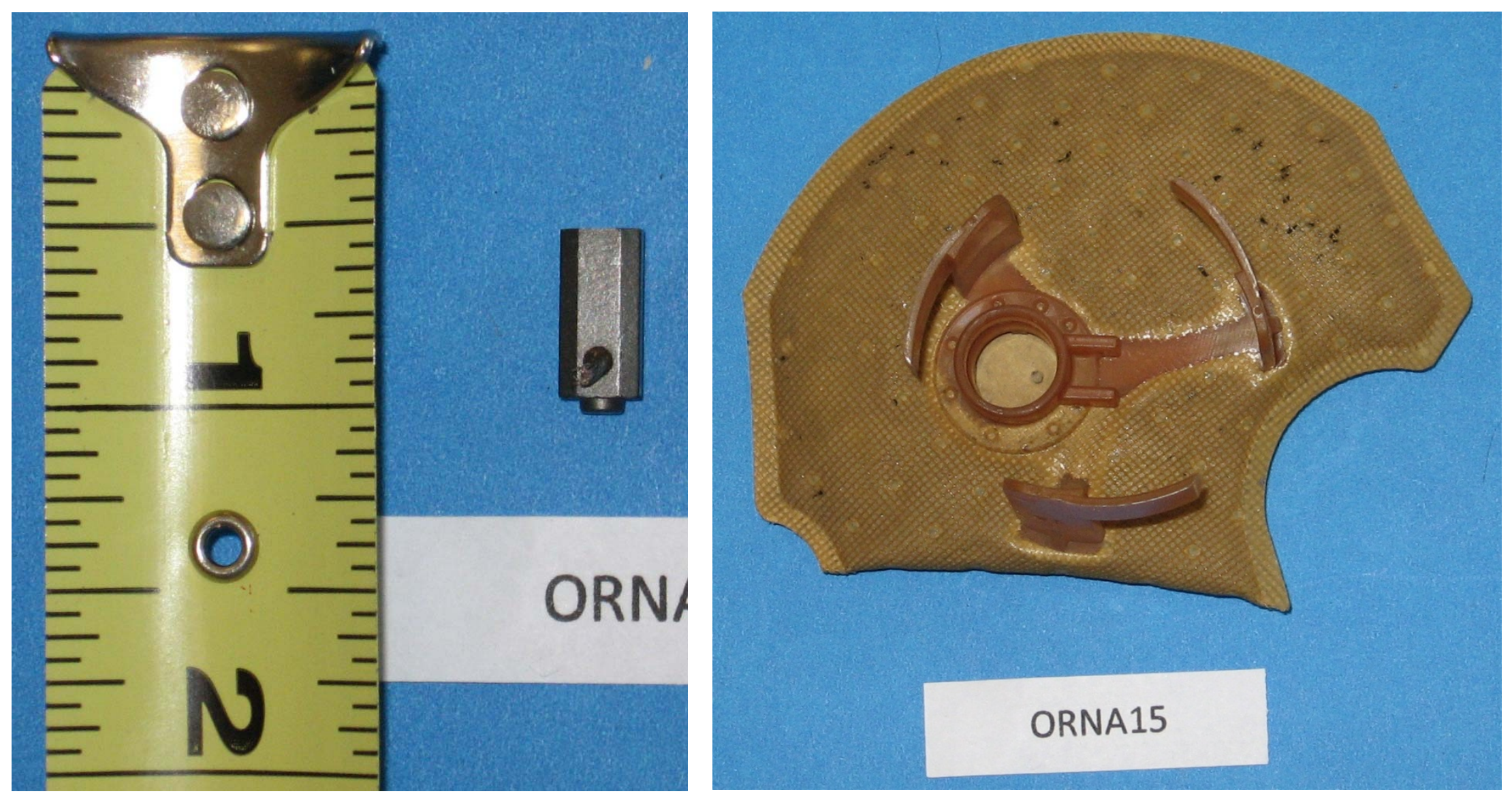

Figure F6. ORNA15 Fuel Pump Sock Filter (Left) and Brushes (Right). 


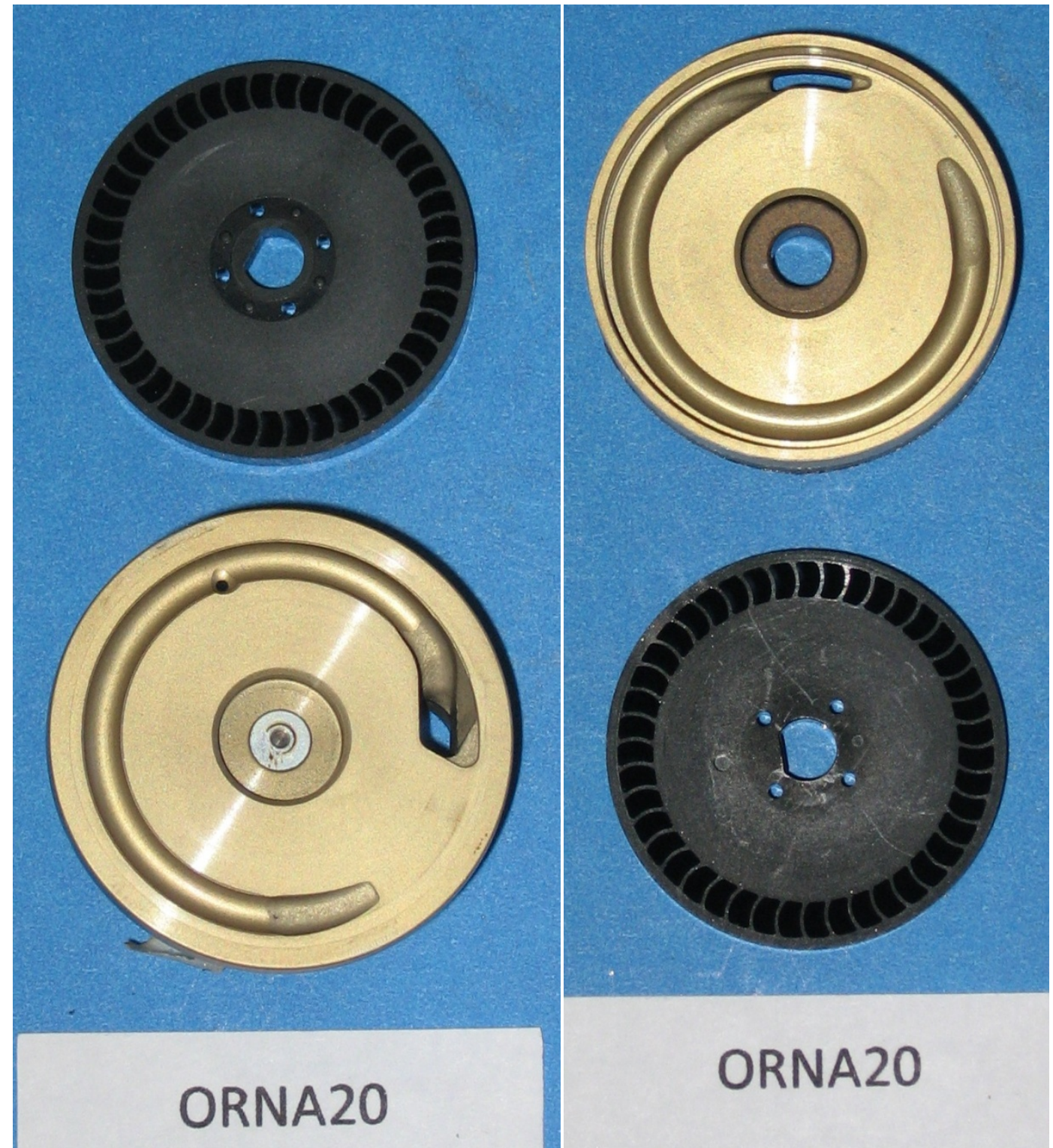

Figure F7. ORNA20 Fuel Pump Impeller with Inlet (Left) and Outlet (Right) Mating Cover. 


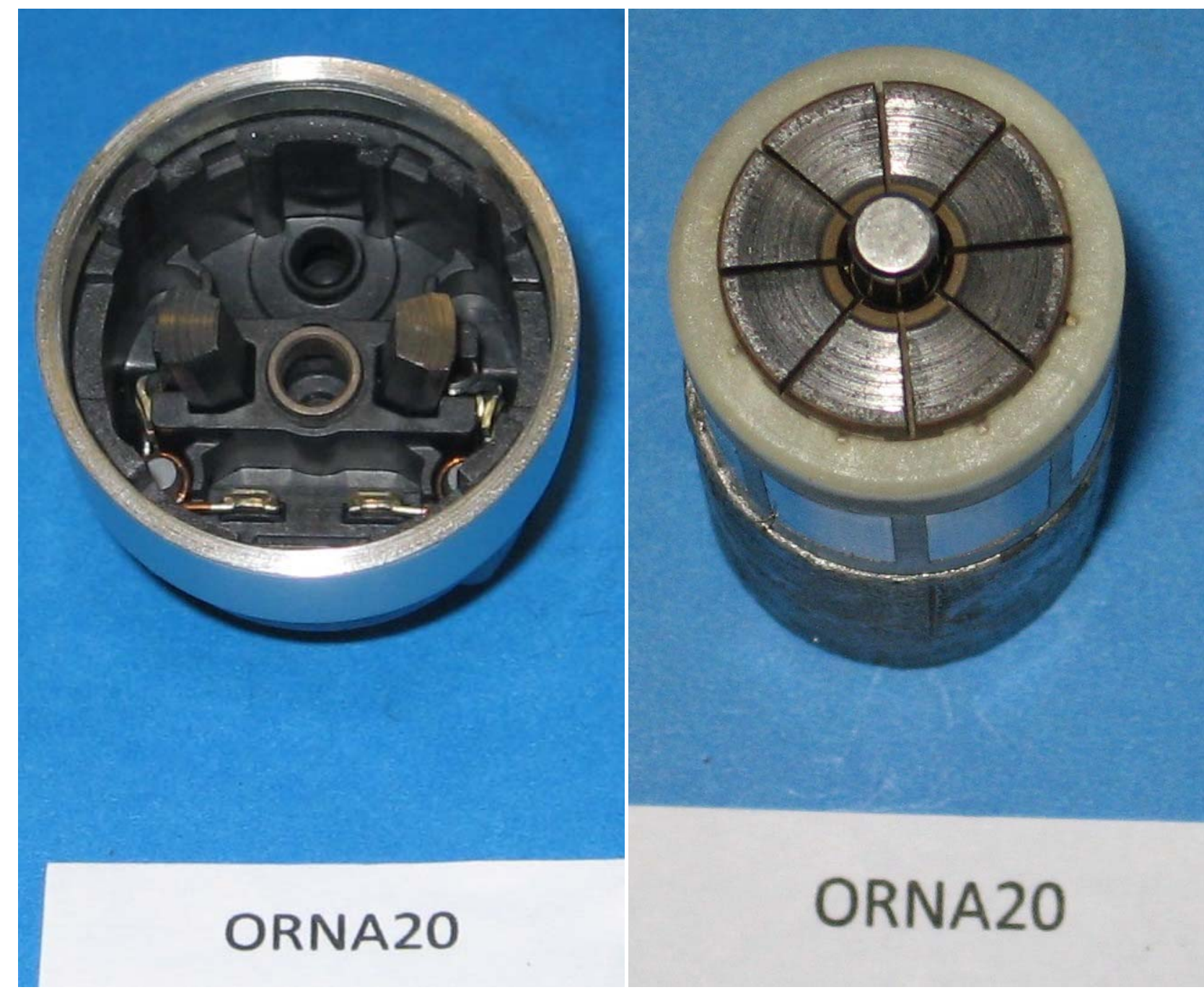

Figure F8. ORNA20 Fuel Pump Brushes (Left) and Commutator (Right). 


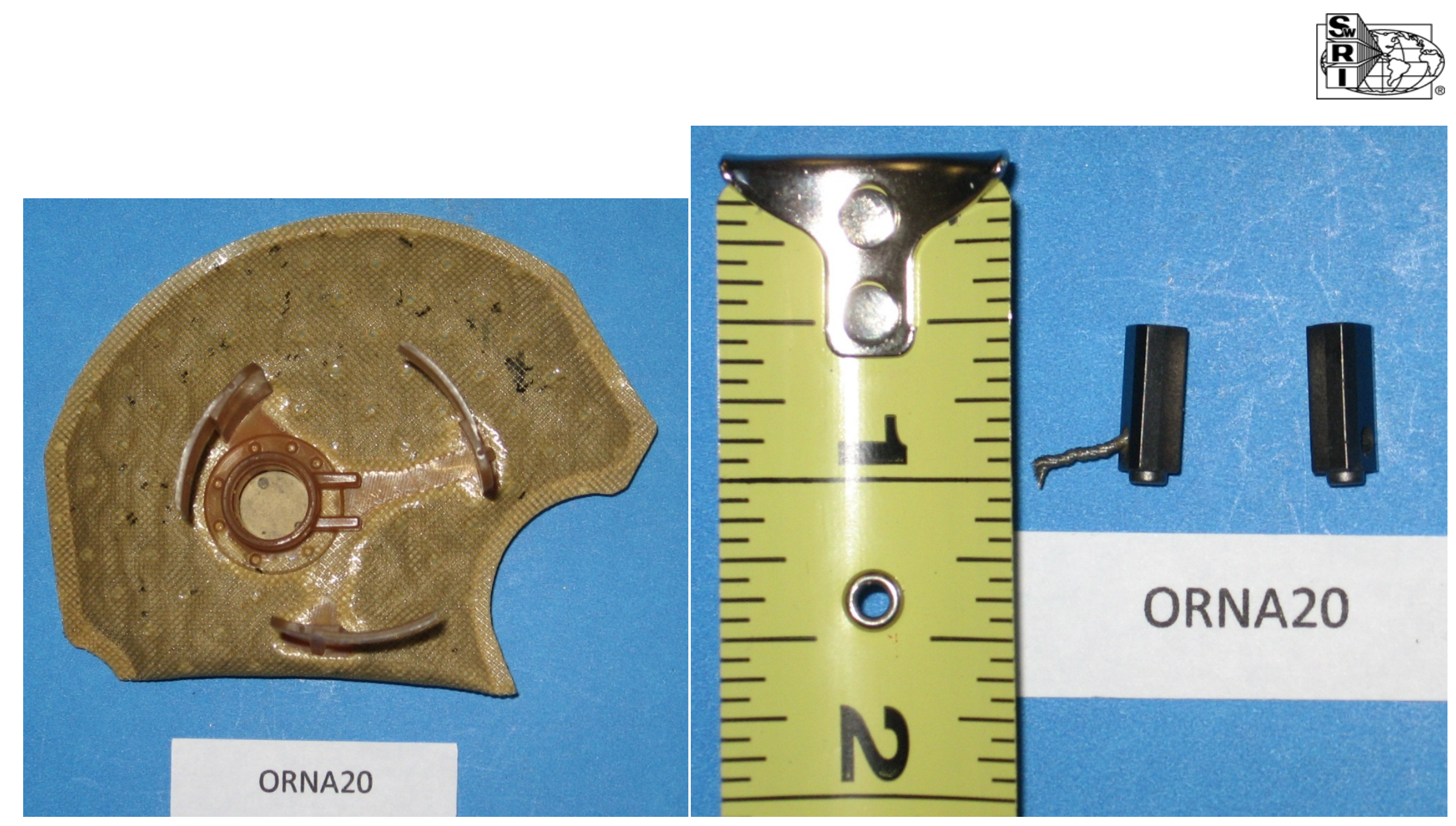

Figure F9. ORNA20 Fuel Pump Sock Filter (Left) and Brushes (Right). 


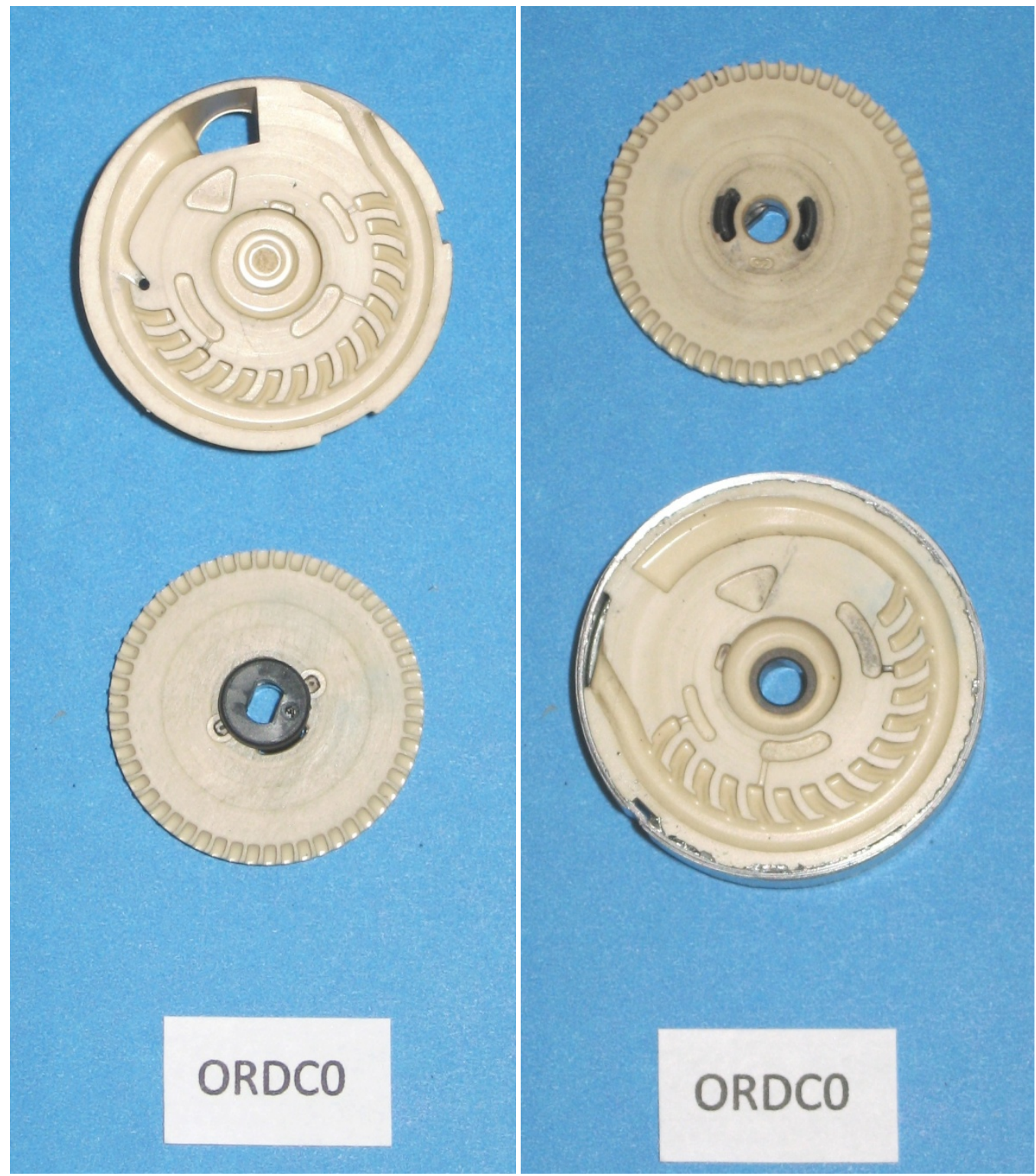

Figure F10. ORDC0 Fuel Pump Impeller with Inlet (Left) and Outlet (Right) Mating Cover. 


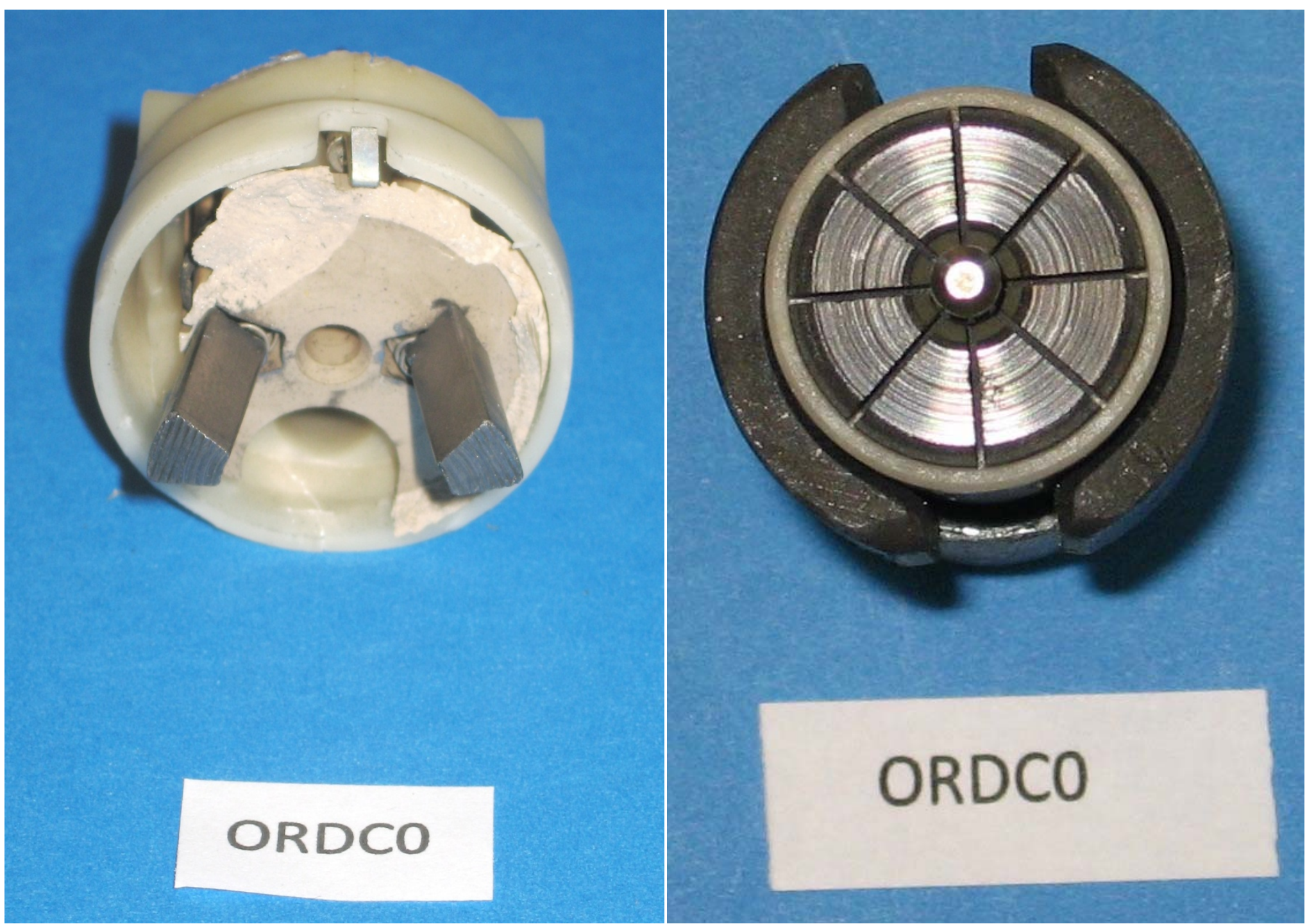

Figure F11. ORDC0 Fuel Pump Brushes (Left) and Commutator (Right). 


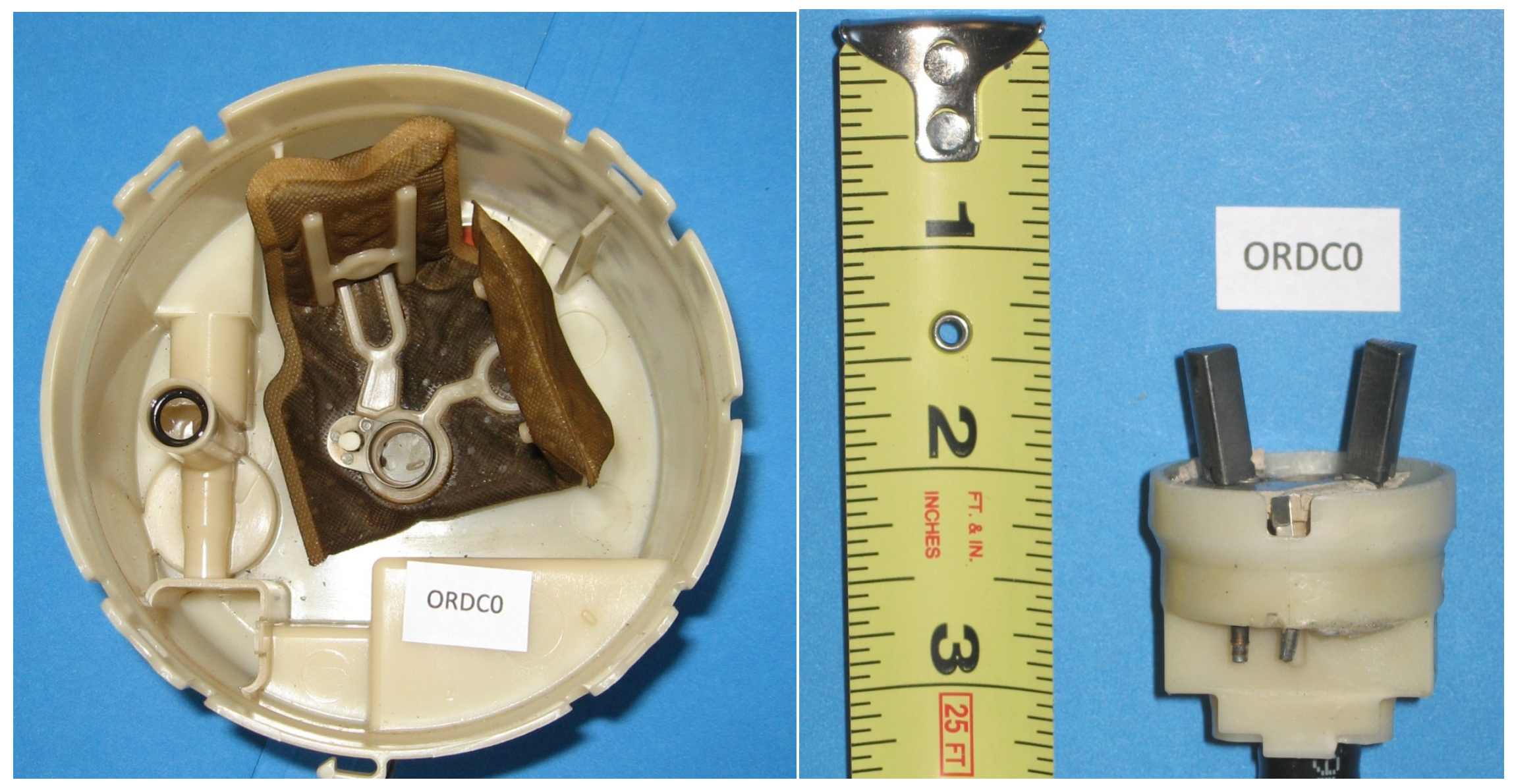

Figure F12. ORDC0 Fuel Pump Sock Filter (Left) and Brushes (Right). 


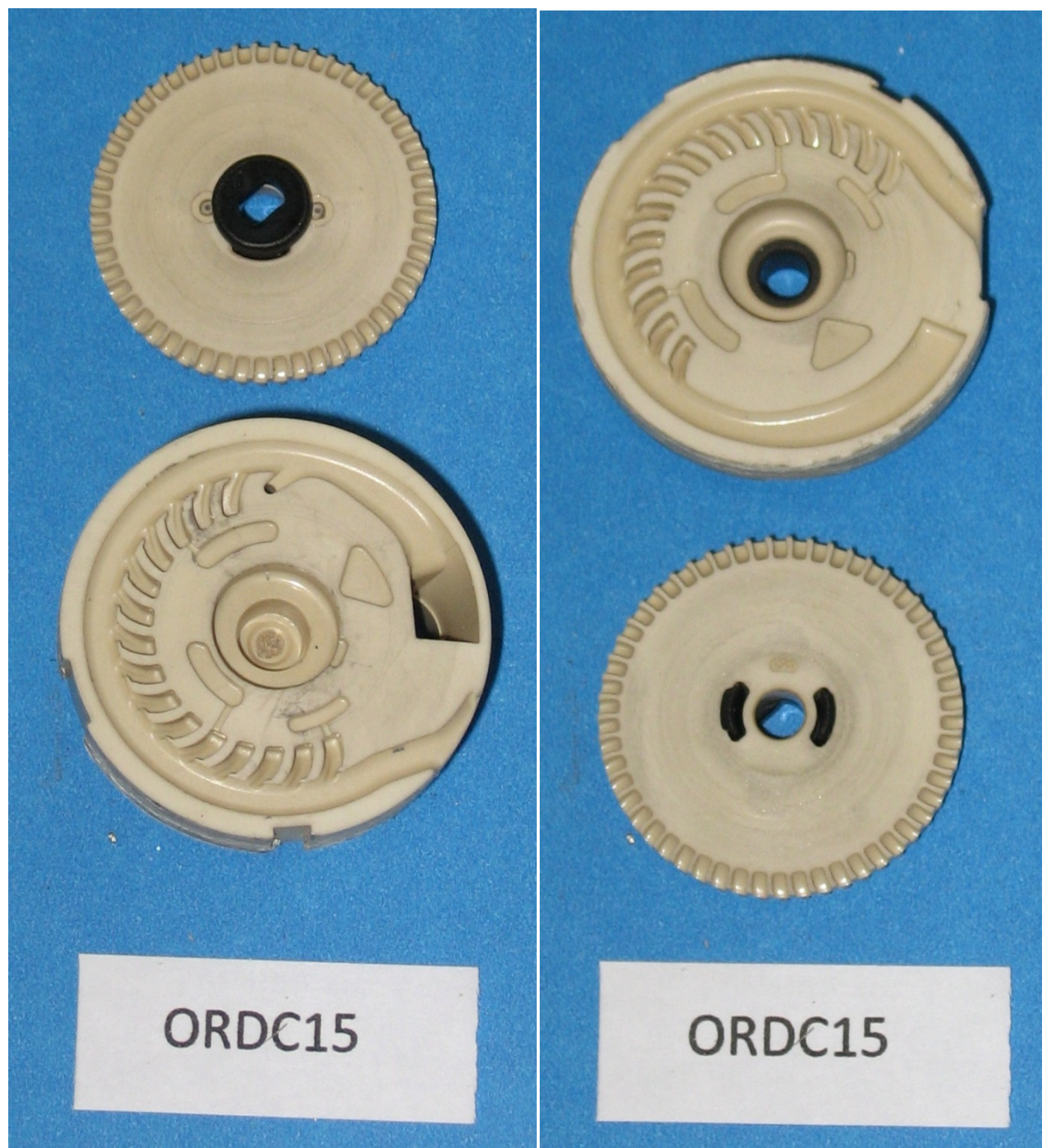

Figure F13. ORDC15 Fuel Pump Impeller with Inlet (Left) and Outlet (Right) Mating Cover. 


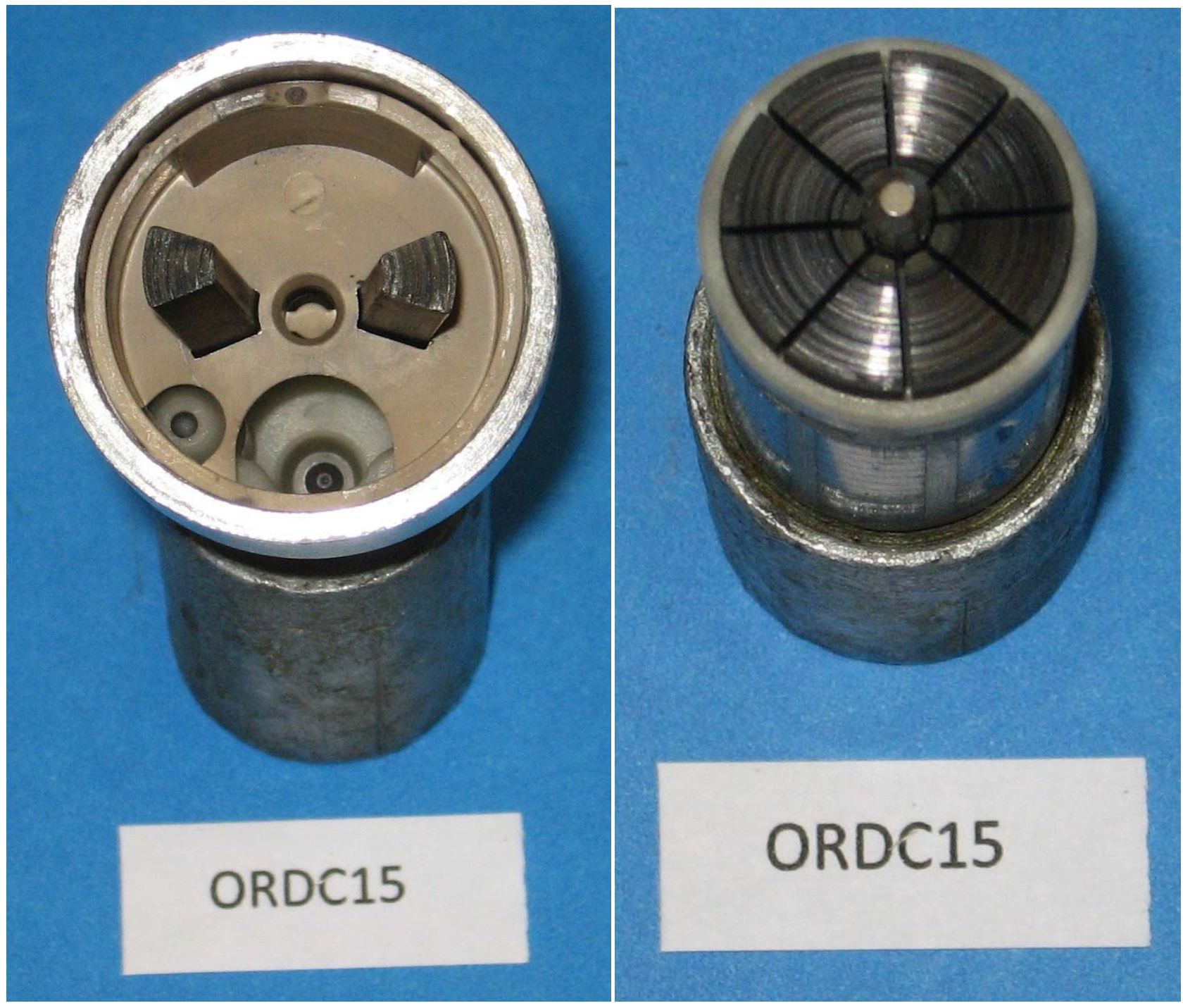

Figure F14. ORDC15 Fuel Pump Brushes (Left) and Commutator (Right). 


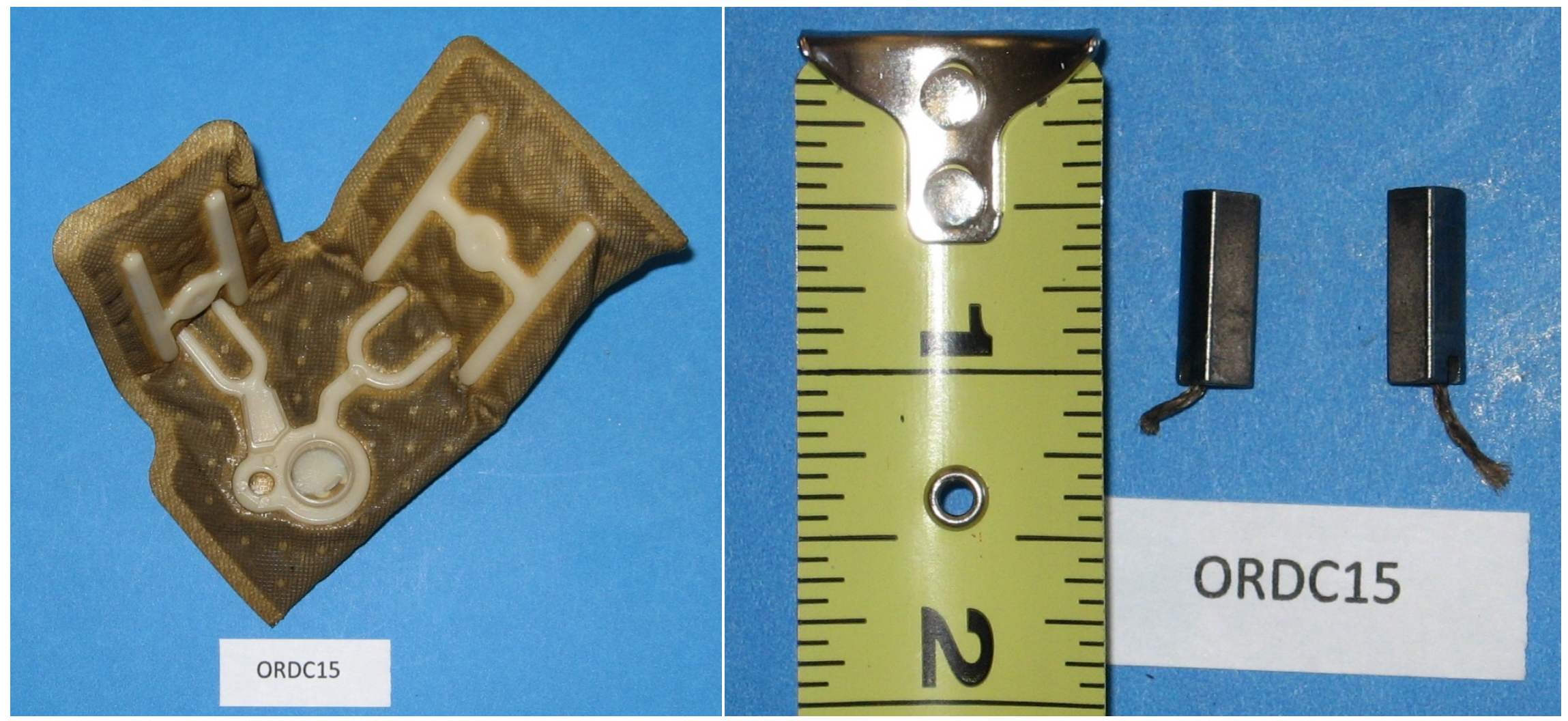

Figure F15. ORDC15 Fuel Pump Sock Filter (Left) and Brushes (Right). 


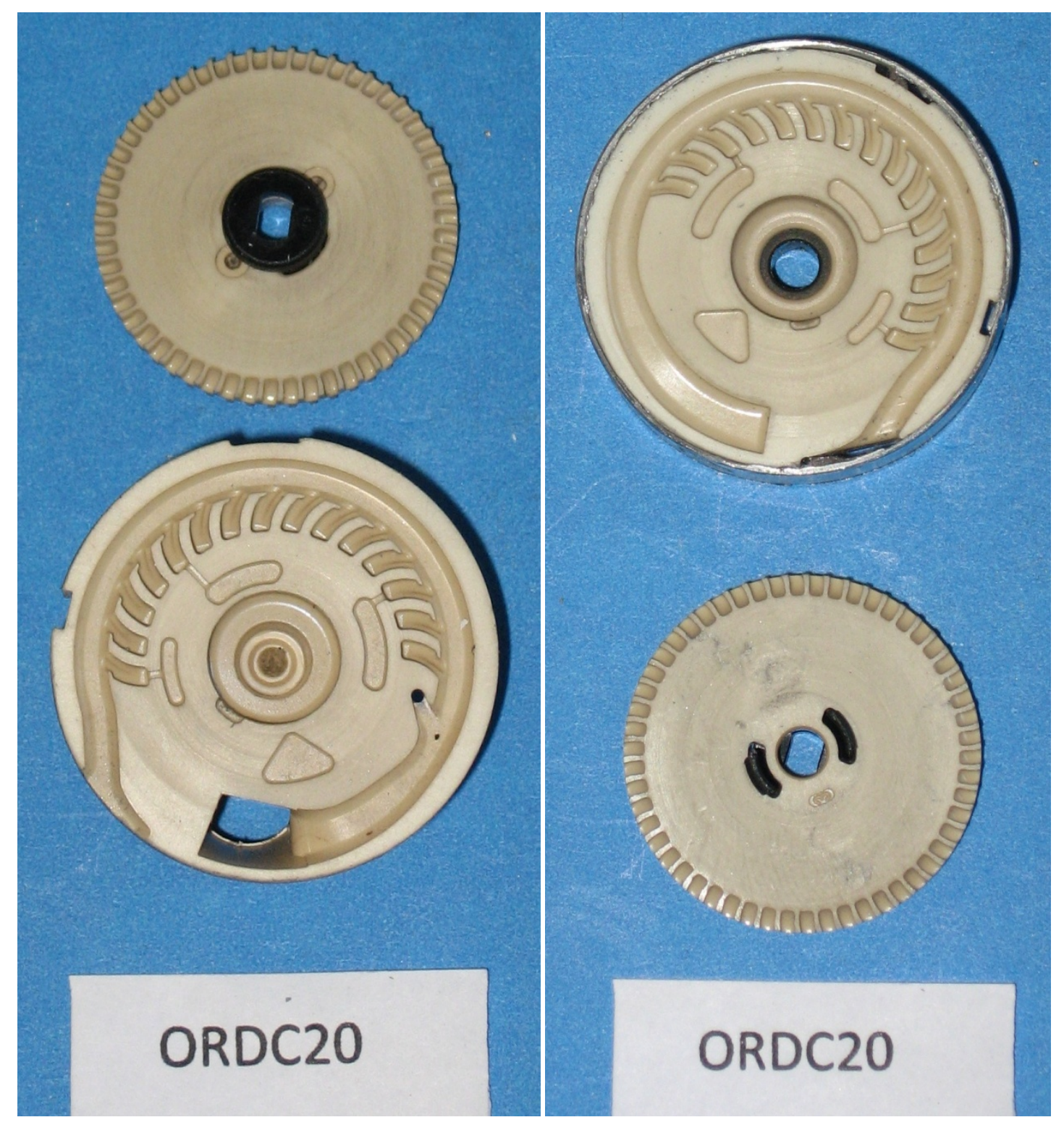

Figure F16. ORDC20 Fuel Pump Impeller with Inlet (Left) and Outlet (Right) Mating Cover. 


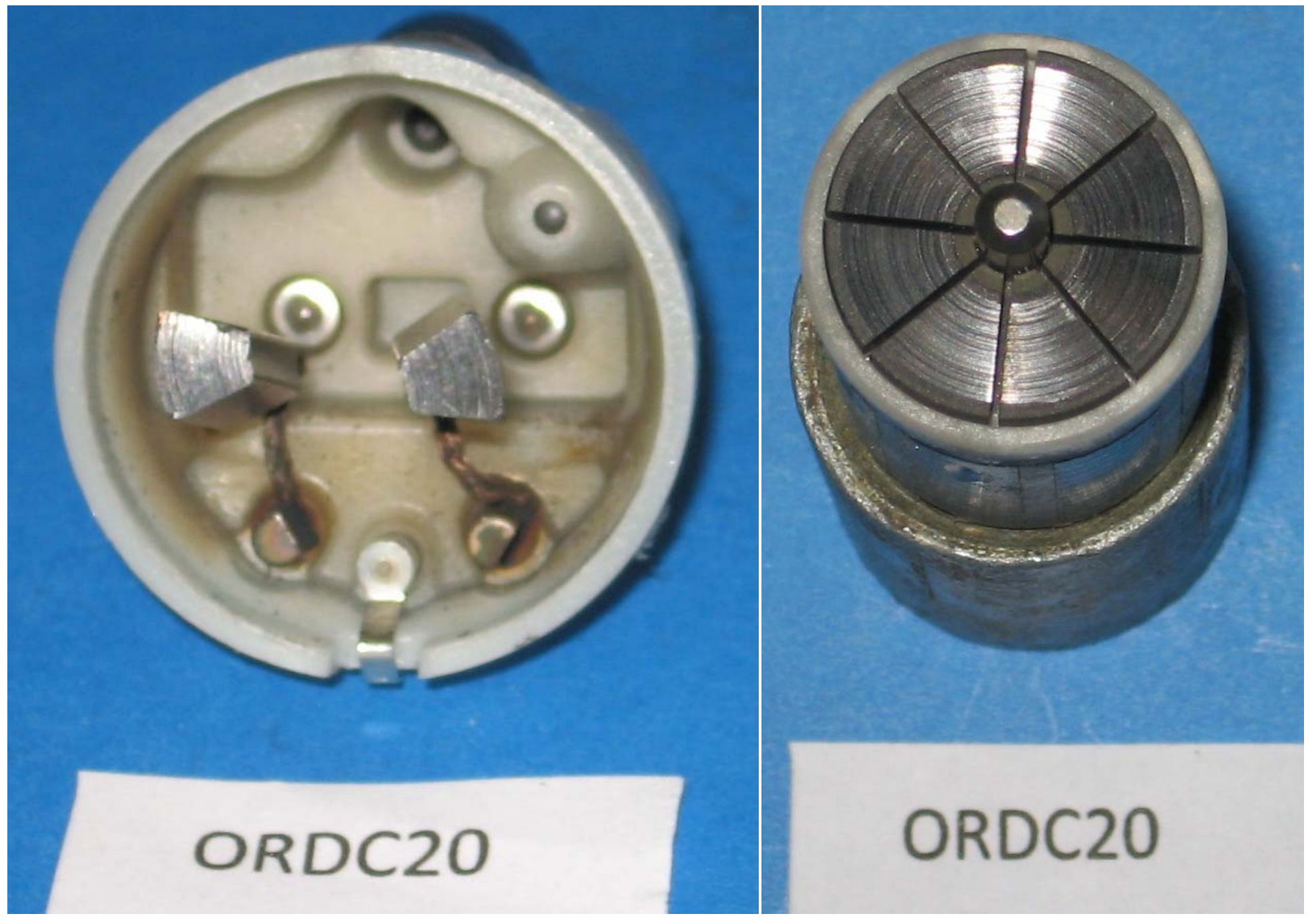

Figure F17. ORDC20 Fuel Pump Brushes (Left) and Commutator (Right). 


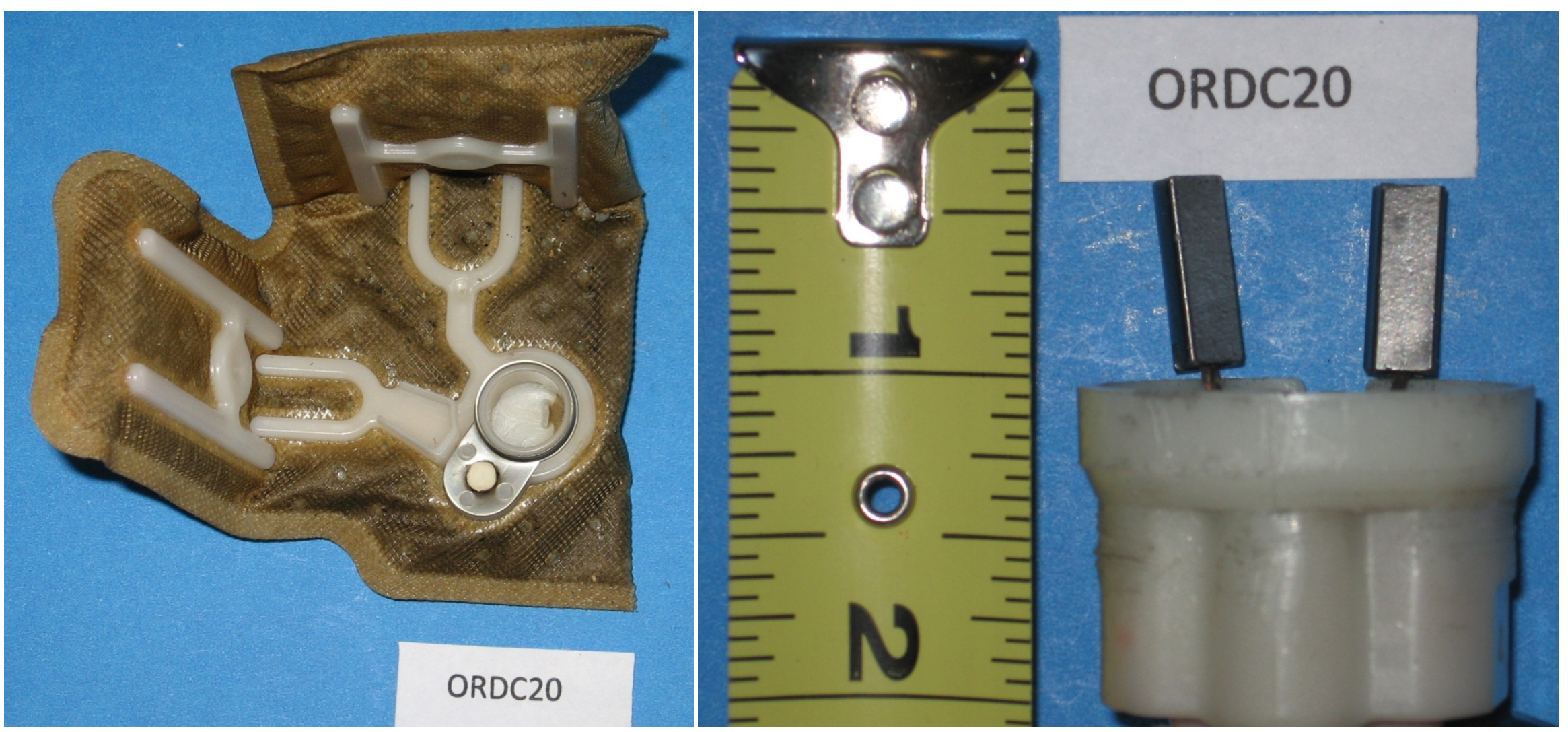

Figure F18. ORDC20 Fuel Pump Sock Filter (Left) and Brushes (Right). 


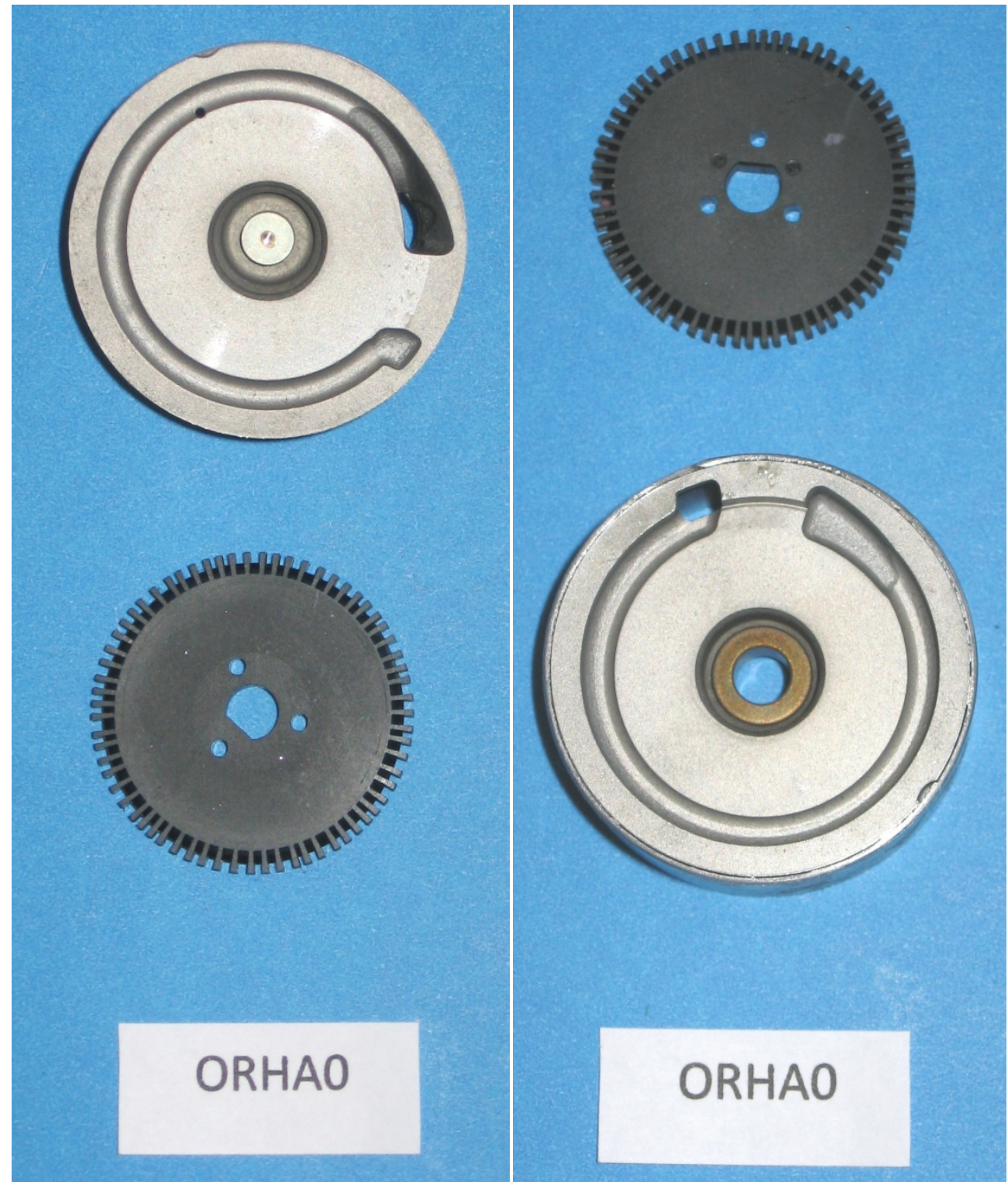

Figure F19. ORHA0 Fuel Pump Impeller with Inlet (Left) and Outlet (Right) Mating Cover. 


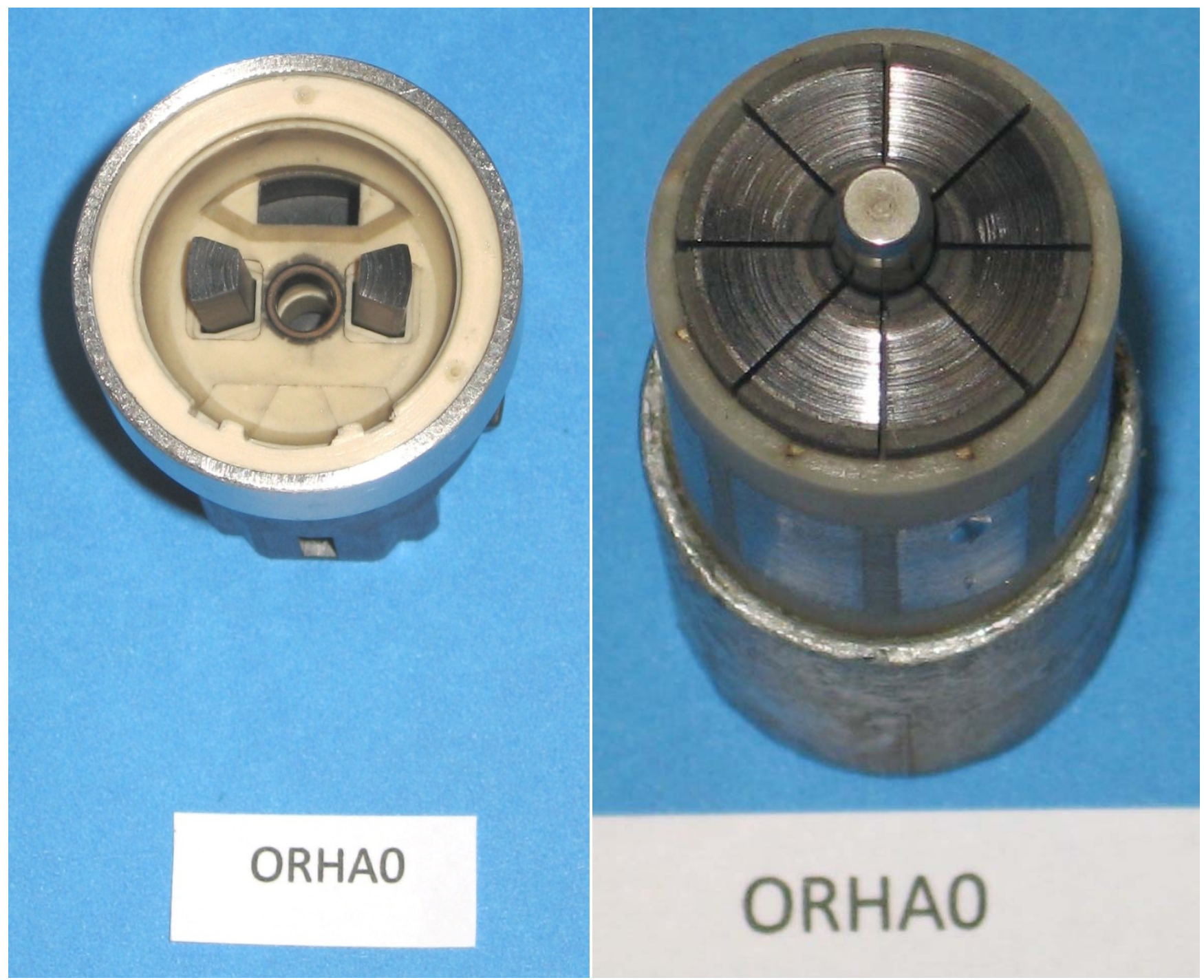

Figure F20. ORHA15 Fuel Pump Brushes (Left) and Commutator (Right). 


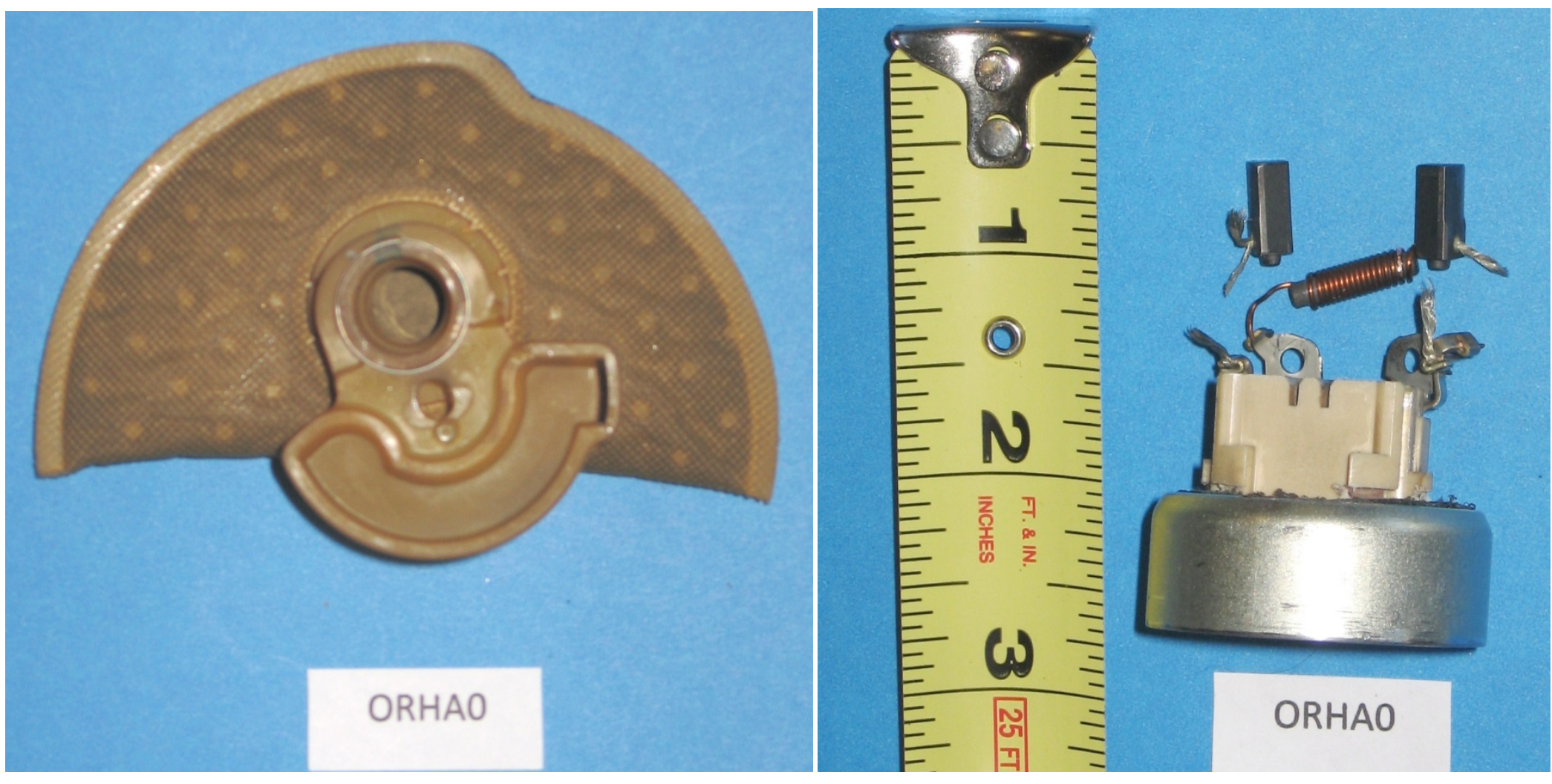

Figure F21. ORHA0 Fuel Pump Sock Filter (Left) and Brushes (Right). 


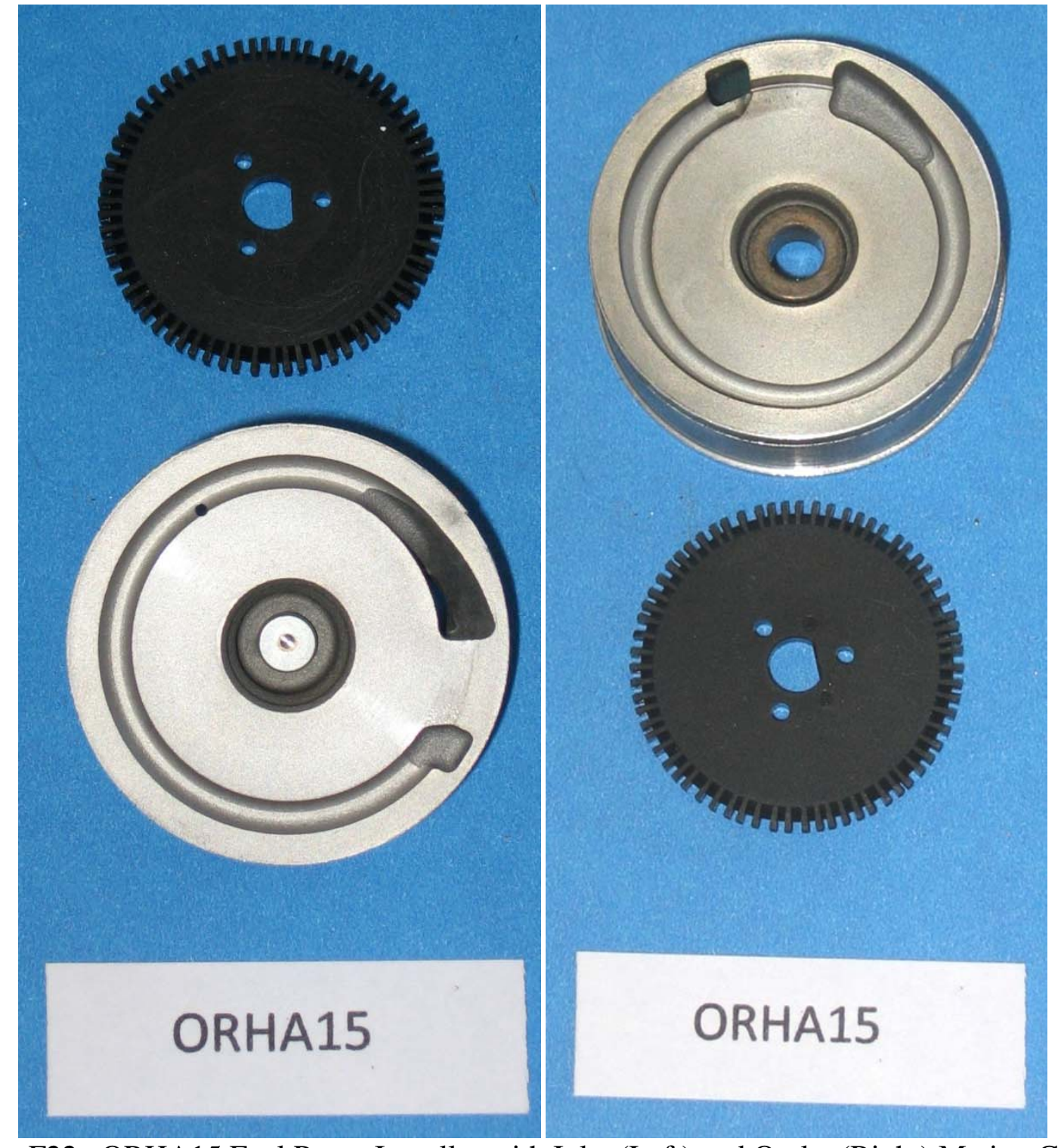

Figure F22. ORHA15 Fuel Pump Impeller with Inlet (Left) and Outlet (Right) Mating Cover. 


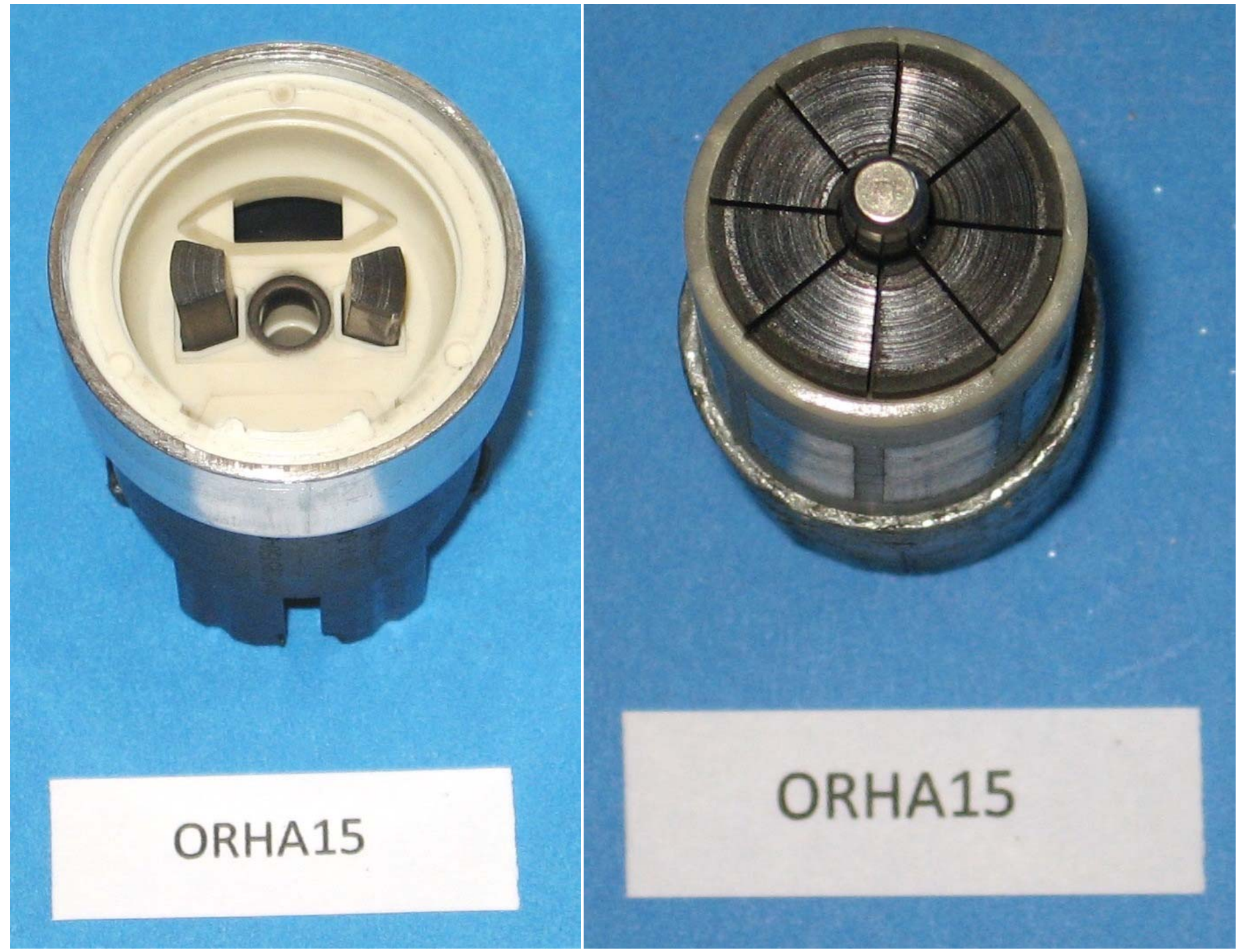

Figure F23. ORHA15 Fuel Pump Brushes (Left) and Commutator (Right). 


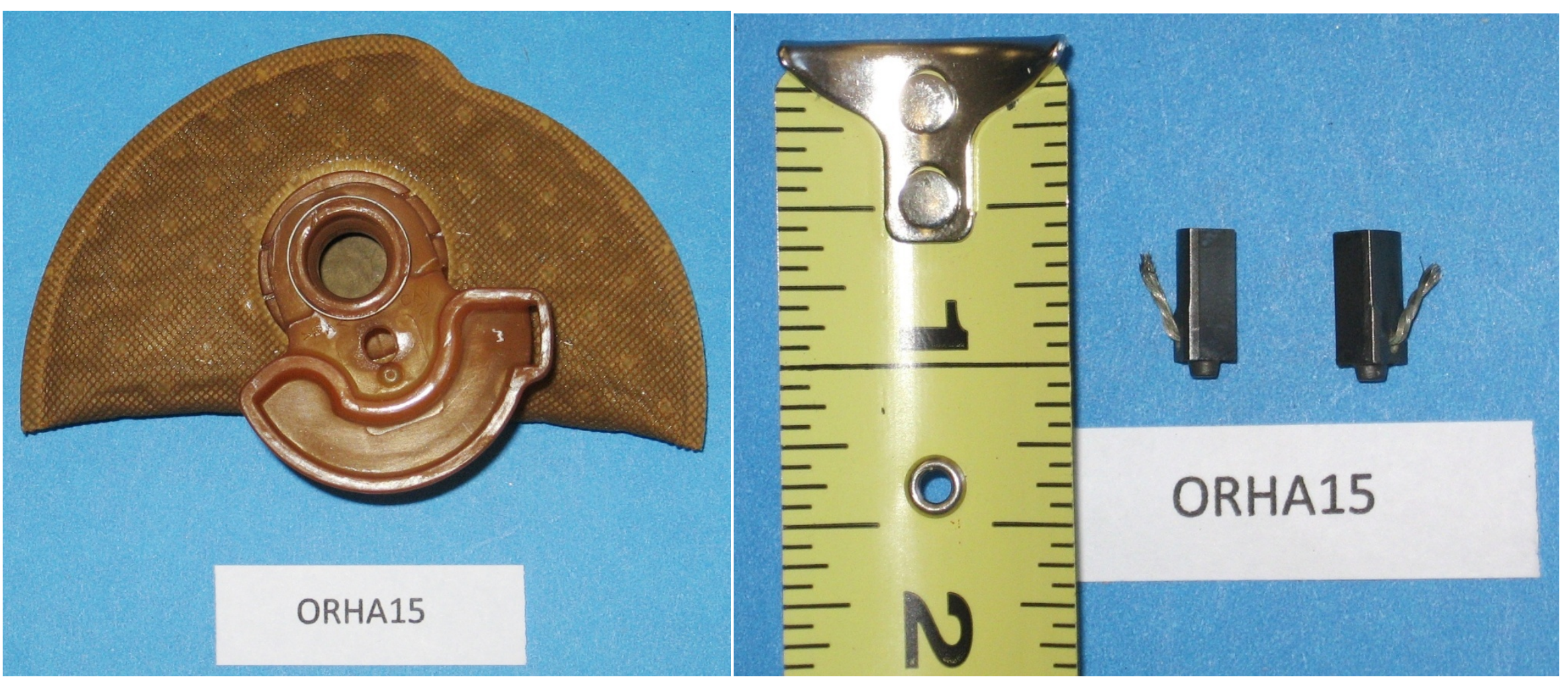

Figure F24. ORHA15 Fuel Pump Sock Filter (Left) and Brushes (Right). 


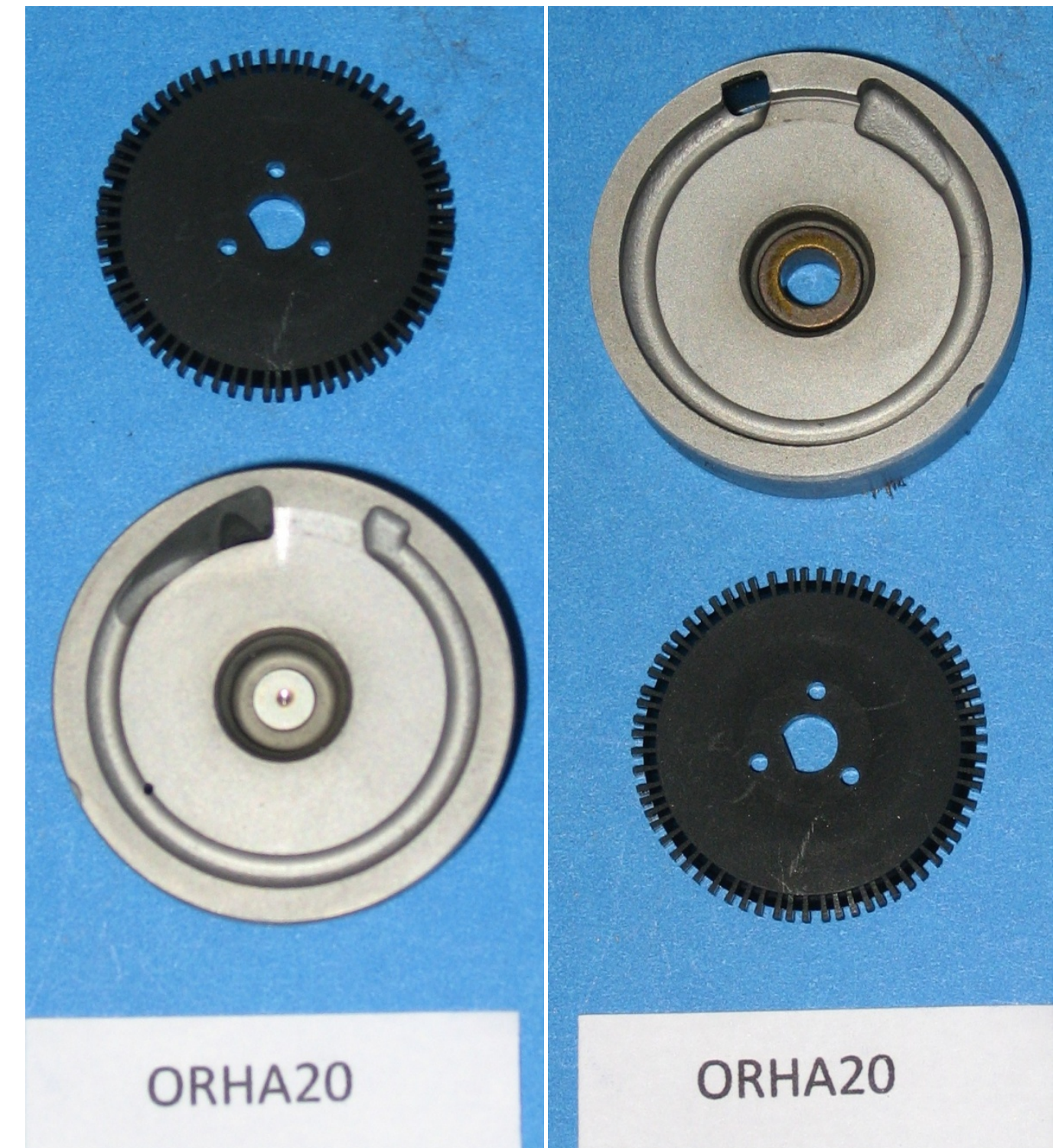

Figure F25. ORHA20 Fuel Pump Impeller with Inlet (Left) and Outlet (Right) Mating Cover. 


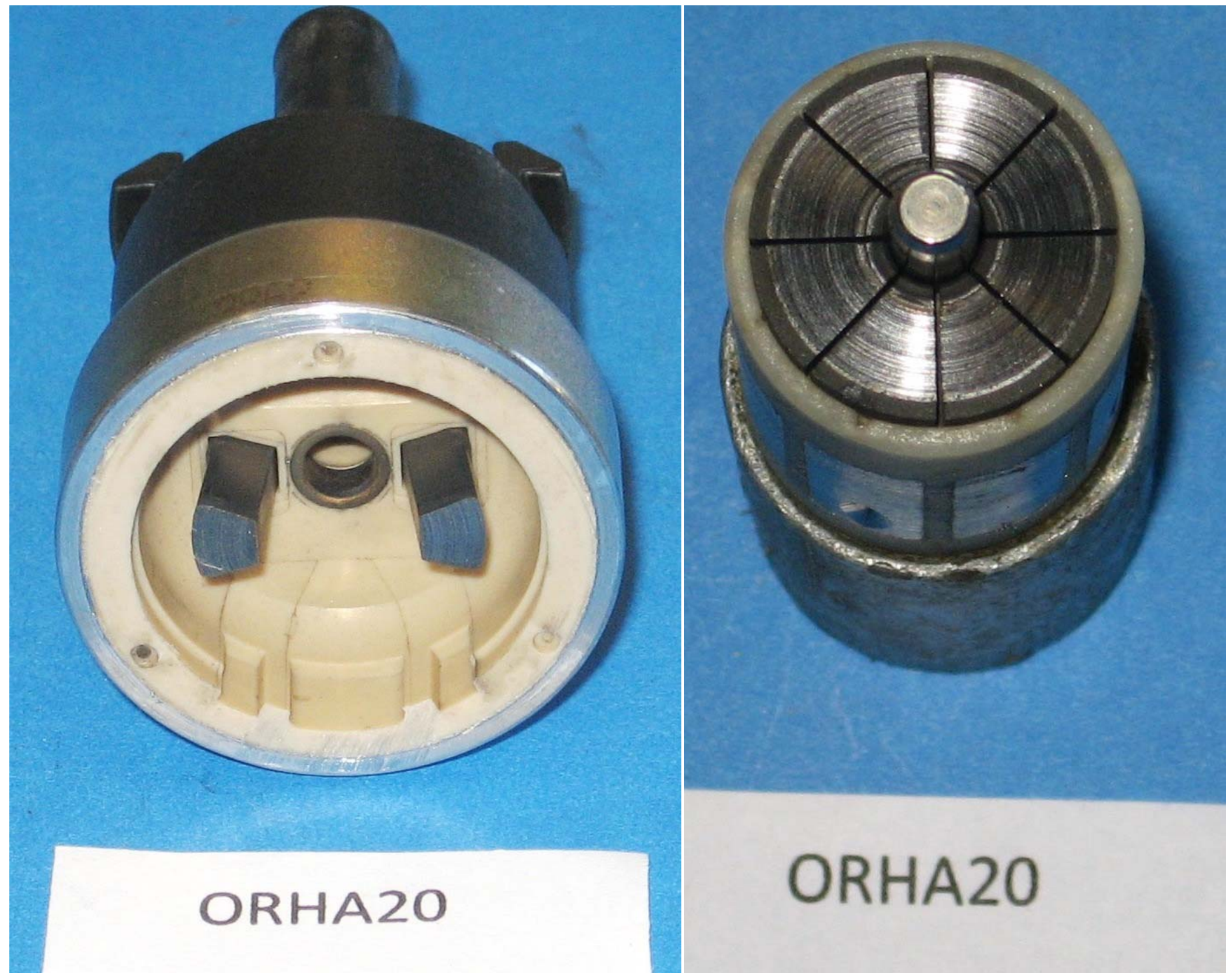

Figure F26. ORHA20 Fuel Pump Brushes (Left) and Commutator (Right). 


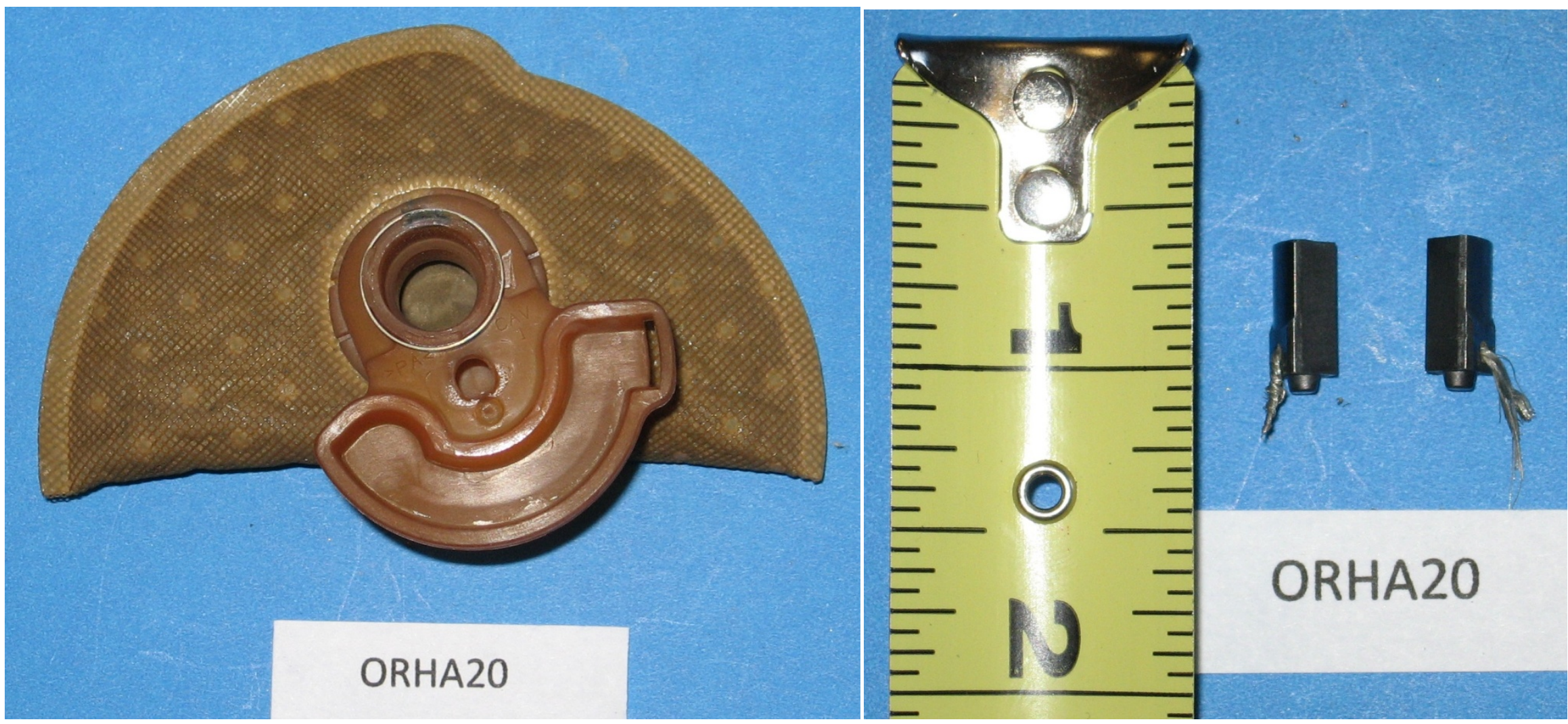

Figure F27. ORHA20 Fuel Pump Sock Filter (Left) and Brushes (Right). 


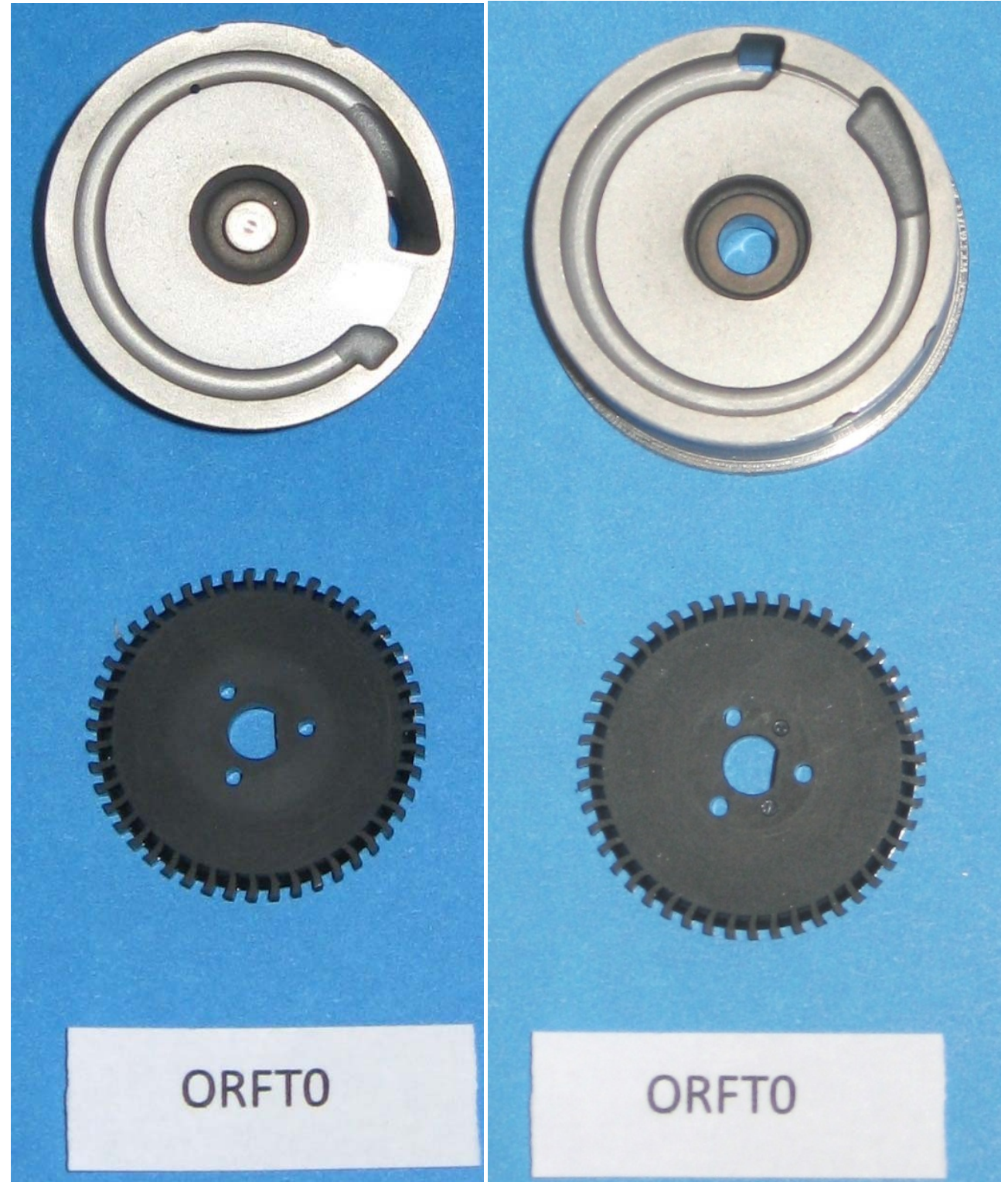

Figure F28. ORFT0 Fuel Pump Impeller with Inlet (Left) and Outlet (Right) Mating Cover. 


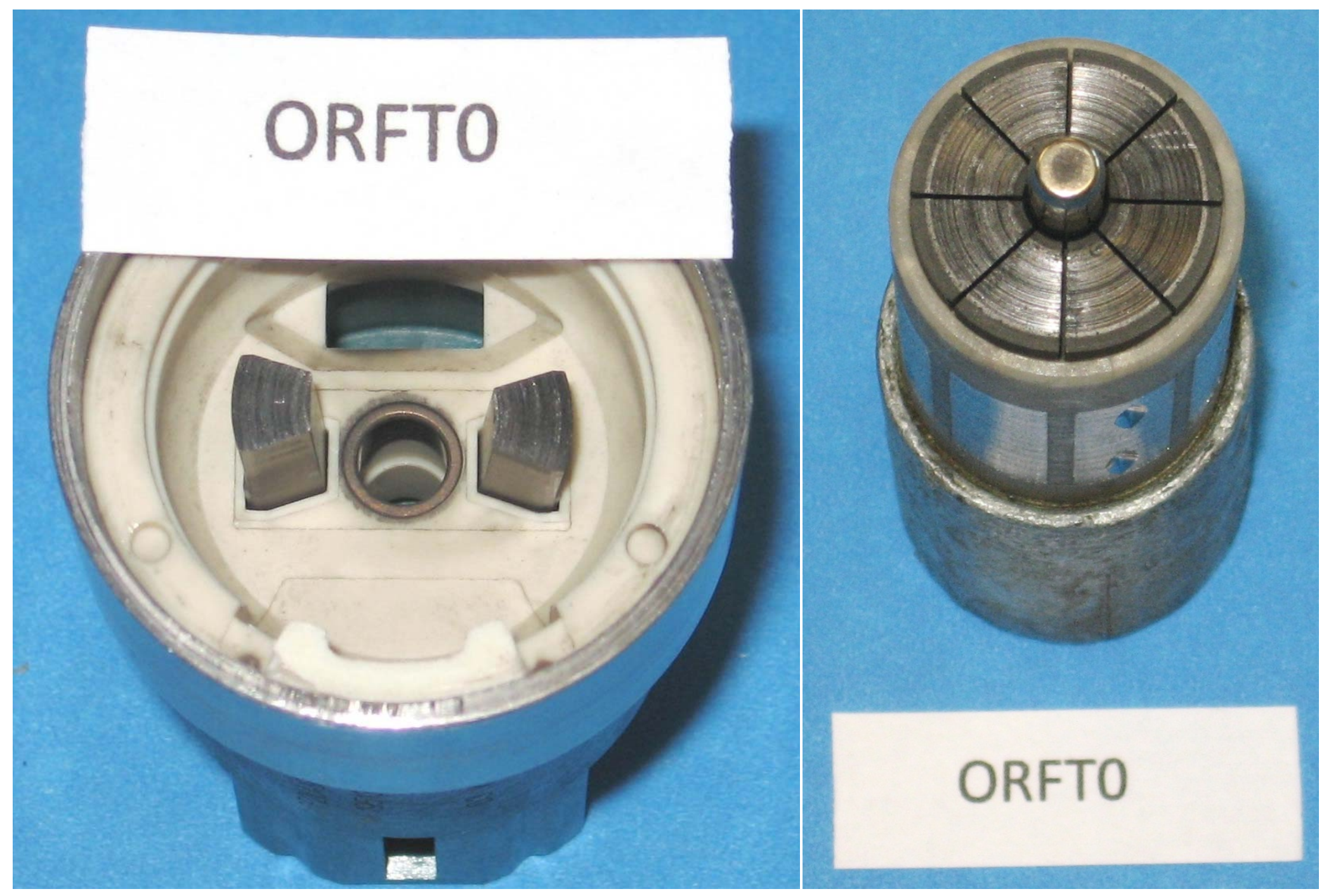

Figure F29. ORFT0 Fuel Pump Brushes (Left) and Commutator (Right). 


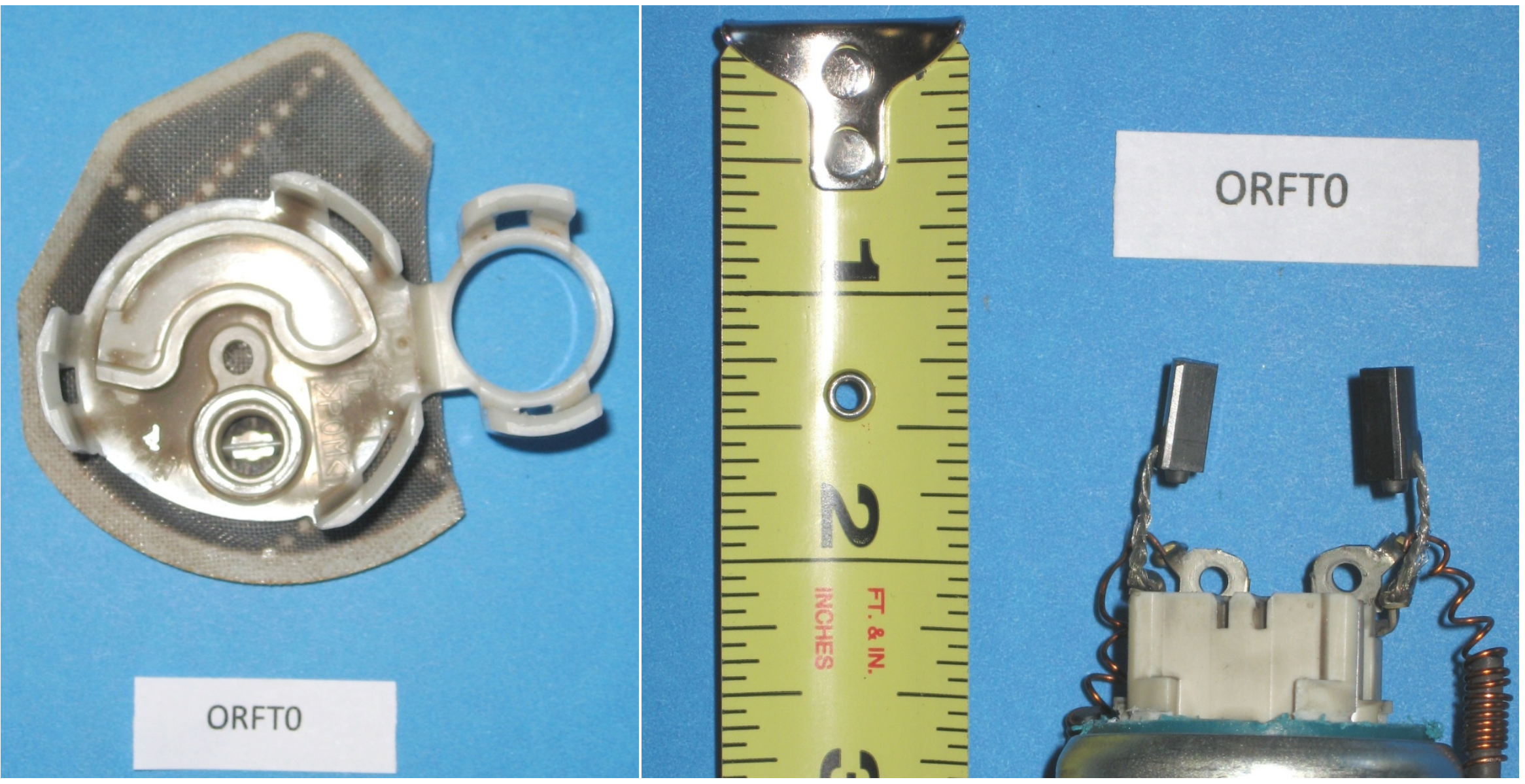

Figure F30. ORFT0 Fuel Pump Sock Filter (Left) and Brushes (Right). 


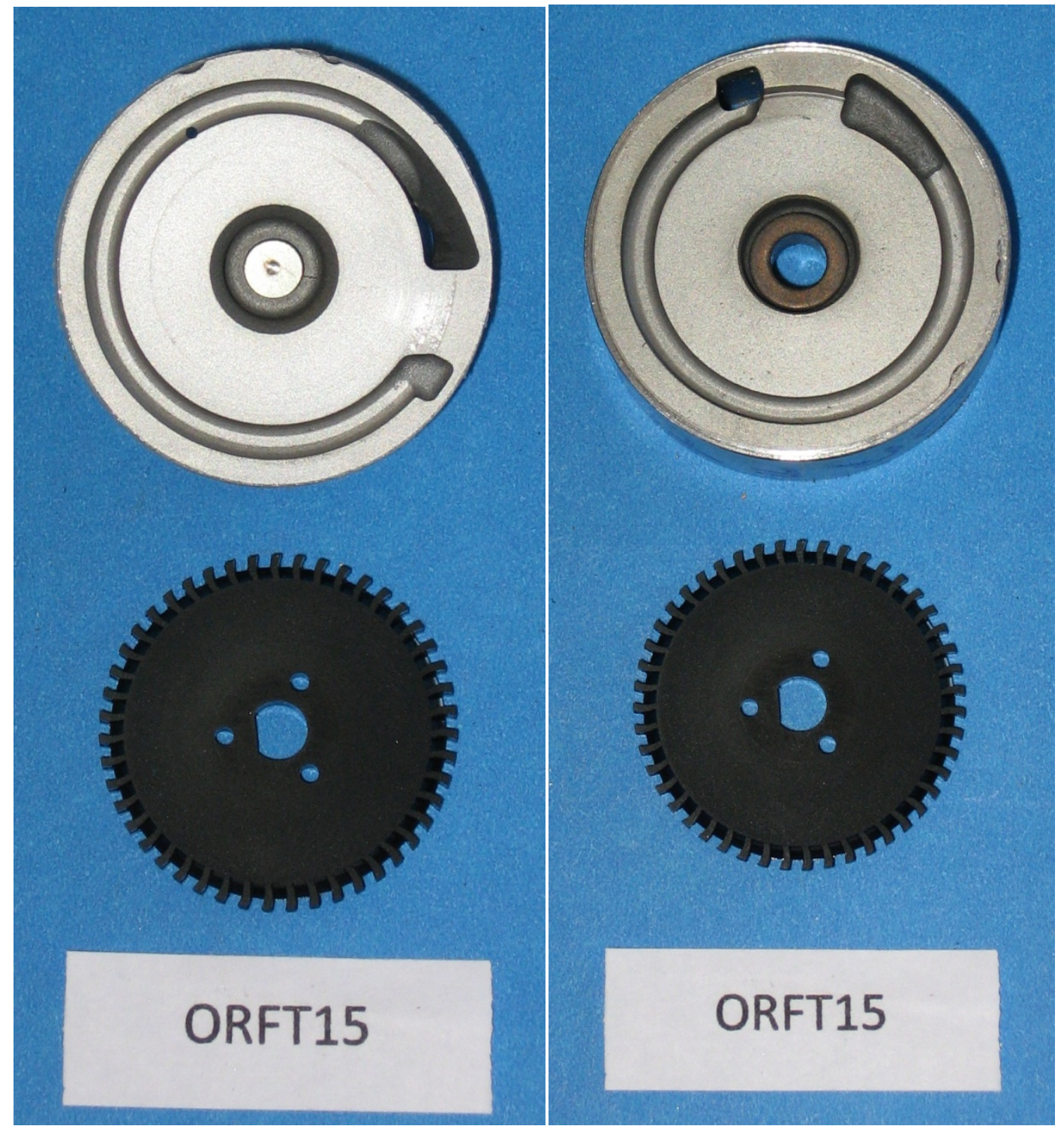

Figure F31. ORFT15 Fuel Pump Impeller with Inlet (Left) and Outlet (Right) Mating Cover. 


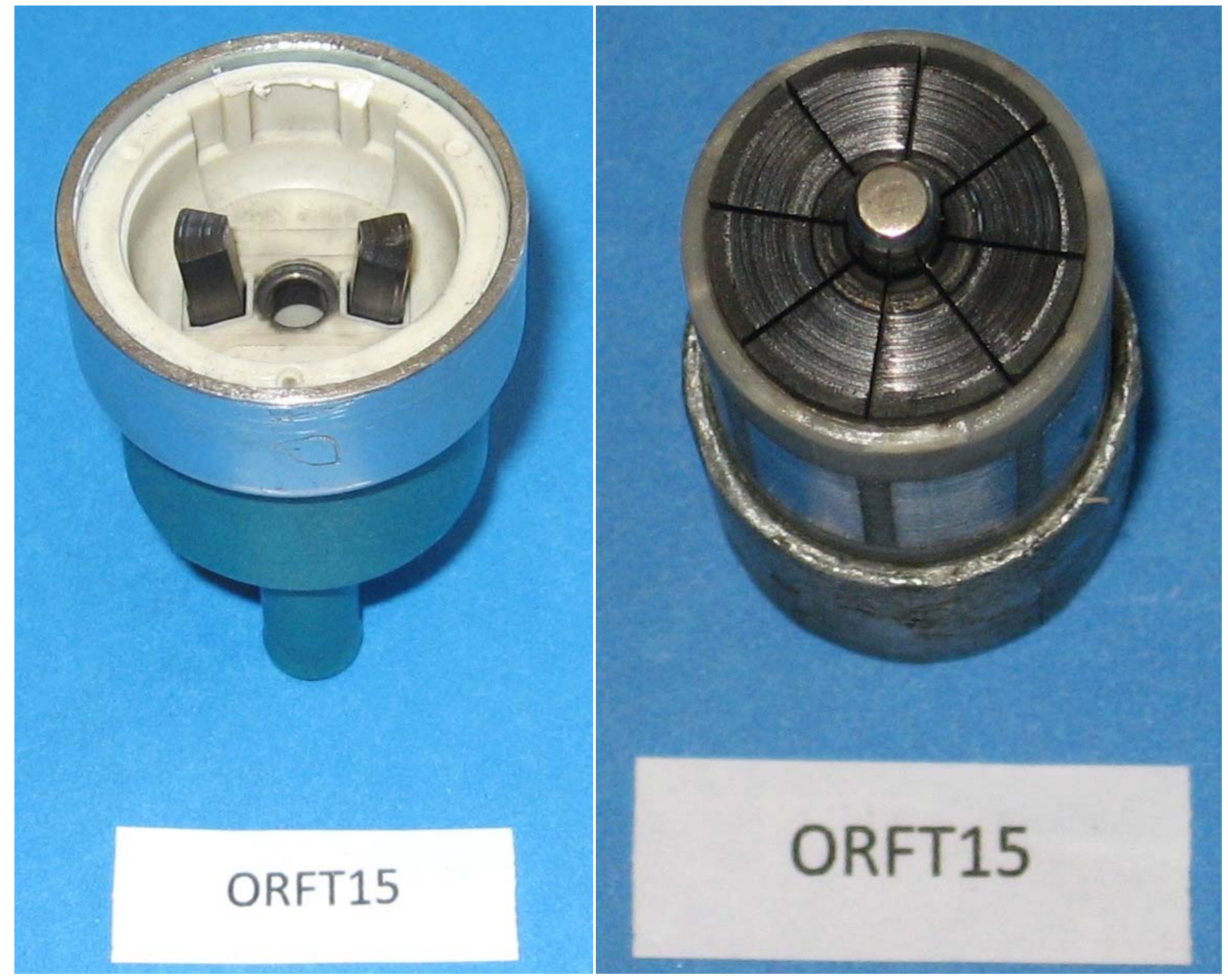

Figure F32. ORFT15 Fuel Pump Brushes (Left) and Commutator (Right). 


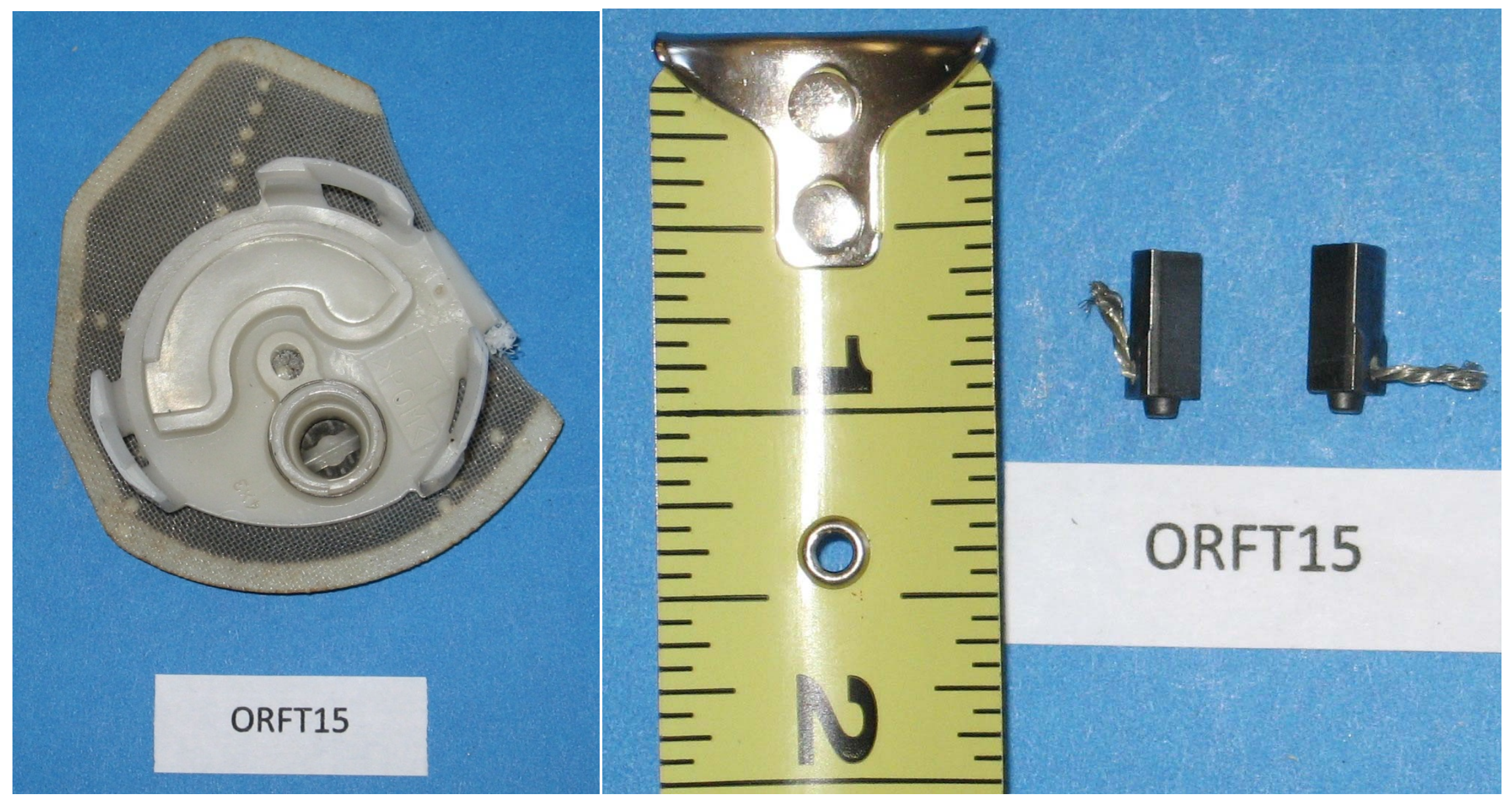

Figure F33. ORFT15 Fuel Pump Sock Filter (Left) and Brushes (Right). 


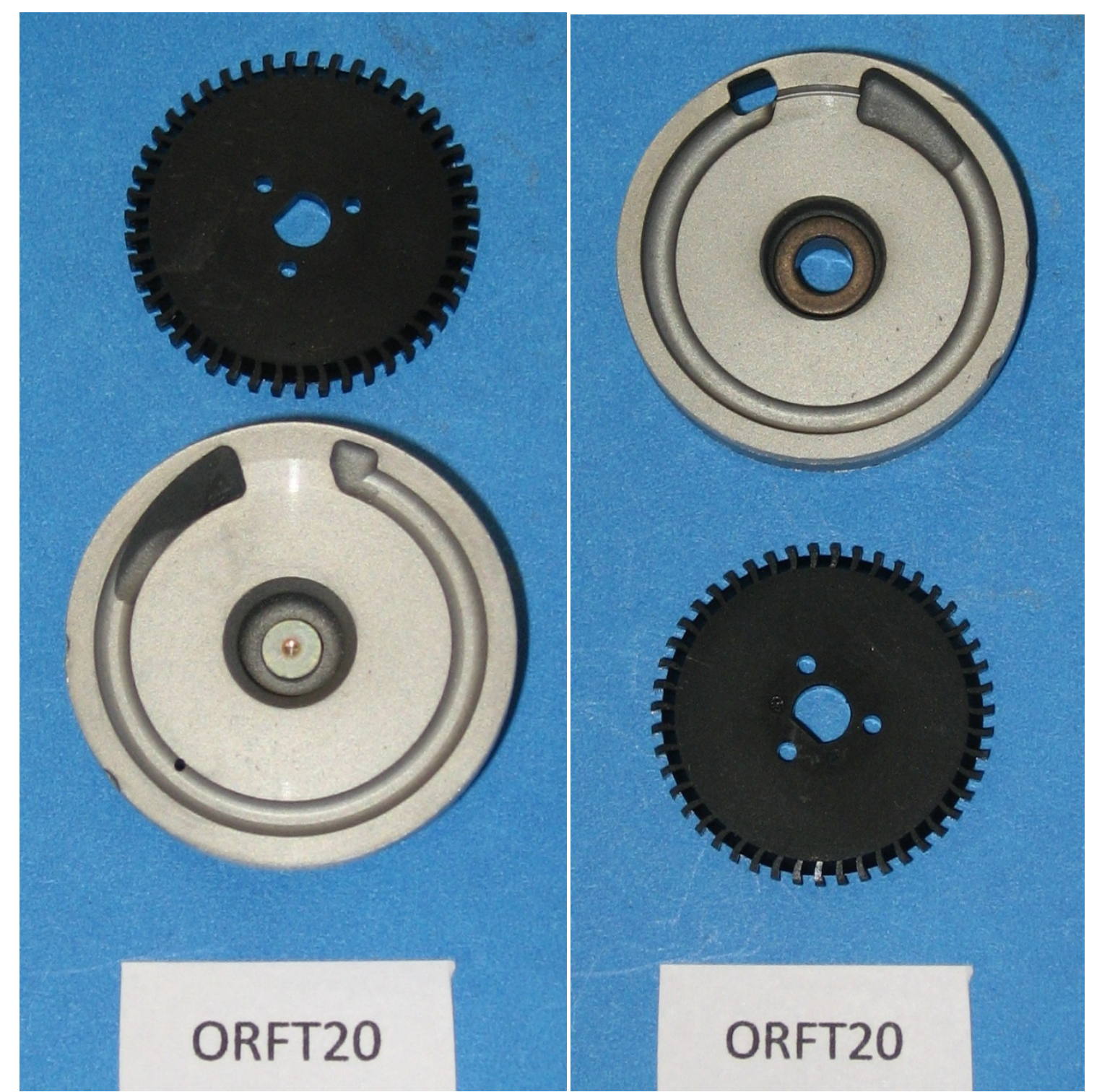

Figure F34. ORFT20 Fuel Pump Impeller with Inlet (Left) and Outlet (Right) Mating Cover. 


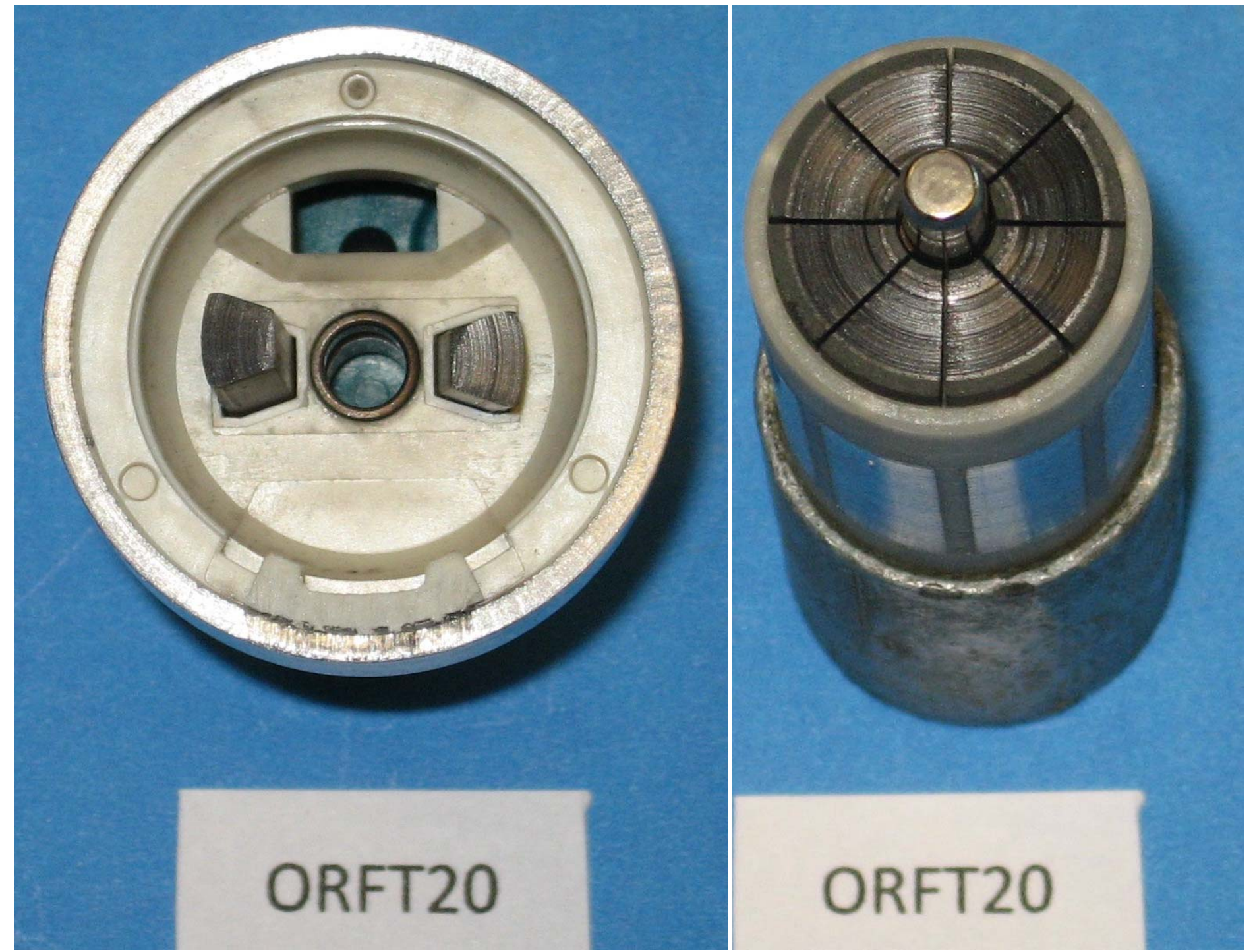

Figure F35. ORFT20 Fuel Pump Brushes (Left) and Commutator (Right). 


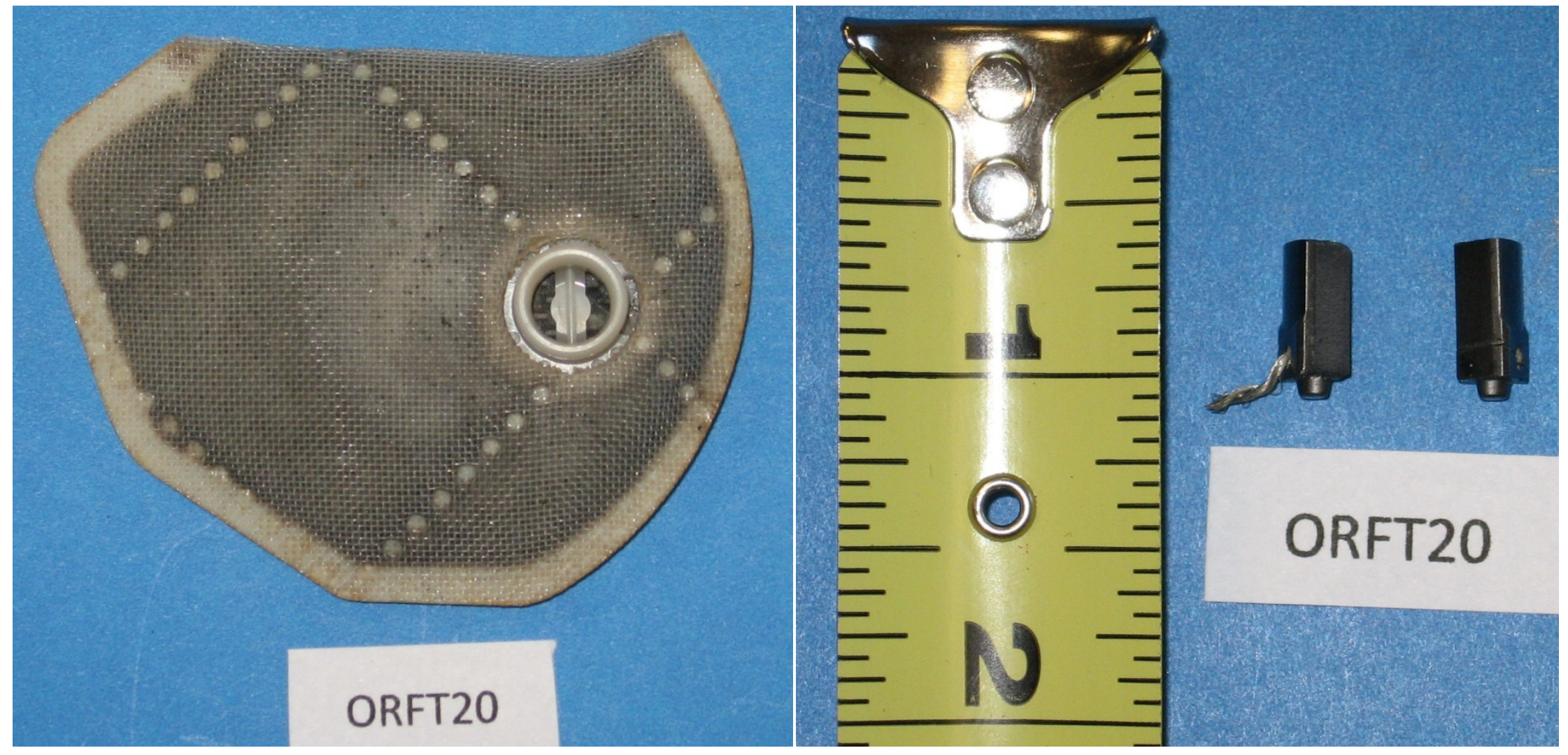

Figure F36. ORFT20 Fuel Pump Sock Filter (Left) and Brushes (Right). 


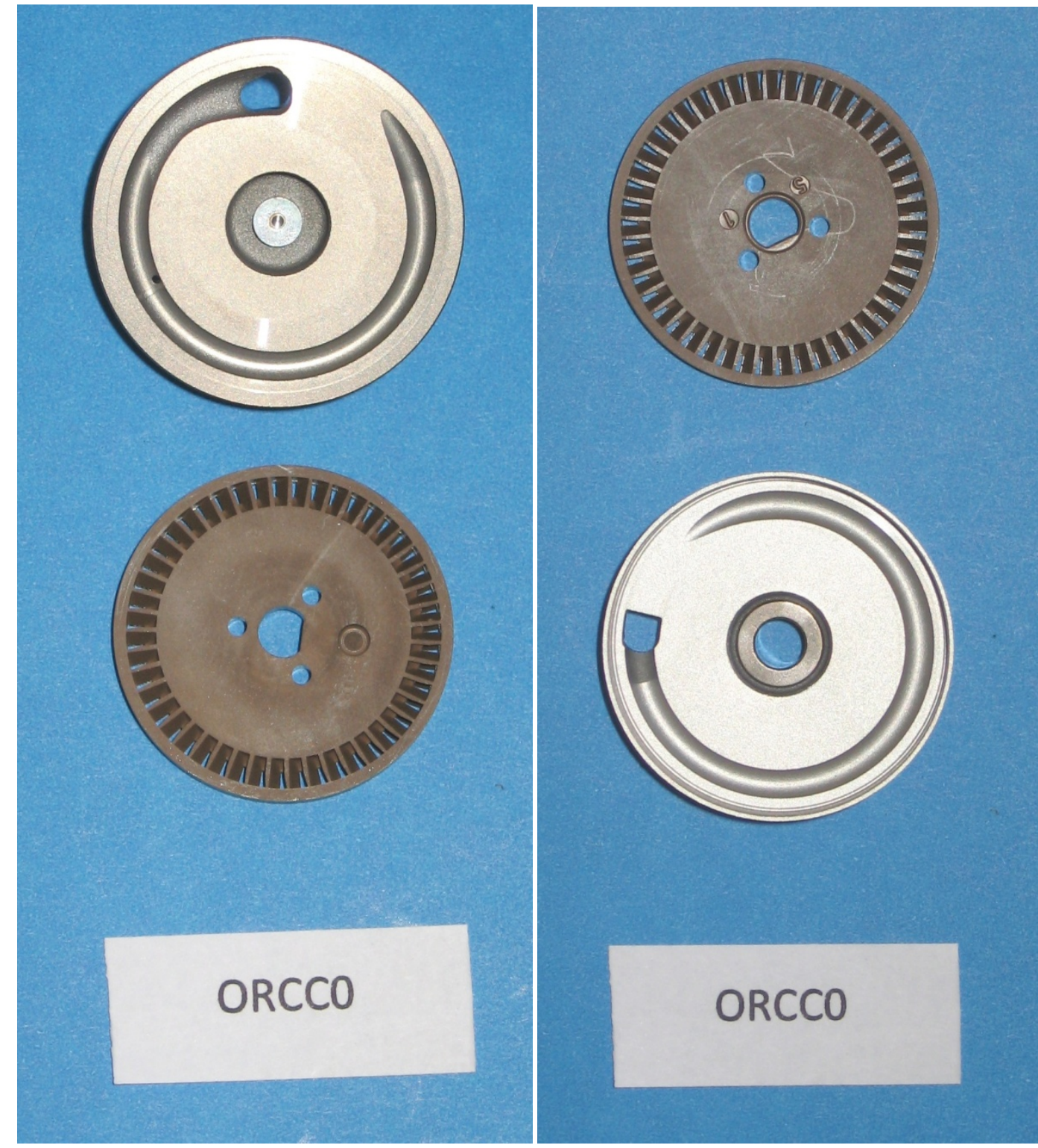

Figure F37. ORCC0 Fuel Pump Impeller with Inlet (Left) and Outlet (Right) Mating Cover. 


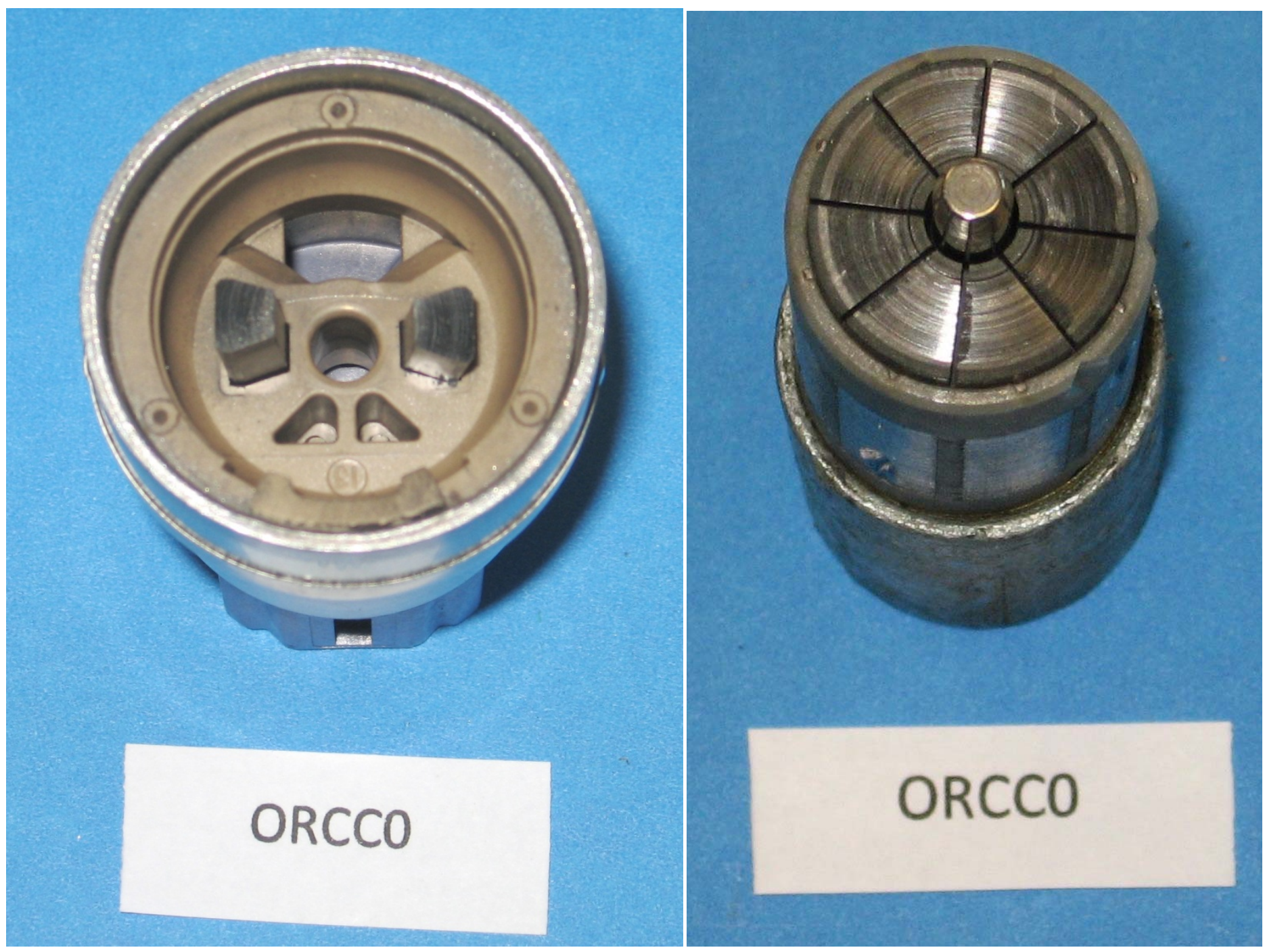

Figure F38. ORCC0 Fuel Pump Brushes (Left) and Commutator (Right). 


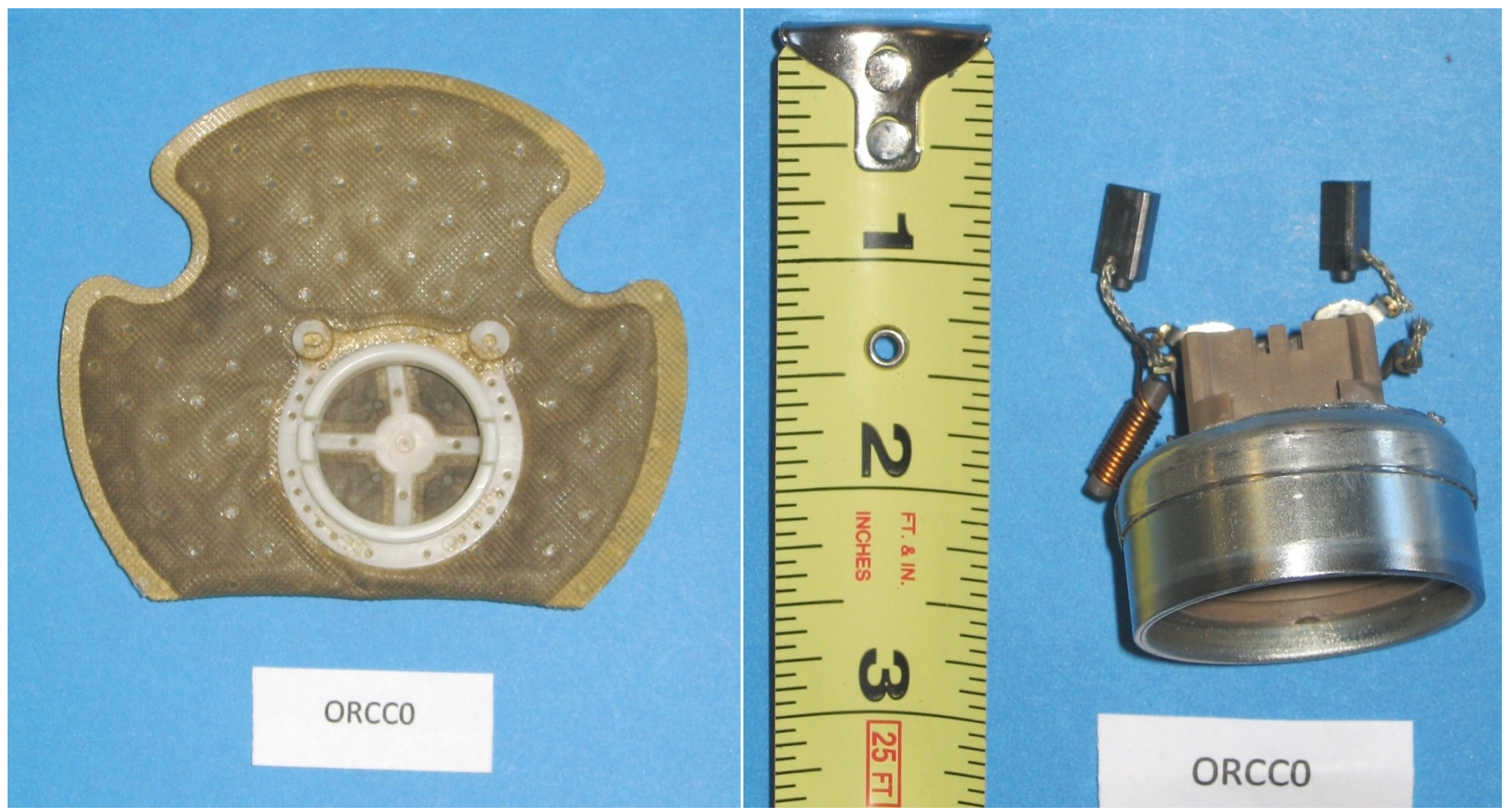

Figure F39. ORCC0 Fuel Pump Sock Filter (Left) and Brushes (Right). 


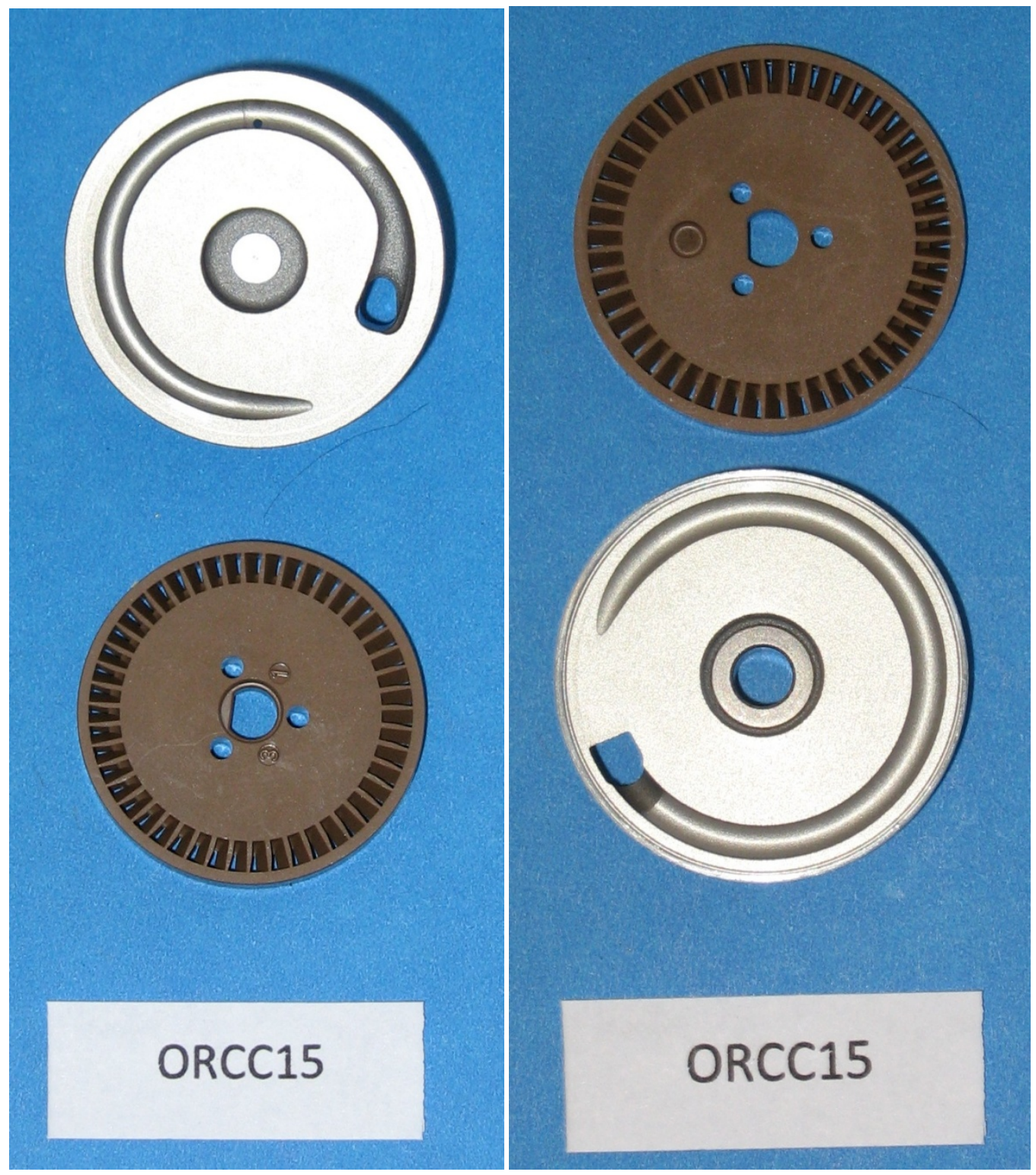

Figure F40. ORCC15 Fuel Pump Impeller with Inlet (Left) and Outlet (Right) Mating Cover. 


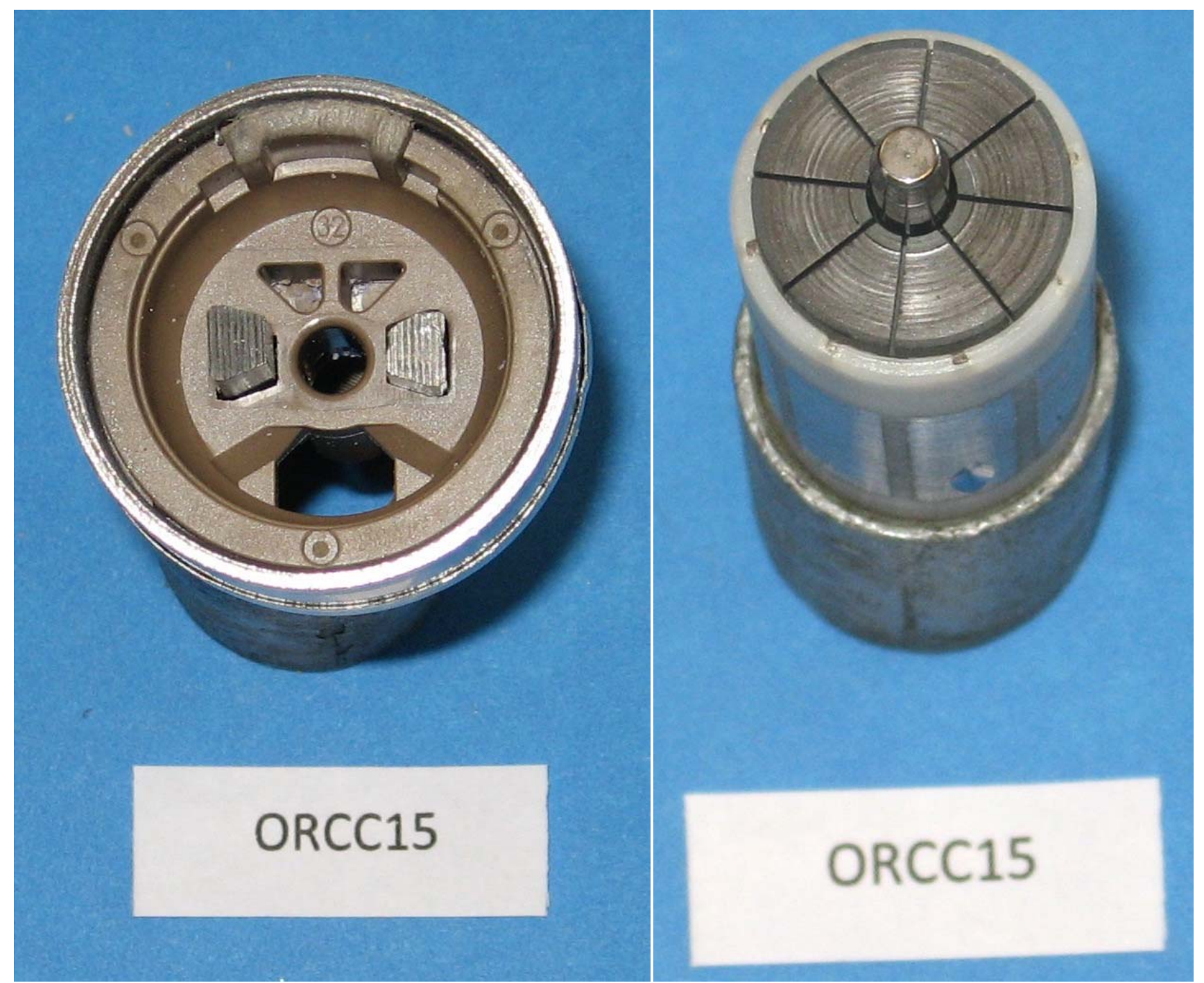

Figure F41. ORCC15 Fuel Pump Brushes (Left) and Commutator (Right). 


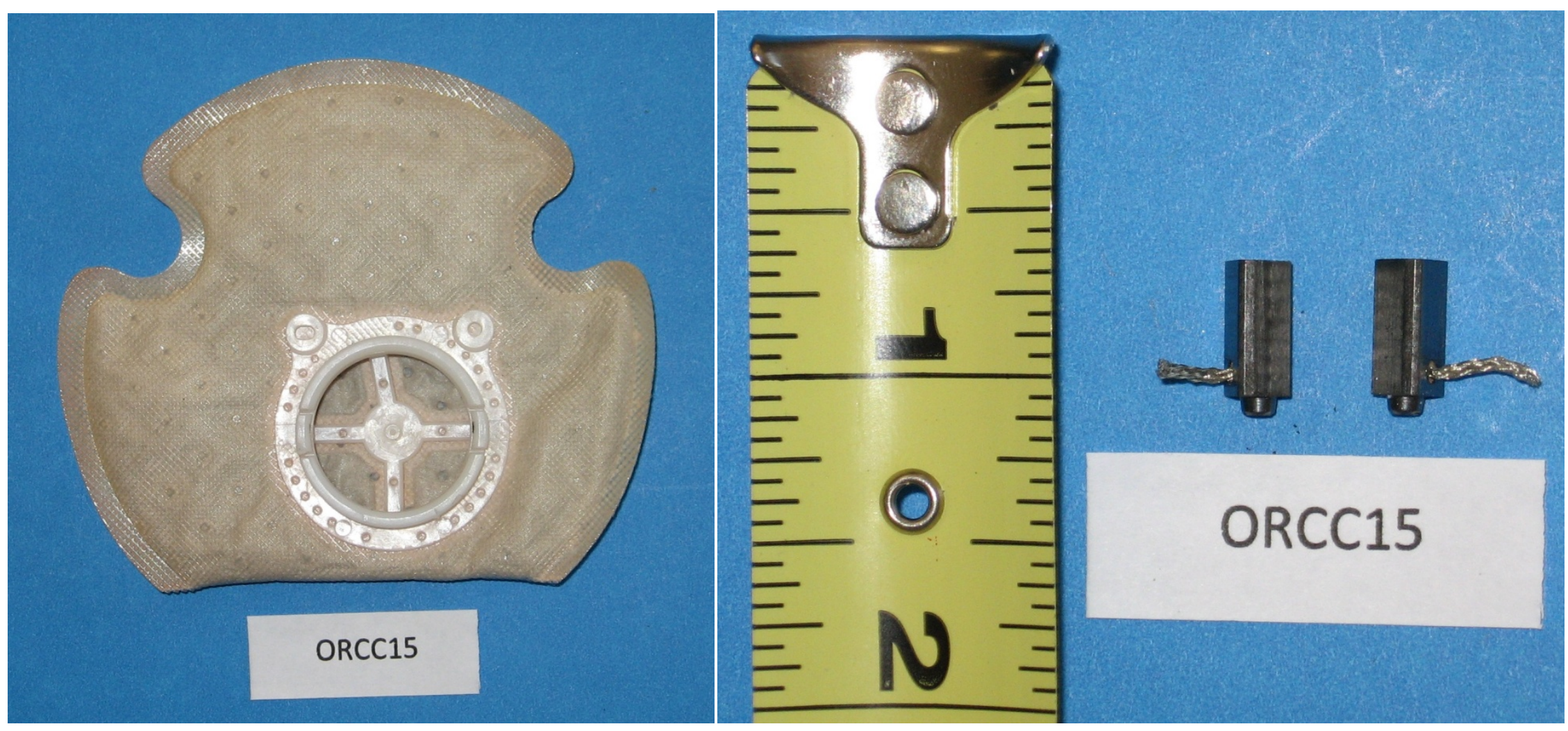

Figure F42. ORCC15 Fuel Pump Sock Filter (Left) and Brushes (Right). 


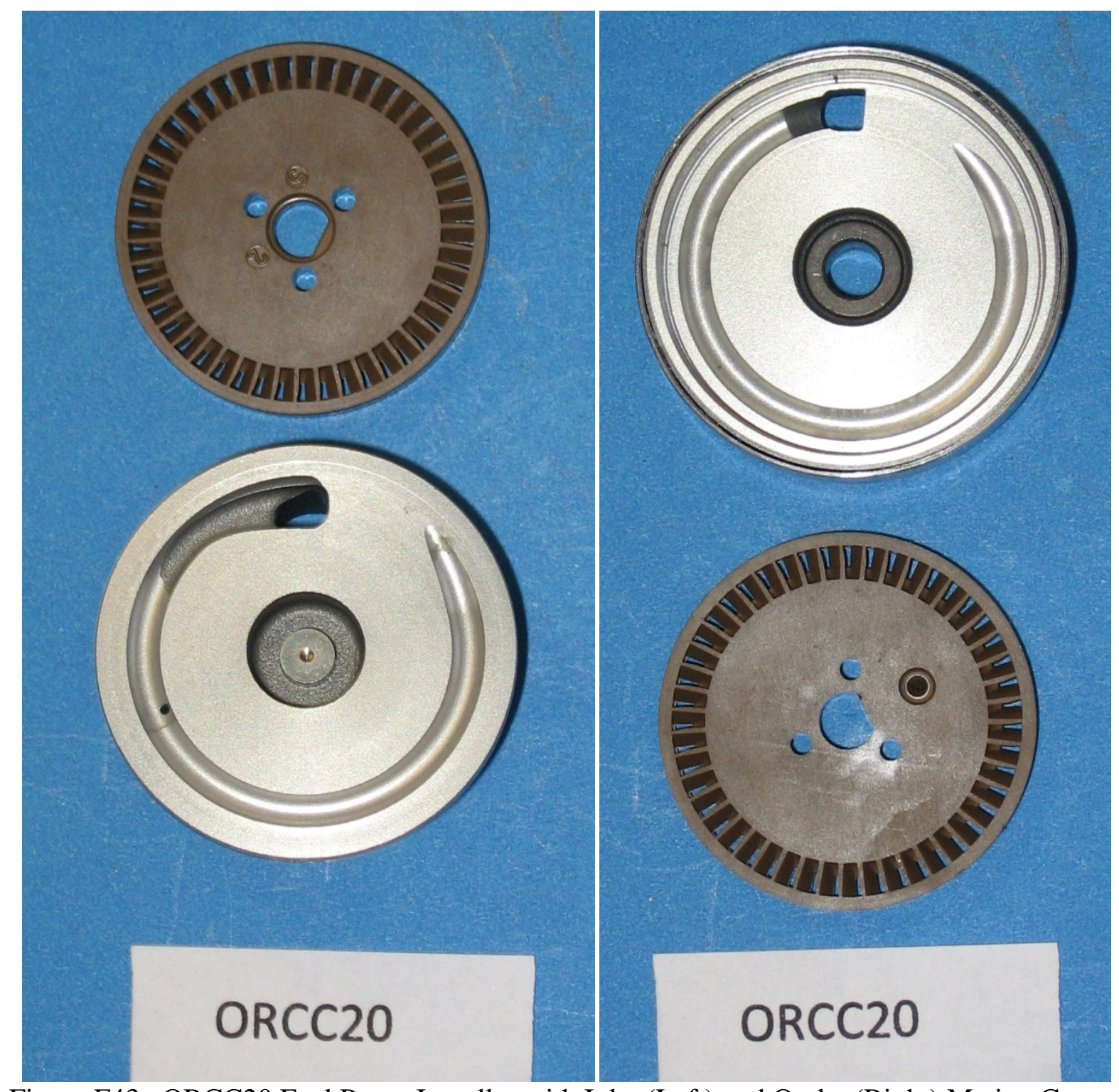

Figure F43. ORCC20 Fuel Pump Impeller with Inlet (Left) and Outlet (Right) Mating Cover. 


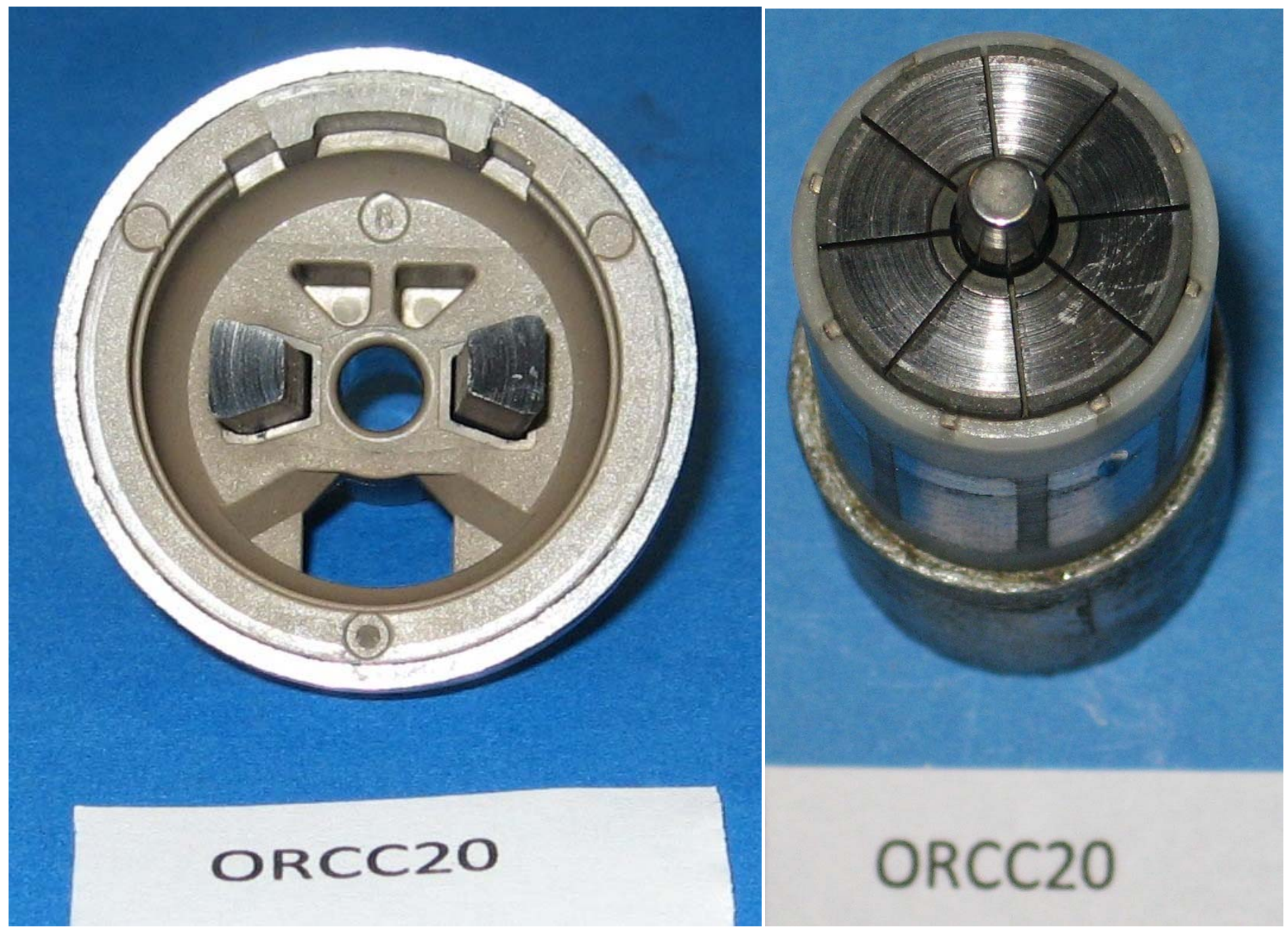

Figure F44. ORCC20 Fuel Pump Brushes (Left) and Commutator (Right). 


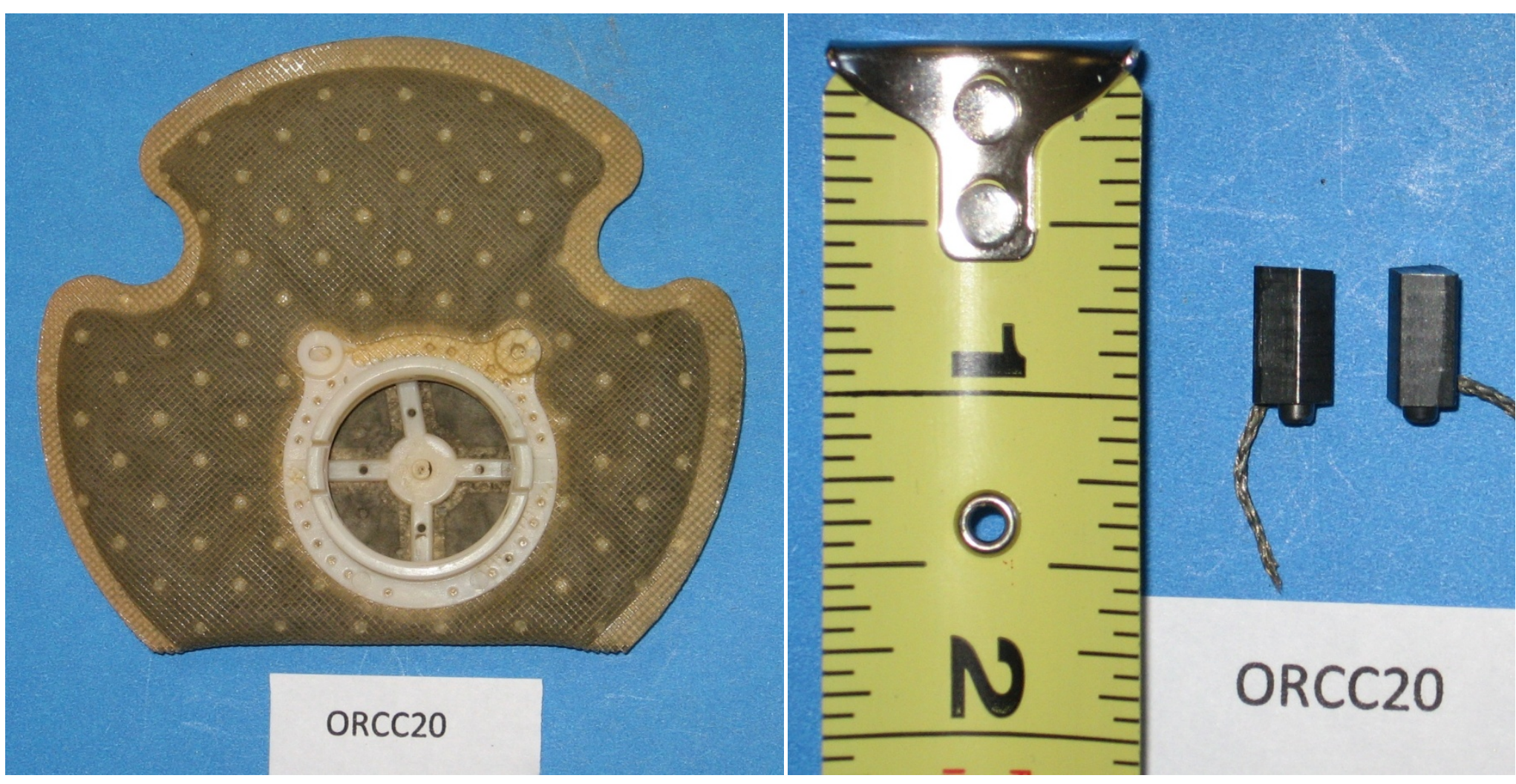

Figure F45. ORCC20 Fuel Pump Sock Filter (Left) and Brushes (Right). 


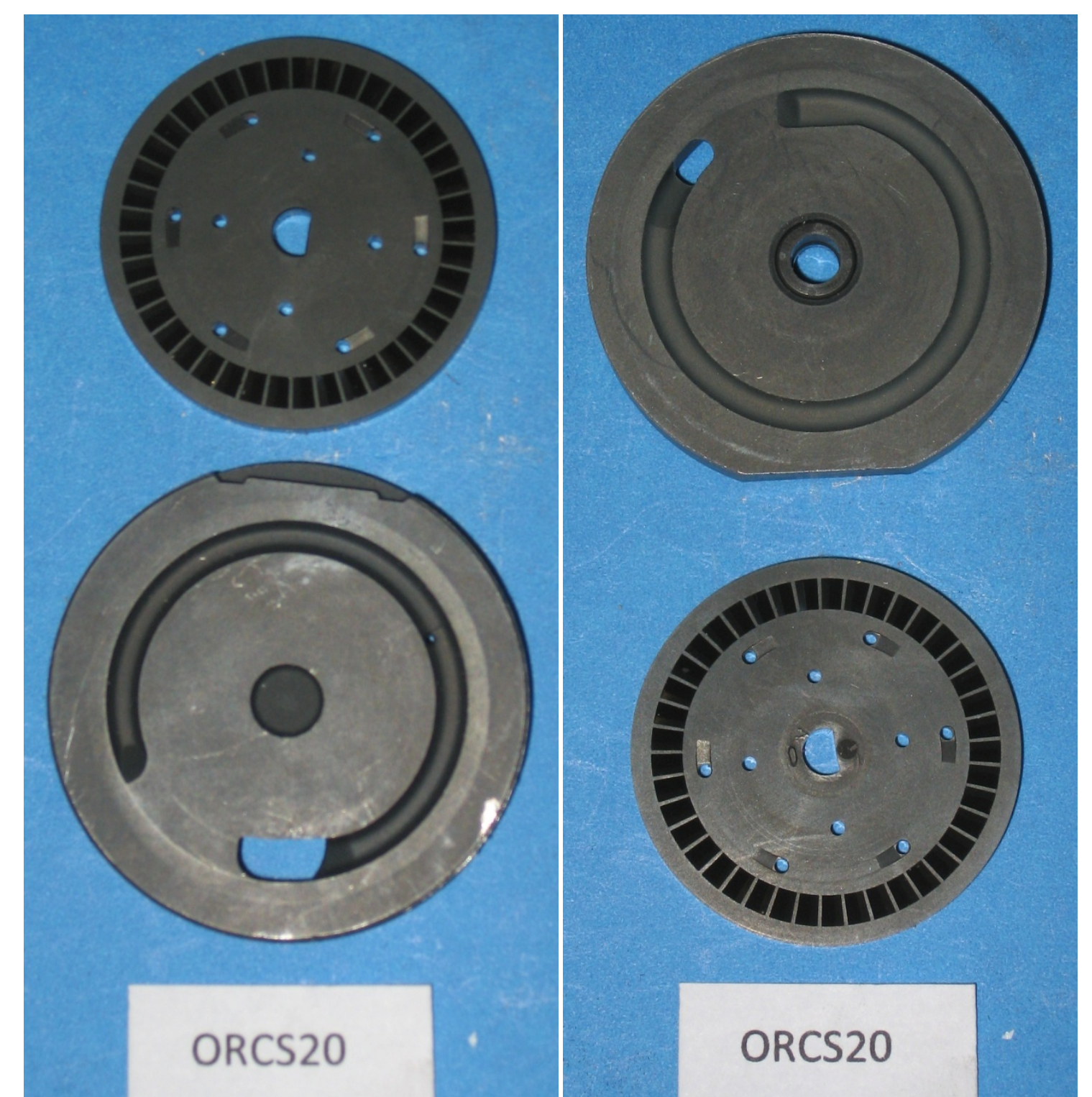

Figure F46. ORCS20 Fuel Pump Impeller with Inlet (Left) and Outlet (Right) Mating Cover. 


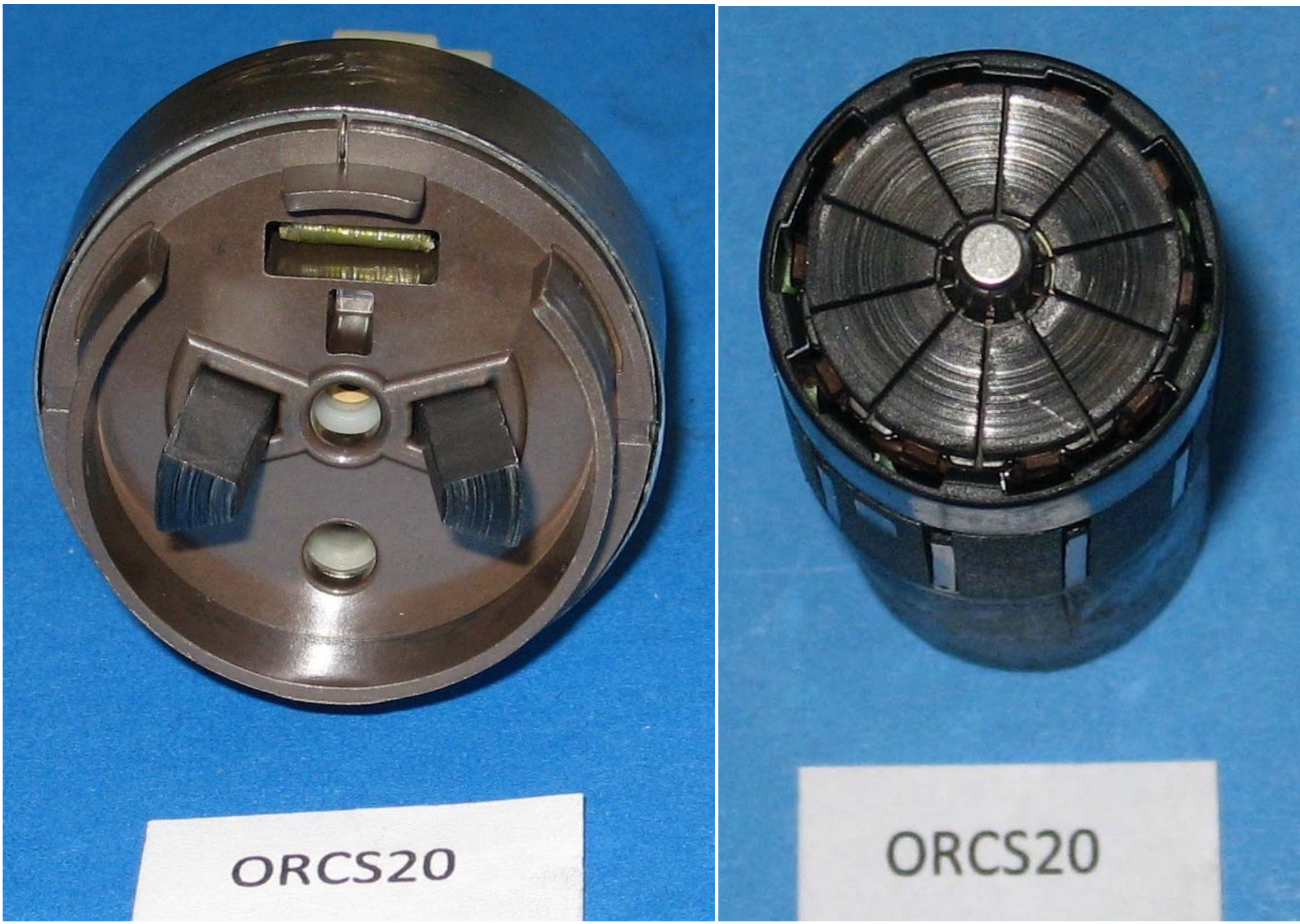

Figure F47. ORCS20 Fuel Pump Brushes (Left) and Commutator (Right). 


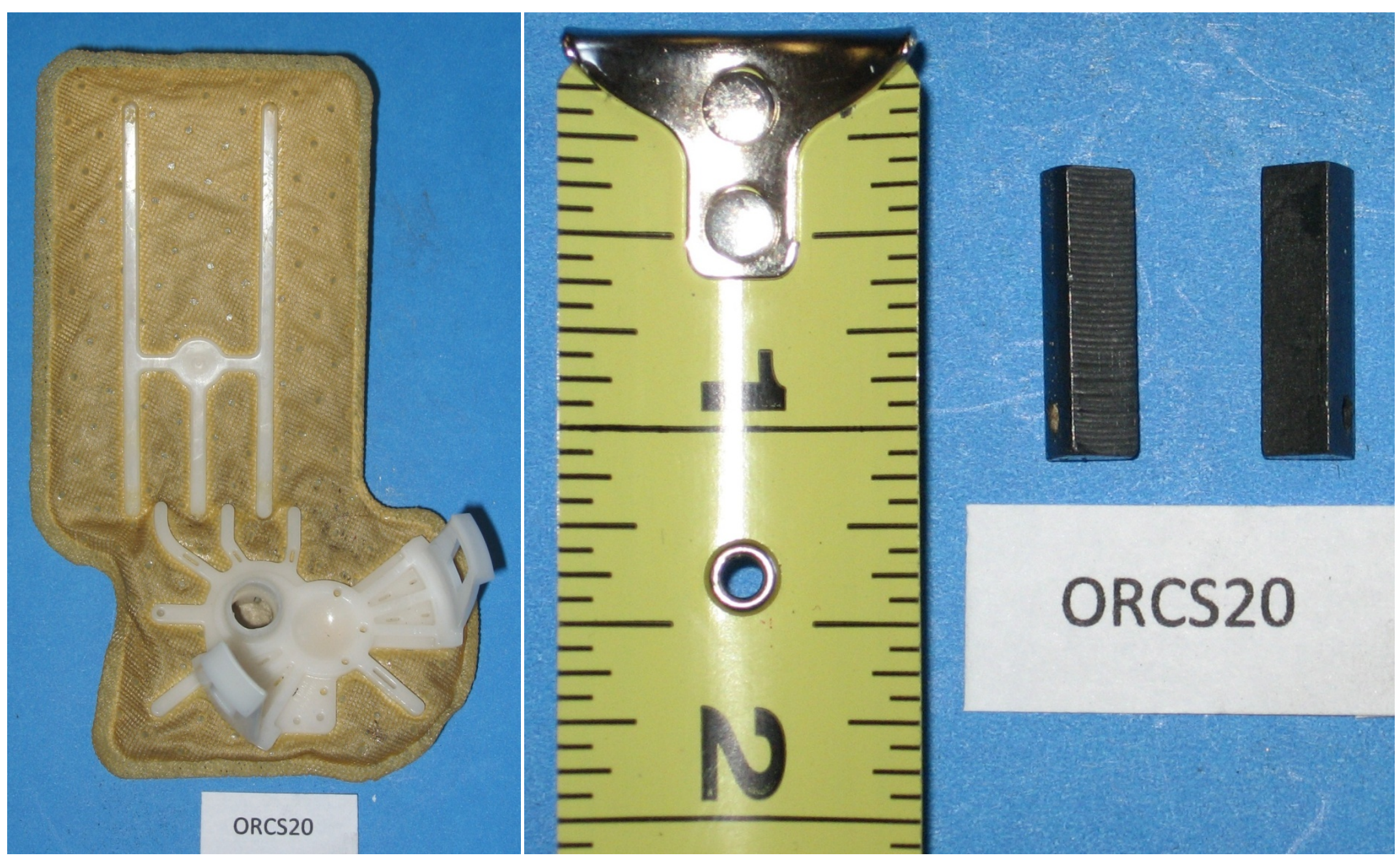

Figure F48. ORCS20 Fuel Pump Sock Filter (Left) and Brushes (Right). 


\section{Appendix G}

\section{Fuel Injector Flow Rates}



Nissan Altima

\begin{tabular}{|c|c|c|r|r|r|r|}
\hline & & & \multicolumn{4}{|l|}{} \\
\cline { 5 - 7 } Vehicle & Date & Cylinder & $\begin{array}{l}75 \% \text { DC } \\
\text { (gr/10sec) }\end{array}$ & $\begin{array}{l}35 \% \text { DC } \\
\text { (gr/10sec) }\end{array}$ & $\begin{array}{l}25 \% \text { DC } \\
(\mathrm{gr} / 10 \mathrm{sec})\end{array}$ & $\begin{array}{l}\text { Average } \\
(\mathrm{gr} / 10 \mathrm{sec})\end{array}$ \\
\hline ORNA0 & $7 / 22 / 2010$ & 1 & 29.6000 & 14.3550 & 10.4525 & 18.1358 \\
\hline ORNA0 & $7 / 22 / 2010$ & 2 & 29.6150 & 14.3100 & 10.4475 & 18.1242 \\
\hline ORNA0 & $7 / 22 / 2010$ & 3 & 29.6300 & 14.3900 & 10.5250 & 18.1817 \\
\hline ORNA0 & $7 / 22 / 2010$ & 4 & 29.2525 & 14.1125 & 10.3700 & 17.9117 \\
\hline ORNA15 & $7 / 30 / 2010$ & 1 & 29.6350 & 14.3200 & 10.5925 & 18.1825 \\
\hline ORNA15 & $7 / 30 / 2010$ & 2 & 29.6875 & 14.3800 & 10.4700 & 18.1792 \\
\hline ORNA15 & $7 / 30 / 2010$ & 3 & 29.6975 & 14.3575 & 10.5150 & 18.1900 \\
\hline ORNA15 & $7 / 30 / 2010$ & 4 & 29.7650 & 14.4525 & 10.5100 & 18.2425 \\
\hline ORNA20 & $9 / 22 / 2010$ & 1 & 29.6050 & 14.3575 & 10.5425 & 18.1683 \\
\hline ORNA20 & $9 / 22 / 2010$ & 2 & 29.8025 & 14.4800 & 10.6075 & 18.2967 \\
\hline ORNA20 & $9 / 22 / 2010$ & 3 & 29.8675 & 14.4950 & 10.6175 & 18.3267 \\
\hline ORNA20 & $9 / 22 / 2010$ & 4 & 29.5800 & 14.2775 & 10.4775 & 18.1117 \\
\hline
\end{tabular}




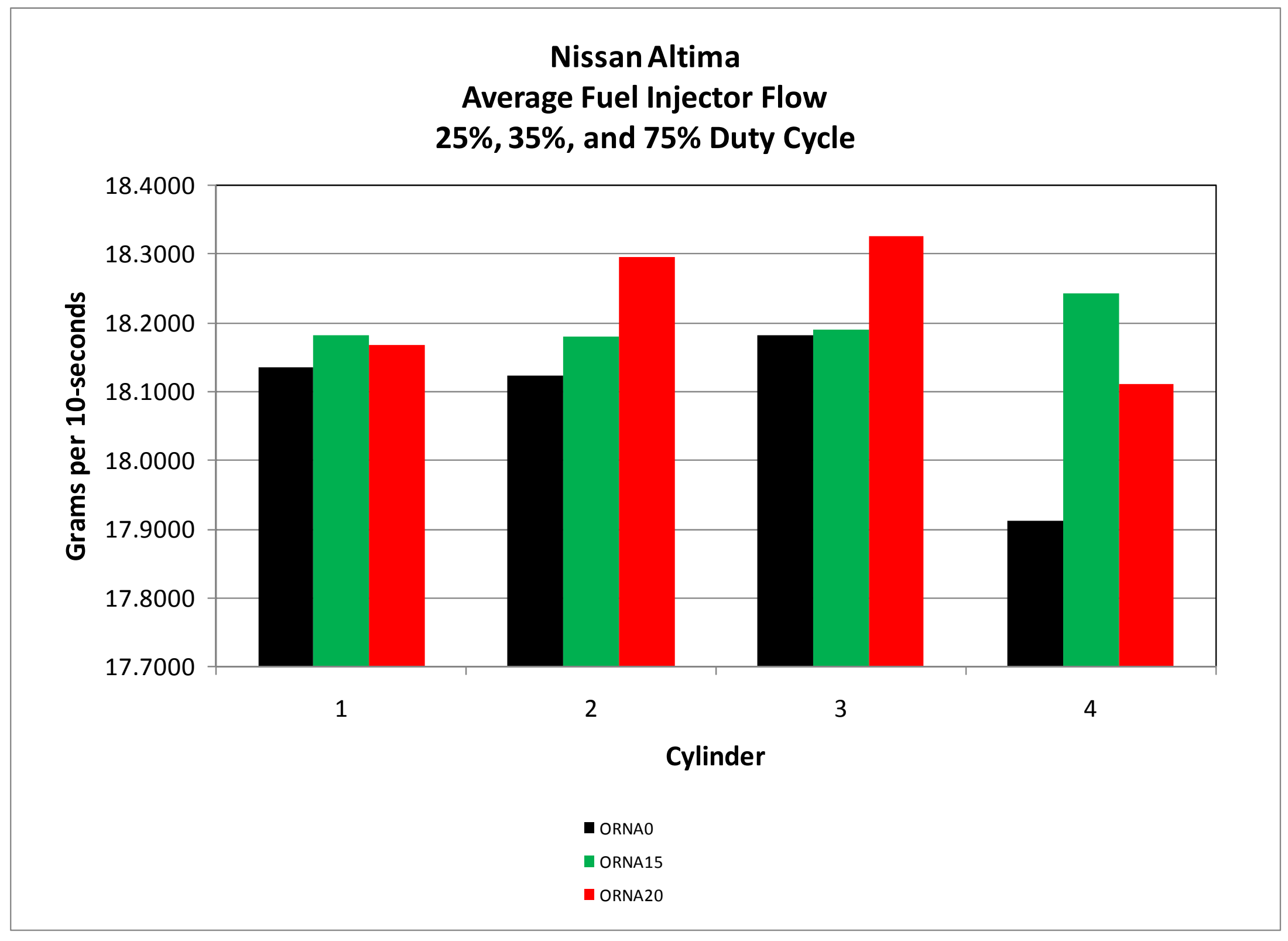




\section{Chevrolet Cobalt}

\begin{tabular}{|c|c|c|r|r|r|r|}
\hline & & & \multicolumn{4}{l|}{} \\
\cline { 4 - 7 } Vehicle & Date & Cylinder & $\begin{array}{l}75 \% \text { DC } \\
\text { (gr/10sec) }\end{array}$ & $\begin{array}{l}35 \% \text { DC } \\
(\mathrm{gr} / 10 \mathrm{sec})\end{array}$ & $\begin{array}{l}25 \% \text { DC } \\
(\mathrm{gr} / 10 \mathrm{sec})\end{array}$ & $\begin{array}{l}\text { Average } \\
(\mathrm{gr} / 10 \mathrm{sec})\end{array}$ \\
\hline ORCC0 & $8 / 5 / 2010$ & 1 & 25.6475 & 12.6675 & 9.3625 & 15.8925 \\
\hline ORCC0 & $8 / 5 / 2010$ & 2 & 25.8725 & 12.8550 & 9.5025 & 16.0767 \\
\hline ORCC0 & $8 / 5 / 2010$ & 3 & 25.8925 & 12.7400 & 9.4400 & 16.0242 \\
\hline ORCC0 & $8 / 5 / 2010$ & 4 & 25.7475 & 12.6750 & 9.3275 & 15.9167 \\
\hline ORCC15 & $8 / 5 / 2010$ & 1 & 26.3075 & 13.1800 & 9.7725 & 16.4200 \\
\hline ORCC15 & $8 / 5 / 2010$ & 2 & 26.0700 & 12.9925 & 9.6200 & 16.2275 \\
\hline ORCC15 & $8 / 5 / 2010$ & 3 & 26.3050 & 13.1800 & 9.8300 & 16.4383 \\
\hline ORCC15 & $8 / 5 / 2010$ & 4 & 26.0850 & 12.9775 & 9.6350 & 16.2325 \\
\hline ORCC20 & $9 / 22 / 2010$ & 1 & 25.8450 & 12.9350 & 9.6000 & 16.1267 \\
\hline ORCC20 & $9 / 22 / 2010$ & 2 & 25.6750 & 12.7900 & 9.4500 & 15.9717 \\
\hline ORCC20 & $9 / 22 / 2010$ & 3 & 26.0575 & 13.0050 & 9.5775 & 16.2133 \\
\hline ORCC20 & $9 / 22 / 2010$ & 4 & 26.0750 & 13.0150 & 9.6525 & 16.2475 \\
\hline
\end{tabular}




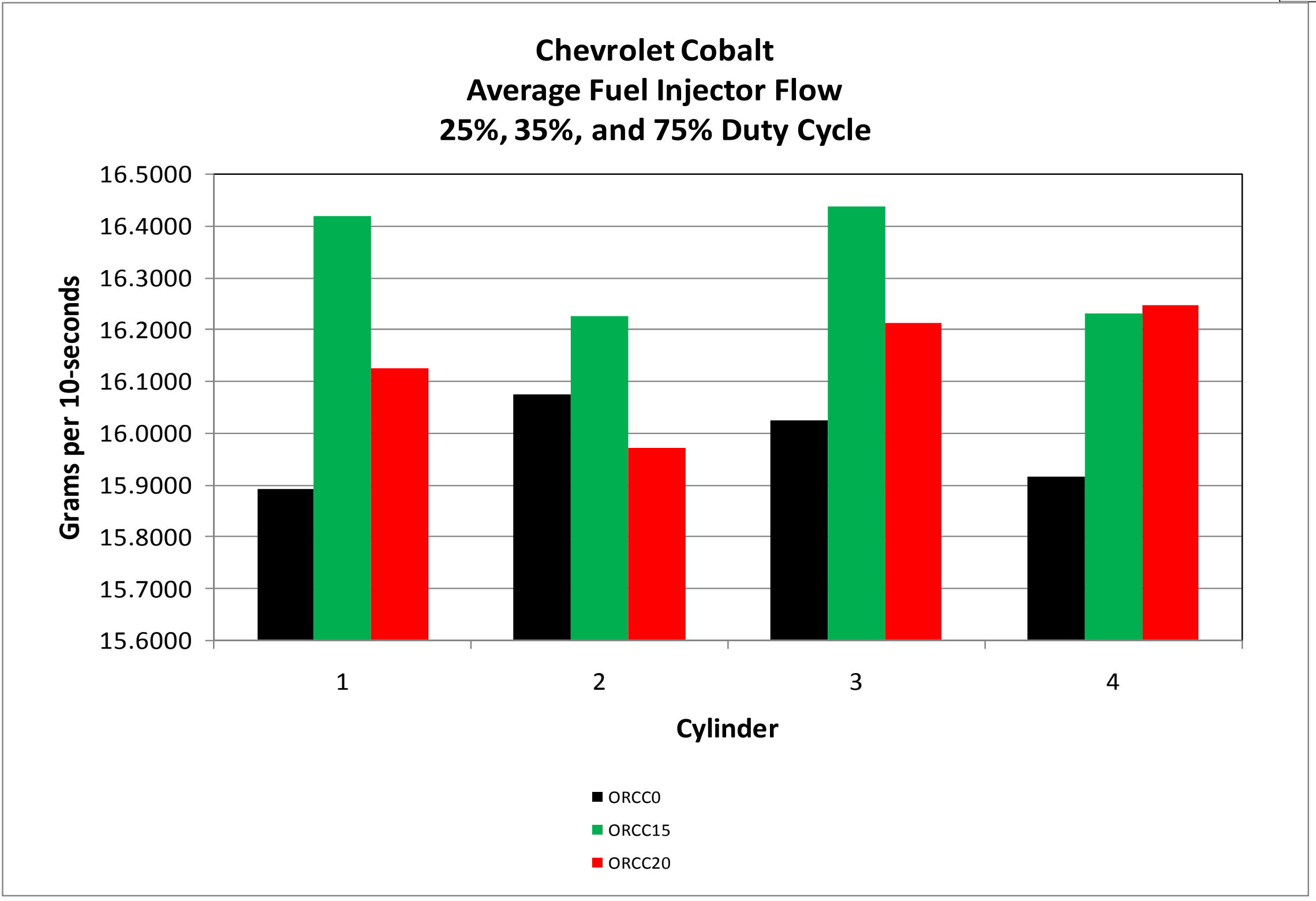


Ford Taurus

\begin{tabular}{|c|c|c|r|r|r|r|}
\hline & & & \multicolumn{5}{|l|}{} \\
\cline { 4 - 7 } Vehicle & Date & Cylinder & $\begin{array}{l}75 \% \text { DC } \\
\text { (gr/10sec) }\end{array}$ & $\begin{array}{l}35 \% \text { DC } \\
\text { (gr/10sec) }\end{array}$ & $\begin{array}{l}25 \% \text { DC } \\
\text { (gr/10sec) }\end{array}$ & $\begin{array}{l}\text { Average } \\
\text { (gr/10sec) }\end{array}$ \\
\hline ORFT0 & $8 / 5 / 2010$ & 1 & 26.9475 & 12.9100 & 9.4175 & 16.4250 \\
\hline ORFT0 & $8 / 5 / 2010$ & 2 & 26.9525 & 12.9350 & 9.4750 & 16.4542 \\
\hline ORFT0 & $8 / 5 / 2010$ & 3 & 26.9425 & 12.9675 & 9.4425 & 16.4508 \\
\hline ORFT0 & $8 / 5 / 2010$ & 4 & 26.9450 & 12.8750 & 9.4150 & 16.4117 \\
\hline ORFT0 & $8 / 5 / 2010$ & 5 & 26.9425 & 12.8425 & 9.3675 & 16.3842 \\
\hline ORFT0 & $8 / 5 / 2010$ & 6 & 27.0600 & 12.9200 & 9.4375 & 16.4725 \\
\hline ORFT15 & $8 / 6 / 2010$ & 1 & 26.7475 & 12.8625 & 9.4000 & 16.3367 \\
\hline ORFT15 & $8 / 6 / 2010$ & 2 & 26.6850 & 12.8475 & 9.3850 & 16.3058 \\
\hline ORFT15 & $8 / 6 / 2010$ & 3 & 26.6925 & 12.8125 & 9.3800 & 16.2950 \\
\hline ORFT15 & $8 / 6 / 2010$ & 4 & 26.6925 & 12.7950 & 9.3050 & 16.2642 \\
\hline ORFT15 & $8 / 6 / 2010$ & 5 & 26.7750 & 12.8175 & 9.3125 & 16.3017 \\
\hline ORFT15 & $8 / 6 / 2010$ & 6 & 26.8475 & 12.8475 & 9.3450 & 16.3467 \\
\hline ORFT20 & $9 / 24 / 2010$ & 1 & 27.2175 & 13.1000 & 9.5725 & 16.6300 \\
\hline ORFT20 & $9 / 24 / 2010$ & 2 & 26.9775 & 12.9875 & 9.4900 & 16.4850 \\
\hline ORFT20 & $9 / 24 / 2010$ & 3 & 26.8650 & 12.8925 & 9.4125 & 16.3900 \\
\hline ORFT20 & $9 / 24 / 2010$ & 4 & 26.8750 & 12.9575 & 9.4175 & 16.4167 \\
\hline ORFT20 & $9 / 24 / 2010$ & 5 & 27.0100 & 12.9425 & 9.4300 & 16.4608 \\
\hline ORFT20 & $9 / 24 / 2010$ & 6 & 27.0775 & 12.9300 & 9.4250 & 16.4775 \\
\hline
\end{tabular}




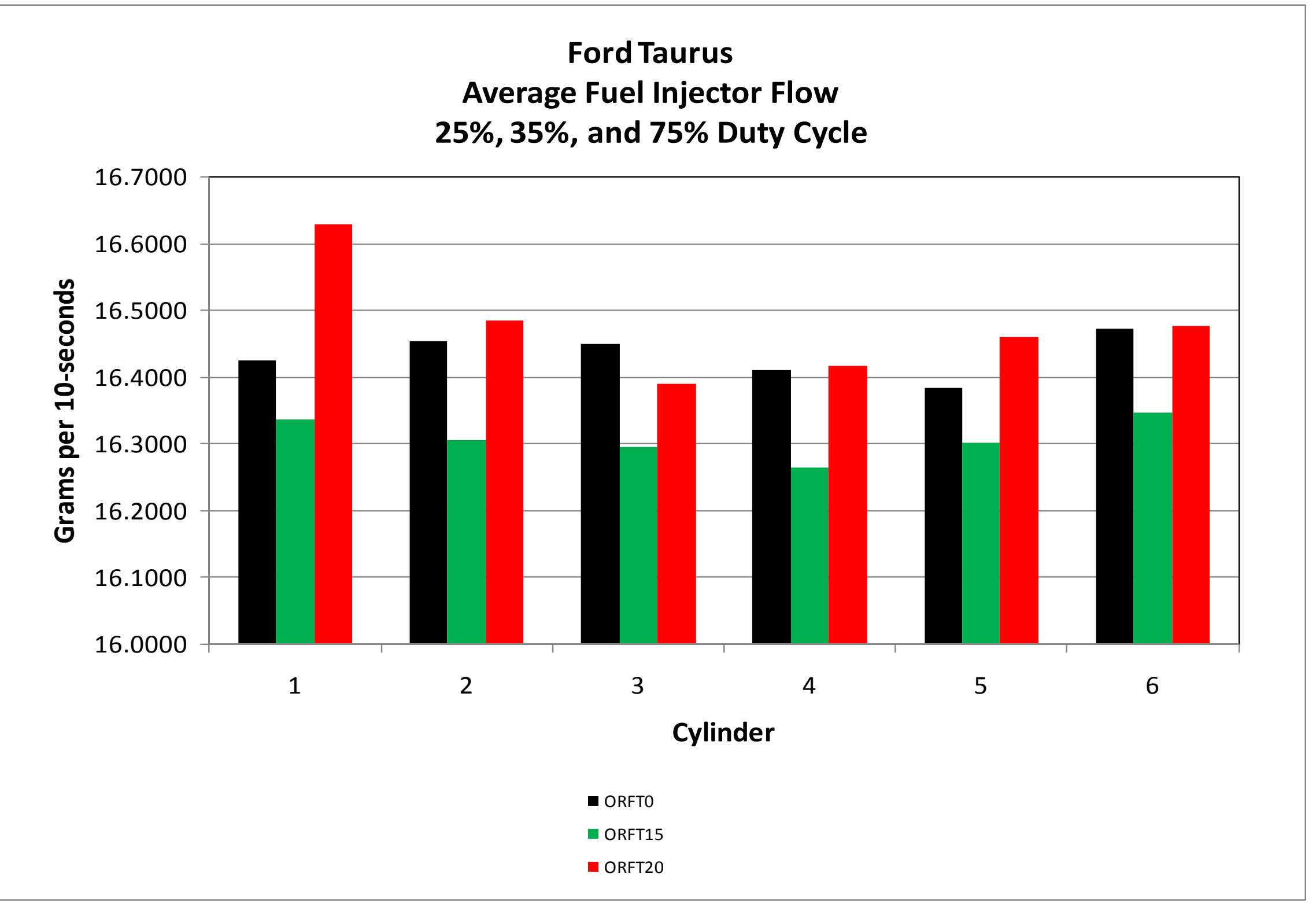


Honda Accord

\begin{tabular}{|c|c|c|r|r|r|r|}
\hline & & & \multicolumn{4}{|l|}{} \\
\cline { 4 - 7 } Vehicle & Date & Cylinder & $\begin{array}{l}75 \% \text { DC } \\
\text { (gr/10sec) }\end{array}$ & $\begin{array}{l}35 \% \text { DC } \\
(\mathrm{gr} / 10 \mathrm{sec})\end{array}$ & $\begin{array}{l}25 \% \text { DC } \\
(\mathrm{gr} / 10 \mathrm{sec})\end{array}$ & $\begin{array}{l}\text { Average } \\
(\mathrm{gr} / 10 \mathrm{sec})\end{array}$ \\
\hline ORHA0 & $8 / 6 / 2010$ & 1 & 23.5600 & 11.3150 & 8.2675 & 14.3808 \\
\hline ORHA0 & $8 / 6 / 2010$ & 2 & 23.5000 & 11.2425 & 8.1625 & 14.3017 \\
\hline ORHA0 & $8 / 6 / 2010$ & 3 & 23.6550 & 11.3350 & 8.2600 & 14.4167 \\
\hline ORHA0 & $8 / 6 / 2010$ & 4 & 23.6450 & 11.2575 & 8.1975 & 14.3667 \\
\hline ORHA15 & $8 / 6 / 2010$ & 1 & 23.5475 & 11.2000 & 8.1375 & 14.2950 \\
\hline ORHA15 & $8 / 6 / 2010$ & 2 & 23.5150 & 11.1700 & 8.1375 & 14.2742 \\
\hline ORHA15 & $8 / 6 / 2010$ & 3 & 23.4300 & 11.1575 & 8.1300 & 14.2392 \\
\hline ORHA15 & $8 / 6 / 2010$ & 4 & 23.7525 & 11.2325 & 8.2000 & 14.3950 \\
\hline ORHA20 & $9 / 22 / 2010$ & 1 & 23.5375 & 11.2325 & 8.1700 & 14.3133 \\
\hline ORHA20 & $9 / 22 / 2010$ & 2 & 23.3575 & 11.1475 & 8.1375 & 14.2142 \\
\hline ORHA20 & $9 / 22 / 2010$ & 3 & 23.5125 & 11.2275 & 8.1950 & 14.3117 \\
\hline ORHA20 & $9 / 22 / 2010$ & 4 & 23.5250 & 11.2175 & 8.1775 & 14.3067 \\
\hline
\end{tabular}




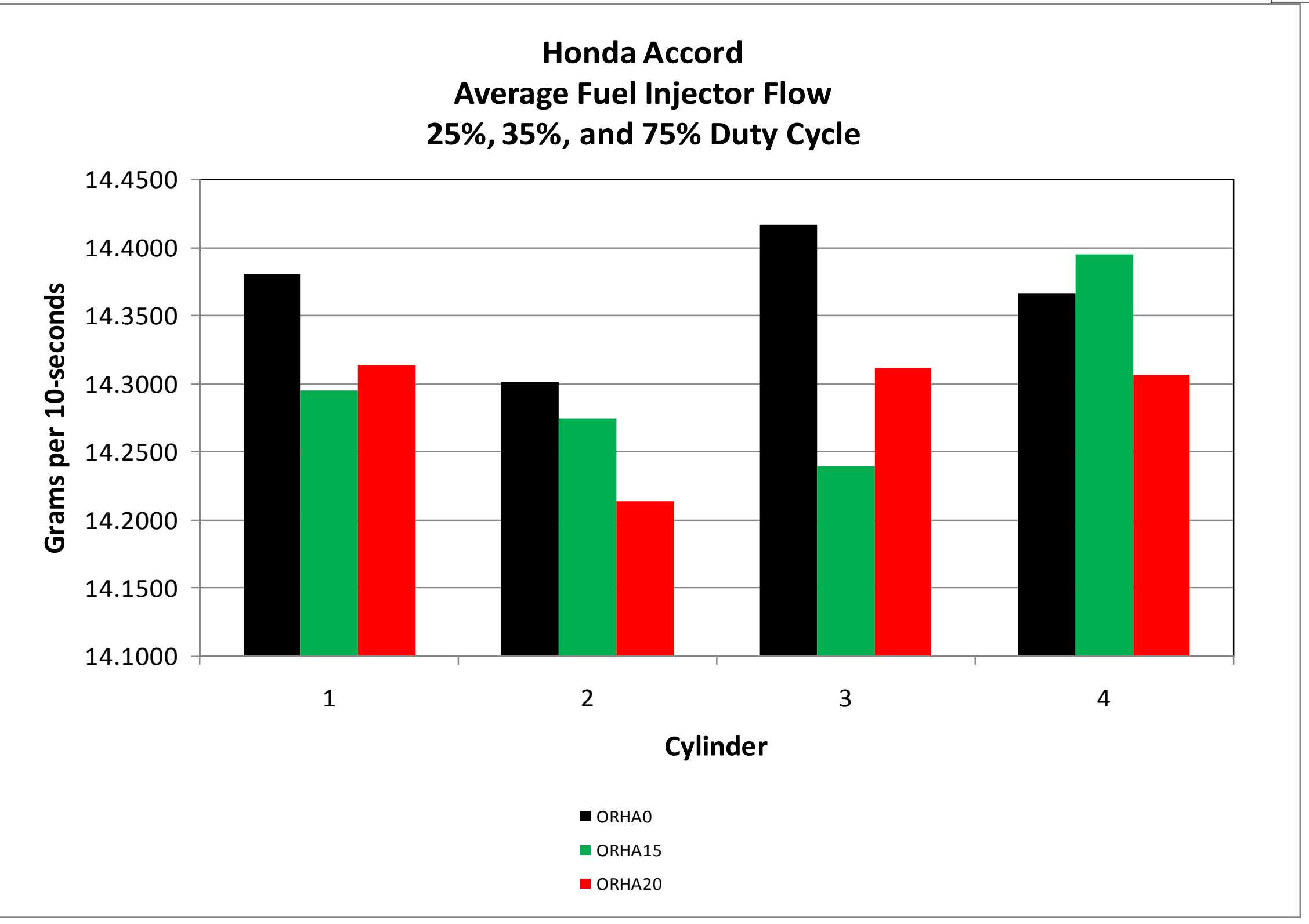

G-8 of 12 
Dodge Caravan

\begin{tabular}{|c|c|c|c|c|c|c|}
\hline ORDC0 & $8 / 9 / 2010$ & 1 & 23.8650 & 11.6100 & 8.5925 & 14.6892 \\
\hline ORDC0 & $8 / 9 / 2010$ & 2 & 23.5250 & 11.4625 & 8.4950 & 14.4942 \\
\hline ORDC0 & $8 / 9 / 2010$ & 3 & 23.5400 & 11.4575 & 8.4400 & 14.4792 \\
\hline ORDC0 & $8 / 9 / 2010$ & 4 & 23.8275 & 11.6150 & 8.6100 & 14.6842 \\
\hline ORDC0 & $8 / 9 / 2010$ & 5 & 23.8350 & 11.6125 & 8.5850 & 14.6775 \\
\hline ORDC0 & $8 / 9 / 2010$ & 6 & 23.7475 & 11.5750 & 8.5200 & 14.6142 \\
\hline ORDC15 & $8 / 9 / 2010$ & 1 & 23.7575 & 11.5925 & 8.6025 & 14.6508 \\
\hline ORDC15 & $8 / 9 / 2010$ & 2 & 23.7950 & 11.6250 & 8.6200 & 14.6800 \\
\hline ORDC15 & $8 / 9 / 2010$ & 3 & 23.6175 & 11.5500 & 8.5175 & 14.5617 \\
\hline ORDC15 & $8 / 9 / 2010$ & 4 & 24.1100 & 11.8525 & 8.8050 & 14.9225 \\
\hline ORDC15 & $8 / 9 / 2010$ & 5 & 23.8600 & 11.7925 & 8.7750 & 14.8092 \\
\hline ORDC15 & $8 / 9 / 2010$ & 6 & 24.0475 & 11.8475 & 8.8300 & 14.9083 \\
\hline ORDC20B & $9 / 24 / 2010$ & 1 & 23.6975 & 11.5875 & 8.5900 & 14.6250 \\
\hline ORDC20B & $9 / 24 / 2010$ & 2 & 23.4775 & 11.4800 & 8.4900 & 14.4825 \\
\hline ORDC20B & $9 / 24 / 2010$ & 3 & 23.8700 & 11.6825 & 8.6725 & 14.7417 \\
\hline ORDC20B & $9 / 24 / 2010$ & 4 & 23.8325 & 11.6400 & 8.5950 & 14.6892 \\
\hline ORDC20B & $9 / 24 / 2010$ & 5 & 23.9200 & 11.6650 & 8.6350 & 14.7400 \\
\hline ORDC20B & $9 / 24 / 2010$ & 6 & 23.7300 & 11.6100 & 8.5425 & 14.6275 \\
\hline
\end{tabular}




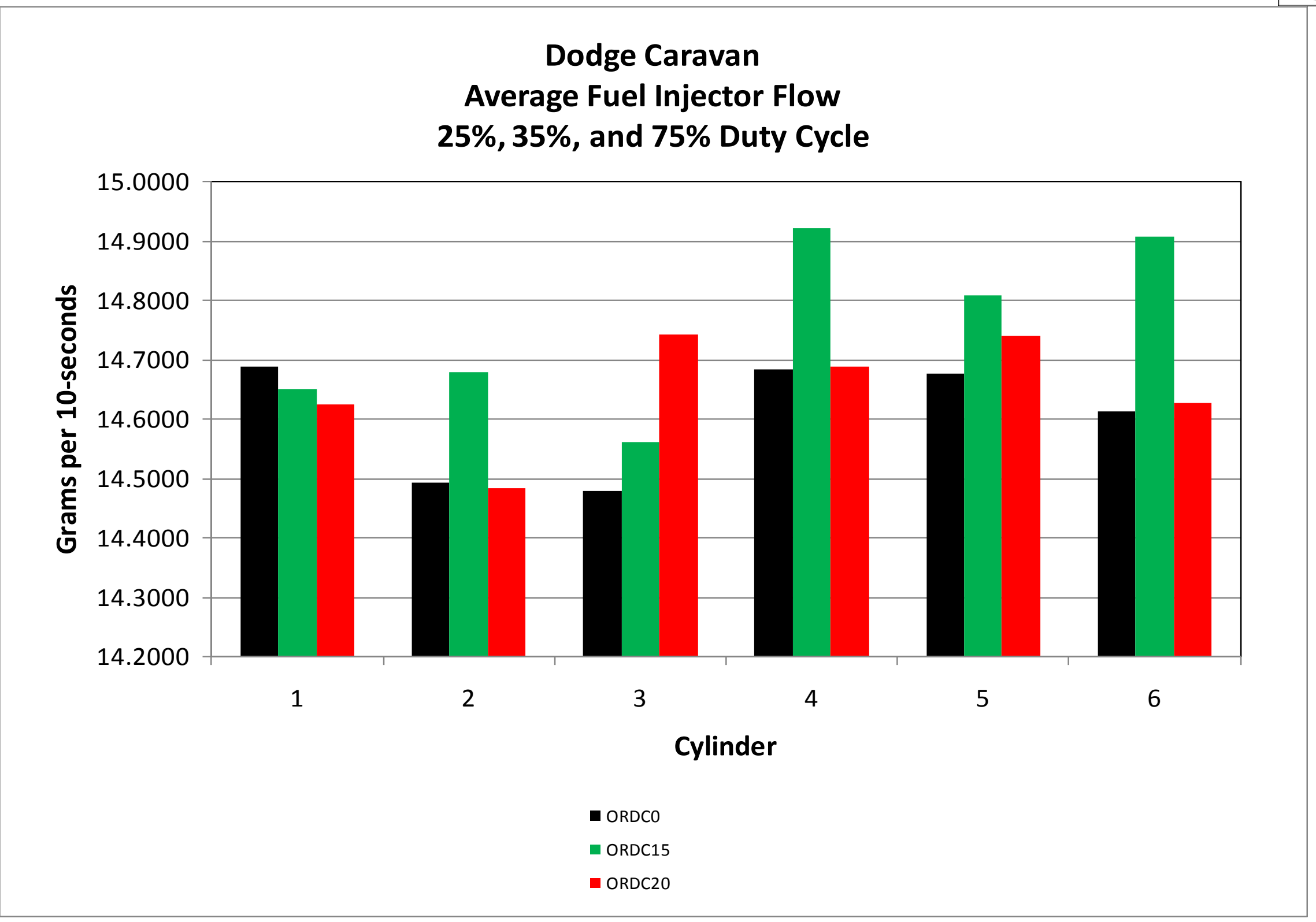

G-10 of 12 
Chevrolet Silverado

\begin{tabular}{|c|c|c|c|c|c|c|}
\hline & & & \multicolumn{3}{|l|}{} \\
\cline { 3 - 7 } Vehicle & Date & Cylinder & $\begin{array}{l}75 \% \text { DC } \\
\text { (gr/10sec) }\end{array}$ & $35 \%$ DC & $25 \%$ DC & Average \\
& & & & (gr/10sec) & (gr/10sec) \\
\hline ORCS0 & $8 / 10 / 2010$ & 1 & 23.6850 & 11.5500 & 8.5050 & 14.5800 \\
\hline ORCS0 & $8 / 10 / 2010$ & 2 & 23.7175 & 11.6475 & 8.5525 & 14.6392 \\
\hline ORCS0 & $8 / 10 / 2010$ & 3 & 23.8500 & 11.7050 & 8.6300 & 14.7283 \\
\hline ORCS0 & $8 / 10 / 2010$ & 4 & 23.7000 & 11.4775 & 8.4525 & 14.5433 \\
\hline ORCS0 & $8 / 10 / 2010$ & 5 & 23.6850 & 11.5850 & 8.4675 & 14.5792 \\
\hline ORCS0 & $8 / 10 / 2010$ & 6 & 23.6600 & 11.4500 & 8.3850 & 14.4983 \\
\hline ORCS0 & $8 / 10 / 2010$ & 7 & 23.8100 & 11.5725 & 8.4675 & 14.6167 \\
\hline ORCS0 & $8 / 10 / 2010$ & 8 & 23.6775 & 11.5550 & 8.4850 & 14.5725 \\
\hline ORCS15 & $8 / 10 / 2010$ & 1 & 23.7150 & 11.6175 & 8.5325 & 14.6217 \\
\hline ORCS15 & $8 / 10 / 2010$ & 2 & 23.7475 & 11.6600 & 8.5575 & 14.6550 \\
\hline ORCS15 & $8 / 10 / 2010$ & 3 & 23.6550 & 11.5975 & 8.5050 & 14.5858 \\
\hline ORCS15 & $8 / 10 / 2010$ & 4 & 23.7450 & 11.6450 & 8.5650 & 14.6517 \\
\hline ORCS15 & $8 / 10 / 2010$ & 5 & 23.7825 & 11.5875 & 8.4700 & 14.6133 \\
\hline ORCS15 & $8 / 10 / 2010$ & 6 & 23.5225 & 11.5550 & 8.4650 & 14.5142 \\
\hline ORCS15 & $8 / 10 / 2010$ & 7 & 23.5275 & 11.4125 & 8.4075 & 14.4492 \\
\hline ORCS15 & $8 / 10 / 2010$ & 8 & 23.7500 & 11.5175 & 8.4100 & 14.5592 \\
\hline ORCS20 & $9 / 27 / 2010$ & 1 & 23.7750 & 11.6175 & 8.6125 & 14.6683 \\
\hline ORCS20 & $9 / 27 / 2010$ & 2 & 23.8900 & 11.7075 & 8.5975 & 14.7317 \\
\hline ORCS20 & $9 / 27 / 2010$ & 3 & 23.7175 & 11.5525 & 8.5225 & 14.5975 \\
\hline ORCS20 & $9 / 27 / 2010$ & 4 & 23.8600 & 11.6675 & 8.6600 & 14.7292 \\
\hline ORCS20 & $9 / 27 / 2010$ & 5 & 23.7900 & 11.5825 & 8.5600 & 14.6442 \\
\hline ORCS20 & $9 / 27 / 2010$ & 6 & 23.9150 & 11.6125 & 8.5750 & 14.7008 \\
\hline ORCS20 & $9 / 27 / 2010$ & 7 & 23.8150 & 11.5675 & 8.5025 & 14.6283 \\
\hline ORCS20 & $9 / 27 / 2010$ & 8 & 24.0425 & 11.7325 & 8.6675 & 14.8142 \\
\hline
\end{tabular}




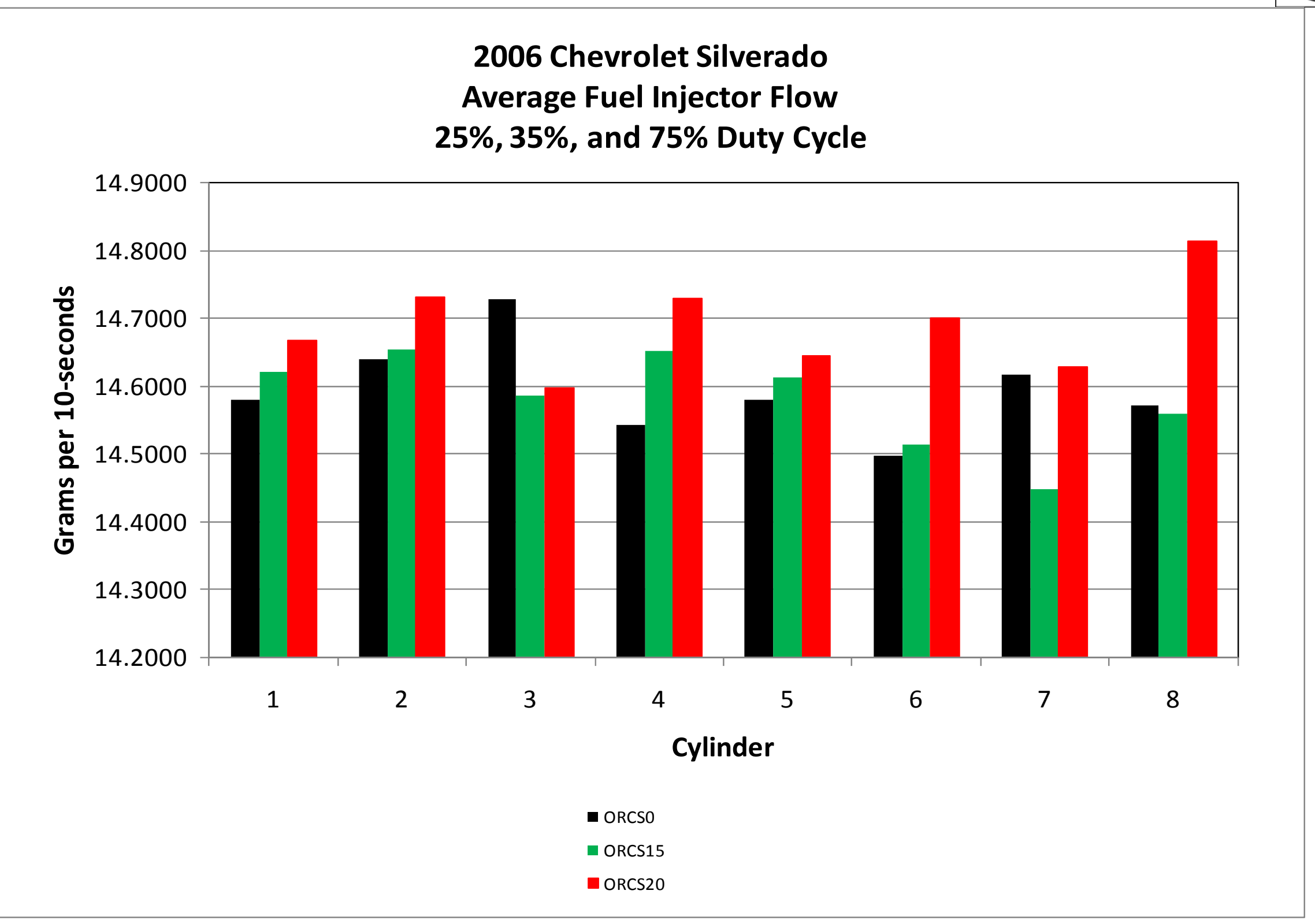

G-12 of 12 
Appendix H

Visual Inspection Rating of Valve Seals 



\section{Oak Ridge National Laboratory Proposal 08-58845 Powertrain Inspection Intake Valve Seal Rating}

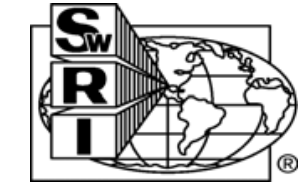

Date:

11-Aug-10

Technician:

JR

Valve seals rated prior to removal from cylinder head

Ratings conducted in accordance with CRC Manual 21

\begin{tabular}{|c|c|c|c|c|c|}
\hline \multicolumn{6}{|c|}{ ORHAO } \\
\hline Cylinder \# & Intake & Sludge & Wear & Condition & Varnish \\
\hline Front & 1 & Light & Trace & Good & Medium \\
\hline 1 & 2 & Light & Trace & Good & Medium \\
\hline \multirow{2}{*}{2} & 3 & Light & Trace & Good & Medium \\
\hline & 4 & Light & Trace & Good & Medium \\
\hline \multirow{2}{*}{3} & 5 & Light & Trace & Good & Medium \\
\hline & 6 & Light & Trace & Good & Medium \\
\hline \multirow[b]{2}{*}{ Rear } & 7 & Light & Trace & Good & Medium \\
\hline & 8 & Light & Trace & Good & Medium \\
\hline
\end{tabular}

\begin{tabular}{|c|c|c|c|c|c|}
\hline \multicolumn{6}{|c|}{ ORHA15 } \\
\hline Cylinder \# & Intake & Sludge & Wear & Condition & Varnish \\
\hline Front & 1 & Light & Trace & Good & None \\
\hline 1 & 2 & Light & Trace & Good & None \\
\hline \multirow{2}{*}{2} & 3 & Light & Trace & Good & None \\
\hline & 4 & Light & Trace & Good & None \\
\hline \multirow{2}{*}{3} & 5 & Light & Trace & Good & None \\
\hline & 6 & Light & Trace & 1 cut on top of seal & None \\
\hline 4 & 7 & Light & Trace & Good & None \\
\hline Rear & 8 & Light & Trace & Good & None \\
\hline
\end{tabular}




\section{Oak Ridge National Laboratory Proposal 08-58845 Powertrain Inspection Intake Valve Seal Rating}

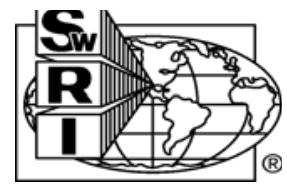

Date:

22-Sep-10

Technician:

$\mathrm{JR}$

Valve seals rated prior to removal from cylinder head

Ratings conducted in accordance with CRC Manual 21

\begin{tabular}{|c|c|c|c|c|c|}
\hline \multicolumn{6}{|c|}{ ORHA20 } \\
\hline Cylinder \# & Intake & Sludge & Wear & Condition & Varnish \\
\hline Front & 1 & Trace & Light & Good & Light \\
\hline 1 & 2 & Trace & Light & Good & Light \\
\hline \multirow{2}{*}{2} & 3 & Trace & Light & Good & Light \\
\hline & 4 & Trace & Light & Good & Light \\
\hline \multirow{2}{*}{3} & 5 & Trace & Light & Good & Light \\
\hline & 6 & Trace & Light & Good & Light \\
\hline \multirow[b]{2}{*}{ Rear } & 7 & Trace & Light & Good & Light \\
\hline & 8 & Trace & Light & Good & Light \\
\hline
\end{tabular}



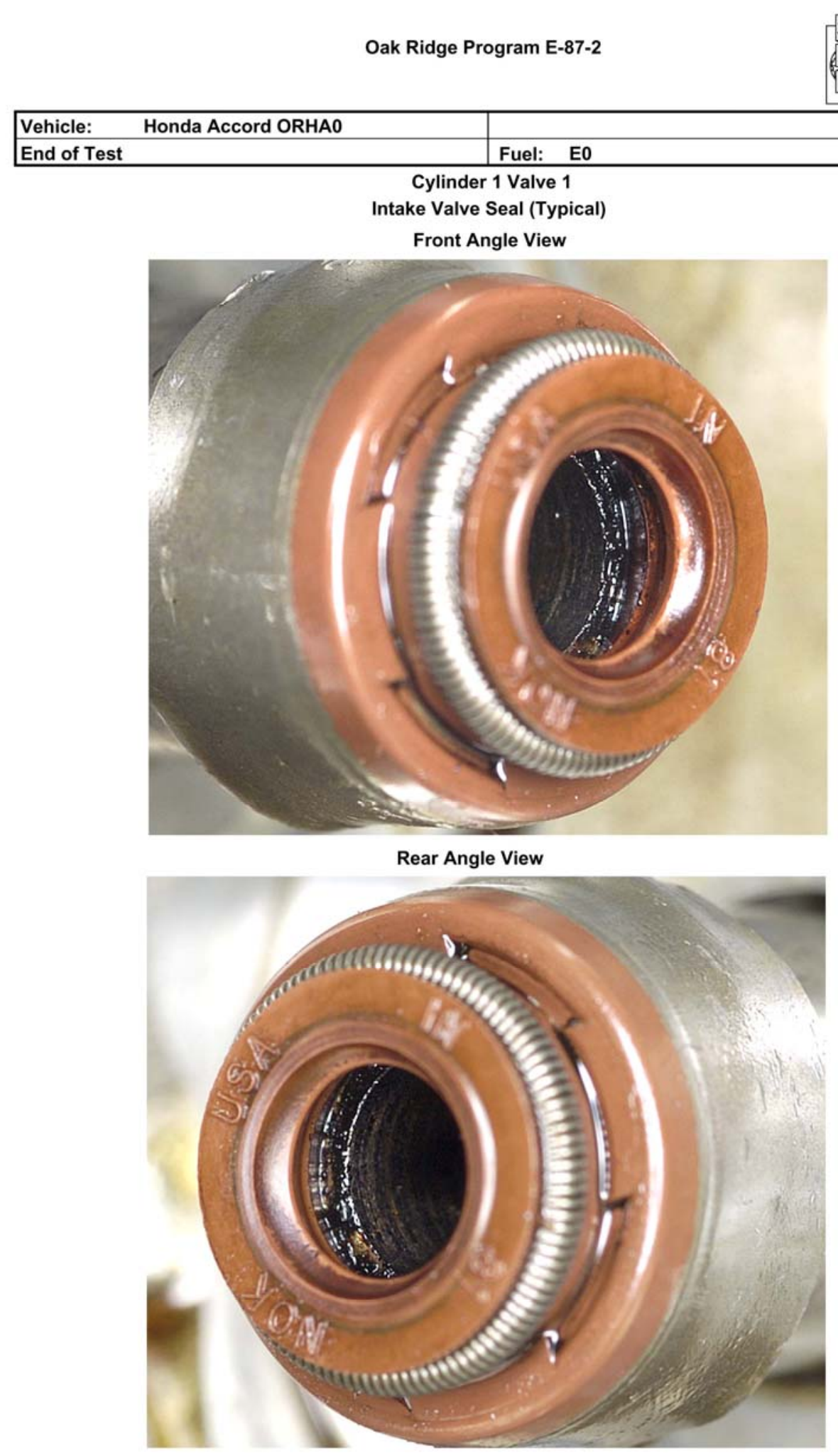

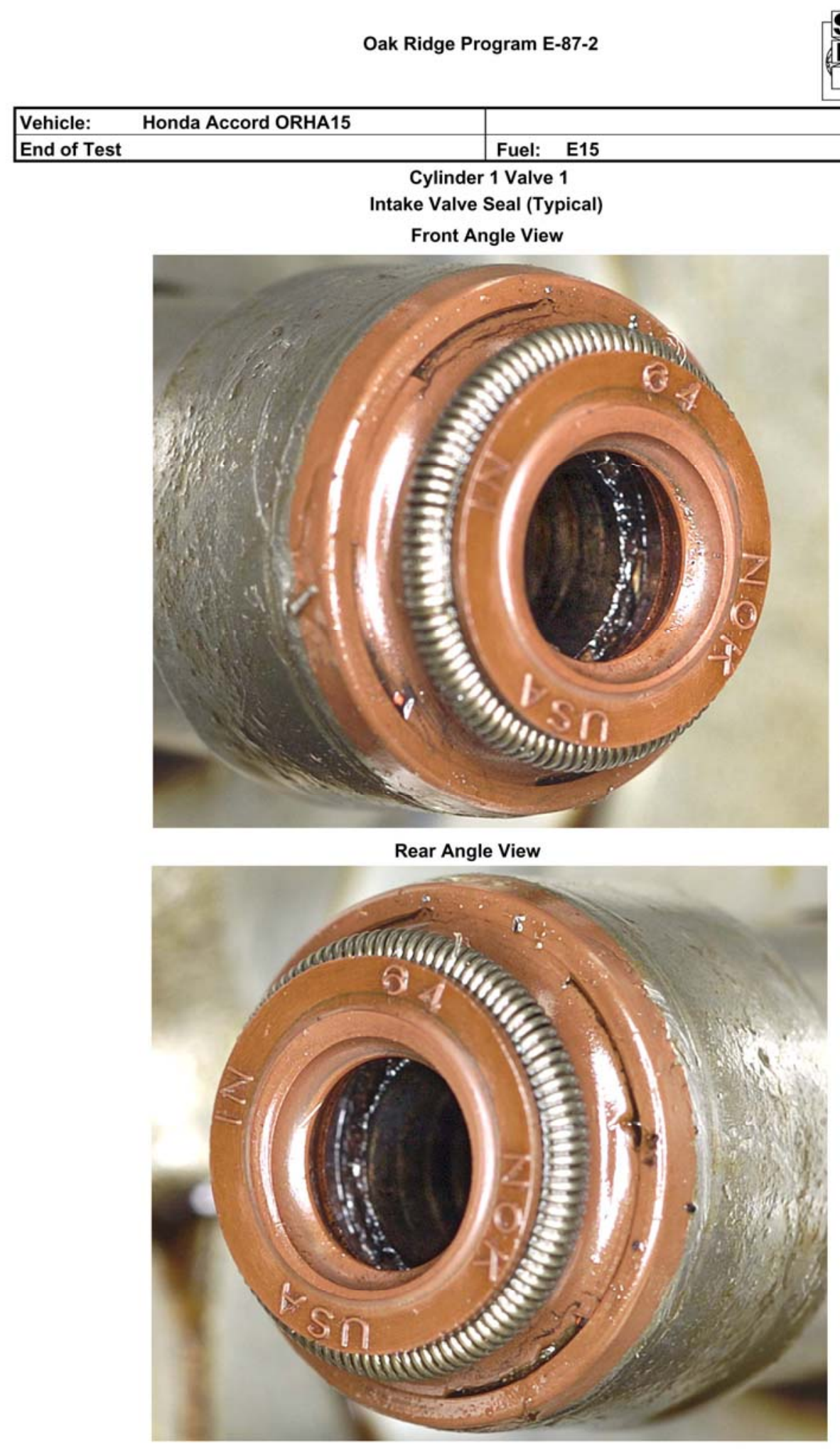

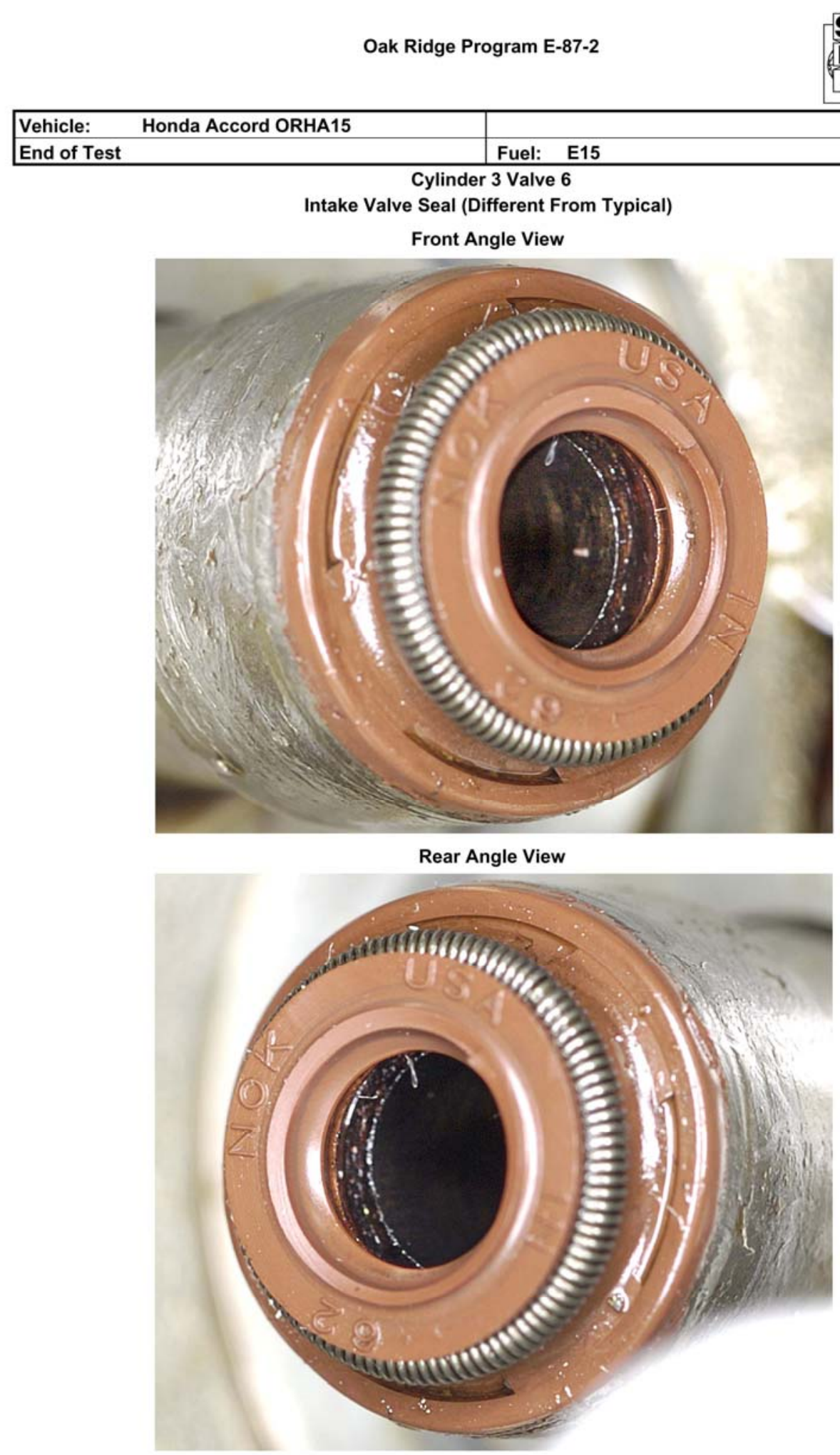


\section{Oak Ridge National Laboratory Proposal 08-58845 Powertrain Inspection Intake Valve Seal Rating}

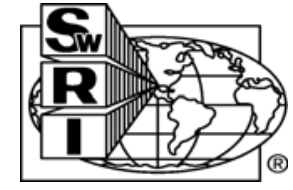

Date:

24-Aug-10

Technician:

$\mathrm{JR}$

Valve seal removal from cylinder head was necessary for rating Ratings conducted in accordance with CRC Manual 21

\begin{tabular}{|c|c|c|c|c|c|}
\hline \multicolumn{6}{|c|}{ ORCCO } \\
\hline Cylinder \# & Intake & Sludge & Wear & Condition & Varnish \\
\hline Front & 1 & Medium & Medium/Heavy & Good & None \\
\hline 1 & 2 & Medium & Medium/Heavy & Good & None \\
\hline \multirow{2}{*}{2} & 3 & Medium & Medium & Good & None \\
\hline & 4 & Light/Medium & Medium/Heavy & Good & None \\
\hline \multirow{2}{*}{3} & 5 & Light/Medium & Medium/Heavy & Good & None \\
\hline & 6 & Light/Medium & Medium/Heavy & Good & None \\
\hline \multirow[b]{2}{*}{ Rear } & 7 & Light/Medium & Medium & Good & None \\
\hline & 8 & Light/Medium & Medium/Heavy & Good & None \\
\hline
\end{tabular}

\begin{tabular}{|c|c|c|c|c|c|}
\hline \multicolumn{6}{|c|}{ ORCC15 } \\
\hline Cylinder \# & Intake & Sludge & Wear & Condition & Varnish \\
\hline Front & 1 & Medium & Medium/Heavy & Good & None \\
\hline 1 & 2 & Medium & Medium/Heavy & Good & None \\
\hline \multirow{2}{*}{2} & 3 & Medium & Medium & Good & None \\
\hline & 4 & Light/Medium & Medium/Heavy & Good & None \\
\hline \multirow{2}{*}{3} & 5 & Light/Medium & Medium/Heavy & Good & None \\
\hline & 6 & Light/Medium & Medium/Heavy & Good & None \\
\hline \multirow[b]{2}{*}{ Rear } & 7 & Medium & Medium/Heavy & Good & None \\
\hline & 8 & Light/Medium & Medium/Heavy & Good & None \\
\hline
\end{tabular}




\section{Oak Ridge National Laboratory Proposal 08-58845 Powertrain Inspection Intake Valve Seal Rating}

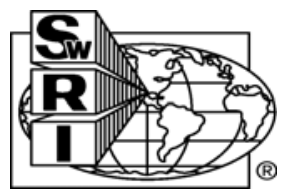

Date:

15-Sep-10

Technician:

$\mathrm{JR}$

Valve seal removal from cylinder head was necessary for rating Ratings conducted in accordance with CRC Manual 21

\begin{tabular}{|c|c|c|c|c|c|}
\hline \multirow{2}{*}{ Cylinder \# } & Intake & Sludge & Wear & Condition & Varnish \\
\hline \multirow{2}{*}{ Front } & 1 & Light/Medium & Trace/Light & Good & Light \\
\cline { 2 - 6 } 1 & 2 & Light/Medium & Trace/Light & Good & Trace/Light \\
\hline \multirow{2}{*}{2} & 3 & Light/Medium & Trace/Light & Good & Trace \\
\cline { 2 - 6 } & 4 & Light/Medium & Trace/Light & Good & Trace \\
\cline { 2 - 6 } & 5 & Light/Medium & Trace/Light & Good & Trace/Light \\
\hline \multirow{2}{*}{4} & 6 & Light/Medium & Trace/Light & Good & Trace \\
\cline { 2 - 6 } & 7 & Light/Medium & Trace/Light & Good & Trace \\
\hline \multirow{2}{*}{ Rear } & 8 & Light/Medium & Trace/Light & Good & Trace \\
\hline
\end{tabular}




\section{Oak Ridge National Laboratory \\ Proposal 08-58845 Powertrain Inspection Intake Valve Seal Rating}

Date:

24-Aug-10

Technician:

$J R$

Valve seal removal from cylinder head was necessary for rating Ratings conducted in accordance with CRC Manual 21

\begin{tabular}{|c|c|c|c|c|c|}
\multicolumn{2}{|c|}{} & & & & \\
Cylinder \# & Intake & Sludge & Wear & Condition & Varnish \\
\hline \multirow{2}{*}{ Front } & 1 & Light/Medium & Trace/Light & Cracking & None \\
\cline { 2 - 6 } 1 & 2 & Light/Medium & Trace/Light & Cracking & None \\
\hline \multirow{2}{*}{2} & 3 & Light/Medium & Trace/Light & Cracking & None \\
\cline { 2 - 6 } & 4 & Light/Medium & Trace/Light & Cracking & None \\
\hline \multirow{2}{*}{3} & 5 & Light/Medium & Trace/Light & Cracking & None \\
\cline { 2 - 6 } & 6 & Light/Medium & Trace/Light & Cracking & None \\
\hline \multirow{2}{*}{ Rear } & 7 & Light/Medium & Trace/Light & Cracking & None \\
\cline { 2 - 6 } & 8 & Light/Medium & Trace/Light & Cracking & None \\
\hline
\end{tabular}

\begin{tabular}{|c|c|c|c|c|c|}
\hline \multicolumn{6}{|c|}{ ORNA15 } \\
\hline Cylinder \# & Intake & Sludge & Wear & Condition & Varnish \\
\hline Front & 1 & Light/Medium & Trace/Light & Cracking & None \\
\hline 1 & 2 & Light/Medium & Trace/Light & Cracking & None \\
\hline \multirow{2}{*}{2} & 3 & Light/Medium & Trace/Light & Cracking & None \\
\hline & 4 & Light/Medium & Trace/Light & Cracking & None \\
\hline \multirow{2}{*}{3} & 5 & Light/Medium & Trace/Light & Cracking & None \\
\hline & 6 & Light/Medium & Trace/Light & Cracking & None \\
\hline \multirow[t]{2}{*}{4} & 7 & Light/Medium & Trace/Light & Cracking & None \\
\hline & 8 & Light/Medium & Trace/Light & Cracking & None \\
\hline
\end{tabular}




\section{Oak Ridge National Laboratory \\ Proposal 08-58845 Powertrain Inspection Intake Valve Seal Rating}

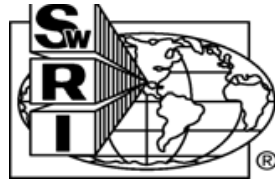

Date:

16-Sep-10

Technician:

JR

Valve seal removal from cylinder head was necessary for rating Ratings conducted in accordance with CRC Manual 21

\begin{tabular}{|c|c|c|c|c|c|}
\hline \multicolumn{6}{|c|}{ ORNA20 } \\
\hline Cylinder \# & Intake & Sludge & Wear & Condition & Varnish \\
\hline Front & 1 & Light/Medium & Trace/Light & Good & None \\
\hline 1 & 2 & Light/Medium & Trace/Light & Good & None \\
\hline \multirow{2}{*}{2} & 3 & Light/Medium & Trace/Light & Good & None \\
\hline & 4 & Light/Medium & Trace/Light & Good & None \\
\hline \multirow{2}{*}{3} & 5 & Light/Medium & Trace/Light & Good & None \\
\hline & 6 & Light/Medium & Trace/Light & Good & None \\
\hline \multirow[b]{2}{*}{ Rear } & 7 & Light/Medium & Trace/Light & Good & None \\
\hline & 8 & Light/Medium & Trace/Light & Good & None \\
\hline
\end{tabular}


Oak Ridge National Laboratory

Proposal 08-58845 Powertrain Inspection

Intake Valve Seal Rating

Date:

23-Aug-10

Technician:

$\mathrm{JR}$

Valve seals rated prior to removal from cylinder head

Ratings conducted in accordance with CRC Manual 21

Seals are black in color, therefore varnish cannot be rated

\begin{tabular}{|c|c|c|}
\hline & \multicolumn{2}{|c|}{ ORCSO } \\
\hline Cylinder \# & Sludge & Wear \\
\hline 1 & Light to Medium & Trace to Light \\
\hline 2 & Light to Medium & Trace to Light \\
\hline 3 & Light to Medium & Trace to Light \\
\hline 4 & Light to Medium & Trace to Light \\
\hline 5 & Light to Medium & Trace to Light \\
\hline 6 & Light to Medium & Trace to Light \\
\hline 7 & Light to Medium & Trace to Light \\
\hline 8 & Light to Medium & Trace to Light \\
\hline
\end{tabular}

\begin{tabular}{|c|c|c|}
\hline & \multicolumn{2}{|c|}{ ORCS15 } \\
\hline Cylinder \# & Sludge & Wear \\
\hline 1 & Light to Medium & Trace to Light \\
\hline 2 & Light to Medium & Trace to Light \\
\hline 3 & Light to Medium & Trace to Light \\
\hline 4 & Light to Medium & Trace to Light \\
\hline 5 & Light to Medium & Trace to Light \\
\hline 6 & Light to Medium & Trace to Light \\
\hline 7 & Light to Medium & Trace to Light \\
\hline 8 & Light to Medium & Trace to Light \\
\hline
\end{tabular}




\section{Oak Ridge National Laboratory Proposal 08-58845 Powertrain Inspection Intake Valve Seal Rating}

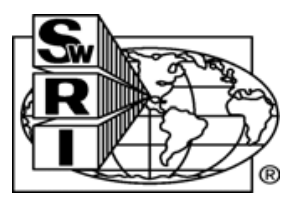

Date: 17-Sep-10

Technician: $\mathrm{JR}$

Valve seals rated prior to removal from cylinder head Ratings conducted in accordance with CRC Manual 21 Seals are black in color, therefore varnish cannot be rated

\begin{tabular}{|c|c|c|}
\hline & \multicolumn{2}{|c|}{ ORCS20 } \\
\hline Cylinder \# & Sludge & Wear \\
\hline 1 & Light to Medium & Trace to Light \\
\hline 2 & Light to Medium & Trace to Light \\
\hline 3 & Light to Medium & Trace to Light \\
\hline 4 & Light to Medium & Trace to Light \\
\hline 5 & Light to Medium & Trace to Light \\
\hline 6 & Light to Medium & Trace to Light \\
\hline 7 & Light to Medium & Trace to Light \\
\hline 8 & Light to Medium & Trace to Light \\
\hline
\end{tabular}




\section{Oak Ridge National Laboratory Proposal 08-58845 Powertrain Inspection Intake Valve Seal Rating}

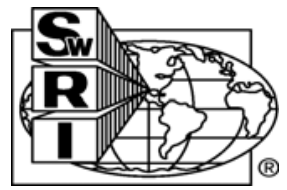

Date: 20-Aug-10

Technician:

$\mathrm{JR}$

Valve seals rated prior to removal from cylinder head

Ratings conducted in accordance with CRC Manual 21

Seals are black in color, therefore varnish cannot be rated

TOP OF SEAL EDGE: chipping or chunking on edge is normal with new seal

\begin{tabular}{|c|c|c|}
\hline & \multicolumn{2}{|c|}{ ORDC0 } \\
\hline Cylinder \# & Sludge & Wear \\
\hline 1 & Light to Medium & Trace to Light \\
\hline 2 & Light to Medium & Trace to Light \\
\hline 3 & Light to Medium & Trace to Light \\
\hline 4 & Light to Medium & Trace to Light \\
\hline 5 & Light to Medium & Trace to Light \\
\hline 6 & Light to Medium & Trace to Light \\
\hline
\end{tabular}

\begin{tabular}{|c|c|c|}
\hline & \multicolumn{2}{|c|}{ ORDC15 } \\
\hline Cylinder \# & Sludge & Wear \\
\hline 1 & Light to Medium & Trace to Light \\
\hline 2 & Light to Medium & Trace to Light \\
\hline 3 & Light to Medium & Trace to Light \\
\hline 4 & Light to Medium & Trace to Light \\
\hline 5 & Light to Medium & Trace to Light \\
\hline 6 & Light to Medium & Trace to Light \\
\hline
\end{tabular}




\section{Oak Ridge National Laboratory Proposal 08-58845 Powertrain Inspection Intake Valve Seal Rating}

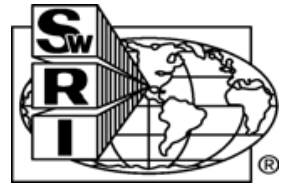

Date: 13-Sep-10

Technician:

$\mathrm{JR}$

Valve seals rated prior to removal from cylinder head

Ratings conducted in accordance with CRC Manual 21

Seals are black in color, therefore varnish cannot be rated

TOP OF SEAL EDGE: chipping or chunking on edge is normal with new seal

\begin{tabular}{|c|c|c|}
\hline & \multicolumn{2}{|c|}{ ORDC20B } \\
\hline Cylinder \# & Sludge & Wear \\
\hline 1 & Light to Medium & Trace to Light \\
\hline 2 & Light to Medium & Trace to Light \\
\hline 3 & Light to Medium & Trace to Light \\
\hline 4 & Light to Medium & Trace to Light \\
\hline 5 & Light to Medium & Trace to Light \\
\hline 6 & Light to Medium & Trace to Light \\
\hline
\end{tabular}




\section{Oak Ridge National Laboratory Proposal 08-58845 Powertrain Inspection Intake Valve Seal Rating}

Date: 25-Aug-10

Technician:

$\mathrm{JR}$

Valve seal removal from cylinder head was necessary for rating

Ratings conducted in accordance with CRC Manual 21

Seals are black in color, therefore varnish cannot be rated

\begin{tabular}{|c|c|c|c|}
\hline \multicolumn{2}{|c|}{ ORFT0 } \\
\hline \multirow{2}{*}{1} & 1 & Sludge & Wear \\
\cline { 2 - 5 } & 2 & Light/Medium & Light \\
\hline \multirow{3}{*}{2} & 3 & Light/Medium & Light \\
\cline { 2 - 5 } & 4 & Light/Medium & Light \\
\hline \multirow{3}{*}{3} & 5 & Light/Medium & Light \\
\cline { 2 - 5 } & 6 & Light/Medium & Light \\
\hline \multirow{2}{*}{4} & 7 & Light/Medium & Light \\
\cline { 2 - 5 } & 8 & Light/Medium & Light \\
\hline \multirow{2}{*}{5} & 9 & Light/Medium & Light \\
\cline { 2 - 5 } & 10 & Light/Medium & Light \\
\hline \multirow{2}{*}{6} & 11 & Light/Medium & Light/Medium \\
\hline
\end{tabular}

\begin{tabular}{|c|c|c|c|}
\hline \multicolumn{5}{|c|}{ ORFT15 } \\
\hline \multirow{2}{*}{ Cylinder \# } & Intake & Sludge & Wear \\
\hline \multirow{2}{*}{1} & 1 & Light/Medium & Light \\
\cline { 2 - 5 } & 2 & Light/Medium & Light \\
\hline \multirow{2}{*}{2} & 3 & Light/Medium & Light \\
\cline { 2 - 5 } & 4 & Light/Medium & Light \\
\hline \multirow{3}{*}{3} & 5 & Light/Medium & Light \\
\cline { 2 - 5 } & 6 & Light/Medium & Light \\
\hline \multirow{3}{*}{4} & 7 & Light/Medium & Light \\
\cline { 2 - 5 } & 8 & Light/Medium & Light \\
\hline \multirow{2}{*}{5} & 9 & Light/Medium & Light \\
\cline { 2 - 5 } & 10 & Light/Medium & Light \\
\hline \multirow{2}{*}{6} & 11 & Light/Medium & Light \\
\cline { 2 - 5 } & 12 & Light/Medium & \\
\hline
\end{tabular}




\section{Oak Ridge National Laboratory Proposal 08-58845 Powertrain Inspection Intake Valve Seal Rating}

Date: 22-Sep-10

Technician:

$\mathrm{JR}$

Valve seal removal from cylinder head was necessary for rating Ratings conducted in accordance with CRC Manual 21 Seals are black in color, therefore varnish cannot be rated

\begin{tabular}{|c|c|c|c|}
\hline \multicolumn{4}{|c|}{ ORFT20 } \\
\hline \multirow{2}{*}{ Cylinder \# } & Intake & Sludge & Wear \\
\hline \multirow{2}{*}{1} & 1 & Light/Medium & Light \\
\cline { 2 - 5 } & 2 & Trace/Light & Light \\
\hline \multirow{3}{*}{2} & 3 & Trace/Light & Light \\
\cline { 2 - 5 } & 4 & Trace/Light & Light \\
\hline \multirow{3}{*}{3} & 5 & Light/Medium & Light \\
\cline { 2 - 5 } & 6 & Trace/Light & Light \\
\hline \multirow{3}{*}{4} & 7 & Trace/Light & Light \\
\cline { 2 - 5 } & 8 & Trace/Light & Light \\
\hline \multirow{2}{*}{5} & 9 & Trace/Light & Light \\
\cline { 2 - 5 } & 10 & Light/Medium & Light \\
\hline \multirow{2}{*}{6} & 11 & Trace/Light & Light \\
\cline { 2 - 5 } & 12 & Trace/Light & \\
\hline \multirow{2}{*}{} & & & \multicolumn{3}{|c|}{} \\
\hline
\end{tabular}





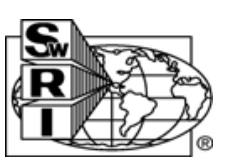

Appendix I

Evaporative Canister Working Capacity Test Results 



\section{TABLE I-1. EVAPORATIVE EMISSIONS CANISTER WORKING CAPACITY}

\begin{tabular}{|c|c|c|c|c|c|c|c|c|c|c|}
\hline $\begin{array}{c}\text { Test Vehicle } \\
\text { Set }\end{array}$ & $\begin{array}{l}\text { Canister } \\
\text { ID }\end{array}$ & $\begin{array}{c}\text { Carbon } \\
\text { Volume }^{\mathrm{a}} \\
\text { (liters) }\end{array}$ & $\begin{array}{l}\text { Butane or } \\
\text { Gasoline } \\
\text { Testing }\end{array}$ & $\begin{array}{c}\text { Test Dates } \\
\text { (2010) }\end{array}$ & $\begin{array}{c}\text { Cycle } 9 \\
\text { Break- } \\
\text { through } \\
\text { (+2 grams) }\end{array}$ & $\begin{array}{c}\text { Cycle } 10 \\
\text { Break- } \\
\text { through } \\
\text { (+2 grams) }\end{array}$ & $\begin{array}{c}\text { Cycle } 14 \\
\text { Break- } \\
\text { through } \\
\text { (+2 grams) }^{\mathrm{e}}\end{array}$ & $\begin{array}{c}\text { Cycle } 15 \\
\text { Break- } \\
\text { through } \\
\text { (+2 grams) }\end{array}$ & $\begin{array}{c}\text { Working } \\
\text { Capacity }^{\text {b,c }} \\
\text { (grams) }^{\text {(grams }}\end{array}$ & $\begin{array}{c}\text { Specific } \\
\text { Working } \\
\text { Capacity, g/L } \\
\text { (grams/liter } \\
\text { canister } \\
\text { volume) }\end{array}$ \\
\hline \multirow{3}{*}{$\begin{array}{l}2008 \text { Nissan } \\
\text { Altima }\end{array}$} & ORNA00 & \multirow{3}{*}{2.05} & \multirow{3}{*}{$\begin{array}{l}\text { Tested with Butane } \\
\text { for } 15 \text { Cycles }\end{array}$} & 09/07 - 09/12 & 90.29 & 104.87 & 108.31 & 108.12 & 108.22 & 52.79 \\
\hline & ORNA15 & & & $09 / 16-09 / 21$ & 99.04 & 100.45 & 103.50 & 102.67 & 103.09 & 50.29 \\
\hline & ORNA20 & & & $09 / 22-09 / 26$ & 79.40 & 84.15 & 93.70 & 93.31 & 93.51 & 45.61 \\
\hline \multirow{3}{*}{$\begin{array}{l}2006 \text { Chevrolet } \\
\text { Cobalt }\end{array}$} & ORCC00 & \multirow{3}{*}{2.2} & \multirow{3}{*}{$\begin{array}{l}\text { Tested with Butane } \\
\text { for } 15 \text { Cycles }\end{array}$} & $09 / 21-09 / 25$ & 84.50 & 81.37 & 86.68 & 88.44 & 87.56 & 39.80 \\
\hline & ORCC15 & & & $09 / 12-09 / 16$ & 95.59 & 94.04 & 97.16 & 96.83 & 97.00 & 44.09 \\
\hline & ORCC20 & & & $09 / 26-09 / 30$ & 88.53 & 88.62 & 91.06 & 91.43 & 91.25 & 41.48 \\
\hline \multirow{3}{*}{$\begin{array}{l}2007 \text { Honda } \\
\text { Accord }\end{array}$} & ORHA00 & \multirow{3}{*}{2.5} & \multirow{3}{*}{$\begin{array}{l}\text { Tested with Butane } \\
\text { for } 15 \text { Cycles }\end{array}$} & $09 / 23-09 / 27$ & 101.1 & 98.9 & 99.1 & 98.3 & 98.7 & 39.5 \\
\hline & ORHA15 & & & $09 / 23-09 / 27$ & 95.6 & 92.1 & 95.8 & 93.2 & 94.5 & 37.8 \\
\hline & ORHA20 & & & $10 / 07-10 / 12$ & 97.4 & 91.9 & 94.9 & 92.2 & 93.6 & 34.4 \\
\hline \multirow{3}{*}{$\begin{array}{l}2007 \text { Dodge } \\
\text { Caravan }\end{array}$} & ORDC00 & \multirow{3}{*}{2.7} & \multirow{3}{*}{$\begin{array}{l}\text { Tested with } \\
\text { Gasoline for } \\
10 \text { Cycles }\end{array}$} & $09 / 26-09 / 27$ & 173.1 & 172.9 & \multirow{3}{*}{\multicolumn{2}{|c|}{ d }} & 173.00 & 64.07 \\
\hline & ORDC15 & & & $09 / 23-09 / 24$ & 173.6 & 172.4 & & & 173.00 & 64.07 \\
\hline & ORDC20 2 & & & $09 / 27-09 / 28$ & 166.2 & 169.0 & & & 167.70 & 62.11 \\
\hline \multirow{3}{*}{$\begin{array}{l}2008 \text { Ford } \\
\text { Taurus }\end{array}$} & ORFT00 & \multirow{3}{*}{2.7} & \multirow{3}{*}{$\begin{array}{l}\text { Tested with Butane } \\
\text { for } 15 \text { Cycles }\end{array}$} & $09 / 23-09 / 28$ & 90.4 & 99.0 & 97.6 & 95.8 & 96.7 & 35.8 \\
\hline & ORFT15 & & & $09 / 23-09 / 27$ & 103.3 & 93.8 & 104.3 & 97.7 & 101.0 & 37.4 \\
\hline & ORFT20 & & & $10 / 07-10 / 14$ & 96.7 & 94.6 & 107.6 & 102.2 & 104.9 & 38.9 \\
\hline \multirow{3}{*}{$\begin{array}{l}2006 \text { Chevrolet } \\
\text { Silverado }\end{array}$} & ORCS00 & \multirow{3}{*}{3.3} & \multirow{3}{*}{$\begin{array}{l}\text { Tested with } \\
\text { Gasoline for } \\
10 \text { Cycles }\end{array}$} & $09 / 25-09 / 26$ & 156.7 & 156.9 & \multirow{3}{*}{\multicolumn{2}{|c|}{ d }} & 156.80 & 47.52 \\
\hline & ORCS15 & & & $09 / 24-09 / 25$ & 161.6 & 162.2 & & & 161.90 & 49.06 \\
\hline & ORCS20 & & & $09 / 27-09 / 27$ & 160.9 & 162.4 & & & 161.65 & 48.98 \\
\hline \multicolumn{11}{|c|}{$\begin{array}{l}\text { a } \text { Canister volumes provided by the vehicle manufacturers. } \\
{ }^{\mathrm{b}} \text { Butane working capacity (BWC): BWC = (Cycle } 14+\text { Cycle 15) } \\
{ }^{\mathrm{c}} \text { Gasoline working capacity (GWC): GWC = (Cycle } 9+\text { Cycle 10) } \\
\text { d Not applicable for GWC tests. GWC test complete at } 10 \text { cycles. }\end{array}$} \\
\hline
\end{tabular}




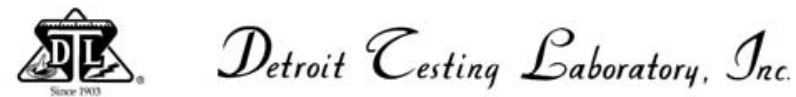

GENERAL DATA SHEET

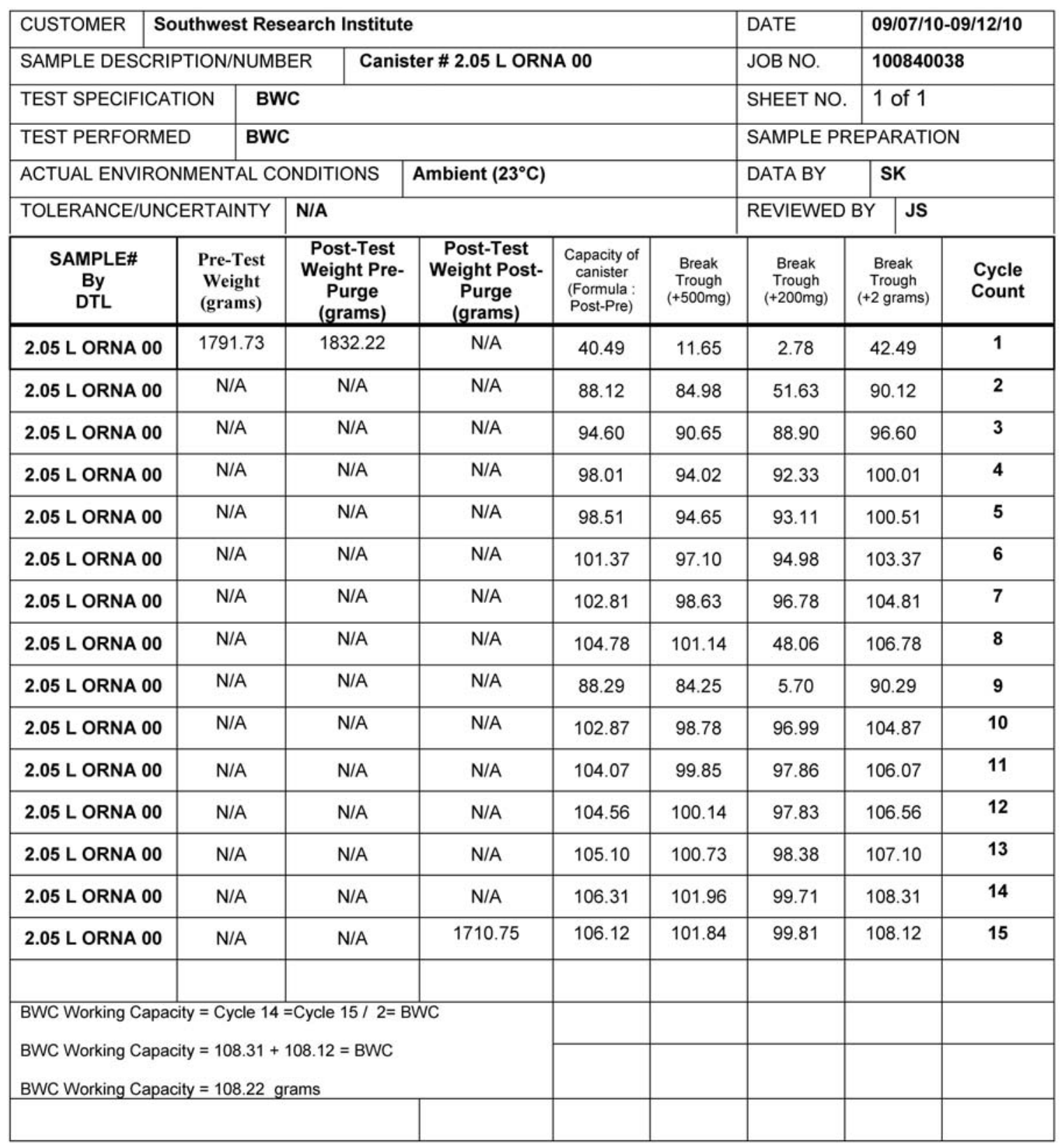

QCF-1041 REVISED 3/29/00 


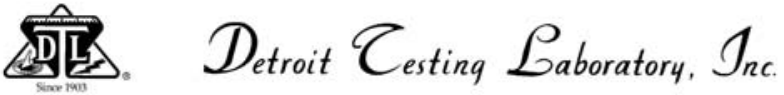

GENERAL DATA SHEET

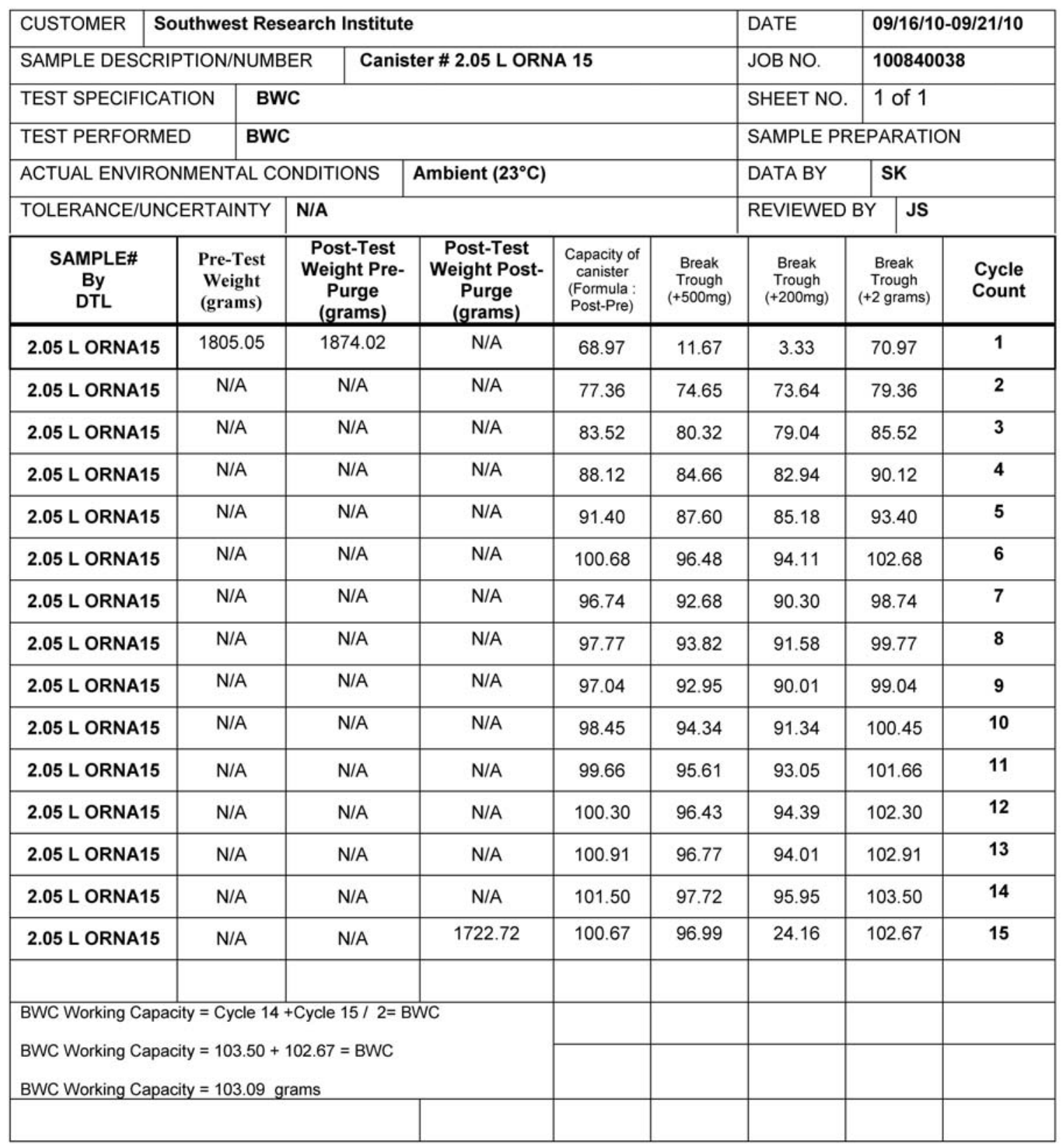

QCF-1041 REVISED 3/29/00 


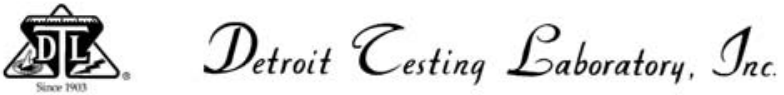

GENERAL DATA SHEET

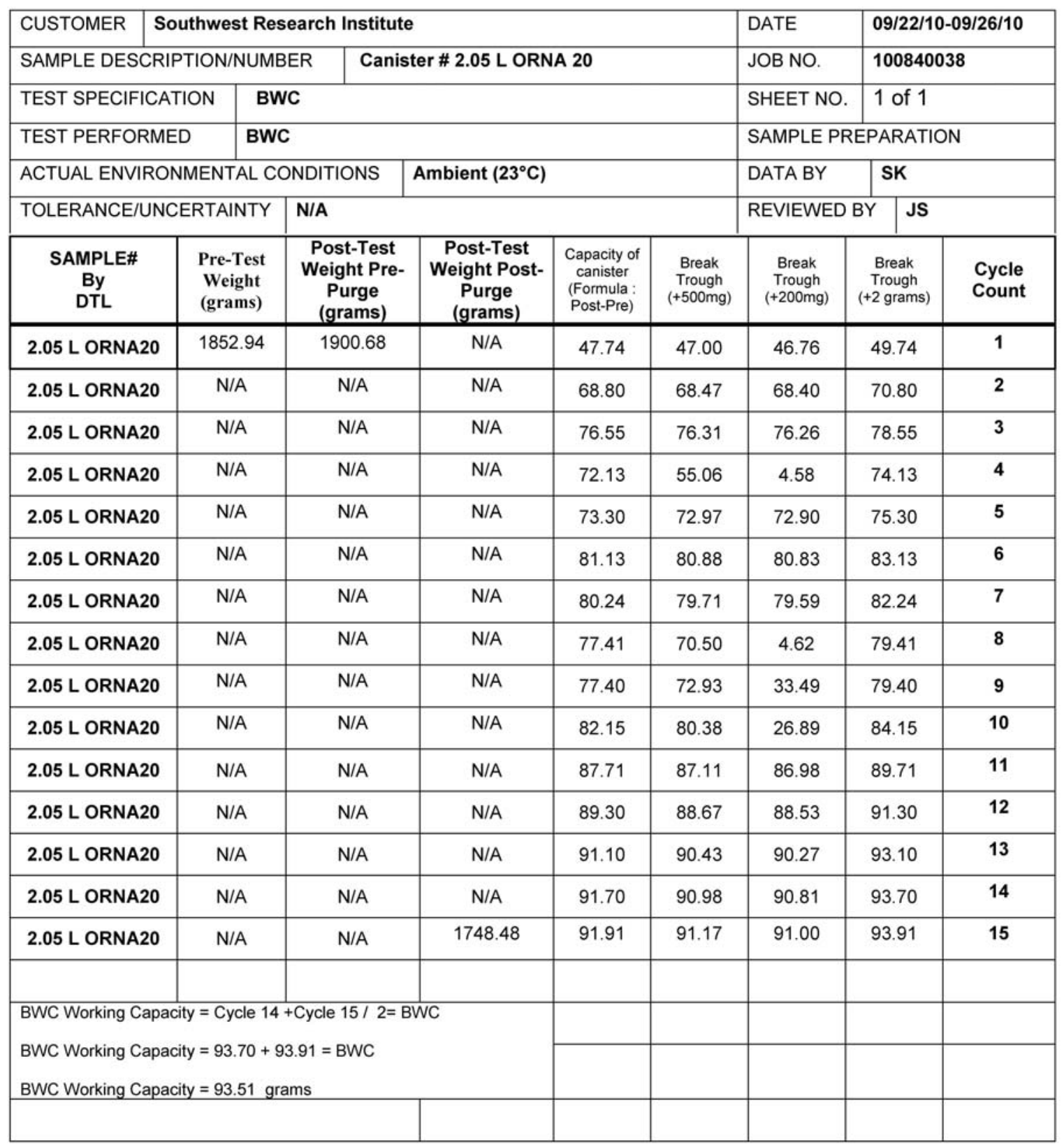

QCF-1041 REVISED 3/29/00 


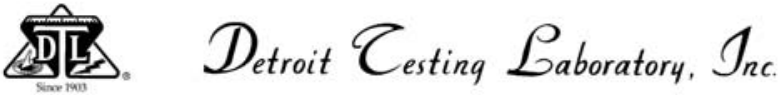

GENERAL DATA SHEET

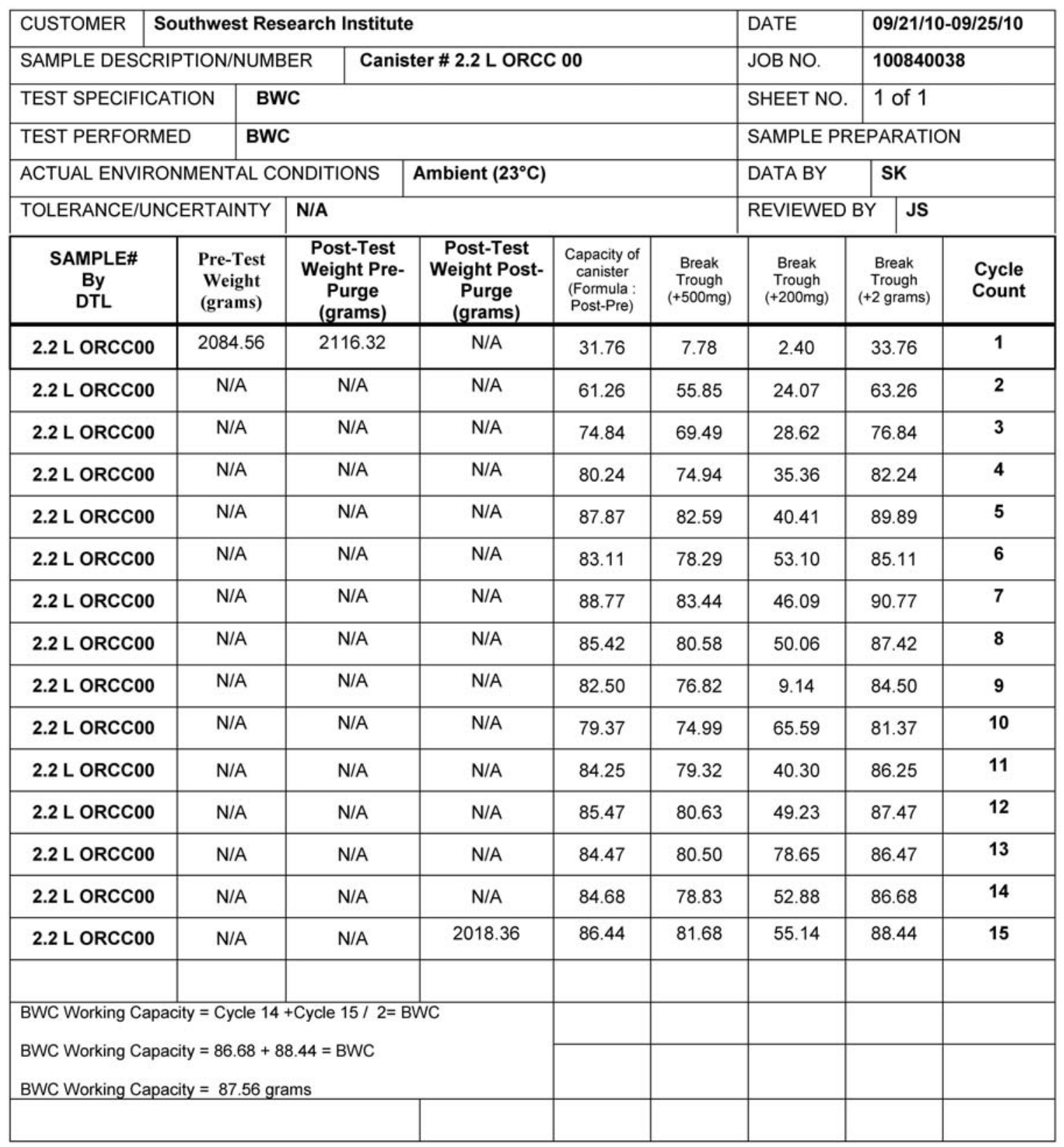

QCF-1041 REVISED 3/29/00 


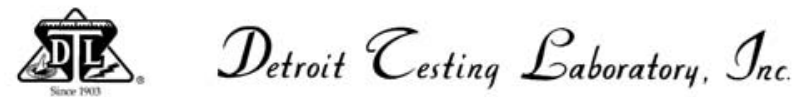

GENERAL DATA SHEET

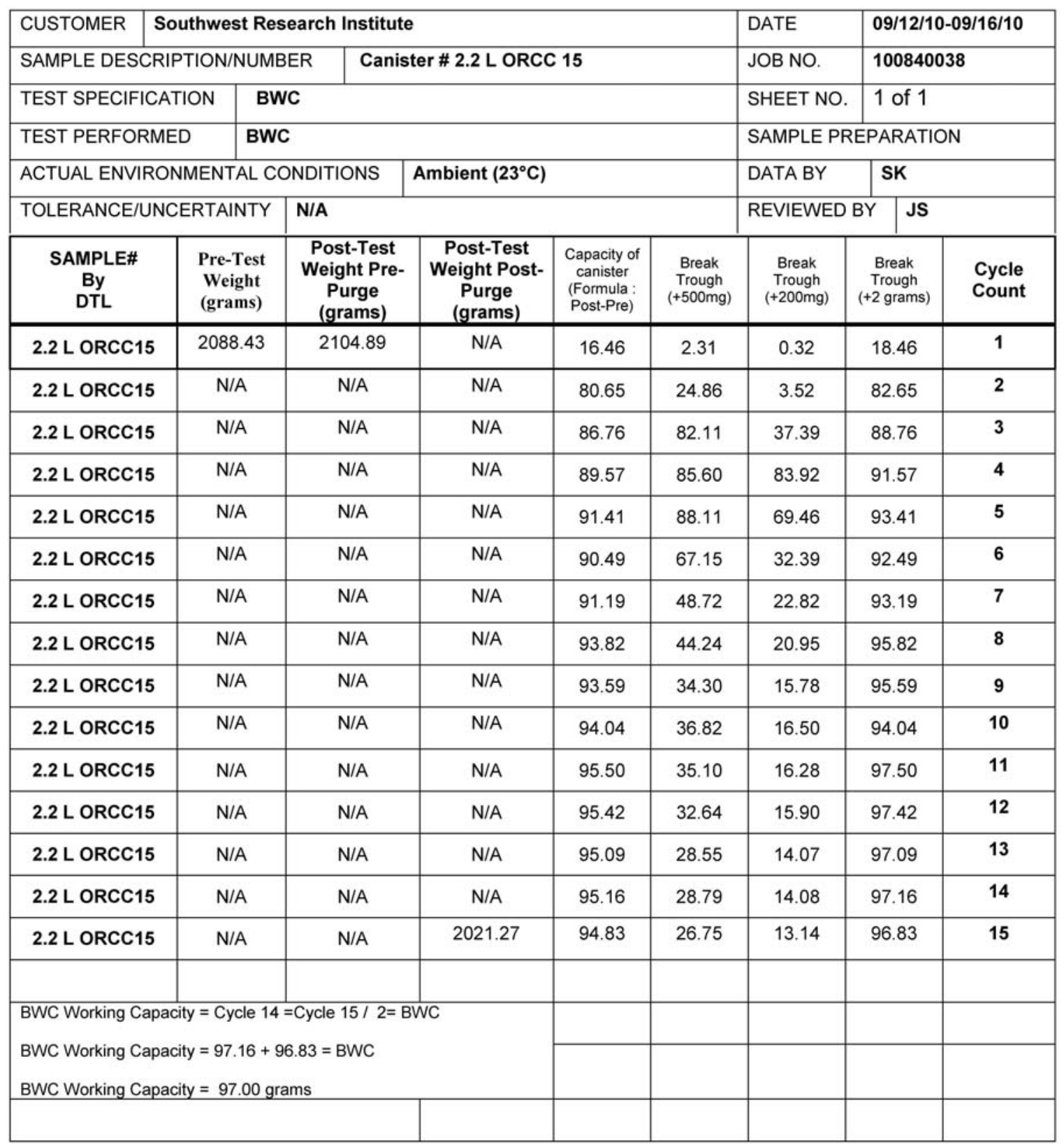

QCF-1041 REVISED 3/29/00 


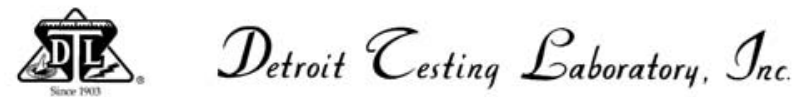

GENERAL DATA SHEET

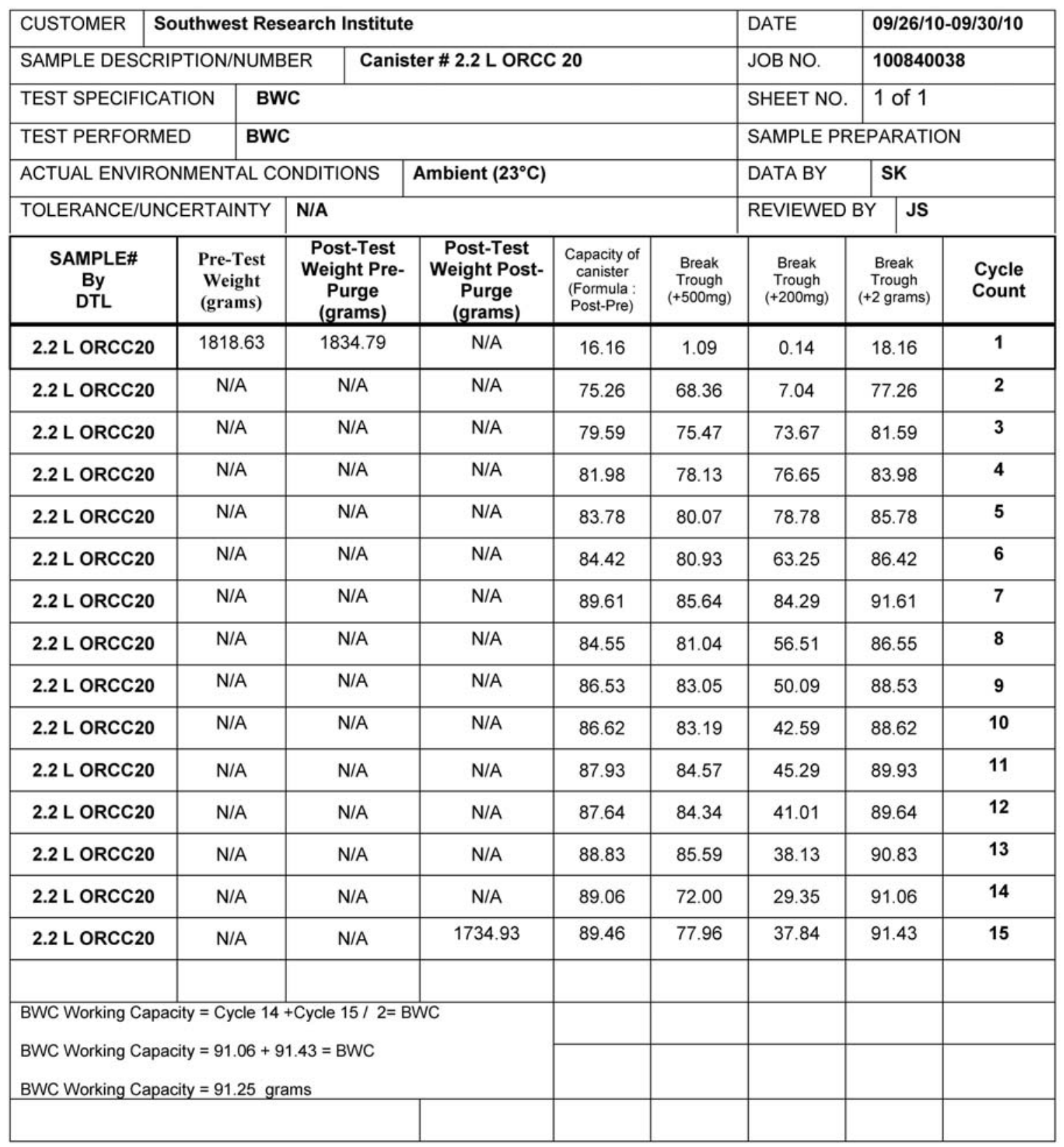

QCF-1041 REVISED 3/29/00 


\section{ENVIRONMENTAL TESTING CORP. Canister Purge - Load Test Summary Vehicle Set: 2007 Honda Accords}

\begin{tabular}{|c|c|c|c|c|c|c|c|}
\hline $\begin{array}{c}\text { CANISTER ID } \\
\text { NO. }\end{array}$ & $\begin{array}{c}\text { CYCLE } \\
\text { NO. }\end{array}$ & $\begin{array}{l}\text { PRE-TEST } \\
\text { WT. (g) }\end{array}$ & $\begin{array}{c}\text { AFTER-PURGE } \\
\text { WT. (g) }\end{array}$ & $\begin{array}{c}\text { AFTER-LOAD } \\
\text { WT. (g) }\end{array}$ & $\begin{array}{c}\text { TEST } \\
\text { START } \\
\text { DATE }\end{array}$ & $\begin{array}{l}\text { TEST ID } \\
\text { NO. }\end{array}$ & $\begin{array}{l}\text { CANISTER } \\
\text { LOAD (g) }\end{array}$ \\
\hline \multirow[t]{14}{*}{ ORHAOO } & 1 & 2804.9 & 2775.5 & 2866.7 & 23-Sep & 911409 & 91.2 \\
\hline & 2 & 2866.7 & 2774.2 & 2863.4 & 23-Sep & 911422 & 89.2 \\
\hline & 3 & 2863.4 & 2769.6 & 2865.6 & 24-Sep & 911423 & 96.0 \\
\hline & 4 & 2865.6 & 2768.7 & 2856.3 & 24-Sep & 911427 & 87.6 \\
\hline & 5 & 2856.3 & 2762.6 & 2849.2 & 24-Sep & 911428 & 86.6 \\
\hline & 6 & 2849.2 & 2759.3 & 2858.9 & 25-Sep & 911435 & 99.6 \\
\hline & 7 & 2858.9 & 2761.5 & 2858.1 & 25-Sep & 911436 & 96.6 \\
\hline & 8 & 2858.1 & 2758.8 & 2859.9 & 25-Sep & 911437 & 101.1 \\
\hline & 9 & 2859.9 & 2758.5 & 2857.4 & 25-Sep & 911438 & 98.9 \\
\hline & 10 & 2857.4 & 2756.3 & 2855.4 & 26-Sep & 911439 & 99.1 \\
\hline & 11 & 2855.4 & 2755.3 & 2852.7 & 26-Sep & 911440 & 97.4 \\
\hline & 12 & 2852.7 & 2753.1 & 2853.0 & 26-Sep & 911441 & 99.9 \\
\hline & 13 & 2853.0 & 2753.1 & 2852.2 & 27-Sep & 911442 & 99.1 \\
\hline & 14 & 2852.2 & 2751.9 & 2850.2 & 27-Sep & 911443 & 98.3 \\
\hline
\end{tabular}

\begin{tabular}{|c|c|c|c|c|c|c|c|}
\hline $\begin{array}{c}\text { CANISTER ID } \\
\text { NO. }\end{array}$ & $\begin{array}{c}\text { CYCLE } \\
\text { NO. }\end{array}$ & $\begin{array}{l}\text { PRE-TEST } \\
\text { WT. (g) }\end{array}$ & $\begin{array}{c}\text { AFTER-PURGE } \\
\text { WT. (g) }\end{array}$ & $\begin{array}{l}\text { AFTER-LOAD } \\
\text { WT. (g) }\end{array}$ & $\begin{array}{c}\text { TEST } \\
\text { START } \\
\text { DATE }\end{array}$ & $\begin{array}{c}\text { TEST ID } \\
\text { NO. }\end{array}$ & $\begin{array}{c}\text { CANISTER } \\
\text { LOAD (g) }\end{array}$ \\
\hline \multirow[t]{14}{*}{ ORHA15 } & 1 & 3219.9 & 3193.6 & 3276.0 & 23-Sep & 911414 & 82.4 \\
\hline & 2 & 3276.0 & 3187.7 & 3274.3 & 23-Sep & 911424 & 86.6 \\
\hline & 3 & 3274.3 & 3183.7 & 3269.7 & 24-Sep & 911425 & 86.0 \\
\hline & 4 & 3269.7 & 3186.5 & 3263.2 & 24-Sep & 911429 & 76.7 \\
\hline & 5 & 3263.2 & 3172.6 & 3263.0 & 24-Sep & 911430 & 90.4 \\
\hline & 6 & 3263.0 & 3171.4 & 3264.9 & 25-Sep & 911457 & 93.5 \\
\hline & 7 & 3264.9 & 3171.6 & 3265.0 & 25 -Sep & 911458 & 93.4 \\
\hline & 8 & 3265.0 & 3168.8 & 3264.4 & 25-Sep & 911459 & 95.6 \\
\hline & 9 & 3262.4 & 3166.2 & 3258.3 & 25 -Sep & 911460 & 92.1 \\
\hline & 10 & 3258.3 & 3163.5 & 3261.5 & $26-\mathrm{Sep}$ & 911461 & 98.0 \\
\hline & 11 & 3261.5 & 3163.2 & 3256.0 & 26-Sep & 911462 & 92.8 \\
\hline & 12 & 3256.0 & 3159.1 & 3257.0 & 26-Sep & 911463 & 97.9 \\
\hline & 13 & 3257.0 & 3159.0 & 3254.8 & 27-Sep & 911464 & 95.8 \\
\hline & 14 & 3254.8 & 3159.1 & 3252.3 & 27-Sep & 911465 & 93.2 \\
\hline
\end{tabular}




\section{ENVIRONMENTAL TESTING CORP. Canister Purge - Load Test Summary Vehicle Set: 2007 Honda Accords}

\begin{tabular}{|c|c|c|c|c|c|c|c|}
\hline $\begin{array}{l}\text { CANISTER ID } \\
\text { NO. }\end{array}$ & $\begin{array}{l}\text { CYCLE } \\
\text { NO. }\end{array}$ & $\begin{array}{l}\text { PRE-TEST } \\
\text { WT. (g) }\end{array}$ & $\begin{array}{c}\text { AFTER-PURGE } \\
\text { WT. (g) }\end{array}$ & $\begin{array}{l}\text { AFTER-LOAD } \\
\text { WT. (g) }\end{array}$ & $\begin{array}{c}\text { TEST } \\
\text { START } \\
\text { DATE }\end{array}$ & $\begin{array}{l}\text { TEST ID } \\
\text { NO. }\end{array}$ & $\begin{array}{c}\text { CANISTER } \\
\text { LOAD (g) }\end{array}$ \\
\hline \multirow[t]{14}{*}{ ORHA20 } & 1 & 2798.4 & 2764.1 & 2843.4 & 7 -Oct & 911619 & 79.3 \\
\hline & 2 & 2843.4 & 2754.3 & 2835.7 & 8-Oct & 911620 & 81.4 \\
\hline & 3 & 2833.8 & 2748.4 & 2839.6 & 8 -Oct & 911622 & 91.2 \\
\hline & 4 & 2839.6 & 2742.6 & 2833.6 & 9-Oct & 911666 & 91.0 \\
\hline & 5 & 2833.6 & 2739.0 & 2831.2 & 9-Oct & 911623 & 92.2 \\
\hline & 6 & 2829.3 & 2732.2 & 2825.7 & 9-Oct & 911635 & 93.5 \\
\hline & 7 & 2831.2 & 2737.2 & 2829.3 & 9-Oct & 911636 & 92.1 \\
\hline & 8 & 2825.7 & 2729.6 & 2827.0 & 10-Oct & 911637 & 97.4 \\
\hline & 9 & 2827.0 & 2729.4 & 2821.3 & $10-\mathrm{Oct}$ & 911638 & 91.9 \\
\hline & 10 & 2821.3 & 2726.9 & 2822.8 & $10-\mathrm{Oct}$ & 911639 & 95.9 \\
\hline & 11 & 2814.3 & 2723.9 & 2821.1 & 11-Oct & 911641 & 97.2 \\
\hline & 12 & 2821.1 & 2724.0 & 2820.4 & 11-Oct & 911642 & 96.4 \\
\hline & 13 & 2820.4 & 2723.5 & 2818.4 & 12-Oct & 911643 & 94.9 \\
\hline & 14 & 2818.4 & 2724.3 & 2816.5 & 12-Oct & 911644 & 92.2 \\
\hline
\end{tabular}

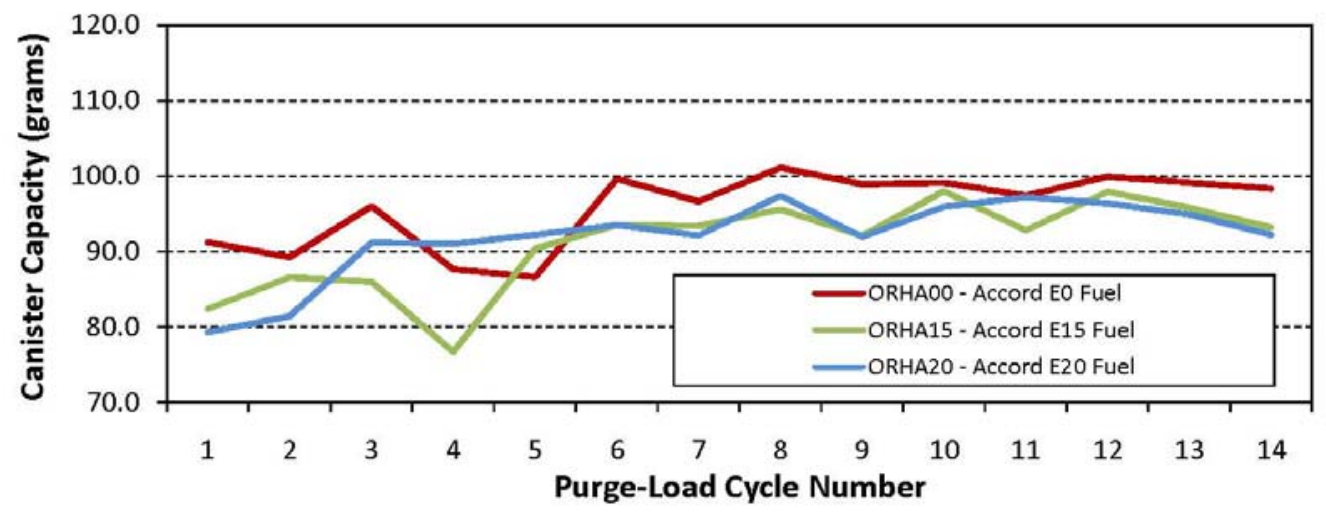




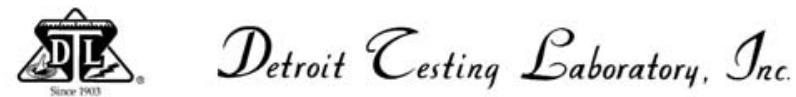

GENERAL DATA SHEET

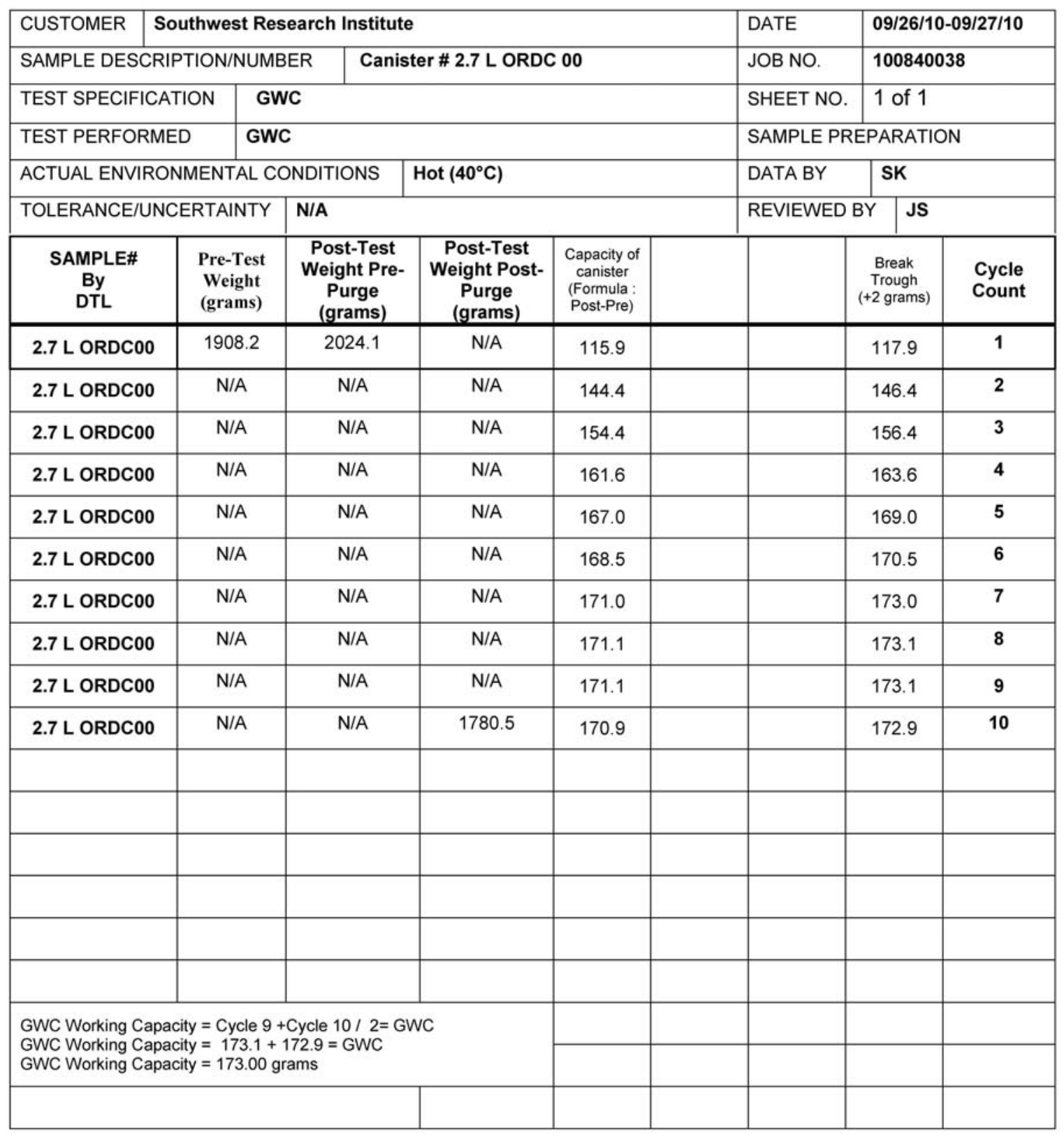

QCF-1041 REVISED 3/29/00 


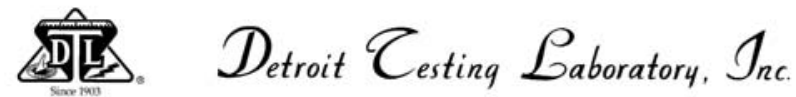

GENERAL DATA SHEET

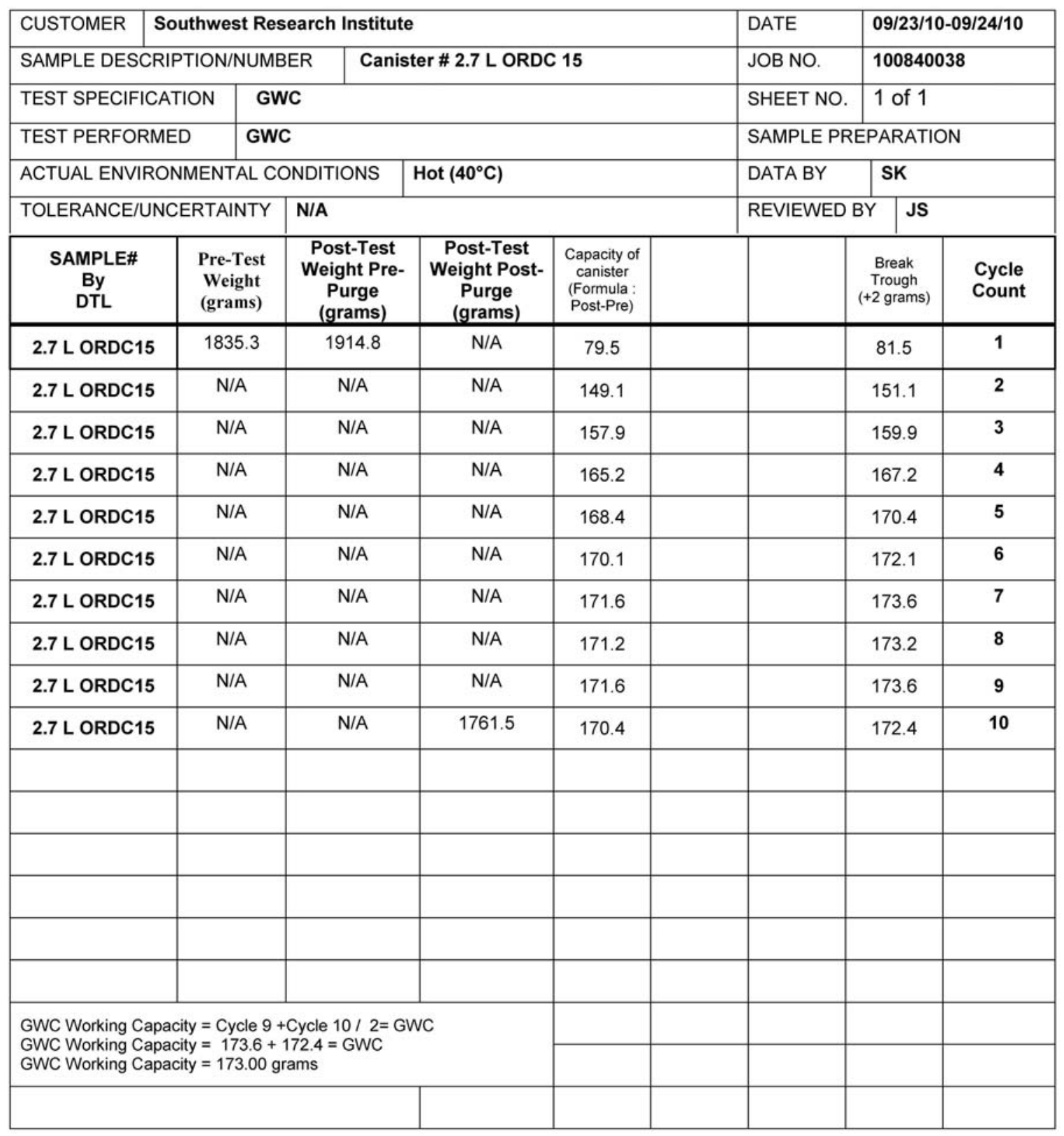

QCF-1041 REVISED 3/29/00 


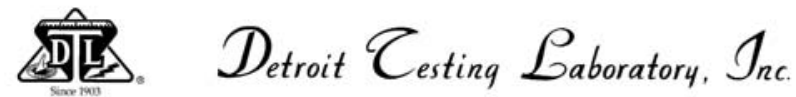

GENERAL DATA SHEET

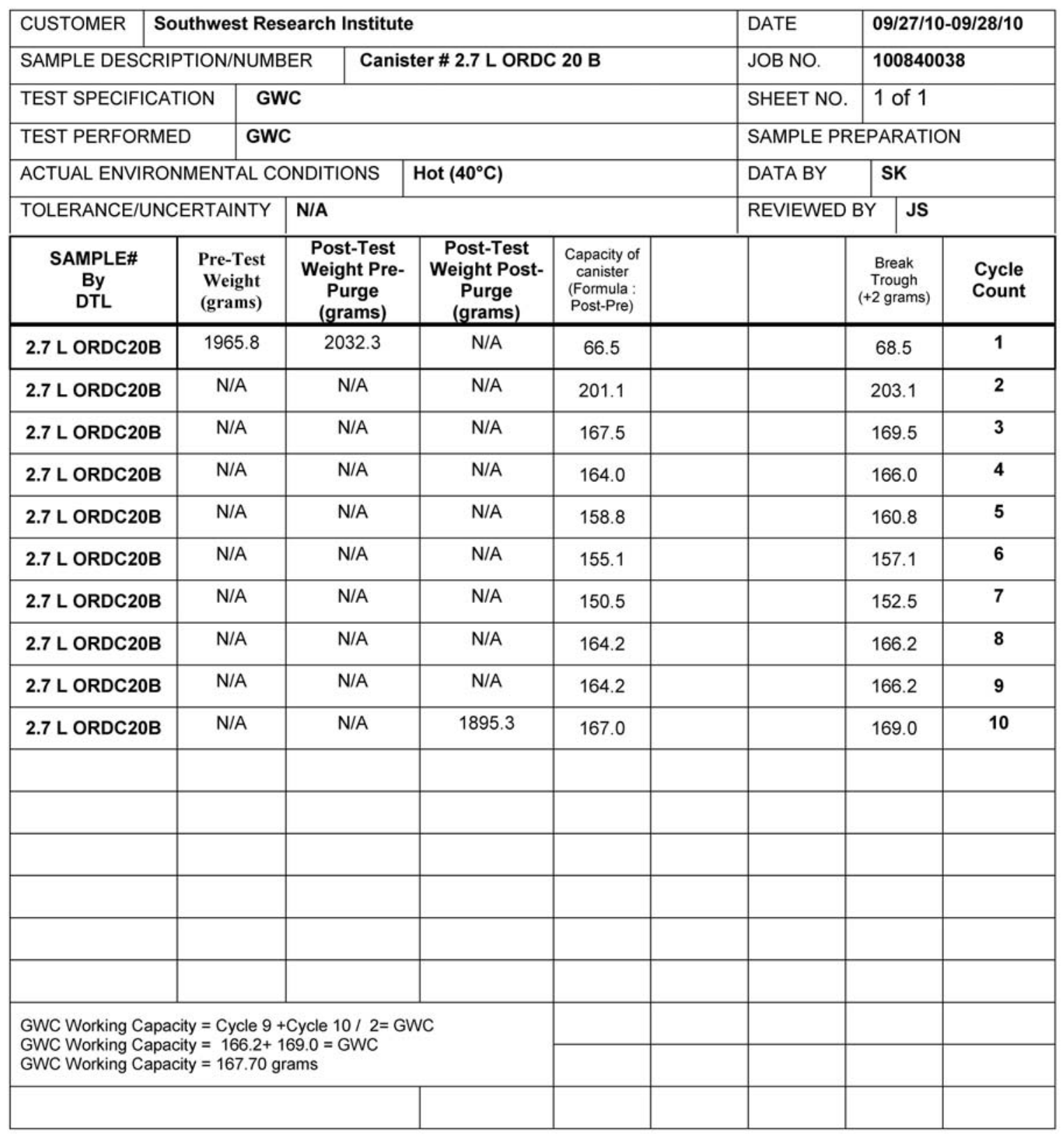

QCF-1041 REVISED 3/29/00 


\section{ENVIRONMENTAL TESTING CORP. Canister Purge - Load Test Summary Vehicle Set: 2008 Ford Taurus}

\begin{tabular}{|c|c|c|c|c|c|c|c|}
\hline $\begin{array}{c}\text { CANISTER ID } \\
\text { NO. }\end{array}$ & $\begin{array}{c}\text { CYCLE } \\
\text { NO. }\end{array}$ & $\begin{array}{c}\text { PRE-TEST } \\
\text { WT. (g) }\end{array}$ & $\begin{array}{c}\text { AFTER-PURGE } \\
\text { WT. (g) }\end{array}$ & $\begin{array}{c}\text { AFTER-LOAD } \\
\text { WT. (g) }\end{array}$ & $\begin{array}{c}\text { TEST } \\
\text { START } \\
\text { DATE }\end{array}$ & $\begin{array}{c}\text { TEST ID } \\
\text { NO. }\end{array}$ & $\begin{array}{l}\text { CANISTER } \\
\text { LOAD (g) }\end{array}$ \\
\hline \multirow[t]{14}{*}{ ORFT0 } & 1 & 2166.7 & 2153.4 & 2249.3 & 23-Sep & 911416 & 95.9 \\
\hline & 2 & 2249.3 & 2154.4 & 2247.0 & 23-Sep & 911418 & 92.6 \\
\hline & 3 & 2247.0 & 2149.2 & 2242.5 & 24-Sep & 911419 & 93.3 \\
\hline & 4 & 2242.5 & 2147.3 & 2242.2 & 24-Sep & 911431 & 94.9 \\
\hline & 5 & 2242.2 & 2152.6 & 2241.8 & 25-Sep & 911432 & 89.2 \\
\hline & 6 & 2241.8 & 2143.5 & 2239.8 & 25-Sep & 911467 & 96.3 \\
\hline & 7 & 2239.8 & 2148.5 & 2239.2 & 25-Sep & 911468 & 90.7 \\
\hline & 8 & 2239.2 & 2148.7 & 2239.1 & 26-Sep & 911469 & 90.4 \\
\hline & 9 & 2239.1 & 2139.8 & 2238.8 & 26-Sep & 911470 & 99.0 \\
\hline & 10 & 2170.5 & 2138.1 & 2234.0 & 27-Sep & 911472 & 95.9 \\
\hline & 11 & 2234.0 & 2141.4 & 2234.8 & 27-Sep & 911473 & 93.4 \\
\hline & 12 & 2234.8 & 2136.8 & 2233.2 & 27-Sep & 911474 & 96.4 \\
\hline & 13 & 2233.2 & 2132.7 & 2230.3 & 28-Sep & 911475 & 97.6 \\
\hline & 14 & 2230.3 & 2137.2 & 2233.0 & 28-Sep & 911476 & 95.8 \\
\hline
\end{tabular}

\begin{tabular}{|c|c|c|c|c|c|c|c|}
\hline $\begin{array}{c}\text { CANISTER ID } \\
\text { No. }\end{array}$ & $\begin{array}{c}\text { CYCLE } \\
\text { No. }\end{array}$ & $\begin{array}{c}\text { PRE-TEST } \\
\text { WT. (g) }\end{array}$ & $\begin{array}{c}\text { AFTER-PURGE } \\
\text { WT. (g) }\end{array}$ & $\begin{array}{l}\text { AFTER-LOAD } \\
\text { WT. (g) }\end{array}$ & $\begin{array}{l}\text { TEST } \\
\text { START } \\
\text { DATE }\end{array}$ & $\begin{array}{c}\text { TEST ID } \\
\text { No. }\end{array}$ & $\begin{array}{c}\text { CANISTER } \\
\text { LOAD (g) }\end{array}$ \\
\hline \multirow[t]{14}{*}{ ORFT15 } & 1 & 2244.9 & 2214.2 & 2299.7 & 23-Sep & 911417 & 85.5 \\
\hline & 2 & 2299.7 & 2207.9 & 2297.8 & 23-Sep & 911420 & 89.9 \\
\hline & 3 & 2299.8 & 2201.4 & 2289.4 & 24-Sep & 911421 & 88.0 \\
\hline & 4 & 2289.4 & 2195.3 & 2291.5 & 24-Sep & 911433 & 96.2 \\
\hline & 5 & 2291.5 & 2193.4 & 2294.1 & 24-Sep & 911434 & 100.7 \\
\hline & 6 & 2294.1 & 2193.7 & 2287.4 & 25-Sep & 911446 & 93.7 \\
\hline & 7 & 2287.4 & 2188.3 & 2284.1 & 25-Sep & 911447 & 95.8 \\
\hline & 8 & 2284.1 & 2185.7 & 2289.0 & 25-Sep & 911448 & 103.3 \\
\hline & 9 & 2189.0 & 2189.4 & 2283.2 & 25-Sep & 911449 & 93.8 \\
\hline & 10 & 2283.2 & 2185.0 & 2283.6 & 26-Sep & 911450 & 98.6 \\
\hline & 11 & 2295.0 & 2188.8 & 2284.3 & 26-Sep & 911452 & 95.5 \\
\hline & 12 & 2284.3 & 2182.8 & 2280.2 & 27-Sep & 911453 & 97.4 \\
\hline & 13 & 2280.2 & 2180.0 & 2284.3 & 27-Sep & 911454 & 104.3 \\
\hline & 14 & 2284.3 & 2180.7 & 2278.4 & 27-Sep & 911455 & 97.7 \\
\hline
\end{tabular}




\section{ENVIRONMENTAL TESTING CORP. Canister Purge - Load Test Summary \\ Vehicle Set: 2008 Ford Taurus}

\begin{tabular}{|c|c|c|c|c|c|c|c|}
\hline $\begin{array}{l}\text { CANISTER ID } \\
\text { NO. }\end{array}$ & $\begin{array}{l}\text { CYCLE } \\
\text { NO. }\end{array}$ & $\begin{array}{l}\text { PRE-TEST } \\
\text { WT. (g) }\end{array}$ & $\begin{array}{l}\text { AFTER-PURGE } \\
\text { WT. (g) }\end{array}$ & $\begin{array}{l}\text { AFTER-LOAD } \\
\text { WT. (g) }\end{array}$ & $\begin{array}{l}\text { TEST } \\
\text { START } \\
\text { DATE }\end{array}$ & $\begin{array}{l}\text { TEST ID } \\
\text { No. }\end{array}$ & $\begin{array}{l}\text { CANISTER } \\
\text { LOAD (g) }\end{array}$ \\
\hline \multirow[t]{14}{*}{ ORFT-20 } & 1 & 2245.2 & 2221.5 & 2309.0 & 7-Oct & 911614 & 87.5 \\
\hline & 2 & 2309.0 & 2218.6 & 2302.9 & 8-Oct & 911615 & 84.3 \\
\hline & 3 & 2303.5 & 2211.1 & 2300.9 & $8-\mathrm{Oct}$ & 911616 & 89.8 \\
\hline & 4 & 2300.9 & 2204.7 & 2298.5 & 9-Oct & 911617 & 93.8 \\
\hline & 5 & 2298.5 & 2200.7 & 2294.1 & 9-Oct & 911618 & 93.4 \\
\hline & 6 & 2294.1 & 2198.2 & 2299.2 & 9-Oct & 911625 & 101.0 \\
\hline & 7 & 2299.2 & 2197.9 & 2293.0 & 10-Oct & 911626 & 95.1 \\
\hline & 8 & 2293.0 & 2193.7 & 2290.4 & $10-\mathrm{Oct}$ & 911627 & 96.7 \\
\hline & 9 & 2266.7 & 2189.6 & 2284.2 & 11-Oct & 911628 & 94.6 \\
\hline & 10 & 2284.2 & 2195.3 & 2289.4 & 11-Oct & 911629 & 94.1 \\
\hline & 11 & 2289.4 & 2191,0 & 2289.0 & $11-\mathrm{Oct}$ & 911630 & 98.0 \\
\hline & 12 & 2289.0 & 2188.9 & 2290.2 & 12-Oct & 911631 & 101.3 \\
\hline & 13 & 2280.5 & 2183.3 & 2290.9 & $13-$ Oct & 911632 & 107.6 \\
\hline & 14 & 2290.9 & 2186.9 & 2289.1 & 14-Oct & 911634 & 102.2 \\
\hline
\end{tabular}

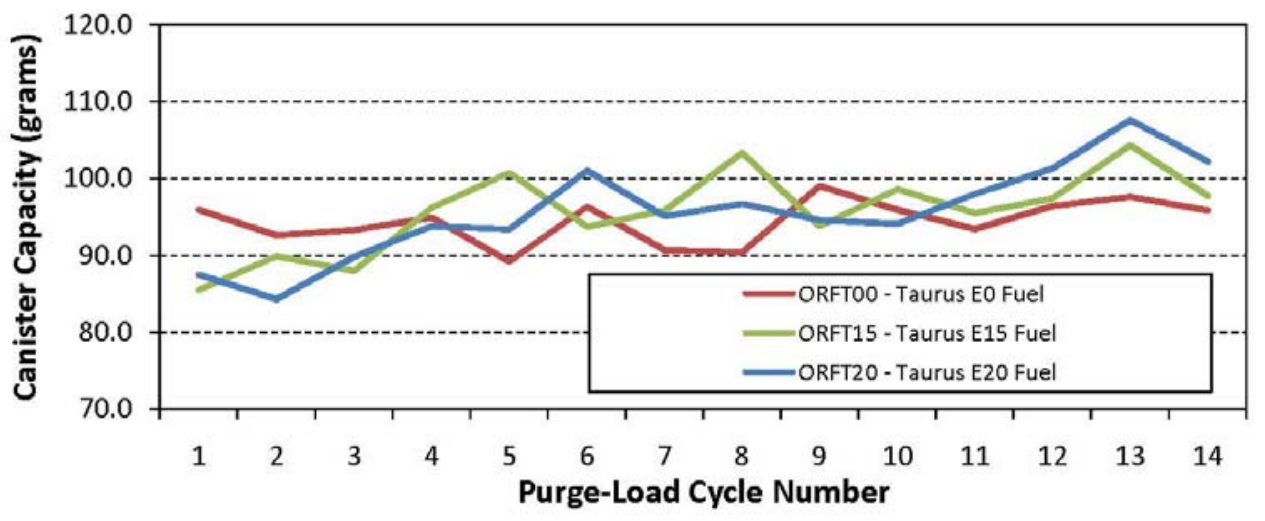




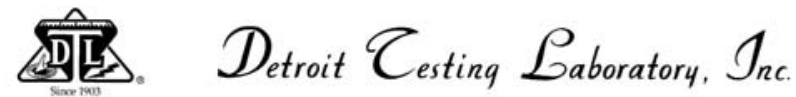

GENERAL DATA SHEET

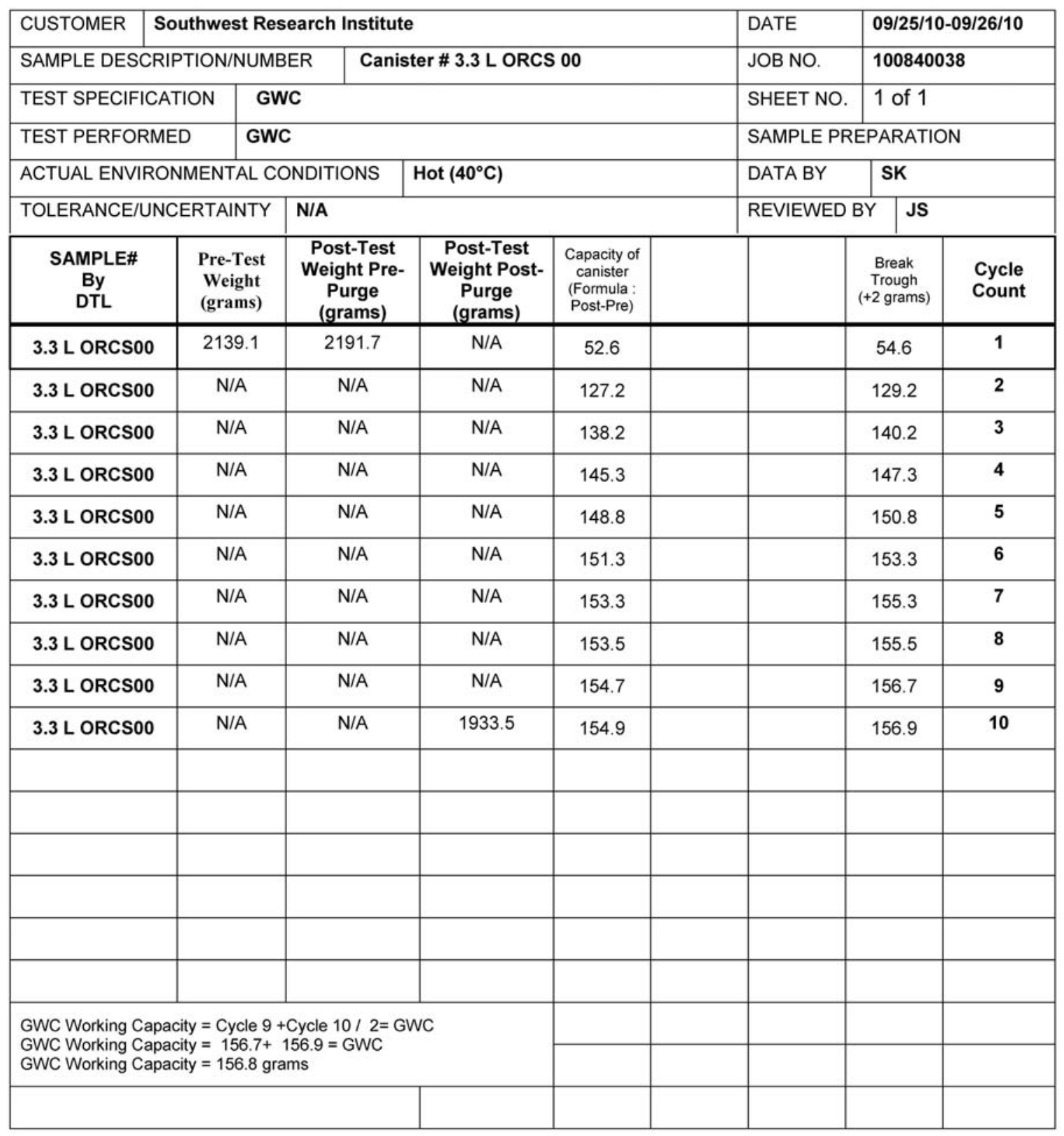

QCF-1041 REVISED 3/29/00 


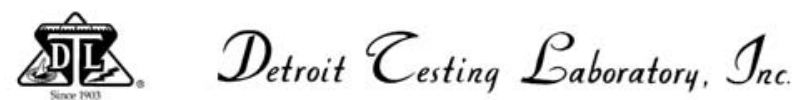

GENERAL DATA SHEET

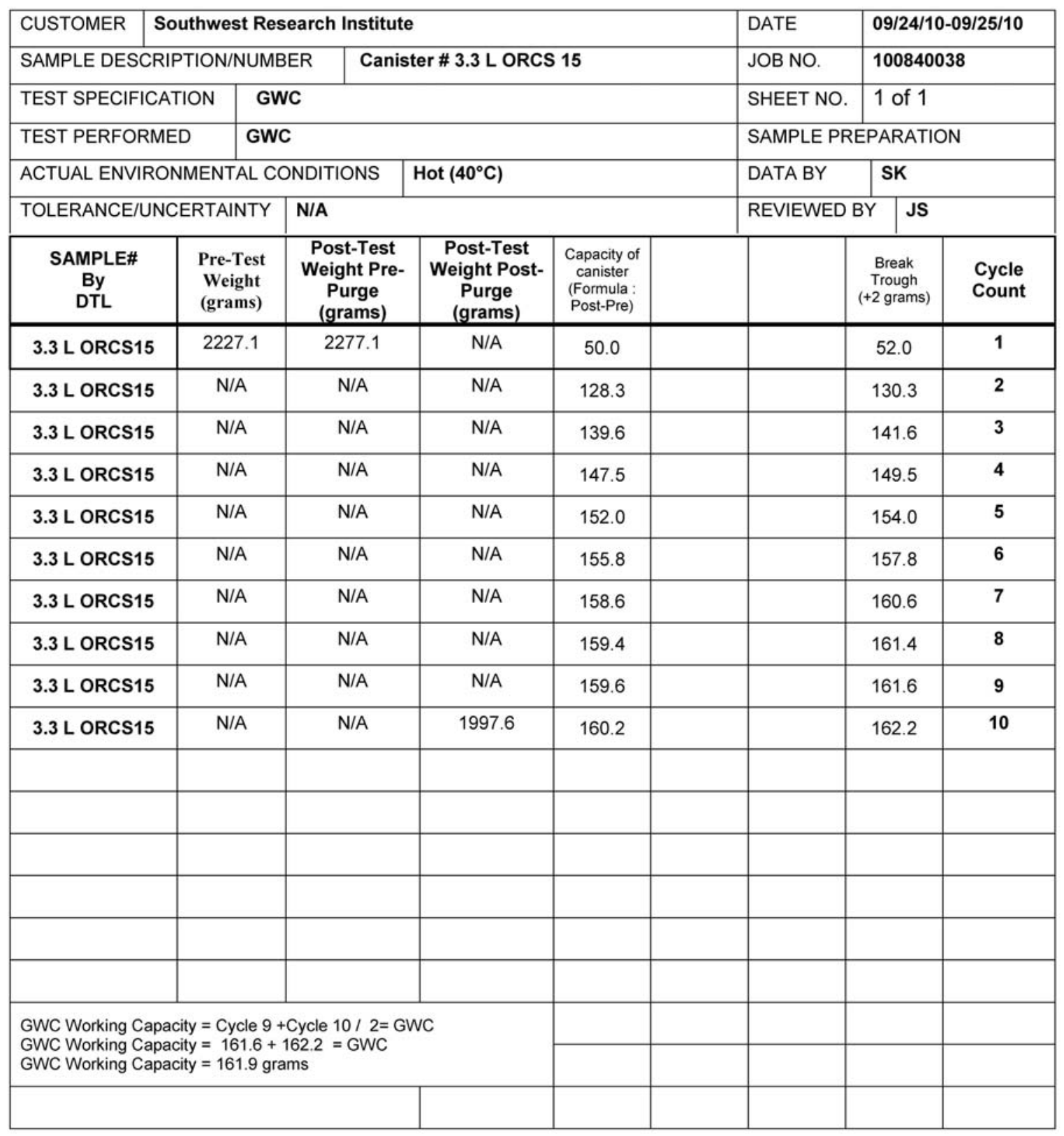

QCF-1041 REVISED 3/29/00 


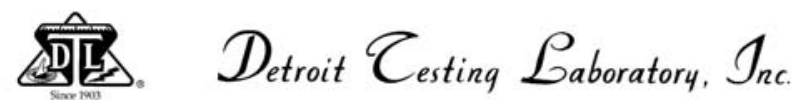

GENERAL DATA SHEET

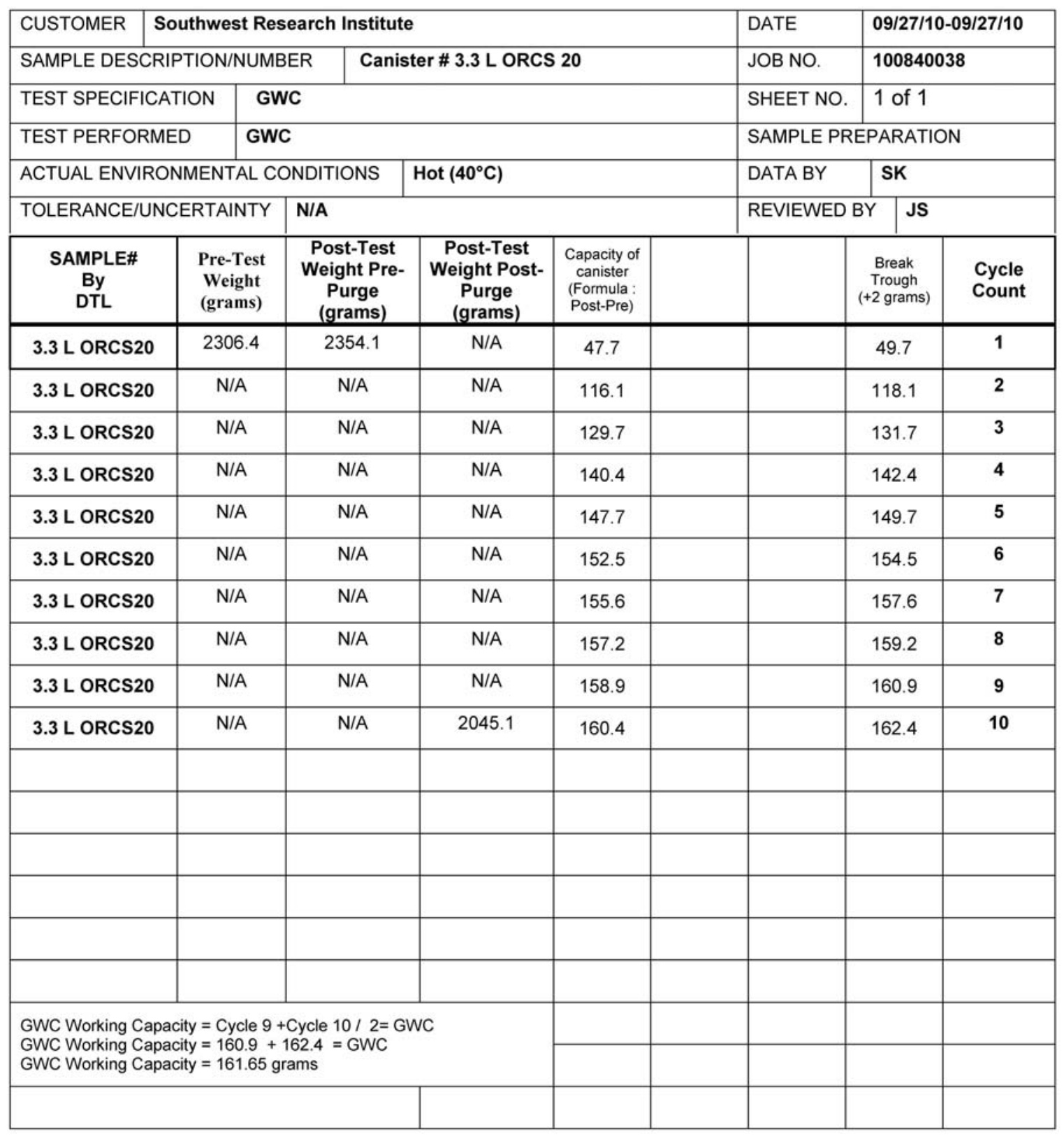

QCF-1041 REVISED 3/29/00 

Appendix $\mathbf{J}$

Visual Inspection of Fuel Tanks, Fuel Lines and Evaporative Emissions Lines 



\section{Chevrolet Cobalt}

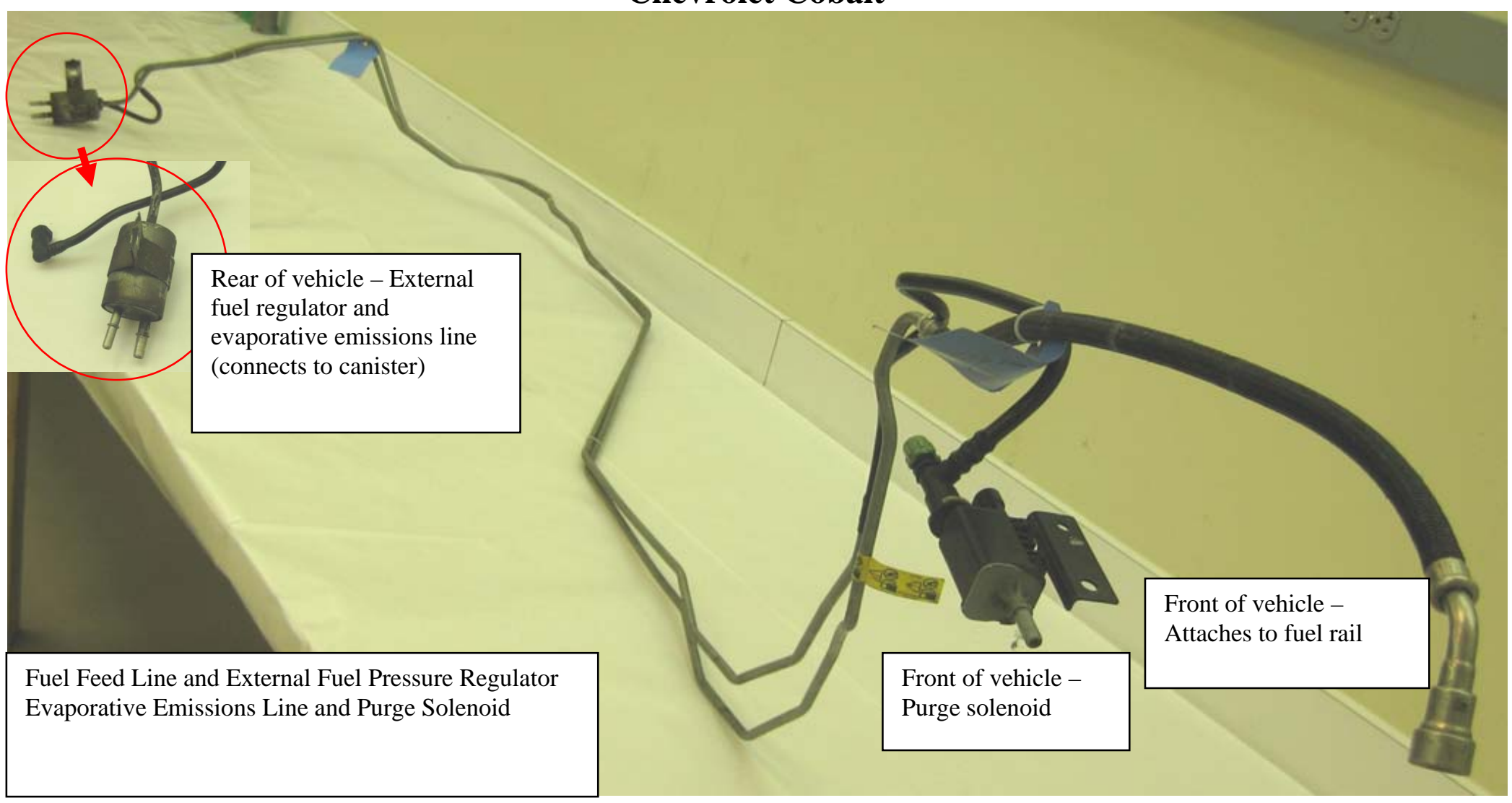

Fuel Feed Line

\begin{tabular}{|c|l|l|}
\hline \multirow{2}{*}{ Vehicle } & \multicolumn{2}{|c|}{ Visual Rating Comments } \\
\cline { 2 - 3 } & Front of Vehicle & Rear of Vehicle \\
\hline ORCC0 & $\begin{array}{l}\text { 15\% white deposits, 85\% clean } \\
\text { O-ring rating: Good condition }\end{array}$ & $\begin{array}{l}\text { Small diameter line }-2 \% \text { white deposit, 98\% clean } \\
\text { Large diameter line }-100 \% \text { clean }\end{array}$ \\
\hline ORCC15 & $\begin{array}{l}100 \% \text { white deposit } \\
\text { O-ring rating: Good condition }\end{array}$ & $\begin{array}{l}\text { Small diameter line }-100 \% \text { rust } \\
\text { Large diameter line }-10 \% \text { rust, 90\% clean }\end{array}$ \\
\hline ORCC20 & $\begin{array}{l}\text { 100\% white deposit } \\
\text { O-ring rating: Good condition }\end{array}$ & $\begin{array}{l}\text { Small diameter line }-5 \% \text { rust, } 95 \% \text { clean } \\
\text { Large diameter line }-100 \% \text { rust }\end{array}$ \\
\hline
\end{tabular}




\section{Chevrolet Cobalt}

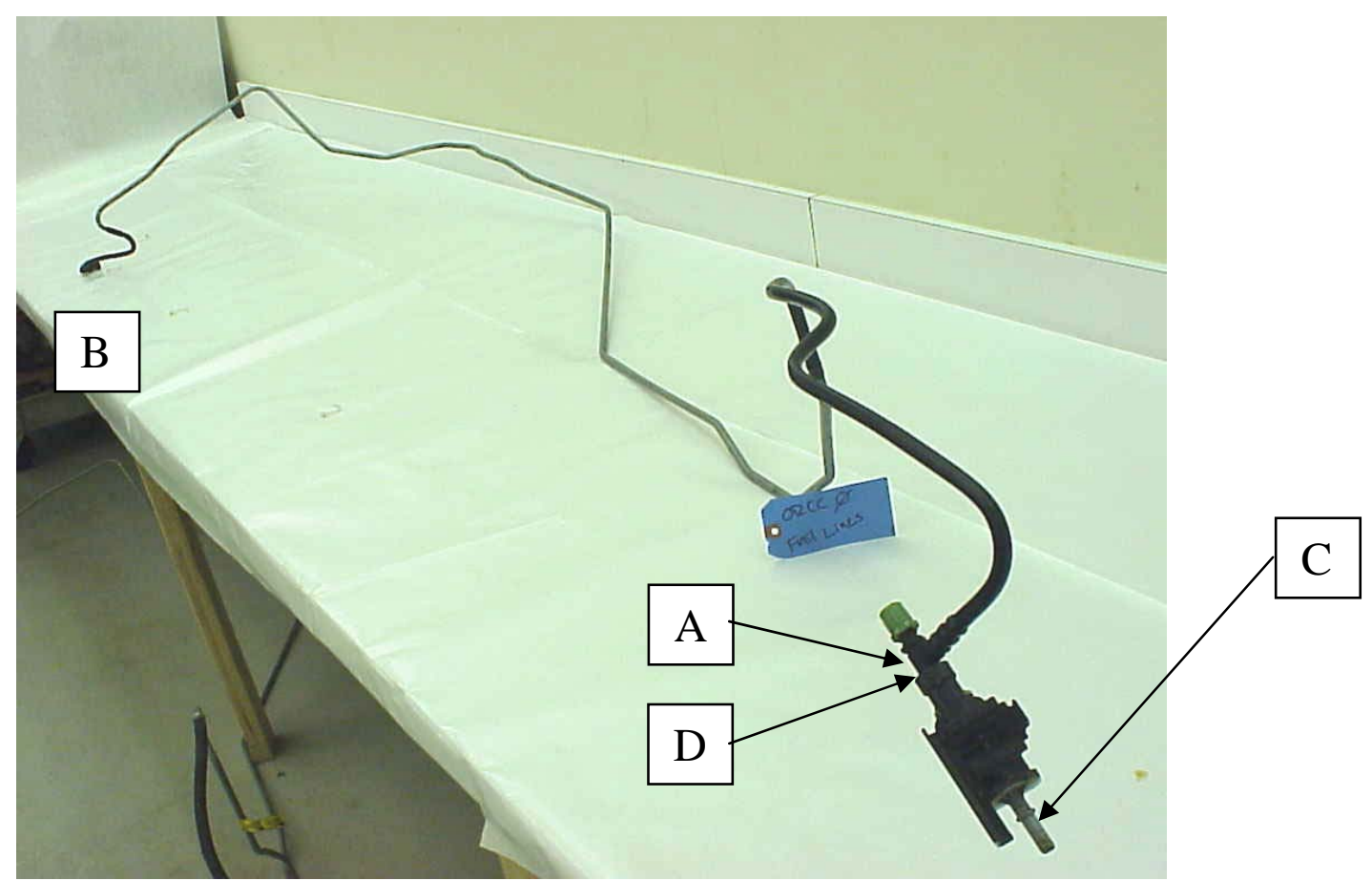

Evaporative Emissions Line

\begin{tabular}{|c|l|l|}
\hline \multirow{2}{*}{ Vehicle } & \multicolumn{2}{|c|}{ Visual Rating Comments } \\
\cline { 2 - 3 } & Front of Vehicle [A] & Rear of Vehicle [B] \\
\hline ORCC0 & $\begin{array}{l}\text { Plastic hose rating: 90\% white deposit, 10\% clean } \\
\text { O-ring rating: Good condition }\end{array}$ & $\begin{array}{l}\text { Plastic line rating: 10\% white deposit, 90\% clean } \\
\text { O-ring rating: Good condition }\end{array}$ \\
\hline ORCC15 & $\begin{array}{l}\text { Plastic hose rating: Wet fuel present } \\
\text { O-ring rating: Good condition }\end{array}$ & $\begin{array}{l}\text { Hose rating: 100\% clean } \\
\text { O-ring rating: Good condition }\end{array}$ \\
\hline ORCC20 & $\begin{array}{l}\text { Plastic hose rating: 5\% brown deposit, 95\% clean } \\
\text { O-ring rating: Good condition }\end{array}$ & $\begin{array}{l}\text { Plastic hose rating - 10\% white deposit, 90\% clean } \\
\text { O-ring rating: Good condition }\end{array}$ \\
\hline \multicolumn{2}{|l|}{} \\
\hline ORCC0 & Front of Purge Solenoid [C] & Rear of Vehicle [D] \\
\hline ORCC15 & $100 \%$ clean & $100 \%$ trace white deposit \\
\hline ORCC20 & $100 \%$ clean & $100 \%$ clean \\
\hline
\end{tabular}




\section{Chevrolet Silverado}

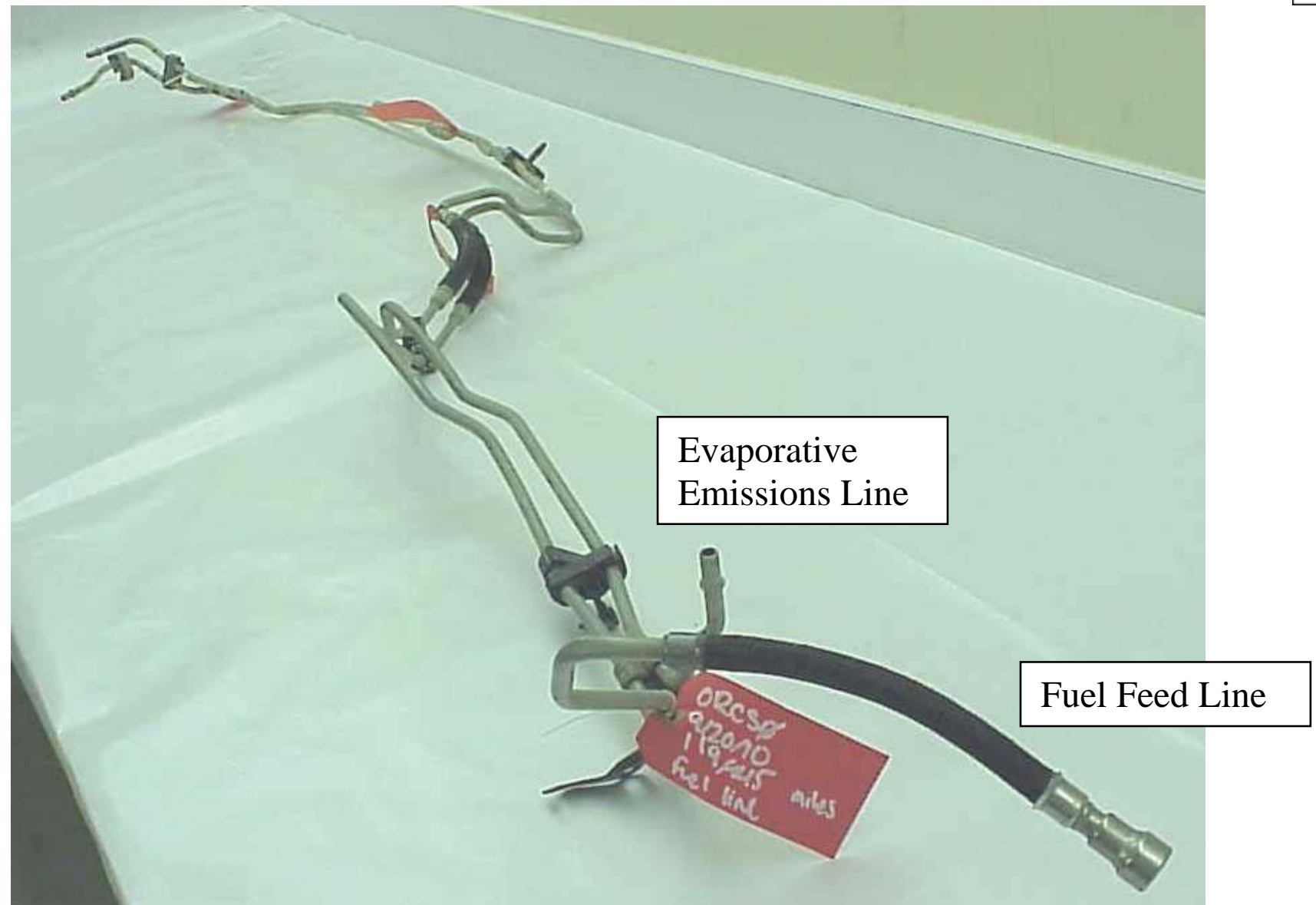

Fuel Feed Line

\begin{tabular}{|c|l|l|}
\hline \multirow{2}{*}{ Vehicle } & \multicolumn{1}{|c|}{ Visual Rating Comments } \\
\cline { 2 - 3 } ORCS0 & $\begin{array}{l}\text { Front of Vehicle } \\
\text { Hose, 10\% white deposit } \\
\text { O-ring (yellow), good condition - (black) good condition }\end{array}$ & Rear of Vehicle \\
\hline ORCS15 & $\begin{array}{l}\text { Hose, 100\% clean } \\
\text { O-ring (yellow), good condition - (black) good condition }\end{array}$ & $5 \%$ rust, 95\% clean \\
\hline ORCS20 & $\begin{array}{l}\text { Hose, 100\% clean } \\
\text { O-ring (yellow), good condition - (black) good condition }\end{array}$ & 95\% clean, 5\% white deposits \\
\hline
\end{tabular}




\section{Chevrolet Silverado}

\section{Evaporative Emissions Line}

\begin{tabular}{|c|l|l|}
\hline \multirow{2}{*}{ Vehicle } & \multicolumn{2}{|c|}{ Visual Rating Comments } \\
\cline { 2 - 3 } & Front of Vehicle & Rear of Vehicle \\
\hline ORCS0 & $90 \%$ clean, 10\% white deposits & $100 \%$ rust deposits \\
\hline ORCS15 & $100 \%$ clean & $100 \%$ rust deposits \\
\hline ORCS20 & $\begin{array}{l}30 \% \text { clean, 10\% white deposits, 60\% light gray } \\
\text { deposits }\end{array}$ & $100 \%$ rust deposits \\
\hline
\end{tabular}




\section{Dodge Caravan}

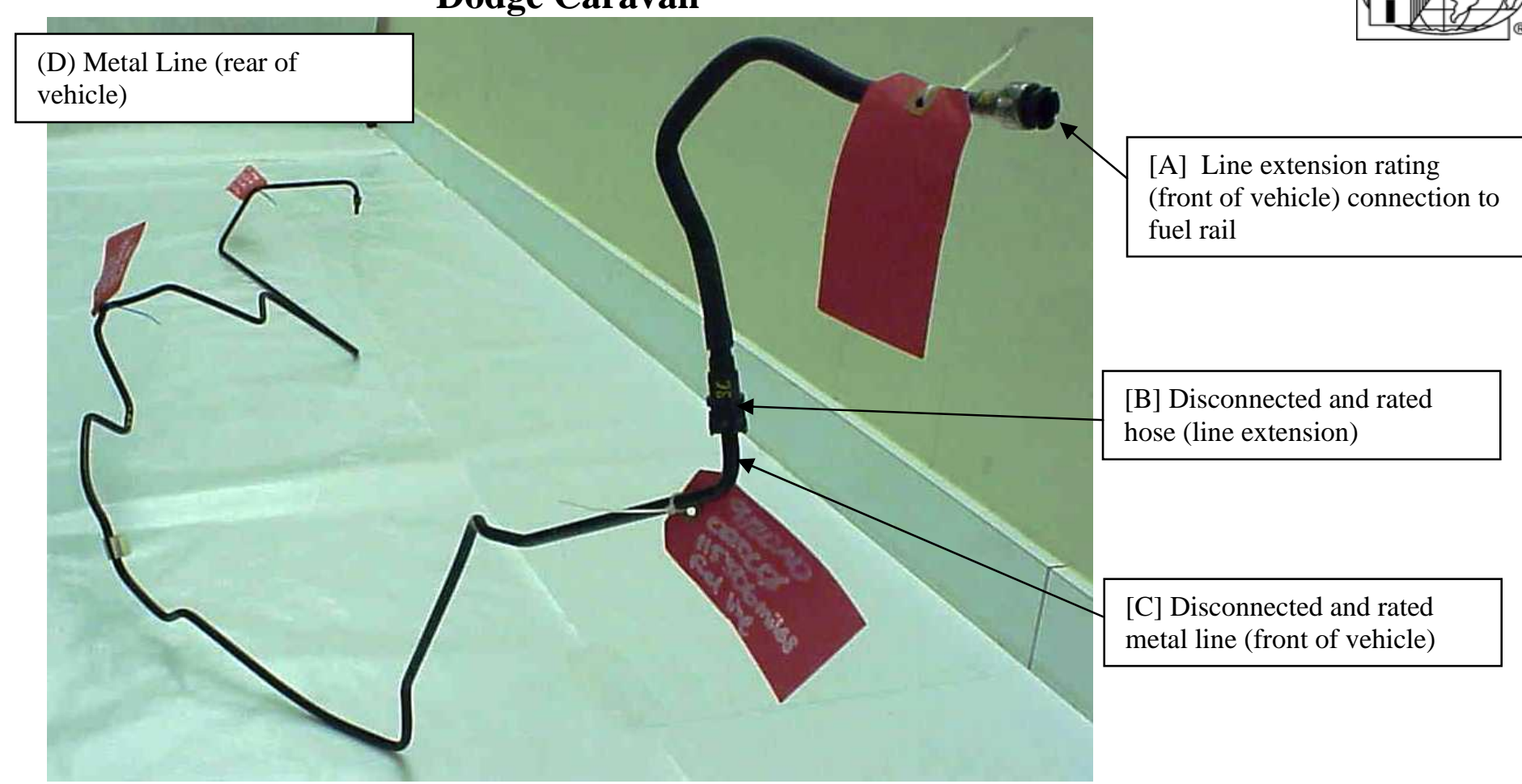

Fuel Feed Line Metal Line

\begin{tabular}{|c|l|l|}
\hline \multirow{2}{*}{ Vehicle } & \multicolumn{2}{|c|}{ Visual Rating Comments } \\
\cline { 2 - 3 } & [C] Front of Vehicle (attaches to the line extension) & [D] Rear of Vehicle \\
\hline ORDC0 & $100 \%$ clean & $10 \%$ rust, $90 \%$ clean \\
\hline ORDC15 & $100 \%$ clean & $20 \%$ rust, $80 \%$ clean \\
\hline ORDC20B & $100 \%$ clean & $100 \%$ clean \\
\hline
\end{tabular}

Fuel Feed Line - Line Extension

\begin{tabular}{|c|l|l|}
\hline Vehicle & \multicolumn{2}{|c|}{ Visual Rating Comments } \\
\cline { 2 - 4 } & [A] Front of Vehicle (connection to the fuel rail) & [B] Rear of Vehicle \\
\hline ORDC0 & $80 \%$ white deposits, 20\% rust - O-ring good condition & $100 \%$ clean, O-ring in good condition \\
\hline ORDC15 & $75 \%$ white deposits, 25\% rust - O-ring good condition & $100 \%$ clean, O-ring in good condition \\
\hline ORDC20B & $90 \%$ white deposits, 10\% rust - O-ring good condition & $100 \%$ clean, O-ring in good condition \\
\hline
\end{tabular}




\section{Dodge Caravan}

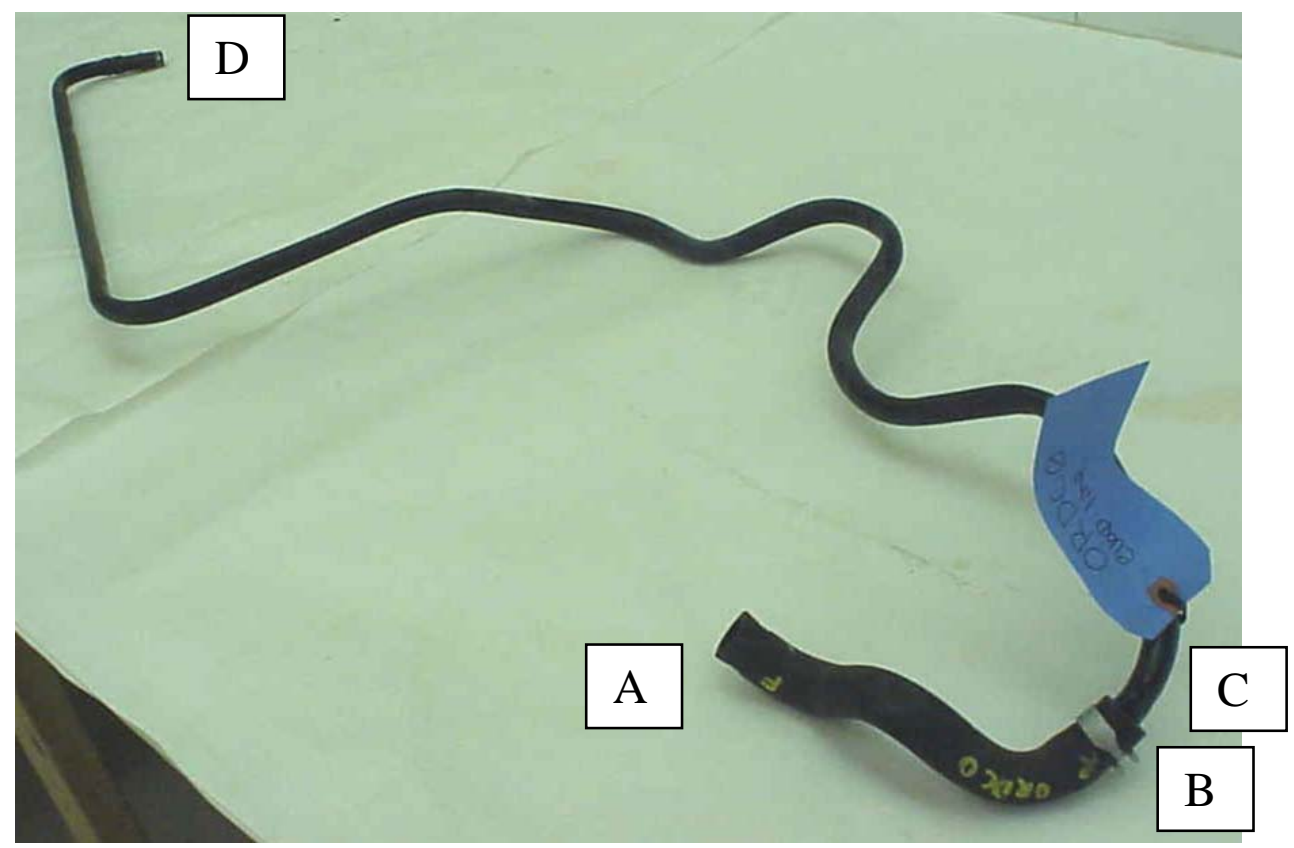

Evaporative Emissions Line - Rubber Hose

\begin{tabular}{|c|l|l|}
\hline \multirow{2}{*}{ Vehicle } & \multicolumn{2}{|c|}{ Visual Rating Comments } \\
\cline { 2 - 3 } & {$[\mathrm{A}]$ Front of Vehicle } & {$[\mathrm{B}]$ Rear of Hose } \\
\hline ORDC0 & $10 \%$ white deposit, 90\% clean & $15 \%$ white deposit, 85\% clean \\
\hline ORDC15 & $2 \%$ white deposit, 98\% clean & $5 \%$ white deposit, 95\% clean \\
\hline ORDC20B & $1 \%$ white deposit, 99\% clean & $100 \%$ clean \\
\hline
\end{tabular}

Evaporative Emissions Line

\begin{tabular}{|c|l|l|}
\hline \multirow{2}{*}{ Vehicle } & \multicolumn{2}{|c|}{ Visual Rating Comments } \\
\cline { 2 - 3 } & [C] Front of Vehicle (attaches to the line extension) & [D] Rear of Vehicle \\
\hline ORDC0 & $90 \%$ white deposit, 10\% clean & $97 \%$ clean, 3\% debris \\
\hline ORDC15 & $43 \%$ white deposit, 57\% clean & $1 \%$ white deposit, 99\% clean \\
\hline ORDC20B & $1 \%$ white deposit, 99\% clean & $10 \%$ clean \\
\hline
\end{tabular}



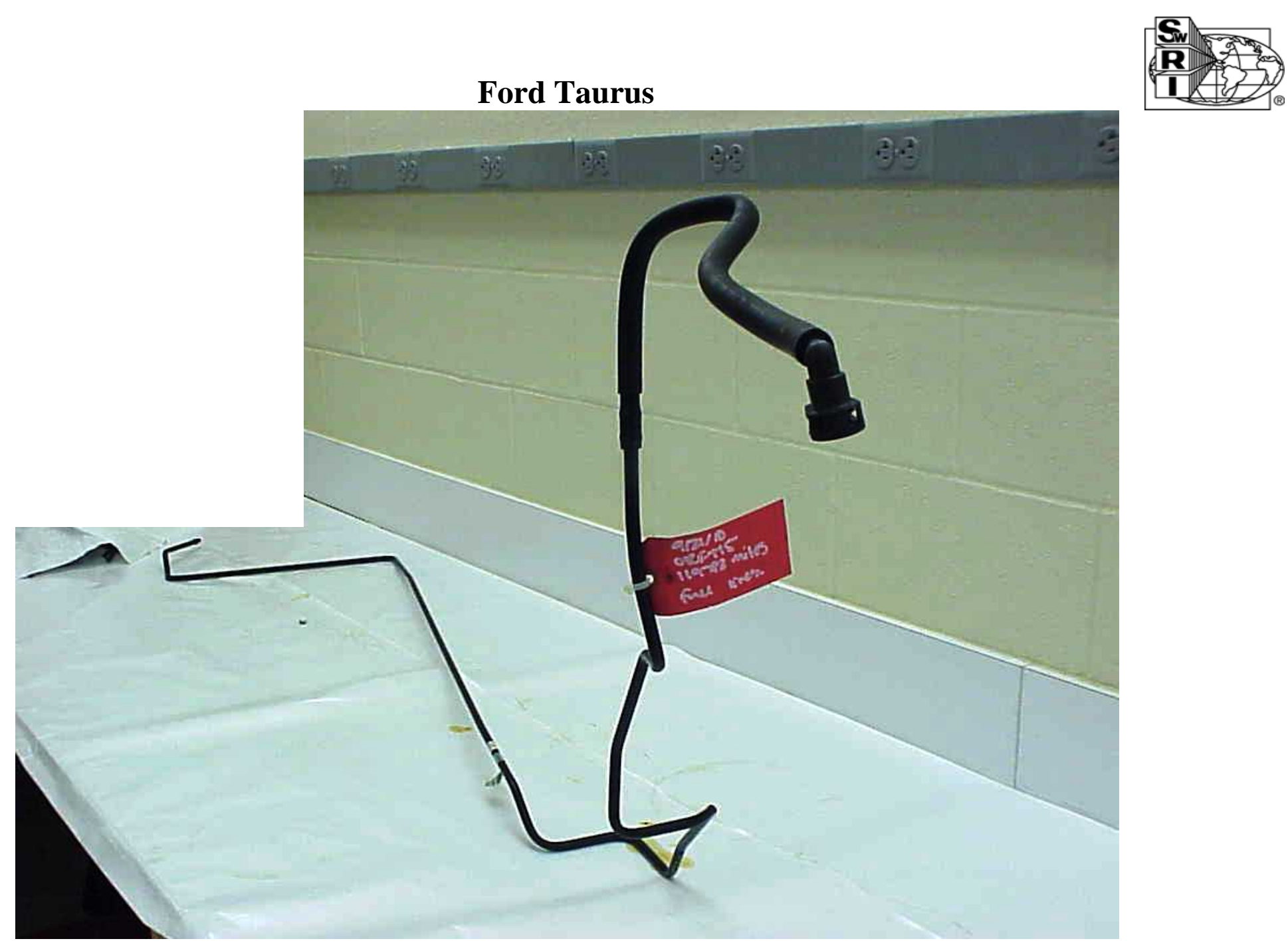

Fuel Feed Line

\begin{tabular}{|c|l|l|}
\hline \multirow{2}{*}{ Vehicle } & \multicolumn{2}{|c|}{ Visual Rating Comments } \\
\cline { 2 - 3 } & Front of Vehicle & Rear of Vehicle \\
\hline ORFT0 & $100 \%$ clean, o-ring: good condition & $2 \%$ rust: $98 \%$ clean \\
\hline ORFT15 & $100 \%$ clean, o-ring: good condition & $100 \%$ clean \\
\hline ORFT20 & $100 \%$ clean, o-ring: good condition & $2 \%$ rust: $98 \%$ clean \\
\hline
\end{tabular}




\section{Ford Taurus}

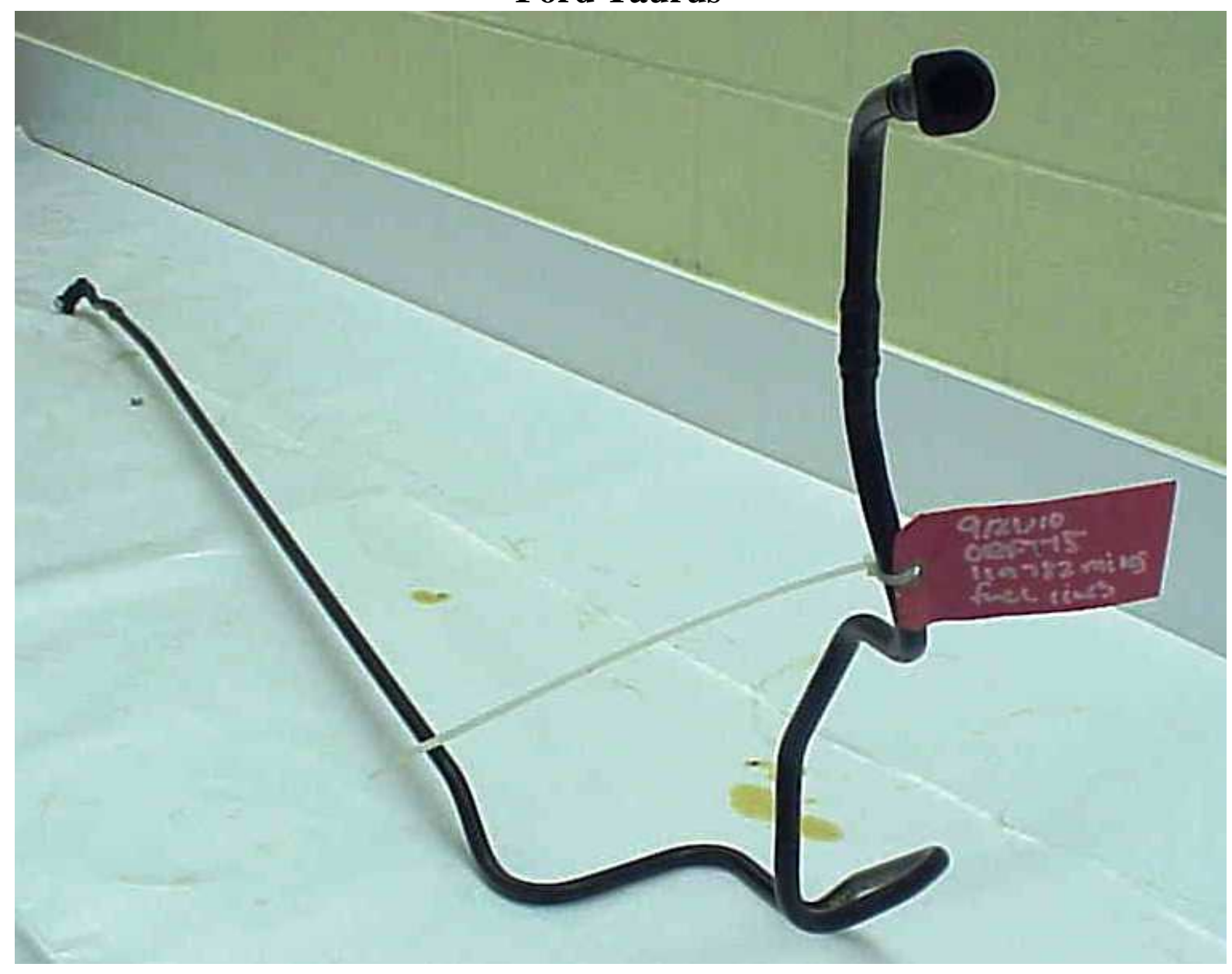

Evaporative Emissions Line

\begin{tabular}{|l|l|l|}
\hline \multirow{2}{*}{ Vehicle } & \multicolumn{2}{|c|}{ Visual Rating Comments } \\
\cline { 2 - 3 } & Front of Vehicle & Rear of Vehicle \\
\hline ORFT0 & $100 \%$ white deposit - O-ring in good condition & $100 \%$ clean, O-ring in good condition \\
\hline ORFT15 & $100 \%$ clean, O-ring in good condition & $100 \%$ clean, O-ring in good condition \\
\hline ORFT20 & $100 \%$ clean, O-ring in good condition & $100 \%$ clean, O-ring in good condition \\
\hline
\end{tabular}




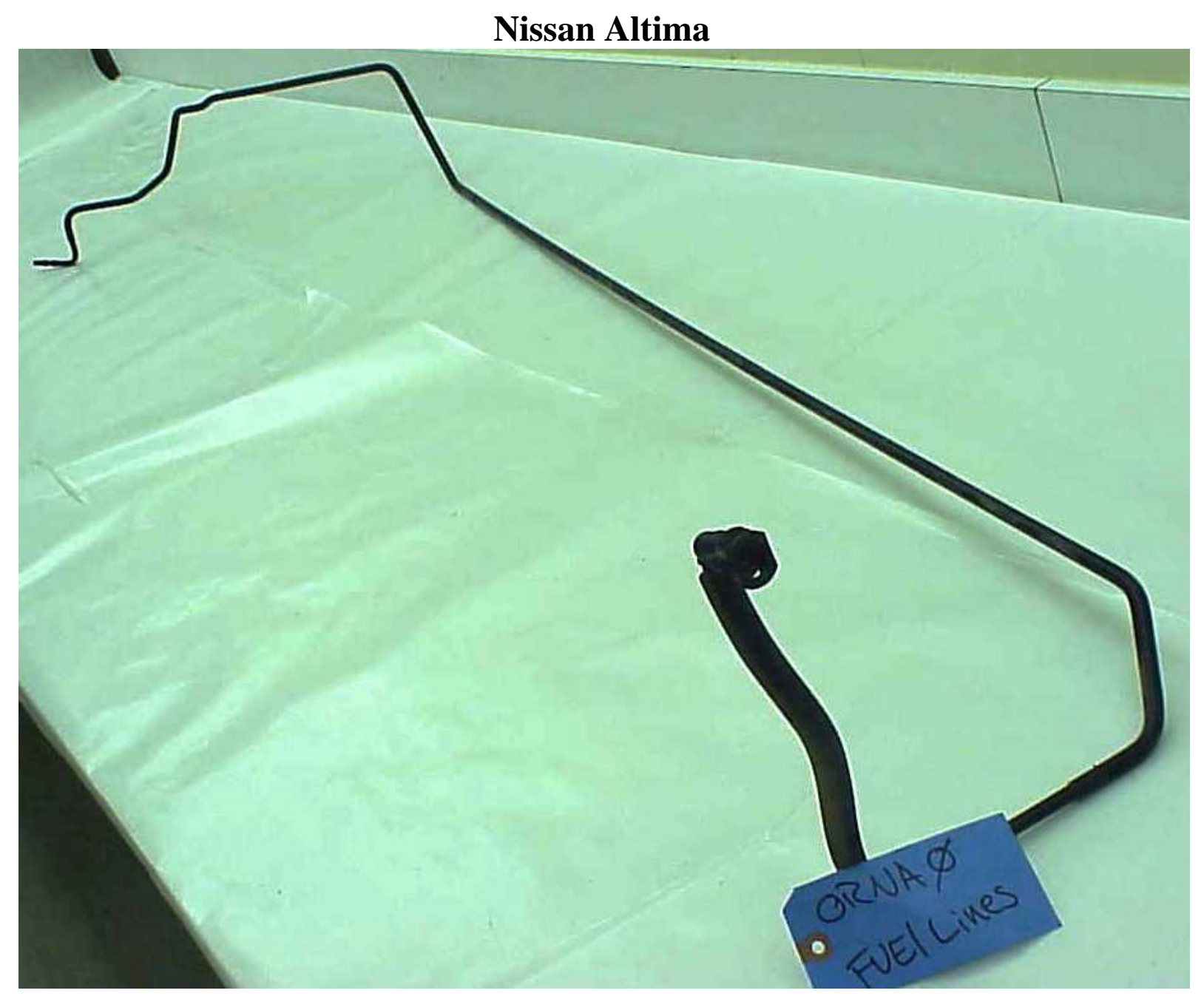

Fuel Feed Line

\begin{tabular}{|c|l|l|}
\hline \multirow{2}{*}{ Vehicle } & \multicolumn{2}{|c|}{ Visual Rating Comments } \\
\cline { 2 - 3 } & Front of Vehicle & Rear of Vehicle \\
\hline ORNA0 & Hose rating, 100\% clean, o-ring in good condition & $100 \%$ clean, no deposits \\
\hline ORNA15 & Hose rating, 100\% clean, o-ring in good condition & $100 \%$ clean, no deposits \\
\hline ORNA20 & Hose rating, 100\% clean, o-ring in good condition & $100 \%$ clean, no deposits \\
\hline
\end{tabular}




\section{Nissan Altima}

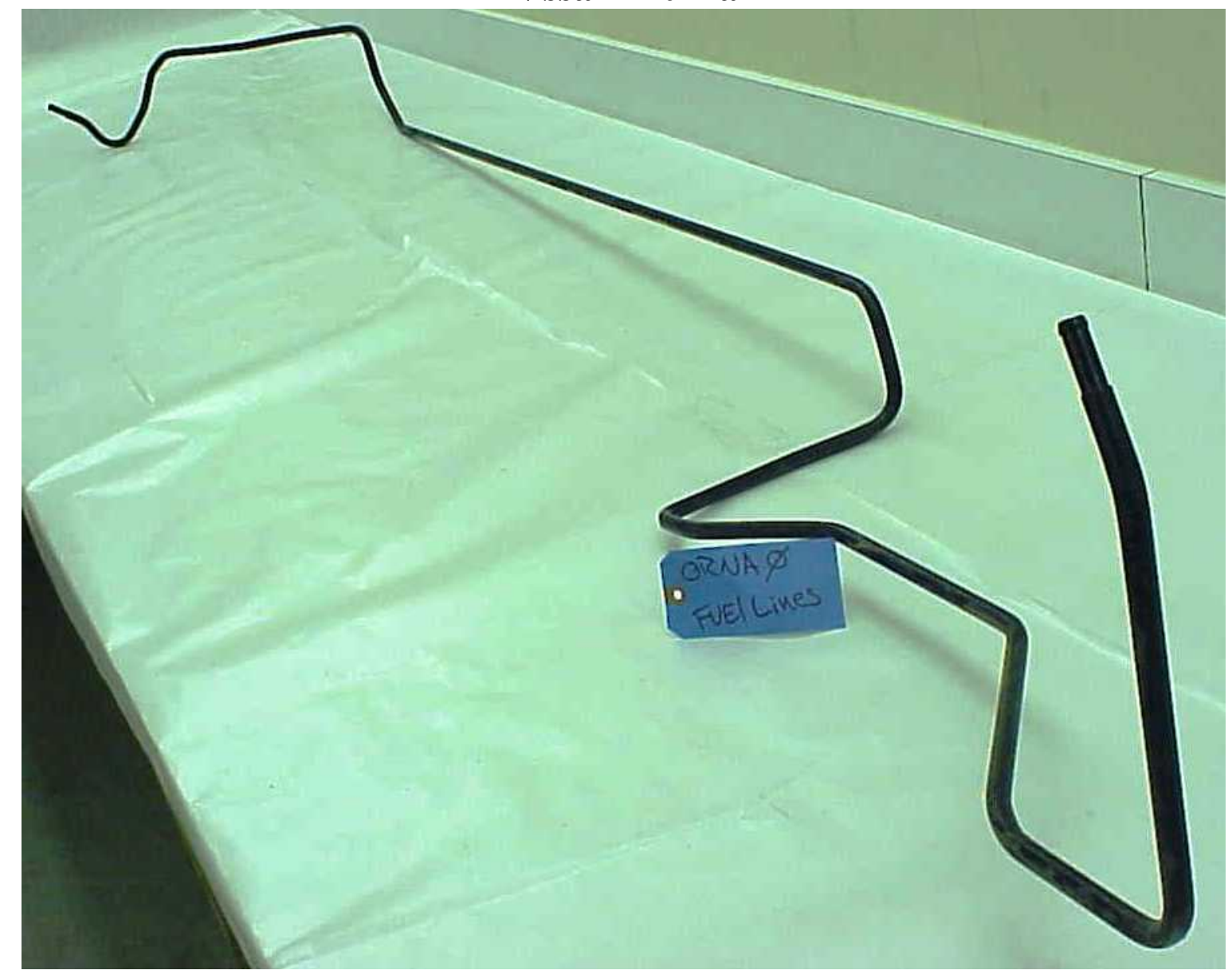

Evaporative Emissions Line

\begin{tabular}{|c|l|l|}
\hline \multirow{2}{*}{ Vehicle } & \multicolumn{2}{|c|}{ Visual Rating Comments } \\
\cline { 2 - 3 } & Front of Vehicle & Rear of Vehicle \\
\hline ORNA0 & $100 \%$ clean & $100 \%$ clean \\
\hline ORNA15 & $100 \%$ clean & $100 \%$ clean \\
\hline ORNA20 & $100 \%$ clean & $3 \%$ white deposit, 97\% clean \\
\hline
\end{tabular}


Honda Accord

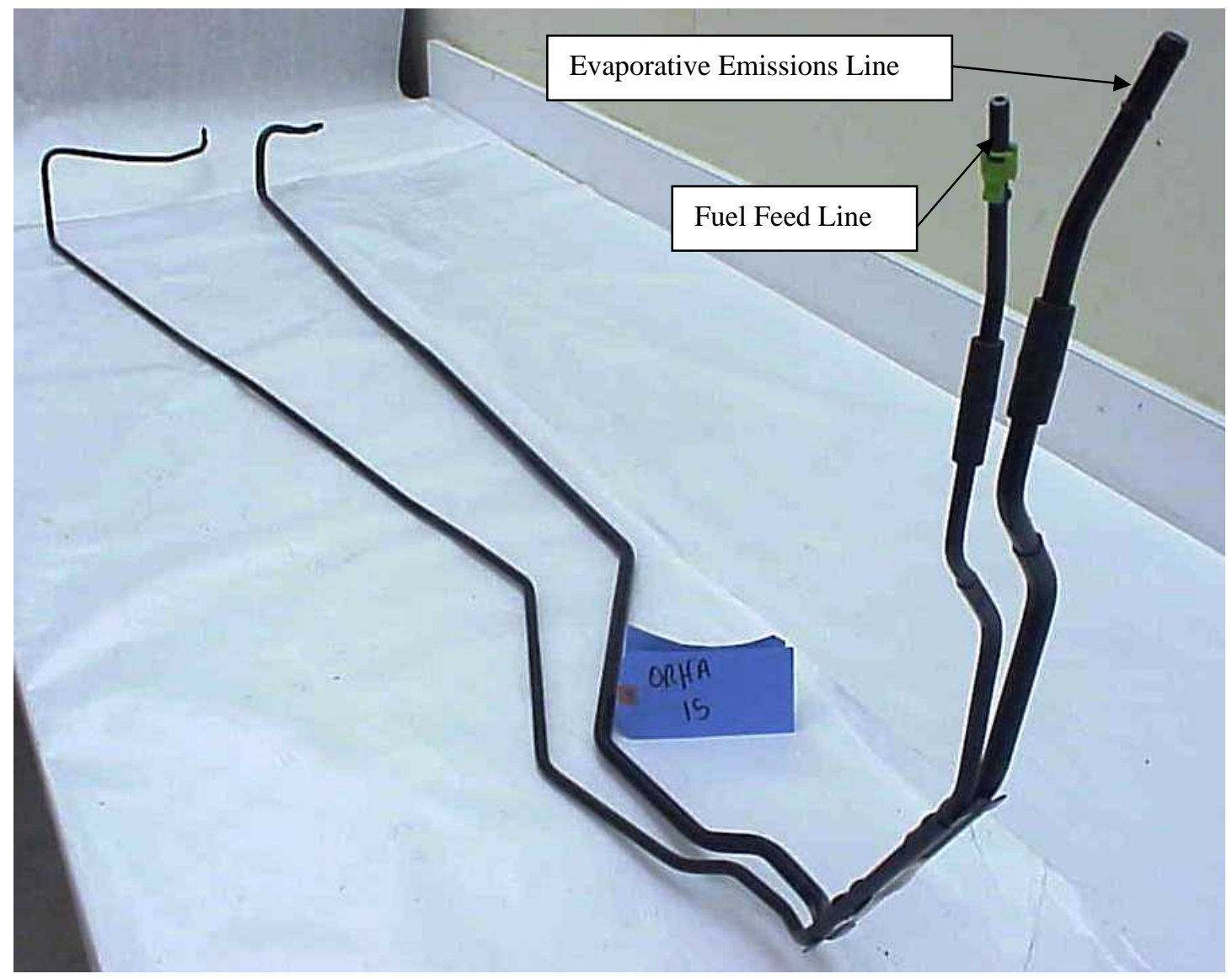

Fuel Feed Line

\begin{tabular}{|c|l|l|}
\hline \multirow{2}{*}{ Vehicle } & \multicolumn{2}{|c|}{ Visual Rating Comments } \\
\cline { 2 - 3 } & Front of Vehicle & Rear of Vehicle \\
\hline ORHA0 & $100 \%$ clean & $100 \%$ clean \\
\hline ORHA15 & $100 \%$ clean & $100 \%$ clean \\
\hline ORHA20 & $100 \%$ clean & $100 \%$ clean \\
\hline
\end{tabular}

$\mathrm{J}-11$ of 24 
Honda Accord

Evaporative Emissions Line

\begin{tabular}{|c|l|l|}
\hline \multirow{2}{*}{ Vehicle } & \multicolumn{2}{|c|}{ Visual Rating Comments } \\
\cline { 2 - 3 } & Front of Vehicle & Rear of Vehicle \\
\hline ORHA0 & $100 \%$ clean & $2 \%$ white deposit, 98\% clean \\
\hline ORHA15 & $100 \%$ clean & $5 \%$ rust deposits, 95\% clean \\
\hline ORHA20 & $100 \%$ clean & $5 \%$ rust deposits, 95\% clean \\
\hline
\end{tabular}




\section{Chevrolet Cobalt Fuel Tank Ratings}

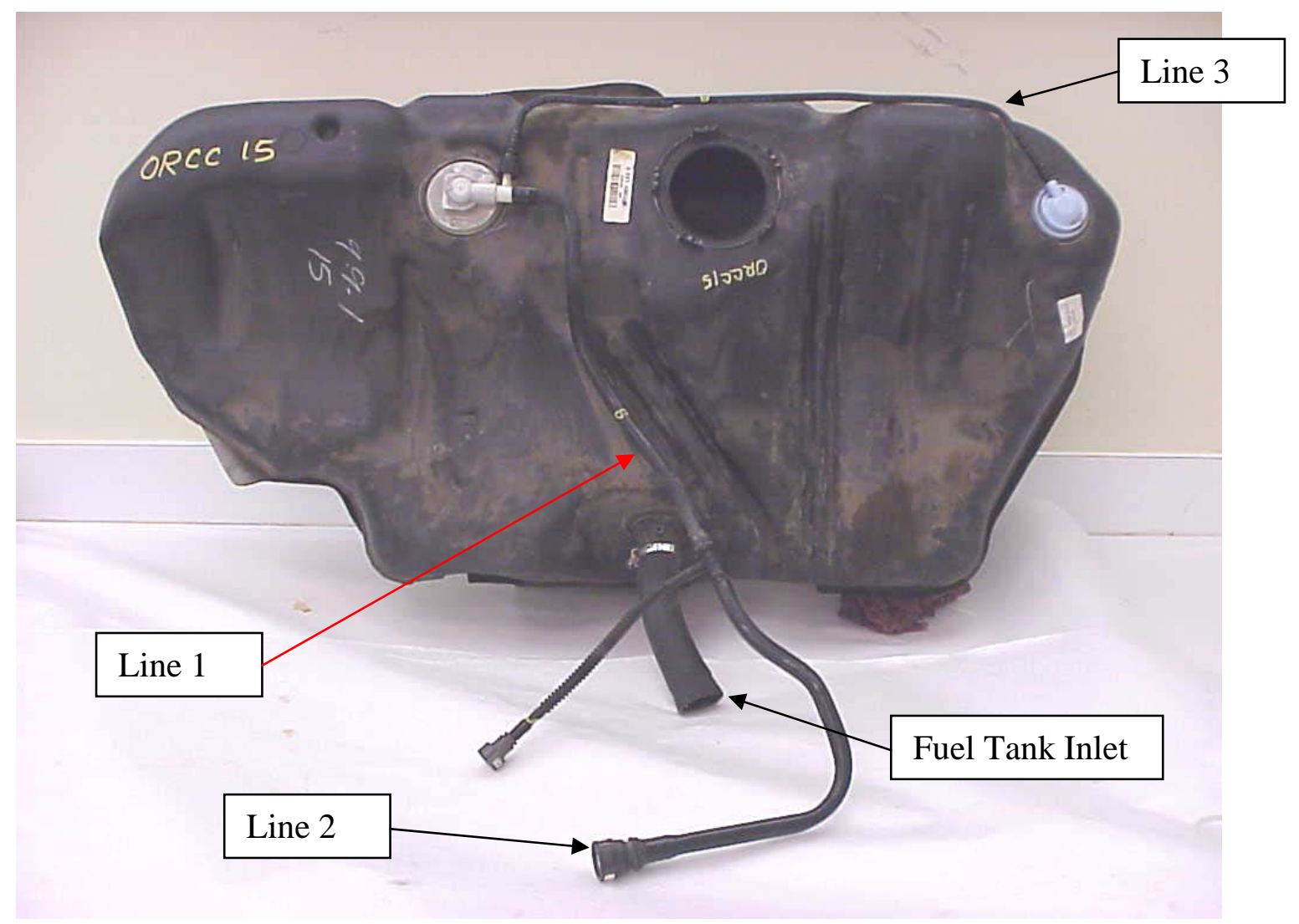

\begin{tabular}{|l|l|l|l|}
\hline \multirow{2}{*}{ Vehicle } & \multicolumn{3}{|c|}{ Visual Rating Comments } \\
\cline { 2 - 4 } & Inside Tank Rating & Fuel Tank Inlet & Tank Inlet Rubber Hose \\
\hline ORCC0 & $4 \%$ debris, 96\% clean & $100 \%$ clean & $100 \%$ clean \\
\hline ORCC15 & $4 \%$ debris, 96\% clean & $100 \%$ clean & $2 \%$ debris, 98\% clean \\
\hline ORCC20 & $5 \%$ debris, 95\% clean & $98 \%, 2 \%$ debris & Missing \\
\hline
\end{tabular}




\section{Chevrolet Cobalt Fuel Tank Ratings}

\begin{tabular}{|l|l|l|l|}
\hline \multicolumn{1}{|c|}{ Line } & \multicolumn{1}{|c|}{ ORCC0 } & \multicolumn{1}{c|}{ ORCC15 } & \multicolumn{1}{c|}{ ORCC20 } \\
\hline \multirow{2}{*}{ Line 1} & 3\% white deposits, 97\% clean & $\begin{array}{l}\text { 3\% white deposits, 2\% debris, } \\
\text { 95\% clean }\end{array}$ & $10 \%$ debris, 90\% clean \\
\cline { 2 - 4 } O-rings & Good condition & Good condition & Good condition \\
\hline \multirow{3}{*}{ O-ring 2} & $12 \%$ debris, 88\% clean & $1 \%$ debris, 99\% clean & $\begin{array}{l}\text { 2\% debris,3\% white deposits, } \\
95 \% \text { clean }\end{array}$ \\
\cline { 2 - 4 } Line 3 & Good condition & Good condition & Good condition \\
\hline \multirow{2}{*}{ O-rings } & $\begin{array}{l}\text { 3\% white deposits, 5\% debris, } \\
\text { 92\% clean }\end{array}$ & $7 \%$ debris, 93\% clean & debris, 90\% clean \\
\cline { 2 - 4 } & Good condition & Good condition & Good condition \\
\hline
\end{tabular}




\section{Chevrolet Silverado Fuel Tank Ratings}

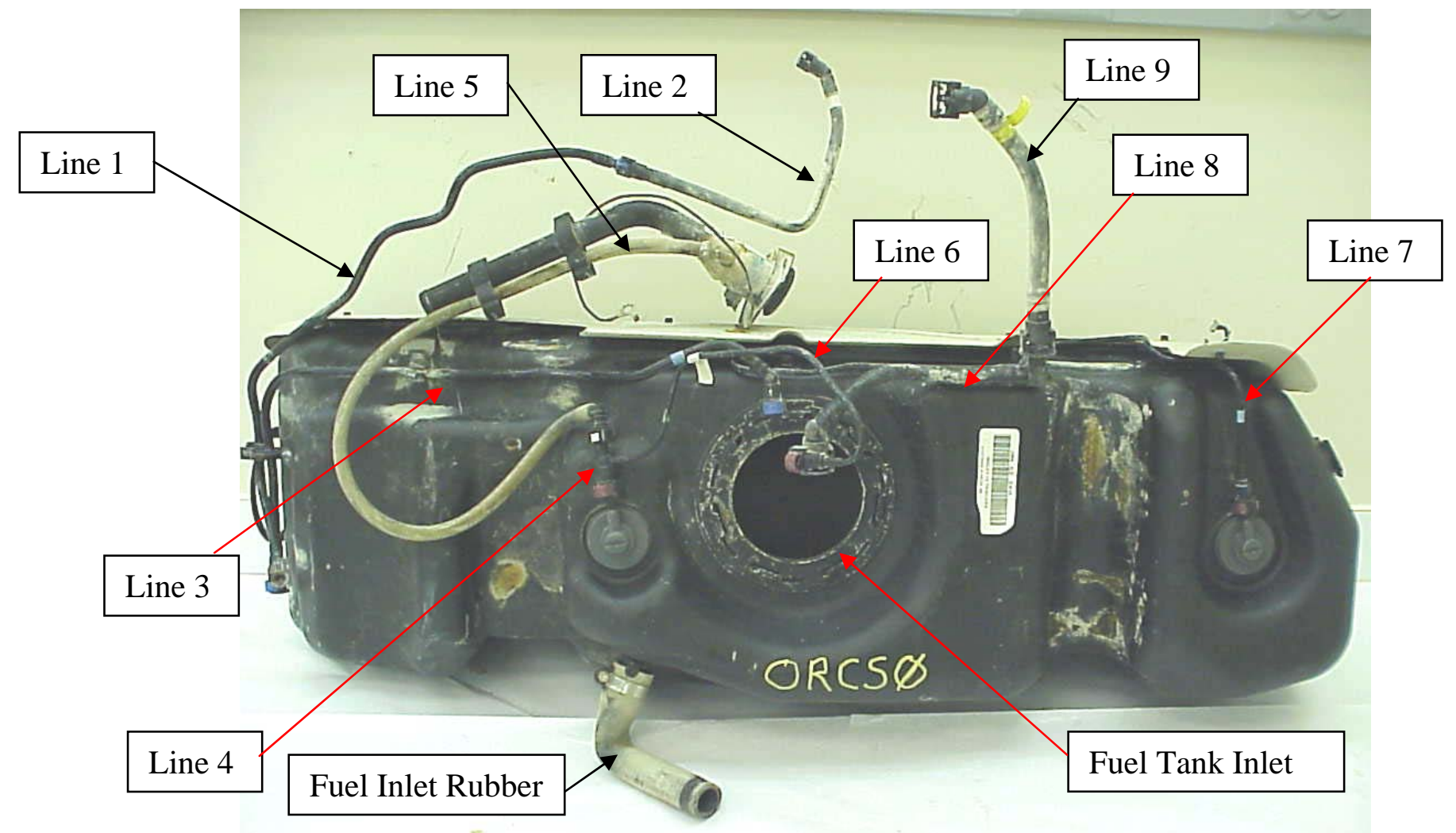

\begin{tabular}{|c|l|l|l|l|}
\hline \multirow{2}{*}{ Vehicle } & \multicolumn{3}{|c|}{ Visual Rating Comments } \\
\cline { 2 - 5 } & \multicolumn{1}{|c|}{ Inside Tank Rating } & $\begin{array}{c}\text { Fuel Tank } \\
\text { Inlet }\end{array}$ & $\begin{array}{c}\text { Tank Inlet } \\
\text { Rubber Hose }\end{array}$ & \multicolumn{1}{|c|}{ Gasoline Pump Line } \\
\hline ORCS0 & $\begin{array}{l}\text { 5\% rust, 5\% debris, } \\
90 \% \text { clean }\end{array}$ & $\begin{array}{l}\text { 5\% deposits, } \\
95 \% \text { clean }\end{array}$ & $\begin{array}{l}100 \% \text { clean } \\
\text { clean debris, 2\% rust, 93\% }\end{array}$ \\
\hline ORCS15 & 5\% debris, 95\% clean & $100 \%$ clean & $100 \%$ clean & Missing \\
\hline ORCS20 & 5\% debris, 95\% clean & $100 \%$ clean & $100 \%$ clean & Missing \\
\hline
\end{tabular}


Chevrolet Silverado Fuel Tank Ratings

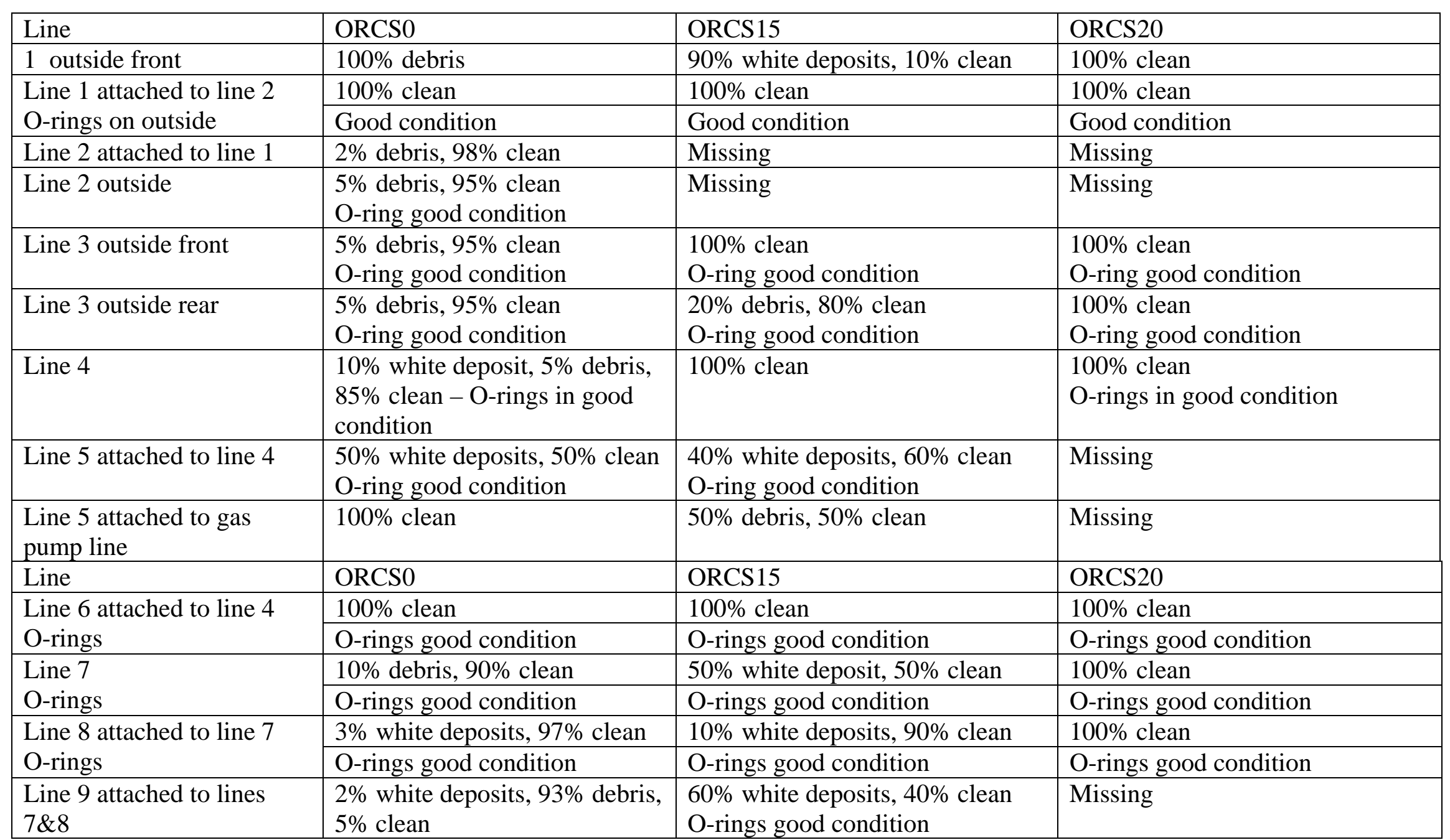




\section{Dodge Caravan Fuel Tank Ratings}

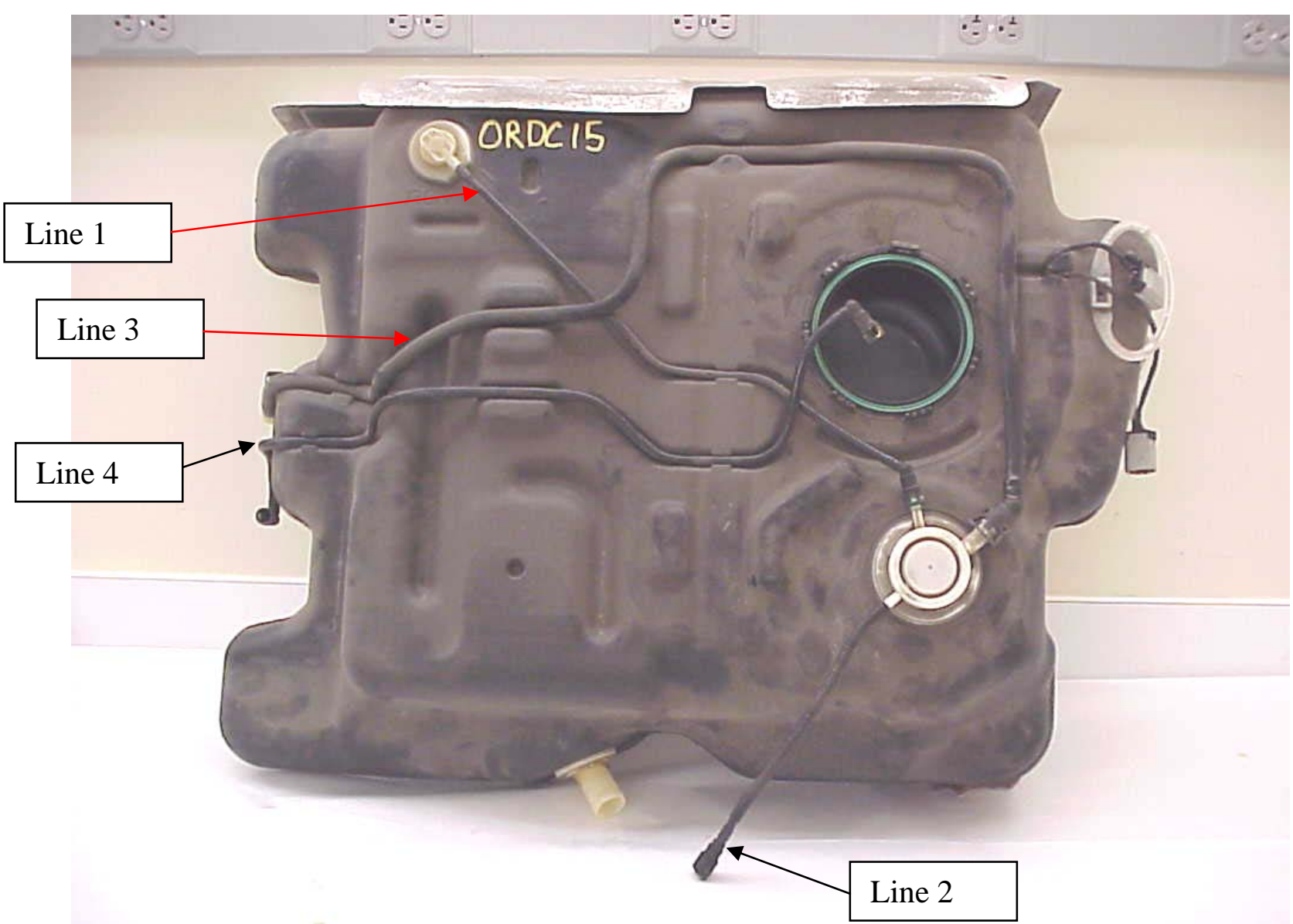

\begin{tabular}{|c|l|l|l|}
\hline \multirow{2}{*}{ Vehicle } & \multicolumn{3}{|c|}{ Visual Rating Comments } \\
\cline { 2 - 4 } & Inside Tank Rating & Fuel Tank Inlet & Fuel Pump Module O-Ring \\
\hline ORDC0 & $1 \%$ debris, 1\% rust, 98\% clean & $100 \%$ clean & Good condition \\
\hline ORDC15 & $1 \%$ debris, 99\% clean & $100 \%$ clean & Good condition \\
\hline ORDC20 & $2 \%$ debris, 98\% clean & $1 \%$ debris, 99\% clean & Missing \\
\hline
\end{tabular}


Dodge Caravan Fuel Tank Ratings

\begin{tabular}{|c|c|c|c|}
\hline Line & ORDC0 & ORDC15 & ORDC20 \\
\hline \multirow{2}{*}{$\begin{array}{l}\text { Line } 1 \\
\text { O-Rings }\end{array}$} & 100\% white deposits & $40 \%$ white deposits \& $60 \%$ clean & 20\% white deposits \& $80 \%$ clean \\
\hline & Good condition & Good condition & Good condition \\
\hline \multirow{2}{*}{$\begin{array}{l}\text { Line } 2 \\
\text { O-Ring }\end{array}$} & $100 \%$ clean & $100 \%$ clean & $100 \%$ clean \\
\hline & Good condition & Good condition & Good condition \\
\hline \multirow{2}{*}{$\begin{array}{l}\text { Line } 3 \\
\text { O-Rings }\end{array}$} & 20\% deposit and 80\% clean & $30 \%$ white deposit and 70\% clean & $100 \%$ clean \\
\hline & Good condition & Good condition & Good condition \\
\hline \multirow{2}{*}{$\begin{array}{l}\text { Line } 3 \text { Outside } \\
\text { O-Ring }\end{array}$} & 10\% white deposits & $100 \%$ clean & 3\% debris, $97 \%$ clean \\
\hline & Good condition & Good condition & Good condition \\
\hline \multirow{2}{*}{$\begin{array}{l}\text { Line } 4 \\
\text { O-Rings }\end{array}$} & $100 \%$ clean & $100 \%$ clean & $100 \%$ clean \\
\hline & Good condition & Good condition & Good condition \\
\hline \multirow{2}{*}{$\begin{array}{l}\text { Line } 4 \text { Outside } \\
\text { O-Ring }\end{array}$} & $100 \%$ clean & $100 \%$ clean & $100 \%$ clean \\
\hline & Good condition & Good condition & Good condition \\
\hline
\end{tabular}




\section{Ford Taurus Fuel Tank Ratings}

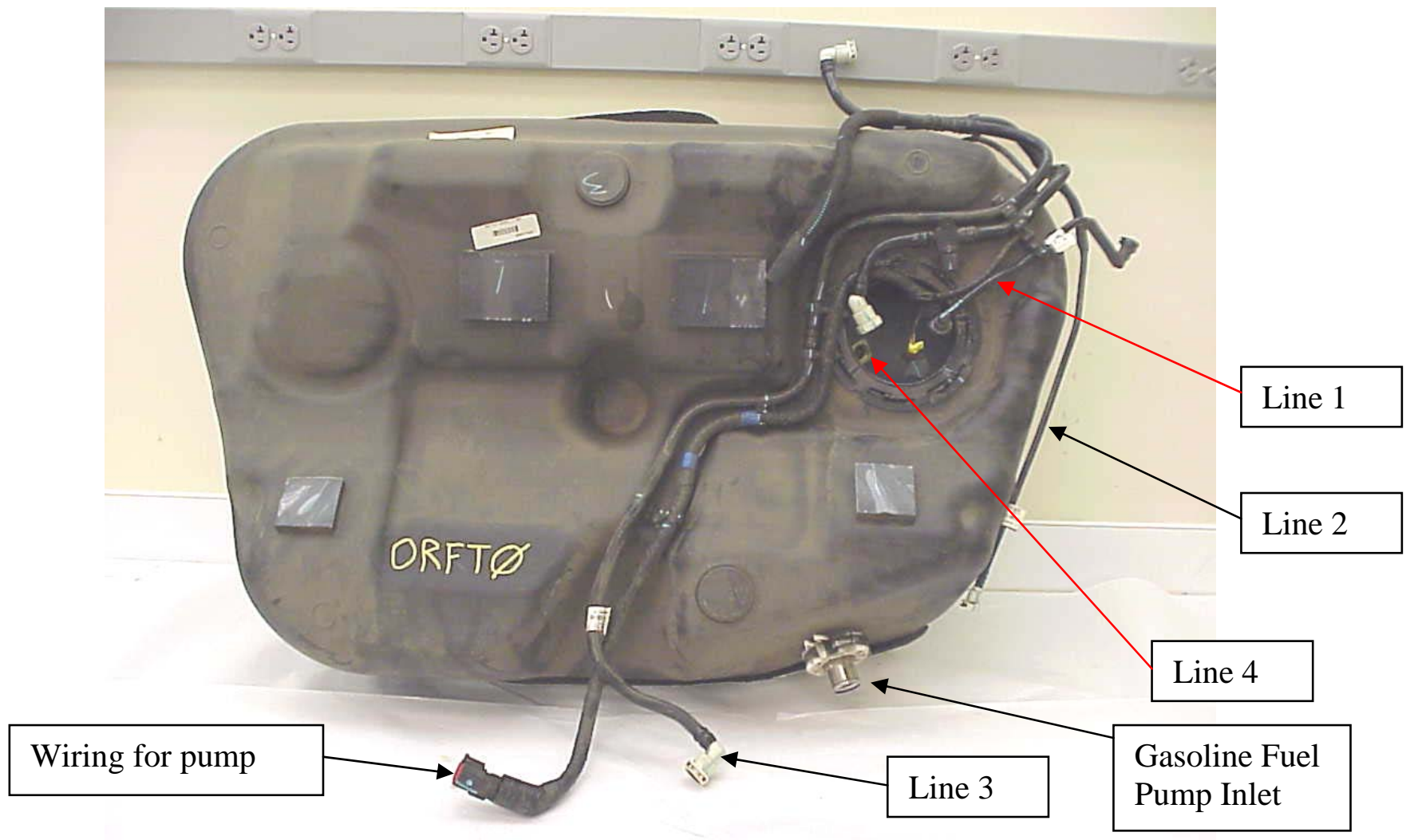

\begin{tabular}{|c|l|l|}
\hline \multirow{2}{*}{ Vehicle } & \multicolumn{2}{|c|}{ Visual Rating Comments } \\
\cline { 2 - 3 } & Inside Tank Rating & Fuel Pump Inlet Line \\
\hline ORFT0 & $1 \%$ debris, 99\% clean & $100 \%$ clean \\
\hline ORFT15 & $100 \%$ clean & $100 \%$ clean \\
\hline ORFT20 & $100 \%$ clean & $100 \%$ clean \\
\hline
\end{tabular}




\section{Ford Taurus Fuel Tank Ratings}

\begin{tabular}{|c|c|c|c|}
\hline Line & ORFT0 & ORFT15 & ORFT20 \\
\hline \multirow{2}{*}{$\begin{array}{l}\text { Line } 1 \\
\text { O-ring }\end{array}$} & $100 \%$ clean & Missing & Missing \\
\hline & Good condition & Missing & Missing \\
\hline Line 1 outside of tank & $5 \%$ rust and $95 \%$ clean & Missing & Missing \\
\hline \multirow{2}{*}{$\begin{array}{l}\text { Line } 2 \text { to open hole } \\
\text { O-rings }\end{array}$} & $100 \%$ clean & 1\% debris, 99\% clean & $100 \%$ clean \\
\hline & Good condition & Good condition & Good condition \\
\hline \multirow{2}{*}{$\begin{array}{l}\text { Line } 2 \text { front side } \\
\text { O-rings }\end{array}$} & $100 \%$ clean & $100 \%$ clean & $100 \%$ clean \\
\hline & Good condition & Good condition & Good condition \\
\hline \multirow{2}{*}{$\begin{array}{l}\text { Line } 2 \text { rear side } \\
\text { O-rings }\end{array}$} & 8\% debris, $92 \%$ clean & $10 \%$ debris, $90 \%$ clean & $100 \%$ clean \\
\hline & Good condition & Good condition & Good condition \\
\hline \multirow{2}{*}{$\begin{array}{l}\text { Line } 3 \text { to front side } \\
\text { O-ring }\end{array}$} & $30 \%$ debris, $70 \%$ clean & $30 \%$ debris, $70 \%$ clean & Missing \\
\hline & Good condition & Good condition & Missing \\
\hline \multirow{2}{*}{$\begin{array}{l}\text { Line } 3 \text { going to rear side } \\
\text { O-ring }\end{array}$} & 5\% white deposits, 95\% debris & 100\% deposits & Missing \\
\hline & Good condition & Good condition & Missing \\
\hline \multirow{2}{*}{$\begin{array}{l}\text { Line } 4 \\
\text { O-ring }\end{array}$} & $100 \%$ clean & $100 \%$ clean & 2\% debris, $98 \%$ clean \\
\hline & Good condition & Good condition & Good condition \\
\hline
\end{tabular}




\section{Nissan Altima Fuel Tank Ratings}

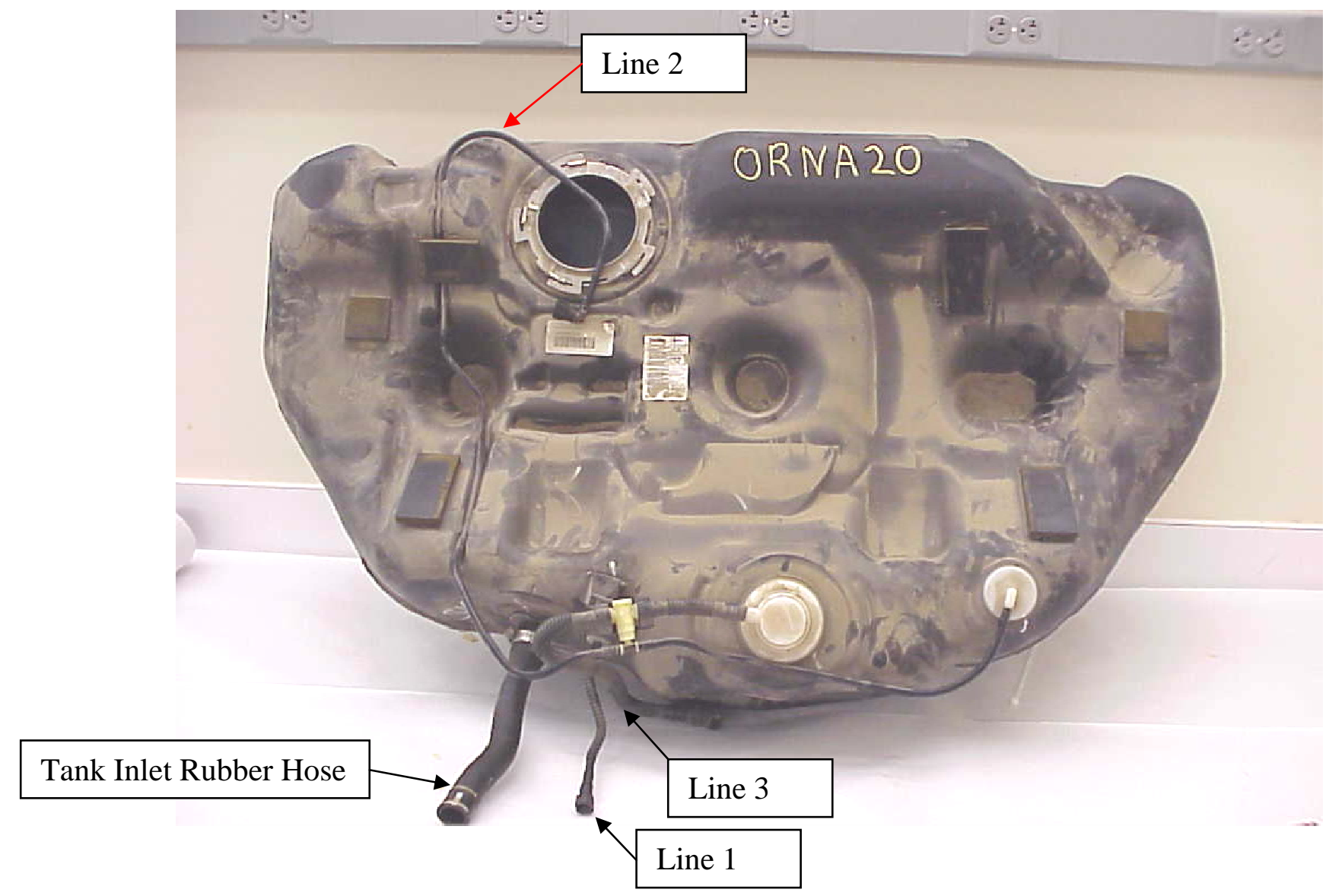

\begin{tabular}{|c|l|l|l|l|}
\hline \multirow{2}{*}{ Vehicle } & \multicolumn{4}{|c|}{ Visual Rating Comments } \\
\cline { 2 - 5 } & Inside Tank Rating & O-Ring & Gasoline Tank Inlet & Tank Inlet Rubber Hose \\
\hline ORNA0 & $1 \%$ debris, 99\% clean & Good condition & $100 \%$ clean & $100 \%$ clean \\
\hline ORNA15 & $100 \%$ clean & Good condition & $100 \%$ clean & $100 \%$ clean \\
\hline ORNA20 & $1 \%$ debris, 99\% clean & Good condition & $100 \%$ clean & $100 \%$ clean \\
\hline
\end{tabular}


Nissan Altima Fuel Tank Ratings

\begin{tabular}{|l|l|l|l|}
\hline \multicolumn{1}{|c|}{ Line } & \multicolumn{1}{|c|}{ ORNA0 } & \multicolumn{1}{c|}{ ORNA15 } & \multicolumn{1}{c|}{ ORNA20 } \\
\hline \multirow{2}{*}{$\begin{array}{l}\text { Line } 1 \text { outside of tank } \\
\text { O-ring }\end{array}$} & $90 \%$ clean, 10\% debris & $100 \%$ clean & $90 \%$ clean, 10\% debris \\
\cline { 2 - 4 } $\begin{array}{l}\text { Line 2 outside of tank } \\
\text { O-rings }\end{array}$ & Good condition & Good condition & Good condition \\
\cline { 2 - 4 } $\begin{array}{l}\text { Line 3 outside of tank } \\
\text { O-rings }\end{array}$ & Good condition & $100 \%$ clean & Goon clean \\
\cline { 2 - 4 } & G9\% clean, 1\% debris & Good condition & $100 \%$ clean \\
\hline
\end{tabular}




\section{Honda Accord Fuel Tank Ratings}

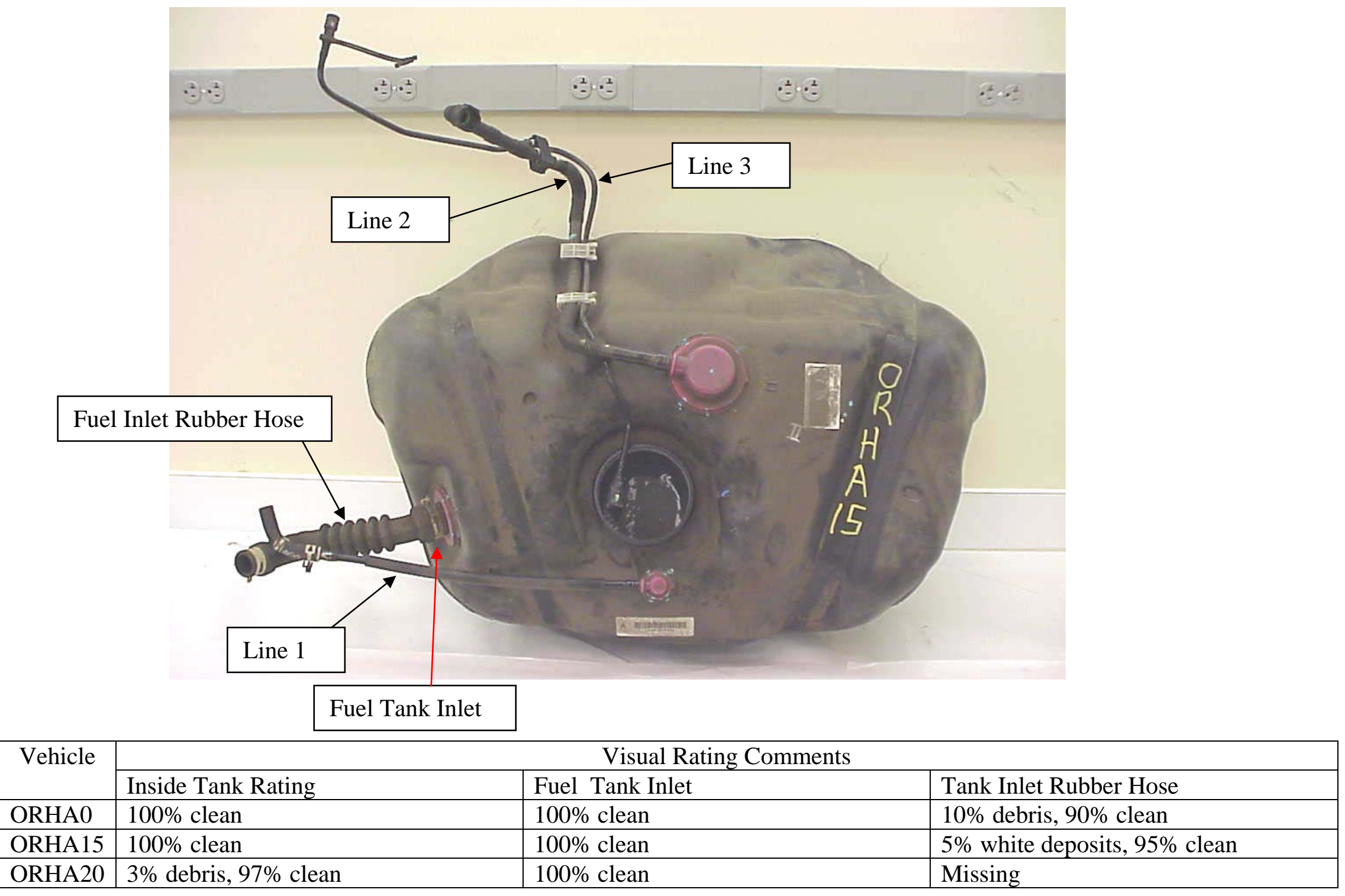




\section{Honda Accord Fuel Tank Ratings}

\begin{tabular}{|c|c|c|c|}
\hline Line & ORHA0 & ORHA15 & ORHA20 \\
\hline \multirow{2}{*}{$\begin{array}{l}\text { Line } 1 \\
\text { Rubber hose }\end{array}$} & $100 \%$ clean & $100 \%$ clean & $100 \%$ clean \\
\hline & 5\% debris, $95 \%$ clean & $100 \%$ clean & $100 \%$ clean \\
\hline \multirow{2}{*}{$\begin{array}{l}\text { Line } 2 \\
\text { O-rings }\end{array}$} & 2\% white deposits, $98 \%$ clean & $100 \%$ clean & 2\% white deposits, $98 \%$ clean \\
\hline & Good condition & Good condition & Good condition \\
\hline 3 going to open hole & Missing & $\begin{array}{l}100 \% \text { clean } \\
\text { O-rings in good condition }\end{array}$ & $\begin{array}{l}100 \% \text { clean } \\
\text { O-rings in good condition }\end{array}$ \\
\hline $\begin{array}{l}3 \text { going to outside } \\
\text { front }\end{array}$ & Missing & $\begin{array}{l}100 \% \text { clean } \\
\text { O-rings in good condition }\end{array}$ & $\begin{array}{l}100 \% \text { clean } \\
\text { O-rings in good condition }\end{array}$ \\
\hline
\end{tabular}

